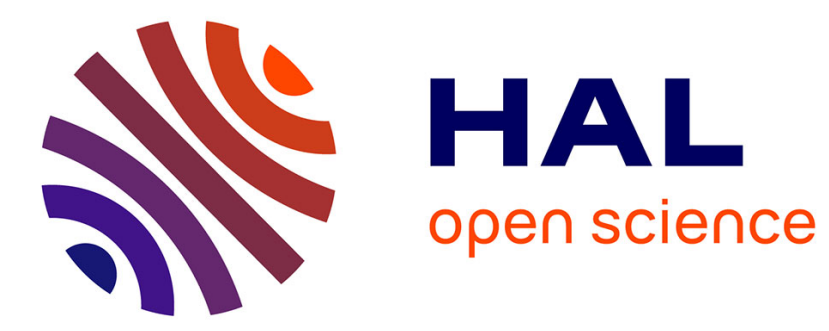

\title{
Alcidedorbignya inopinata, a basal pantodont (Placentalia, Mammalia) from the early Palaeocene of Bolivia: anatomy, phylogeny and palaeobiology
}

Christian De Muizon, Guillaume Billet, Christine Argot, Sandrine Ladevèze, Florent Goussard

\section{To cite this version:}

Christian De Muizon, Guillaume Billet, Christine Argot, Sandrine Ladevèze, Florent Goussard. Alcidedorbignya inopinata, a basal pantodont (Placentalia, Mammalia) from the early Palaeocene of Bolivia: anatomy, phylogeny and palaeobiology. Geodiversitas, 2015, 37, pp.397 - 634 . 10.5252/g2015n4a1 . hal-01455642

\section{HAL Id: hal-01455642 https://hal.science/hal-01455642}

Submitted on 3 Feb 2017

HAL is a multi-disciplinary open access archive for the deposit and dissemination of scientific research documents, whether they are published or not. The documents may come from teaching and research institutions in France or abroad, or from public or private research centers.
L'archive ouverte pluridisciplinaire HAL, est destinée au dépôt et à la diffusion de documents scientifiques de niveau recherche, publiés ou non, émanant des établissements d'enseignement et de recherche français ou étrangers, des laboratoires publics ou privés. 


\title{
Alcidedorbignya inopinata, a basal pantodont (Placentalia, Mammalia) from the early Palaeocene of Bolivia: anatomy, phylogeny and palaeobiology
}

\author{
Christian de MUIZON \\ Guillaume BILLET \\ Christine ARGOT \\ Sandrine LADEVÈZE \\ Florent GOUSSARD \\ CR2P UMR 7207 (MNHN, CNRS, UPMC, Sorbonne-Université), \\ Département Histoire de la Terre, Muséum national d'Histoire naturelle, \\ case postale 38, 57 rue Cuvier, F-75231 Paris cedex 05 (France) \\ muizon@mnhn.fr
}

Published on 31 December 2015

Muizon C. de, Billet G., Argot C., Ladevèze S. \& Goussard F. 2015. - Alcidedorbignya inopinata, a basal pantodont (Placentalia, Mammalia) from the early Palaeocene of Bolivia: anatomy, phylogeny and palaeobiology. Geodiversitas 37 (4): 397-634. http://dx.doi.org/10.5252/g2015n4a1

\begin{abstract}
Alcidedorbignya inopinata Muizon \& Marshall, 1987 is a basal pantodont (Placentalia, Mammalia) of small body size, from the early Palaeocene of the Santa Lucia Formation at Tiupampa, Bolivia. Tiupampa is the type locality for the Tiupampan, a South American Land Mammal age (SALMA), which is assigned an age equivalent to the basal Torrejonian 1 of North America (c. $65 \mathrm{Ma}$ ). Alcidedorbignya is known by exceptionally preserved specimens, which are described here. The two major specimens are an almost complete skeleton (MHNC 8372) and a partial skull (MHNC 8399), the former representing one of the best-preserved fossil placentals from the early Palaeocene and probably the oldest placental skeleton that is so completely known. These specimens are also the first eutherian skulls and skeleton ever discovered at Tiupampa, a locality which has yielded numerous metatherian skulls and partial skeletons. The remarkable preservation of the two skulls allows a detailed description of the cranial anatomy with well-identified sutures and foramina, including those of the auditory region. Through CT scanning of the skulls, the arterial and venous circulation pattern in the basicranium as well as the bony labyrinth of the inner ear were tentatively reconstructed. A thorough description of the postcranial skeleton of MHNC 8372 is also provided. Among pantodonts, Alcidedorbignya presents the closest morphological similarities with Pantolambda, the oldest and earliest diverging North American pantodont (known by skulls and skeletons), from the late early Palaeocene (Torrejonian 2 and 3) of New Mexico. Alcidedorbignya is one-third the size, much more gracile, and clearly exhibits more plesiomorphic features than Pantolambda. It is also at least $3 \mathrm{Ma}$ older. Interesting similarities are also observed between the skull of Alcidedorbignya and several "condylarths", such as Maiorana, Baioconodon, Arctocyon, and Arctocyonides. The basicranium of Alcidedorbignya is also similar to that of some extant afrotheres (e.g., Tenrec) or Lipotyphla (e.g.,
\end{abstract}


KEY WORDS

Pantodonta, early Palaeocene,

Bolivia,

Functionnal anatomy,

Phylogeny,

Palaeobiology.
Solenodon), but most of these similarities may represent placental symplesiomorphies. In fact, the cranial anatomy of Alcidedorbignya, beyond the simple thorough description of a basal pantodont, sheds light on the cranial anatomy of placentals from the earliest Paleocene, previously unknown in this detail. The postcranial skeleton of MHNC 8372 together with some isolated specimens referred to A. inopinata, is compared to adequate morphofunctional models (e.g., Solenodon, Dendrohyrax, Sciurus), which indicates that it was a moderately agile, plantigrade, generalized terrestrial mammal with good climbing ability (scansorial) and occasionally capable of standing in a bipedal position. The scutiform ungual phalanges were probably bearing nail-like hooves (or primate-like nails) and because of the absence of claws, fossorial habits are unlikely.

A parsimony analysis of a data matrix including 72 taxa and 426 characters (cranial and postcranial) has been undertaken with TNT and Paup 4b10. The matrix includes several stem eutherians and representatives of the four major clades of Placentalia: Laurasiatheria, Euarchontoglires, Xenarthra, and Afrotheria. Furthermore, because one of the major aims of this analysis is to test possible close phylogenetic relationships of Alcidedorbignya inopinata with the five orders of South American "ungulates", 12 taxa belonging to the Notoungulata, Astrapotheria, Litopterna, Pyrotheria and Xenungulata, were included in the matrix. Two analyses were performed: one unconstrained and one with a backbone constraint based on the latest molecular phylogenies. One of the main results of these analyses is the high level of homoplasy detected by the low retention index (RI close to 0.5; CI inferior to 0.2), which indicates that half of the similarities present in the matrix are interpreted as homoplasies. Nevertheless, some interesting observations emerge. In both analyses, all members of Pantodonta are closely related and included in the Placentalia, and the Eocene "cimolestid" taxon Didelphodus, which has been regarded as closely related to pantodonts, is their sister-group. In the constrained analysis, the Pantodonta are monophyletic and included in the Laurasiatheria; Didelphodus and the pantodonts are clustered with the Tillodontia, either as their sister group or included in a clade also comprising some "condylarths". In the unconstrained analysis the xenungulate Carodnia is included within the Pantodonta as the sister group of Coryphodon, this clade being part of a large basal polytomy of placental lineages. Both analyses agree with a northern origin of the South American pantodont Alcidedorbignya as it is nested within northern taxa. Furthermore, no close relationship of Alcidedorbignya with South American endemic ungulates is supported, except for pantodont-xenungulate affinities in the unconstrained analysis. In the constrained analysis the five groups of South American endemic "ungulates" are gathered in a single clade included in the laurasiatheres but not directly related to pantodonts. Finally, the rather apical position of Alcidedorbignya in both analyses may be considered as an argument for a pre-K-Pg placental diversification, as this taxon is dated at c. $65 \mathrm{Ma}$, nearly coincident with the end of the Cretaceous.

\section{RÉSUMÉ}

Alcidedorbignya inopinata, un pantodonte (Placentalia, Mammalia) basal du Paléocène inférieur de Bolivie: anatomie, phylogénie et paléobiologie.

Alcidedorbignya inopinata Muizon \& Marshall, 1987 est un pantodonte (Placentalia, Mammalia) basal de petite taille du Paléocène inférieur de la Formation Santa Lucia à Tiupampa (Bolivie), la localité type du Tiupampien, un âge de mammifères continentaux sud-américains (SALMA) qui est considéré comme un équivalent de la base du Torréjonien 1 d'Amérique du Nord (c. $65 \mathrm{Ma}$ ). Alcidedorbignya est connu par des spécimens exceptionellement preservés qui sont décrits dans ce travail. Les deux principaux spécimens sont un squelette presque complet (MHNC 8372) et un crâne partiel (MHNC 8399), le premier constituant probablement un des squelettes de placentaire fossile les mieux conservés connus dans le Paléocène inférieur et probablement le plus ancien squelette de placentaire aussi complet connu. Ces spécimens sont aussi les premiers crânes et squelettes d'euthériens découverts à Tiupampa, une localité qui a livré, par ailleurs, de nombreux crânes et squelettes partiels de métathériens. La conservation remarquable des deux crânes permet une description détaillée de l'anatomie crânienne avec une parfaite identification des sutures et des foramens, entre autres, concernant la région auditive. L'analyse microtomograhique par RX des crânes a permis une reconstitution des circulations artérielle et veineuse dans la base du crâne ainsi que du labyrinthe osseux de l'oreille interne. Une description exhaustive du squelette post-crânien de MHNC 8372 est également présentée. Parmi les pantodontes, Alcidedorbignya présente les ressemblances les plus étroites avec Pantolambda, le plus ancien (connu par des crânes et des squelettes partiels) et le plus basal des pantodontes nord-américains provenant de la partie supérieure du Paléocène inférieur (Torréjonien 2 et 3) du Nouveau-Mexique. Alcidedorbignya est trois fois plus petit, plus gracile, et présente des caractères anatomiques nettement plus plésiomorphes que Pantolambda. Il est aussi plus ancien d'au moins 3 Ma. D'intéressantes ressemblances sont également observées entre le crâne d'Alcidedorbignya et ceux de plusieurs "condylarthres" tels que Maiorana, Baioconodon, Arctocyon et Arctocyonides. Le basicrâne d'Alcidedorbignya ressemble aussi à celui d'Afrotheria (e.g., Tenrec) ou de Lipotyphla actuels 
(e.g., Solenodon), mais la plupart de ces ressemblances représentent probablement des symplésiomorphies de placentaires. En fait, l'anatomie crânienne d'Alcidedorbignya, au-delà de la simple description d'un pantodonte basal, apporte des lumières sur l'anatomie crânienne des placentaires du Paléocène basal, jusqu'alors inconnue de façon aussi détaillée. Le squelette post-crânien de MHNC 8372 avec quelques os isolés rapportés à Alcidedorbignya, est comparé avec soin avec des modèles morpho-fonctionnels adéquats (e.g., Solenodon, Dendrohyrax, Sciurus), ce qui permet de conclure qu'il était un mammifère terrestre généralisé, plantigrade, modérément agile, avec de bonnes capacités à grimper et occasionnellement capable d'adopter une position bipède. Les phalanges unguéales scutiformes portaient très probablement des sabots à morphologie d'ongles (ou des ongles de type primates) et, en raison de l'absence de griffe, un comportement fouisseur est considéré comme improbable.

Une analyse de parcimonie d'une matrice de données comportant 426 caractères (crâniens et post-crâniens) et 72 taxons a été réalisée avec TNT et Paup 4b10. La matrice inclut plusieurs euthériens souches, et des représentants des quatre grands clades de Placentalia: Laurasiatheria, Eurachontoglira, Xenarthra et Afrotheria. De plus, puisque l'un des buts majeurs de cette analyse est de tester la possibilité d'une relation phylogénétique étroite d'Alcidedorbignya inopinata avec l'un des cinq ordres d' " ongulés» endémiques sud-américains, 12 taxons appartenant aux Notungulata, Astrapotheria, Litopterna, Pyrotheria et Xenungulata ont été inclus dans la matrice. Deux analyses ont été réalisées, l'une non-contrainte et l'autre contrainte par les phylogénies moléculaires les plus récentes. Un des résultats principaux de ces analyses réside dans le haut degré d'homoplasie mis en évidence par le faible indice de rétention (RI proche de 0,5 ; CI inférieur à 0,2 ). Cette valeur signifie que la moitié des ressemblances observées sont interprétées comme des synapomorphies et donc que l'autre moitié constitue des homoplasies. Néanmoins quelques observations intéressantes peuvent être faites. Dans les deux analyses, les Pantodonta sont inclus dans les Placentalia et le "cimolestidé» éocène Didelphodus, qui a été considéré comme étroitement apparenté aux pantodontes, est leur groupe frère. Dans l'analyse contrainte les Pantodonta sont monophylétiques et inclus dans les laurasiathères; Didelphodus et les pantodontes sont regroupés avec les Tillodontia, soit en tant que groupe frère, soit inclus dans un clade comprenant également des «condylarthres». Dans l'analyse non-contrainte, le xénongulé Carodnia est inclus dans les Pantodonta en tant que groupe frère de Coryphodon, ce clade formant partie d'une vaste polytomie basale des lignées de placentaires. Les deux analyses sont en accord avec une origine nord-américaine du pantodonte sudaméricain Alcidedorbignya puisqu'il est enraciné au sein de taxons du Nord. De plus, aucune relation étroite avec les "ongulés» endémiques sud-américains n'est mise en évidence à l'exception d'une relation avec les xénongulés, comme cela est observé dans l'analyse non-contrainte. Dans l'analyse contrainte, les cinq groupes d' «ongulés» endémiques sud-américains sont réunis en un seul clade inclus dans les laurasiathères mais non reliés directement aux pantodontes. Enfin, la position relativement apicale d'Alcidedorbignya dans les deux analyses peut être considérée comme un argument en faveur d'une diversification des placentaires, antérieure à la limite K-Pg puisque ce taxon date d'environ $65 \mathrm{Ma}$, soit presque contemporaine de la fin du Crétacé.

\section{INTRODUCTION}

Pantodonts are generally large-sized herbivorous to omnivorous mammals from the Palaeogene, which have been placed in their own order Pantodonta Cope, 1873, a small group that includes less than 25 genera (McKenna \& Bell 1997). Pantodonta have been considered by McKenna $\&$ Bell (1997) as a suborder of the order Cimolesta and Rose (2006) considered Pantodonta and Tillodontia to be orders of the mirorder Cimolesta. The affinities of pantodonts have long been a matter of debate. They have been regarded as close relatives of the Dinocerata and placed with them in the superorder Amblypoda (Cope 1875), but this grouping was not accepted by Simpson (1937), who recognized two separate orders and, therefore, invalidated the Amblypoda. Later, pantodonts were regarded as closely related to tillodonts (Chow \& Wang 1979; Lucas 1998a; Rose 2006; contra Gazin 1953) or to didelphodonts (Lucas 1982,
1984, 1993, 1998b) and included in the order Cimolesta (McKenna 1975; McKenna \& Bell 1997). Pantodonts apparently do not belong to the grandorder Ungulata (Lucas 1993; McKenna \& Bell 1997), although this has not been confirmed by a parsimony analysis. Pantodonts are traditionally diagnosed by a distinctive dental synapomorphy, the double V-shaped morphology of the upper premolars, a character that has not been observed so far in other eutherians (Lucas 1982, 1998b; Muizon \& Marshall 1992).

Most of the pantodonts are from the Palaeogene of North America and Asia, but one cosmopolitan laurasian genus, Coryphodon, is also known in the early Eocene of Europe. So far, a single species, Alcidedorbignya inopinata, has been recorded from southern continents. It comes from the early Palaeocene of the Santa Lucía Formation at Tiupampa (Bolivia). Alcidedorbignya is one of the oldest known pantodonts (Muizon \& Marshall 1992; Gelfo et al. 2009) and possibly the second oldest, since a single upper molar of pantodont 
has been recorded in slightly older beds (Puercan 3) from Montana (Wilson 2014; see below, Phylogenetic Analysis section). The occurrence of a single species of pantodont in the Palaeogene of South America raises the question of the fate of this taxon with respect to the problem of the origin of the South American "ungulates". The Tertiary of South America has yielded abundant remains of several groups of eutherians commonly referred to as the "South American ungulates" or meridiungulates (McKenna 1975; McKenna \& Bell 1997). These groups are: the notoungulates, the litopterns, the astrapotheres, the pyrotheres and the xenungulates. It is generally agreed that at least some of these groups (and possibly all of them) have their origins in North American eutherian radiations. A temporary land connection between the Americas during the late Cretaceous or early Palaeocene would have permitted southward migrations of the ancestral taxa of some or all the South American "ungulates". A relationship has been proposed between North American mioclaenids and South American litopterns (Muizon \& Cifelli 2000); and a relationship of pyrotheres, xenungulates, and dinoceratans, has been suggested by Lucas (1993). Furthermore, relationships of notoungulates, pyrotheres, and astrapotheres with afrotheres have also been proposed by Agnolin \& Chimento (2011); see also O'Leary et al. (2013). However, some of their arguments have been challenged by Billet \& Martin (2011) and Kramarz \& Bond (2014). Therefore, the phylogenetic origin of the South American "ungulates" remained virtually unresolved, until recent proteomic analyses in Notoungulata and Litopterna suggest affinities with the order Perissodactyla (Welker et al. 2015; Buckley 2015), thereby confirming potential northern origins for some South American ungulates. In this context the occurrence in the basal Palaeocene of central South America of a representative of a typically laurasian group, the pantodonts, provides some new hope for understanding the affinities of the South American ungulates, or at least, some of them. Initially described on the basis of dental remains only (Muizon \& Marshall 1992), Alcidedorbignya inopinata did not suggest any obvious affinities of the South American "ungulates" with the pantodonts. It was, therefore, hypothesized that pantodonts migrated to South America by the Late Cretaceous or earliest Palaeocene and rapidly disappeared there with no descendants. However, the exceptionally complete new specimens presented below, with remarkably preserved cranial remains provide the opportunity to make detailed comparison of the cranial anatomy of pantodonts to the South American "ungulates" and to evaluate their possible relationships with South American "ungulates".

The Tiupampa local fauna and locality are the reference for the Tiupampan age, the oldest South American land mammal age. It is regarded here as early Palaeocene in age (contra Marshall et al. 1997 and Sempere et al. 1997; Pascual \& Ortiz-Jaureguizar 2007) following Muizon (1998) and Gelfo et al. (2009).

The locality of Tiupampa has yielded an abundant and diverse mammal fauna including metatherians and eutherians. More than 26 taxa have been recorded, metatherians being slightly more abundant taxonomically than eutherians. An updated faunal list is presented below. Metatherian classification follows McKenna \& Bell (1997), Kirsch et al. (2007), Ladevèze \& Muizon (2007), Horovitz et al. (2009).

The Tiupampa mammal faunal assemblage is the oldest of the Tertiary of South America. It is noteworthy however, that one metatherian (represented by a single lower molar) from the earliest Paleocene of Argentina has been regarded as the oldest Tertiary mammal of South America (Goin et al. 2006). Given its age, the Tiupampa mammal fauna is remarkable in terms of taxonomic diversity; 23 taxa have been already described; three other new ones are undescribed. This fauna is also exceptional in terms of preservation since more than 40 skulls and partial skeletons of three metatherians have been discovered at Tiupampa (Muizon 1992; Marshall et al. 1995; Muizon et al. 1997; Muizon 1998; Ladevèze et al. 2011). The other metatherians are known by a few partial skulls and mainly by partial jaws and by teeth (Muizon 1992 and unpublished data). The eutherians are known by abundant "condylarth" jaws and teeth and, during the past decade, some skulls and a complete skeleton referred to the pantodont Alcidedorbignya inopinata have been discovered. These latter specimens are presented below and the adult individuals are thoroughly described in this work.

\section{FAUNAL LIST}

\section{Infraclass METATHERIA}

Supercohort NOTOMETATHERIA Kirsch, Lapointe \& Springer, 1997

Order SPARASSODONTA Ameghino, 1894

Family MAYUlESTIDAE Muizon, 1994 Allqokirus australis Marshall \& Muizon, 1988

Mayulestes ferox Muizon, 1994

Family PUCADELPHYIDAE Muizon, 1998

Pucadelphys andinus Marshall \& Muizon, 1988 Andinodelphys cochabambensis Marshall \& Muizon, 1988

Family JASKHADELPHYIDAE Muizon, 1992 Jaskhadelphys minutus Marshall \& Muizon, 1988

Order DIDELPHIMORPHIA (Gill, 1872)

Family Peradectidae (Crochet, 1979) Peradectes cf. $P$. austrinum

Family incertae sedis Incadelphys antiquus Marshall \& Muizon, 1988 Mizquedelphys pilpinensis Marshall \& Muizon, 1988 Szalinia gracilis Muizon \& Cifelli, 2001 Tiulordia floresi Marshall \& Muizon, 1988

Order PAUCITUBERCULATA Ameghino, 1897

Suborder POLYDOLOPIMORPHIA (Marshall, 1987)

Family POLYDOLOPIDAE Ameghino, 1897

Roberthoffstetteria nationalgeographica Marshall, Muizon \& Sigé, 1983

Superorder AUSTRALIDELPHIA Szalay, 1982

Order MICROBIOTHERIA Ameghino, 1889

Family MicrobiotheriIdAe Ameghino, 1887 Khasia cordillierensis Marshall \& Muizon, 1988 
Infraclass EUTHERIA

Family Mioclaenidae Osborn \& Earle, 1895

Molinodus suarezi Muizon \& Marshall, 1987

Tiuclaenus minutus Muizon \& Marshall, 1987

Tiuclaenus cotasi Muizon \& Cifelli, 2000

Tiuclaenus robustus Muizon \& Cifelli, 2000

Pucanodus gagnieri Muizon \& Marshall, 1991

Andinodus boliviensis Muizon \& Marshall, 1987

Simoclaenus sylvaticus Muizon \& Cifelli, 2000

Order CIMOLESTA McKenna, 1975

Family PaLaeoryctidae Winge, 1917

cf. Cimolestes sp.

Family indet.

Gen. and sp. indet.

Order PANTODONTA COPE, 1873

Family ALCIDEDORBIGNYIDAE Muizon \& Marshall, 1992

Alcidedorbignya inopinata Muizon \& Marshall, 1987

Order NOTOUNGULATA Roth, 1903

Family HeNRICOSBORNIIDAE Ameghino, 1901

or OldFieldThomasiIdae Simpson, 1945

Gen. and sp. indet. (Muizon 1992).

\section{MATERIAL AND METHODS}

\section{INSTITUTIONAL ABBREVIATIONS}

ACM Amherst College Museum, Amherst, Massachusetts;

AMNH American Museum of Natural History, New York;

FMNH Field Museum of Natural History, Chicago;

IVPP Institute of Vertebrate Paleontology and Paleoanthropology, Chinese Academy of Sciences, Beijing;

MACN Museo Argentino de Ciencias Naturales "Bernardino Rivadavia", Buenos Aires;

MHNC Museo de Historia Natural "Alcide d'Orbigny", Cochabamba;

MNHN.F Muséum national d'Histoire naturelle, Paris, Collection de Paléontologie;

MNHN-AC Muséum national d'Histoire naturelle, Paris, Collection d'Anatomie Comparée;

PLV Geological Institute, Catholic University, Louvain;

PSS-MAE Paleontological and stratigraphy Section (Geological Institute), Mongolian Academy of Sciences, Ulaan Baatar;

PVL Colección de Paleontología de Vertebrados Instituo Miguel Lillo, Universidad Nacional de Tucumán;

RH Robert Hoffstetter collection of Recent vertebrates, in the MNHN;

UCMP Museum of Paleontology, University of California, Berkeley;

UMZC Museum of Zoology Cambridge;

UFRJ Universidad Federal do Estado de Rio de Janeiro, Rio de Janeiro;

UM University of Michigan, Museum of Paleontology, Ann Arbor, Michigan;

USNM United States National Museum, Smithsonian Institution, Washington DC;

YPFB Yacimientos petrolíferos fiscales de Bolivia;

YPM-PU Princeton University collection housed in the Yale Peabody Museum, Yale University, New Haven;

ZPAL Paleontological Institute of the Polish Academy of Sciences, Warsaw.

DESCRIPTION AND COMPARISON

Muizon \& Marshall (1992) described the upper and lower dentition of Alcidedorbignya inopinata on the basis of several upper and lower jaws of adults and juveniles. Affinities of the Bolivian pantodont were analyzed and relationships of pantodonts to other groups of mammals were discussed on the basis of dental characters. The new specimens mentioned above are remarkably complete, with exquisitely preserved skulls. In this paper we describe the new pantodont specimens from Tiupampa, focusing on the cranial and postcranial anatomy of the adults. The two available adult skulls (MHNC 8372 and 8399) will be thoroughly described and isolated upper or lower jaws will be included when they provide supplementary information. The juvenile skulls will be considered if they provide complementary information not available on the adult specimens (i.e. the ectotympanic of MHNC 8423). They will be described elsewhere, in a developmental perspective, in a work in progress. Dentition will be considered only where the new specimens provide supplementary information to the description published by Muizon \& Marshall (1992). This will essentially concern the incisors, which will be described before the skull. Any new data concerning the position of the teeth in their respective bones they will be treated with the description of the appropriate bones.

The description is partly comparative and is based, mainly, but not solely, on the most primitive pantodonts (essentially Pantolambda, Bemalambda, and Harpyodus). Comparison with other eutherians will be treated in the comparison section except for some basal eutherians, which have been thoroughly described in authoritative monographs and which present a condition approaching the eutherian plesiomorphic condition especially as far as the basicranium is concerned. Justification of our choice is the fact that these taxa - namely Leptictis (Novacek 1986), Zalambdalestes (Wible et al. 2004), and Maelestes (Wible et al. 2009) - have been useful (sometimes critical) to interpret the cranial anatomy of Alcidedorbignya.

In the comparison section we compare the major cranial and postcranial characters of Alcidedorbignya and discuss their distribution and, when relevant, the state present in the Bolivian pantodont. A list of characters that are included in the parsimony analysis presented below is provided in Appendix 1. Not all the characters discussed in the comparison section will be automatically included in the parsimony analysis and not all the characters included in the analysis will be discussed in the comparison.

Appendix 2 provides a list of the taxa included in the analysis. This list also indicates the material of fossil and Recent mammals available to us (original specimen with catalogue numbers, casts, photos, publications). Justification of our taxa selection for the comparison is given below. This list does not include all the taxa used in the comparison section.

The comparison presented below does not pretend to represent an exhaustive study of all eutherian groups but rather is a survey of several interesting characters found in pantodonts (especially in Alcidedorbignya), briefly discussing their distribution within Eutheria. Therefore, the comparison with pantodonts will be extended to a number of non-pantodont eutherians, in addition to the non-therian taxon Vincelestes and the early-diverging metatherians from Tiupampa (Puca- 
delphys, Andinodelphys, and Mayulestes), for which we have at hand a remarkably complete set of specimens, and which are the oldest nearly completely preserved representatives of the infraclass. In the discussion of postcranial characters comparison was also made with the Recent didelphids Caluromys and Metachirus.

Occasional comparisons will also be made with multituberculates (e.g., Catopsalis, Nemegtbaatar, Kryptobaatar, Chulsanbaatar), but no taxon of this order will be included in the parsimony analysis.

Muizon \& Marshall (1992) documented the great dental similarities existing between Alcidedorbignya inopinata and Pantolambda bathmodon from the Torrejonian of North America, although, in terms of molar morphology, the former is clearly more primitive than the latter. Pantolambda is approximately three times larger than Alcidedorbignya. The discovery of more complete material of Alcidedorbignya confirms that Pantolambda is also the North American pantodont that is the most similar to the South American taxon. The following study therefore will be focused on a comparison between these two American taxa. The best-preserved specimen of Pantolambda (AMNH 16663), a subcomplete skeleton described by Matthew (1937), the skull of which we had at hand during this study, will be the primary base for comparisons. This specimen is especially interesting because its skull has been further prepared. Especially its basicranium has been prepared by acid etching by one of us (CM) and probably represents the only North American early Palaeocene pantodont with such a perfectly preserved auditory region, including the ectotympanic. Because the anatomy of this specimen is highly relevant to the study and interpretation of the skull of Alcidedorbignya, we present below, in Appendix 3, a full description and interpretation of the auditory region of AMNH 16663. Because the dentition of Alcidedorbignya is also similar to that of Harpyodus, we thus consider H. euros, the holotype of which is a partial maxilla with P4-M2 (Qiu \& Li 1977), and H. decorus, the holotype of which is a skull (Wang 1979). Harpyodus decorus is dentally almost identical to $H$. euros, and this is the reason why Muizon \& Marshall (1992) rejected Van Valen's assignment of the species to a new genus (Wanglia) (Van Valen 1988; McKenna \& Bell 1997). Other pantodonts considered in the comparison are Haplolambda, Caenolambda (Gazin 1956), and Bemalambda (Zhou et al. 1977).

Alcidedorbignya will also be extensively compared to earlydiverging eutherian taxa from Mongolia, which have been thoroughly described in earlier works (Kielan-Jaworowska 1981; Wible et al. 2001; Wible et al. 2004; Wible et al. 2009), and which exhibit numerous plesiomorphic anatomical features. These taxa are: Prokennalestes, Asioryctes, Kennalestes, Zalambdalestes, Barunlestes, and Maelestes. Occasional comparison also will be made with Kulbeckia (Ekdale et al. 2004). Deltatherium and Didelphodus have been suggested as potentially related to pantodonts (Simons 1960; McKenna 1975; Van Valen 1978, 1988; Zhou et al. 1977; Chow \& Wang 1979; Lucas 1982, 1993, 1998b; Muizon \& Marshall 1992; Rose 2006). Other taxa have been included in the Cimolesta with the pantodonts (McKenna \& Bell 1997; Rose 2006) and are represented by reasonably complete cranial remains. These include the palaeoryctids Palaeoryctes, Eoryctes and Pararyctes and the pantolestans Aphronorus, Pantolestes, and Palaeosinopa. When appropriate they have been also included in the comparison below.

The tillodonts have been regarded as closely related to the pantodonts (Muizon \& Marshall 1992; Lucas 1993, 1998a) and in some cases they have even been included in the Pantodonta (Chow \& Wang 1979). We have, therefore, included in the comparison and parsimony analysis the two best know tillodonts represented by well-preserved skulls: Azygonyx and Trogosus. Azygonyx is a taxon morphologically very close to Esthonyx, which has been regarded as a junior synonym (Lucas 1998a). In this paper, we tentatively retain the genus Azygonyx, which exclusively refers to the only complete skull of Esthonychinae (UM 68511); and in the parsimony analysis we associated the two genera (see Appendix 2) considering that, being so closely related, their characters are almost identical and their differences are of specific rather than generic value. The most primitive tillodont, Lofochaius, has not been included in the analysis because the only skull known of this genus (for which we only had access to a partial cast) is very poorly preserved and apparently not fully prepared.

Pantodonts have often been referred to as ungulate-like mammals (Simpson 1945; Szalay 1977; Van Valen 1978 1988), but their relationships with ungulates have never been really established and were rejected by McKenna \& Bell (1997), Zhou \& Wang (1979), and Lucas (1993, 1998a). Nevertheless, a thorough comparison with archaic ungulates ("Condylarthra") is presented below, especially with the two oldest known "condylarth" genera known by complete skulls (Maiorana and Baioconodon). Other archaic ungulate genera compared to Alcidedorbignya are: Hyopsodus, Meniscotherium, Phenacodus, Arctocyon, Arctocyonides, Pleuraspidotherium. Three early-diverging artiodactyls (Dichobune, Acotherulum, and Diacodexis) will also be compared to Alcidedorbignya.

Comparison with the South American "ungulates" will include: 1) xenungulates (Carodnia and Etayoa);2) notoungulates (Oldfieldthomasia, Notopithecus, Protypotherium, Paedotherium, Hegetotherium, Posnankytherium, Puelia, Notostylops, Archaeohyrax, Scarrittia, Leontinia, Periphragnis, Adinotherium, and an isolated notoungulate petrosal MNHN.F.BRD23 described by Billet \& Muizon (2013);3) litopterns Miguelsoria (teeth and referred petrosals; Billet et al. 2015), Protolipterna (teeth and postcranial elements referred to this genus by Cifelli 1983a; Bastos \& Bergqvist 2007), Diadiaphorus, Protherotherium, Macrauchenia); 4) astrapotheres (Astrapotherium, Astraponotus, and Trigonostylops); and 5) the pyrothere Pyrotherium.

Among Recent taxa, the lipotyphlans and tenrecoids generally retain a relatively plesiomorphic cranial anatomy and are therefore suitable for comparison with the very plesiomorphic anatomy of Alcidedorbignya. The following taxa will be included in our comparisons: the lipotyphlans Solenodon, Erinaceus, Echinosorex, and Suncus, and the tenrecoids Tenrec, Potamogale, Microgale, Hemicentetes, Oryzorictes, and Setifer. Scandentia will also be considered including the genera Tupaia and Ptilocercus and paenungulates represented by Procavia and Dendrohyrax. 
In the discussion on the postcranial skeleton some interesting comparisons were also made with Recent rodents (e.g., Sciurus, Ratufa, Callosciurus, Marmota, Cynomys, Dipus), lagomorphs (e.g., Lepus, Oryctolagus), and carnivores (e.g., Ailurus, Nasua, Potos, Martes), and paenungulates (e.g., Procavia and Dendrohyrax).

Some basal carnivores that retain a relatively unspecialized basicranium provide useful comparison: Protictis and Cynodictis. The latter genus was chosen because we have at hand remarkably preserved skulls of these genera from the basal Oligocene of the Quercy (France).

Xenarthra are represented by Kuntinaru (the oldest dasypodid known by skulls), Dasypus, and the pilosan Tamandua.

Therefore, our selection of comparative taxa includes representatives of the four major clades of Placentalia: Laurasiatheria, Euarchontoglires, Xenarthra, and Afrotheria.

The comparison of the endocast of MHNC 8372 will be with internal molds of pantodonts (e.g., Pantolambda, Leptolambda, Coryphodon [Edinger 1956; Lucas 1984; and CT scan of AMNH 16663]). Concerning Leptolambda, it is noteworthy that the specimen (FMNHP 20075) illustrated by Edinger (1956: fig. 18[right]) and referred by this author to Haplolambda has been referred by Lucas (1984) to Leptolambda and will be our reference specimen for comparison. Furthermore, as evidenced by Lucas (1984), the endocast of Coryphodon elephantopus figured by Cope (1877a), is poorly preserved and includes part of the nasal cavity. Therefore, in our following comparison we will refer to Lucas (1984), who provides a description and a corrected illustration of the better-preserved endocast of C. "hamatus" figured by Marsh $(1877,1884)$. Other comparisons will be with dinoceratans (e.g., Uintatherium; Edinger 1964), notoungulates (e.g., Rhynchippus, Eurygenium [Dozo \& Martínez 2015], Nesodon, Homalodotherium, Typotheriopsis, undetermined Notohippidae [Patterson 1937], Rhyphodon, Notostylops [Simpson 1933]), "condylarths" (e.g., Arctocyon, Arctocyonides [Russell \& Sigogneau 1965], Pleuraspidotherium [Edinger 1956; Russell \& Sigogneau 1965], Meniscotherium [Gazin 1965], Phenacodus [Edinger 1956]); basal eutherians (e.g., Barunlestes [Kielan-Jaworowska \& Trofimov 1986], Kennalestes, Zalambdalestes [Kielan-Jaworowska 1984], Leptictis [Novacek 1986]). Comparison will be also with recent CT-images of cladotherians (e.g., Vincelestes [Macrini et al. 2007a]), basal metatherians (e.g., Pucadelphys [Macrini et al. 2007b]), multituberculates (e.g., Kryptobaatar [Macrini 2006]), "condylarths" (Hyopsodus; Orliac et al. 2012a), basal artiodactyls (Diacodexis; Orliac \& Gilissen 2012, Orliac et al. 2012b), and recent taxa of interest (see description and references cited thereafter). Among extant mammals, lipotyphlans and more particularly tenrecids have a relatively primitive brain and are often used as a basis for comparison in studies of the mammalian brains (Stephan et al. 1991). Pending CT images of additional comparative taxa (e.g., other pantodonts, "condylarths" other than Hyopsodus), we will restrict our consideration of the evolution of endocranial features in eutherians to a discussion about the distribution of endocranial features in major eutherian clades. A list of the genus and species names cited in the text with authorship and year is available in Appendix 4.
Anatomical terminology for the skull essentially follows Wible $(2008,2011)$ and Wible \& Spaulding (2013) unless otherwise specified (e.g., Appendix 5). Postcranial skeleton anatomical terminology essentially follows Evans \& de Lahunta (2012). Muscular terminology and muscle attachments and functions are based on Howell (1928), Davis (1964), Bryden (1971), Piérard (1971), Evans \& de Lahunta (2012), and Barone (2000). Terminology for the vascular and nervous system, as well as for their passageways, is from the studies of MacPhee (1981); Wible (1987, 1993, 2008, 2009, 2010, 2011, 2012); Wible \& Spaulding (2013); and Evans \& de Lahunta (2012). Most of this terminology corresponds to anglicized terms of Nomina Anatomica Veterinaria (2005), hereafter abbreviated NAV.

\section{Phylogenetic ANALYsis}

Taxonomic sample

In order to formulate hypotheses for the relationships of Alcidedorbignya and Pantodonta within placentals, we have performed a phylogenetic analysis based on a reworked data matrix of Wible et al. (2009). The taxonomic sampling has been modified according to this objective. We have removed poorly known taxa that were not considered members of Placentalia by Wible et al. (2009) (e.g., Bobolestes, Lainodon, etc). In return, several well-known extinct placental taxa from the Paleogene, whose remains were mostly directly available in the collections of the MNHN or available through loans (e.g., Pleuraspidotherium, Didelphodus, Cynodictis, Arctocyon, Arctocyonides, Baioconodon, Maiorana), were added to the matrix in order to explore potential relationships with pantodonts. Extant taxa present in the matrix of Wible et al. (2009) have been retained here. Furthermore, as no pantodont other than Alcidedorbignya has been discovered in South America so far, several authors have considered that it simply represents an abortive attempt to colonize the continent by the order Pantodonta (Muizon \& Marshall 1992; Gelfo et al. 2009; Woodburne et al. 2014). However, no detailed phylogenetic analysis has been undertaken with respect to this suggestion. In order to test this hypothesis through the analysis of the phylogenetic affinities of Alcidedorbignya with other South American taxa, we have also included 12 representatives of the five orders of South American native ungulates: Notoungulata, Litopterna, Astrapotheria, Pyrotheria and Xenungulata. These additions to the sample led to the construction of a matrix containing 72 taxa, with many traditional placental orders being represented (see list of taxa and sources in Appendix 2). As in the analysis of Wible et al. (2009), only the extant orders Pholidota, Perissodactyla, Chiroptera, Dermoptera and Sirenia are absent from our matrix.

The stem therian mammal Vincelestes (e.g., Rougier et al. 2012) was included as an a priori outgroup, following Wible et al. (2009). Vincelestes is represented by nearly complete specimens and previous analysis placed it as a taxon very close to the base of the therian clade (Rougier et al. 2012). However, we are conscious that it is, in some aspects, a highly derived taxon, which may introduce confusion in 
an analysis exclusively focused on intra-eutherian relationships. In order to try to minimize this bias, other out-group taxa are considered. The early-diverging metatherians Deltatheridium, Pucadelphys, and Mayulestes have been scored to fulfill this function.

With two exceptions, terminal taxa were scored at the genus level; material on which observations are based are detailed in Appendix 2. The exceptions just noted are two terminal taxa correspond however to clusters of two genera, which are believed to be very close: Azygonyx and Esthonyx (see above) on the one hand (Gingerich \& Gunnell 1979; Gingerich 1989; McKenna \& Bell 1997; Lucas 1998a), and Adapis and Magnadapis on the other (Godinot \& Couette 2008).

The sources used for the taxa include Wible et al. (2009) (see below), direct observation of specimens or casts, and on rare occasion observation of illustrations in past publications (see sources in Appendix 2). We have thus generally been able to observe most of the currently documented anatomy of each taxon.

\section{Character sampling and scoring}

Our character list is largely based on that of Wible et al. (2009) and we have voluntarily kept modifications to a minimum. The few modifications that have been made to the original characters of Wible et al. have generally been done in order to provide more precise criteria for scoring the various states (e.g., ex-character 150 of Wible et al. [noted as $150 \mathrm{~W}$ here]), to avoid confusing statements or possible overlap between the states (e.g., 149W), or to eliminate the distinction of trivial and/or artefactual variations (e.g., $188 \mathrm{~W}$; the opening of the major palatine foramina is often observed more anteriorly than they probably originally were, due to breaks in the thin ventral wall, especially in fossils). Moreover, some characters have been deleted and a justification for this is provided in the character list (see Appendix 1). New characters have also been added to the matrix; these stem from new observations made in this study and/or in recent contributions (see references cited in the character list, Appendix 1). This has led to the construction of a datamatrix containing 426 characters.

For the taxa and characters in common with those of Wible et al. (2009), the scores have been unchanged unless stated otherwise (see italic notes in the list of characters in Appendix 1). When the definition (the content) of a character has been significantly modified from the original version of Wible et al. (which is indicated in Appendix 1), the changes made to the original scores are not detailed. For some characters however, only the wording (and not the content) has been modified, which is then specified as "modified 'text only". Scoring of the new and modified characters and new taxa was undertaken by CM and GB.

\section{Phylogenetic analysis}

The parsimony analyses were carried out in TNT (Goloboff et al. 2008) (traditional search, 100 replications, tree bisection and reconnection [TBR] swapping algorithm) and PAUP 4b10 (Swofford 2002) (heuristic search, 100 replications, TBR swapping), with multistate taxa set to "uncertainty" and zero-length branches set to collapse if their minimum length is zero ("amb-"). The main analysis treated some multistate characters as ordered (which is indicated in the character list). Finally, the Bremer indices were calculated with TNT on the one hand and TreeRot v.2 (Sorenson 1999) and Paup 3.1.1 (Swofford 1993), both run inside SheepShaver, on the other hand.

An analysis with a backbone constraint forcing the monophyly of the four major extant placental clades (Xenarthra, Afrotheria, Euarchontoglires, Laurasiatheria; see Results and Discussion) was also undertaken. We have set this constraint such that the extant members and fossil representatives of extant orders are forced to be included within the corresponding molecular clade (e.g., the fossil artiodactyls and fossil carnivorans were forced into a Laurasiatheria clade).

A two-tailed Templeton test has been performed with PAUP $4 \mathrm{~b} 10$ to statistically assess the hypothesis supporting the monophyly of the four major extant clades of placentals obtained with a backbone constraint by comparing the constrained most parsimonious trees (MPTs) with the unconstrained MPTs (Templeton 1983). Character reconstruction was made with PAUP 4 b10.

\section{MeAsurements}

Measurements of most of the specimens are given in tables included in the description of the skull or of the postcranial skeleton. They represent more an indication of size than a precise size because almost all the specimens have variably suffered some postmortem distortion. This is especially true for the postcranial skeleton of MHNC 8372. Concerning the bones of this specimen with bilateral symmetry (vertebrae, skull) when one side of the element was missing or damaged, half of the measurement was taken and was multiplied by 2 . In that case, the number indicated has been adjoined with an "e" for estimate. Several length measurements of the vertebral centra of MHNC 8372 were also estimated because they were missing one epiphysis. However the length of this missing element could be evaluated from the other epiphysis of the vertebra or from the epiphysis of the adjacent vertebra. In the Tables, $\mathrm{R}=$ right and $\mathrm{L}=$ left.

\section{SUPPLEMENTARY DATA}

Supplementary data are available at the following addresses: - http://sciencepress.mnhn.fr/en/periodiques/geodiversitas/37/4/ alcidedorbignya-inopinata-additional-files (in English); - http://sciencepress.mnhn.fr/fr/periodiques/geodiversitas/37/4/ alcidedorbignya-inopinata-fichiers-additionnels (in French).

Supplementary data contain the following files:

\section{PARSIMONY ANALYSIS FILES}

- The nexus file of the data matrix;

- The list of synapomorphies distributed on the strict consensus tree of the unconstrained analysis. 


\section{D PDF FILES}

- File 1: 3D rendering of CT data of the skull of Alcidedorbignya inopinata (MHNC 8372) in pdf format (Figs 22, 30, $32,39,47,54,57,58)$.

- File 2: 3D rendering of CT data of the skull of Alcidedorbignya inopinata (MHNC 8399) in pdf format (Figs 44, 48). - File 3: Pdf file of the 3D photograph of the auditory region of Alcidedorbignya inopinata (MHNC 8372)(Fig. 36).

- File 4: Pdf file of the 3D photograph of the auditory region of Alcidedorbignya inopinata (MHNC 8399) (Figs 37, 38).

- File 5: 3D rendering of CT data of the skeleton of Alcidedorbignya inopinata (MHNC 8372) in arboreal locomotion (Fig. 117).

- File 6: 3D rendering of CT data of the skeleton of Alcidedorbignya inopinata (MHNC 8372) in terrestrial locomotion (Fig. 118).

- File 7: 3D rendering of CT data of the left ectotympanic and malleus of Pantolambda bathmodon (AMNH 16663) (Fig. 126);

- File 8: 3D rendering of CT data of the postcranial skeleton of Alcidedorbignya inopinata (MHNC 8372).

\section{SPECIMENS, TAPHONOMY AND BIOLOGY}

\section{THE NEW PANTODONT SPECIMENS}

Pantodonta are represented at Tiupampa by a single species, which is, by far, the most abundant placental mammal of the fauna. Most of the specimens are isolated teeth or jaws, which provide a good knowledge of the dentition of this species. The dental anatomy of Alcidedorbignya was thoroughly described and illustrated by Muizon \& Marshall (1992). The holotype of Alcidedorbignya is a partial maxilla bearing P4-M3 (Fig. 1). Virtually no remains of the bony skull of Alcidedorbignya were known until 1999. That year, a nearly complete articulated skeleton (MHNC 8372) of Alcidedorbignya was discovered at the site called "the quarry" by Muizon \& Marshall (1992). This site had already yielded several articulated skeletons of marsupials: Pucadelphys andinus (Marshall \& Muizon 1988; Marshall et al. 1995; Ladevèze et al. 2011), Mayulestes ferox (Muizon 1994, 1998), and Andinodelphys cochabambensis (Muizon et al. 1997; Muizon \& Argot 2003). However, the pantodont skeleton described here is the first placental skeleton discovered at Tiupampa. The skeleton is remarkably complete and all the bones, except some vertebrae, have been separated (Fig. 2). Without doubt, MHNC 8372 represents one of the best and most complete early Palaeocene mammal skeletons ever discovered (Gelfo et al. 2009). The skull is especially complete and undistorted. The only fragments missing are the left premaxilla and the posterior third of the right promontorium. The mandible is only missing the i1s. The skull is broken into two parts almost along the sagittal plane. This accidental breakage, which occurred during discovery (Fig. 3), has allowed an excellent preparation and observation of the internal anatomy of the braincase. Because of the exceptional preservation of this specimen, it

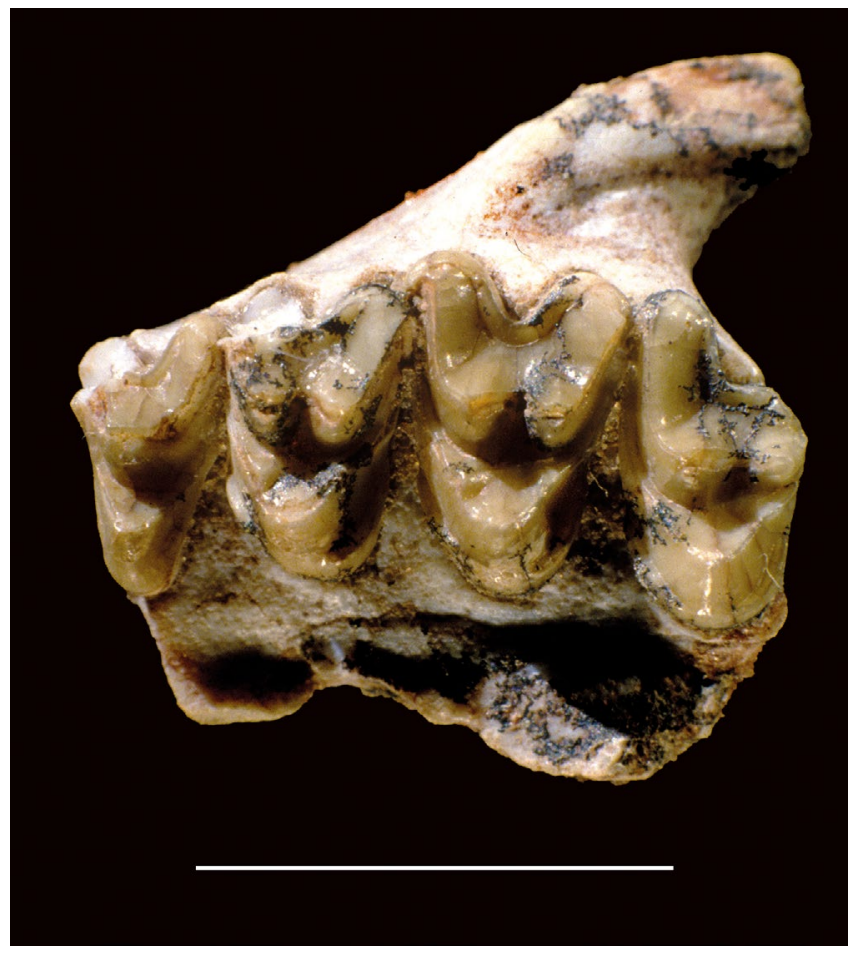

FIG. 1. - Holotype of Alcidedorbignya inopinata: left partial maxilla with P4-M3, YPFB Pal 6121 . Scale bar: $1 \mathrm{~cm}$.

has been possible to securely refer several isolated petrosals to Alcidedorbignya. Some of these petrosals are even better preserved than those of the MHNC 8372 and provide invaluable data on the structure and anatomy of the middle and inner ear, as well as on the passageway of surrounding vessels and nerves. A second skull of Alcidedorbignya was discovered in 2009. It is an isolated skull partially weathered (MHNC 8399). The dorsal face of the rostrum and most of the left maxilla have been washed away. However, the braincase and the basicranium are exquisitely preserved and provide a welcome complement to MHNC 8372.

Furthermore, fossil belonging to three juvenile individuals have also been discovered (Fig. 4). One is a partial skull and articulated mandible with some tiny and poorly ossified post-cranial elements belonging to a very young individual (MHNC 8373) (Fig. 4A). It has all deciduous teeth erupted except dp2, which is still in the crypt. No molars are erupted but $\mathrm{m} 1$ is in crypt. This specimen was discovered during the preparation of the skeleton MHNC 8372 mentioned above. MHNC 8373 was positioned between the hind limbs of the adult and could represent either a new born or a near term foetus of MHNC 8372 (see discussion below).

Another juvenile skull and partial skeleton (MHNC 8416) belongs to an older individual, which still retains its deciduous dentition but has erupted M1s and $\mathrm{m} 1 \mathrm{~s}$ and unerupted M2s and $\mathrm{m} 2 \mathrm{~s}$, still in the crypts. Part of the right forelimb (humerus, radius, and ulna) and some ribs of this specimen are also preserved (Fig. 4C).

The third juvenile skull is that of an individual intermediate in age between the first two (MHNC 8423). It is a 


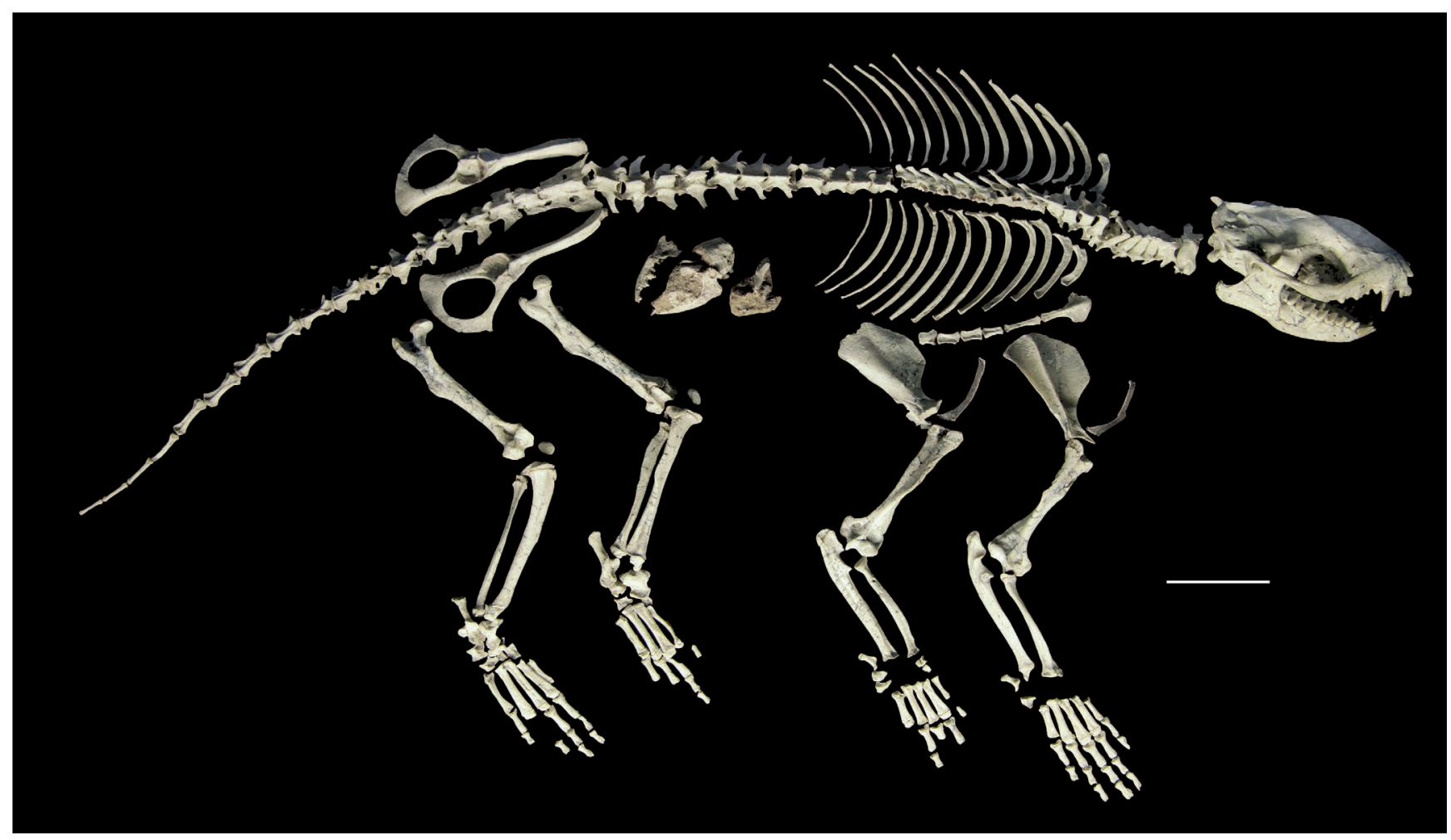

FIG. 2. - Alcidedorbignya inopinata, skeleton MHNC 8372. Scale bar: $2 \mathrm{~cm}$.

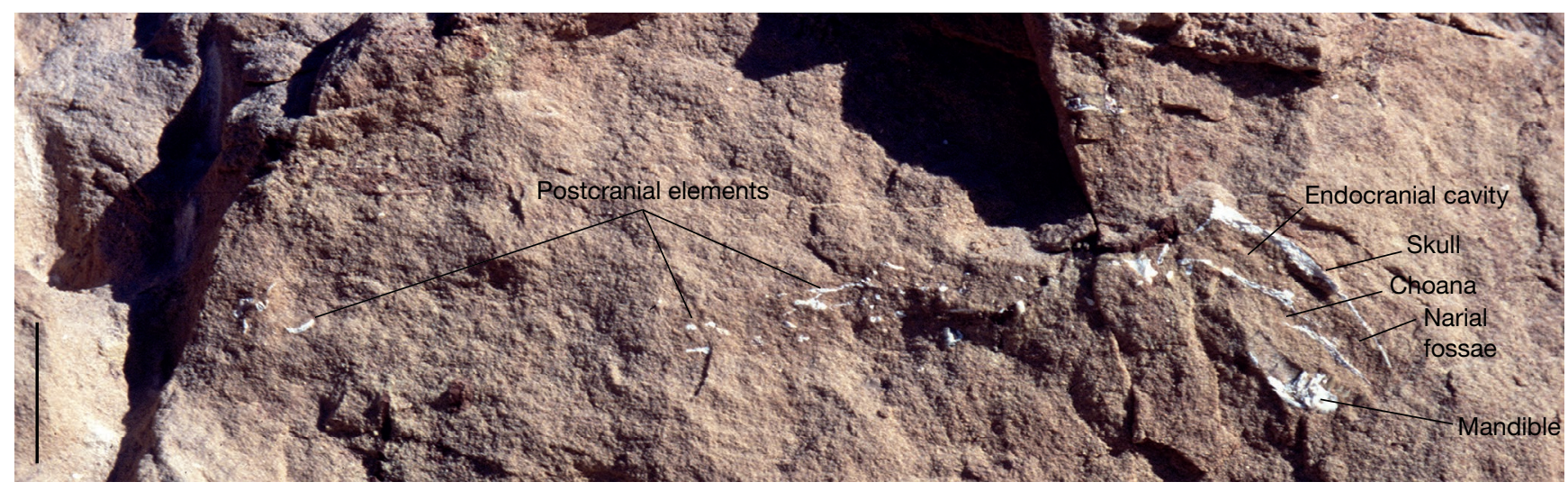

FIG. 3. - Section of the skull of MHNC 8372 (Alcidedorbignya inopinata) when discovered in the "quarry" of Tiupampa in July 1999. Scale bar: 3 cm.

partial skull (missing part of the occiput and premaxillae) with mandible. All deciduous cheek teeth and lower incisors are functional. Nothing can be said about the upper incisors as the premaxillae are missing, but it is likely that they were also functional. M1s and $\mathrm{m} 1 \mathrm{~s}$ are erupting. This specimen is undistorted and preserves the ectotympanic and part of the malleus in situ and in a position close to anatomical (Fig. 4B).

Furthermore, several partial to nearly complete upper and lower jaws of adults and juveniles have been discovered since the first monograph by Muizon \& Marshall (1992). These provide important new information on individual variation, sexual dimorphism, and sequence of dental eruption.
TAPHONOMY AND PALAEOBIOLOGY

The skeleton described here (MHNC 8372) is that of a young adult. All the teeth are fully erupted and almost unworn. However, the epiphyses of the limb bones are not fused to the diaphyses, which indicates a not fully-grown individual. Furthermore, an interesting taphonomic observation can be made. The specimen was discovered with most of the vertebrae and ribs still articulated and the limb bones only slightly displaced. This indicates that the animal was buried very rapidly (as were the marsupial skeletons mentioned above), probably because of a catastrophic flood, and experienced no significant transport. In this context, the discovery of the skull and partial skeleton of a very young individual (the foetus or newborn individual mentioned above MHNC 8373) of the 


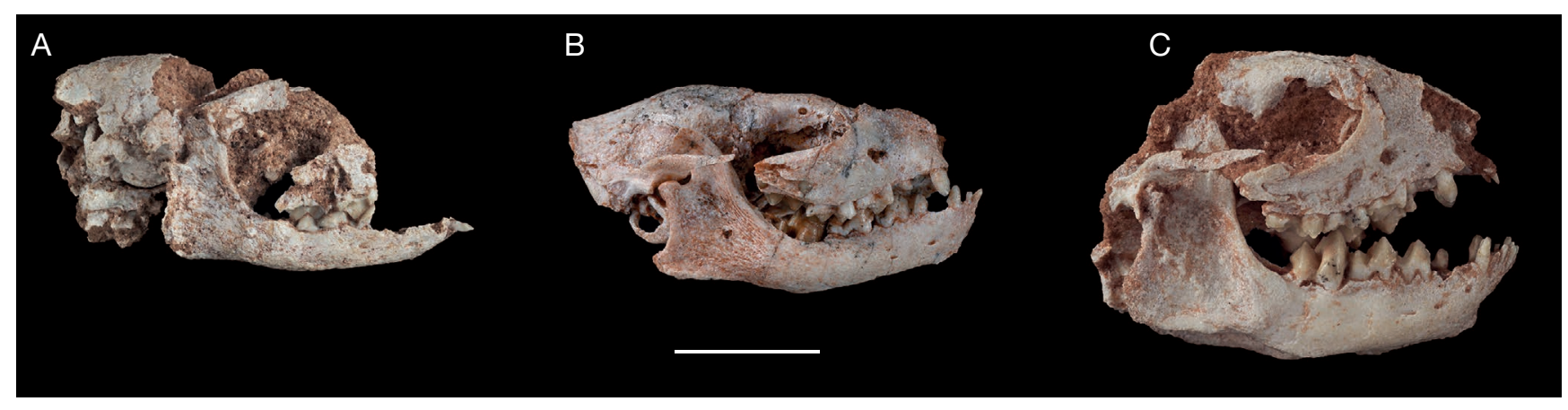

FIG. 4. - Juvenile skulls of Alcidedorbignya inopinata: A, MHNC 8373; B, MHNC 8423; C, MHNC 8416. Scale bar: $1 \mathrm{~cm}$.

same species positioned between the limb bones of the young adult skeleton could indicate that the latter was a female. The juvenile specimen has the DP4s erupted and bearing hardly discernible wear facets. The M1s are still in their crypts, fully mineralized, but were not close to erupting. Gingerich et al. (2009: table 4) provided an overview of the development and mineralization of deciduous and permanent teeth at birth compared to the developmental maturity categories of Langer (2008) in several Recent mammals. Some of the most precocial mammals (category IV: eyes open, haired, nidifugous at birth) also have all deciduous teeth erupted at birth and the crown of M1 half or fully mineralized. This condition is observed in several ungulates (Dama, Giraffa, Hexaprotodon) and is the dental development stage of the juvenile skull mentioned above. Therefore, the juvenile skeleton found between the limbs of the adult skeleton described here could reasonably be that of a near-term foetus. Alternatively, it could also constitute a slightly later stage around birth, like a newborn individual. Nevertheless, the fact that such a poorly ossified skeleton is so well preserved (for instance, the ectotympanics and ear ossicles are still articulated) might be better explained if it was protected within the uterus of the female. In this context, it is considered here to belong to a perinatal stage, most likely close before birth. As mentioned above, this specimen will be described from a developmental perspective in a work in progress.

It is also noteworthy that remains of juveniles are very abundant in the fauna of the "quarry" at Tiupampa. For the pantodonts, which are the most abundant eutherian specimens, jaws of skulls are distributed among 33 juveniles and 35 adults. Juvenile remains at Tiupampa not only belong to pantodonts but also to some "condylarths" and several marsupials. As stated by Muizon \& Cespedes (in press) the Tiupampa locality called "the quarry" was probably a pond set aside a river (for example, a depression on a floodplain) or an ox-bow that was flooded almost instantaneously by a wave of water and sediment, owing to a crevasse splay, probably during a breeding season. It is therefore not surprising that some juveniles (weaker than adults) and a possibly gravid female (MHNC 8372), more vulnerable given their condition, could have been trapped more easily than other individuals. Furthermore, the greatest abundance of articulated skeletons is observed by far, in the sample of the metatherian Pucadel-

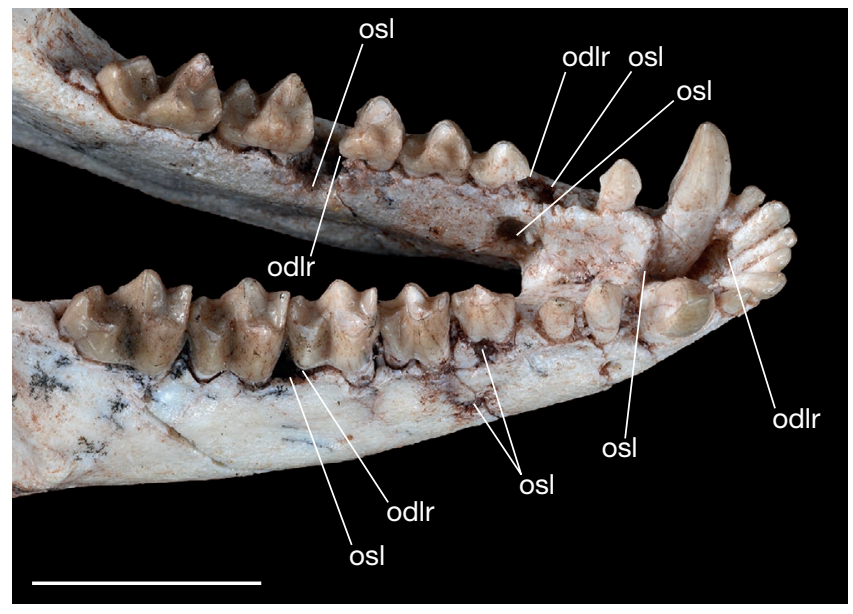

FIG. 5. - Pathological osteolysis and odontolysis on MHNC 8399. Abbreviations: odlr, odontolysis of root; osl, ostelolysis. Scale bar: $1 \mathrm{~cm}$.

phys andinus, which is known by at least 35 partial and subcomplete skulls and skeletons. This has been interpreted by Marshall et al. (1995), Ladevèze et al. (2011), and Muizon \& Céspedes (in press) as related to the fact that this species was sheltering in burrows and were burried during a flood. This interpretation is unlikely to apply to Alcidedorbignya, since most of the specimens (several hundreds) are isolated teeth or jaws, thus indicating important scattering of the remains. This interpretation is corroborated by the fact that, in contrast to the condition observed concerning Pucadelphys remains, only one articulated skeleton of Alcidedorbignya has been discovered. This would indicate that fossorial habits are unlikely for the Tiupampa pantodont.

In this context some pathological observations of the other adult skull (MHNC 8399) are of interest. The skull of this individual presents numerous bony lesions on the mandible, which are likely to be the result of osteolysis and odontolysis during life due to gumboils or tumors (Fig. 5). These infections led to destruction of several roots of the lower teeth. One large osteolytic cavity is present in the mandibular symphysis behind the incisors, which had lost the basal part of their roots; one is present on the medial side of the alveolar border of the right p3, which had lost its posterior root; another one is present slightly below the medial alveolar border of left p3, which had 


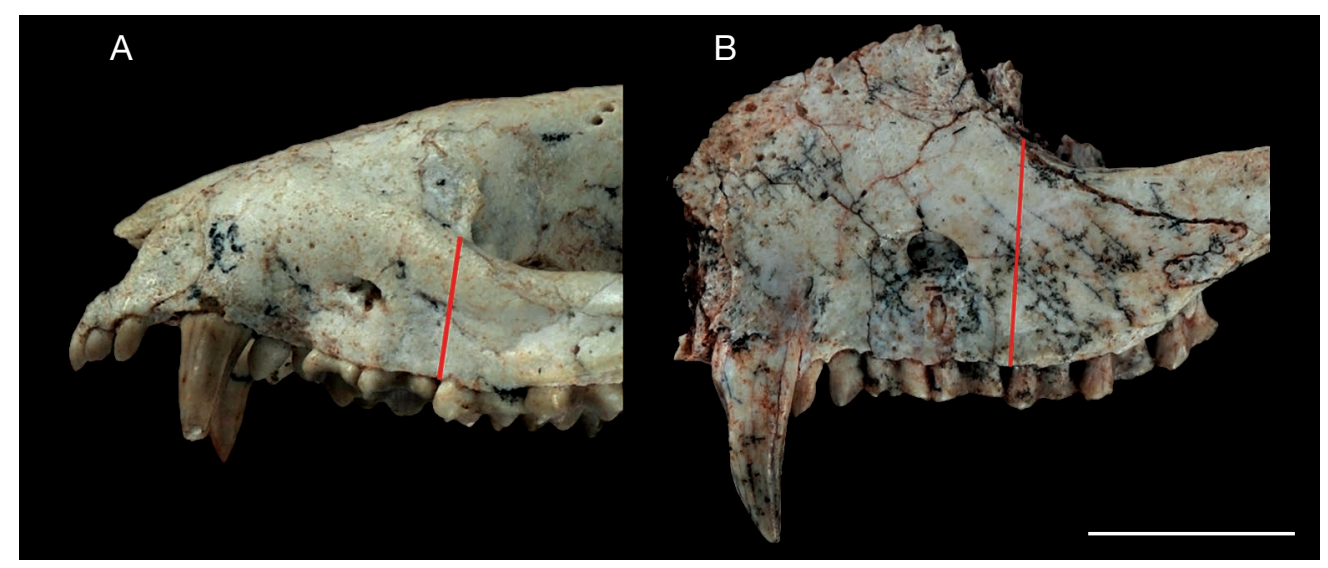

FIG. 6. - Lateral view of the maxilla a of Alcidedorbignya inopinata showing the difference in the elevation of the snout (red bar = maxilla height in Fig. 7): A, female (MHNC 8372, right side reversed); B, male (MHNC 8400). Scale bar: $1 \mathrm{~cm}$.

TABLE 1. - Measurements of the elevation of the rostrum, proportions of upper canine and M2 in selected specimens of Alcidedorbignya inopinata (see Fig. 7A). Abbreviations: F, female; HtMx, height of the maxilla from the anterolabial angle of $\mathrm{M} 1$ to anterior end of jugal; LC, anteroposterior length of upper canine; LM2, anteroposterior length of M2; M, male; WC, labiolingual width of upper canine; WM2, labiolingual width of M2.

\begin{tabular}{lcclllll}
\hline \multicolumn{1}{c}{$\begin{array}{c}\text { Inferred } \\
\text { Specimen }\end{array}$} & sex & HtMx & LC & WC & HC & WM2 & LM2 \\
\hline YPFB Pal 6121 & F & 8.92 & & & & 7.3 & 4.7 \\
YPFB Pal 6165 & M & 10.2 & & & & & \\
MHNC 1210 & F & 7.9 & & & & 8.1 & 5.1 \\
MHNC 8372 & F & 7 & 3.4 & 3.4 & 7.2 & 8.63 & 5.3 \\
MHNC 8399 & F & 7.8 & 3.75 & 3 & 7.75 & 7.5 & 4.9 \\
MHNC 8400 & M & 11.4 & 4.4 & 4.1 & 10 & 7.9 & 4.8 \\
MHNC 8401 & M & 11.6 & 4.28 & 4 & & 7.5 & 5 \\
MHNC 8402 & F & 8.6 & & & & 8.3 & 5.6 \\
MHNC 8403 & F & $7.7 e$ & 3.4 & 3 & 5.2 & 7.7 & 4.8 \\
MHNC 8404 & M & 10.9 & & & & 8.4 & 5.5 \\
MHNC 8405 & M & 10.1 & & & & $7.3 e$ & $4.6 e$ \\
MHNC 8406 & F & 8.1 & & & & 7.8 & 4.8 \\
MHNC 8407 & M & 10.4 & & & & 8 & 5 \\
MHNC 8408 & F & 7.5 & & & & $8.1 e$ & $5.3 e$ \\
\hline
\end{tabular}

lost its anterior root; another on the alveolar borders of left $\mathrm{m} 1$, which had lost its posterior root; another is on the lateral alveolar border of right $\mathrm{m} 1$, which had lost most of posterior root (and the anterior root was undergoing excavation); and one at the lateral alveolar border of the left $\mathrm{m} 3$, the anterior root of which is distinctly corroded. Moreover, a large pathological osteolytical cavity is present on the right side of the palate medial to P4-M2. The teeth of this specimen are only slightly worn and indicate a relatively young adult. Given the amount and the size of the spots showing bone and root resorption (at least eight on the mandible and a large one on the maxilla) it is clear that this animal was suffering severe infection, which could have affected its whole body. Therefore it is not impossible that this individual died of a generalised infection, possibly septicaemia. It is also possible that this individual had been affected by these infections and was seriously weakened and more vulnerable than other healthier individuals to a sudden catastrophic flood event.

\section{SEXUAL DIMORPHISM}

Given the abundance of juvenile remains relative to adults (see above) it is possible that all the individuals were living close to the locality where they were fossilized and probably were contemporaneous (or nearly so). It is therefore reasonable to suspect that they could belong to the same population. A similar hypothesis has been made for the metatherian Pucadelphys andinus, a stem-metatherian from the same locality known by 35 individuals, 22 of which are represented by nearly complete skulls and associated postcrania (Ladevèze et al. 2011). Evidence of sexual dimorphism and social behaviour has been given for that species mainly based on the skull size and on the relative size of the canines and sagittal crests. In the case of Alcidedorbignya inopinata, although nearly 50 jaws have been collected, only two adult skulls are known and such criteria are not easy to identify in order to investigate possible sexual dimorphism. We have, however, been able to select three potentially sexually dimorphic characters observable on relatively complete upper jaws. One measurement seems to separate a sample of 14 maxillaries into two distinct clusters. The measurement is the distance from the anterolabial alveolar angle of M1 to the anterior extremity of the jugal on the anteroventral edge of the orbit (Fig. 6). This measurement almost corresponds to a vertical line from the anterolabial angle of M1 to the edge of the orbit and provides an indication on the elevation of the maxilla in this region, and therefore of the whole rostrum and nasal cavity. In some cases the anterior edge of the jugal is not preserved itself, but marks of the suture are present and a reasonably accurate evaluation can be made. On one specimen (MHNC 8403) the anterior edge of the orbit is damaged and the position of the anterior end of the jugal has been estimated. However, given the position of this specimen on the graph of Figure 7A, it is unlikely that it could have belonged to the other cluster of measurements. As seen in Table 1, in the cluster with an elevated rostrum the measurement spans from 10.1 to $11.6 \mathrm{~mm}$ (with a range of 1.5) and in the cluster with a low rostrum the span is 
A

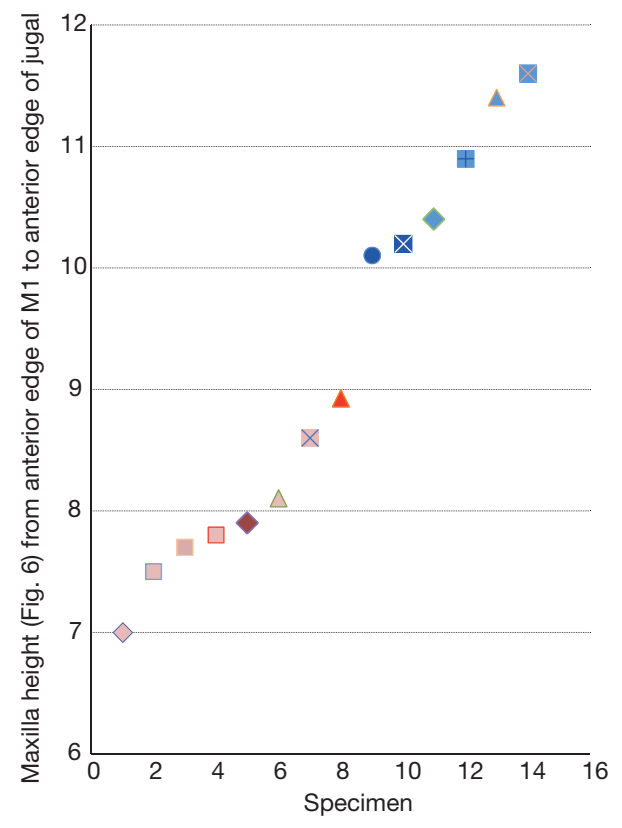

C

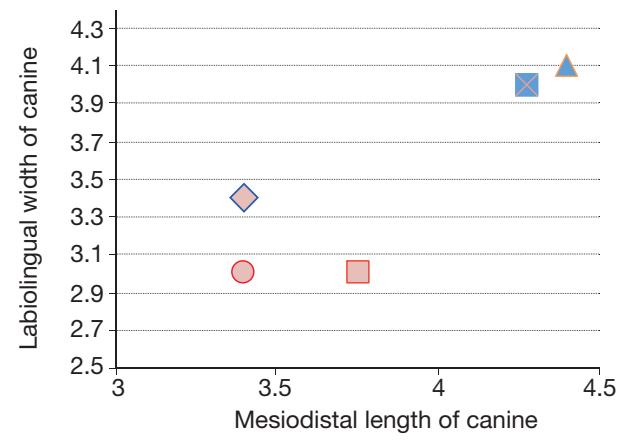

B

$\triangle$ MHNC 8372
$\square$ MHNC 8408
MHNC 8406

$X$ MHNC 8402

$\square$ MHNC 8399

A YPFB Pal 6121

MHNC 8405

$\triangle$ YPFB Pal 6365

MHNC 8407

I MHNC 8404

MHNC 8400

MHNC 8401

MHNC 8403e

MHNC 1210

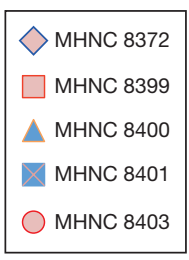

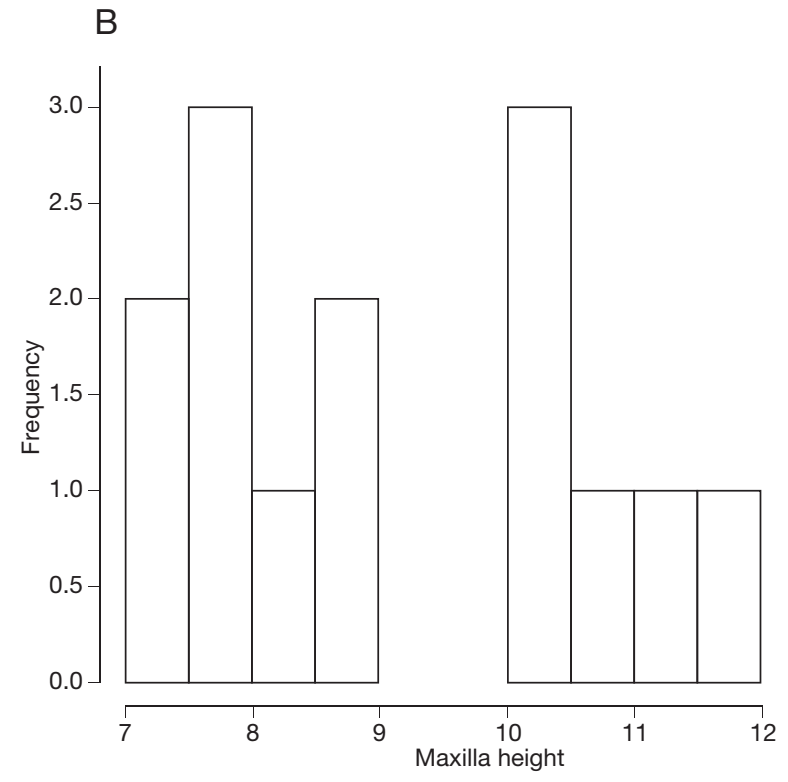

$\mathrm{D}$

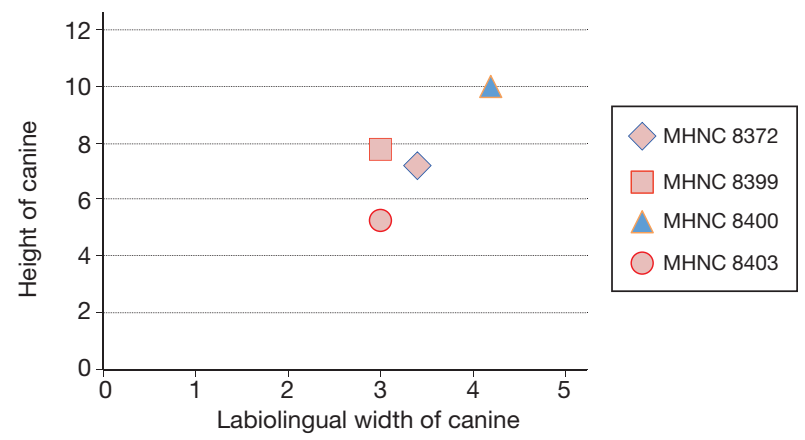

$\mathrm{E}$

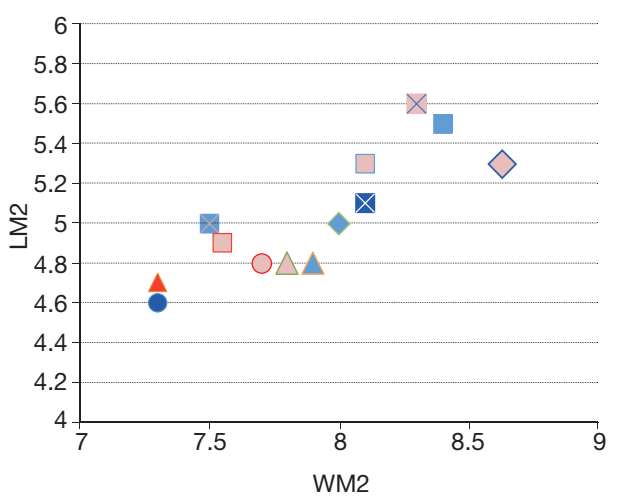

\begin{tabular}{|l|}
$\triangle$ MHNC 8372 \\
$\square$ MHNC 8399 \\
$\triangle$ MHNC 8400 \\
$\square$ MHNC 8401 \\
X MHNC 8402 \\
MHNC 8403 \\
MHNC 8404 \\
MHNC 8405 \\
$\triangle$ MHNC 8406 \\
MHNC 8407 \\
$\square$ MHNC 8408 \\
$\triangle$ YPFB Pal 6121 \\
\hline
\end{tabular}

FIG. 7. - Intraspecific variation in the elevation of the snout in Alcidedorbignya inopinata, which is considered here as related to sexual dimorphism: A, scatter plot showing the distribution of maxilla height from the anterolabial angle of M1 to the anterior end of the jugal on the anterior edge of the orbit; $\mathbf{B}$, histogram showing the bimodal distribution of maxilla height; $\mathbf{C}$, scatter plot showing the distribution of the width and length of the upper canine according to inferred sex of the individuals; D, scatter plot showing the distribution of the height and width of the upper canine according to inferred sex of the individuals; $\mathbf{E}$, scatter plot showing the distribution of the proportions of the M2 of Alcidedorbignya inopinata as compared to inferred sex of the individuals. Colors: blue filling (males); pink or red filling (females). Abbreviations: LM2, mesiodistal length of M2; WM2, labiolingual width of M2.

from 7 to 8.9 (with a range of 1.9). The diagram presented in Figure 7B clearly shows the bimodal distribution of the two clusters of measurements, which are well separated by a gap of $1.1 \mathrm{~mm}$. We hypothesize that the cluster with the elevated rostrum could represent males and that with low rostrum represent females (Fig. 6). 
The other two characters refer to the size of the upper canine, one of the most significant features related to sexual dimorphism in mammals. Among the 14 maxillaries measured above, five preserve the canine. Four of them have a complete to subcomplete canine and one has only the cross-section of the canine preserved. In Table 1 and Figure $7 \mathrm{C}$ the sections of the five canines are compared. Although the sample is small, the distribution perfectly matches the hypothesized male and female clusters observed on the elevation of the rostrum. The two males have the most robust canine and the three females have more slender ones. Comparison of canine lengths yields a less clear pattern, but it is compatible with the hypothesized sexual dimorphism (Table 1; Fig. 7D). Because the elevation of the rostrum in some individuals of our sample is probably related to the greater size of the canine, the concordance of the data on maxilla elevation and canine size strengthens our hypothesis on sexual dimorphism in Alcidedorbignya inopinata.

Given this hypothesis of sexual dimorphism in Alcidedorbignya, we have compared the size proportion of M2 (length vs. width) in the sample of 14 maxillae to the assumed sex distribution. Figure 7E shows that proportions of M2 are clearly random and independent of our tentative sex referrals. In this respect, it is noteworthy that the smallest female (MHNC 8372) has among largest M2s of our sample.

\section{SYSTEMATIC PALAEONTOLOGY}

\section{Order PANTODONTA Cope, 1873}

Family AlCidedorbignYidAe Muizon \& Marshall, 1992

Genus Alcidedorbignya Muizon \& Marshall, 1987

TYPE SPECIES. - Alcidedorbignya inopinata.

DiagnOSIS. - Because the genus is monospecific, its diagnosis is that of the type species.

\section{Alcidedorbignya inopinata Muizon \& Marshall, 1987}

Alcidedorbignya inopinata Muizon \& Marshall, 1987c: 205.

Holotype. - A partial maxilla with P4-M3 (YPFB Pal 6121).

Hypodigm. - The hypodigm is that given in Muizon \& Marshall (1992), to which we add the following specimens: MHNC 8372 , an almost complete skeleton, which is missing only the left upper incisors, the two ils, a few phalanges and some carpal and tarsal bones and a few caudal vertebrae; MHNC 8373, a partial skull with complete braincase and mandibles of a very young individual, possibly a near-term foetus or a new-born individual (some postcranial elements are preserved); MHNC 8399, a partial skull with the braincase and basicranium perfectly preserved; the rostrum has been partly weathered and part of the left maxilla and the dorsal bones of the rostrum are missing; the right maxilla and premaxilla and the dentaries are almost complete; the specimen also includes half of the atlas; MHNC 8416, a partial skull (with crushed basicranium but the two petrosals well-preserved) and mandibles of a juvenile individual, with a partial right limb, and some ribs; MHNC 8423 a partial skull and mandibles of a juvenile missing part of the occipital region and bearing right $\mathrm{dC}$, erupting P1s, DP2s-DP4s, erupting $\mathrm{M} 1 s ; \mathrm{di} 2 \mathrm{~s}$, right di3, dcs, $\mathrm{p} 1 \mathrm{~s}$ in crypt, dp2s-dp4s, and erupting m1s; MHNC 8400, a left maxilla (with C-M3) with jugal and lacrimal; MHNC 8401 a right maxilla with roots of C-P2 and P3-M3; MHNC 8402, associated left maxilla and dentary, with P1-M3 and p3-m3; MHNC 8403, associated right maxilla and dentary, with C-M3 and right i2-trigonid of $\mathrm{m} 3$ and left i1-C; MHNC 8404, left maxilla with P3-M3; MHNC 8405, left maxilla with root of canine and P1-M3; MHNC 8406 left maxilla with root of P2 and P3-M3; MHNC 8407, left maxilla with roots of P4, partial M1 and M2-M3; MHNC 8408, maxilla with P4-M1 and partial M2; MHNC 8409, right dentary with c-m3, MHNC 8410, fragment of left dentary with trigonid of $\mathrm{m} 2$ and $\mathrm{m} 3$; MHNC 8411, fragment of right dentary with $\mathrm{m} 2-\mathrm{m} 3$; MHNC 8412, fragment of right dentary with $\mathrm{m} 2-\mathrm{m} 3$; MHNC 8413, fragment of left dentary with talonid of $\mathrm{m} 2$ and $\mathrm{m} 3$; MHNC 8414 , left dentary with roots of p3-m1 and weathered m2-m3; MHNC 8415, m3; MHNC 8359; right periotic; MHNC 8360, left periotic; MHNC 8361, right periotic; MHNC 8362, left periotic; MHNC 8419, right periotic; MHNC 8420, right periotic; MHNC 8421, left periotic; MHNC 8422, right periotic (pars cochlearis); MHNC 8423, a sub-complete skull and mandibles of a juvenile, missing most of the occipital and the premaxillae.

GeOlOGICAL SETTING AND AGE. - The Tiupampa mammals occur in the middle section of the Santa Lucia Formation (Marshall et al. 1997). This section has been referred to the early Palaeocene by Gayet et al. (1992), Bonaparte et al. (1993), and Muizon (1998). However, an early late Palaeocene age has been suggested by Marshall et al. (1997) and Pascual \& Ortiz-Jaureguizar (2007). Recently, Gelfo et al. (2009) have reassessed the age of the Tiupampa mammal fauna and concluded that it is of early Palaeocene age. The Tiupampa mammal-bearing beds are regarded by these authors as a probable equivalent of the late Puercan (Pu3) of North America and would therefore be as old as 64 to $64.5 \mathrm{Ma}$. This hypothesis is based on the fact that the Tiupampa mammal-bearing beds are included in a single reversed stratigraphic series, which is likely to correspond to Chron 28r (see Gelfo et al. 2009 for discussion). However, when Gelfo et al. (2009) wrote their paper, the reference for calibration of Chron 28r was Lofgren et al. (2004), and the correlation was with the late Puercan (Pu3). New dates and calibration of the NALMAs have slightly modified the age and position of Chron 28r. According to Wilson $(2013,2014)$ and Sprain et al. (2014) Chron $28 \mathrm{r}$ is totally included in the base of the early Torrejonian (To1) and its absolute age is bracketed between $c .65 \mathrm{Ma}$ and 64.866. This represents the very base of the early Torrejonian (To1), which spans from 65.118 Ma to $c$. 63.5 Ma. In other words, the absolute age for the Tiupampa fauna is $c .65 \mathrm{Ma}$.

\section{EMENDED DIAGNOSIS}

This following extensive diagnosis presents a list of the major morphological features of the skeleton and teeth of Alcidedorbignya inopinata and represents, in fact, a summarized description. Below a differential diagnosis is also provided.

\section{General morphology}

Small size by pantodonts standards; tail, long, $70 \%$ of the head and body length.

\section{Teeth}

Dental formula I3/3, C1/1, P4/4, M3/3; incisors spatulate; I1 distinctly smaller than I2-3; contralateral I1s widely separated from each other; $\mathrm{i} 1<\mathrm{i} 2>\mathrm{i} 3$; C and c large, protruding, splayed labially; P1-2 and p1-2 single rooted and proodont 
(i.e. single elevated cusp); P3-4 and p3-4 semimolariform; P3-4 with strongly V-shaped paracone and protocone (double V-shaped); M1-3 with distinct pre- and postcingula; paraconule well developed; metaconule weak to indistinct; protocone anteroposteriorly broad; paracone and metacone separated at base (i.e. not connate); M1 with paracone and metacone subequal in size; M2 with paracone slightly higher than metacone; $\mathrm{M} 3$ with paracone distinctly higher and much more voluminous than metacone; M1-2 with moderately dilambdodont ectoloph (i.e. slightly V-shaped centrocrista); well-developed wing-like preparacrista (including parastyle) and postmetacrista (including metastyle); well-developed stylar shelf approximately $30 \%$ of tooth width on M2; no mesostyle; M3 with wing-like preparacrista (including parastyle) and virtually no postmetacrista; lower molars increase in size posteriorly with $\mathrm{m} 1$ distinctly smaller than $\mathrm{m} 2$; trigonids wider than talonids; protoconid and metaconid subequal in size, paraconid smaller; antero- and posterocingulid weakly developed; talonid basined; $\mathrm{m} 3$ talonid elongate.

\section{Skull}

Dome-shaped skull in lateral view, with the nasals sloping anteriorly; greatest width of the skull at the level of the posterior root of the zygomatic arch, slightly anterior to the level of the anterior edge of the dentary-squamosal articulation; rostrum approximately twice as wide at base (level of the anterior edge of the orbits) as at apex; in dorsal view nasals distinctly wider posteriorly; frontal-parietal suture on sagittal plane located at mid-length of the skull; numerous foramina for temporal rami on the parietal and at the parietal-squamosal suture; small sagittal crest; bilobate nuchal crest strongly protruding posteriorly; palate narrow (less than two M2 widths); long parallel-sided basipharyngeal canal (approximately $20 \%$ of the total length of the skull); large triangular ectopterygoid process of the alisphenoid; small posterior opening of the alisphenoid canal opening just anterior to the foramen ovale; anterior end of the canal opening internally just posterior to the foramen rotundum; foramen rotundum and sphenorbital fissure separated but adjacent one to the other and opening anteriorly in the same common fossa; large ascending process of the palatine in the orbit; large descending process of the frontal posterior to the palatine process; maxilla does not contribute to the lateral wall of the skull; two large diploic frontal foramina above the orbit; double lacrimal foramen; large wing of the lacrimal on face; jugal-maxilla suture bifurcated; jugal-lacrimal contact; zygomatic arch relatively slender; postglenoid process narrow and elevated (more elevated than wide), approximately half the transverse width of the glenoid cavity; postglenoid foramen on the medial edge of postglenoid process; presence of a tubercle (postglenoid eminence) on the posteromedial edge of the foramen; relatively flat promontorium with a distinct sigmoid groove for the internal carotid artery; promontorium totally separated from the alisphenoid, basioccipital and basisphenoid; deep notch for the internal carotid artery in the posterolateral angle of the basisphenoid; apex of the promontorium truncated; epitympanic recess opened ventrally and formed by the squamosal laterally and the petrosal medially; large foramen for the ramus superior of the stapedial artery opening in the petrosal; triangular post-promontorial tympanic sinus posterior to the external aperture of the cochlear fossula; spur-like medial caudal tympanic process on posterior angle of postpromontorial tympanic sinus; conical subarcuate fossa; vestigial anterior lamina of the petrosal; presence of an intracranial cavity (the petrosquamosal fossa) for the passage of the ramus superior of the stapedial artery and its tributary vessels and, capsuloparietal emissary vein and tributaries; long and deep groove on cerebral face of the braincase for the orbitotemporal artery and vein; large mastoid exposure on occiput; large post-temporal foramen in the squamosal petrosal suture; ectotympanic horseshoe-shaped, with distinct styliform process ventrally; anterior crus of the ectotympanic with a double articulation with squamosal laterally and petrosal medially.

\section{Dentary}

Stout body with a roughly constant height; symphysis fused in adults; ramus triangular with a large masseteric fossa, coronoid process recurved posteriorly and almost as wide at apex as at base; strong and salient coronoid crest at anterior border of masseteric fossa; condyloid process well above occlusal plane; angular process extends posterior to the level of the condyle; angular process hook-like but robust and almost as long as high; anteroposteriorly oriented crest on medial face of the angular process for the insertion of the pterygoideus medialis.

\section{Postcranial skeleton}

Axis with anteroposteriorly elongated neural process; 13 thoracic vertebrae, 9 lumbar vertebrae, 4 sacral vertebrae, and approximately 18 caudal vertebrae; long neural processes of the thoracic vertebrae; scapula with a large triangular supraspinatus fossa and a narrow and deep infraspinatus fossa; spine elevated; acromion anteriorly and distally projected; humerus with greater tubercle slightly higher than the head; deltopectoral crest limited to proximal half of the bone; very robust, long, and medially projecting medial epicondyle; well-developed epicondylar ridge; medial crest of the humeral trochlea projecting more distally than the lateral; ulna bent anteriorly and medially; deep fossa for the flexor muscles on medial face of olecranon and diaphysis; trochlear notch wide open; angle between humeral and radial facets (in anterodistal view) obtuse; radius with ovalshaped proximal epiphysis; centrale present; five anterior digits with short metacarpals; no size reduction of any digit; ungual phalanges with robust palmar tubercle; innominate with a distinctly everted ilium and a ventrolaterally deflected anteroventral spine; acetabulum relatively shallow and open; femur with a spherical head; greater trochanter as high as the head; lesser trochanter triangular, blade-like, and posteromedially oriented; small third trochanter; distal epiphysis as wide as deep; tibia with a salient tibial crest and tubercle; fibula with a well-developed interosseous crest on its proximal half; tibial trochlea on the astragalus relatively flat and angle between the medial and lateral tibial facets 
TABLE 2. - Measurements of the upper and lower teeth of Alcidedorbignya inopinata (MHNC 8372 and 8399).

\begin{tabular}{|c|c|c|}
\hline Upper teeth & MHNC 8372 & MHNC 8399 \\
\hline I1 (right) mesiodistal length & 1.43 & 1.08 \\
\hline I1 (right) labiolingual width & 1.02 & 0.92 \\
\hline I2 (right) mesiodistal length & 1.44 & 1.36 \\
\hline I2 (right) labiolingual width & 1.02 & 1.13 \\
\hline I3 (right) mesiodistal length & 1.71 & 1.41 \\
\hline I3 (right) labiolingual width & 1.35 & 1.18 \\
\hline $\mathrm{C}$ (right) mesiodistal length & 3.4 & 3.75 \\
\hline C (right) labiolingual width & 3.4 & 3 \\
\hline $\mathrm{C}$ height & 6.94 (left) & 7.8 (right) \\
\hline P1 (right) mesiodistal length & 1.86 & 1.79 \\
\hline P1 (right) labiolingual width & 1.59 & 1.21 \\
\hline P2 (right) mesiodistal length & 2.21 & 1.65 \\
\hline P2 (right) labiolingual width & 1.64 & 1.16 \\
\hline P3 (right) mesiodistal length & 3.6 & 3.60 \\
\hline P3 (right) labiolingual width & 5.08 & 3.83 \\
\hline P4 (right) mesiodistal length & 4.06 & 3.60 \\
\hline P4 (right) labiolingual width & 5.93 & 5.21 \\
\hline M1 (right) mesiodistal length & 4.9 & 4.44 \\
\hline M1 (right) labiolingual width & 7.55 & 6.44 \\
\hline M2 (right) mesiodistal length & 5.3 & 4.9 \\
\hline M2 (right) labiolingual width & 8.63 & 7.55 \\
\hline M3 (right) mesiodistal length & 3.8 & 3.91 \\
\hline M3 (right) labiolingual width & 8.07 & 4.46 \\
\hline Lower teeth & MHNC 8372 & MHNC 8399 \\
\hline i1 (right) mesiodistal length & - & 0.89 \\
\hline i1 (right) labiolingual width & - & 0.82 \\
\hline i2 (right) mesiodistal length & 1.52 & 1.24 \\
\hline i2 (right) labiolingual width & 1.24 & 1.19 \\
\hline i3 (right) mesiodistal length & 1.42 & 0.97 \\
\hline i3 (right) labiolingual width & 1.19 & 0.92 \\
\hline c (right) mesiodistal length & 2.94 & 2.65 \\
\hline c (right) labiolingual width & 3.24 & 2.61 \\
\hline c (right) height & 5.34 & 5.1 \\
\hline p1 (right) mesiodistal length & 2.06 & 1.86 \\
\hline p1 (right) labiolingual width & 1.87 & 1.5 \\
\hline p2 (right) mesiodistal length & 1.76 & 1.66 \\
\hline p2 (right) labiolingual width & 1.49 & 1.29 \\
\hline p3 (right) mesiodistal length & 3.49 & 2.84 \\
\hline p3 (right) labiolingual width & 2.28 & 1.97 \\
\hline p4 (right) mesiodistal length & 4.26 & 3.50 \\
\hline p4 (right) labiolingual width & 3.12 & 2.52 \\
\hline m1 (right) mesiodistal length & 4.5 & 4.01 \\
\hline m1 (right) trigonid labiolingual width & 3.83 & 3.19 \\
\hline m1 (right) talonid labiolingual width & 3.03 & 3.01 \\
\hline m2 (right) mesiodistal length & 5.51 & 4.39 \\
\hline m2 (right) trigonid labiolingual width & 4.75 & 3.68 \\
\hline m2 (right) talonid labiolingual width & 3.69 & 3.21 \\
\hline m3 (right) mesiodistal length & 6.39 & 5.52 \\
\hline m3 (right) trigonid labiolingual width & 4.55 & 3.69 \\
\hline m3 (right) talonid labiolingual width & 3.03 & 2.93 \\
\hline
\end{tabular}

more than $120^{\circ}$; angle between lateral tibial facet and fibular facet close to $90^{\circ}$; astragalus with a distinct cuboid facet on the head; calcaneofibular facet present on the calcaneus lateral to ectal facet; well-developed peroneal process of the calcaneus; calcaneus with a strongly oblique (in dorsal view) cuboid facet, which faces distolaterally; presence of a supplementary articular facet for the astragalus on the medial edge of the cuboid facet; five pedal digits slightly longer than the manual ones, ungual phalanges cleft, with distal half expanded transversely (scutiform) in dorsal view and deflected palamarly or plantarly in lateral view, with robust plantar tubercle.

\section{DIFFERENTIAL DIAGNOSIS}

Alcidedorbignya inopinata is similar in size to Harpyodus euros and $H$. decorus but it is much smaller than all the other Pantodonta.

Alcidedorbignya inopinata differs from all the other Pantodonta in having single rooted P2 and p2, and a hook-like angular process of the dentary.

Alcidedorbignya inopinata differs from all the other Pantodonta except Bemalambda in having a distinct (although short) neck of the astragalus.

It further differs from Pantolambda bathmodon in the following characters: size approximately 60\% (not 40\% as stated by Muizon \& Marshall 1992) smaller, lack of a mesostyle on the upper molars, broader stylar shelf, posteriorly protruding bilobate nuchal crest, a horse-shoe shaped ectotympanic (not expanded medially as in Pantolambda), more gracile limb bones, ulna distinctly bent anteriorly and medially, obtuse angle between the anterior edge of the radial and trochlear notches of the ulna (acute angle in Pantolambda), much less developed and less everted anteroventral iliac spine, medial condyle of the femur similar in size to the lateral one (i.e. not projecting posteriorly), presence of a well-developed interosseous crest on the medial edge of the fibula, presence of a distinct (although short) neck of the astragalus, cleft pedal ungual phalanges (In pantolambda the anterior pedal phalanges are cleft; the posterior are not), distal half of ungual phalanges deflected palmarly or plantarly.

Alcidedorbignya inopinata further differs from Harpyodus decorus and $H$. euros in the well-separated paracone and metacone, the V-shaped centrocrista and the much thinner postcingulum, whereas in Harpyodus the cusps are connate at base, the postcingumlum is much thicker and the centrocrista is straight.

Alcidedorbignya inopinata further differs from Bemalamb$d a$ in the following characters: size approximately $75 \%$ smaller, well-separated paracone and metacone at base (not connate), lack of the anteroposterior compression of the upper molars, lack of the great enlargement of the metaconid observed in Bemalambda, much lower sagittal crest, bilobate nuchal crest, lack of a well-developed postorbital process, much lower sagittal crest, dorsoventrally lower dentary in its anterior half, condyloid process located well above the level of the occlusal plane of the molars, much longer neural process of the axis, presence of 13 thoracic, nine lumbar, and four sacral vertebrae (respectively 17T, 8L, and $3 \mathrm{~S}$ in Bemalambda), more gracile limb bones, ulna distinctly bent anteriorly and medially, presence of a much less developed and everted anteroventral iliac spine, medial condyle of the femur similar in size to the lateral (i.e. not projecting posteriorly as in Bemalambda), and presence of a well-developed interosseous crest on the medial edge of the fibula.

NB: some of the above-mentioned morphological differences between Alcidedorbignya and larger pantodonts may be related to allometric phenomenons, which yet remain to be investigated (e.g., robustness of limb bones). 


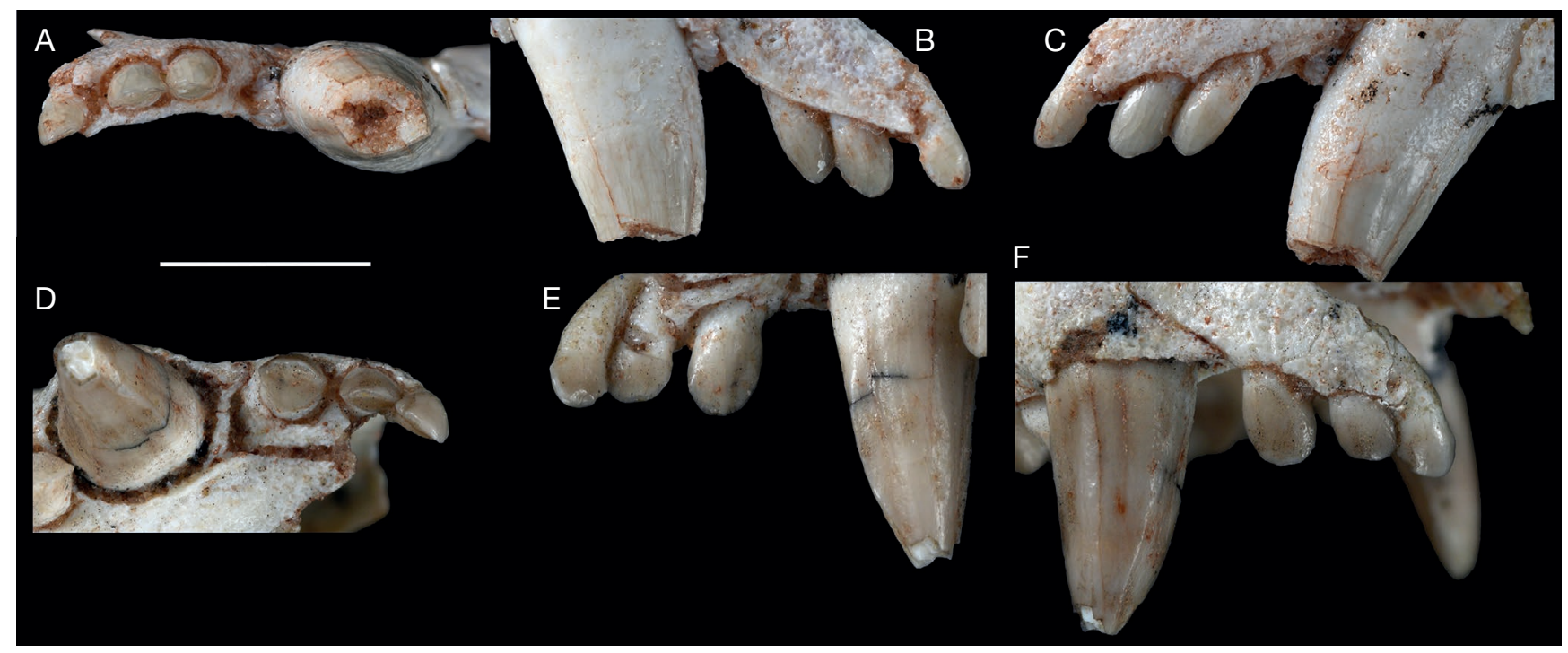

FIG. 8. - Upper incisors of Alcidedorbignya inopinata: A, MHNC 8399 (left incisors), occusal view; B, MHNC 8399 (left incisors), lingual view; C, MHNC 8399 (left incisors), labial view; D, MHNC 8372 (right incisors), occusal view; E, MHNC 8372 (right incisors), lingual view; F, MHNC 8372 (right incisors), labial view. Scale bar: 5 mm.

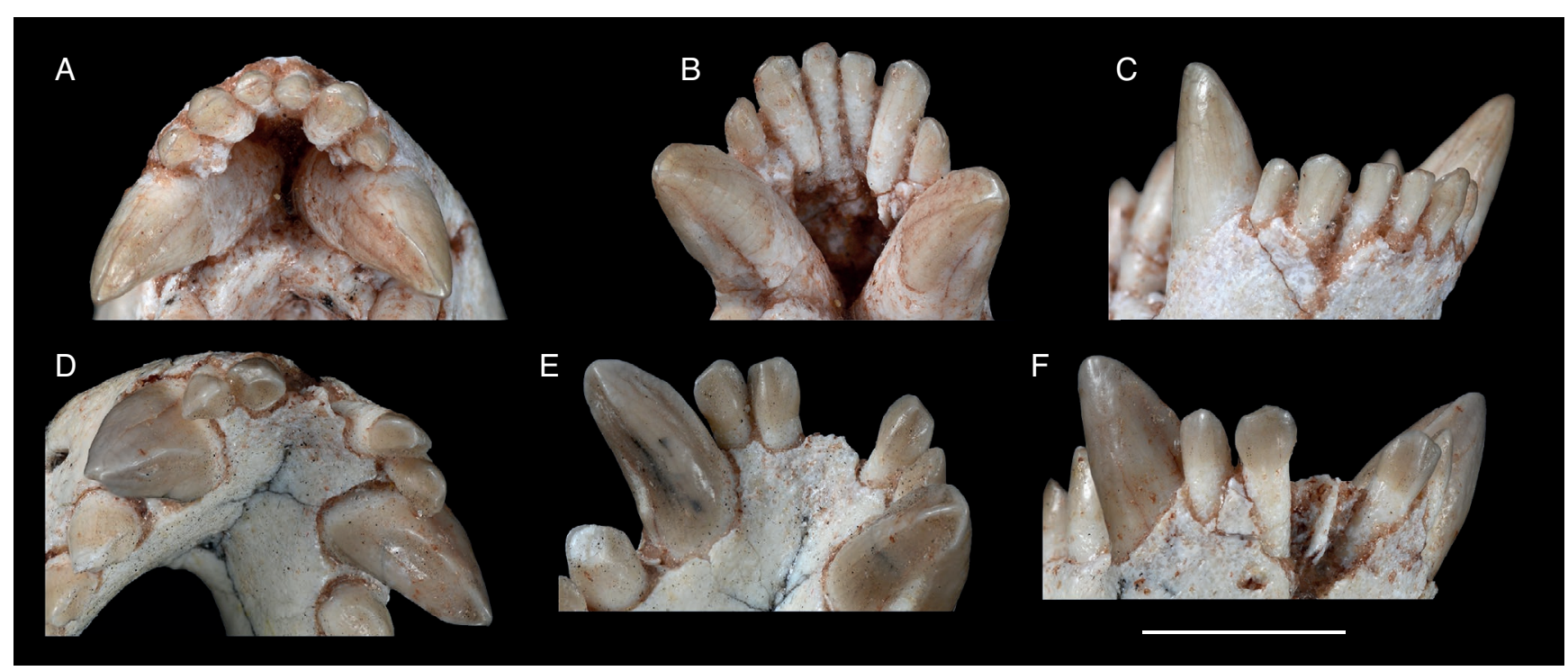

FIG. 9. - Lower incisors of Alcidedorbignya inopinata: A, MHNC 8399, occusal view; B, MHNC 8399, lingual view; C, MHNC 8399 labial view; D, MHNC 8372 occusal view; E, MHNC 8372, lingual view; F, MHNC 8372, labial view. Scale bar: 5 mm.

\section{UNAMBIGUOUS APOMORPHIES}

OF THE TAXON ALCIDEDORBIGNYA

These apomorphies are based on our cladistic analysis (unconstrained; see Phylogenetic Analysis section). None of them is unique to Alcidedorbignya but they show homoplastic evolution with some other placentals in our sample. They are: ultimate premolar precingulum present (character 47 , state 0 ), prominent paraconule mid-way or closer to paracone on upper molars (character 98, state 2), trigonid moderately long $<75 \%$ relative to talonid and $>50 \%$ tooth length (character 126, state 1 ), slightly obtuse or right angle between anterior border of coronoid process of mandible and horizontal alveolar border of cheek teeth (character 143, state 2), anterior extension of masseteric fossa on the body of the dentary, below the last molars (character 146, state 1), angular process of mandible hook-like with width of base subequal to (or wider than) length (character 152, state 1), nasal foramina present (character 179, state 0 ), large external wing of the facial process of the lacrimal on the rostrum at the anterior edge of the orbit (character 184, state 0 ), double lacrimal foramen (character 187 , state 0 ), posterior border of hypophyseal fossa (located just anterior to basisphenoid-basioccipital suture) anterior to or roughly even with to posterior edge of foramen ovale (character 263 , state 0 ), ringlike extotympanic (character 336, state 0 ), 13 or fewer thoracic vertebrae (character 365 , state 0 ), greater trochanter higher than femoral head (character 392, state 2), longest axis of ectal facet of calcaneum oriented anteromedial to posterolateral (character 416, state 0 ), cuboid facet of calcaneum much deeper (dorsoplantar) than wide (mediolateral) (character 425, state 0). 


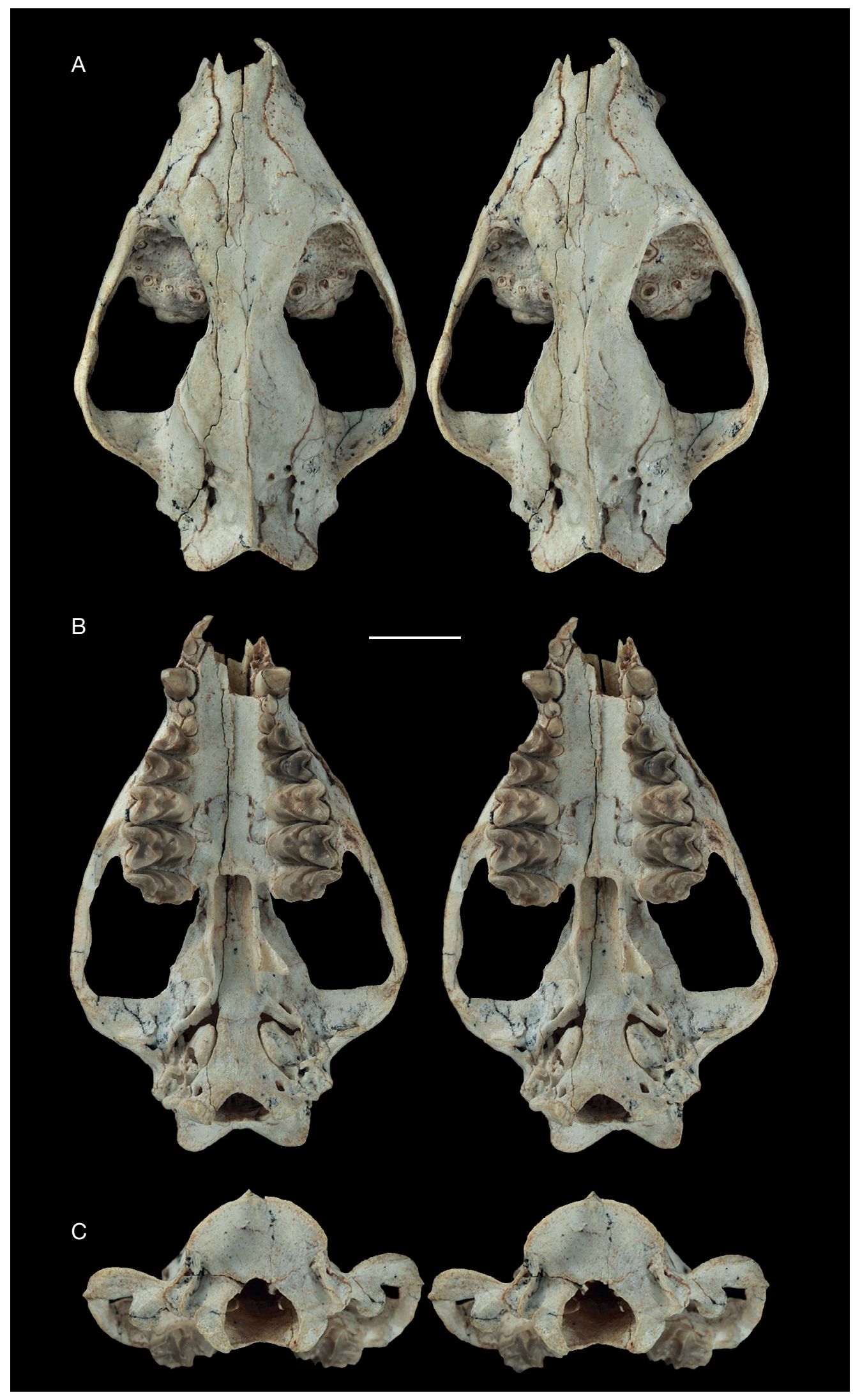

FIG. 10. - Stereophotographs of the skull of Alcidedorbignya inopinata (MHNC 8372): A, dorsal view; B, ventral view; C, occipital view. Scale bar: $1 \mathrm{~cm}$. 


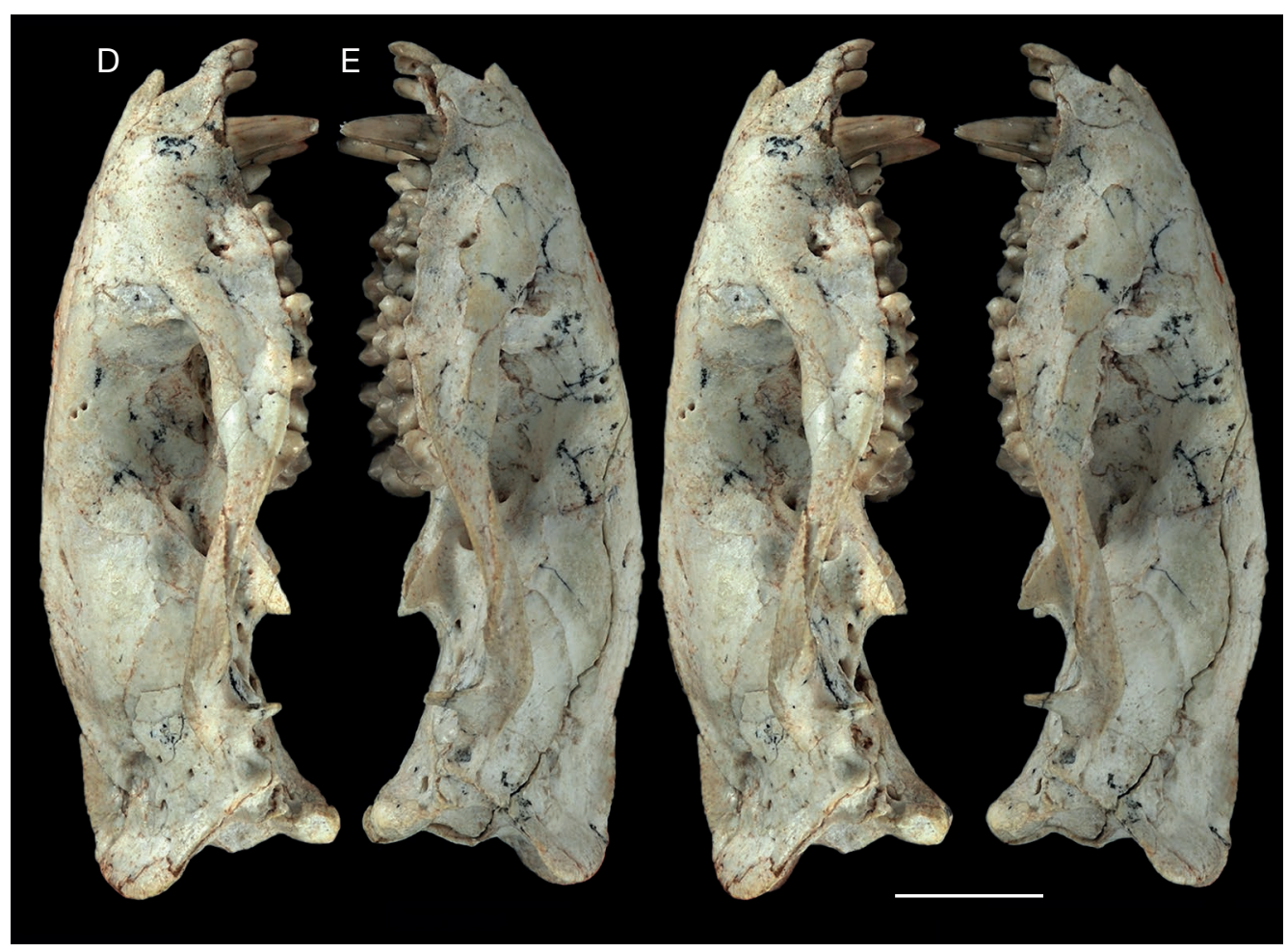

FIG. 10 (continuation). - Stereophotographs of the skull of Alcidedorbignya inopinata (MHNC 8372): D, right lateral view; $\mathbf{E}$, left lateral view. Scale bar: $1 \mathrm{~cm}$.

\section{COMPARATIVE DESCRIPTION}

\section{SKULL}

\section{Dentition}

As mentioned above, the dental morphology of Alcidedorbignya has been thoroughly described and discussed by Muizon \& Marshall (1992) and the new specimens described here add little to the knowledge of the dental anatomy of the Bolivian pantodont. Therefore most of the dental anatomy will not be considered here. However, measurements of the teeth of the two specimens are given below (Table 2). Furthermore, some data on the incisors, well-preserved on the two specimens MHNC 8372 and 8399, allow us to complete the previous description. Muizon \& Marshall (1992) described one partial premaxilla bearing a broken I2. The two adult specimens bear the three right upper incisors (Fig. 8). The teeth increase in size from I1 to I3 on MHNC 8372 but on MHNC 8299 I2 and $\mathrm{I} 3$ are subequal in size. They have a low crown, which is often as wide as high. They are spatulate and present a sharp crest, which forms the edge of the crown apically from its mesial to its distal base and which divides it into a lingual and a labial face. The lingual edge of I1 and I 2 is slightly concave on MHNC 8399 and slightly convex on MHNC 8372. The labial face is strongly convex. On I3, the lingual side of the crown bears a vertical thick rounded ridge, which extends from the base of the crown to its apex.

The lower incisors have been described by Muizon \& Marshall (1992) based on specimen MHNC 1229, which bears heavily worn teeth. Unworn incisors are preserved on MHNC 8372 (i2s and i3s) and on MHNC 8399 (all six lower incisors). The teeth are roughly similar to the upper incisors, being spatulate and bearing a sharp apical crest from the mesial to the distal edge (Fig. 9). The major difference from the upper incisors is their relative size. The i1 is the smallest and the $\mathrm{i} 2$ is the largest of the three incisors, i3 being distinctly smaller than i2. No i1 is preserved on MHNC 8372 but both are present on MHNC 8399. These teeth have a relatively flat lingual face and a distinctly convex labial side. The i 2 and i 3 bear a thick and rounded lingual ridge, which extends form the tip of the crown to its base. The six incisors are tightly appressed against each other and they are not separated by diastemata.

\section{Bony skull}

General features. Of the two adult skulls of Alcidedorbignya that have been recovered, one is remarkably complete (MHNC 8372; Figs 10-12) and is almost undistorted with well-preserved proportions. The other skull (MHNC 8399) is incomplete but allows some measurements (Fig. 13). The skull of Alcidedorbignya is very small for a pantodont, which are generally mediumsized to large mammals. It is approximately $38 \%$ as long and $36 \%$ as wide as the skull of Pantolambda, the smallest North American pantodont. The skull of Alcidedorbignya is roughly the same length as that of Harpyodus decorus Wang, 1979 (early Palaeocene of China), but its proportions are more massive and it is relatively much wider. Although the anterior tip of the premaxillae of MHNC 8372 is missing, the right I1 is preserved and provides a good approximation of the total length of the skull. The bizygomatic width/maximum length ratio of the skull MHNC 8372 of Alcidedorbignya is $37.53 / 55.3 \mathrm{~mm}=0.678$. 

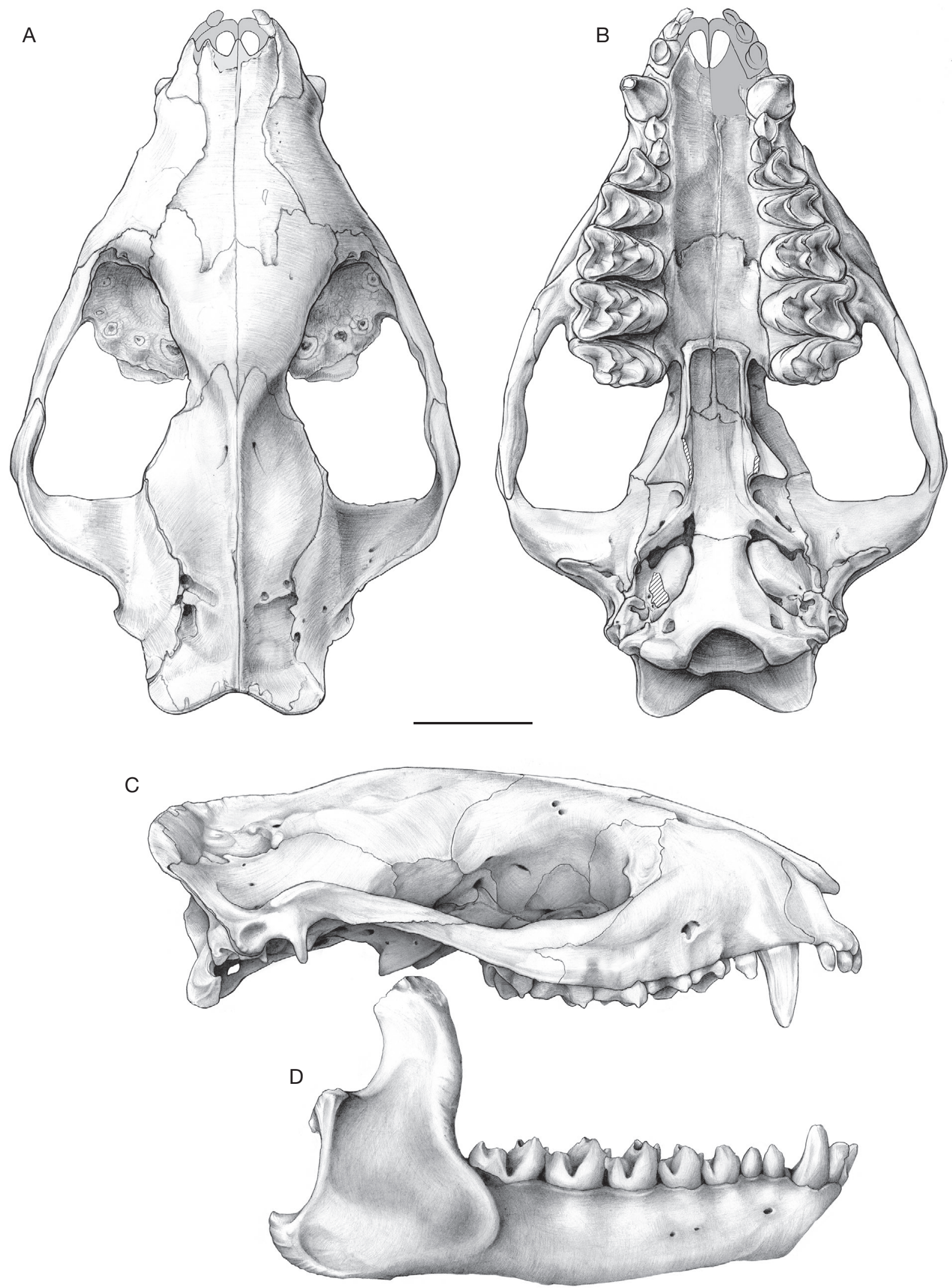

FIG. 11. - Pencil drawings of the skull and mandible of Alcidedorbignya inopinata (MHNC 8372) emphasizing the suture lines. Part of the right premaxilla, left premaxilla and left upper incisors have been reconstructed: A, dorsal view; B, ventral view; C, skull in lateral view; $\mathbf{D}$, mandible in lateral view. Scale bar: $1 \mathrm{~cm}$. 


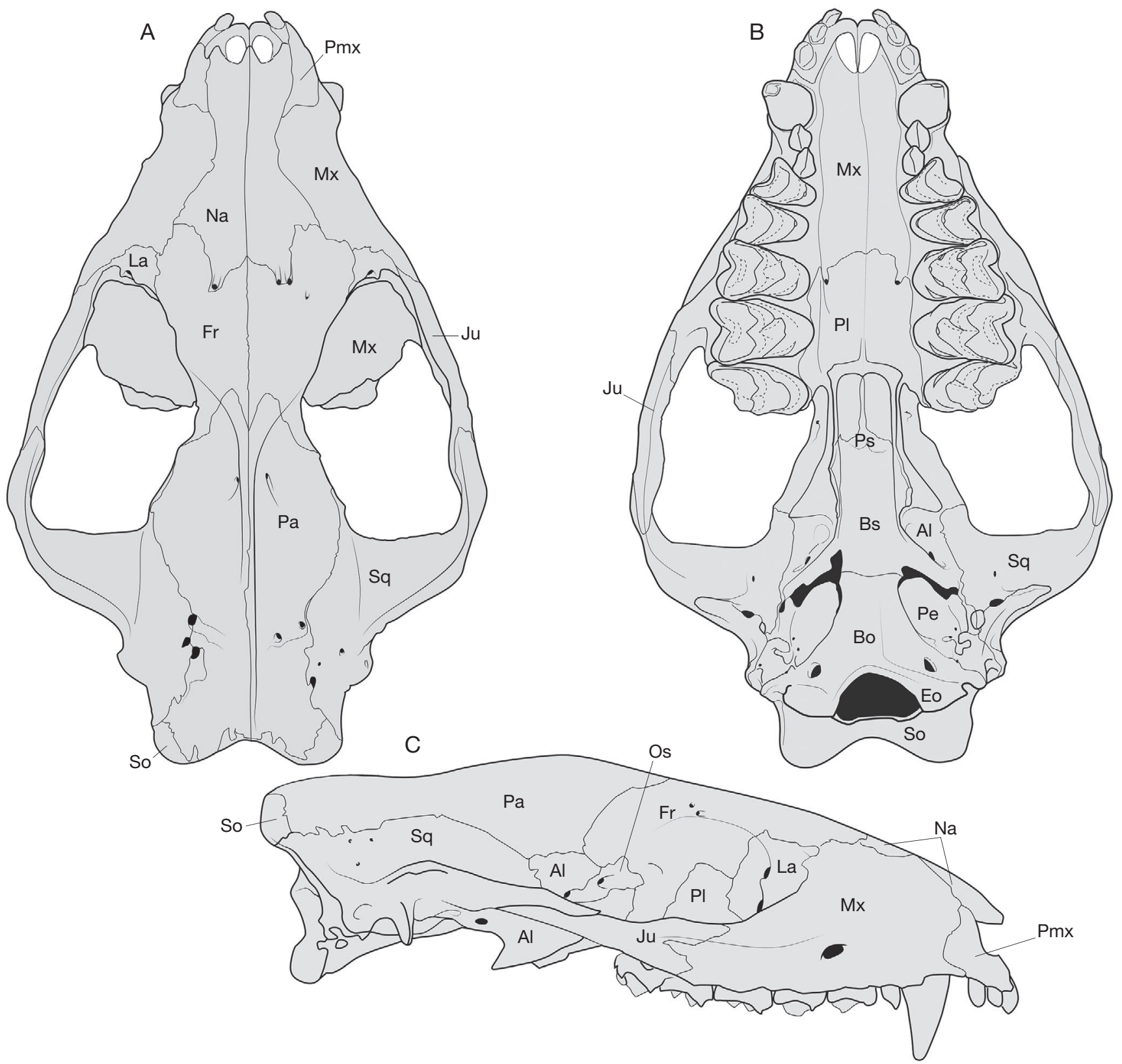

FIG. 12. - Diagram of the skull of Alcidedorbignya inopinata (MNHN 8372) with bone labels. Abbreviations: Al, alisphenoid; Bo, basioccipital; Bs, basisphenoid; Eo, exoccipital; Fr, frontal; Ju, jugal; La, lacrimal; Mx, maxilla; Na, nasal; Os, orbitosphenoid; Pa, parietal; Pe, petrosal; PI, palatine; Pmx, premaxilla; Ps, presphenoid; So, supraoccipital; Sq, squamosal.

The second skull (MHNC 8399) is apparently not (or very little) distorted and complete enough to provide a reasonable idea of its proportions. Because the left zygomatic process of the squamosal is missing, its bizygomatic width has been estimated by doubling the width of the right half of the skull. The estimated bizygomatic width is therefore $18.25 \mathrm{~mm} \times 2=36.5 \mathrm{e} \mathrm{mm}$; the length of the skull is $59.75 \mathrm{~mm}$. The ratio is slightly lower than that of MHNC 8372 being 36.5/59.75 = 0.610. For Harpyodus decorus, measurements were taken from photographs (provided by S. Lucas) of the skull figured by Wang (1979), which is apparently undistorted (or little distorted). The length of the skull of $H$. decorus has been estimated because the premaxillae of the holotype are not completely preserved. A rough estimate of the skull length could approach $62 \mathrm{~mm}$ and the width is $22 \mathrm{~mm}$. These measurements provide a much smaller width/length ratio of c. 0.35 . The ratio of Alcidedorbignya is 94\% (for MHNC 8372) and $74 \%$ (for MHNC 8399) greater than that of H. decorus, a difference, which is regarded as significant in spite of the approximation made for the Chinese species. Measurements for Pantolambda bathmodon are also estimated because none of the skulls known are complete or undistorted. The skull of AMHN 16663 is not distorted transversely but has suffered some anteroposterior compression. However, this compression can be estimated from the posterior displacement of the posterior end of the jugal relatively to its articulation with the squamosal, which is close to $17 \mathrm{~mm}$. The actual condylobasal length of AMNH 
TABLE 3. - Skull proportions of early pantodonts.

\begin{tabular}{lccc}
\hline Specimen & $\begin{array}{c}\text { Max width } \\
\text { of skull }\end{array}$ & $\begin{array}{c}\text { Max. length } \\
\text { of skull }\end{array}$ & $\begin{array}{c}\text { W/L } \\
\text { ratio }\end{array}$ \\
\hline $\begin{array}{c}\text { Alcidedorbignya inopinata } \\
\text { (MHNC 8372) }\end{array}$ & $37.53 \mathrm{~mm}$ & $55.3 \mathrm{~mm}$ & 0.678 \\
$\begin{array}{c}\text { Alcidedorbignya inopinata } \\
\text { (MHNC 8399) }\end{array}$ & 59.75 & $18.25 \times 2=36.5 \mathrm{e}$ & 0.610 \\
$\begin{array}{c}\text { Bemalambda nanhsiungensis } \\
\text { (IVPP-V 4118) }\end{array}$ & $145 \mathrm{e}$ & $211 \mathrm{e}$ & 0.68 \\
$\begin{array}{c}\text { Harpyodus decorus IVPP-V } \\
\text { (5035) }\end{array}$ & 22 & $62 \mathrm{e}$ & 0.35 \\
$\begin{array}{l}\text { Pantolambda bathmodon } \\
\text { (AMNH 16663) }\end{array}$ & $105 \mathrm{e}$ & $157 \mathrm{e}$ & 0.67 \\
\hline
\end{tabular}

TABLE 4. - Relative length of the rostrum in some pantodonts, tillodonts and the "condylarth" Maiorana. Length in $\mathrm{mm}$.

\begin{tabular}{|c|c|c|c|}
\hline Taxon & $\begin{array}{l}\text { Length } \\
\text { Rostrum }\end{array}$ & $\begin{array}{l}\text { Length } \\
\text { Skull }\end{array}$ & LRo/LSk \\
\hline $\begin{array}{l}\text { Alcidedorbignya inopinata } \\
\text { (MHNC 8372) }\end{array}$ & 22.1 & 55.3 & 0.399 \\
\hline $\begin{array}{l}\text { Alcidedorbignya inopinata } \\
\text { (MHNC 8399) }\end{array}$ & 22.68 & 59.75 & 0.379 \\
\hline $\begin{array}{l}\text { Pantolambda bathmodon } \\
\text { (AMNH16663) }\end{array}$ & 51 & 157 & 0.32 \\
\hline $\begin{array}{l}\text { Pantolambda bathmodon } \\
\text { (AMNH 16665) }\end{array}$ & 56 & 171.2 & 0.327 \\
\hline $\begin{array}{l}\text { Bemalambda nanhsiungensis } \\
\text { (IVPP-V- 4118) }\end{array}$ & $67.8 \mathrm{e}$ & $211 e$ & 0.34 \\
\hline $\begin{array}{l}\text { Haplolambda quinni } \\
\text { (FMNH 15542) }\end{array}$ & 96 & $280 \mathrm{e}$ & 0.355 \\
\hline $\begin{array}{l}\text { Caenolambda pattersoni } \\
\text { (USNM 21036) }\end{array}$ & $110 \mathrm{e}$ & 326 & 0.337 \\
\hline $\begin{array}{l}\text { Barylambda faberi } \\
\text { (FM P 14944) }\end{array}$ & $97.5 \mathrm{e}$ & $315 e$ & 0.309 \\
\hline $\begin{array}{l}\text { Titanoides primaevus } \\
\text { (FM P 15520) }\end{array}$ & 127.5 & 377.5 & 0.337 \\
\hline $\begin{array}{l}\text { Coryphodon elephantopus } \\
\text { (USNM 111) }\end{array}$ & 165 & 426 & 0.388 \\
\hline Coryphodon testis (AMNH 2867) & 187 & 506 & 0.37 \\
\hline Azygonyx ancylion (UM 68511) & $55 \mathrm{e}$ & $150 \mathrm{e}$ & 0.36 \\
\hline $\begin{array}{l}\text { Maiorana noctiluca } \\
\text { (YPM-PU 14111) }\end{array}$ & $26.4 \mathrm{e}$ & $61.8 \mathrm{e}$ & 0.42 \\
\hline
\end{tabular}

16663 as preserved is $140 \mathrm{~mm}$ and therefore the estimated total length of the skull is $140+17=157 \mathrm{~mm}$. The bizygomatic width of the skull is $105 \mathrm{~mm}$ and therefore the width-length ratio is $105 / 157=c .0 .67$. In spite of the approximation it is clear that the skull proportions of Alcidedorbignya are extremely close to those of Pantolambda and very different from those of Harpyodus. We did not have access to the specimens of Bemalambda from the early Palaeocene of Nanxiong Basin, Guangdong, China. However, some measurement of Bemalambda nanhsiungensis (IVPP-V 4118) could be taken from Zhou et al. (1977: pl. X, fig. 1). Although we are conscious that these measurements are approximate (the photograph of the specimen is reduced by $2 / 3$ ), they should not (or little) affect the W/L ratio. With estimated length of $211 \mathrm{~mm}$ and width of $145 \mathrm{~mm}$ and a ratio of 0.68 , although much larger, Bemalambda shows skull proportions quite similar to those of Alcidedorbignya (although the Chinese taxon is much larger). Skull size and proportions of Bemalambda are almost identical to those of Pantolambda (Table 3).
In Alcidedorbignya, the widest part of the skull is slightly anterior to the glenoid cavity. From this point, the skull tapers forward. In other words, from this point, the edges of the skull are almost straight and converge anteriorly, contrary to the condition in Pantolambda, in which the lateral edges of the snout at its base are strongly concave between the canine and the anterior foramen of the infraorbital canal. This is due, in part, to the inflation of the ascending process of the maxilla (see below, description of the nasal cavity) of Alcidedorbignya, while this region is distinctly concave in Pantolambda. The skull of MHNC 8372 is $55.3 \mathrm{~mm}$ long from the occipital condyle to the anterior edge of the base of the crown of I1, which is regarded as a point very close to the anterior border of the premaxilla. The length of the snout from the anterior edge of the orbit to the anterior base of the crown of I1 is $22.1 \mathrm{~mm}$. In Pantolambda (AMNH 16663), the snout is $51 \mathrm{~mm}$ long and the estimated length of the skull from condyles to anterior tip of premaxillae is $157 \mathrm{~mm}$ (see above). In this specimen (AMHN 16663), the skull has suffered some anteroposterior compression, which can be evaluated to approximately $17 \mathrm{~mm}$, as noted above. The snout/skull length ratio of Alcidedorbignya (MHNC 8372) is 0.398 and that of Pantolambda is 0.32 . Therefore, the rostrum of the Alcidedorbignya skulls described here is c. 23\% (MHNC 8372) and c. 17\% (MHNC 8399) relatively longer than that of Pantolambda bathmodon (AMHN 16663) (see Table 4). In spite of the variation observed between the two skulls of Alcidedorbignya, it appears that this genus has a relatively long rostrum by pantodont standards.

The snout of Alcidedorbignya tapers anteriorly as in Pantolambda and Bemalambda. In other pantodonts (e.g., Titanoides, Haplolambda, Caenolambda, Barylambda) the muzzle slightly flares anteriorly. This condition indicates a relatively much smaller nasal cavity in Alcidedorbignya (but see below description of the internal aspect of the maxilla in the nasal cavity).

The orbitotemporal fossa is relatively short as in Pantol$a m b d a$, thus differing from the condition in Haplolambda, Caenolambda, and Titanoides.

The dorsal surface of the skull of Alcidedorbignya is regularly convex from the apex of the premaxilla to the occipital crest, being slightly domed at the level of the frontals. Among pantodonts, this condition is also present in Pantolambda (although not so pronounced in this genus). Alcidedorbignya differs from the other pantodonts, in which the dorsal surface of the skull is relatively flat, especially in the nasofrontal region.

The zygomatic arches are not regularly convex in dorsal view but are somewhat angled. They have a long anterior portion, which is sub-rectilinear and strongly oblique with an orientation closer to anteroposterior than to transverse. The short posterior portion is approximately half the length of the anterior one and its orientation is closer to transverse than to anteroposterior. The junction of the two portions is a rounded angle located at the anterior rectilinear edge of the glenoid fossa. An angled zygomatic arch is apparently absent in Harpyodus and Bemalambda but is present in Haplolambda and to a lesser extent in Pantolambda. The angle of the arch is more distinctly posterior in Alcidedorbignya than in the other pantodonts. 


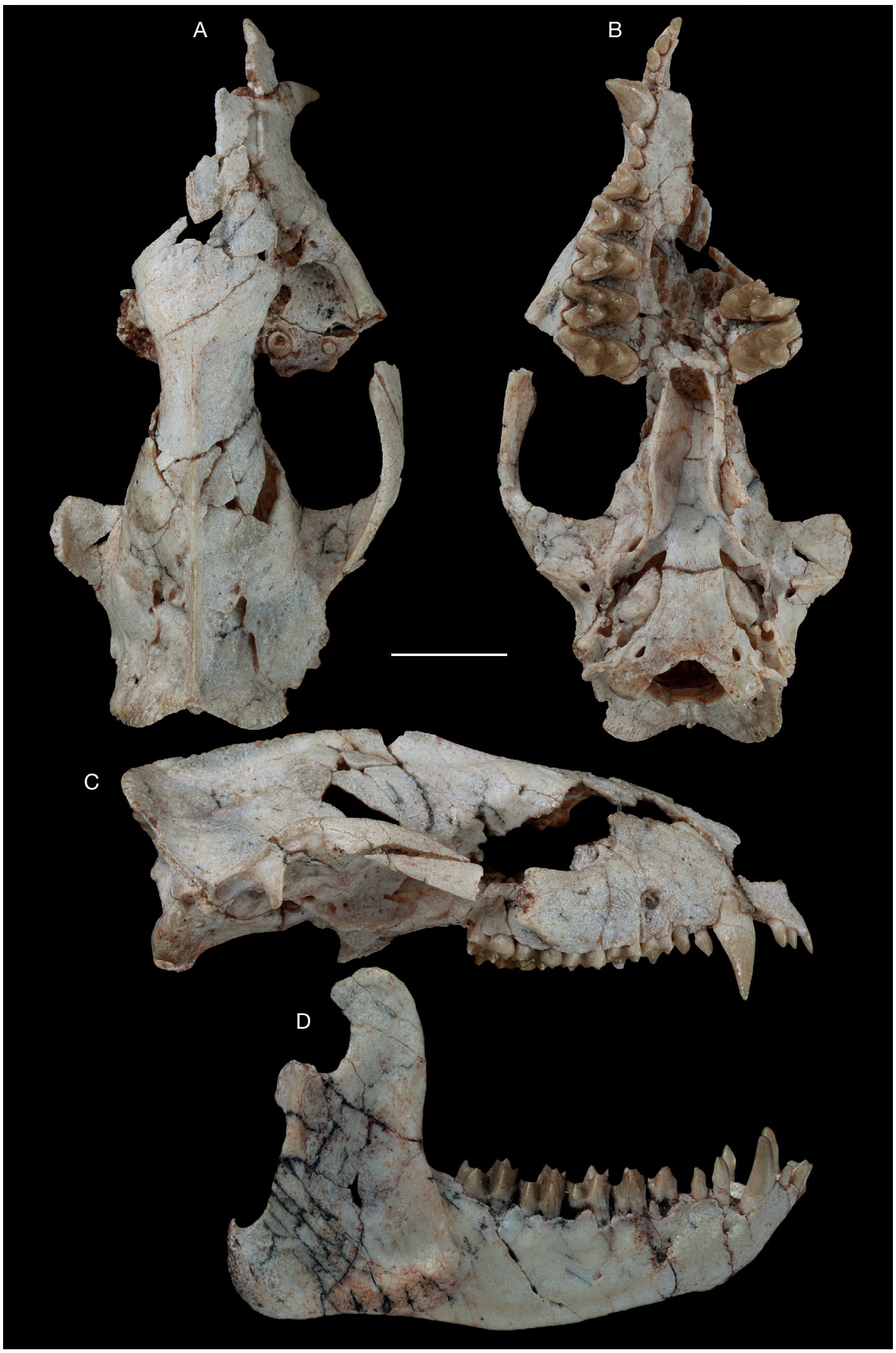

FIG. 13. - Partial skull of Alcidedorbignya inopinata (MHNC 8399): A, dorsal view; B, ventral view; C, right lateral view; $\mathbf{D}$, right lateral view of the mandible. Scale bar: $1 \mathrm{~cm}$. 


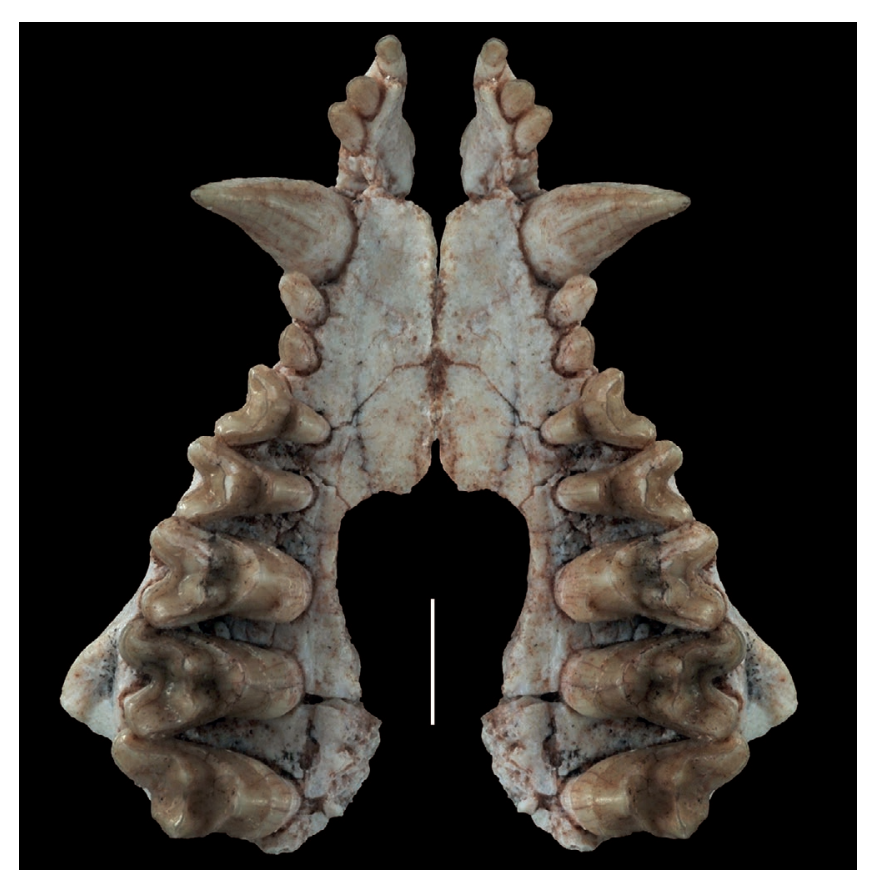

FIG. 14. - Photo montage of the anterior part of the palate of Alcidedorbignya inopinata: MHNC 8399 with the left maxilla and premaxilla reconstructed with the mirror image of the right ones (as preserved). This montage shows that the upper incisors and premaxillae of Alcidedorbignya inopinata did not contact anteromedially and were widely separated. Scale bar: $1 \mathrm{~cm}$.

Premaxilla. As a whole, the premaxilla of Alcidedorbignya is more slender than in Pantolambda. In lateral view, the premaxilla has an anteroposteriorly large, but dorsoventrally short posterodorsal process (Marshall \& Muizon 1995; Muizon 1998). It articulates with the nasal and the maxilla. In lateral view the suture with the maxilla starts ventrally just posterior to the posterior edge of I3. In this section it is slightly oblique. It ascends posteriorly but remains close to the alveolar edge of the canine for half its length. Then, it forms a wide curve and runs dorsally towards the nasal. The posterodorsal process narrows at its apex and is wedged between the maxilla and the nasal, being posterodorsally oriented. The dorsal edge of the posterodorsal process is almost straight along the nasal and anterodorsally concave on the lateral border of the nasal aperture. At the anterior end of the Na-Pmx suture the dorsal edge of the premaxilla exhibits a small angulation $\left(c .170^{\circ}\right)$. This angle is approximately $120^{\circ}$ in Pantolambda and Caenolambda. This condition is an indication that the premaxillae of Alcidedorbignya are more projected anteriorly than in the two other genera. Furthermore, the upper incisors of Alcidedorbignya are slightly but distinctly procumbent, while they are sub-vertical in Pantolambda. The alveolar border of the premaxilla slopes anteroventrally in lateral view, when the occlusal plane of the cheek teeth is horizontal. Because of this condition the apex of I1 is more ventral than that of I 2 and the latter is more ventral than that of I3. The wide contact of the premaxillae with the nasals, observed in Alcidedorbignya and Pantolambda, is also present in Caenolambda. In most other pantodonts the premaxillae have no contact with the nasals. The condition of Harpyodus decorus is unknown, as the premaxilla is not preserved on the only skull known for this species.
The posterodorsal process of the premaxilla does not extend far posteriorly between the nasal and the maxilla. Its posterior apex is dorsal to the anterior edge of the canine. A similar condition is observed in Pantolambda and in all North American pantodonts.

The anterior part of the premaxilla, which bears the incisors, is incomplete, and the portion of bone that covers the anterior edge of I1 is missing on MHNC 8372. On MHNC 8399 the anterior portion of both premaxillae is preserved and the alveoli of the I1 are almost complete. Because this specimen is partly damaged, the left premaxilla, incisors, canine and part of the maxilla form a fragment that has no contact with the rest of the skull. Although the rostrum and palate of this specimen are not completely preserved it is possible to reconstruct this part of the skull using symmetry, because the median suture of the right maxilla is preserved (Fig. 13). According to this reconstruction, as illustrated on Figures 11 and 12, it is likely that the anterior tips of the premaxillae were not in contact and the I1s were widely separated. This condition is also shown on the photo montage presented on Figure 14, with the mirror image of the right maxilla and premaxilla of MHNC 8399. Therefore, the anterior end of the palate, between the incisors probably presented a V-shaped notch as is observed, for instance, in some species of the hedgehog Erinaceus (CM and GB pers. obs.). On the single specimen of Pantolambda preserving the upper incisors, the premaxillae distinctly extend further medially than the alveolar border of the I1 and contact each other medially at their anteriormost apex, without any marked notch, contrary to the probable condition of Alcidedorbignya. However, the I1s of Pantolambda are widely separated medially as in Alcidedorbignya (Fig. 15). This is also the case in later diverging pantodonts such as Titanoides (Simons, 1960) and Coryphodon (CM pers. obs.).

In ventral view, the horizontal portion of the premaxilla that bears the incisors is long in ventral view and low (in lateral view) relative to the condition in Pantolambda. It becomes lower anteriorly and is very slender. This anterior narrowing is concomitant with the presence of a distinct angle on the anterodorsal edge of this portion, defining the lateral border of the nasal aperture. Such an angle is absent on the premaxillary of Pantolambda and other pantodonts.

On the palate of MHNC 8372, very little of the premaxilla is preserved and on the right side only. The alveolus of I3 is complete. At the level of this alveolus, the premaxilla is very narrow and does not contribute to the palate. The palatal process of the premaxilla (i.e. the portion of the premaxilla posterior to I3) is short and is closely appressed against the thin anterior border of the alveolus of the canine, a condition regarded as a metatherian synapomorphy by Rougier et al. (1998) and Wible et al. (2009). No specimen entirely preserves the incisive foramina, which usually are present in the premaxillae or between the premaxillae and maxillae (but see below in the maxillary section).

Nasal. The nasals are anteroposteriorly elongated and wide posteriorly. They project anteriorly overhanging the nasal aperture, and posteriorly they reach the level of the anterior edge of the orbitotemporal fossa. The nasals are, however, much shorter than 


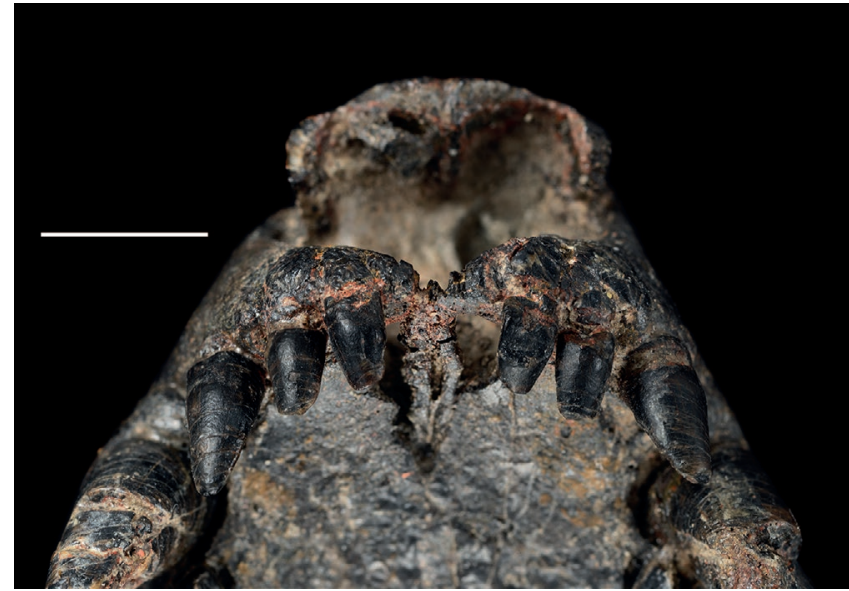

FIG. 15. - Anterior part of the palate of Pantolambda bathmodon (AMHN 16663), showing the widely separated I1s. Scale bar: $1 \mathrm{~cm}$.

in most other pantodonts, in which they consistently extend more posteriorly than the anterior edge of the orbitotemporal fossa. They articulate with the maxillae laterally, with the premaxillae anterolaterally, and with the frontals posteriorly. The suture with the maxilla is strongly concave laterally in its anterior half. In other words, in dorsal view, the nasals are distinctly wider anteriorly than in their median portion. The nasals are distinctly waisted being much wider posteriorly than anteriorly. They are almost twice as wide posteriorly as in the median region. At the anterior end of the nasal the lateral angle of the bone projects anteriorly and the medial angle is well posterior to it. However on MHNC 8372, the unique adult specimen preserving this structure, a part of the medial angle of the bone is probably missing. On MHNC 8416, a skull of a juvenile specimen, the anterior end of the left nasal is completely preserved. The nasal is deeply $\mathrm{V}$-shaped anteriorly and the lateral branch of the $\mathrm{V}$ is slightly longer than the medial one (Fig. 16). Therefore, the anterior border of both nasals in this juvenile specimen, which together form the dorsal roof of the nasal aperture, is markedly W-shaped with the anteriormost point of the inter-nasal suture being slightly posterior to the lateral angle of the nasals. This condition differs from that of adult specimens of Pantolambda and Haplolambda, in which the notch in each nasal is not as deep as in the juvenile specimen of Alcidedorbignya and in which the medial angle of the nasals is anterior to the lateral angle and strongly protrudes anteriorly. Therefore, in these genera, the anterior edge of each nasal is roughly convex anteriorly.

The nasals are the narrowest just above a point of the maxilla between P1 and P2. Posterior to this point, the nasal-maxilla suture diverges posterolaterally and, when reaching the frontal, abruptly takes a transverse medial direction and then forms a large curve, which places it again in an anteroposterior direction. In other words, the posterior third of the nasal presents a salient lateral process, the anterior border of which is convex and the posterior border concave. The posterior processes of the nasals are narrower than the lateral processes and are separated in the sagittal plane by the small anterior processes of the frontals, wedged between them. In other words the

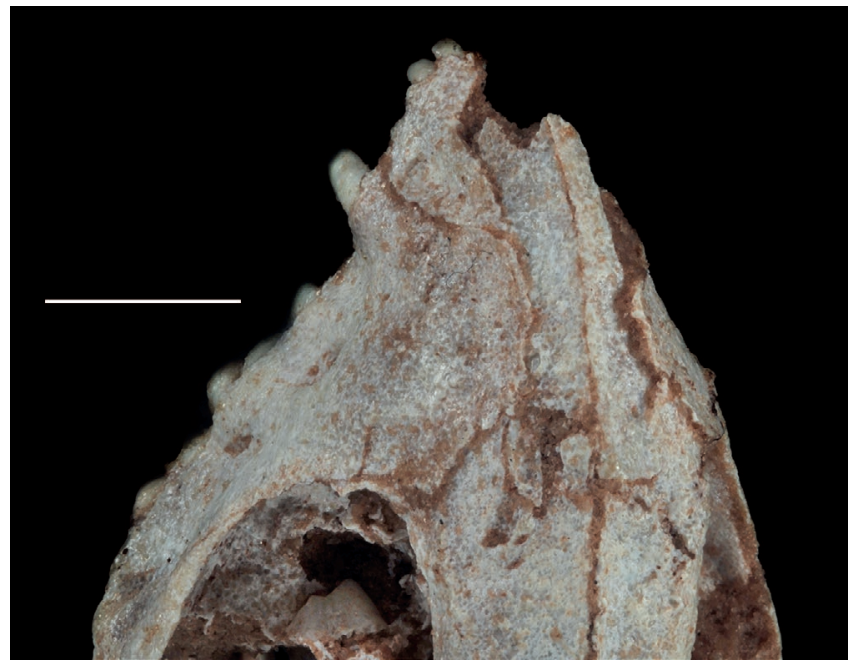

FIG. 16. - Dorsal view of the skull of a juvenile individual of Alcidedorbignya inopinata (MHNC 8416), which preserves the anterior tip of the left nasal. Scale bar: $5 \mathrm{~mm}$.

fronto-nasal suture forms an $\mathrm{M}$ in dorsal view, with the rostrum pointed downward. The lateral process of the nasal is present but smaller in Pantolambda and Titanoides but it is absent in Haplolambda and Caenolambda. The small anterior process of the frontal observed in Alcidedorbignya is absent in Pantolambda and Haplolambda.

The nasal of Alcidedorbignya does not contact the lacrimal although the bones are approximated. The nasals of Alcidedorbignya are much wider posteriorly than in any other pantodonts, in which they tend to be more parallel sided (excepted in Pantolambda and Titanoides).

A well-developed nasal foramen is present on the right side of MHNC 8372 at the level of the posterior flaring of the bone and anteroposteriorly aligned with the anterior opening of the infraorbital canal. The left nasal only bears two much smaller foramina in the flared posterior portion of the bone.

Maxilla. The maxilla occupies most of the lateral side of the snout. Its vertical wall is strongly convex and inflated between the anterior extremity of the zygomatic arch and the posterodorsal process of the premaxilla. On both specimens (MHNC 8372 and 8399), the medial side of the bone (within the the nasal cavity) presents a distinct fossa at the level of the inflation observed on the lateral face (external to the nasal cavity). This fossa is regarded here as a maxillary sinus, which is described below with the nasal cavity). In the other pantodonts, the lateral edge of the snout is generally either concave or flat. In Alcidedorbignya, this lateral inflation probably compensated for the smaller size of the nasal cavity due to the anterior tapering of the snout when compared to other pantodonts. Ventral to this inflation and dorsal to the alveolar border of P1-2, the maxilla is deeply excavated, probably because of the extreme reduction of these premolars, especially P2, which is single-rooted and single-cusped in Alcidedorbignya. Such a fossa is absent in Pantolambda in which P2 has two roots, and bears a large paracone with parastyle, metastyle, and a small protocone. 


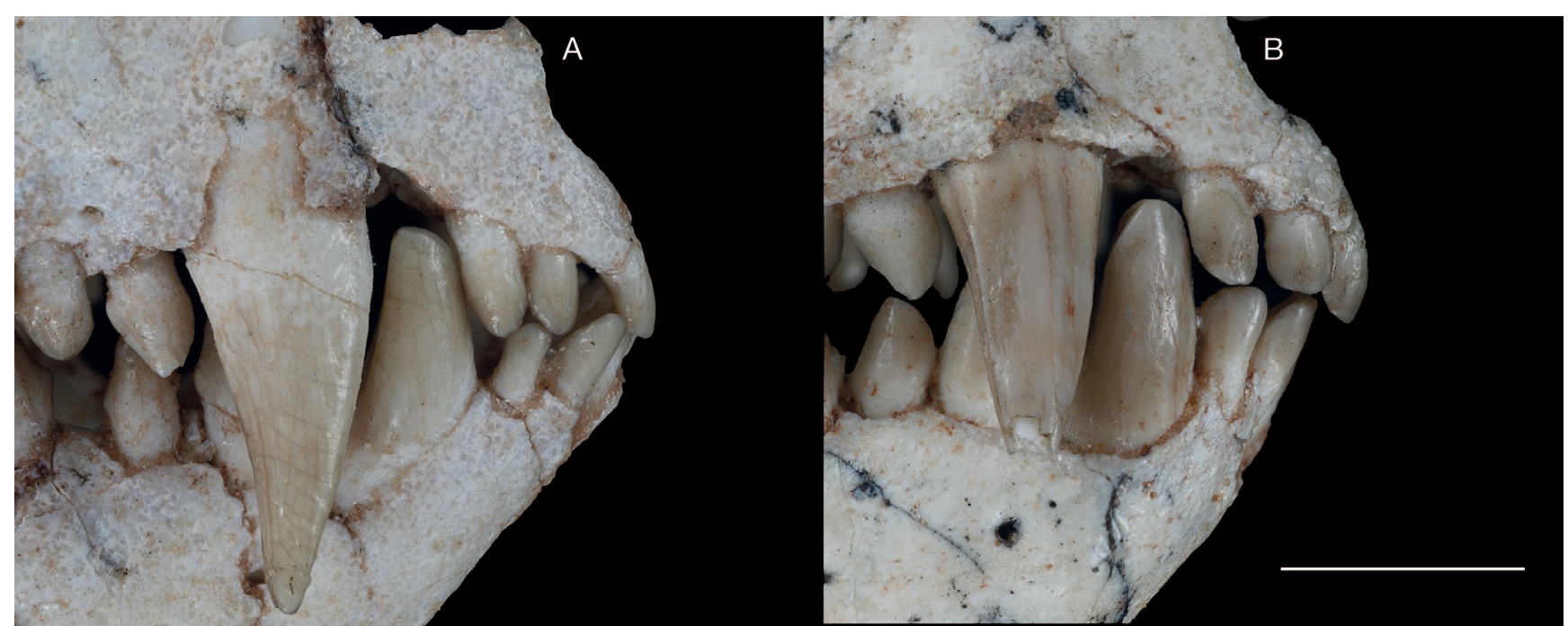

FIG. 17. - Lateral view of the snout of Alcidedorbignya inopinata showing the occlusion of the upper and lower canines: A, MHNC 8399; B, MHNC 8372. Scale bar: $5 \mathrm{~mm}$.

The anterior suture of the maxilla with the premaxilla is strongly sigmoid, more so than in Pantolambda. At the ventral end of the lateral premaxilla-maxilla suture, the maxilla has a small anteriorly projecting process, which abuts against the posterior edge of the alveolus of $\mathrm{I} 3$ and forms the anterolateral alveolar border of the canine. Because of this process a small diastema is present between $\mathrm{I} 3$ and $\mathrm{C}$. The lower canine perfectly fits in this space and does not project dorsolaterally above the alveolar border of the maxilla. In contrast, during occlusion, the upper canine extends ventrally below the alveolar border of the dentary and approximately reaches, in height, the level of the anterior mental foramen (Fig. 17).

The anterior opening of the infraorbital canal is relatively small and almost invariably located above the metastyle of $\mathrm{P} 3$ and parastyle of $\mathrm{P} 4$ in the 10 adult specimens preserving this character (MHNC 8372, 8399, 8400, 8401, 8402, $8404,8405,8406,8407$ and 8408) (Fig. 18). It is similar in this respect to Haplolambda; the anterior opening of the infraorbital canal (= infraorbital foramen) is slightly more posterior than in Pantolambda, in which it lies above the ectoflexus of P3. The infraorbital canal conveys the infraorbital artery and infraorbital nerve (i.e. maxillary nerve in the infrorbital canal; CN V2); the former supplies blood to the upper lip and anterior part of the snout and the latter provide inervation of the eyelid, upper lip, vibrissae, and part of the nasal vestibule (Davis 1964; Evans \& de Lahunta 2012). Its anterior opening is a single foramen but, on both sides of the skull MHNC 8372, on the medial wall of the foramen and anterior to it, are two small posterolaterally opening foramina, which probably carried nerves and vessels into the maxillary sinus and anterior upper teeth. This condition is present in most (but not all) of the specimens in which this region is adequately preserved. It is especially distinct on MHNC 8400 , in which the more dorsal of the two foramina is particularly large (Fig. 18B). The maxilla forms the anterior root of the zygomatic arch and, in lateral view, has a bifurcated suture, which interdigitates with a matching anterior end of the jugal (MHNC 8372, 8399, 8400). The ventral edge of this root is formed by a short process of the maxilla, above which is a small and anteriorly-directed short process of the jugal. More dorsal is a posteriorly-directed long process of the maxilla dorsally covered, in turn, by a long process of the jugal, which forms the dorsal edge of the zygomatic arch (Fig. 18). The ventral jugal process of the maxilla, which appears to be very small in lateral view, extends posteriorly (in ventral view) on the medial side of the zygomatic arch, within the orbit. It extends further posteriorly than does the dorsal process of the same bone and is both thicker and dorsoventrally taller. In fact, along most of its length (in medial view), the ventral process of the maxilla is almost as high as the jugal. Its apex is rounded and is at the level of the metacone of M3. In lateral view, the vertical wall of the maxilla on the rostrum presents some variation in height at the level of the anterior root of the zygomatic arch (from anterior edge of M1 to anterior edge of the jugal). It varies from 10.1 to $11.6 \mathrm{~mm}$ in six MHNC specimens and from $7 \mathrm{~mm}$ to $8.92 \mathrm{~mm}$ in eight other MHNC specimens. This variation is interpreted here as related to sexual dimorphism and has been further detailed above in relation to dental size and proportions (see above in the "Specimens, taphonomy, and biology" section).

On the palate, the maxilla is slightly arched (in transverse section), while it is almost flat in the other pantodonts (e.g., Pantolambda, Haplolambda). It is narrow compared to most other pantodonts, especially in its anterior region. Alcidedorbignya is, however, very similar in this respect to Harpyodus.

On the posterior region of the palate, the maxilla-palatine suture almost contacts the medial edge of $\mathrm{M} 3$ and $\mathrm{M} 2$, and turns medially at the level of the protocone of M1 (Fig. 19). Approximately $1.5 \mathrm{~mm}$ medial to the protocone of M1 is a well-developed major palatine foramen. It is the anterior opening of the major palatine canal, which is located in the 

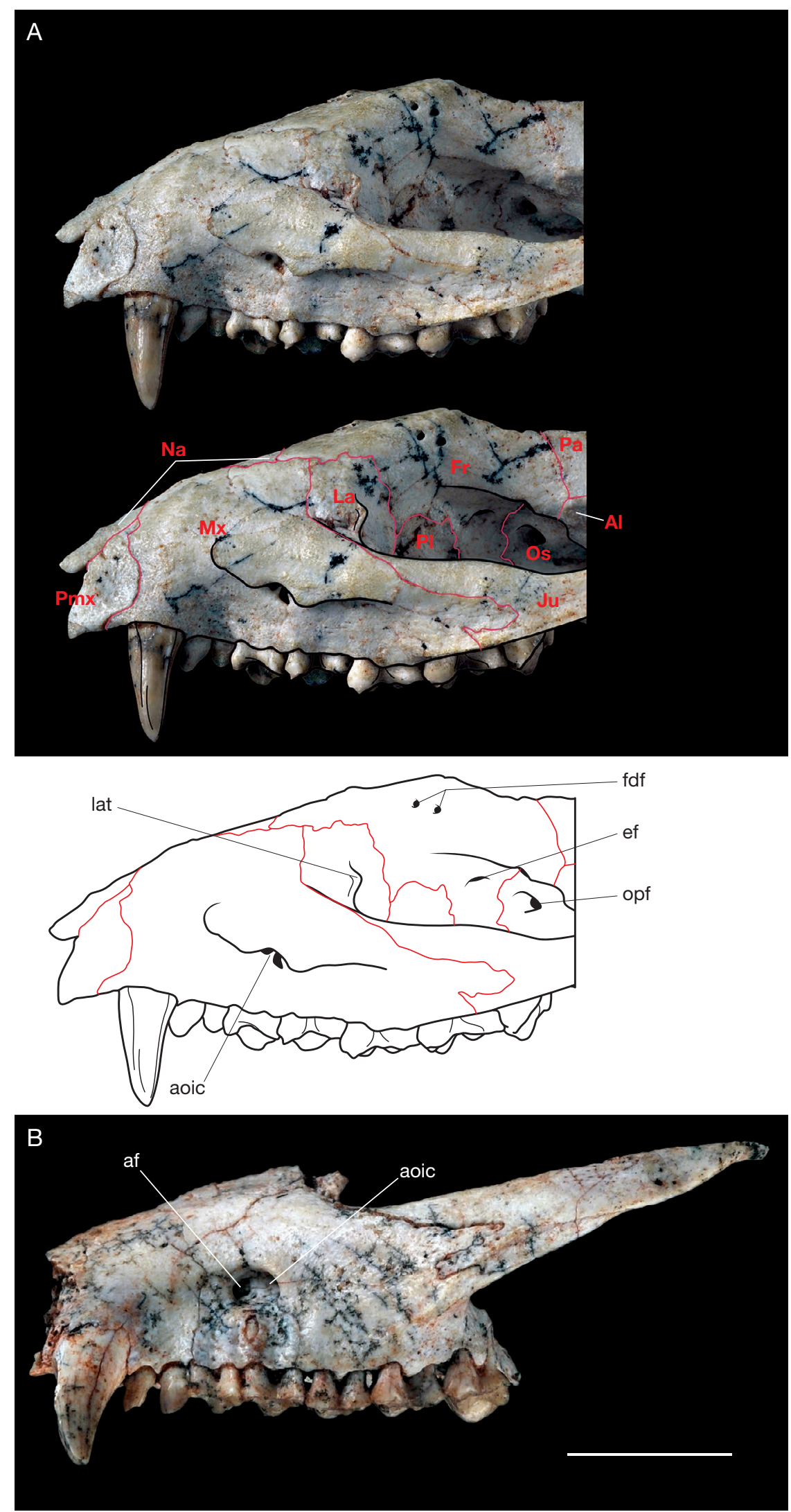

FIG. 18. - Lateral view of the rostrum of Alcidedorbignya inopinata showing the interdigitated suture of the maxilla and the jugal: A, MHNC 8372 ; B, MHNC 8400. Abbreviations: af, anterior foramen possibly for the passage of a branch of the infraorbital artery entering the nasal cavity (see maxilla section in the description); Al, alisphenoid; aoic, anterior opening of the infraorbital canal; ef ethmoidal foramen; fdf, frontal diploic foramina; Fr, frontal; Ju, jugal; La, lacrimal; lat, lacrimal tubercle; Mx, maxilla; Na, nasal; opf, optic foramen; Os, orbitosphenoid; Pa, parietal, PI, palatine; Pmx, premaxilla. Scale bar: $1 \mathrm{~cm}$. 
maxilla-palatine suture and transmits the major palatine nerve and vessels (Wible et al. 2004; Evans \& de Lahunta 2012). From this foramen a shallow groove runs anteriorly up to the anterior edge of the alveolus for the canine. At this level the palatine groove is very close to the alveolar border. The anterior process of the maxilla is preserved on the right side only of MHNC 8372. Its anteromedial edge forms the lateral border of the incisive foramen. As preserved it provides an indication of the size of the foramen (Fig. 19). The posterior edge of the incisive foramen, as reconstructed (Figs 12, 13), extends well posterior to the level of the posterior edge of the I3. The incisive foramen of Alcidedorbignya was therefore slightly larger than in Pantolambda, where its posterior edge is anterior to the posterior edge of I3. The anterior process of the maxilla is longer and narrower than in Pantolambda, but, as in this genus, its apex is posteromedial to I2.

The maxilla forms the floor of the orbit and strongly projects posteriorly in such a way that M3, M2, and part of M1 are located below the eye. Most of M3 is even posterior to the posterior edge of the palate (i.e. the ventral edge of the choanae). The posteriorly-projecting posterior part of the alveolar process of the maxilla forms a true lobe (in MHNC 8372), which is separated by deep notches from the zygomatic arch laterally, and from the perpendicular processes of the palatines (see below) medially, by deep notches. A similar condition is also present in Pantolambda, Haplolambda, and Caenolambda, in which the floor of the orbit is formed by the molar-bearing part of the alveolar process of the maxilla. However, in these genera the notches are not as deep as in Alcidedorbignya (MHNC 8372), being almost absent in Haplolambda (the medial one only) and Caenolambda (both notches). However, in Alcidedorbignya this condition varies. In MHNC 8399 the medial notches, which actually correspond to the minor palatine notches (see below), are not as deep as in MHNC 8372. In MHNC 8400 and 8401 there is apparently no true medial notch and the M3 has a more anterior position in relation to the posterior edge of the palate. Similarly the lateral notch in MHNC 8372 reaches anteriorly to the level of the metastyle of $\mathrm{M} 2$, while in MHNC 8399 it does not extend anterior to the level of the parastyle of M3. In MHNC 8400 and 8401 the lateral notch is even more posteriorly located, being at the level of the metacone of M3. In fact, it is noteworthy that the two specimens, which apparently have no medial notch and a shallow lateral notch (MHNC 8400 and 8401), have relatively worn teeth indicating that they represent ontogenetically much older individuals than the two others, which have almost unworn teeth. MHNC 8372 is the youngest specimen of the four considered and it has the deepest notches. Therefore, the depth of the medial and lateral notches and the relative position of $\mathrm{M} 3$ relative to the posterior edge of the palate probably vary with the relative anterior migration of the molars related to the postnatal ontogenetic growth of the skull.

On the floor of the orbit the maxilla is perforated by the roots of the posterior molars. On MHNC 8372 the three roots of M3 and the medial and posterolabial roots of M2 distinctly perforate the surface of the floor of the orbit. On MHNC 8399 the roots of M3 and the lingual root only of M2 pierce the maxilla dorsally. This character may also be related to the ontogenetic age of the individual, because on MHNC 8400 only the medial and posteromedial roots of M3 perforate the floor of the orbit and only the medial root of M3 in MHNC 8401. These two specimens are relatively old individuals as indicated by the heavy wear of the their teeth. In Pantolambda (AMNH 16630) only the medial root of M3 is visible on the floor of the orbit. This feature is apparently absent in other pantodonts.

Within the orbit, the maxilla articulates with the jugal at the anterior third of the zygomatic arch, but shows some variation. The posterior apex of the zygomatic process of the maxilla (the medial contribution of the maxilla to the zygomatic arch), extends far beyond the posterior edge of the palate on MHNC 8400. This process is smaller on MHNC 8372, where its posterior apex barely reaches the level of the posterior edge of the palate posteriorly. Here again, this condition may be related to the postnatal allometric growth of the skull because MHNC 8372 represents a young adult and MHNC 8400 is an old one. However, because MHNC 8400 is a male and MHNC 8372 a female (see above), this difference may also be the result of a greater robustness of the zygomatic arch in the former. At the anterior border of the orbit, the dorsal suture of the maxilla turns medially and remains approximately horizontal (as in Pantolambda). In this region, the maxilla articulates with the lacrimal dorsally. The lacrimal-maxilla suture is located dorsal to the maxillary foramen, the posterior opening of the infraorbital canal. Further medially, the suture turns posteriorly and lies, with the palatine, on the medial orbital wall. The lacrimal-maxilla suture is oriented anteroposteriorly and is approximately horizontal. It runs along and ventrolateral to the sphenopalatine foramen (see below). Further posteriorly, the palatine-maxilla suture extends along the lateral side of the minor palatine notch and passes on the palate. The maxilla has no ascending process in the orbit and does not contact the frontal in the orbit (Fig. 20). It does however contact the frontal on the dorsal face of the rostrum (see frontal section).

Turbinals and nasal cavity. The nasal cavity is organized in different spaces, each of them housing a peak of complexity of specific turbinals. The nasal fossa is therefore subdivided according to turbinals and the roots by which they are attached to the lateral nasal wall. The nasal capsule of mammals is traditionally divided into three spaces that are routes of airflow: anterior (pars anterior), posterior (posterior cupula) and lateral (recessus lateralis or pars intermedia, linked to the maxillary sinus) (Maier 2000; Rossie 2006; Smith \& Rossie 2008). This tripartite nasal capsule observed in foetal stages becomes increasingly complex in later developmental stages with processes of folding, ossifications, and pneumatisations (see Smith \& Rossie 2008). The pars anterior projects posteriorly as the semicircular crest and gives rise to portions of the maxilloturbinal and nasoturbinal, the pars intermedia produces structures of the recessus lateralis (including one 


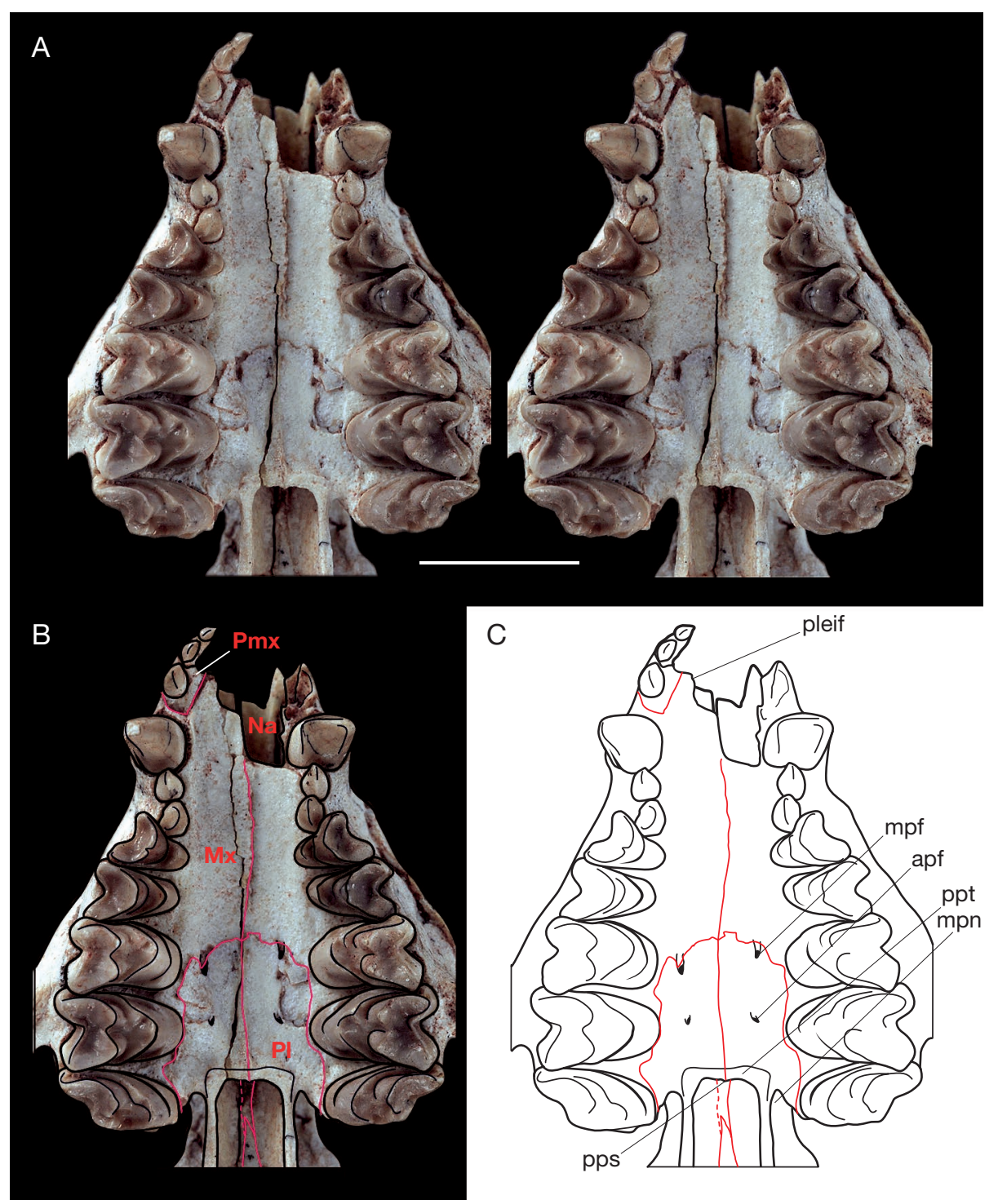

FIG. 19. - Ventral view of the palate of Alcidedorbignya inopinata (MHNC 8372): A, stereophotograph; B, schematic drawing with bone sutures on photo; C, schematic drawing with captions. Abbreviations: apf, accessory palatine foramina; $\mathbf{m p f}$, major palatine foramen; mpn, minor palatine notch; Mx, maxilla; Na, nasal; PI, palatine; pleif, posterolateral edge of the incisive foramen; Pmx, premaxilla; pps, postpalatine spine, ppt postpalatine torus. Scale bar: $2 \mathrm{~cm}$.

or more frontoturbinals), and the pars posterior gives rise to the ethmoturbinal complex, housed in the olfactory recess (=ethmoturbinal or sphenoidal recess) and the lateral part of transverse lamina (Smith \& Rossie 2008; Van Valkenburgh et al. 2014). Here we will follow the terminology of Smith \& Rossie (2008) to describe the spaces of the nasal cavity in adult forms, which can be divided into three recesses: olfactory, maxillary, and frontal, the two latter forming the lateral recess (Smith \& Rossie 2008: fig. 2, table 2). For the purpose of describing an adult morphology of the nasal cavity and its osseous and cartilaginous components, the positional terminology for turbinals is of use (e.g., Macrini 2014), although such structures are better understood through studies of embryonic stages (see Maier \& Ruf 2014). Here, we will therefore use a turbinal terminology that reflects anatomical articulation in adults (but see Smith \& Rossie 2008, for a comparison of terminologies for structures and spaces of the nasal fossa). The maxilloturbinal articulates with the maxilla and premaxilla, the nasoturbinal with the nasal bone (and with the more posterior ethmoid to a variable extent), the frontoturbinals with the frontal, and the ethmoturbinals with the ethmoid.

Turbinals - The nasal cavity of Alcidedorbignya is surrounded by the premaxillae, maxillae, palatines, nasals, and frontals. The nasal cavity of MHNC 8372 was completely filled with matrix, in which few internal nasal bony laminae are observable through CT scanning (Fig. 21). Remarkably, however, the primary laminae from which the maxilloturbinals originated (Fig. 21C-F) are well preserved bilaterally and are separated by a partly preserved nasal midline septum, which subdivides the cavity. The lower edge of the nasal septum is continuous with the vomer. The lateral nasal wall usually bears four sets 

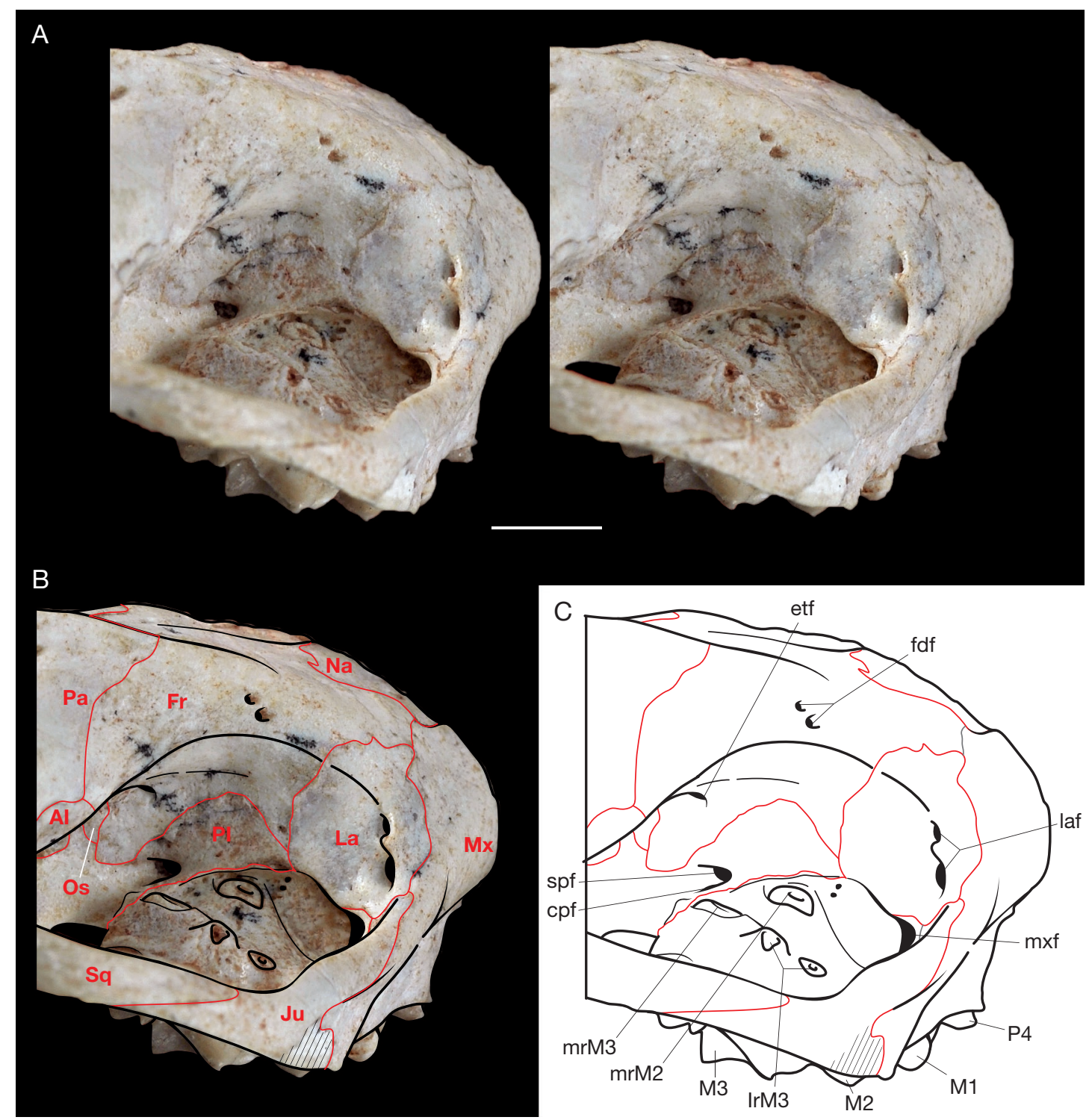

FIG. 20. - Posterodorsolateral view of the right side of the skull of Alcidedorbignya inopinata (MHNC 8372) showing the anterior region of the orbit. A, stereophotograph; B, schematic drawing with bone sutures on photo; C, schematic drawing with captions. Abbreviations: Al, alisphenoid; cpf, caudal palatine foramen; etf, ethmoidal foramen; fdf, frontal diploic foramen; Fr, frontal; Ju, jugal; La, lacrimal; laf, lacrimal foramen; IrM3, lateral roots of M3; mrM2, medial root of M2; mrM3, medial root of M3; mxf, maxillary foramen; Mx, maxilla; Na, nasal; Os, orbitosphenoid; Pa, parietal; PI, palatine; spf, sphenopalatine foramen; Sq, squamosal. Scale bar: $1 \mathrm{~cm}$.

of turbinals in mammals: ethmoturbinals, nasoturbinals, frontoturbinals, and maxilloturbinals. However, the CT-images of the nasal cavity of Alcidedorbignya exhibit only tiny bony remnants of osseous turbinals throughout the nasal region; these were too fragmentary to reconstruct. Assuming that turbinals were likely mostly cartilaginous or very thin bones in life, it makes sense that they are not preserved for their main part in this fossil specimen. Their description will therefore focus on the identification of bony ridges that are likely to have been sites of attachment for putative cartilaginous turbinals, and on the fossae, which in some cases received the turbinals. As mentioned above, the primary laminae of the maxilloturbinals are clearly visible on the floor of the nasal cavity and lie medial to the roots of the M1 and M2 (Fig. 21C-F). They arise vertically from the posterior floor of the cavity with no anterior attachment on the maxilla or premaxilla. Therefore, most of the concha of the maxilloturbinal, which probably extended anteriorly up to the bony nares, was overhanging the floor of the cavity. Connecting to this anchor on the maxilla, and more obvious on the left side of the nasal cavity in the coronal plane, are fragments of a thin lamina, which is roughly horizontal and which we interpret as part of the maxilloturbinal (Fig. 21D).

The area of attachment of the nasoturbinal is visible on the nasal bone and can be followed through the CT slices in coronal plane (Fig. 21A-D) (see below). More posteriorly in the nasal cavity, on a coronal slice at a level with the anterior part of the M2, a more complex pattern of scroll laminae is visible apposed onto the frontal bone and is interpreted as a possible frontoturbinal (Fig 21E). On the same CT slice, at the 

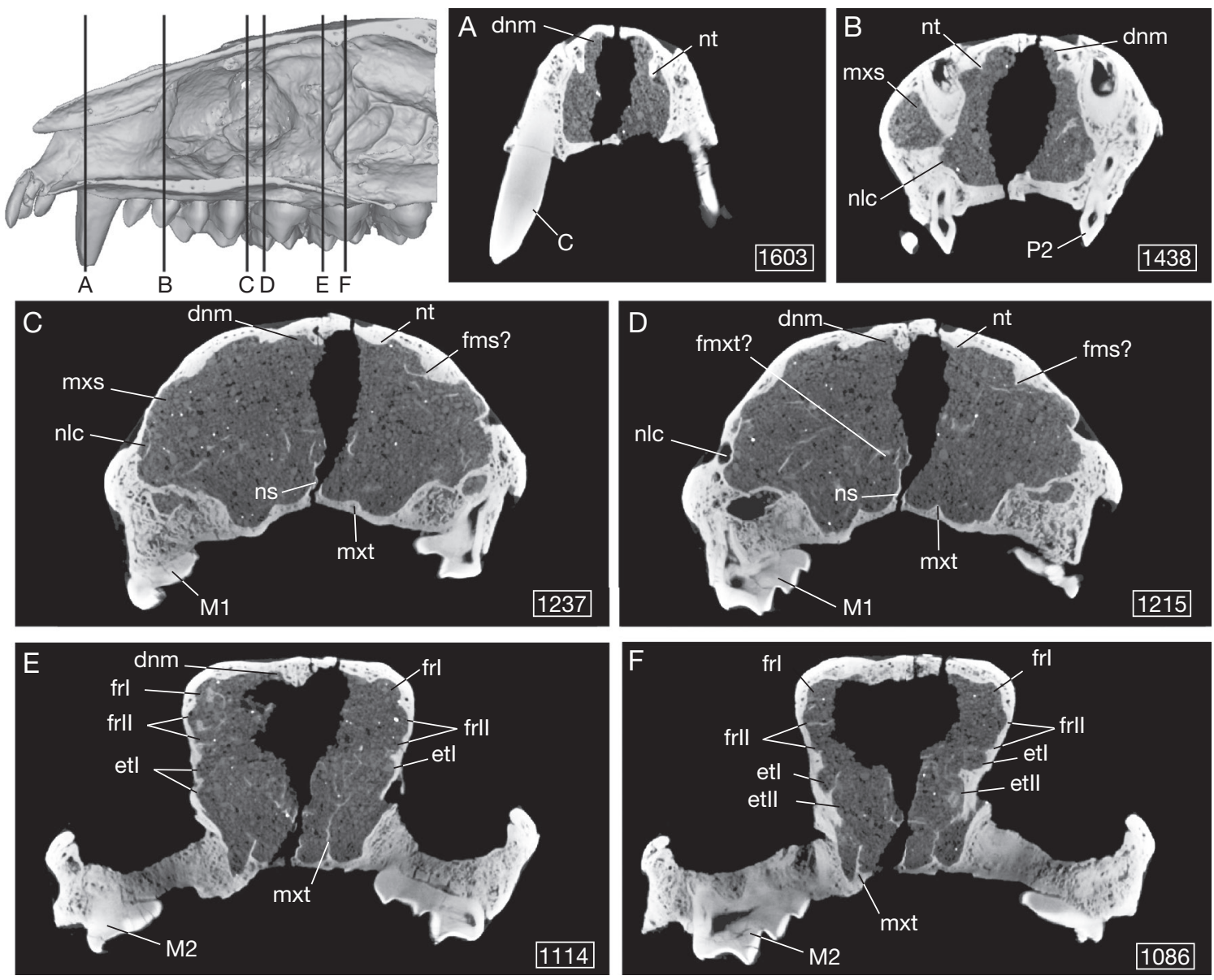

FIG. 21. - Internal nasal structures and spaces of Alcidedorbignya inopinata (MHNC 8372) identified on six CT-slices in coronal view (A to F). Each cutting plane is indicated in the 3D-model of the right lateral wall of the nasal cavity (top left, septum removed). On the bottom right corner of each CT-slice is indicated the position of the slice within the sequence of 1812 images (resolution: on binned images, voxel size $=0.033 \mathrm{~mm}$ ). Abbreviations: C, canine; dnm, dorsal nasal meatus; etl, fossa for ethmoturbinal I; etIl, fossa for ethmoturbinal II; fms?, probable area of attachment of the frontomaxillary septum; frl fossa for frontoturbinal I; frlI, fossa for frontoturbinal II; fmxt?, possible fragments of the maxilloturbinal; M1, first upper molar; mxs, maxillary sinus; mxt, primary lamina of the maxilloturbinal; nlc, nasolacrimal canal; ns, nasal septum; nt, primary lamina of the nasoturbinal; P2, second upper premolar. Not to scale.

level of the ethmoid, a small scroll-like pattern recognizable as a typical snailing of osseous ethmoturbinals (as illustrated in Monodelphis by Rowe et al. (2005), or Caluromys by Macrini (2014), or Carcinella by Koenigswald et al. (2009; Fig. 21F) is visible but cannot be more precisely identified.

Paranasal recesses and turbinal fossae - The nasal cavity (Fig. 22) shows well-demarcated and deeply excavated spaces (especially visible on the right side of the skull) that we described as paranasal recesses, although some may be secondary pneumatisations within the paranasal sinuses (Maier 2000; Rossie 2005).

The posteriormost space, covered by the frontal, is the ethmoidal fossa, which contained the olfactory bulbs and which is anteriorly bounded by a small remnant of the cribriform plate. This peripheral edge of the cribriform plate separates the nasal cavity from the braincase. Anterior and ventral to the ethmoidal fossa is the olfactory recess, made of four distinct fossae, which contained the four large ethmoturbinals, well discriminated by their imprints on the bone (Fig. 22A). The fourth ethmoturbinal is the most posterior one, and the first ethmoturbinal is the most anterior and lateral. It is bordered dorsolaterally by a frontoturbinal fossa (see below). The fourth, third, and second ethmoturbinal fossae are distinctly oval-shaped and arranged in parallel in posterodorsal to anteroventral orientation, oblique to the palatal axis. The first ethmoturbinal is more anteriorly extended than the three others and corresponds to a roughly triangular fossa. Its dorsal edge is oriented roughly anteroposteriorly, parallel to the palatal axis. The fourth ethmoturbinal is inside a small and deep pocket, which may be considered as the sphenoidal recess, as is the case in Solenodon (Wible 2008). Dorsal to the ethmoturbinal I fossa are two anteroposteriorly elongated oval-shaped fossae, much larger than the fossa of ethmoturbinal II and III. They are interpreted here as fossae for the frontoturbinals. They are separated by a marked septum. They are adjacent, parallel to each other, and roughly anteroposteriorly oriented. The dorsal frontoturbinal fossa is longer and wider than the ventral one and extends onto the roof of the nasal cavity, on the medial edge of the posterior region of the nasoturbinal fossa. At its anteroventral end and at its contact with the maxillary sinus (see below) is an elevated area, which probably represents 
an attachment structure for the bone or, more likely, for the frontomaxillary septum, a horizontal lamina that separates the maxillary and frontal recesses. Its anteromedial area is excavated by a small but deep fossula that is separated from the posterior elongated oval-shaped portion of the dorsal frontoturbinal fossa.

On the dorsal region of the nasal cavity, on the ventral side of the nasal is an elongated and narrow fossa, the dorsal nasal meatus (Novacek 1986). This meatus extends posteriorly to the cribriform plate. The lateral edge of the nasal forms a rounded crest that extends posteriorly on approximately half the length of the bone reaching the level of the posterior edge of the canine root. At this level it is interrupted but extends posteriorly in a thin crest, which continues posteriorly to the frontal almost reaching the cribriform plate. The whole ridge is regarded as the attachment zone of the nasoturbinal. In tenrecids the nasoturbinal is generally a simple, ventrally oriented blade in its anterior part, but this bone tends to be more complex and expands transversely in its posterior region. In Alcidedorbignya, it is therefore possible that the anterior region of the nasoturbinal was a simple blade oriented downward. The posterior part of the nasoturbinal was probably housed in the fossa in the roof of the nasal cavity, which corresponds to the expanded triangular posterior part of the nasal.

The lateral recess is a very wide excavated area that starts just anterior to the cribriform plate and olfactory recess and that expands to the pyriform opening and occupies the whole lateral wall of the nasal cavity. Here we consider that in Alcidedorbignya, as in the plesiomorphic eutherian condition (Maier 2000), the lateral recess comprises one upper (frontal) recess and one lower (maxillary) recess. The frontal recess received the frontoturbinals, as discussed above, and is made of three to four distinct fossae. These fossae are deep but not hypertrophied and we do not regard them as sinuses. The frontal recess is posterolateral to the nasoturbinal and anterolateral to the olfactory recess (which houses the ethmoturbinals). The maxillary recess is clearly divided into upper and lower compartments. The upper compartment is so deeply excavated in its anterior part that we regard it as a maxillary sinus. The maxillary sinus lies in the maxilla, beneath the frontal recess. It is clearly observable on the lateral aspect of the snout, in the inflation of the maxilla between the canine and the anterior root of the zygoma, and above the anterior opening of the infraorbital canal (see above, description of the rostrum and maxilla). The maxillary sinus is made of three fossae. The larger is anterior and abuts against the very salient volume formed by the root of the canine in the anterolateral region of the nasal cavity. It faces posteromedially and exclusively excavates the maxilla. Posterior to it is a much smaller circular fossa, which faces medially and excavates the lacrimal. Ventral to this fossa is a deep pit in the floor of the nasal cavity, which excavates both the maxilla and the lacrimal, and which may be regarded as part of the maxillary sinus as well, due to its consequent pneumatisation (Fig. 22B). The two small posterior fossae of the maxillary sinus are separated by a short rounded ridge, which houses a canal for the passageway of the nasolacrimal duct. This canal opens anteriorly in a groove, which received the nasolacrimal duct (Fig. 22C). This groove is strongly oblique and extends medially to the posterior edge of the canine alveolus. The passageway of the nasolacrimal duct (canal and groove) runs from the internal rim of the orbit above M1 to a more ventromedial position above the P1. It separates the upper and lower parts of the maxillary recess. The lower maxillary recess presents a lateral and a medial deep fossa separated by an elevated median area corresponding to the medial roots of P4 and M1. The lateral fossa is the deep pit in the floor of the nasal cavity, which we regard as part of the maxillary sinus (see above). This pit is roughly the same size as the lacrimal fossa above and faces dorsally. The medial fossa is anteroposteriorly elongate and runs along the lateral edge of the primary lamina of the maxilloturbinal.

Anteriorly, the bony nares (the pyriform opening) are relatively narrow because of the extremely salient volumes of the canine alveoli. In other words, the nasal cavity is very wide in its central region because of the hypertrophied maxillary sinuses and very narrow anteriorly because of the thickness of the canine roots.

Palatine. The palatines are well developed and their horizontal processes (Wible 2011) form almost the posterior third of the bony palate. As mentioned above, they almost contact the medial edge of the molars and occupy the total width of the palate at the level of M2-M3 (Fig. 19). A similar condition is present in Pantolambda although in this genus the palatines are not so approximated laterally to the molars as they are in Alcidedorbignya. The major palatine foramina, present at the suture with the maxillary, have been discussed in the section describing the maxilla. Posterior to these foramina, at the level of the anterior edge of the protocone of M2, is a pair of small accessory palatine foramina (see Wible \& Rougier 2000: 77 for a review of the palatine foramina in mammals).

The posterior border of the horizontal process of the palatines forms the ventral edge of the choanae. It is slightly thickened and forms a weakly developed but distinct postpalatine torus (as in Pantolambda and Haplolambda). It is almost straight (incipiently double arched) with a minute posterior nasal spine (caudal nasal spine, Evans \& de Lahunta 2012). It differs from Pantolambda and Caenolambda, in which the torus is biconcave with a well-developed posterior nasal spine in the latter. Just medial to the protocone of M3 the minor palatine notch is developed as a deep groove (but see above in the maxilla section for variation), which is the result of the opening of the minor palatine canal. The plesiomorphic condition observed in many early-diverging mammals is a minor palatine canal (postpalatine canal of Novacek 1986; see also Wible \& Rougier 2000: 77; Wible et al. 2004, 2009) totally enclosed in the maxilla and palatine. A deep minor palatine notch is also observed in Harpyodus decorus. A notch is also present in Pantolambda, but it is shallower than in Alcidedorbignya. There is hardly a notch in Haplolambda and it is absent in Caenolambda. In Alcidedorbignya inopinata the medial edge of the minor palatine notch forms a small laterally projecting spine, which partly overhangs it ventromedially (smaller on MHNC 8372 than on MHNC 8399). This spine is a relict of the ventral bridge of the minor 

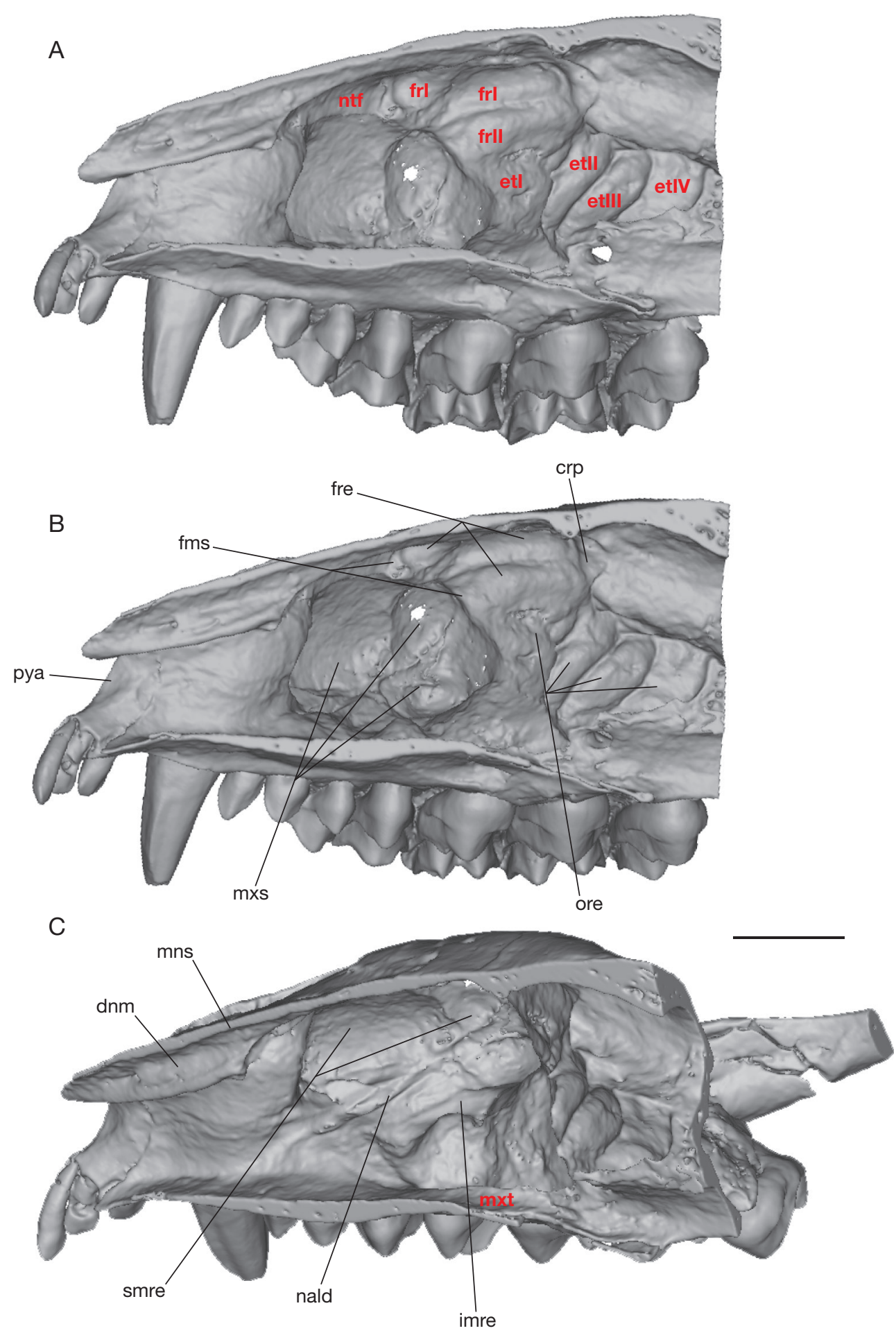

FIG. 22. - Identification of internal spaces in the right lateral wall of the nasal cavity of the 3D-model of Alcidedorbignya inopinata (MHNC 8372, septum removed). The spaces that received in life the scroll-like turbinals are delimited by bony laminae which are observable througth CT-slices (Fig. 21). Three main spaces are described in the text and illustrated here from posterior to anterior: olfactory recess (for ethmoturbinals etl to etIV - etl being the anterior projection of the posterior cupula that extends onto the lateral recess), frontal recess (for frontoturbinals frl and frll), maxillary recess (divided into superior and inferior maxillary spaces, for maxilloturbinal); the last two being part of the lateral recess (which also contained the nasoturbinal nt). A, ventromedial view; $\mathbf{B}$, medial view; C, dorsomedial view. Abbreviations : crp, cribriform plate; dnm, dorsal nasal meatus; etl, fossa for ethmoturbinal l; etll, fossa for ethmoturbinal II; etIll, fossa for ethmoturbinal III; etIV, fossa for ethmoturbinal IV; fms, area of attachment of frontomaxillary septum; frl fossa for frontoturbinal I; frlI, fossa for frontoturbinal II; fre, frontal recess; imre, inferior maxillary recess; $\mathbf{m n s}$, midline nasal septum; $\mathbf{m x s}$, maxillary sinus; $\mathbf{m x t}$, primary lamina of maxilloturbinal; nald, nasolacrimal duct; $\mathbf{n t f}$, fossa for nasoturbinal; ore, olfactory recess; pya, pyriform aperture; smre, superior maxillary recess. Scale bar: $5 \mathrm{~mm}$. The 3D rendering of CT data is available in pdf format at the address http://sciencepress.mnhn.fr/en/periodiques/geodiversitas/37/4/alcidedorbignya-inopinata-additional-files. 
palatine foramen or canal, which is closed in several groups of early-diverging mammals (see comparisons below).

The choanae open at the level of the anterior edge of M3 on MHNC 8372 (similar to Pantolambda and Haplolambda) and slightly posterior to the level of the protocone of M3 on MHNC 8399. The roof of the choanae is mainly formed by the palatines but the bones are not in contact in the sagittal plane, and are separated by the presphenoid. In the orbitotemporal fossa, the perpendicular process of the palatine (as opposed to the horizontal process contributing to the palate) forms approximately the anterior half of the lateral wall of the basipharyngeal canal, posterior to the aperture of the choanae (Wible 2011). In the anterior half of the basipharyngeal canal, the ventral edge of the lateral wall (in ventral view) is a single straight crest that splits more posteriorly into the ento- and ecto-pterygoid crests. At the anterior edge of these diverging crests, the posterior end of the palatine is wedged between the hamular process of the pterygoid medially (entopterygoid crest of the pterygoid) and the ectopterygoid process of the alisphenoid laterally, this condition being more easily observable on MHNC 8399, in which the sutures are not fused (Fig. 23B). The region of the basipharyngeal canal of Alcidedorbignya is less elongated than in Haplolambda and Caenolambda but similar to Pantolambda.

On its posterolateral aspect, the perpendicular process of the palatines has a suture with the alisphenoid close to the level of the ventral edge of the common foramen for the sphenorbital fissure and the foramen rotundum (but see below). On MHNC 8372 the palatine suture runs dorsally along the medial edge of this common foramen and, at mid-height of the foramen, leaves the alisphenoid, reaches the orbitosphenoid anteriorly and has a small contribution to the ventromedial edge of the optic foramen (Fig. 24A, B). From this point, the dorsal border of the palatine runs subhorizontally along the ventral edge of the orbitosphenoid and reaches the posterior edge of the orbital process of the frontal. At this point, the palatine is partially fused with the orbitosphenoid and the suture between the two bones is indicated by a small ridge rather than by a distinct interdigitated articulation. When reaching the frontal, the palatine suture turns ventrally and reaches the ventral edge of the orbital process of the frontal. From this point, the fronto-palatine suture runs horizontally along the ventral edge of the orbital process of the frontal and turns anterodorsally at the level of the sphenopalatine foramen, where it forms a well-developed ascending orbital process of the palatine (Wible et al. 2009) in the anteromedial region of the orbitotemporal fossa (Fig. 25). This process is slightly wider than high and occupies the ventral half of the medial wall of the orbit. The dorsal edge of the orbital process of the palatine is regularly convex and excavates the anteroventral edge of the descending orbital process of the frontal. However, some variation occurs as the fronto-palatine suture actually penetrates the sphenopalatine foramen on the left side of the skull of MHNC 3872 and, as a consequence, the anteroventral angle of the orbital process of the frontal also contributes to the dorsal edge of the foramen. On the right side of the skull the sphenopalatine foramen is totally enclosed in the palatine (Fig. 25).
In the orbit, the anteroventral edge of the palatine articulates with the maxilla. The anteriormost extremity of the palatine in the orbit also has a small articulation with the lacrimal and a large contact with the frontal dorsally.

The sphenopalatine foramen is well developed and is located in a small fossa, which opens posteriorly into a short but deep sphenopalatine groove. Anteromedially, the sphenopalatine foramen opens into the posterior region of the nasal cavity. In life, this foramen conducted the sphenopalatine artery and vein and the caudal nasal nerve from the orbitotemporal fossa to the nasal cavity (Wible 2008, 2011; Evans \& de Lahunta 2012) (Fig. 26). The caudal palatine foramen (caudal opening of the major palatine canal; Wible 2011; Evans \& de Lahunta 2012) opens onto the lateral side of the sphenopalatine groove, posteroventral to the sphenopalatine foramen and anterior to the minor palatine notch. It is well separated from the sphenopalatine foramen, but distinctly opens in a common recess. This foramen is likely to have transmitted the major palatine nerve and vessels to the hard palate through the major palatine foramen at the anterior edge of the palatine-maxilla suture (Wible 2011; Evans \& de Lahunta 2012).

Lacrimal. The lacrimal of Alcidedorbignya is well developed. It has a large facial process, which is slightly more than half the surface of the orbital wing. The facial process is slightly convex and the orbital wing concave. Two lacrimal foramina are located at the anteriormost point of the orbit, above the anterodorsal angle of the zygomatic arch (Fig. 20). They are roughly one above the other in lateral view. The ventral one is the largest and is oval-shaped with the long axis oriented ventrolaterally-dorsomedially. The dorsal one is triangular and is located dorsal and slightly medial to the ventral one; it lies on the medial side of the well-developed lacrimal tubercle. In Pantolambda a large ventral foramen is present with a robust lacrimal tubercle. Apparently Pantolambda lacks a dorsal lacrimal foramen. The edge of the lacrimal, posteroventral to the ventral lacrimal foramen has a small contact with the jugal. The lacrimal articulates antero- and posteroventrally with the maxilla, and dorsally with the frontal. It also has a small suture with the palatine at its posteromedial angle. The condition in the other pantodonts is unclear because the sutures of this bone are apparently obliterated early in ontogeny. However, in Pantolambda (AMNH 16663), although displaced because of the anteroposterior compression of the skull, the left lacrimal is relatively well preserved and distinctly presents a narrow facial process.

Frontal. The frontal occupies most of the skull roof above the orbit. Its length on the sagittal plane is approximately $25 \%$ of the total length of the skull. It is longer than in Pantolambda, in which the relative length of the frontal on the roof of the skull is less than $15 \%$. On the roof of the skull, the frontal of Alcidedorbignya faces anterodorsally and is slightly oblique in lateral view. A similar condition is observed in Pantolambda while in other pantodonts the frontal faces dorsally and the dorsal face of the skull is generally subhorizontal (Harpyodus, 

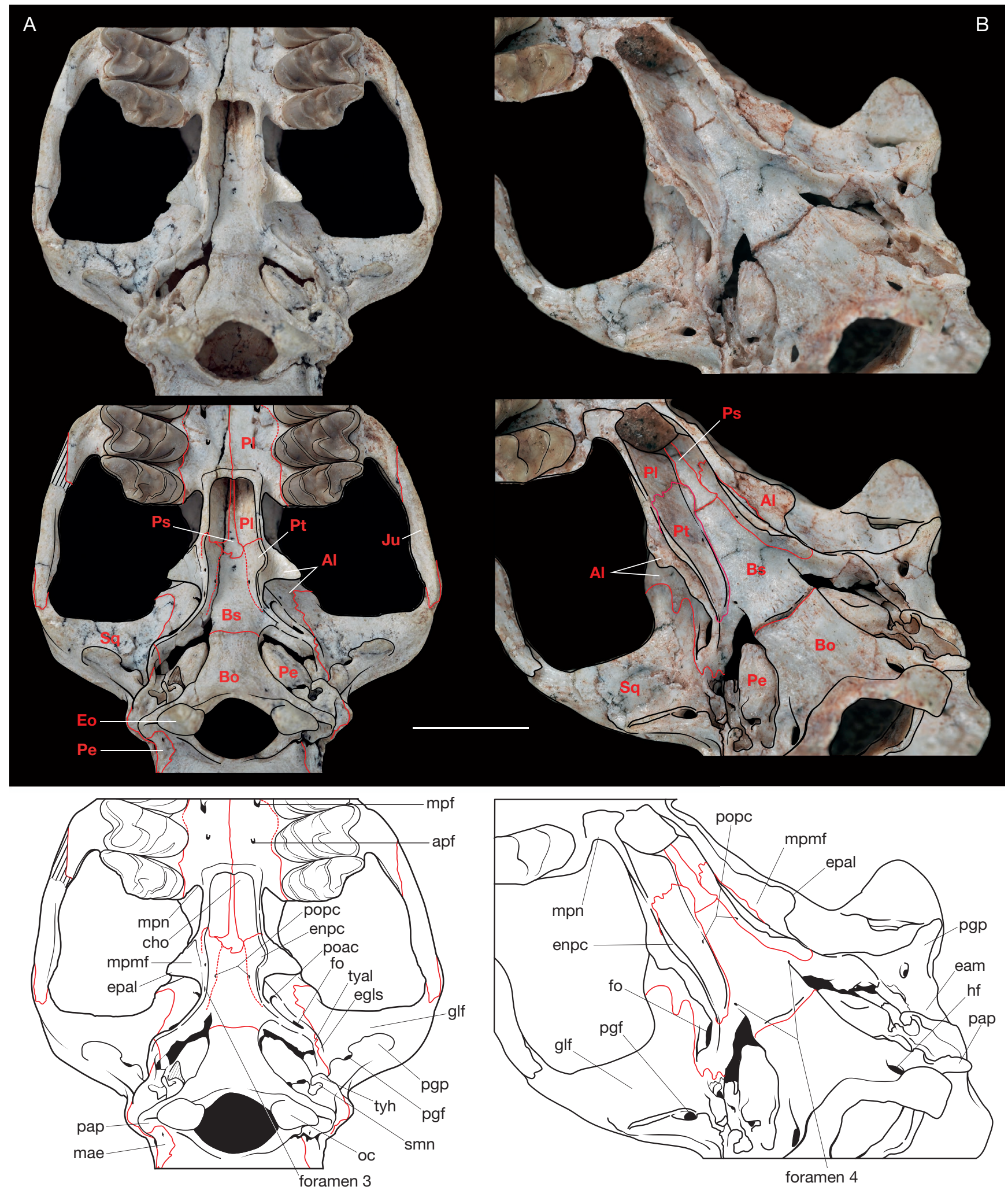

FIG. 23. - Ventral view of the skull of Alcidedorbignya inopinata showing the basipharyngeal canal and its lateral walls with the posterior opening of the pterygoid canals: A, MHNC 8372; B, MHNC 8399. Abbreviations: Al, alisphenoid; apf, accessory palatine foramen; Bo, basioccipital; Bs, basisphenoid; cho, choanae; eam, external auditory meatus; enpc, entopterygoid crest; Eo, exoccipital; epal, ectopterygoid process of the alisphenoid; egls, entoglenoid process of the squamosal; fo, foramen ovale; foramen 3, see pterygoid section in the description; foramen $\mathbf{4}$, see basisphenoid section in the description; glf, glenoid fossa; hf, hypoglossal foramen; Ju, jugal; mae, mastoid exposure of the petrosal; mpf, major palatine foramen; mpmf, fossa for the medial pterygoid muscles; mpn, minor palatine notch; oc occipital condyle; pap, paroccipital process of the exoccipital; Pe, petrosal; pgf, postglenoid foramen; pgp, postglenoid process; PI, palatine; poac, posterior opening of the alisphenoid canal; popc, posterior opening of the pterygoid canal; Ps, presphenoid; Pt, pterygoid; smn, stylomastoid notch; Sq, squamosal; tyal, tympanic process of the alisphenoid; tyh, typmanohyal. Scale bar: $1 \mathrm{~cm}$. 

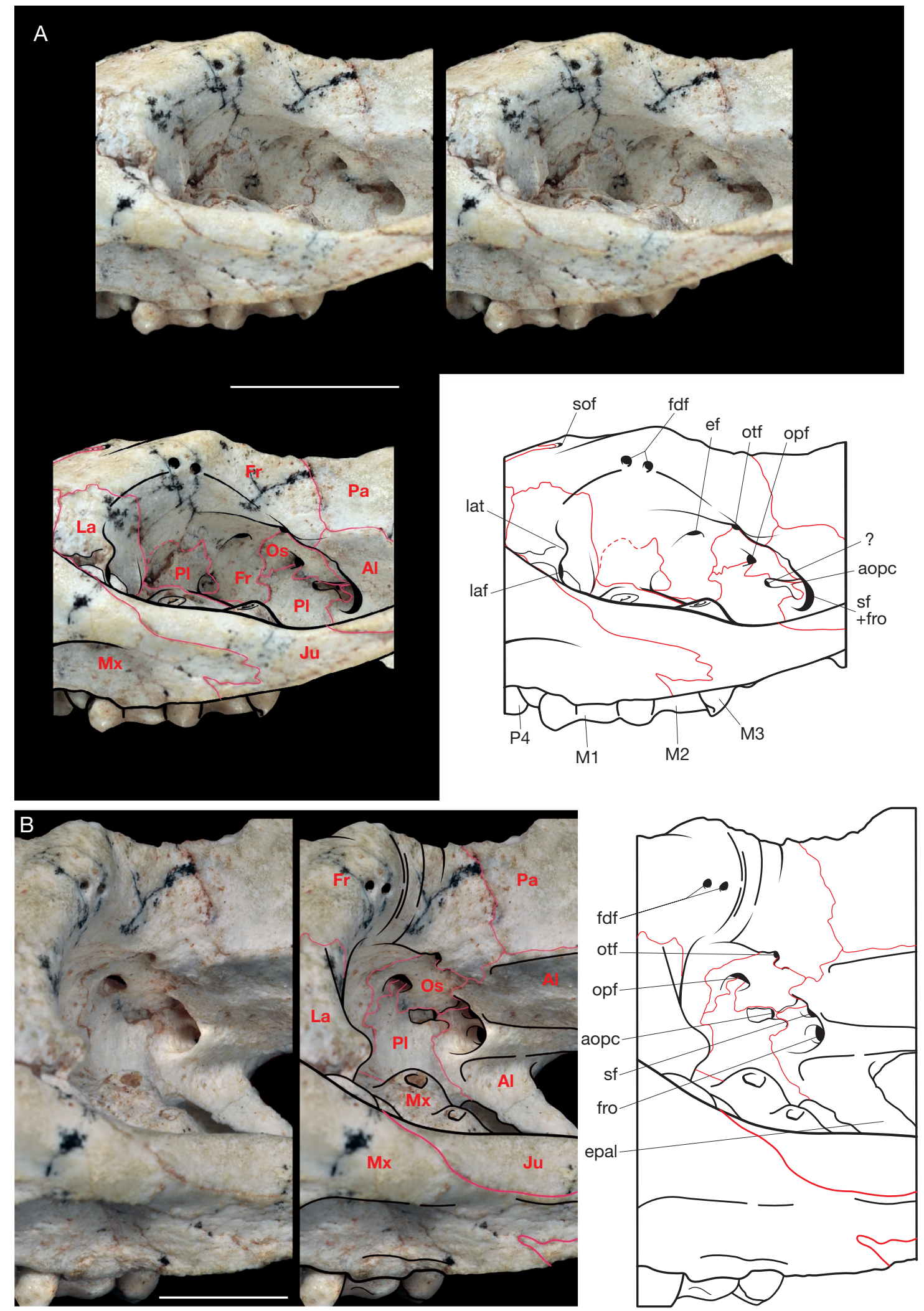

FIG. 24. - Anterolateral view of the orbitotemporal fossa of Alcidedorbignya inopinata showing the relationships of the palatine, alisphenoid, and frontal and the anterior opening of the pterygoid canal (stereophotographs, schematic drawing with bone sutures on photo, and schematic drawing with captions): A, MHNC 8372 (left side) ; B, MHNC 8372 (left side) with a slightly more anterior orientation in order to see the septum between the sphenorbital fissure and the foramen rotundum. Abbreviations: Al, alisphenoid; aopc, anterior opening of the pterygoid canal; ef, ethmoidal foramen; epal, ectopterygoid process of the alisphenoid; fdf, frontal diploic foramina; Fr, frontal; fro, foramen rotundum; Ju, jugal; La, lacrimal; laf, lacrimal foramen; lat, lacrimal tubercle; Mx, maxilla; Os, orbitosphenoid; opf, optic foramen; otf, orbitotemporal foramen; Pa, parietal; PI, palatine; sof, supraorbital foramen; sf, sphenorbital fissure. Scale bars: A, $1 \mathrm{~cm}$; B, $5 \mathrm{~mm}$. 

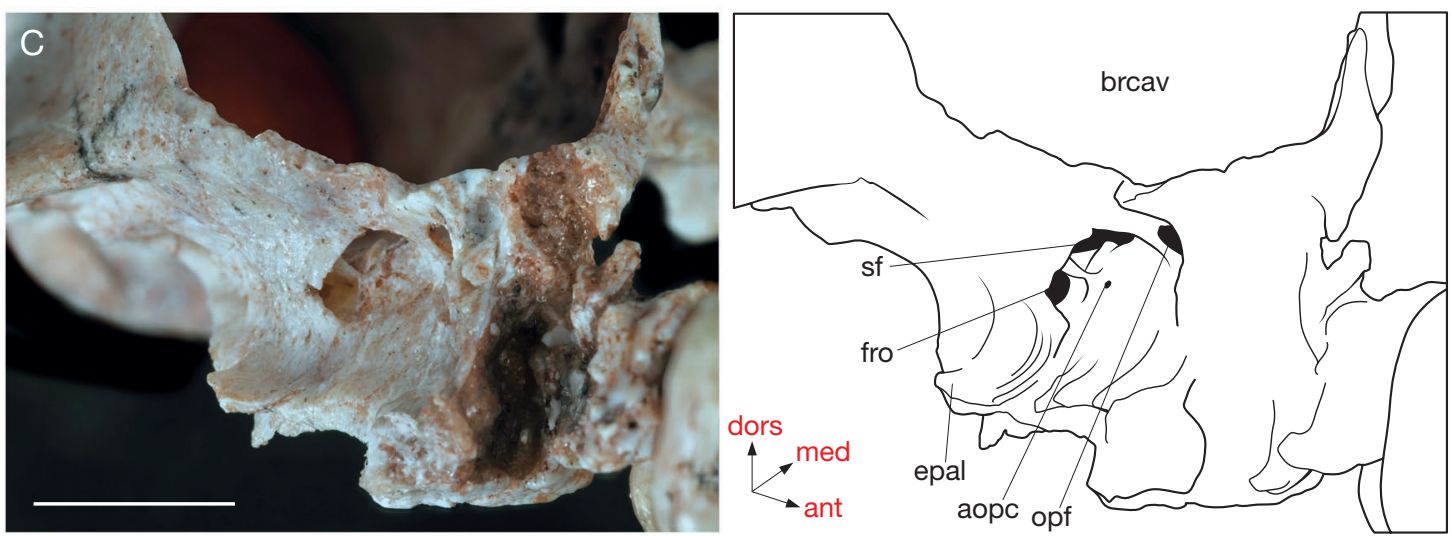

FIG. 24 (continuation). - Anterolateral view of the orbitotemporal fossa of Alcidedorbignya inopinata showing the relationships of the palatine, alisphenoid, and frontal and the anterior opening of the pterygoid canal (stereophotographs, schematic drawing with bone sutures on photo, and schematic drawing with captions): C, MHNC 8399 (right side). Abbreviations: ant, anterior; aopc, anterior opening of the pterygoid canal; brcav, brain cavity; dors, dorsal; epal, ectopterygoid process of the alisphenoid; fro, foramen rotundum; med, medial; opf, optic foramen; sf, sphenorbital fissure. Scale bar: C, $5 \mathrm{~mm}$.

Caenolambda, Haplolambda, Coryphodon). This condition is not as obvious in Bemalambda because of the development of a prominent sagittal crest.

The whole naso-frontal suture is W-shaped and follows the morphology of the posterior and lateral processes of the nasal (the suture is described above, in the nasal section) (Figs 10A; 11A). In the sagittal plane, the frontals bear a broad anterior process that protrudes anteriorly between the posterior processes of the nasals. At the anteromedial angle of each frontal is a tiny spur, which penetrates even deeper between the nasals. A broad anterior process of the frontals is present in many other pantodonts (Pantolambda, Haplolambda, Bemalambda) but the anterior spur of Alcidedorbignya is apparently absent in other pantodonts. The frontal-maxillary suture is limited (Fig. 20), a condition which is correlated with the width of the posterior end of the nasals and the relatively wide exposure of the lacrimal on the face. As a consequence, the lacrimalmaxillary suture is longer.

Posteriorly, in the sagittal plane, the frontals have a narrow posterior process. The two processes form an isosceles triangle, which is wedged between the parietals. The posterior processes of the frontals are approximately three times longer and wider at base than the anterior spurs. Sagittal posterior processes of the frontals are also present in Pantolambda, but in this genus they are much shorter and more slender than in Alcidedorbignya.

The frontal has a large orbital process, which descends ventrally within the orbit. This descending process extends ventrally to the lowest part of the orbit and either reaches or almost reaches the sphenopalatine foramen (see above). This descending orbital process has an anteroposterior length that represents one-third of the total length of the frontal on the skull roof. It is located approximately at mid-length of the frontal anteroposterior extension. In the middle region of the descending orbital process is a large ethmoidal foramen, which opens posteroventrally, well anterior and slightly dorsal to the optic foramen. The ethmoidal foramen is totally enclosed in the frontal, in the middle of the descending orbital process, and well separated from the frontal-orbitosphenoid suture (Figs 20, 25). The condition in Pantolambda is difficult to determine because of the crushing of the bones of the orbitotemporal fossa of AMNH 16663, which was available during this study. In this specimen a small foramen, anterodorsal to the optic foramen, is probably the ethmoidal foramen and opens in the orbital process of the frontal. The condition of other pantodonts could not be checked during this study.

The descending orbital process of the frontal articulates with the ascending process of the palatine anteriorly and ventrally, and with the orbitosphenoid posteriorly. The posterolateral part of the frontal within the orbitotemporal fossa has a small contact with the alisphenoid and, medial to this point, has a long and anteriorly concave suture with the parietal.

There is no well-developed postorbital crest or processes, but rather a tuberosity that overhangs the posterodorsal region of the orbit. It is located above and slightly posterior to the root of the protocone of M2, which is visible in the orbital floor. This postorbital tuberosity is quite anteriorly located within the orbitotemporal fossa, and delineates a reduced anteroposterior extent of the orbit, as in Pantolambda and Haplolambda. Two small foramina are present on the posterodorsal edge of the orbit slightly anterior to the postorbital tuberosity (Fig. 20, 25). These foramina likely conveyed the frontal diploic vein, an emissary of the dorsal cerebral vein/dorsal sagittal sinus or a vein issuing from the frontal diploë (Thewissen 1989; Evans \& de Lahunta 2012; Wible et al. 2004). These foramina are called supraorbital foramina by Novacek (1986) and Marshall \& Muizon (1995), frontal foramina by Evans \& de Lahunta, (2012), foramina for the frontal diploic vein by Wible \& Rougier (2000), Wible (2003, 2011) and Wible et al. (2009), and frontal diploic foramina by Thewissen (1989). In this paper we retain the terminology "frontal diploic foramen". The frontal generally bears a single foramen but in Leptictis two foramina are present (Novacek 1986), as is observed in Alcidedorbignya. The posterior frontal diploic foramen of Alcidedorbignya is slightly lower than the anterior on the 


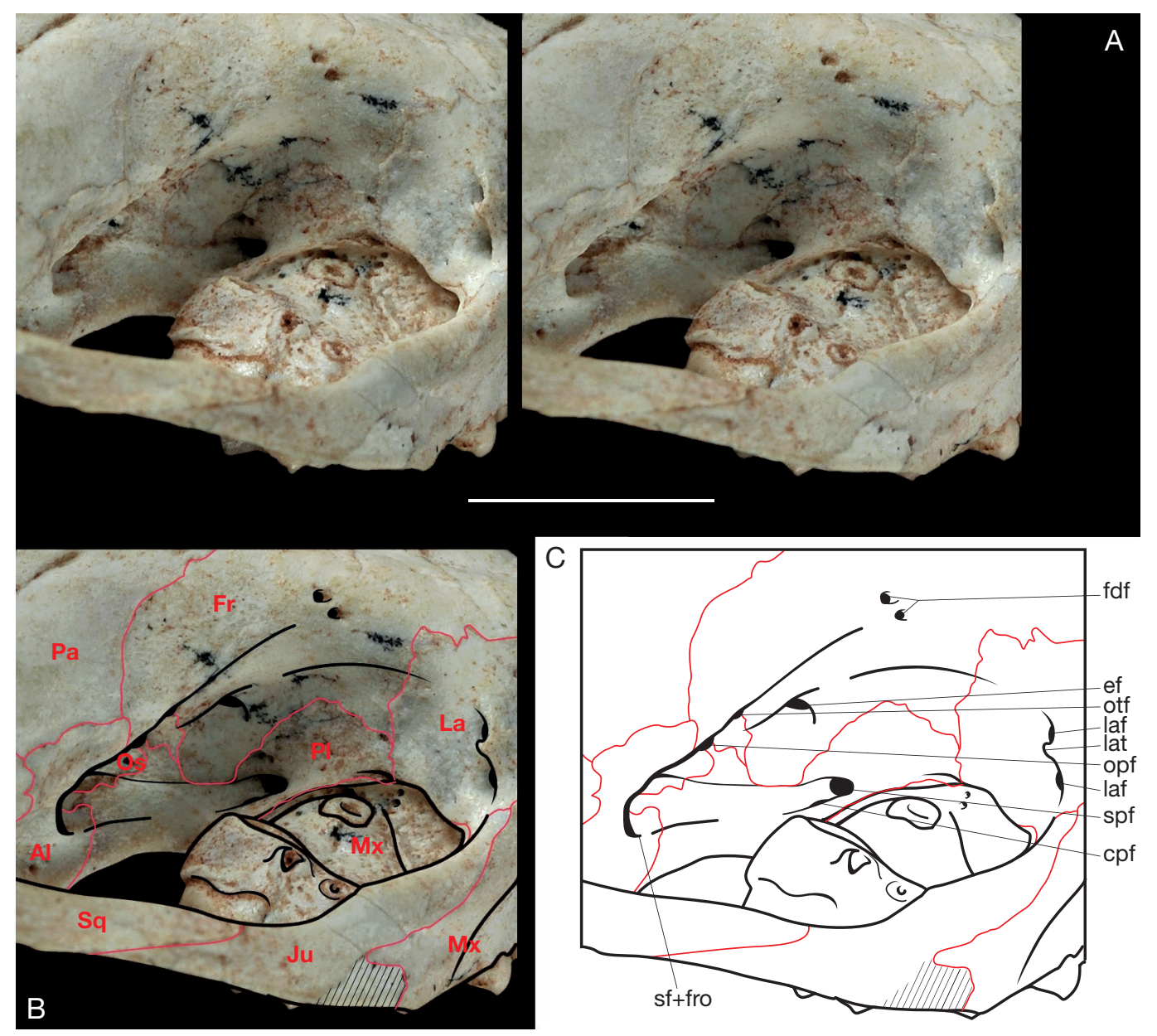

FIG. 25. - Posterolateral view of the orbit of Alcidedorbignya inopinata (MHNC 8372) showing the relationships of the palatine, maxilla, lacrimal and frontal: A, stereophotograph; B, schematic drawing with bone sutures on photo; C, schematic drawing with captions. Abbreviations: Al, alisphenoid; cpf, caudal palatine foramen; ef, ethmoidal foramen; fdf, frontal diploic foramina; Fr, frontal; Ju, jugal; fro, foramen rotundum; La, lacrimal; laf, lacrimal foramen; lat, lacrimal tubercle; Mx, maxilla; Os, orbitosphenoid; opf, optic foramen; otf, orbitotemporal foramen; Pa, parietal; PI, palatine; sf, sphenorbital fissure; spf, sphenopalatine foramen; Sq, squamosal. Scale bar: $1 \mathrm{~cm}$.

left side, while this condition is reversed on the right side. Both foramina open anteriorly. However, the number of these foramina is variable in our sample of Alcidedorbignya skulls: only one frontal diploic foramen is present on the left side of the juvenile skull MHNC 8416, while on the other juvenile MHNC 8423 two foramina are present on the right side and three on the left side. Two foramina are present on the adult skull MHNC 8399 one on each side. Postorbital tuberosities and frontal foramina are possibly present in Pantolambda, while eminences or processes are well developed in Caenolambda, Haplolambda, Bemalambda, and Harpyodus decorus.

Anteriorly, close to the posterior or frontal process of the nasals, are well-developed supraorbital foramina (Barone 1997). On the right side of MHNC 8372 are two (or three - see below) anteriorly-opened foramina. The medial foramen is in the naso-frontal suture and extends anteriorly in a shallow groove that reaches the nasal foramen. Immediately lateral to this foramen is another supraorbital foramen, which pierces the frontal only. It extends anteriorly in a short groove, which apparently joins the groove of the medial foramen. Posterolateral to the second foramen is a third foramen located approximately mid-way between the edge of the orbit and the naso-frontal suture. It is absent on the left side of the specimen. We are doubtful that this foramen conducted any large vessels or nerve and hence should be regarded as a supraorbital foramen and rather consider it as a nutrient foramen. On the left side of MHNC 8372 a single large anteriorly opening supraorbital foramen is present. It opens just posterior to the naso-frontal suture at the apex of the posterior process of the nasal. It also extends anteriorly on the dorsal surface of the nasal in a shallow groove, which vanishes at the level of the nasal foramen of the right nasal. As observed on a CT scan of MHNC 8372, these foramina extend posteriorly in a canal, which vanishes posteriorly in the diploë or spongy structure of the frontal, as is observed in the case of the more posteroventrally located frontal diploic foramina. On MHNC 8399 a single foramen is present on the right side, which opens in the frontal, and two are present on the left side, of which only the medial one opens in the naso-frontal suture. Two supraorbital foramina are observable on the juvenile skull 


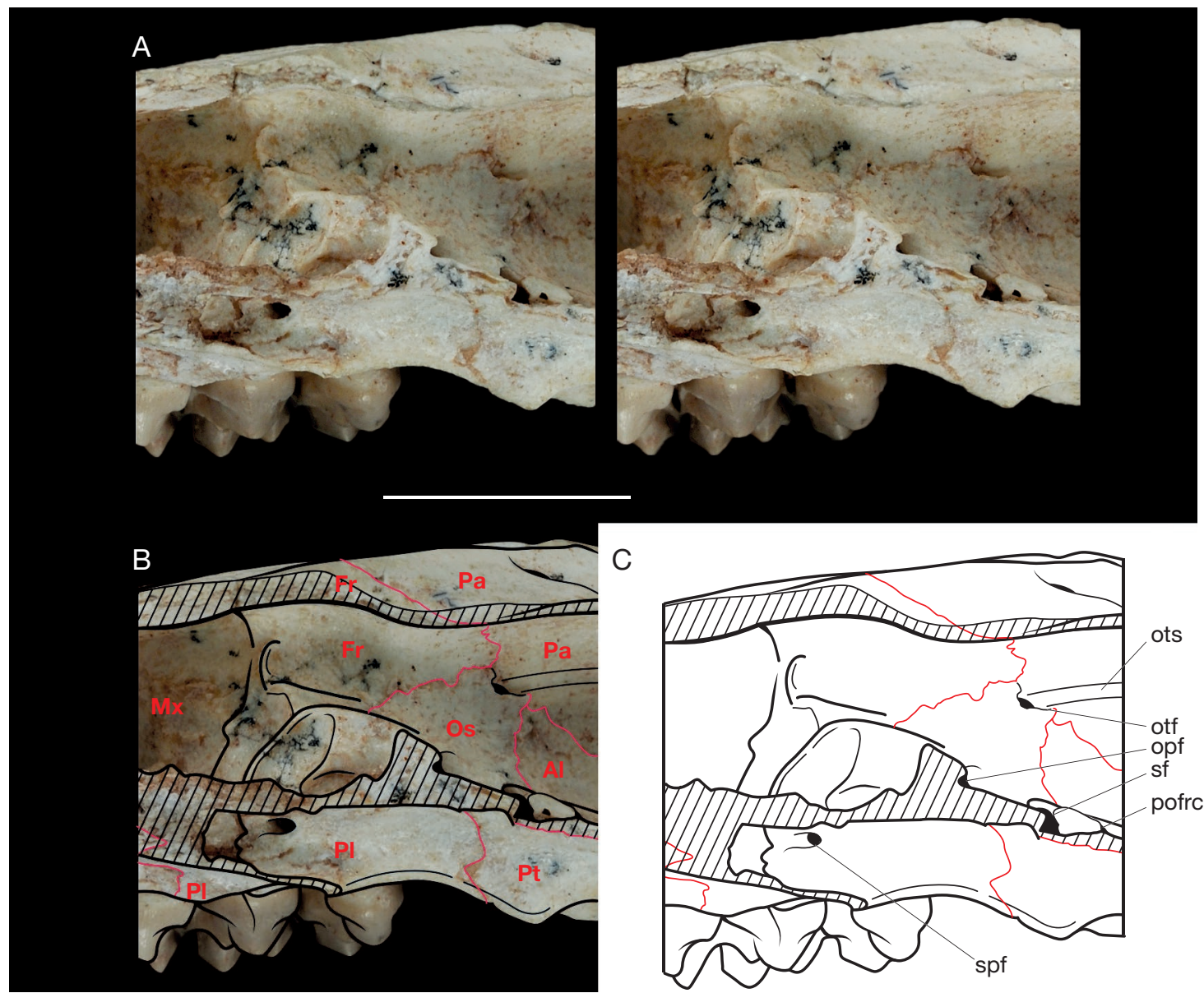

FIG. 26. - Internal view of the skull of Alcidedorbignya inopinata showing the opening of the sphenopalatine canal in the nasal cavity (MHNC 8372). A, stereophotograph; B, schematic drawing with bone sutures on photo; C, schematic drawing with captions. Abbreviations: Al, alisphenoid; Fr, frontal; Ju, jugal; fro, foramen rotundum; La, lacrimal; Mx, maxilla; Os, orbitosphenoid; opf, optic foramen; otf, orbitotemporal foramen; ots, orbitotemporal sulcus; Pa, parietal; PI, palatine; pofrc, posterior opening of the foramen rotundum canal; Pt, pterygoid; spf, sphenopalatine foramen; sf, sphenorbital fissure. Scale bar: $1 \mathrm{~cm}$.

MHNC 8423, a medial one in the naso-frontal suture and a lateral one, as on MHNC 8372, but in the frontal. At least two supraorbital foramina are observed on both sides of the juvenile skull MHNC 8416. Two supraorbital foramina are present on both sides in Pantolambda (AMNH 16665) and are located in the frontal slightly posterior to the naso-frontal suture.

Jugal. The jugal is a relatively thick bone especially in its anterior portion, where it is slightly concave dorsally (in lateral view) (Figs 17, 18). This concavity is distinctly more pronounced in Pantolambda, while the bone is almost straight in Haplolambda, Coryphodon, Barylambda, and Titanoides (Simons, 1960). Its anterior articulation with the maxillary is bifurcated and has been described above in the maxillary section. The anterodorsal branch is much longer than the anteroventral and reaches the lacrimal anteriorly, just posterolateral to the lacrimal foramen. The anteroventral branch is short and is level with the metacone of M2. In its middle portion, just posterior to the apex of the ventral jugal process of the maxilla, the jugal has a roughly semicircular section. It is almost flat laterally and convex medially (MHNC 8372 and 8400). The height of the jugal reduces progressively posteriorly and the bone becomes very low where it contacts the zygomatic process of the squamosal. The posterior end of the jugal is very thin and extends almost until the glenoid cavity, but does not reach it (MHNC 8372, 8399, and MHNC 8400). It does however reach the level of the anterolateral edge of the transverse posterior root of the zygomatic arch. The overall morphology of the jugal of Alcidedorbignya is very similar to that of Pantolambda.

Parietal (Figs 27-29). The parietal forms slightly more than half of the length of the dorsal surface of the skull. The major characteristic of the parietals is the conspicuous sagittal crest formed at the junction of the two bones. The crest is present all along their suture. At the anterior end of the parietal is a well-developed anterior process. The anterior processes of the parietals diverge and between them are wedged the posterior processes of the frontals. This condition is similar to that of Pantolambda. The parietals form the dorsal and anterolateral roof of the braincase and their anterior end is at the level of the contact between M2 and M3 on MHNC 8372. In its anterior and middle portion the parietal of Alcidedorbignya is strongly convex laterally, while it is flat or even slightly con- 

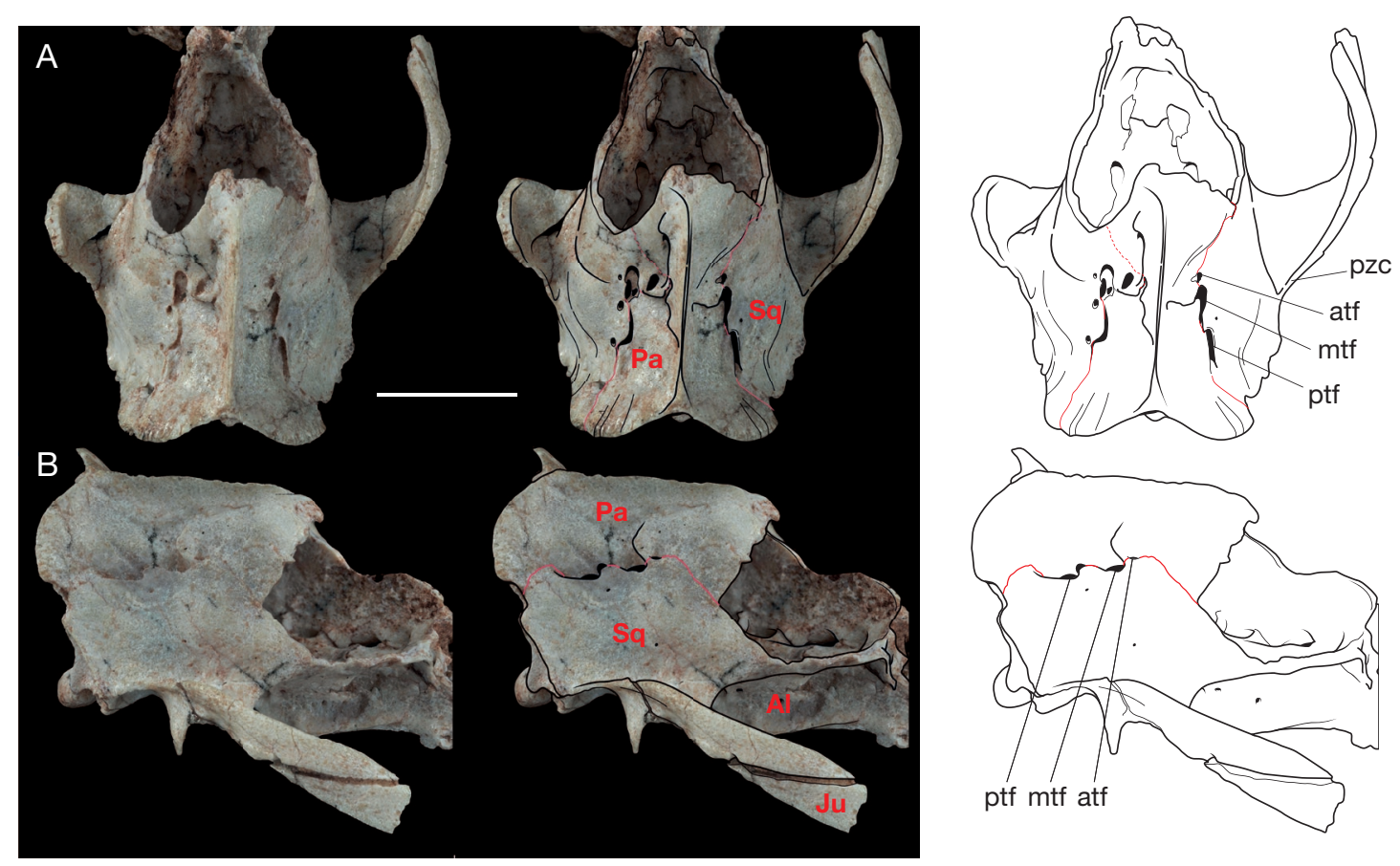

FIG. 27. - Dorsal and lateral views of the parietal of Alcidedorbignya inopinata (MHNC 8399) showing the temporal foramina (photograph, schematic drawing with bone sutures on photo, and schematic drawing with captions): A, dorsal view; B, lateral view. Abbreviations: Al, alisphenoid; atf, anterior temporal foramen; Ju, jugal; mtf, median temporal foramen; Pa, parietal; ptf, posterior temporal foramen; pzc, postzygomatic crest; Sq, squamosal. Scale bar: $1 \mathrm{~cm}$.

cave in the other pantodonts. It has a long contact with the alisphenoid anteroventrally. The more posterior suture with the squama of the squamosal is strongly convex dorsally. The posterodorsal region of the parietal forms a deep fossa, thus indicating a powerful development of the temporal muscle. The depth of this parietal fossa is emphasized by the elevation of the nuchal crest. The development of the temporal muscle is probably related to the large temporal foramina (foramina for rami temporales of the stapedial artery; Wible \& Gaudin 2004; Giannini et al. 2006; Wible 2008) present in the parietal in the vicinity of the squamosal-parietal suture. On the two skulls there are three temporal foramina (anterior, median and posterior) on each side of the skull; however their size and morphology vary. On MHNC 8372 the anterior foramen is relatively small on the right side while it is at least three times larger on the left. The median foramen is three times larger than the anterior on the right side while it is three times smaller on the left. The posterior foramen is relatively small on the right side and approximately the size of the small anterior right foramen; on the left side the posterior foramen is large and approximately the size of the large anterior left foramen. All three foramina pierce the parietal but their lateral edge is very thin and appressed against the squamosal. On the right side of MHNC 8372 a small foramen is present dorsal to the anterior temporal foramen and approximately similar in size. CT-images show that it communicates with the canal leading to the previously described anterior temporal foramen. Thus, it conveyed a ramification of the anterior temporal vessels as is observed on the reconstruction of the temporal arteries (see below Figures 47 and 48). No such foramen is observed on the left side but a deep and wide groove is present in the same place. In MHNC 8399 temporal foramina are also present but the anterior pair is smaller than the median one and the posterior one is slit-like. Ventrolateral to these foramina, are other smaller accessory foramina piercing the squamosal. On the left side, three small foramina are present, each one posterolateral to the large parietal temporal foramen (Fig. 27). On the right side only one very small foramen is present, posterolateral to the median large parietal temporal foramen. On MHNC 8372 a single similar foramen is present only on the right side of the skull anterolateral to the posterior temporal foramen.

Dorsomedial to the large parietal temporal foramina is a large irregular fossa excavated in the parietal, which may have corresponded to a blood sinus supply for the temporalis muscle. Its development is variable since on MHNC 8372 the right fossa is clearly larger than the left one. On MHNC 8399 the two fossae are equally developed.

The canals of the temporal foramina open internally into a single large cavity excavated between the parietal, squamosal and petrosal, dorsal to the foramen for the ramus superior of the stapedial artery in the petrosal, and at the level of the anterior region of the sulcus for the superior petrosal sinus on the petrosal, anterodorsal to the fossa subarcuata. This fossa is described below and named petrosquamosal fossa, in the petrosal section. This set of parietal temporal foramina is for the rami temporales of the stapedial artery. Generally, in eutherians, these rami arise from the arteria diploëtica magna, the anastomotic posterior branch of the ramus superior of the stapedial artery (sensu Wible 1987), which runs in the suture 

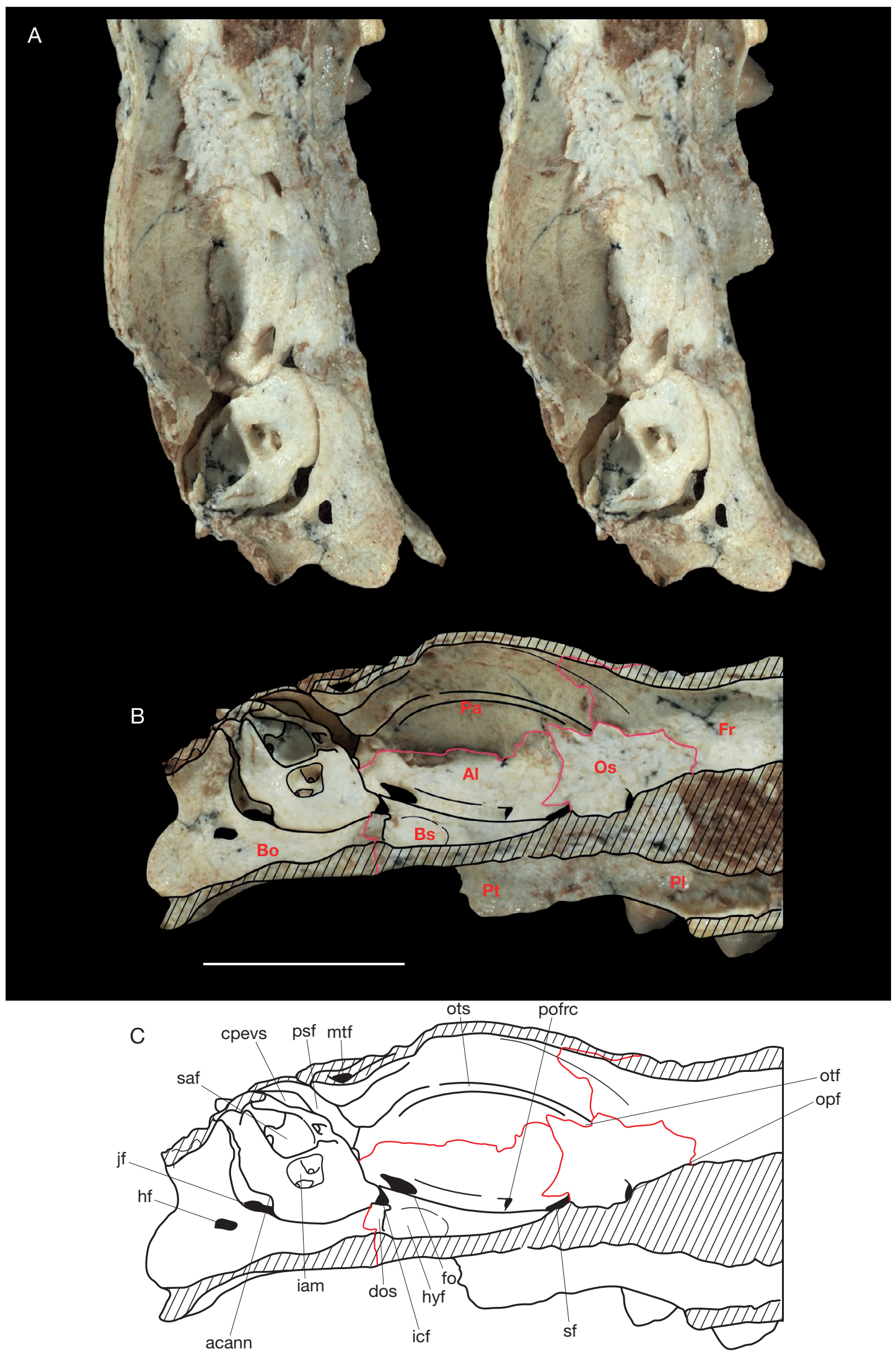

FIG. 28. - Medial view of the left internal aspect of the braincase of Alcidedorbignya inopinata (MHNC 8372), showing the internal composition of the wall of the skull: A, stereophotograph; $\mathbf{B}$, schematic drawing with bone sutures on photo; $\mathbf{C}$, schematic drawing with captions. Abbreviations: acann, notch housing the external aperture of the cochlear canaliculus; Al, alisphenoid; Bo, basioccipital; Bs, basisphenoid; cpevs, sulcus for the capsuloparietal emissary vein; dos, dorsum sellae; fo, foramen ovale; hf, hypoglossal foramen; hyf, hypophyseal fossa; iam, internal acoustic meatus; icf, internal carotid foramen; jf, jugular foramen; mtf, median temporal foramen; opf, optic foramen; Os, orbitosphenoid; otf, orbitotemporal foramen; ots, orbitotemporal sulcus; Pa, parietal; PI, palatine; pofrc, posterior opening of the foramen rotundum canal; psf, petrosquamosal fossa; Pt, pterygoid; saf, subarcuate fossa; sf, sphenorbital fissure. Scale bar: $1 \mathrm{~cm}$. 

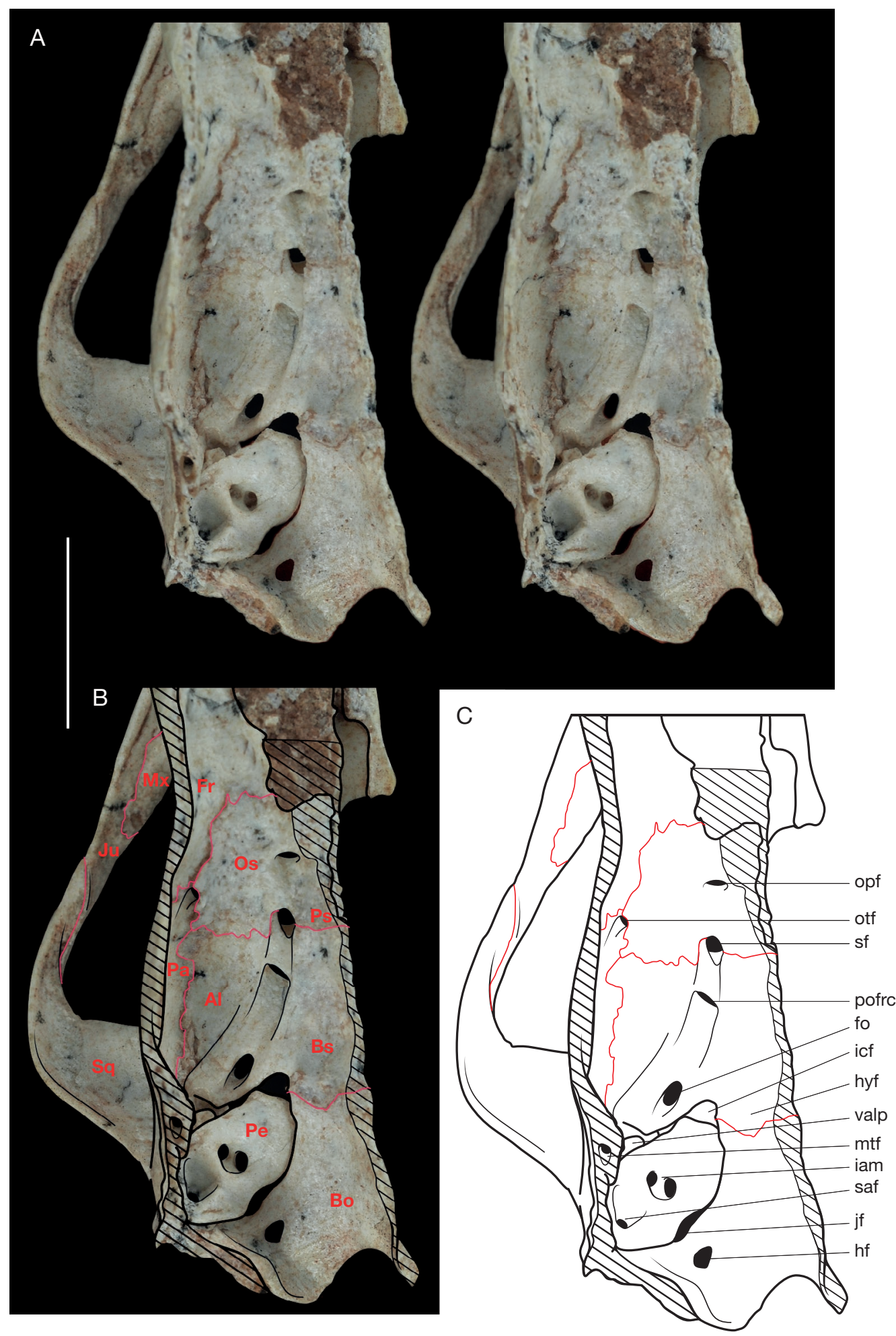

FIG. 29. - Dorsomedial view of the floor of the braincase of Alcidedorbignya inopinata (MHNC 8372), showing the course of the trigeminal nerve: A, stereophotograph; B, schematic drawing with bone sutures on photo; C, schematic drawing with captions. Abbreviations: Al, alisphenoid; Bo, basioccipital; Bs, basisphenoid; fo, foramen ovale; Fr, frontal; hf, hypoglossal foramen; hyf, hypophyseal fossa; iam, internal acoustic meatus; icf, internal carotid foramen; jf, jugular foramen; Ju, jugal; mtf, median temporal foramen; Mx, maxilla; opf, optic foramen; Os, orbitosphenoid; otf, orbitotemporal foramen; Pa, parietal; Pe, petrosal; pofrc, posterior opening of the foramen rotundum canal; Ps, presphenoid; saf, subarcuate fossa; sf, sphenorbital fissure; Sq, squamosal; valp, vestigial anterior lamina of the petrosal. Scale bar: $1 \mathrm{~cm}$.

between the squamosal and the petrosal (Wible et al. 2004, 2009). However, the CT-images clearly indicate that the rami temporales of Alcidedorbignya were not all emitted by the arteria diploëtica magna (the posterior division of the ramus superior of the stapedial artery). Instead, in both specimens, the anterior, at least, was emitted by the anterior division 
of the ramus superior, the orbitotemporal artery (see below Figures 47 and 48; Billet \& Muizon 2013). According to the course of its canal, which is visible on CT-images, the median ramus was probably variably emitted by the orbitotemporal artery (the anterior division of the ramus superior) (in MHNC 8399 ) or by the arteria diploëtica magna (the posterior division of the ramus superior) (in MNHC 8372). However, on the left side of MHNC 8372 (see below Fig. 47), the canals of the anterior and median foramina converge internally and the vessels they conveyed might therefore have been confluent. If this was the case, the single artery and vein resulting from this confluence are likely to have connected to the base of the sulcus for the orbitotemporal artery (the anterior division of the ramus superior), the orbitotemporal sulcus. This confluence has not been observed on the right side of the skull, where the median temporal foramen is closer to the inferred passageway of the arteria diploëtica (the posterior division of the ramus superior) magna than to that of the orbitotemporal artery (the anterior division of the division superior), and we favour (for the right side) a connection of the median temporal ramus to the arteria diploëtica magna rather than to the orbitotemporal artery. In MHNC 8399, although the anterior and median temporal foramina and associated canals are not confluent internally, they seem to both connect to the posterior origin of the sulcus for the orbitotemporal artery, as observed from CT-images. On both specimens the posterior ramus temporalis apparently arose from the base of the course of the arteria diploëtica magna just after its divergence from the ramus superior and before its entrance into the squamosal-petrosal suture (see below). Therefore, the condition in Alcidedorbignya may have been variable in the fusion of the anterior and median temporal branches, but constant in the connection of the anterior branch to the orbitotemporal artery and of the posterior branch to the arteria diploëtica magna.

The accompanying veins of the rami temporales probably proceeded from the vena diploëtica magna, which has its origin in the capsuloparietal emissary vein (superior petrosal sinus of Novacek 1986) (see Wible 1993 and below in the petrosal section). In Pteropus, the veins accompanying the rami temporales connect to the capsuloparietal emissary vein anteriorly and the vena diploëtica magna posteriorly in the post-temporal canal (Giannini et al. 2006). In Pantolambda the foramina for rami temporales are present but relatively much smaller than in Alcidedorbignya and pierce the squamosal externally. Numerous foramina are also present on the parietal and/or squamosal of Caenolambda, Barylambda and Titanoides, which could be nutrient foramina or true foramina for the rami temporales.

On the intracranial surface of the parietal is a long and ventrally bowed groove, which runs anteroposteriorly on its whole length: the orbitotemporal groove (Wible 2011), labelled ophthalmic sulcus in Novacek (1986). Posteriorly, it runs from a point dorsoanterolateral to the canal for the ramus superior (see below) and, anteriorly, it enters a canal (the orbitotemporal canal) at the triple junction of the parietal, frontal, and orbitosphenoid (Figs 28, 29). The orbitotemporal

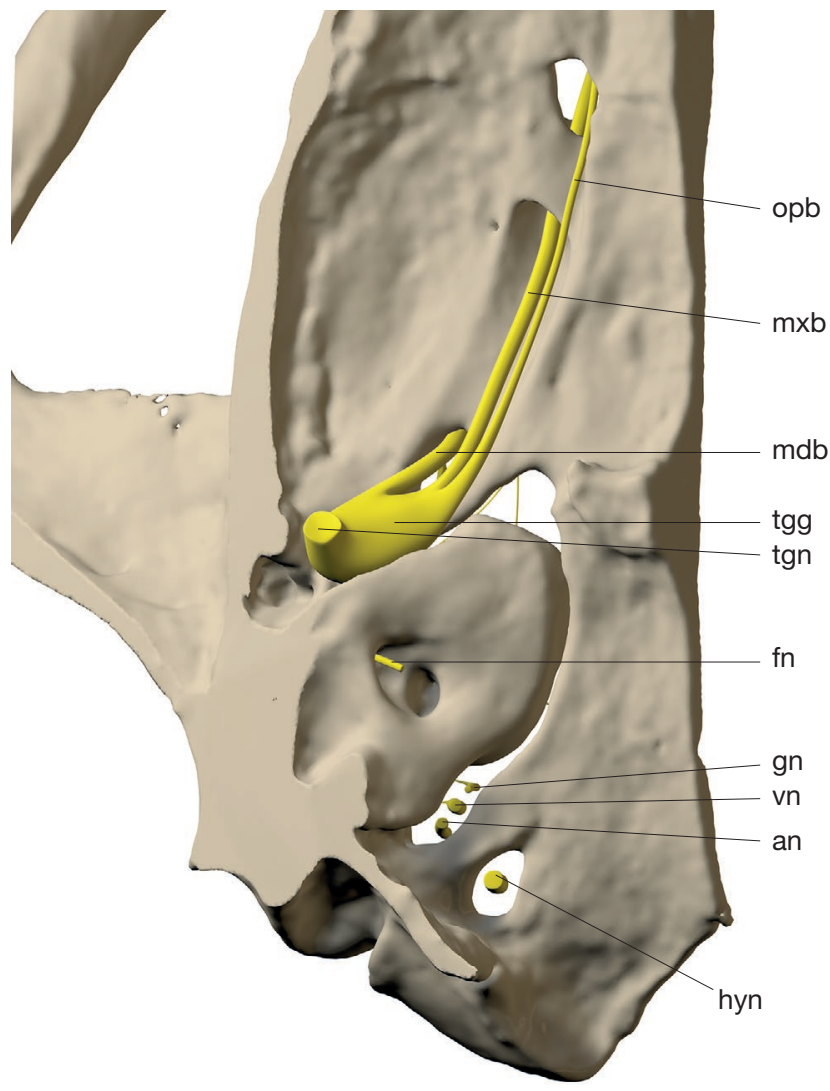

FIG. 30. - Reconstruction of the three branches of the trigeminal nerve (CN V) of Alcidedorbignya inopinata (MHNC 8372) on a surface rendering of CT data. Abbreviations: an, accessory nerve; fn, facial nerve (CN VII); gn, glossopharyngeal nerve; hyn, hypoglossal nerve; mdb, mandibular branch (CN V3); $\mathbf{m x b}$, maxillary branch (CN V2); opb, ophtalmic branch (CN V1); tgg, trigeminal ganglion; tgn, trigeminal nerve; vn vagus nerve. The $3 D$ rendering of $C T$ data is available in pdf format at the address http://sciencepress.mnhn.fr/en/periodiques/ geodiversitas/37/4/alcidedorbignya-inopinata-additional-files.

groove conveys the anterior division of the ramus superior of the stapedial artery or orbitotemporal artery and accompanying vein (Wible 1987, 2010; Billet \& Muizon 2013). The orbitotemporal artery exits the skull through the anterior foramen of the orbitotemporal canal, in the orbit in the suture between the frontal and the orbitosphenoid (see below). It is distinctly dorsal to and slightly anterior to the optic foramen and posterior to the ethmoid foramen (Figs 24, 25, 31, 32).

Posteriorly, on the dorsal side of the skull, the parietal does not cover the occipital crest totally and participates only in its medial two-thirds. The lateral rounded angle of the occipital crest is formed only by the occipital, with no parietal or squamosal contribution. This condition is clear on MHNC 8372 , in which the parietal-occipital suture is still visible (Figs 11, 12). It is not observable on MHNC 8399, in which the bones are fused (Fig. 27).

Squamosal. The squama of the squamosal is large. It is long and elevated, and forms more than half the height of the lateral wall of the braincase at the level of the posterior root of the zygomatic arch. It bears several small nutrient foramina, which are distinct (in their smaller size and irregular distribu- 
tion) from the large foramina for the rami temporales of the stapedial artery. Posteriorly, the squama articulates with the pars mastoidea of the petrosal in its ventral $80 \%$ and with the occipital at its posterodorsal angle. This condition is difficult to observe on MHNC 8399 because of the fusion of bones in this region of the skull. In both specimens, MHNC 8372 and 8399, the squamosal does not cover the anterior face of the rounded lateral angle of the occipital crest. As a consequence, the occipital outcrops dorsally on the posterodorsolateral angle of the skull. The posterior root of the zygomatic arch is excavated dorsally by a deep fossa, located at the posterior end of the orbitotemporal fossa, dorsal to the glenoid fossa. It is wider than long (slightly narrower on MHNC 8399 than on 8372) and forms a right triangle, the anterior edge of which is straight and perpendicular to the sagittal plane of the skull. In other words, the posterior extremity of the orbitotemporal fossa, in dorsal view, is relatively straight and transverse. A similar condition is present in Pantolambda, although the dorsal fossa of the posterior root of the zygoma is less excavated than in the Bolivian genus. The condition in Haplolambda differs, as the posterior root of the zygomatic arch is anteriorly tilted and oblique relative to the sagittal plane. The postzygomatic crest is the posterior extension of the dorsal edge of the zygomatic process of the squamosal (Novacek 1986: fig. 17). In Alcidedorbignya it is very salient and oblique, at an angle of approximately $45^{\circ}$ to the sagittal plane, in dorsal view. It extends posteriorly above the postglenoid process and the anterior part of the external auditory meatus. It extends posterodorsally and fades on the posterior region of the squama but does not reach the occipital crest. The condition of Alcidedorbignya is similar to that of Pantolambda but differs from that of Haplolambda, in which the dorsal fossa of the posterior root of the zygomatic arch is more elongated anteroposteriorly (clearly longer than wide), with a deeply concave anterior edge, rather than roughly straight and transverse as in Alcidedorbignya, and the postzygomatic crest is continuous with the occipital crest. The zygomatic process of the squamosal of Alcidedorbignya is relatively straight and slender. It tapers regularly anteriorly and has a long suture with the jugal on more than two-thirds of its length.

In the dorsal fossa of the posterior root of the zygomatic arch and in the ventral region of the squama adjacent to this fossa are numerous small to medium-sized vascular foramina. There are nine medium-sized foramina in the left fossa and five in the right in MHNC 8372. On the left squama of MHNC 8372, approximately nine medium-sized foramina are present, while there are five on the right side. Ten to 15 small foramina are also present on both sides. Such foramina are also present in MHNC 8399 but less abundant. Some of them, as we have observed on CT images, likely transmitted vessels from the arteria diploëtica magna for irrigation of the temporalis muscle. However, in order to restrict the use of "foramina for rami temporales" to the three large openings in the vicinity of the parietal-squamosal suture we regard these foramina as accessory foramina of the temporalis complex (Wible \& Gaudin 2004; Giannini et al. 2006), even if most of them did not transmit vessels supplied by the stapedial system, but probably represent squamosal nutrient foramina receiving tiny vessels from the external carotid artery system (Giannini et al. 2006; Evans \& de Lahunta 2012).

Ventrally, the glenoid fossa is flat on its anterolateral half. It is slightly excavated posteromedially, a condition, which is accentuated by the anterior concavity of the subvertical postglenoid process (Wible et al. 2009; retroarticular process in Evans \& de Lahunta 2012). The latter is higher than wide and slightly concave anteriorly. On MHNC 8372 it borders the posterior edge of the medial half of the glenoid articular surface. It is in a more median position on MHNC 8399. Medial to the medial edge of the postglenoid process is a large postglenoid foramen (Wible et al. 2009; retroarticular foramen in Evans \& de Lahunta 2012), for the passage of the capsuloparietal emissary vein (vena emissaria capsuloparietalis; Gelderen 1924), which connects the transverse sinus to the postglenoid vein (retroarticular vein in Evans \& de Lahunta 2012). The posteromedial edge of the foramen is thickened and presents a small, elongated eminence (it is sharper on MHNC 8399, probably because of better preservation). This postglenoid eminence (new term) forms the lateral border of a wide sulcus, the fissura Glaseri (fissura petrotympanica, NAV), which transmits the chorda tympani and, often (but not in the case of Alcidedorbignya and Pantolambda - see below in the petrosal section), the ramus inferior of the stapedial artery (Klaauw 1931:164; MacPhee 1981; Novacek 1986; Giannini et al. 2006; Wible 2008). The chorda tympani is a branch of the facial nerve (CN VII), which exits the middle ear via the fissura Glaseri and connects to the lingual nerve (a branch of the mandibular nerve - CN V3) in the orbitotemporal fossa (Evans \& de Lahunta 2012). The postglenoid eminence forms the anterior border of the dorsal part of the external auditory meatus, the ventral part being formed by the ectotympanic (see below). Given its position, it could bear the articulation for the lateral portion of the anterior crus of the ectotympanic (see below in Appendix 3; ectotympanic of Pantolambda). In fact, on MHNC 8399, which represents a slightly older individual than MHNC 8372, the bony structures are better marked and, on the medial edge of the eminence, at the anteroventral edge of the squamosal portion of the epitympanic recess, is a distinct elongated cupula, which could have received the articulation of the lateral portion of the anterior crus of the ectotympanic (Fig. 38C). The major axis of this cupula is anteroventrally-posterodorsally oriented. Such a position is identical to that seen in Pantolambda, which also bears a small postglenoid eminence posteromedial to the postglenoid foramen, and in which the medial edge of this eminence is excavated by a distinct concave facet (see below in Appendix 3) for the lateral part of the anterior crus of the ectotympanic (see below Figs 36-38, 124). Therefore, it is likely that the medial edge of the postglenoid eminence of Alcidedorbignya also received the lateral part of the anterior crus of the ectotympanic.

The medial edge of the fissura Glaseri is an elevated wall of the squamosal, which articulates anteriorly with another elevation of the alisphenoid. Both structures articulate tightly 


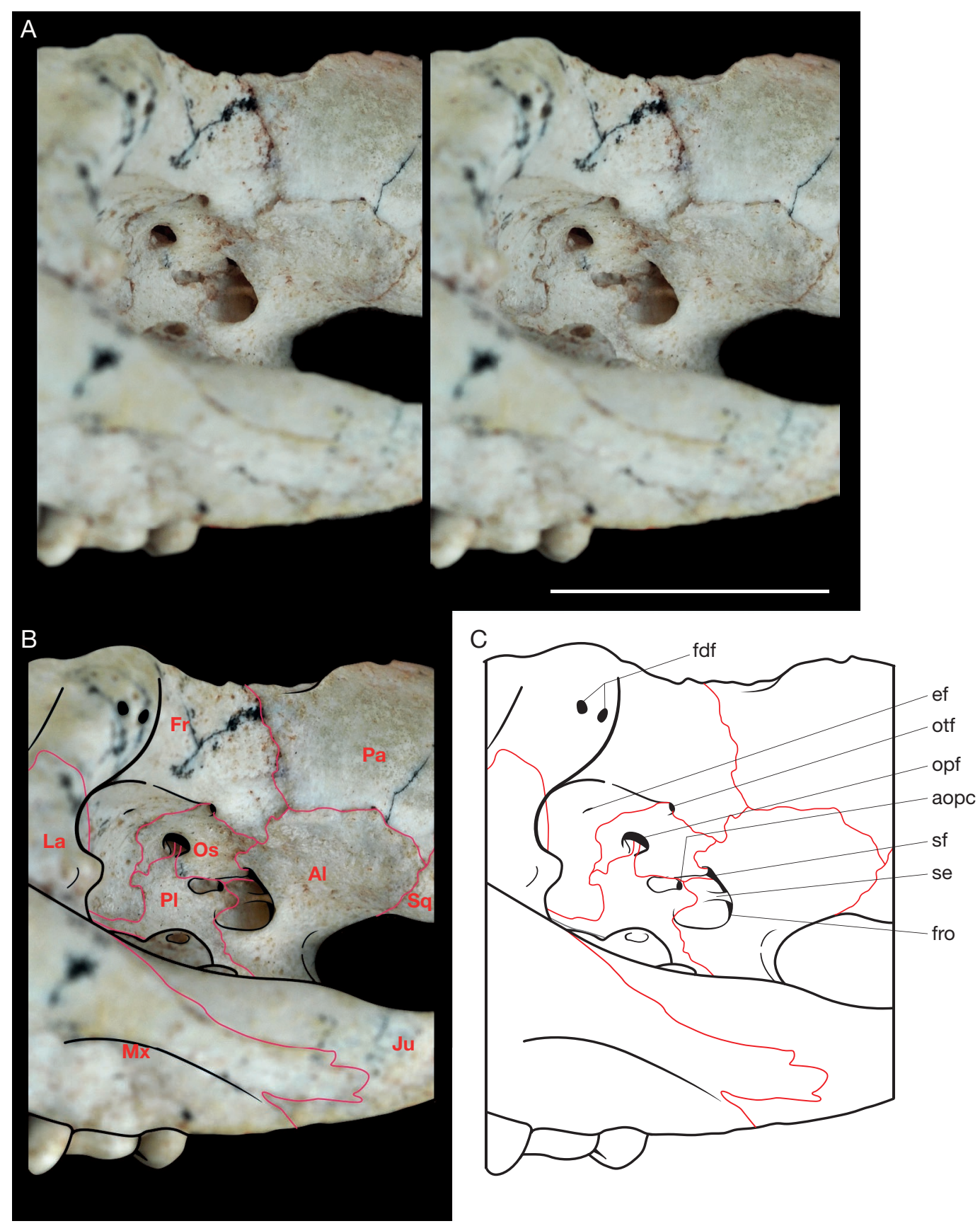

FIG. 31. - Anterolateral view of the orbitotemporal fossa of Alcidedorbignya inopinata (MHNC 8372) showing the foramina of the alisphenoid and orbitosphenoid: A, stereophotograph; B, schematic drawing with bone sutures on photo; C, schematic drawing with captions. Abbreviations: Al, alisphenoid; aopc, anterior opening of the pterygoid canal; ef, ethmoidal foramen; fdf, frontal diploic foramina; Fr, frontal; fro, foramen rotundum; Ju, jugal; La, lacrimal; Mx, maxilla; Os, orbitosphenoid; opf, optic foramen; otf, orbitotemporal foramen; Pa, parietal; PI, palatine; se, septum; sf, sphenorbital fissure; Sq, squamosal. Scale bar: $1 \mathrm{~cm}$.

and form the preotic crest (sensu McDowell 1958; Novacek 1986), which is slightly inflected medially. The anterior portion of the preotic crest is the tympanic process of the alisphenoid (MacPhee 1981) and the posterior is the entoglenoid process of the squamosal (McDowell 1958; Wible et al. 2004). A similar structure is present in Pantolambda and Leptictis although more massive in the former and more elevated in the latter (Novacek 1986). As is observed in Pantolambda the entoglenoid process of the squamosal received part of the medial articulation of the ectotympanic (see Appendix 3 for a description of the ectotympanic of Pantolambda, which permits an interpretation of the articular facets and position of the ectotympanic of Alcidedorbignya). The grooved space between the lateral articulation of the anterior crus of the ectotympanic (on the postglenoid eminence) and the entoglenoid process of the squamosal is the roof of the fissura Glaseri.

From the preotic crest, the squamosal-alisphenoid suture runs anteroposteriorly, dorsolateral to the foramen ovale and, 


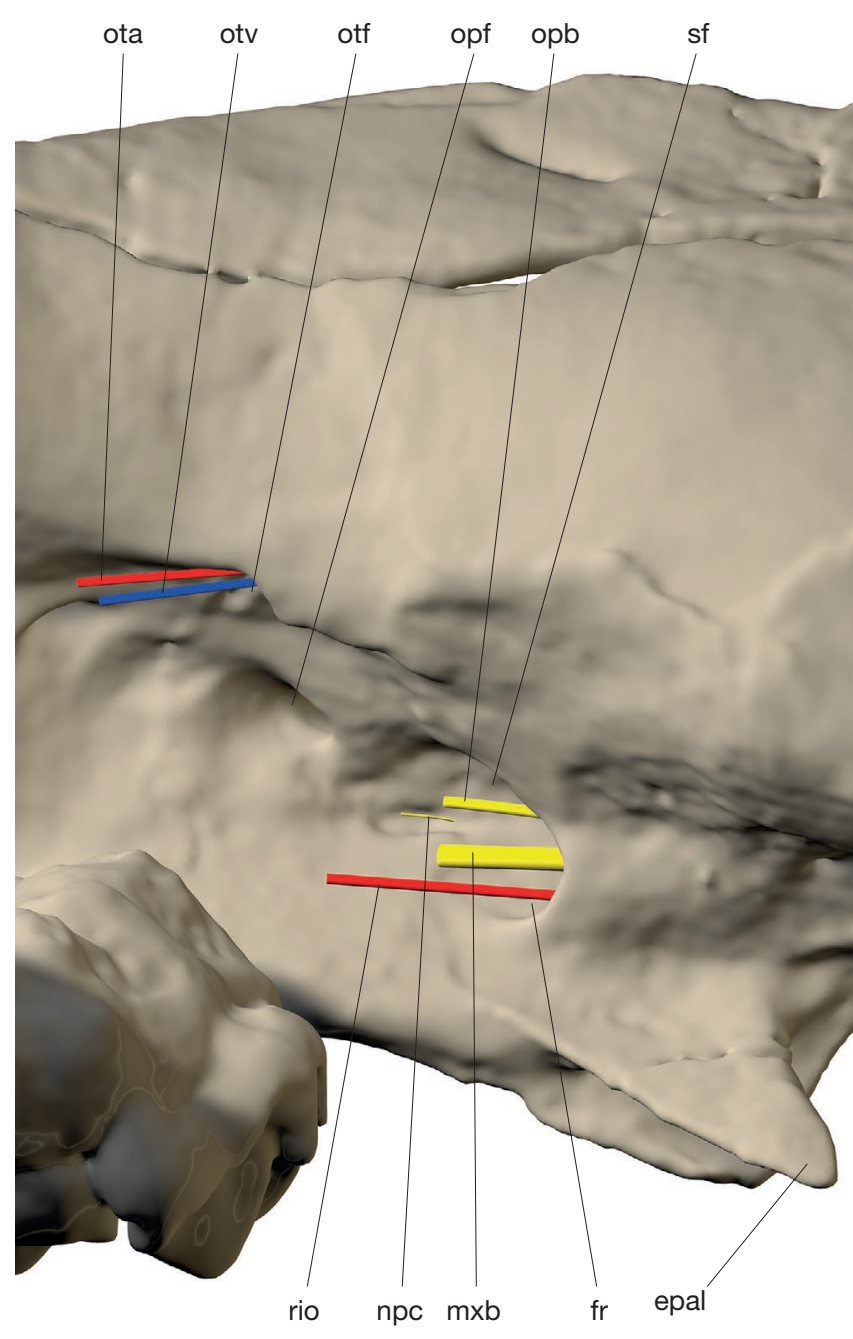

FIG. 32 - Three-dimensional rendering of CT data illustrating lateral view of the orbitotemporal fossa of Alcidedorbignya inopinata (MHNC 8372) showing reconstruction of vessels and nerves reconstruction. Abbreviations: epal, ectopterygoid process of the alisphenoid; $\mathbf{f r}$, foramen rotundum; $\mathbf{m x b}$, maxilla branch of the trigeminal nerve (CN V2); npc, nerve of the pterygoid canal opb, ophthalmic branch of the trigeminal nerve (V1); opf, optic foramen; ota, orbitotemporal artery; otf, orbitotemporal foramen; otv, orbitotempora vein; rio, ramus infraorbitalis; sf, sphenorbital fissure. The 3D rendering of $C T$ data is available in pdf format at the address http://sciencepress.mnhn.fr/en/ periodiques/geodiversitas/37/4/alcidedorbignya-inopinata-additional-files.

at the level of the ectopterygoid process of the alisphenoid, it turns dorsolaterally to reach the triple junction of the alisphenoid-squamosal-parietal.

Posterodorsal to the postglenoid eminence, the squamosal forms the lateral wall of the epitympanic recess in the middle ear cavity. The squamosal portion of the epitympanic recess is deeply excavated and is approximately subequal in volume to the corresponding portion of the petrosal, although slightly shorter (see the petrosal section for description of the epitympanic recess). The crest that forms the ventral edge of the squamosal portion of the epitympanic recess is the dorsal edge of the external acoustic meatus. Dorsal to it and on the lateral side of the squamosal is a subvertical area (more ventrolaterally facing in MHNC 8399 than in MHNC 8372), which is ventral to the postzygomatic crest and posterodorsal to the posterior face of the postglenoid process. This area corresponds to the suprameatal surface of the squamosal (Novacek 1986), which is excavated by a deep suprameatal fossa in Alcidedorbignya. The fossa is absent in the other pantodonts. Furthermore, in Pantolambda, the suprameatal surface is more oblique than in Alcidedorbignya (its orientation is less sub-vertical and it has a stronger horizontal component) as its lateral edge is only slightly dorsal to the medial edge, and in Haplolambda the surface is subhorizontal. The dorsal edge of the suprameatal surface is limited by a rounded crest (the suprameatal crest), which is distinct from, and ventral to, the posterior end of the postzygomatic crest on MHNC 8372. On MHNC 8399 the crests are not as distinctly separated but they clearly diverge posteriorly. The posterior edge of the suprameatal fossa is formed by the post-tympanic process of the squamosal, which is small and tightly appressed against the petrosal, especially against the mastoid process (see below for a definition of the mastoid process). This suprameatal surface does not present any distinct foramen that could be identified as a suprameatal foramen, as is observed, for instance, in many metatherians (Pucadelphys, Andinodelphys, Recent didelphids; Marshall \& Muizon 1995; Wible 2003, pers. obs. of CM and SL) and in some eutherians (e.g., Zalambdalestes, Maelestes, Leptictis, Solenodon [Novacek 1986; Wible et al. 2004, 2009]). However, on both sides of the skull of MHNC 8372, a tiny foramen located in a small fossula is present posterodorsal to the suprameatal surface, dorsal to the posterior end of the suprameatal crest, dorsal to the posttympanic process, and very close to the squamosal-petrosal suture. Nevertheless, some variation exists as no such foramen is observed in MHNC 8399. We have considered the possibility that this foramen could be a suprameatal foramen (Novacek 1986; Wible 2003; Wible et al. 2004). The suprameatal foramen is defined as a medium-sized to small opening perforating the suprameatal surface of the squamosal, below the suprameatal crest. It communicates with the postglenoid foramen and conveys a branch of the ramus posterior of the stapedial artery into the substance of the squamosal (Wible 2008, 2011). The suprameatal foramen may also transmit temporal rami from the ramus superior to the temporalis muscle as in didelphids, the xenarthran Euphractus and probably Zalambdalestes and Maelestes (Wible 2003; Wible \& Gaudin 2004; Wible et al. 2004, 2009). However, this tiny foramen in Alcidedorbignya is located above the suprameatal crest, whereas a suprameatal foramen should theoretically be located below this crest, within the suprameatal fossa. Because of its position, this foramen is not regarded as a suprameatal foramen. We have not been able to observe on the CT-scans a connection with the passageway of the rami temporales and we are inclined to regard this foramen as an accessory foramen of the temporalis complex (Wible $\&$ Gaudin 2004; Giannini et al. 2006), like those observed on the squama of the squamosal and in the fossa of the dorsal face of the posterior root of the zygomatic process (see above). Its absence on MHNC 8399, which indicates variation, reinforces this interpretation. Therefore, we consider that, on the basis of the available skulls, a well-identified suprameatal foramen of the squamosal is absent in Alcidedorbignya. The suprameatal foramen is also absent in all other pantodonts. 

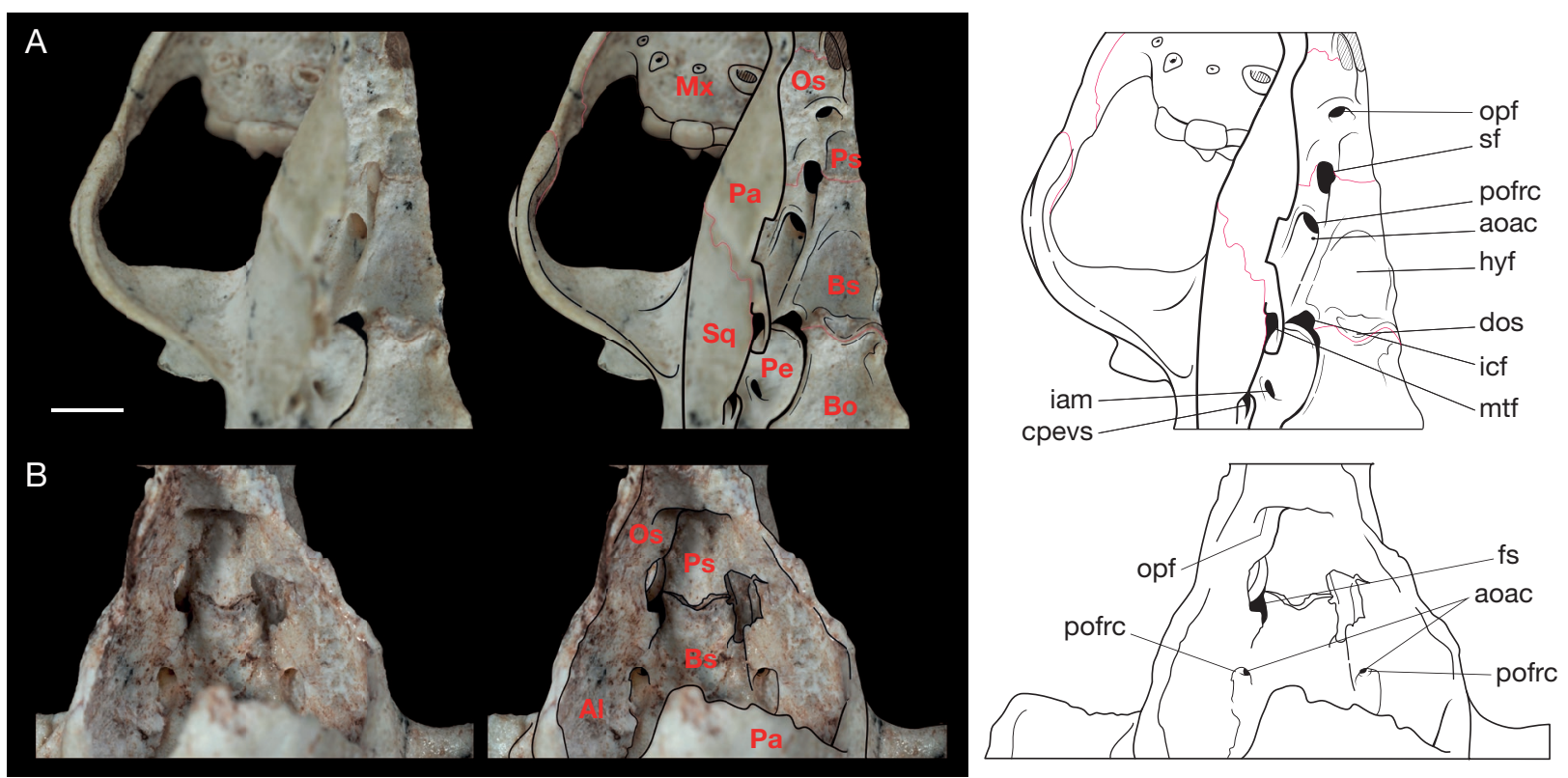

FIG. 33. - Intracranial dorsal view of the braincase of Alcidedorbignya inopinata showing the anterior opening of the alisphenoid canal in the ventromedial wall of the foramen rotundum canal (photograph, schematic drawing with bone sutures on photo, and schematic drawing with captions): A, MHNC 8372; B, MHNC 8399. Abbreviations: Al, alisphenoid; aoac, anterior opening of the alisphenoid canal; Bo, basioccipital; Bs, basisphenoid; cpevs, sulcus for the capsuloparietal emissary vein; dos, dorsum sellae; hyf, hypophyseal fossa; iam, internal auditory meatus; icf, internal carotid foramen; mtf, median temporal foramen; Mx, maxilla; Os, orbitosphenoid; opf, optic foramen; Pa, parietal; Pe, petrosal; pofrc, posterior opening of the foramen rotundum canal; Ps, presphenoid; Sq, squamosal; sf, sphenorbital fissure. Scale bar: $5 \mathrm{~mm}$.

Alisphenoid. The alisphenoid is generally poorly preserved in geologically old mammals and its sutures with adjacent bones are often extremely difficult to observe. In the specimens of Alcidedorbignya described here, the lateral wall of the braincase is perfectly preserved in MHNC 8372, and because the individual is a young adult the sutures are sharply distinct. In MHNC 8399, a slightly older individual, the sutures are partly obliterated and more difficult to observe. A comparison of this region with that of other pantodonts is also difficult because, in this group, the sutures of most of the bones in this region of the skull apparently fused and became indistinct relatively early in ontogeny.

The alisphenoid of Alcidedorbignya is large and forms the anteroventral wall of the braincase (Figs 23-25, 31). One of the major characteristics of the bone is observed in its anteroventral region. Here it is appressed against the lateral edge of the pterygoid and develops a large ventroposterolaterally oriented triangular blade, the ectopterygoid process of the alisphenoid (Novacek 1986; Giannini et al. 2006). This process is thin, sharp, and pointed at its apex. It is approximately as long as wide at the base (slightly shorter in MHNC 8399) and is relatively flat. At its posterolateral corner and medial to the glenoid fossa, the alisphenoid presents a salient subvertical process, the tympanic process of the alisphenoid, which articulates with the entoglenoid process of the squamosal and which forms the anteromedial wall of the preotic crest (sensu Novacek 1986). This tympanic process is anteroposteriorly oriented and bent medially, a condition that is less pronounced in MHNC 8399.
Anterolateral to the preotic crest is a large foramen ovale (NAV) for the passage of the mandibular ramus of the trigeminal nerve (CN V3) and the middle meningeal artery, a branch of the maxillary artery, which is, itself, part of the external carotid system (Figs 23; 34-39). The foramen ovale is ovalshaped, oblique and faces anteroventrally. Its posterior edge is a narrow bridge of bone, which forms the anterior border of the pyriform fenestra in the middle ear cavity and joins the base of the preotic crest to the posterior base of the pterygoid. Anteromedial to the foramen ovale and posterodorsal to the posterior base of the ectopterygoid crest is a smaller posteriorly facing foramen, the posterior foramen of the alisphenoid canal. In many eutherian mammals, it transmits, an anterior continuation of the ramus inferior of the stapedial artery, i.e. the ramus infraorbitalis (Wible 1987), the internal maxillary artery of Novacek (1986), or the maxillary artery of Evans \& de Lahunta (2012; see further details on this canal below). This foramen is oval-shaped and its size approaches one-third of that of the foramen ovale.

On the intracranial side of the alisphenoid, a deep groove runs forward from the foramen ovale to a large posteriorly facing foramen. The latter is the posterior and intracranial foramen of a short canal, which opens externally in the orbitotemporal fossa (Figs 28-32). The anterior opening of this canal is the foramen rotundum (Novacek 1986). It transmitted the maxillary ramus of the trigeminal nerve (CN V2) after its origin from the trigeminal ganglion (see below). It forms a common anteriorly-facing opening with the sphenorbital fissure in the orbitotemporal fossa, the foramen rotundum being ventrolateral to the sphenorbital fissure. The latter 


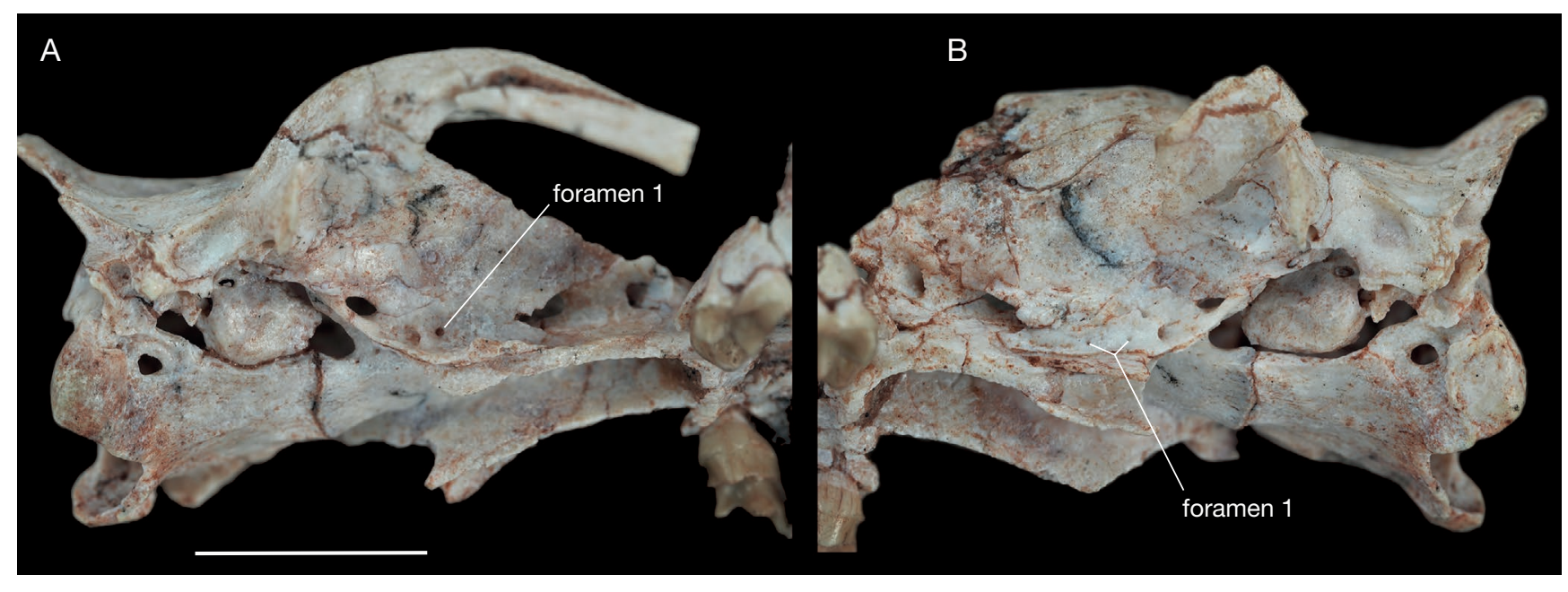

FIG. 34. - Ventrolateral view of the skull of Alcidedorbignya inopinata (MHNC 8399) showing the undetermined foramina discussed in text: A, right side; B, left side. Scale bar: $1 \mathrm{~cm}$.

presumably transmitted the oculomotor (CN III), trochlear (CN IV) and abducens (CN VI) nerves and the ophthalmic division (and also possibly remaining parts of the maxillary branch) of the trigeminal nerve (CN V1) (Novacek 1986). The two foramina, however, are not confluent but adjacent because they are clearly separated by a bony septum even though this separation is internal, being slightly posterior to the lateral wall of the common opening (Fig. 31). The opening of the foramen rotundum is posteroventrolateral to the sphenorbital fissure and is approximately of the same size to slightly smaller in diameter. Therefore, in Alcidedorbignya, the foramen rotundum appears as the anterior opening of a short canal ("foramen rotundum canal") individualized within a common opening with the sphenorbital fissure in the orbitotemporal fossa and observable from the inside of the braincase. This canal is exclusively excavated in the alisphenoid.

Intracranially, at the level of the ventromedial edge of the posterior opening of the "foramen rotundum canal" is a small anteriorly-opening foramen (best visible on the left side of skull of MHNC 8372 and on both sides of MHNC 8399) approximately the same size as the foramen identified above as the posterior opening of the alisphenoid canal (Figs 28-30). It is highly probable that the two foramina are the anterior and posterior openings of the same canal (the alisphenoid canal), which connects to the "foramen rotundum canal" and transmitted the ramus infraorbitalis of the stapedial artery and accompanying vein (Figs 32, 33) to the orbitotemporal fossa (Wible \& Zeller 1994; Wible 2011; Evans \& de Lahunta 2012). In the "foramen rotundum canal", these vessels were thus probably adjacent to the maxillary nerve (CN V2) and the three structures exited the skull through the foramen rotundum ventrolateral to the sphenorbital fissure. Therefore, the alisphenoid canal of Alcidedorbignya has a peculiar morphology because its anterior end opens within the braincase, on the medial edge of the foramen rotundum canal. This condition differs from that generally observed in other mammals (e.g., leptictids [Novacek 1986]). In Panto- lambda the posterior opening of the alisphenoid canal is in the same position as in Alcidedorbignya but it is much larger, even larger than the foramen ovale. The foramen rotundum of Pantolambda opens anteriorly and is nearly coalescent with the sphenorbital fissure, as in Alcidedorbignya. Moreover, because there is no anterior opening of the alisphenoid canal individualised in the orbitotemporal fossa of Pantolambda, it is possible that the anterior opening of the alisphenoid canal is in a similar intracranial position as in Alcidedorbig$n y a$, i.e. in the posteroventromedial region of the "foramen rotundum canal". Furthermore, contrary to the condition in Alcidedorbignya, the foramen rotundum of Pantolambda is much larger than the sphenorbital fissure being approximately twice its size. Therefore, in the Bolivian genus the small size of the posterior opening of the alisphenoid canal and of the foramen rotundum indicates relatively smaller vessels (ramus infraorbitalis of the stapedial artery and vein, maxillary branch of CN V2) than in Pantolambda.

In MHNC 8399, on the right alisphenoid just anterior to the posterior opening of the alisphenoid canal, is a small foramen approximately one-third to half the size of the former. A few millimetres anterior to this foramen, above the anterior end of the ectopterygoid crest, is another small foramen. On the left side of the specimen in a similar position are two symmetrical foramina, although the posterior one is slightly smaller than that of the right side (Fig. 34: foramen 1). Similar foramina are observed on the right side of MHNC 8372 (slightly more ventral relative to the posterior opening of the alisphenoid canal than in MHNC 8399) but surprisingly, they are absent on the left side, although two small nutrient (?) foramina are present in the anterodorsal region of the ectopterygoid crest. We have no interpretation for the specific vessels or nerve that may have been transmitted by the two variable foramina observed in MHNC 8399 and on the right side of MHNC 8372, although they may simply represent nutrient foramina. No such foramina have been observed in Pantolambda, nor described in other eutherian mammals to our knowledge. 
In MHNC 8372, anterodorsolateral to the posterior opening of the alisphenoid canal, is a small but conspicuous circular foramen. It is present on both sides although the right one is slightly smaller than the left (Fig. 35: foramen 2). The small size of the right foramen could be explained by some partial compression during fossilization. It opens perpendicular to the surface of the bone. These small foramina apparently have an opening within the braincase as two small slits are observed on the intracranial face of the alisphenoid at the level of the foramina. Such a foramen is possibly present on the left side of MHNC 8399, but we have not observed it on the right side, which could be an indication of individual variation. As for the above-mentioned foramen 1, we have no interpretation for this additional variable foramen. Again these foramina have not been observed in Pantolambda, nor (to our knowledge) have they been described in other eutherian mammals.

In the orbitotemporal fossa the alisphenoid has a large anterodorsolateral ascending expansion (the orbitotemporal wing), which widely contributes to the anteroventral wall of the braincase and reaches approximately mid-height of the lateral view of the skull (Fig. 31). It has long sutures with the parietal dorsally and the squamosal posteriorly, a short one with the frontal anteriorly, and a moderate one with the orbitosphenoid anteroventrally. Ventral to its orbitotemporal wing, the alisphenoid forms the lateral edge of the common anterior opening of the sphenorbital fissure + foramen rotundum and the lateral wall of the foramen rotundum canal. Ventral to this wall is a deep anteroposteriorly elongate fossa for the pterygoideus lateralis muscle (Barone 1997; Evans \& de Lahunta 2012). The first set of variable foramina described above (Figs 34 , 35) for the alisphenoid are also located in this fossa and could therefore have transmitted vessels for supply of these muscles. From this fossa, the ectopterygoid process of the alisphenoid projects ventrolaterally. Between the ectopterygoid process of the alisphenoid and the entopterygoid crest of the pterygoid is a deep and narrow fossa (Fig. 23) for the pterygoideus medialis muscle (Barone 1997; Evans \& de Lahunta 2012). The alisphenoid-pterygoid suture is not observable on MHNC 8372 because of the fusion of the bones, but is clearly visible in MHNC 8399 in the bottom of the fossa for the pterygoideus medialis muscle, which confirms Novacek's hypothesis that the alisphenoid-pterygoid suture of Leptictis was probably in the bottom of the medial pterygoid muscle fossa (Novacek 1986).

Orbitosphenoid. In lateral view, the orbitosphenoid is a relatively small bone, which forms the medial wall of the sphenorbital fissure and foramen rotundum posteriorly. On MHNC 8372, in this wall, anterior to the common opening of the two foramina, is a tiny anteriorly-oriented foramen. This probably corresponds to the anterior opening of the pterygoid canal, which transmits the pterygoid canal nerve (or Vidian nerve) to the pterygopalatine ganglion (Novacek 1986; MacPhee 1994; Wible 2008; Evans \& de Lahunta 2012) (Figs 24A, B; 31, 32). This foramen opens in a very short groove, which seems to penetrate the orbitosphenoidpalatine suture anteriorly: on both sides of MHNC 8372 a small foramen is visible at the anterior end of the groove. We

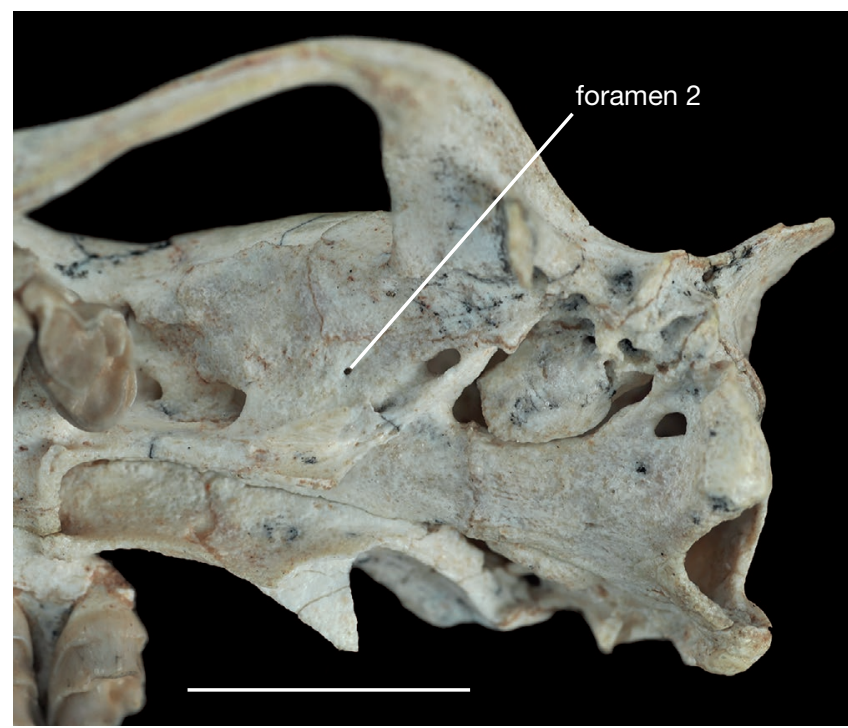

FIG. 35. - Ventrolateral view of the left side of the skull of Alcidedorbignya inopinata (MHNC 8372) showing the undetermined foramen discussed in text. Scale bar: $1 \mathrm{~cm}$.

have no interpretation for this opening. However, we believe that it cannot be for the pterygoid canal nerve because this nerve connects to the pterygopalatine ganglion located in the orbit. The anterior opening of the pterygoid canal is also clearly visible on the right side of MHNC 8399 (Fig. 24C). As in MHNC 8372, it is located slightly anterior to and at the level of the septum which separates the foramen rotundum ventrally and the sphenoidal fissure dorsally. On the left side of MHNC 8399 the septum between the foramina has been partly broken away and the lateral wall of the pterygoid canal is missing but the remaining medial part of the canal is still clearly visible.

Dorsal to the sphenorbital fissure, the orbitosphenoid has a moderately long posterior suture with the alisphenoid. The ventral edge of the bone is relatively straight and subhorizontal; it articulates with the alisphenoid posteriorly (within the sphenorbital fissure) and with the palatine on most of its anterior length. Anteriorly it contacts the descending process of the frontal, a bone with which it has the longest suture dorsally and anteriorly. The most elevated part of the orbitosphenoid is a small ascending process, which forms the ventromedial edge of the orbitotemporal foramen (see below) (Fig. 31).

In the centre of the bone, just below the orbitotemporal foramen is a large circular anteriorly-oriented optic foramen for the passage of the optic nerve (e.g., Wible 2011). This foramen, however, is distinctly smaller than the sphenorbital fissure. There is no suboptic foramen, which represents a plesiomorphic condition (Butler 1956; Novacek 1986).

On the intracranial side of the lateral wall of the braincase the orbitotemporal sulcus enters the suture between the orbitosphenoid and the frontal at a level slightly anterior to the anterior edge of the sphenorbital fissure (Fig. 28). This condition results in a canal running anteriorly between the orbitosphenoid medially and the frontal laterally. This ca- 
nal opens in the orbit through the orbitotemporal foramen (Rougier et al. 1992), at the dorsalmost point of the frontalorbitosphenoid suture. The orbitotemporal sulcus and canal conveys the anterior branch of the ramus superior of the stapedial artery, or orbitotemporal artery, which becomes the ramus supraorbitalis when entering the orbit (Wible 1987, 2010; Rougier et al. 1992; Billet \& Muizon 2013).

The posterior edge of the orbitosphenoid is partly covered by the alisphenoid externally. This condition is clearly observable on MHNC 8372, on the intracranial side of the braincase, in which the orbitosphenoid extends posteriorly at the level of the posterior edge of the sphenorbital fissure (Fig. 29). The intracranial portion of the orbitosphenoid is fused with the presphenoid in its ventromedial region, and covers the alisphenoid and parietal posteriorly. On the anterior three quarters of its length, the intracranial orbitosphenoid widely overlaps the frontal. The anterior extension of the intracranial orbitosphenoid reaches the posterior edge of the fossa for the olfactory lobe of the brain, which fills the space anterior to the postorbital constriction of the skull and posterior to the cribriform plate. It also reaches the posteroventral edge of the cribriform plate. In fact, the cribriform plate of MHNC 8372 is not preserved, but its articulation with the frontal is distinctly observable at the anterior end of the braincase, posterior to the nasal cavity.

Pterygoid. In Alcidedorbignya, the pterygoid is a laminary bone that is closely appressed against the medial side of the ectopterygoid process of the alisphenoid (Fig. 23). The bone extends as a straight continuation from the perpendicular process of the palatine anteriorly to a point medial to the anterior edge of the foramen ovale posteriorly. Most of the bone is made of a large triangular entopterygoid process, which is slightly larger than the ectopterygoid process of the alisphenoid. This process is concave laterally and ends ventrally in a sharp posteriorly projecting hamular process (pterygoid hamulus, NAV). The sutures of the pterygoid are not easily discernible on MHNC 8372 but are distinct on MHNC 8399. Medially, the pterygoid has a large suture with the palatine. This suture is visible in medial view of the lateral wall of the basipharyngeal canal. It is roughly straight and is oriented along an anterodorsal-posteroventral axis. In the roof of the basipharyngeal canal, most of the mediodorsal suture of the pterygoid is with the basisphenoid posteriorly. Anterodorsomedially, the pterygoid has a short suture with the presphenoid. This suture is approximately one fifth the length of the pterygoid-basisphenoid suture. Laterally, the pterygoid has a long suture with the alisphenoid posteriorly and a short one with the palatine anteriory at the anterior end of the ectopterygoid fossa. Dorsally the two pterygoids do not contact in the basipharyngeal canal, being widely separated mainly by the basisphenoid and to a lesser extent by the presphenoid on the anterior third of their length. The pterygoids are also separated medially in Pantolambda and Leptictis (Novacek, 1986) contrary to the condition in Zalambdalestes, in which they contact medially, thus hiding the presphenoid (Wible et al. 2004).
On the medial side of the pterygoid of MHNC 8399, close to the pterygoid-basisphenoid suture (slightly ventrolateral to, and at the level of the anterior third of the basisphenoidpterygoid suture) is a small posteriorly-facing foramen, which is probably the posterior opening of the pterygoid canal (Fig. 23B). This foramen is close to the basisphenoidpresphenoid suture at the level of the anterior one-fourth of the length of the basisphenoid. In MHNC 8372 the pterygoid and basisphenoid are fused and the suture is not easily discernible. However, a similar foramen is also present apparently in a similar position as in MHNC 8399 (Fig. 23A). These foramina are apparently located in the suture between the pterygoid and the basisphenoid as is observed in the dog (Evans \& de Lahunta 2012) and many other eutherian (see below comparison section). On the juvenile skull MHNC 8423 a similar foramen is observable in the same position as in MHNC 8399 on the right side of the specimen piercing the pterygoid close to the basisphenoid-pterygoid suture and at the level of the anterior region of the basisphenoid. The pterygoid canal transmits the nerve of the pterygoid canal (Vidian nerve), which is an anterior extension of the greater petrosal nerve (a branch of the facial nerve) merged with the deep petrosal nerve, an anterior extension of the internal carotid nerve (Evans \& de Lahunta 2012; Wible 2009) (see below, Fig. 39). Further anteriorly, this nerve reaches the pterygopalatine ganglion from which stem three branches for the innervation of the lacrimal, nasal, and palatine glands (Evans \& de Lahunta 2012.). The anterior opening of the pterygoid canal is at the dorsomedial edge of the foramen rotundum in the orbitotemporal fossa (see orbitosphenoid section) (Figs 24B; 31). The course of the pterygoid canal from its posterior to its anterior opening has been followed and verified on the CT scans of MHNC 8399, on the right side of the specimen and on MHNC 8372 on the left side.

On MHNC 8372, at the base of the posterior edge of the entopterygoid crest, approximately at the limit with the posterior preotic crest, is a small foramen, which opens posteriorly (Fig. 23A: foramen 3). On the right side it seems to be in the pterygoid-alisphenoid suture, which separates the ecto- and entopterygoid processes. On the left side, the foramen appears to be in the pterygoid crest and two other smaller foramina appear to be in the alisphenoid-pterygoid suture. No such foramina have been observed in the pterygoid of MHNC 8399 (but in the basisphenoid, see below). These variable pterygoid foramina might be associated with a complex of accessory nutrient vessels that may also be related to the other variable set of nutrient foramina described in the neighbouring alisphenoid (see above). These pterygoid foramina have not been observed in Pantolambda (AMNH 16663) or in Leptictis (Novacek 1986). They are apparently also absent in Zalambdalestes and Maelestes. The ectopterygoid fossa for the medial pterygoid muscles has been described above in the alisphenoid section.

Presphenoid. This narrow bone is clearly shown between vertical plates of the palatines (perpendicular processes) and the anterior fourth of the pterygoids, above and slightly pos- 
terior to the choanae (Fig. 23). The anterior end articulates with the vomer and the vomerian incisure is located just above the postpalatine spine. The short posterior suture with the basisphenoid is shown ventrally, and is especially clear on MHNC 8399. It is also very distinct dorsally, within the braincase. On this side, the suture reaches the medial edge of the sphenoidal fissure, which is formed anteriorly by the presphenoid and posteriorly by the basisphenoid (Fig. 29).

Basisphenoid. Posterior to the presphenoid is the basisphenoid, which is fused to the pterygoids and the alisphenoids on MHNC 8372 but not in MHNC 8399. Its posterior suture with the basioccipital is well marked in both specimens because the fusion of the bones is not complete. The lateral edge of the basisphenoid immediately anterior to this suture forms a small spine, which projects laterally, a condition that is especially pronounced in MHNC 8372. Anterolateral to this spine is a deep notch for the internal carotid artery, which is confluent laterally with the pyriform fenestra. The internal carotid artery therefore entered the cranial cavity at the anterior end of its course on the promontorium, and joined the circle of Willis, which surrounds the hypophysis (Novacek 1986; Wible 1986; Evans \& de Lahunta 2012). Therefore, in Alcidedorbignya the basisphenoid does not totally enclose the internal carotid artery, and the passage for this vessel is open posterolaterally. The condition of Alcidedorbignya is also present in Pantolambda. Lateral to the notch for the internal carotid artery dorsal to the posterior end of the pterygoid, at its contact with the basisphenoid is a deep groove issued from the middle ear cavity for the Eustachian tube (Tuba auditiva, NAV), the auditory tube sulcus (Wible \& Spaulding 2013); (= pharyngeal sulcus of Novacek 1986). This groove also excavates the alisphenoid on the medial side of the medial wall of the foramen ovale in the anterolateral region of the middle ear. This groove extends anteriorly along the dorsolateral angle of the basipharyngeal canal probably also conveyed the pharyngeal nerve, artery and vein (Novacek 1986).

In the anterior concavity of the notch for the internal carotid artery, slightly anteroventral to it, MHNC 8399 exhibits a small foramen facing posteriorly on each side of the skull. The left foramen is smaller than the right one (Fig. 23B: foramen 4). On MHNC 8372 a tiny foramen is also present on the left side of the basisphenoid and this region is not preserved on the right side of the bone. Such foramina are variably present in Solenodon and, in some cases, they are relatively large (MNHN-AC-1932-195, 1902-156, MNHN-AC-14467; AMNH 28271, 28272) and are different from the posterior opening of the pterygoid canal, which, in Solenodon, is a posteriorly (or posteroventrally) facing foramen in the bottom of the carotid notch (Wible 2008). In Alcidedorbignya, these foramina are not regarded as related to the posterior opening of the pterygoid canal, which has been identified more anteriorly in the basipharyngeal groove (see above in the pterygoid section). Furthermore, on the CT scan of MHNC 8399 the foramen extends anterodorsally in a two- to three-mm long canal in the main part of the basisphenoid and then disappears into the bone. A similar condition is observed on MHNC 8372. These observations therefore confirm that these foramina do not represent the posterior opening of the pterygoid canal in Alcidedorbignya and may represent nutrient foramina.

On the internal side of the basisphenoid is the sella turcica, which is made of the hypophyseal fossa (which receives the pituitary gland), the anterior tuberculum sellae, and the posterior dorsum sellae with its caudal clinoid processes (Novacek 1986; Wible 2011). In Alcidedorbignya, the tuberculum sellae is almost indistinct anteriorly where it is expressed as a faint elevation on the presphenoid-basisphenoid suture. Posteriorly, the dorsum sellae is low and forms a small and elevated semicircular basin, the posterior edge of which articulates with the basioccipital (better preserved on MHNC 8372 than 8399). There is no elevated crest as is observed in Leptictis. The hypophyseal fossa is shallow and oval-shaped being wider posteriorly than anteriorly. It is not bordered laterally by conspicuous grooves for the cavernous sinus as in Leptictis (Novacek 1986) or in Ptilocercus (Wible 2011). The lateral edges of the fossa are almost adjacent to the groove for the maxillar ramus of the trigeminal nerve $(\mathrm{CN}$ V2). The hypophyseal fossa is located between the levels of the foramen ovale, posteriorly and the posterior opening of the "canal for the foramen rotundum" anteriorly (Fig. 33A).

The posterior edge of the basisphenoid is convex posteriorly and excavates the anterior edge of the basioccipital both ventrally and dorsally.

No distinct foramen for the craniopharyngeal canal is observable on the ventral side of the bone. When present, the craniopharyngeal canal houses a remnant of the pharyngeal diverticulum from which the pars glandularis of the hypophysis develops (Wible 2008, 2011; Evans \& de Lahunta 2012). It is commonly present in juveniles but generally is lacking in adults.

Petrosal. The petrosal or petromastoid is a crucial bone in the basicranium because it transmits a number of major vessels and nerves, which have proved to provide major indications on the evolutionary history of mammals (e.g., Rougier \& Wible 2006). Therefore, a careful description and interpretation of this bone is a major aspect of the description of the cranial anatomy of Alcidedorbignya. Several (eight) isolated petrosals (MHNC 8359 to 8362 and 8419 to 8422 ) discovered at the same site as the skeleton and the skulls described herein, are referred to Alcidedorbignya. Referral of these isolated specimens to Alcidedorbignya can be made with some confidence, based on comparisons with the petrosals of the two skulls described here (MHNC 8372 and 8399) and on the further prepared basicranium of Pantolambda bathmodon (AMNH 16663), in which the petrosal is perfectly preserved (Appendix 3 ).

Ventral (tympanic) face of the petrosal - The petrosal bone includes two major elements: 1) the pars cochlearis, which includes the promontorium (which houses the cochlea) and the tegmen tympani; and 2) the pars canalicularis, which is 


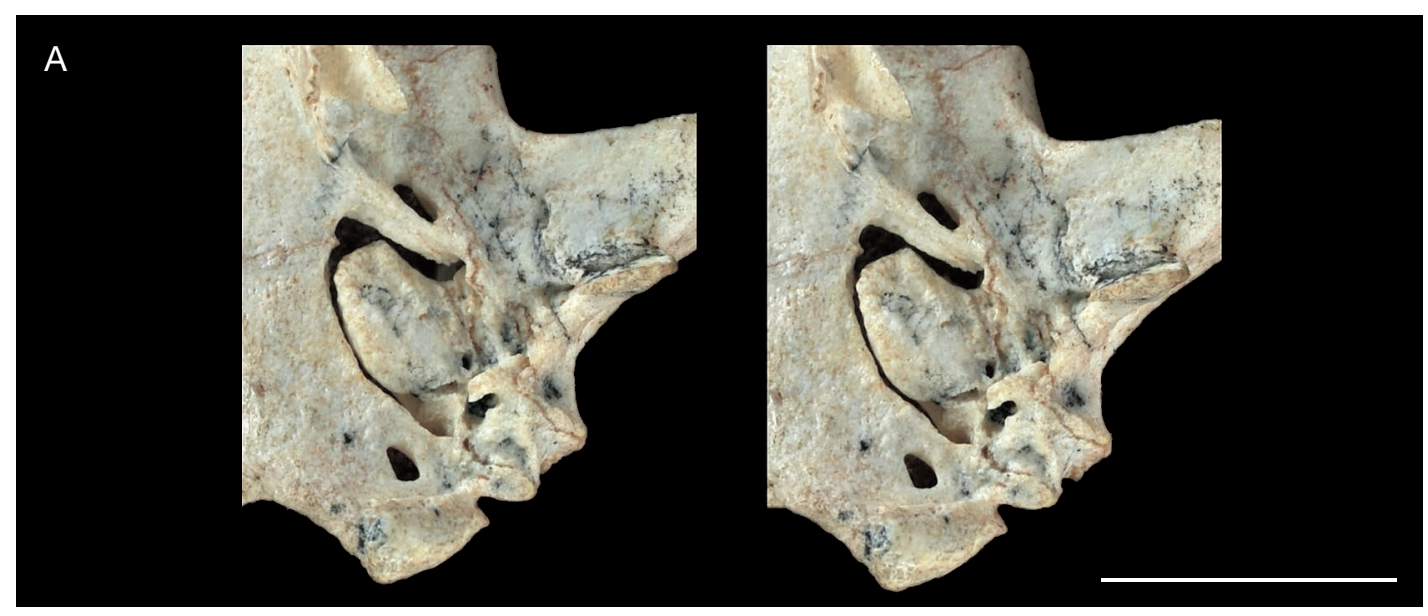

B

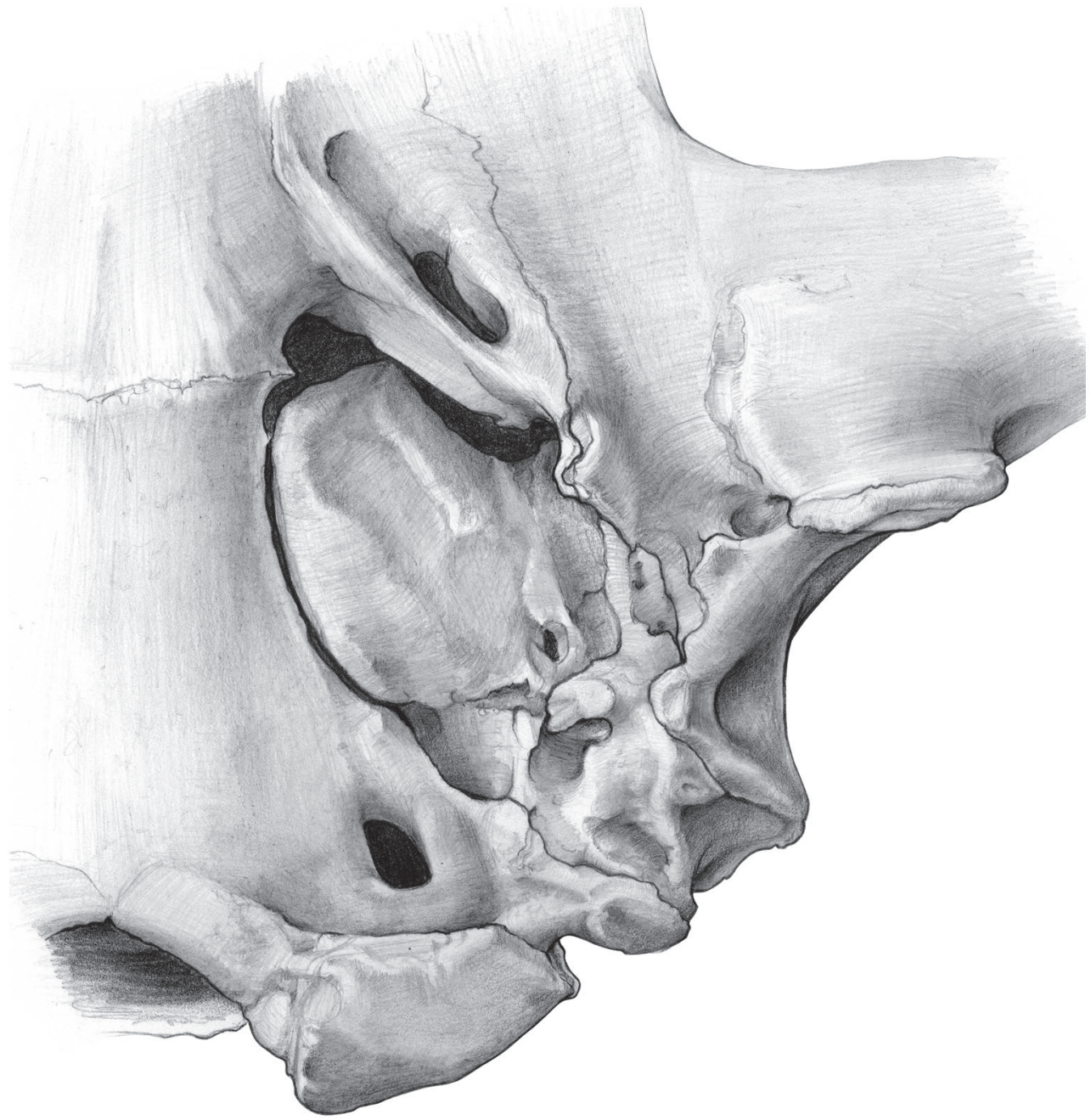

FIG. 36. - Left auditory region of Alcidedorbignya inopinata (MHNC 8372) in ventral view: A, stereophotographs; $\mathbf{B}$, pencil drawing (cracks and breakages of the specimen have been omitted). Scale bar: $1 \mathrm{~cm}$. A 3D photographic pdf file is available at the address http://sciencepress.mnhn.fr/en/periodiques/geodiversitas/37/4/alcidedorbignya-inopinata-additional-files.

lateral and posterodorsal to the pars cochlearis and which includes all the remaining parts of the petrosal: the vestibule and semicircular canals, the facial canal (which conducts the facial nerve from the secondary facial foramen to the stylomastoid notch and posteriorly the stapedial muscle), and the pars mastoidea, which is wedged between the squamosal and the exoccipital posteriorly.

The pars cochlearis of Alcidedorbignya presents a large promontorium, subrectangular in outline and obliquely oriented in relation to the sagittal plane of the skull. It has no contact 
C

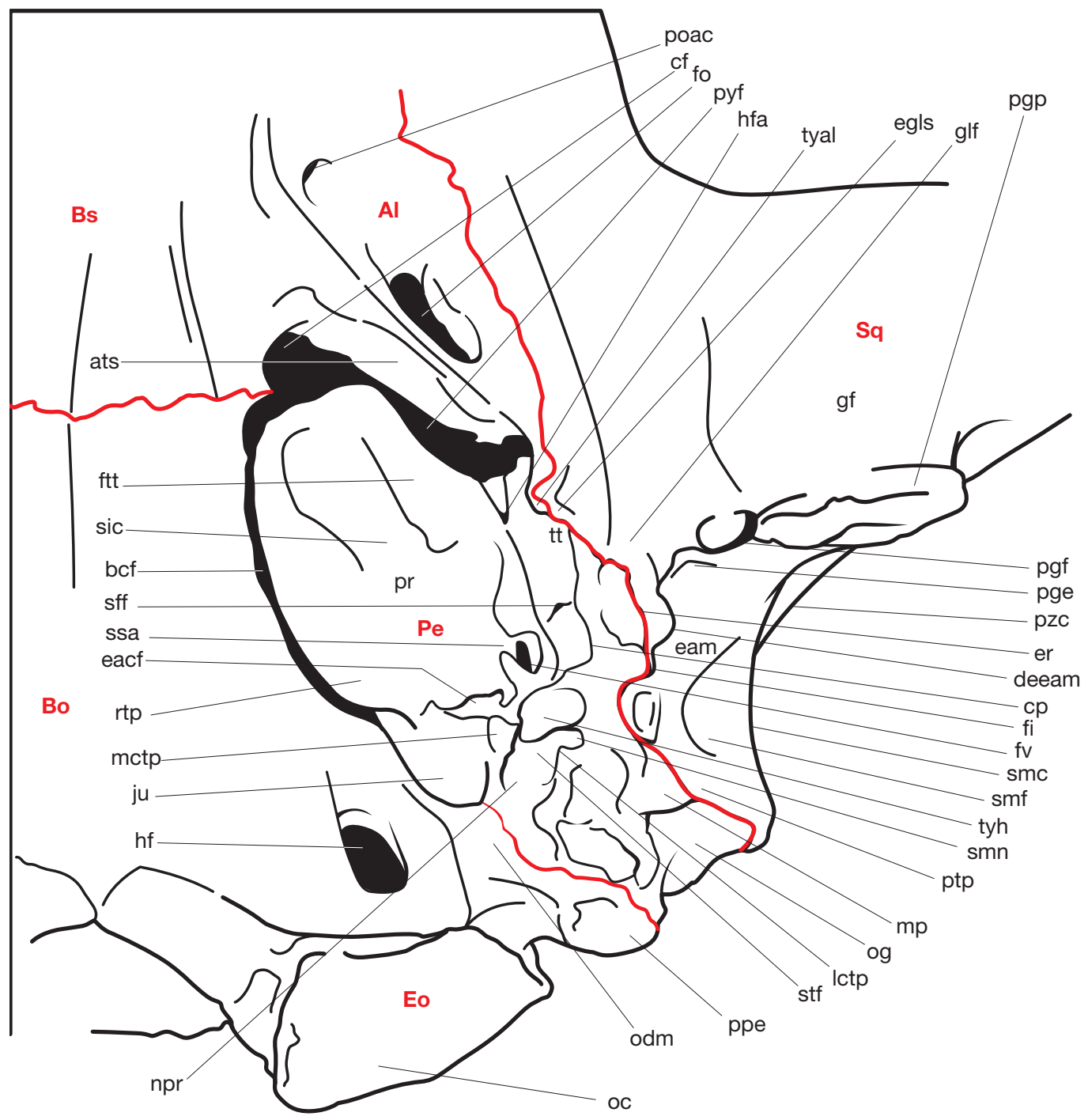

FIG. 36 (continuation). - Left auditory region of Alcidedorbignya inopinata (MHNC 8372) in ventral view: C, line drawings with captions. Abbreviations: Al, alisphenoid; ats, sulcus for the auditory (Eustachian) tube; bcf, basicochlear fissure; Bo, basioccipital; Bs, basisphenoid; cf, carotid foramen; cp, crista parotica; deeam, dorsal edge of the external acoustic meatus; eacf, external aperture of the cochlear fossula; eam, external acoustic meatus; egls, entoglenoid process of the squamosal; Eo, exoccipital; er, epitympanic recess; fi, fossa incudis; fo, foramen ovale; ftt, fossa for tensor tympani muscle; fv, fenestra vestibuli; gf, glenoid fossa; glf, glaserian fissure; hf, hypoglossal foramen; hfa, hiatus Fallopii; ju, jugular foramen; Ictp, lateral caudal tympanic process; mctp, medial caudal tympanic process; mp, mastoid process; npr, notch for the posterior ramus of the stapedial artery; oc, occipital condyle; odm, probable origin of the digastric muscle; og, occipital groove; Pe, petrosal; pge, postglenoid eminence; pgf, postglenoid foramen; pgp, postglenoid process; poac, posterior opening of the alisphenoid canal; ppe, paroccipital process of the exoccipital; pr, promontorium; ptp, postympanic process of the squamosal; pyf, pyriform fenestra; pzc, postzygomatic crest; rtp, rostral typamnic process; sff, secondary facial foramen; sic, sulcus for internal carotid artery; smc, suprameatal crest; smf, suprameatal fossa; smn, stylomastoid notch; Sq, squamosal; ssa, sulcus for the stapedial artery; stf, stapedius fossa; tt, tegmen tympani; tyal, tympanic process of the alisphenoid; tyh, tympanohyal. Scale bar: $1 \mathrm{~cm}$. A 3D photographic pdf file is available at the address http://sciencepress.mnhn.fr/en/periodiques/geodiversitas/37/4/alcidedorbignya-inopinata-additional-files.

with the adjacent anterior and medial bones (alisphenoid, basisphenoid, and basioccipital) and is totally surrounded by a vast cavity, which extends from the hiatus Fallopii anterolaterally to the anteriormost point of the exoccipital-petrosal suture posteromedially. It is possible to divide this cavity into four elements: 1) the pyriform fenestra (not piriform fenestra; see McDowell 1958: 128 for original spelling of this term); 2) the carotid foramen (strictly speaking a notch in the case of Alcidedorbignya); 3) the basicochlear fissure; and 4) the jugular foramen (Figs 36-39).
1) Anterolaterally, a moderately large pyriform fenestra is present. The pyriform fenestra is a significant gap between the auditory capsule and the sphenoid and squamosal bones, which is present in all foetal mammals and some adults (Wible 2008: 392; MacPhee 1981). In Alcidedorbignya, it is preserved in the adult. It is bordered anterolaterally by the alisphenoid and posteriorly and medially by the anterolateral edge of the promontorium. It is an elongate slit and has an oblique anteromedial-posterolateral orientation. Its posterior end is approximately at the level of the anterior third of the 


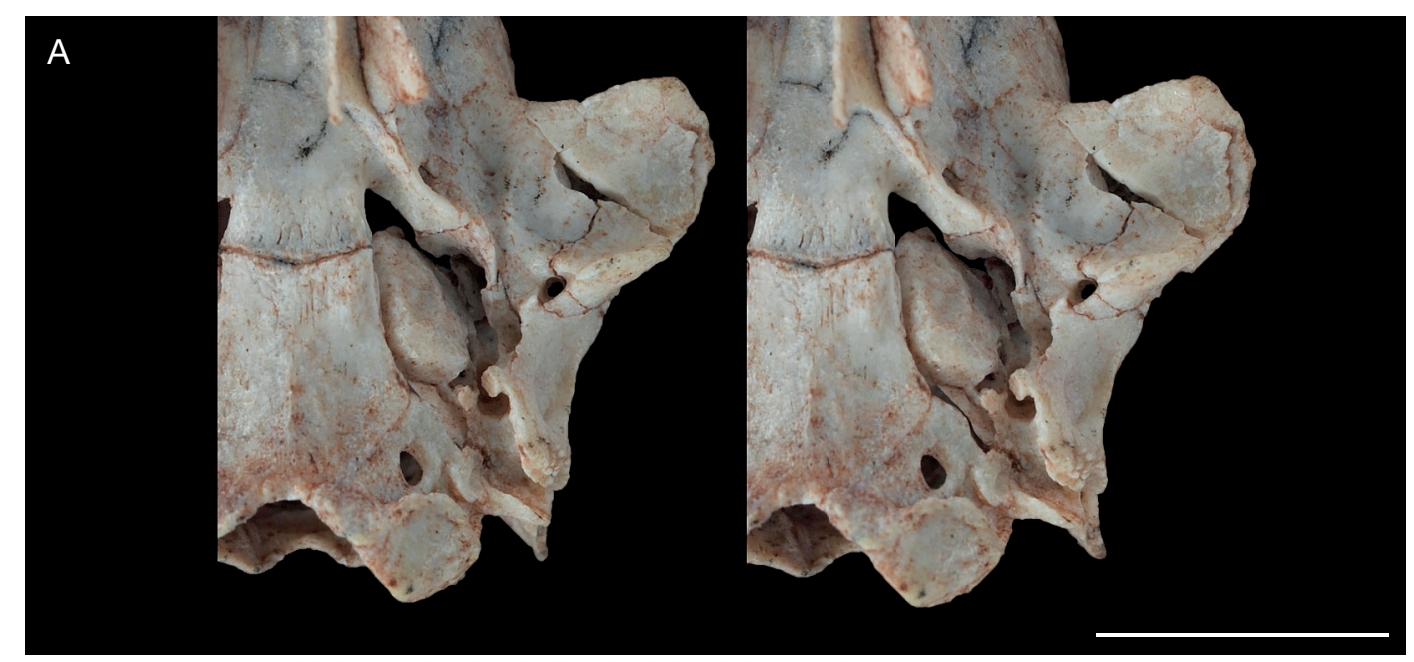

B

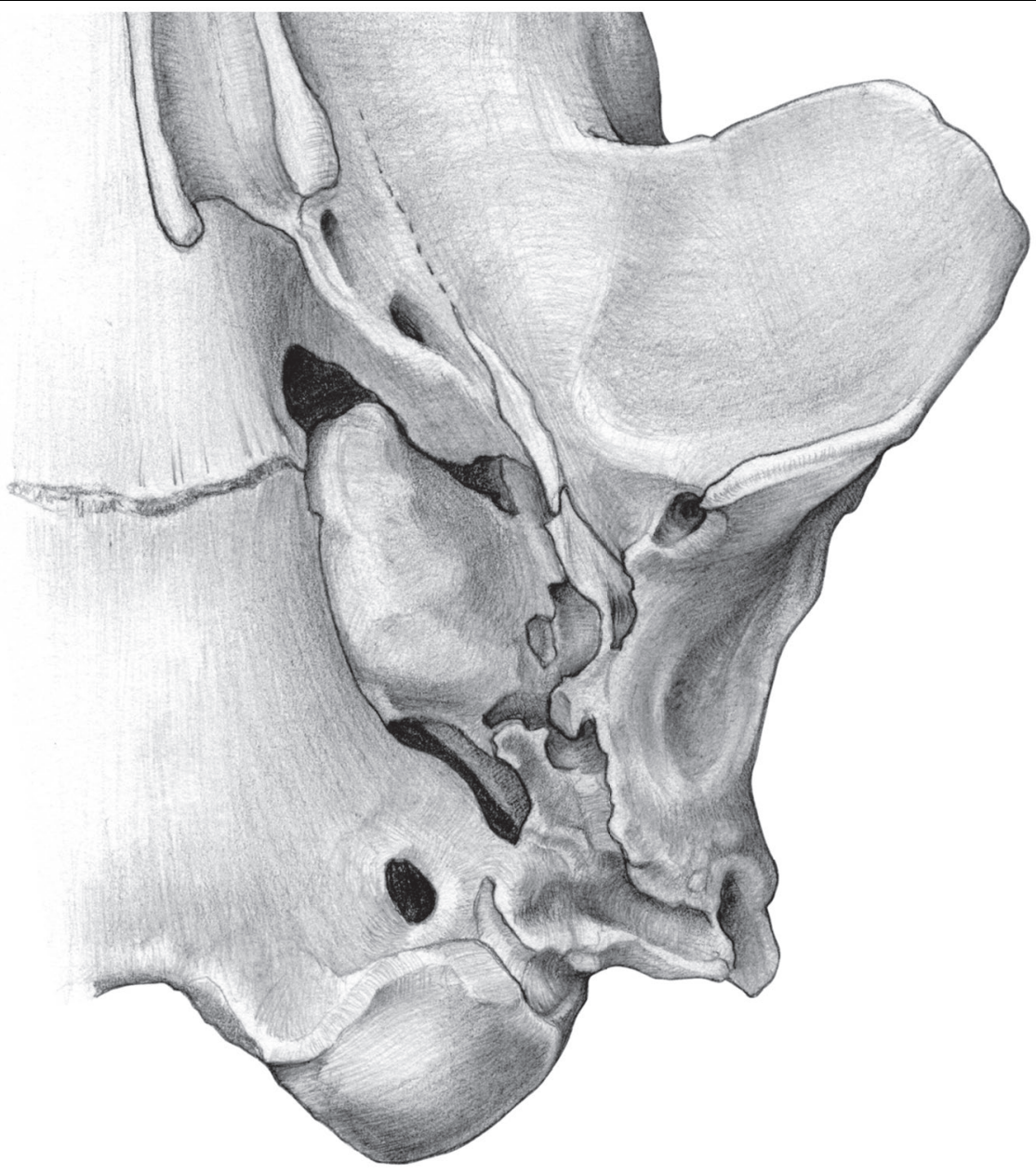

FIG. 37. - Left auditory region of Alcidedorbignya inopinata (MHNC 8399) in ventral view: A, stereophotographs; B, pencil drawing (cracks and breakages of the specimen have been omitted). Scale bar: $1 \mathrm{~cm}$. A 3D photographic pdf file is available at the address http://sciencepress.mnhn.fr/en/periodiques/geodiversitas/37/4/alcidedorbignya-inopinata-additional-files.

promontorium. The pyriform fenestra is the passage for the entrance of the ramus infraorbitalis of the stapedial artery (anterior continuation of the ramus inferior; see below) into the cranial cavity in some mammals presenting an endocranial pathway for this vessel (see Wible et al. 2004: 95, 96). In the case of those taxa, which have an alisphenoid canal, as does
Alcidedorbignya, it is this latter structure that conveys the ramus infraorbitalis (= intramural pathway of Wible et al. 2004; see Wible 2008). The ramus infraorbitalis of the stapedial artery of Alcidedorbignya likely ran in the groove on the medial face of the preotic crest and passed ventral to the lateral edge of the pyriform fenestra on its way to the posterior opening of 


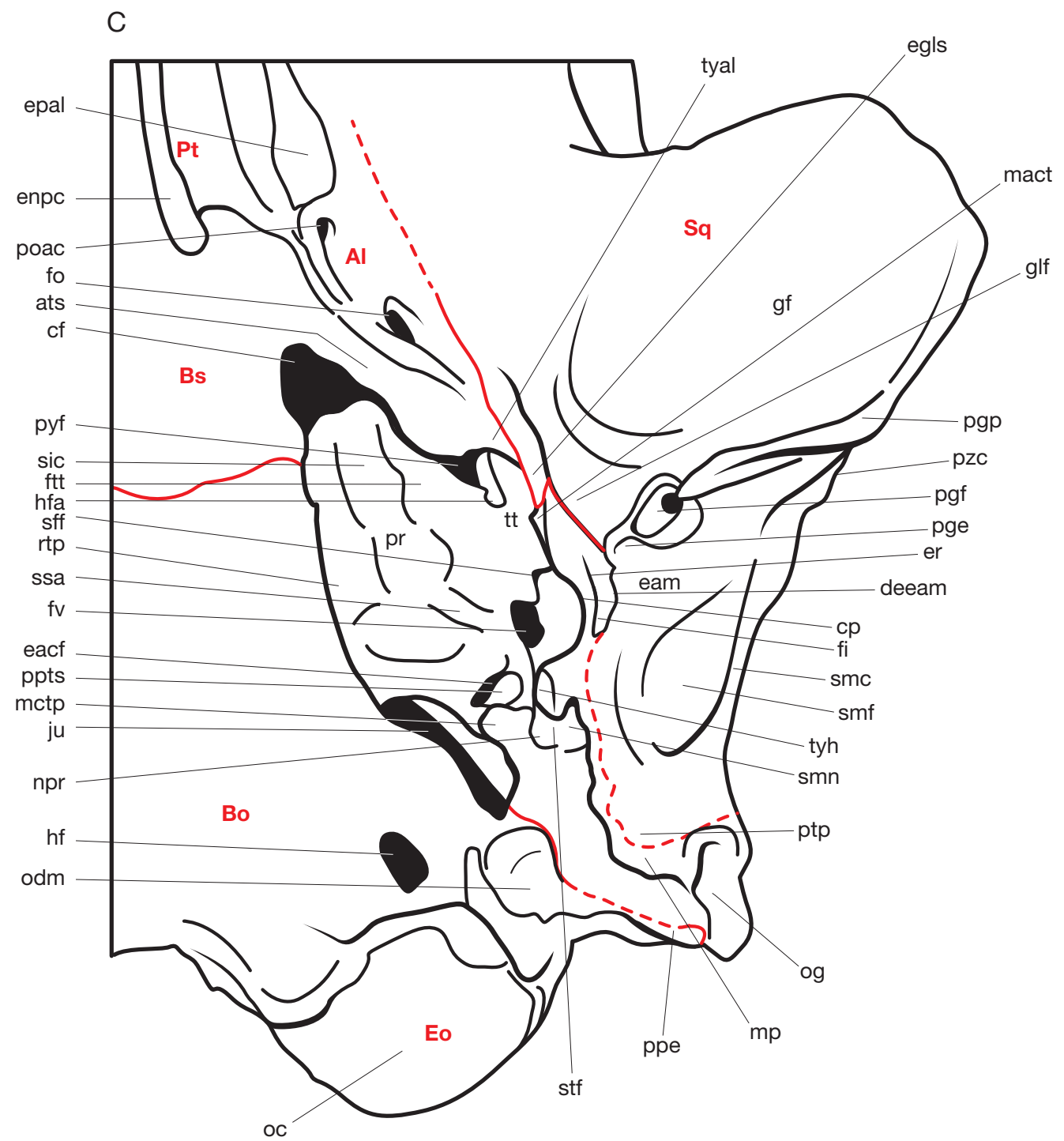

FIG. 37 (continuation). - Left auditory region of Alcidedorbignya inopinata (MHNC 8399) in ventral view: C, line drawings with captions. Abbreviations: Al, alisphenoid; ats, sulcus for the auditory (Eustachian) tube; Bo, basioccipital, Bs, basisphenoid; cf, carotid foramen; $\mathbf{c p}$, crista parotica; deeam, dorsal edge of the external acoustic meatus; eacf, external aperture of the cochlear fossula; eam, external acoustic meatus; egls, entoglenoid process of the squamosal; enpc, entopterygoid crest; Eo, exoccipital; epal, ectopterygoid process of the alisphenoid; er, epitympanic recess; fi, fossa incudis; fo, foramen ovale; ftt, fossa for tensor tympani muscle; fv, fenestra vestibuli; gf, glenoid fossa; glf, Glaserian fissure; hf, hypoglossal foramen; hfa, hiatus Fallopii; ju, jugular foramen; mact, medial articulation of the anterior crus of the ectotympanic; mctp, medial caudal tympanic process; mp, mastoid process; npr, notch for the posterior ramus of the stapedial artery; oc, occipital condyle; odm, probable origin of the digastric muscle; og, occipital groove; pge, postglenoid eminence; pgf, postglenoid foramen; pgp, postglenoid process; poac, posterior opening of the alisphenoid canal; ppe, paroccipital process of the exoccipital; ppts, postpromontorial tympanic sinus; pr, promontorium; Pt, pterygoid; ptp, postympanic process of the squamosal; pyf, pyriform fenestra; pzc, postzygomatic crest; rtp, rostral typamnic process; sff, secondary facial foramen; sic, sulcus for internal carotid artery; smc, suprameatal crest; smf, suprameatal fossa; smn, stylomastoid notch; Sq, squamosal; ssa, sulcus for the stapedial artery; stf, stapedius fossa; tt, tegmen tympani; tyal, tympanic process of the alisphenoid; tyh, tympanohyal. A 3D photographic pdf file is available at the address http://sciencepress.mnhn.fr/en/periodiques/geodiversitas/37/4/alcidedorbignya-inopinata-additional-files.

the alisphenoid canal. The greater petrosal nerve exits the middle ear through the hiatus Fallopii (MacPhee 1981; see below). This nerve likely ran also ventral to the level of the pyriform fenestra, which it crosses diagonally on its way to the posterior opening of the pterygoid canal (see above) (Fig. 39).

2) Anteromedially to the pyriform fenestra is a deep notch in the posterolateral angle of the basisphenoid for the internal carotid artery, which separates the apex of the promontorium from the basisphenoid. Although the carotid foramen is partly confluent with the pyriform fenestra, the shape of the apex of the promontorium (see below) clearly delimi- tates a roughly triangular area for the passage of the internal carotid. The anterolateral edge of the foramen is formed by the alisphenoid, the medial and anterior edges are excavated in the basisphenoid, and the posterolateral side is limited by the apex of the promontorium. The anterolateral angle of the apex of the promontorium is sharp (almost a right angle) and closely approximates the alisphenoid but does not contact it; it partially separates the carotid foramen from the pyriform fenestra (Figs 36, 37).

3) The whole medial edge of the promontorium is separated from the adjacent basioccipital by a narrow slit, constant in width, 


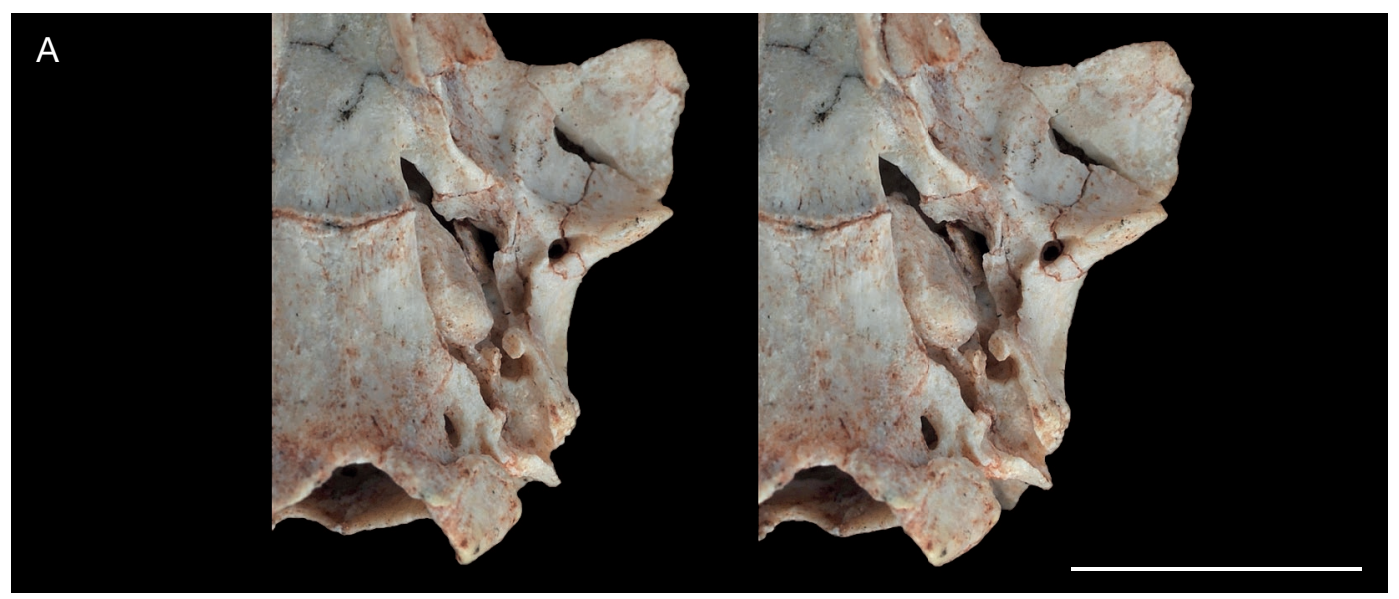

B

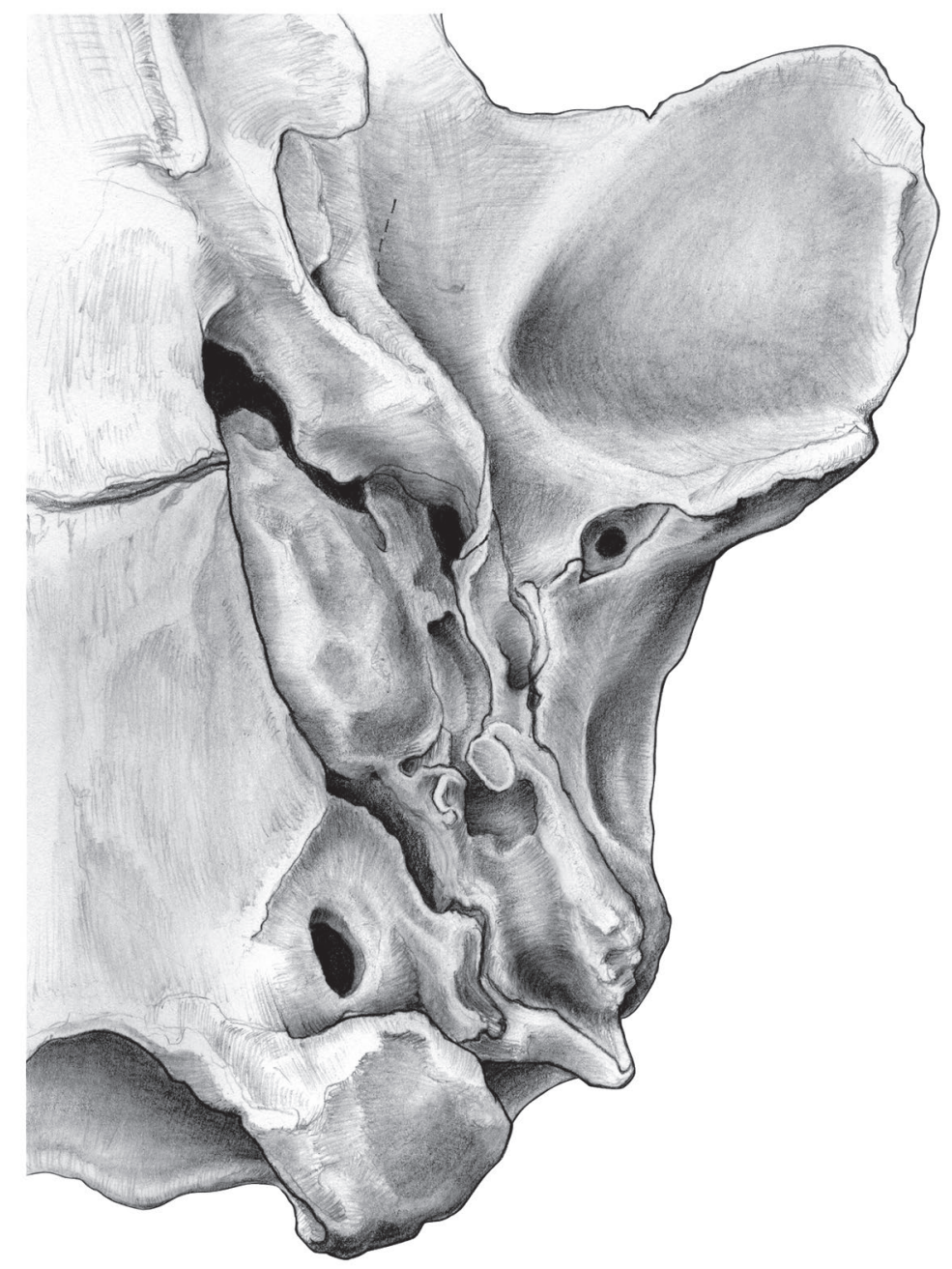

FIG. 38. - Left auditory region of Alcidedorbignya inopinata (MHNC 8399) in ventralmedial view showing the foramen for the ramus superior of the stapedial artery: A, stereophotographs; B, pencil drawing (cracks and breakages of the specimen have been omitted). Scale bar: $1 \mathrm{~cm}$. A 3D photographic pdf file is available at the address $\mathrm{http}: / /$ sciencepress.mnhn.fr/en/periodiques/geodiversitas/37/4/alcidedorbignya-inopinata-additional-files.

the basicochlear fissure (= basicapsular fenestra of MacPhee 1981). As documented by Giannini et al. (2006: 70), the basicochlear fissure is a gap in the chondrocranium between the auditory capsule, laterally and the parachordal plate (central stem) medially (De Beer 1937: 399), bounded by anterior and posterior basicochlear (basicapsular) commissures, if present (MacPhee 1981:
49). In some adult mammals, as in Alcidedorbignya, the fissure is not completely obliterated, resulting in the basicranial exposure of part of the inferior petrosal sinus (McDowell 1958: 202).

And 4) The posteromedial end of the surrounding cavity is formed by an elongated and vaguely sigmoid jugular foramen (posterior lacerate foramen). This foramen extends from the 


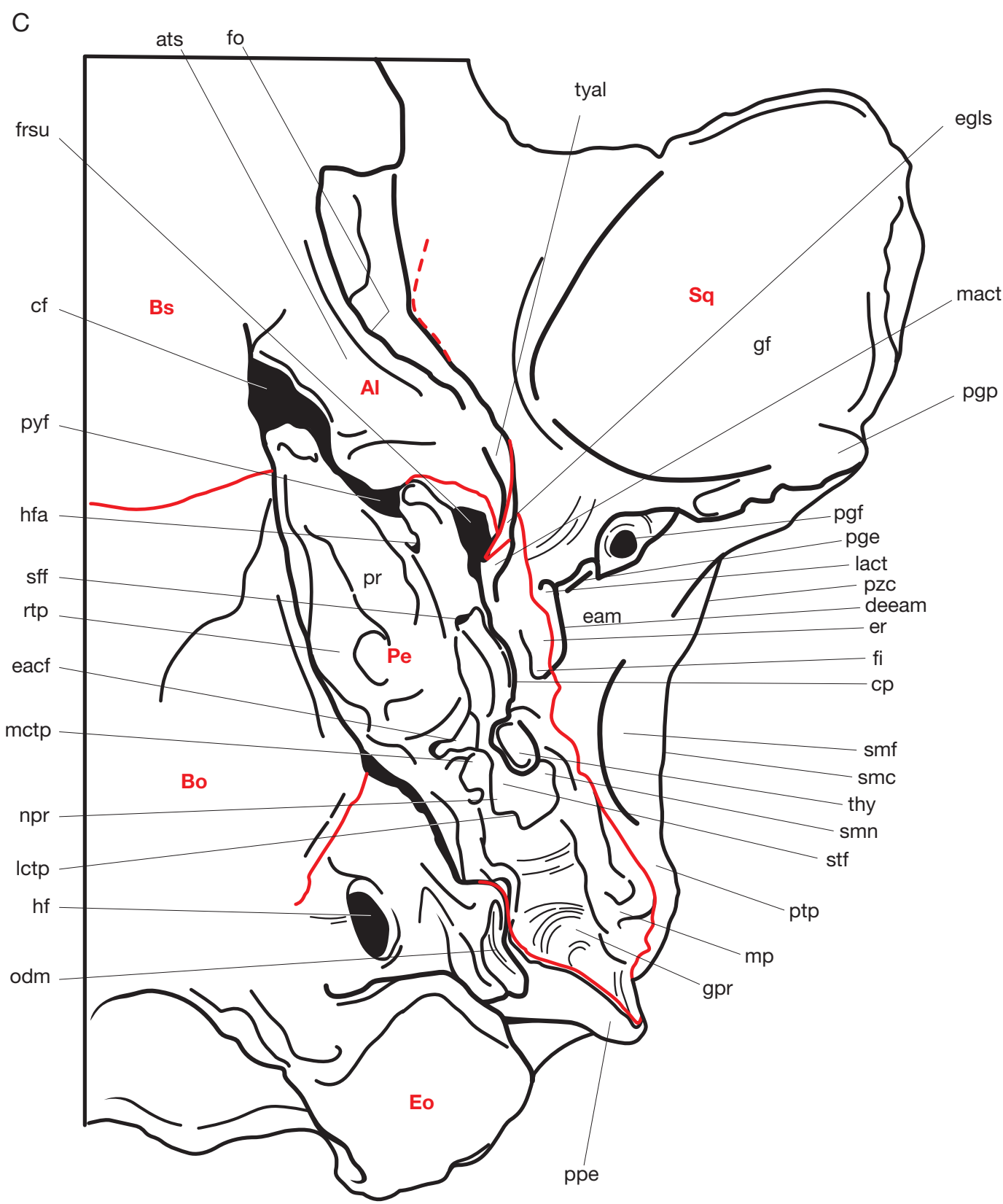

FIG. 38 (continuation). - Left auditory region of Alcidedorbignya inopinata (MHNC 8399) in ventralmedial view showing the foramen for the ramus superior of the stapedial artery: C, line drawings with captions. Abbreviations: Al, alisphenoid; ats, sulcus for the auditory (Eustachian) tube; Bo, basioccipital, Bs, basisphenoid; cf, carotid foramen; $\mathbf{c p}$, crista parotica; deeam, dorsal edge of the external acoustic meatus; eacf, external aperture of the cochlear fossula; eam, external acoustic meatus; egls, entoglenoid process of the squamosal; Eo, exoccipital; er, epitympanic recess; fi, fossa incudis; fo, foramen ovale; frsu, foramen for the superior ramus of the stapedial artery; gf, glenoid fossa; gpr, groove for the passage of the posterior ramus; hf, hypoglossal foramen; hfa, hiatus Fallopii; Ictp, lateral caudal tympanic process; lact, cupula for lateral articulation for the anterior crus of the ectotympanic; mact, medial articulation for the anterior crus of the ectotympanic; mctp, medial caudal tympanic process; mp, mastoid process; npr, notch for the posterior ramus of the stapedial artery; odm, probable origin of the digastric muscle; Pe, petrosal; pge, postglenoid eminence; pgf, postglenoid foramen; pgp, postglenoid process; ppe, paroccipital process of the exoccipital; pr, promontorium; ptp, postympanic process of the squamosal; pyf, pyriform fenestra; pzc, postzygomatic crest; rtp, rostral typamnic process; sff, secondary facial foramen; smc, suprameatal crest; smf, suprameatal fossa; smn, stylomastoid notch; Sq, squamosal; stf, stapedius fossa; tyal, tympanic process of the alisphenoid; tyh, tympanohyal. A 3D photographic pdf file is available at the address http://sciencepress.mnhn.fr/en/periodiques/geodiversitas/37/4/alcidedorbignya-inopinata-additional-files.

level of the external aperture of the cochlear canaliculus (or perilymphatic duct) anteriorly to the anteriormost point of the petrosal-exoccipital suture posteriorly. It is bordered anterolaterally by the petrosal and posteromedially by the exoccipital. Peering through the posterior region of the jugular foramen it is possible to observe, on the petrosal, the sulcus for the sigmoid sinus, which exits the skull via the jugular foramen after joining the inferior petrosal sinus to form the jugular and vertebral veins (MacPhee 1981; Evans \& de Lahunta 2012). Also transmitted by the jugular foramen are the glossopharyngeal (CN IX), vagus $(\mathrm{CN} \mathrm{X})$, and spinal accessory nerves (CN XI) (Evans \& de Lahunta 2012). 
The condition of Pantolambda, with respect to these four cavities, is similar to that of Alcidedorbignya (see below in Appendix 3, Fig. 124). Pantolambda, however, differs from Alcidedorbignya in having a shallower notch in the basisphenoid for the internal carotid artery. In spite of this condition Pantolambda exhibits a proportionally larger cavity at the apex of the promontorium than that of Alcidedorbignya. This cavity forms a single sub-triangular large aperture with the pyriform fenestra. This condition is probably due to the much larger truncation of the apex of the promontorium, which appears as a clear-cut vertical section of the bone. In fact, on AMNH 16663, the anterior edge of the pars cochlearis looks so artificial that it has the appearance of a breakage, but the fact that both sides of the specimens are identical and a careful observation of the potential "section" leaves no doubt that it is the actual morphology of the apex of the promontorium. Because of the enlargement of the confluent carotid foramen + pyriform fenestra, the length of the basicochlear fissure of Pantolambda is relatively reduced, when compared to that of Alcidedorbignya.

The promontorium of Alcidedorbignya is dorsoventrally flattened, being thicker medially than laterally. The medial edge of the promontorium bears a thick, rounded, low ridge, the rostral tympanic process, a periosteal outgrowth of the pars cochlearis (MacPhee 1981). It runs from the ventral border of the fenestra cochleae to the anteromedial angle. It has a strongly roughened surface. It is more pronounced on MHNC 8372 than on 8399 . This process also shows similar variation in its development among the isolated petrosals attributed to Alcidedorbignya.

The apex of the promontorium is squared off and appears somehow truncated along a posteromedial-anterolateral axis. Its anterior edge is at the level of the suture between the basioccipital and the basisphenoid (slightly anterior in MHNC 8399 and slightly posterior in MHNC 8372). The anterolateral angle of the promontorium is much anterior to the anterior limit of the tegmen tympani. As a consequence, the anterolateral border of the promontorium forms the medial edge of the pyriform fenestra. Because of the greater truncation of the apex of the promontorium in Pantolambda the anterior edge of the promontorium is well posterior to the level of the basioccipital-basisphenoid suture (see below, Fig. 124). The anterior edge of the promontorium is almost at the level of the anterior edge of the tegmen tympani laterally (slightly anterior only), and the pyriform fenestra is almost totally merged with the carotid foramen to form the vast triangular opening mentioned above.

Grooves for the internal carotid and stapedial arteries are observable on the promontorium of Alcidedorbignya. They are very shallow but relatively wide. Because of their shallowness, they are not easy to distinguish but observation with lowangle reveals, on both specimens, a sigmoid structure, which slightly excavates the ventral surface of the promontorium on the posterior third of its medial edge (Figs 36, 39). This wide groove ends at the anterior apex of the ventral side of the promontorium, just in front of the notch for the internal carotid artery in the basisphenoid. This condition is also present on isolated petrosals (MHNC 8359, 8360, 8362, 8419, 8421, 8422) referred to Alcidedorbignya (Fig. 40) although they are hardly discernible in juveniles or distorted specimens (8361, 8420 ). We interpret this groove as the mark of the passage of a transpromontorial internal carotid artery (Wible 1986). The fenestra vestibuli is located at the posterolateral angle of the promontorium and receives the footplate of the stapes. It is oval-shaped and its long axis is oriented anteroposteriorly Measurements of long and short axis for the calculation of stapedial ratio (Segall 1970) are: 1.8 for MHNC 8399, 1.7 for MHNC 8360, and 1.65 and 2 for MHNC 8372 (right and left side respectively), (see Table 5). From the medial edge of the fenestra vestibuli, a shallow and narrow groove extends anteromedially and connects to the groove for the transpromontorial internal carotid artery. This is the groove for the stapedial artery, a vessel that passes into the stapedial foramen (Wible 1987) and runs anteromedially in the anterior portion of the facial sulcus and on the ventral surface of the tegmen tympani in Alcidedorbignya (see below). There, it subdivides into superior and inferior rami. The transpromontorial internal carotid and stapedial arteries are the result of the dichotomy of the common carotid artery (Wible 1986; or simply termed internal carotid artery in Wible 1987), which comes from the neck, passes ventral to the exoccipital and runs below the promontorium. In Alcidedorbignya, the common carotid artery enters the promontorium area at the level of a slightly depressed area on the posterior third of its medial edge and the dichotomy of the common carotid artery into stapedial and promontorium arteries is located approximately below the centre of the promontorium as evidenced by the preserved grooves.

The lateral and posterolateral edges of the promontorium are bordered by the facial sulcus, which conveys the facial nerve outside the skull posteriorly (MacPhee 1981). After crossing the petrosal via the facial canal to exit the braincase (see below), the facial nerve enters, via the primary facial foramen, an intrapetrous cavity in which it forms the geniculate ganglion. This cavity is the cavum supracochleare, a roofed space for this ganglion and the common channel from which the hiatus Fallopii (anteriorly) and the secondary facial foramen (posteriorly) open (Wible 2008, 2010). From the geniculate ganglion, two branches are emitted: the posterior continuation of the facial nerve itself, which passes through the secondary facial foramen and runs posteriorly in the facial sulcus and exits the skull via the stylomastoid notch; and the greater petrosal nerve, which runs anteriorly and exits the petrosal through the hiatus Fallopii (e.g., Wible 2008). In Alcidedorbignya, the secondary facial foramen is located anterolateral to the fenestra vestibuli and the hiatus Fallopii opens at the anterolateral edge of the petrosal, which forms the posteromedial border of the pyriform fenestra. The hiatus Fallopii is visible ventrally as it opens slightly posterior to the anterior edge of the petrosal and therefore opens in the middle ear. This condition is clear on MHNC 8372, in which the floor of the cavum supracochleare is fully preserved. The condition in Pantolambda is not easy to determine because of the partial breakage of the floor of the cavum supracochleare. 


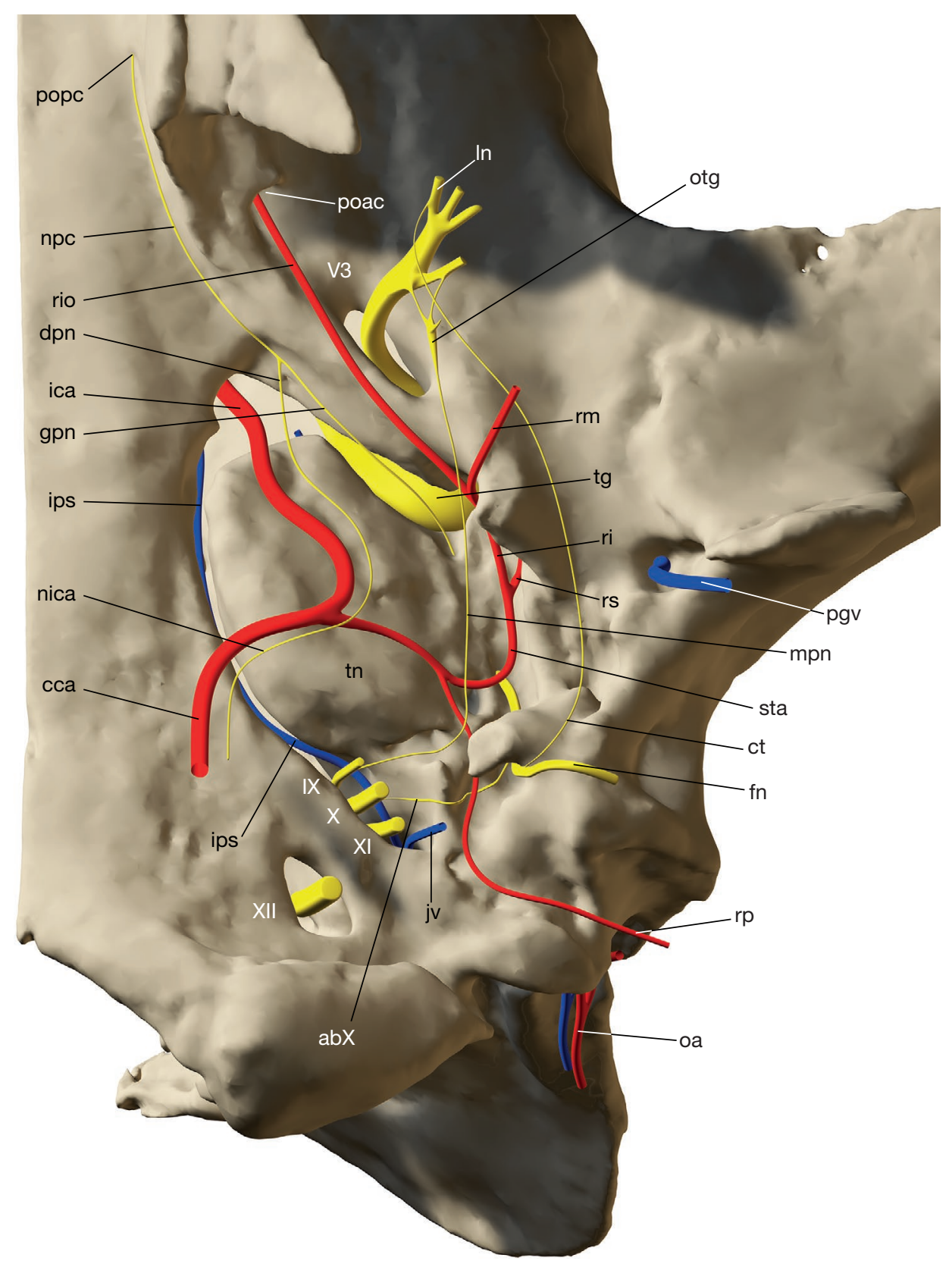

FIG. 39. - Vessels and nerve reconstruction of the left auditory region of Alcidedorbignya inopinata in ventral view (MHNC 8372). Reconstruction of vessels and nerves are superimposed directly on 3D surface rendering of CT data. Abbreviations: abX, auricular branch of the vagus nerve; cca, common carotid artery; ct, chorda tympani; dpn, deep petrosal nerve; fn, facial nerve; gpn, greater petrosal nerve; ica, internal carotid artery; ips, inferior petrosal sinus; jv, jugular vein; In, lingual nerve; mpn, minor petrosal nerve; nica, nerve of the internal carotid artery; npc, nerve of the pterygoid canal; oa, occipital artery; otg, otic ganglion; pgv, postglenoid vein; poac, posterior opening of the alisphenoid canal; popc, posterior opening of the pterygoid canal; ri, ramus inferior; rio, ramus infrorbitalis; $\mathbf{r m}$, ramus mandibularis; rp, ramus posterior; rs, ramus superior; sta, stapedial artery; tg, trigeminal ganglion; tn, tympanic nerve; $\mathbf{V} 3$, mandibular branch of the trigeminal nerve; IX, glossopharyngeal nerve; $\mathbf{X}$, vagus nerve; $\mathbf{X I}$, accessory nerve; $\mathbf{X I I}$, hypoglossal nerve. The 3D rendering of CT data is available in pdf format at the address http://sciencepress.mnhn.fr/en/periodiques/geodiversitas/37/4/alcidedorbignya-inopinata-additional-files.

Nevertheless, it is clear that the hiatus Fallopii of this genus did not open dorsally, within the braincase. It either opened anteriorly in the clear-cut anterior edge of the petrosal; or, more likely, slightly posterior to the anterior edge of the promontorium and therefore within the tympanic cavity (a condition similar to that of Alcidedorbignya). The condition of Alcidedorbignya is also observed in Zalambdalestes, where the hiatus Fallopii opens ventral but very close to the anterior edge of the petrosal. This area of the petrosal anterolateral to the promontorium and which extends from the hiatus Fallopii anteriorly to the anterior edge of the stapedial fossa posteriorly is the tegmen tympani. Wible \& Novacek (1988: 6) provide a useful definition of this structure: "The tegmen tympani is a neomorphic element on the auditory capsule in the therian chondrocranium (Kuhn \& Zeller 1987). It projects anteriorly from the crista parotica (see below), to which the second branchial arch cartilage (Reichert's cartilage) is usually fused, and contributes to the roof of the epitympanic 
recess, the dorsal extension of the middle-ear cavity that lies above the level of the tympanic membrane and contains the incudomalleolar articulation".

The thin blade of bone located between the hiatus Fallopii anteriorly and the secondary facial foramen forms the floor of the cavum supracochleare. Immediately lateral to it, is a large foramen excavated in the petrosal, which penetrates the skull dorsally. This is the foramen for the ramus superior of the stapedial artery, which likely diverged from the ramus inferior at the level of the cavum supracochleare (see above). The foramen is especially well preserved on MHNC 8399 (Fig. 39). It is oval-shaped with the great axis oriented anteromedially-posterolaterally (almost anteroposteriorly). On MHNC 8399, the lateral and medial edges of the foramen do not appear to be fused at the anteromedial end of the long axis but are closely appressed one against the other. This condition is observed on some isolated petrosals (Fig. 40). On MHNC 8372 the two lips are not in contact and are slightly separated, the space being filled by the alisphenoid. On other isolated petrosals (e.g., MHNC 8360, 8362) the two lips do not contact probably because these specimens represent juveniles individuals. In this connection it is worth noting that MHNC 8372, in which the lips are closely approximated but not in contact, is that of a young adult, while MHNC 8399 is apparently a fully-grown adult (see the "Specimens, Taphonomy, and Biology" section). In other isolated petrosals (e.g., MHNC $8361,8419)$ the lips were apparently broken postmortem. As far as it is possible to observe this feature on the specimen of Pantolambda available during this study (AMNH 16663), the foramen is not totally closed either in this genus either. The foramen for the ramus superior of $\mathrm{Al}$ cidedorbignya is located mediodorsal to the preotic crest, with its anterior end at the level of the suture between the tympanic process of the alisphenoid and the entoglenoid process of the squamosal (Figs 38, 39). The close position of the ramus superior foramen to the preotic crest, which exhibits a concavity for the ramus inferior, is in agreement with the proposed division of the stapedial artery close to these structures on the ventral surface of the tegmen tympani (see above).

The anteromedial edge of the foramen for the ramus superior is formed by a blade of the tegmen tympani, which articulates with the alisphenoid anteriorly and, which separates the foramen from the pyriform fenestra anteromedially. Dorsally (endocranially), this blade is excavated by a small fossa which received part of the trigeminal ganglion (Fig. 29; and see below, Figs 43, 49). It corresponds to a vestigial anterior lamina as described by Marshall \& Muizon (1995), Ladevèze \& Muizon (2007), and Billet \& Muizon (2013) (see below; dorsal face of the petrosal). This fossa is generally not preserved on isolated petrosals. The foramen for the ramus superior opens dorsally into a vast cavity of the braincase limited by the squamosal laterally, the petrosal ventrally and ventromedially, and the parietal dorsomedially. We have not found a formal term for such a cavity and therefore have proposed above for it the new term: petrosquamosal fossa (see squamosal section). This fossa is described below with the dorsal (endocranial) face of the petrosal.

In ventral view of the tympanic cavity, from the posterior edge of the foramen for the ramus superior, lateral to the facial sulcus and facing the fenestra vestibuli is an elongate fossa with a deep posterior pit (Figs 36-38). This is the petrosal dorsomedial part of the epitympanic recess. The ventrolateral part of the recess is formed by the squamosal (see above). The petrosal portion of the recess is approximately as wide as the squamosal one but slightly longer. The posterior deep pit of the recess is the fossa incudis, in which attaches the crus breve of the incus (MacPhee 1981). The fossa incudis is almost totally excavated in the petrosal with little squamosal participation. The whole epitympanic recess (petrosal + squamosal portions) receives the incudomalleolar articulation (Klaauw 1931; MacPhee 1981). It is oriented anteroposteriorly and slopes anteroventrally. It is approximately twice as long as wide and opens in a wide groove, which extends the recess anterolaterally. This groove is the fissura Glaseri discussed above in the squamosal section. The anterior end of the petrosal part of the epitympanic recess articulates with the entoglenoid process of the squamosal. Just posterior to this articulation, on its medial edge, the epitympanic recess presents a small, elongated triangular facet, tapering posteriorly and which almost underlies the secondary facial foramen (Figs 37C; 38C). This facet is probably for the lateral articulation of the anterior crus of the ectotympanic (with a possible participation of the rostral process of the malleus). It resembles in shape and position that observed by Wible (2008: fig. 28B) in Solenodon (see below ectotympanic section below). The epitympanic recess of Pantolambda is anteroposteriorly shorter and deeper than in Alcidedorbignya. However, this condition is probably the result of the orientation of the recess, which is apparently more vertical than in Alcidedorbignya.

The medial edge of the recess is a sharp crest, the crista parotica (De Beer 1937), which extends from the posteromedial edge of the preotic crest anteriorly to a knob-like process posteriorly, the tympanohyal. The tympanohyal is an ossification of Reichert's cartilage, which fuses to the petrosal at the crista parotica. For this reason, the crista parotica is said to extend posterior to the tympanohyal (MacPhee 1981; Wible 2008, 2012). The anterior part of the crista parotica is roughly straight to slightly concave in ventral view and strongly concave in lateral view. In ventral to ventromedial view it is roughly parallel to the lateral face of the petrosal. Posterior to the tympanohyal, the crista is straight anteroposteriorly and subvertical. The tympanohyal is short and massive, strongly bent medially and has a large, truncated, and expanded apex for the synchondrostic articulation with the stylohyal. The facet is oval-shaped in MHNC 8372 and more circular in MHNC 8399. This feature is clearly variable in Alcidedorbignya and among the isolated petrosals that preserve this structure, the facet is more circular than oval-shaped on five of eight specimens. This facet faces ventromedially. The tympanohyal partially floors the facial sulcus and, with the posterior part of the crista parotica, it forms the ventral and lateral edges 


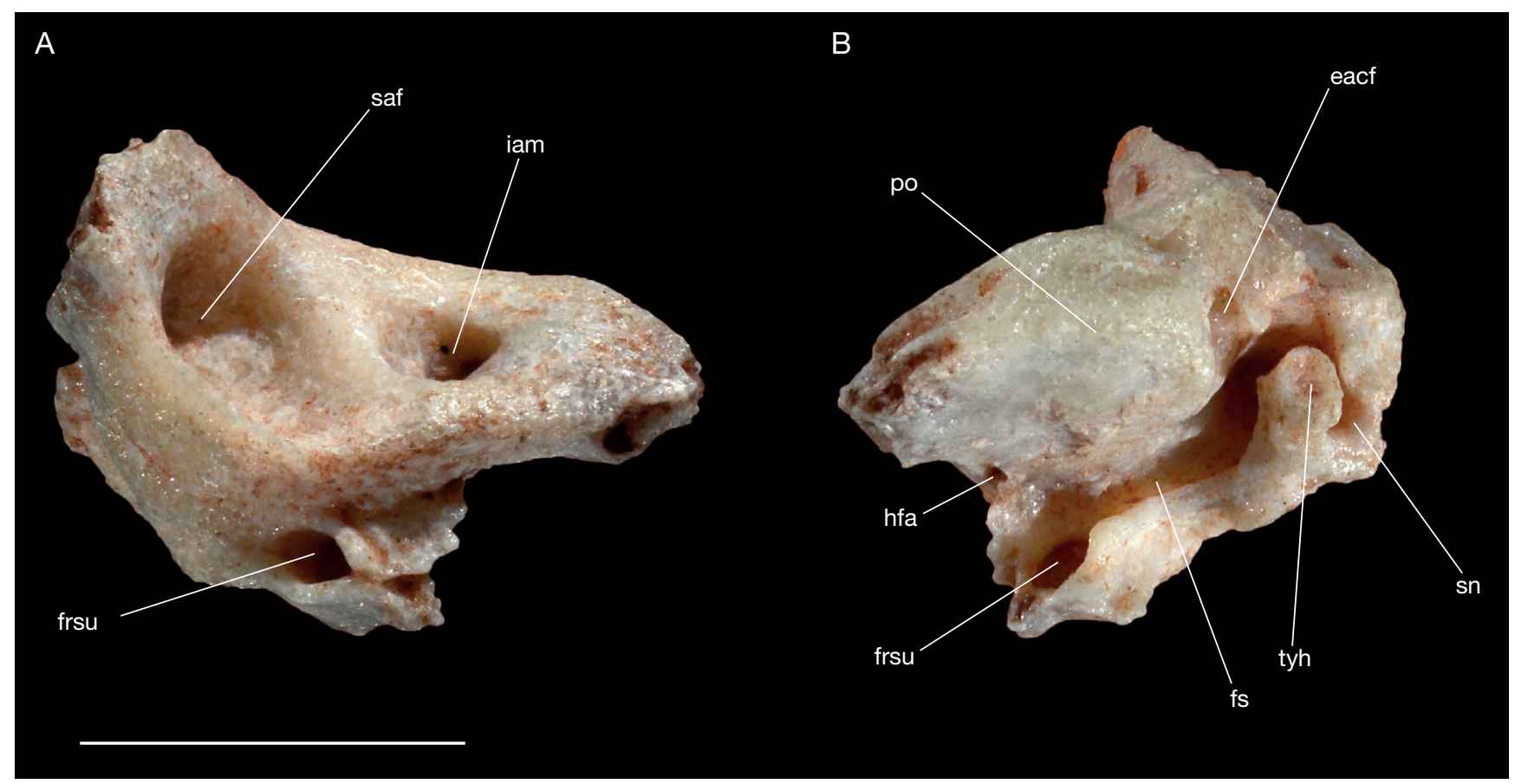

FIG. 40. - Isolated right petrosal of Alcidedorbignya inopinata (MHNC 8359) in: A, posterodorsolateral view; B, anteroventral view. Abbreviations: eacf, external aperture of the cochlear aqueduct; frsu, foramen for the ramus superior of the stapedial artery; fs, facial sulcus; hfa, hiatus Fallopii; iam, internal acoustic meatus; po, promontorium; saf, subarcuate fossa; sn, stylomastoid notch; tyh; tympanohyal. Scale bar: $5 \mathrm{~mm}$.

of the stylomastoid notch, through which the facial nerve exits the skull (Klaauw 1931; Wible 2008). The medial edge of the apex of the tympanohyal almost contacts the medial caudal tympanic process in MHNC 8372 and 8399, while it is closer to the lateral caudal tympanic process in MHNC 8359 (see below a description of the caudal tympanic processes). In Pantolambda bathmodon (AMNH 16663) the apex of the tympanohyal is almost equidistant from both caudal tympanic processes.

Dorsal to the tympanohyal is a deep and large hemispherical fossa, which extends the facial sulcus posteriorly. This fossa receives two elements. Laterally and ventrally one quarter of the cavity is for the passage of the facial nerve, which runs above the tympanohyal and exits the skull through the stylomastoid notch. The remainder of the cavity, dorsal and medial to the facial nerve, corresponds to a rather deep and wide stapedial fossa, which houses the origin of the stapedius muscle (Wible et al. 2001). It is located just behind the fenestra vestibuli and the crista interfenestralis (see below). The breadth of this fossa for the stapedial muscle is clearly more than twice the size of the fenestra vestibuli, which likely represents the plesiomorphic condition among eutherians (Segall 1970; Rougier et al. 1998; Wible et al. 2001).

On the posterior side of the promontorium, anterolateral to the jugular foramen and medial to the tympanohyal and stapedial fossa, is a large opening, the external aperture of the cochlear fossula. In previous works this opening was called the fenestra cochleae or fenestra rotunda (e.g., Novacek 1986; Wible 1990; Marshall \& Muizon 1995; Muizon 1998; Wible et al. 2004; Ladevèze et al. 2010; Ekdale \& Rowe 2011; Billet et al. 2011). An exception is MacPhee (1981), who called this opening the aperture of the fossula of the cochlear fenestra. In fact, as stated by MacPhee (1981: 51), who cites Richany et al. (1975: 92), the distal extremity of the cochlea, the fenestra cochleae (which is closed in life by the secondary tympanic membrane), opens externally on a "funnel-shaped niche, ... which hides it from view". This niche is the cochlear fossula and what is visible on the posterior side of the promontorium is the external aperture of the cochlear fossula, the internal aperture being the fenestra cochleae. Such a nomenclature is used by Wible $(2008 ; 2010 ; 2011)$, Wible et al. (2009), and Billet \& Muizon (2013). In Alcidedorbignya, the external aperture of the cochlear fossula is much larger than the fenestra vestibuli. On MHNC 8372, the external aperture of the cochlear fossula is slightly distorted dorsoventrally but on MHNC 8399 and on some isolated petrosals (MHNC8359, $8360,8362,8420$ and 8421), the apertures are undistorted. The external aperture of the cochlear fossula is oval-shaped and its long axis is oblique relatively to the sagittal and transversal planes of the skull in MHNC 8399 but subtransverse in MHNC 8372. From a ventral perspective, it is at an angle of approximately $90^{\circ}$ to $120^{\circ}$ with the fenestra vestibuli on the two skulls and on most isolated petrosals (the angle is probably the widest in 8399 , where it approaches $120^{\circ}$ ).

The posterodorsal edge of the external aperture of the cochlear fossula extends posteriorly in a wide and roughly triangular (or semicircular) fossa, the postpromontorial sinus (sensu Wible et al. 2009). This sinus is limited posteriorly by a ventrally-projecting sharp process, the medial caudal tympanic process of the petrosal (medial section of the caudal tympanic 

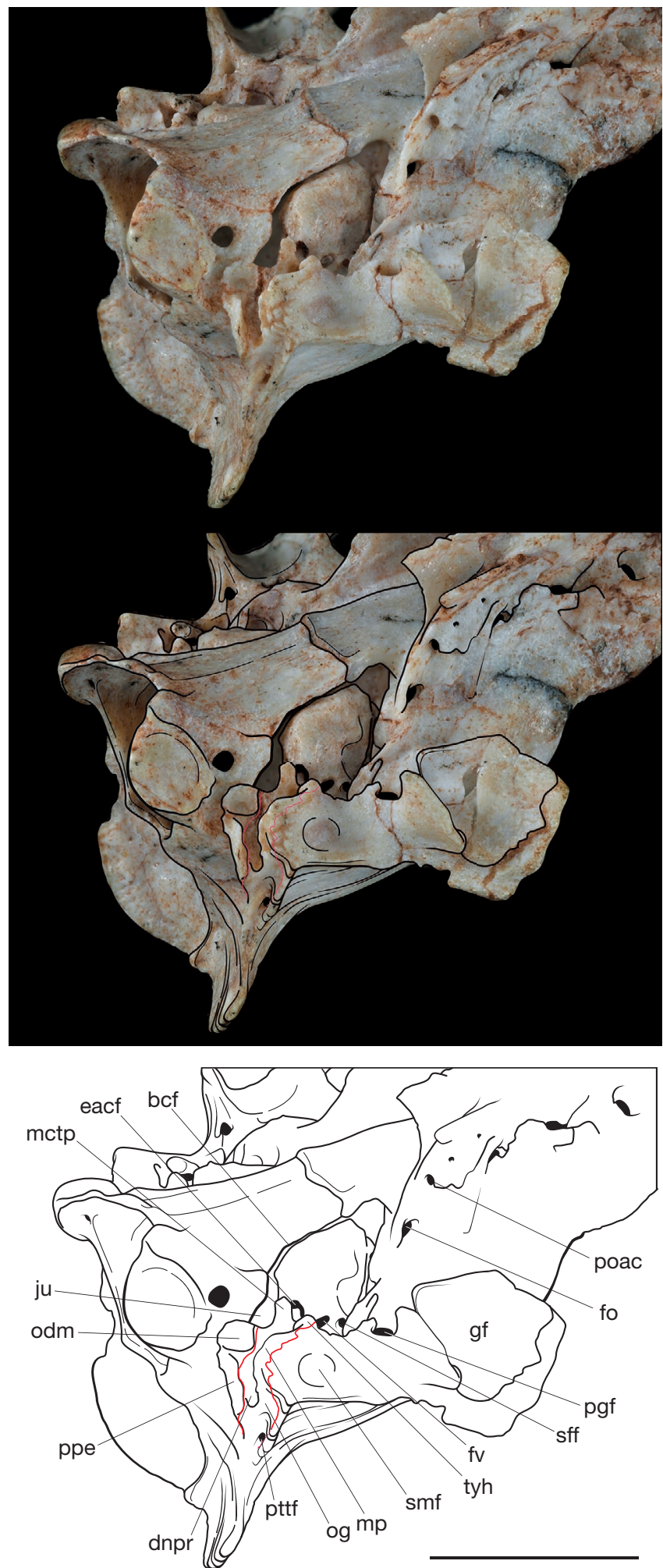

FIG. 41. - Posterolateral view of the skull of Alcidedorbignya inopinata (MHNC 8399) showing the distal notch of the posterior ramus of the stapedial artery (photograph, schematic drawing with bone sutures on photo, and schematic drawing with captions). Abbreviations: bcf, basicochlear fissure; dnpr, distal notch for the posterior ramus of the stapedial artery; eacf, external aperture of the cochlear fossula; fo, foramen ovale; fv, fenestra vestibuli; gf, glenoid fossa; ju, jugular foramen; mctp, medial caudal tympanic process; $\mathbf{m p}$, mastoid process; odm, probable origin of the digastric muscle; og, occipital groove; pgf, postglenoid foramen; poac, posterior opening of the alisphenoid canal; ppe, paroccipital process of the exoccipital; pttf, posttemporal foramen; sff, secondary facial foramen; smf, suprameatal fossa; tyh, tympanohyal. Scale bar: $1 \mathrm{~cm}$. process of MacPhee [1981]). Herein we define the medial caudal tympanic process as the knob or eminence that forms the posterior angle of the roughly triangular (or semicircular) postpromontorial sinus. In this respect we follow MacPhee (1981) rather than Kielan-Jaworowska (1981) and Wible et al. (2004 and 2009) who called it tympanic process. The medial caudal tympanic process is variable in size. In Alcidedorbignya it is roughly triangular in section with sharp angles at its base but is mediolaterally flattened toward its apex. When unbroken (as on left side of MHNC 8399), the medial caudal tympanic process can be up to twice as high as wide at base. However, it is also as high as wide at the base on some isolated petrosals (MHNC 8359, 8360, 8419, 8421), which reflects some individual variation. Furthermore, in four out of eight specimens (the two skulls and six isolated petrosals considered for this character), the medial caudal tympanic process almost contacts the tympanohyal laterally, but in MHNC 8399 and others it is distinctly bent medially (or at least not bent laterally), thus diverging from (or not directed toward) the tympanohyal.

The medial caudal tympanic process is connected to three different crests, posterior, anteromedial and anterolateral to it. On the posterior edge of the process is a sharp crest, which extends posteriorly (and dorsally) and forms the posterolateral edge of the jugular foramen. This crest is straight in ventral view but distinctly concave ventrally in lateral view. Running anteromedially from the medial caudal tympanic process is a short rounded crest that separates the postpromontorial sinus from the jugular foramen. This second crest presents a deep notch on the medial border of the postpromontorial sinus, probably for the passage of the tympanic nerve toward the middle-ear cavity through the postpromontorial sinus (see below). The third crest, anterolateral to the medial caudal tympanic process, extends anterolaterally in a medially bowed crest, which forms the lateral wall of the postpromontorial sinus. It ends at the posteromedial angle of the fenestra vestibuli on MHNC 8399 and 8372 and represents a posterior continuation of the crista interfenestralis, the broad bony bridge that separates the fenestrae of promontorium (see Wible et al. 2004; Ekdale et al. 2004; Billet \& Muizon 2013). On MHNC 8399 a distinct notch is present on the anteromedial side of the latter crest, at its junction with the lateral edge of the cochlear fossula. At the anterolateral corner of the postpromontorial sinus, a distinct groove is present. It leaves the sinus and fades anteriorly but likely passes medially to the fenestra vestibuli. Although MHNC 8372 is not as well preserved as MHNC 8399, the notch (on the medial wall of the postpromontorial sinus) and groove (on the lateral wall) are also probably present on this specimen. This notch and groove are distinctly present on isolated petrosals (MHNC 8360, 8362). On some specimens (MHNC 8359, $8361,8319,8320)$ the notch only is well defined. The notch posteromedial to the external aperture of cochlear fossula and the groove on the lateral corner of the postpromontorial sinus mentioned above, are probably for the passage of the tympanic nerve, an offshoot of the glossopharyngeal nerve (CN IX), which exits the skull at the jugular foramen (MacPhee 1981; Wible 2008, 2010; Evans \& de Lahunta 2012). According to this 
interpretation, the tympanic nerve exits the jugular foramen, passes posterior to the external aperture of the cochlear fossula and ventral to the postpromontorial sinus, runs anteriorly on the crista interfenestralis, passes medial to the fenestra vestibuli, and ventral to the tegmen tympani, where it continues its course anteriorly as the lesser petrosal nerve and joins the otic ganglion, which in turn is connected to the mandibular branch of the trigeminal nerve (CN V3) (MacPhee 1981; Evans \& de Lahunta 2012). Although this hypothesized course of the tympanic nerve in front of the caudal medial tympanic process and crossing the postpromontorial sinus does not correspond to the "standard" pattern described for eutherians (MacPhee 1981: 44), it is quite plausible for Alcidedorbignya as a similar pattern has already been evidenced in Erinaceus (MacPhee 1981: 205) and Dasypus (Wible 2010: fig. 3). A similar condition with a tympanic nerve entering the postpromontorial sinus is also present in Solenodon and Potamogale. This interpretation is also supported by the condition observed in Pantolambda (see below).

The medial caudal tympanic process forms the medial wall of the posterior opening of the tympanic cavity, which is posteroventral to the stapedial fossa. On MHNC 8372 and 8399 , midway between the medial caudal tympanic process and the tympanohyal is a well-developed hump, which may be regarded as a lateral caudal tympanic process (see Appendix 5). It is, however, much lower than the medial process and the tympanohyal. This condition gives to the large posterior opening of the middle ear sulcus (between the medial caudal tympanic process medially and the tympanohyal laterally) a characteristic $\omega$-shaped morphology, of which the lateral concavity is the stylomastoid notch for the facial nerve. Passing this notch, the facial nerve turns abruptly ventrally (to ventrolaterally) in a short groove, which is discernible on the medial face of the posterior continuation of the crista parotica (cf. Wible 2008: fig. 27B). Then it probably shifted laterally and dorsally, passed over (or surrounded posteriorly) the meatal fossa and then spread anteriorly on the superficial regions of the auricular area, face, and snout on the lateral and dorsal surface of the skull (Evans \& de Lahunta 2012). The medial notch of the $\omega$-shaped posterior opening is interpreted here as a probable passageway for a ramus posterior of the stapedial artery, which primarily has a proximal stapedial origin in eutherians and separates from its parent vessel just before this latter reaches the stapes (Wible 1987). It is referred to below as the proximal notch of the ramus posterior. This notch is approximately $30-50 \%$ larger than the stylomastoid notch in the available petrosal specimens attributed to Alcidedorbignya, which would indicate a relatively large vessel. As a matter of fact, MacPhee $(1981: 225,226)$ noted that the ramus posterior is large in tenrecoids and Solenodon (see also Wible 2008: fig. 26 C for Solenodon). This vessel was also observed by McDowell (1958) in Nesophontes and Solenodon and it is present in erinaceomorph embryos, although very reduced (MacPhee 1981)
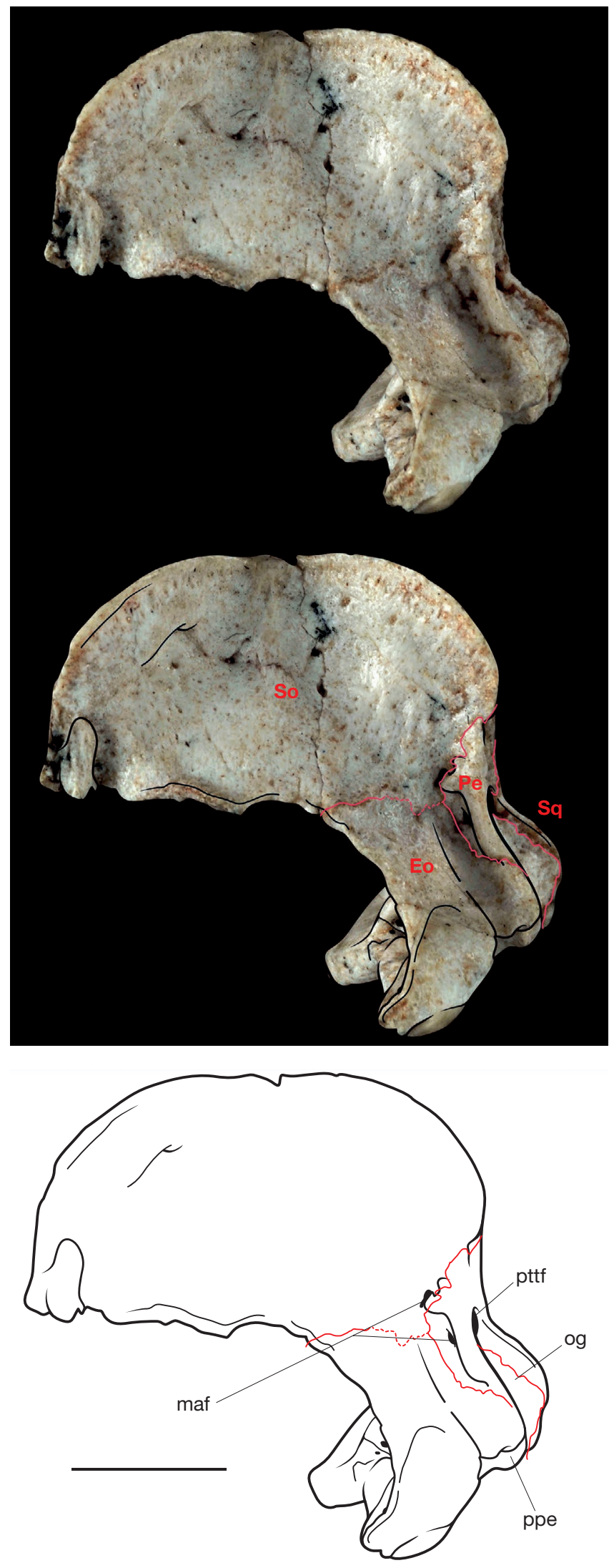

FIG. 42. - Posteromedial view of the right side of the skull of Alcidedorbignya inopinata (MHNC 8372) showing the medially inflected petrosal-exoccipitalsupraoccipital suture (photograph, schematic drawing with bone sutures on photo, and schematic drawing with captions). Abbreviations: Eo, exoccipital; maf, mastoid foramina; Pe, petrosal; ppe, paroccipital process of the exoccipital; og, occipital groove; pttf, posttemporal foramen; So, supraoccipital; Sq, squamosal. Scale bar: $5 \mathrm{~mm}$. 

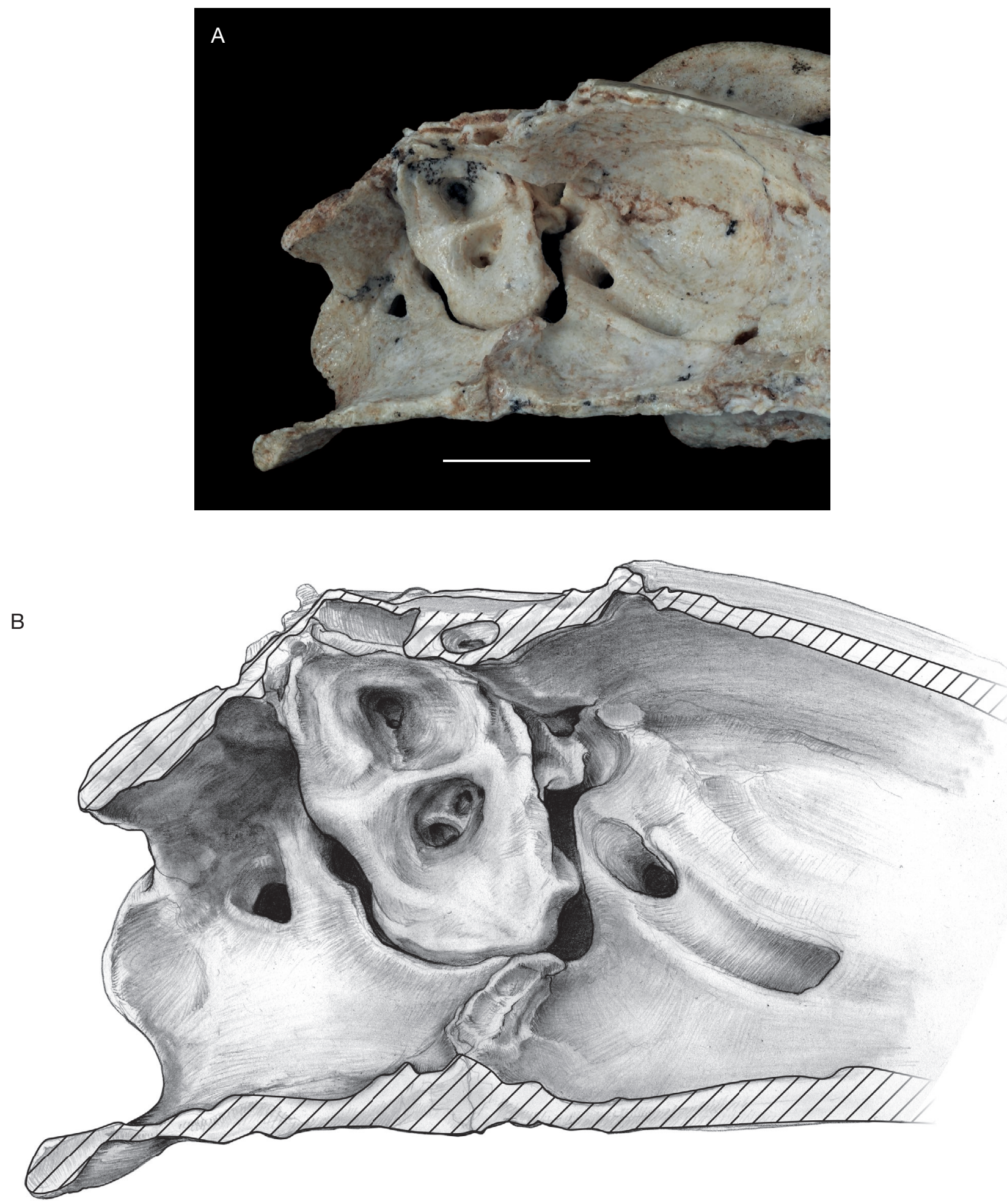

FIG. 43. - Anterodorsomedial view of the left endocranial aspect of the left auditory region of Alcidedorbignya inopinata (MHNC 8372): A, photograph; B, drawing. Scale bar: $A, 5 \mathrm{~mm}$.

The $\omega$-shaped morphology of the above-described posterior opening of the middle ear sulcus is variable and sometimes poorly marked in Alcidedorbignya mainly because the size and distinctness of the lateral caudal tympanic process is variable. In MHNC 8399 the $\omega$-shaped morphology is distinct with a moderately developed lateral caudal tympanic process on the right side but on the left side the process is extremely reduced and the posterior opening of the facial canal has a roughly circular morphology. Furthermore, on some isolated petrosals referred to Alcidedorbignya the process is very faint 


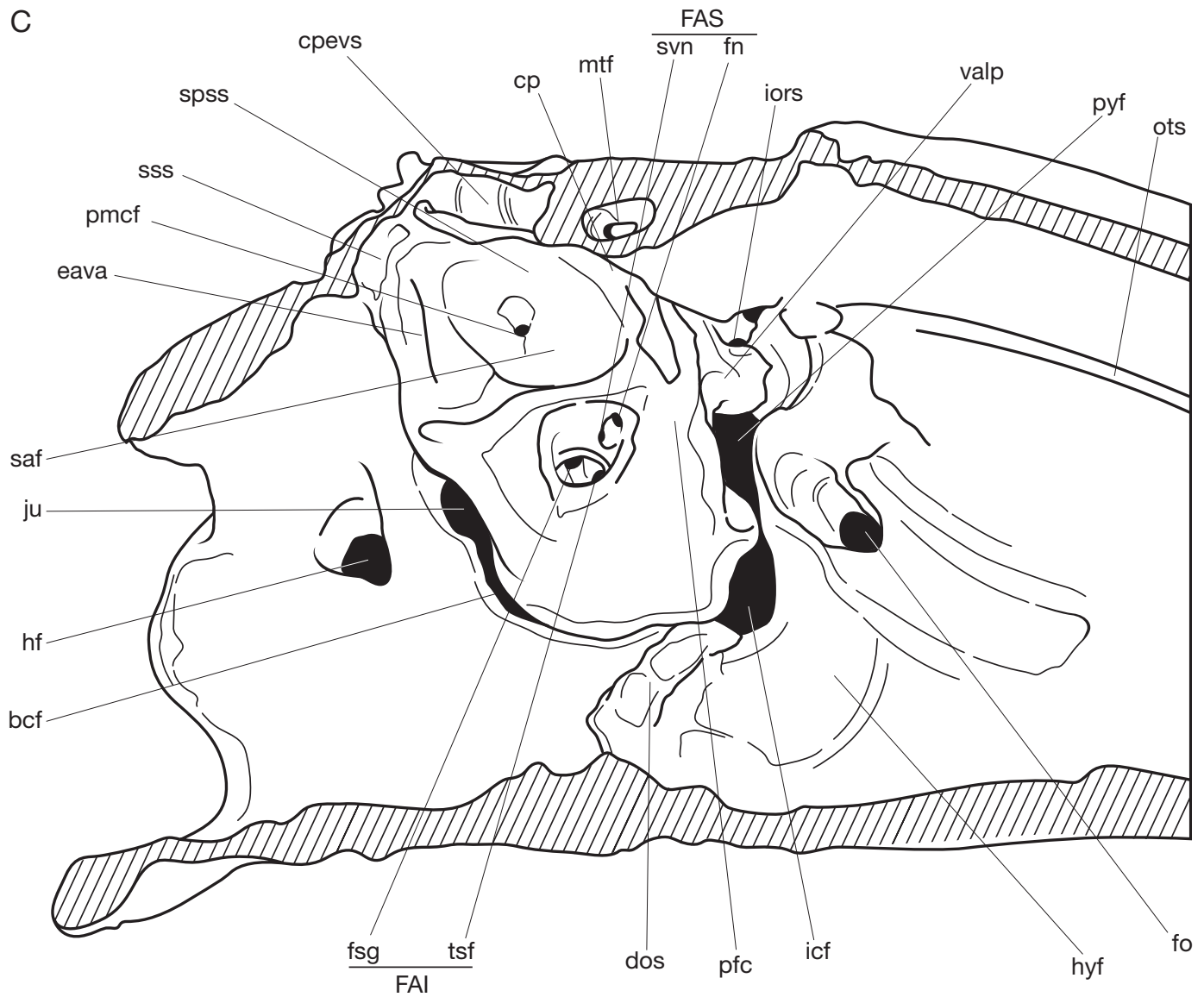

FIG. 43 (continuation). - Anterodorsomedial view of the left endocranial aspect of the left auditory region of Alcidedorbignya inopinata (MHNC 8372): C, schematic diagram with captions. Abbreviations: bcf, basicochlear fissure; $\mathbf{c p}$, crista petrosa; cpevs, sulcus for the capsuloparietal emissary vein; dos, dorsum sellae; FAl, foramen acusticum inferius; eava, external aperture of the vestibular aqueduct; FAS, foramen acusticum superius; fn, canal for the facial nerve; fo, foramen ovale; fsg, foramen singulare for inferior vestibular nerve bundles; hf, hypoglossal foramen; hyf, hypophyseal fossa; icf, internal carotid foramen; iors, intracranial opening of the canal for the ramus superior of the stapedial artery; ju, jugular foramen; mtf, median temporal foramen; ots, orbitotemporal sulcus; pfc, prefacial commissure; pmcf, foramen for the petromastoid canal; pyf, pyriform fenestra; saf, subarcuate fossa; spss, sulcus for the superior petrosal sinus; sss, sulcus for the sigmoid sinus; svn, tractus for the passage of the superior branch of the vestibular nerve; tsf, tractus spiralis foraminosus (canal and cribriform tract for cochlear nerve) valp, vestigial anterior lamina of the petrosal.

and the posterior opening is also roughly circular, while on some others it is well-developed, and together with the tympanohyal almost encloses laterally the stylomastoid notch in a stylomastoid foramen.

In Alcidedorbignya, posterior to the proximal notch of the ramus posterior, the vessel should have continued its course on the occipital mastoid exposure of the petrosal, in a deep and wide groove, which extends posterolaterally up to a ventroantero-dorsoposteriorly oriented crest of the pars mastoidea. This crest joins the mastoid process to the paroccipital process of the exoccipital and presents a notch probably for the passage of the ramus posterior (the distal notch of the ramus posterior) toward the posterolateral angle of the skull (Fig. 41). This notch is especially deep and large in MHNC 8399, where it is almost 50\% larger than the proximal notch. It is relatively shallow in MHNC 8372 and approximates the size of the proximal notch. A similar sulcus for the ramus posterior is also observed on the mastoid exposure of Leptictis with a deep distal notch at the posterolateral angle of the skull. Therefore, although this vessel was not mentioned by Novacek (1986), it was probably present in Leptictis. It is noteworthy that Novacek (1986) regarded this sulcus as the area of origin of the digastricus muscle. However, the origin of the digastricus muscle in mammals is generally on the paroccipital process of the exoccipital and/or on the mastoid process (Howell 1928; Davis 1964; Piérard 1971; Bryden 1971; Barone 1997; Evans \& de Lahunta 2012). In fact, in Leptictis (AMNH FAM 87458 and AMNH 80213), medial to the groove for the ramus posterior is a distinct cupula on the medial edge of the mastoid exposure. This cupula faces the paroccipital process of the exoccipital, which also bears a mark of muscle attachment. We think that these two structures are for the origin of the digastric muscle. A similar condition is observed in Tenrec ecaudatus, in which the digastric muscle has its origin on a flat area of the mastoid exposure just medial to the groove for the ramus posterior and on a deep groove adjacent to this area and excavated in the lateral edge of the paroccipital process of the exoccipital. In Alcidedorbignya the origin of the digastric muscle is apparently mainly on the exoccipital (see below) and with a very small (if any) part on the lateral edge on the mastoid exposure (Figs 37, 38). 


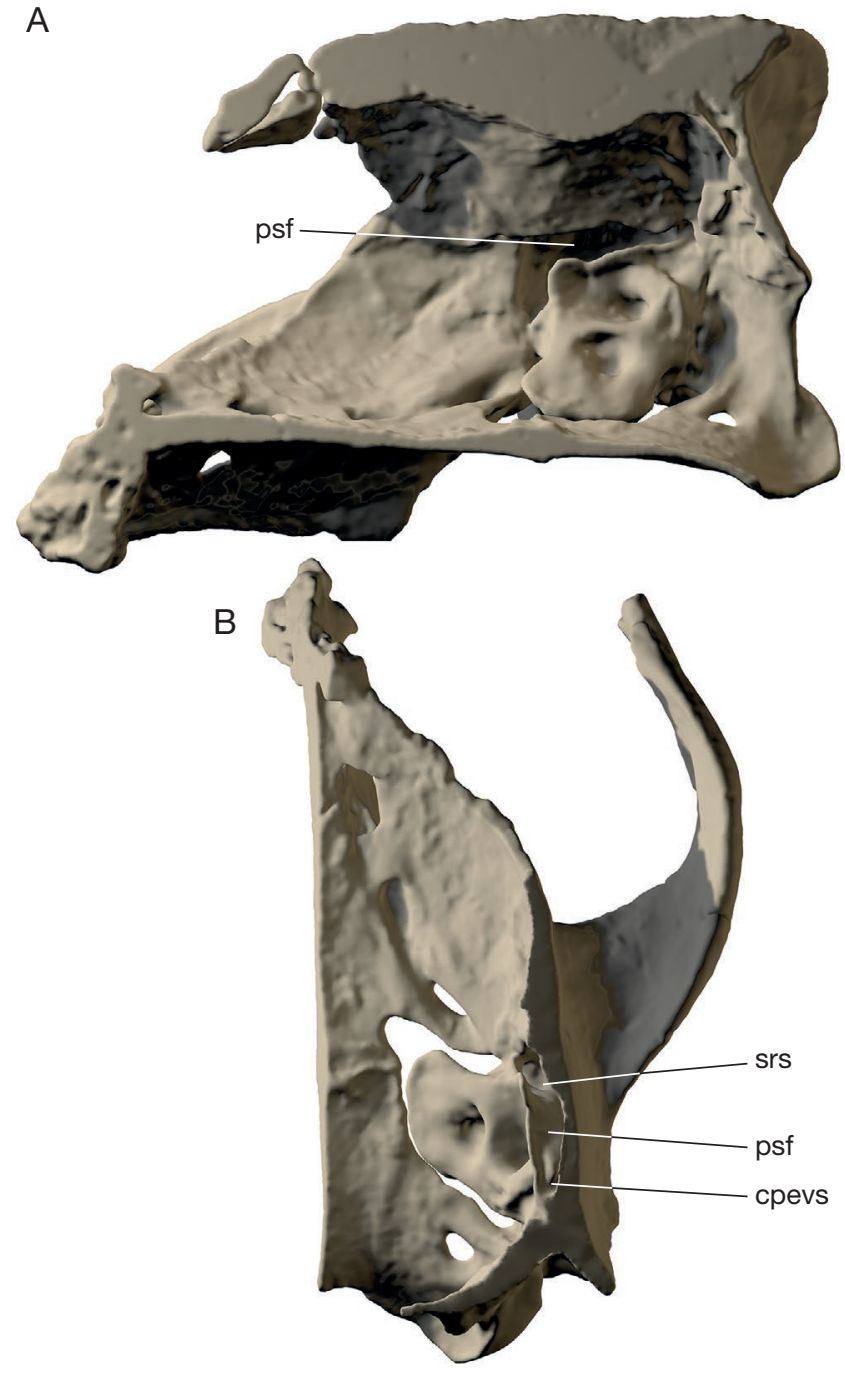

FIG. 44. - Three-dimensional rendering of CT data illustrating the right auditory region of Alcidedorbignya inopinata (MHNC 8399): A, intracranial view showing the petrosquamosal fossa; $\mathbf{B}$, frontal section of the skull at the level of the petrosquamosal fossa. Abbreviations: cpevs, sulcus for the capsuloparietal emissary vein; psf, petrosquamosal fossa; srs, sulcus for the superior ramus of the stapedial artery. The 3D rendering of CT data is available in pdf format at the address http://sciencepress.mnhn.fr/en/periodiques/geodiversitas/37/4/ alcidedorbignya-inopinata-additional-files.

Another possible occupant of the proximal notch for the ramus posterior is the auricular branch of the vagus nerve $(\mathrm{CN}$ $\mathrm{X})$. This branch exits from the jugular ganglion of the vagus nerve in the jugular foramen and "runs laterally across the mastoid region to the stylomastoid foramen, where it meets the facial nerve" (MacPhee 1981: 44; Evans \& de Lahunta 2012). According to MacPhee, this condition represents the "standard" pattern, in which the course of this nervous branch is entirely extratympanic and posterior to the medial caudal tympanic process. In the case of Alcidedorbignya, the connection between the two nerves (auricular and facial) could have followed the standard pattern of MacPhee. The observation of the relative position of the jugular foramen, stylomastoid notch and medial caudal tympanic process in MHNC 8372 and 8399 could favour this interpretation.
However, the auricular branch could also have entered the middle ear via the ramus posterior notch and connected to the facial nerve below the stapedial fossa (Fig. 39). Arguments for this slightly different interpretation are provided by the morphology of some of the isolated petrosals referred to Alcidedorbignya and that of Pantolambda (see below, Appendix 3). In MHNC 8359 and 8360, on the posteromedial face of the petrosal, just anterior to the suture area between the petrosal and the exoccipital is a distinct vertical groove, which probably marked the passage of the vagus nerve $(\mathrm{CN} \mathrm{X})$. This groove meets the base of the medial caudal tympanic process ventrally, where it prints a faint notch on its posterior face. This notch is anterior to the stylomastoid notch and almost level with the tympanohyal (Fig. 39 and below Fig. 50) and could correspond to the passageway of the auricular ramus entering the tympanic cavity. This condition is not so pronounced in other specimens (MHNC 8361). Therefore, the interpretation of an intra-tympanic junction of the auricular branch to the facial nerve is tentatively retained on Fig. 39.

The condition observed in Pantolambda (AMNH 16663) is described in Appendix 3. Because Pantolambda is phylogenetically very close to Alcidedorbignya (Muizon \& Marshall 1992 and below), it provides some possible indication on the interpretation of the passageway of the auricular branch of the vagus nerve in the latter genus. As described below in Appendix 3, in Pantolambda, a distinct groove excavates the medial and posterior edge of the medial caudal tympanic process; this groove is interpreted here as the passageway of the auricular ramus. If this interpretation is correct, this condition clearly indicates that this nerve entered the middle ear and joined the facial nerve probably ventral to the stapedius muscle. Because of the close phylogenetic relationships of Pantolambda and Alcidedorbignya and because of the condition observed on six specimens of Alcidedorbignya (which, either favours or is not incompatible with this interpretation), we are inclined to favour an intratympanic connection of the auricular branch of the vagus nerve to the facial nerve. However, it is noteworthy that the passageway of the auricular branch of the vagus nerve in relation with the medial caudal tympanic process is quite variable in placentals (see MacPhee 1981; Evans \& de Lahunta 2012; Wible 2008, 2010; Wible \& Spaulding 2013) and the hypothesis of an extra-tympanic connection cannot be totally discarded.

Posterolateral to the stylomastoid notch and appressed against the posttympanic process of the squamosal is a low but stout mastoid process (= paroccipital process of the petrosal in Wible et al. 2001; 2004; 2009; mastoid eminence in MacPhee 1981 and Rougier et al. 1992; see Appendix 5). The morphology of the mastoid process of Alcidedorbignya presents some variation as it is distinctly longer and sharper in MHNC 8399 than in MHNC 8372. From the mastoid process a small anteroposteriorly oriented crest is directed posterodorsally (and notched posteriorly for the passage of the ramus posterior; see above); it crosses obliquely the exposed portion of the pars mastoidea, and reaches the posterior edge 

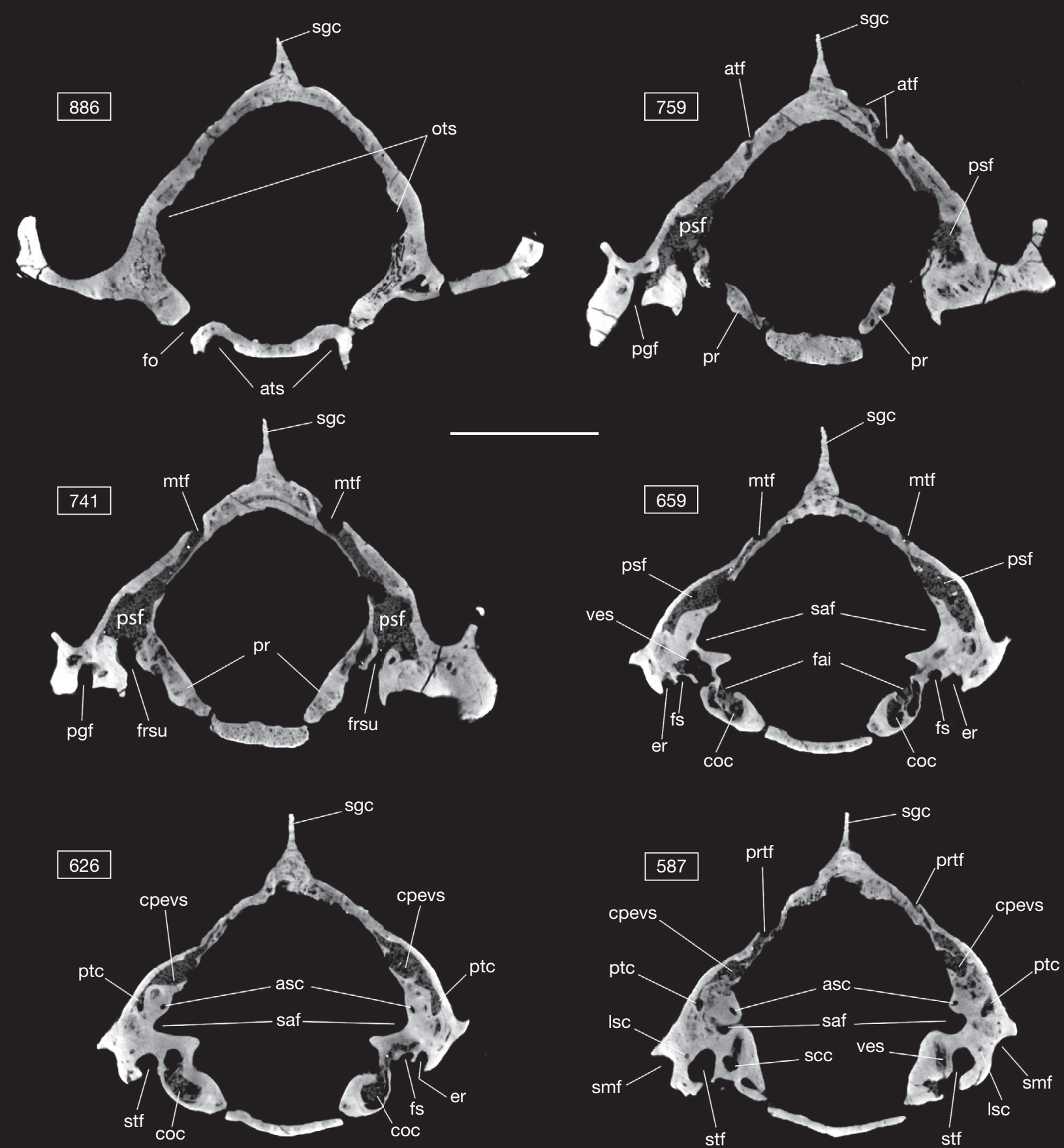

FIG. 45. - CT-slices through the skull of MHNC 8399. The numbers for each illustrated slice refer to the position of the slice within the sequence of 1952 images (resolution binned images, voxel size $=0.024 \mathrm{~mm}$ ). Top of the skull toward the top, right side of the skull toward the left. Note that the slices are slightly oblique relative to the sagittal plane, and therefore the two sides of the skull are not exactly symmetrical on the images. Abbreviations: asc, anterior semicircular canal; atf, anterior temporal foramen; ats, sulcus for the auditory (Eustachian) tube; coc, cochlear canal; cpevs, sulcus of the capsuloparietal emissary vein; er, epitympanic recess; fai, foramen acusticum inferius; fo, foramen ovale; fs, facial sulcus; frsu, foramen for the superior ramus of the stapedial artery; Isc, lateral semicircular canal; mtf, median temporal foramen; ots, orbitotemporal sulcus; pgf, postglenoid foramen; pr, promontory; prtf, posterior temporal foramen; psf, petrosquamosal fossa; ptc, posttemporal canal; saf, subarcuate fossa; scc, secondary common crus; sgc, sagittal crest; smf, suprameatal fossa; stf, stapedial fossa; ves, vestibule. Scale bar: $1 \mathrm{~cm}$.

of the paroccipital process of the exoccipital (= paracondylar process of Wible et al. 2004, 2009) medially (Fig. 37). A small ventrally-facing shallow triangular depression is thus delimited medial to this crest. This forms a posterolaterally oriented groove that received the ramus posterior of the stapedial artery as mentioned above. 
Posterolaterodorsal to this anteroposterior crest, the exposed portion of the pars mastoidea is subvertical and wedged between the squamosal and the exoccipital. It is limited by two anterolateral and posteromedial subvertical crests (but converging dorsally), which enclose a deep sub-vertical groove, the occipital groove, for the occipital artery and vein (see Wible 2010) (Figs 41, 42). The anterolateral crest follows the squamosal-petrosal suture and is in fact the ventral extension of the lambdoid crest. The posteromedial crest extends from the paroccipital process of the exoccipital and follows the exoccipital-petrosal suture on the ventral third of its length. At this point the suture is strongly inflected medially (Fig. 42) until the dorsal apex of the posterior mastoid exposure. As a consequence, the dorsal two-thirds of the crest is formed by the mastoid alone with no participation of the exoccipital. Because of the inflexion of the suture, a vast and roughly semicircular portion of the pars mastoidea of the petrosal is visible on the occipital face of the skull. Several mastoid foramina are observable on the two specimens (MHNC 8372 and 8399). On the right side of MHNC 8372 a well-developed mastoid foramen perforates the petrosal; it located close to the exoccipital suture and slightly below mid-height of the mastoid occipital exposure. On the dorsal part of the exposure is another mastoid foramen located in the suture. On the left side of MHNC 8372 a conspicuous mastoid foramen is present, in the lower part of the exposure and perforating the suture between the petrosal and the exoccipital. Another mastoid foramen is present at mid height of the exposure in the petrosal. On the right side of MHNC 8399 a large mastoid foramen forms a groove on the medial edge of the pars mastoidea in the petrosal-exoccipital suture. A tiny foramen may be also present on the left side of the specimen, slightly dorsal to the right one. In the dog the mastoid foramen transmits the occipital emissary vein, an offshoot of the sigmoid sinus, which drains the occipital muscle (Wible 2008; Evans \& de Lahunta 2012). In this connection, in Alcidedorbignya, there is a well-developed foramen, comparable in size to the mastoid foramen and located at a similar height, is observable intracranially in the petrosal, in the groove for the sigmoid sinus. This foramen could represent the intracranial opening of the canal of the occipital emissary vein. However it has not been possible to follow this canal on the CT scan of MHNC 8399, neither from the mastoid foramen, nor from the intracranial foramen described above. It is therefore possible that this canal was subject to some distortion during fossilisation. Another interpretation would be that the intracranial opening of the canal is not in the petrosal but in the petrosal-exoccipital suture. This interpretation is confirmed by the observation made on an isolated left petrosal (MHNC 8362). On this specimen, the mastoid exposure is reasonably well preserved and on its medial side at the level of the petrosal-exoccipital suture and at approximately the same height as the posttemporal notch laterally, is a notch, which extends anteriorly in a strongly sigmoid groove. This groove ends at the intracranial side of the suture just posterior to the sulcus for the sigmoid sinus. We interpret this groove as the passage of the occipital emissary vein from the sigmoid sinus to the occipital aspect of the skull. The groove was covered medially by the exoccipital forming the canal for the occipital emissary vein. No mastoid foramen was observed on other isolated petrosals (MHNC $8359,8360,8362,8419)$ with a reasonably well-preserved mastoid exposure, neither within the bone nor in the suture. Whatever the interpretation, the comparison of the condition in both skull and in the five petrosals demonstrates that the number and position of the mastoid foramina is subject to strong individual variation.

Dorsally, the pars mastoidea extends further than midheight of the occipital face of the skull. In its dorsal third, at the suture with the squamosal, it presents a well-developed posttemporal foramen for the exit of the diploetic vessels (arteria and vena diploëtica magna; Wible 2008). Because the posttemporal canal is in the petrosal-squamosal suture, the lateral edge of the foramen is formed by the squamosal and its medial edge excavates the petrosal.

Dorsal (endocranial) face of the petrosal. On the endocranial face of the petrosal, the subarcuate fossa is relatively large, conical and moderately deep. The bottom of the fossa faces somewhat anteriorly rather than strictly mediodorsally (Figs 28, 43). It clearly differs from that of Leptictis, which is deeper and which has a constricted opening (i.e. narrower than the greater diameter of the fossa). A small foramen is present in the bottom of this fossa. This foramen is for the petromastoid canal, which transmits the subarcuate artery and vein from the subarcuate fossa to the mastoid cells (Gannon et al. 1988; Krombach et al. 2002; O'Leary 2010).

Posterior and posteromedial to the fossa subarcuata, the sulcus for the sigmoid sinus runs along the suture between the petrosal and the exoccipital. The sigmoid sinus exits the skull through the jugular foramen as the jugular vein (Wible 1990). Before exiting the skull, the jugular vein receives the inferior petrosal sinus anteriorly (MacPhee 1981). This latter sinus runs in a shallow sulcus on the flattened medial edge of the promontorium in the basicochlear fissure or slightly ventral to it (see above), anteromedial to the external aperture of the cochlear fossula and dorsomedial to the cochlear canaliculus. Anteriorly, the inferior petrosal sinus drained from the cavernous sinus, and exited the braincase via the anterior end of the basicochlear fissure just posteromedial to the carotid notch. This course is usual in eutherians with a non-obliterated basicochlear fissure (MacPhee 1981). A similar condition was probably present in Pantolambda, but in this genus the sulcus for the inferior petrosal sinus is best seen more anteriorly, at the anteromedial angle of the promontorium. It is distinctly ventral to the basioccipital.

Below we describe the morphology of the petrosquamosal fossa as presented and defined above. Although this fossa does not refer to the endocranial aspect of the petrosal bone alone (but also to its lateral side) it mainly concerns the endocranial drainage of the ear region of the skull, which we want to consider as a whole (Figs 28, 43-46). We also provide below an interpretation of the reconstruction of the vessels of this region of the skull. The description below refers to MHNC 8399 and MHNC 


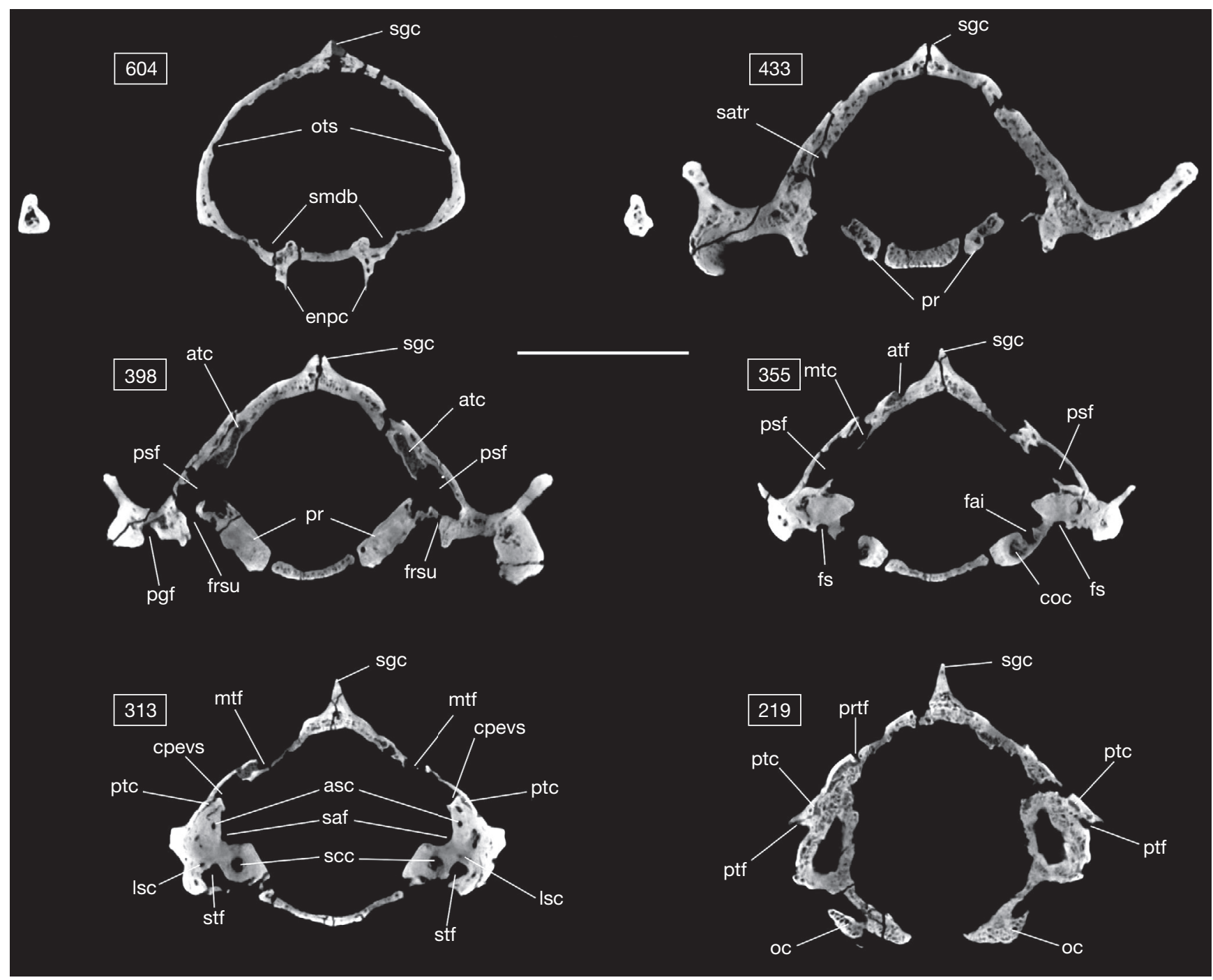

FIG. 46. - CT-slices through the skull of MHNC 8372. The numbers for each illustrated slice refer to the position of the slice within the sequence of 1812 images (resolution: on binned images, voxel size $=0.033 \mathrm{~mm}$ ). Top of the skull toward the top, right side of the skull toward the left. Abbreviations: asc, anterior semicircular canal; atf, anterior temporal foramen; atc, canal for anterior temporal ramus; coc, cochlear canal; cpevs, sulcus of the capsuloparietal emissary vein; enpc, entopterygoid crests; fai, foramen acusticum inferius; frsu, foramen for the ramus superior of the stapedial artery; fs, facial sulcus; Isc, lateral semicircular canal; mtc, canal for median temporal ramus; mtf, median temporal foramen; oc, occipital condyle; ots, orbitotemporal sulcus; pgf, postglenoid foramen; pr, promontory; prtf, posterior temporal foramen; psf, petrosquamosal fossa; ptc, posttemporal canal; ptf, posttemporal foramen; saf, subarcuate fossa; satr, sulcus of anterior temporal ramus; scc, secondary common crus; sgc, sagittal crest; smdb, sulcus of mandibular branch of trigeminal nerve; stf, stapedial fossa. Scale bar: $1 \mathrm{~cm}$.

8372. The fossa of MHNC 8399 is the best-preserved as its medial septum is apparently almost complete (Figs 44, 45). On MHNC 8372 (Fig. 46), the medial septum of the petrosquamosal fossa is incomplete either because of incomplete ossification of the individual (young adult) or because it was partially damaged during fossilisation (less likely). The floor of the fossa is formed by the petrosal. It is excavated by two deep sulci on the dorsolateral face of the petrosal (Figs 43, 44). They are arranged one behind the other and oriented roughly anteroposteriorly. The anterior one is strongly dipping anteriorly and opens ventrally in the foramen for the ramus superior. This was the passageway for the ramus superior leading toward the dorsal region of the petrosal (Fig. 44). The posterior sulcus is subhorizontal (to slightly dipping anteriorly), dorsolateral to the fossa subarcuata, and ends at the posterodorsal angle of the petrosal. It is interpreted as having contained the passage for the capsuloparietal emissary vein (Figs 43, 44). The two sulci are separated by an oblique sharp ridge oriented posterodorsolaterally-anteroventromedially. The ventral part of the medial septum of the petrosquamosal fossa is formed by the medial edge of these sulci. It is a subvertical crest of the petrosal (possibly a posterior extension of the crista petrosa), which extends from the posterodorsalmost point of the petrosal (at the intracranial triple junction between the petrosal, the occipital and the parietal) to the level of the foramen for the ramus superior at the anterolateralmost angle of the fossa subarcuata. This crest actually curves along the anterodorsolateral edge of the fossa subarcuata on MHNC 8399 and its posterior continuation extends into a long subhorizontal dorsolateral edge of the petrosal, much longer than its anterior 


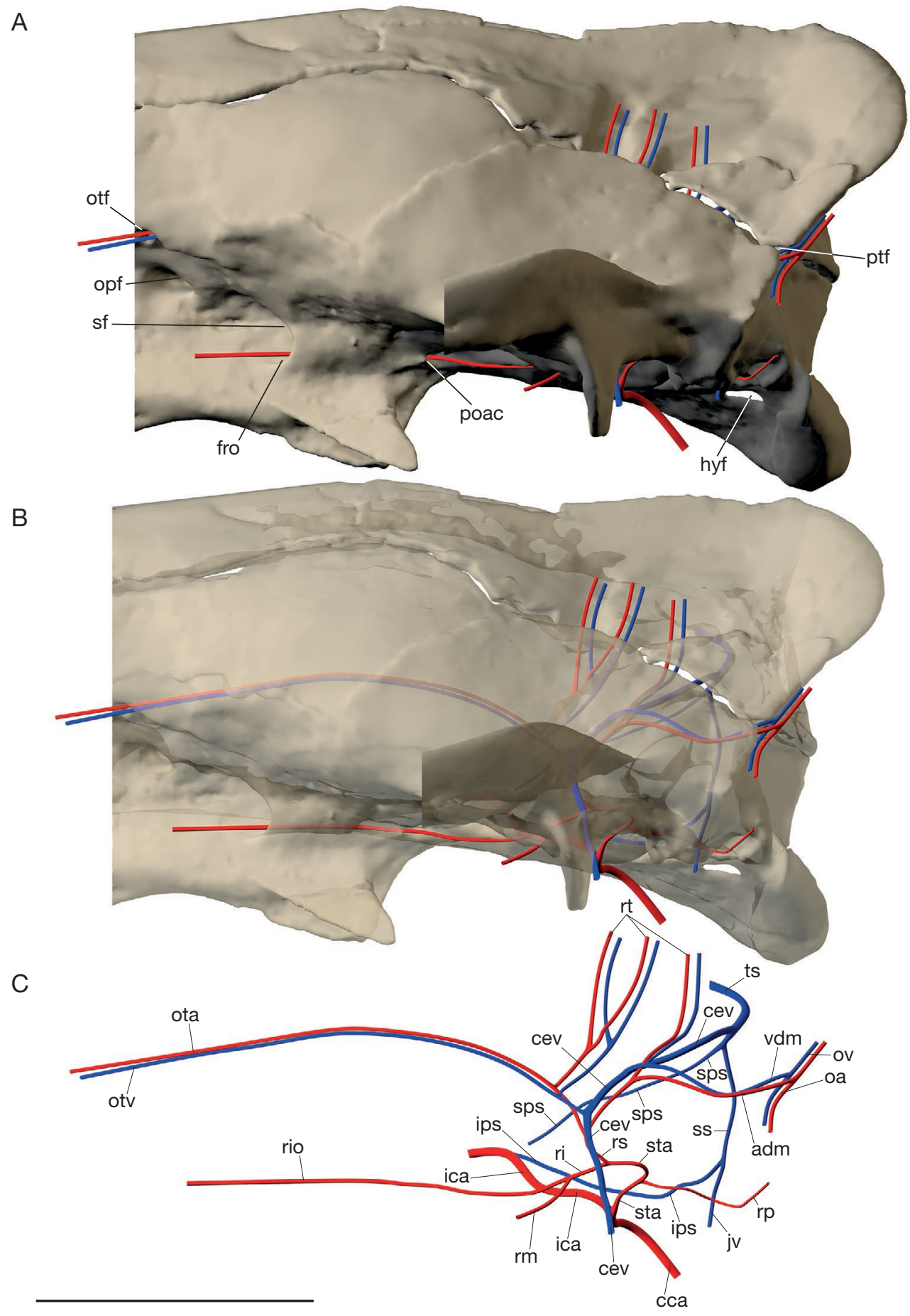

FIG. 47. - Three-dimensional rendering of CT data illustrating the left side of the braincase of Alcidedorbignya inopinata (MHNC 8372) with vessels reconstruction. Vessels courses reconstruction has been performed directly on the slides of the image stack obtained by CT scanning of the skull: A, lateral view without transparency; B, lateral view with transparency; C, vessels only in lateral view. Abbreviations: adm, arteria diploëtica magna; cca, common carotid artery; cev, capsuloparietal emissary vein; fro, foramen rotundum; hyf, hypoglossal foramen; ica, internal carotid artery; ips, inferior petrosal sinus; jv, jugular vein; oa, occipital artery; opf, optic foramen, ota, orbitotemporal artery, otf, orbitotemporal foramen; otv orbitotemporal vein; ov, occipital vein; poac, posterior opening of the alisphenoid canal; ptf, posttemporal foramen; ri, ramus inferior; rio, ramus infrorbitalis; rm, ramus mandibularis; rp, ramus posterior; rs, ramus superior; rt ramus temporalis; sf, sphenorbital fissure; sps, superior petrosal sinus; ss, sigmoid sinus; sta, stapedial artery; ts, transverse sinus; vdm, vena diploëtica magna. Scale bar: $1 \mathrm{~cm}$. The $3 \mathrm{D}$ rendering of CT data is available in pdf format at the address http://sciencepress.mnhn.fr/en/periodiques/geodiversitas/37/4/alcidedorbignya-inopinata-additional-files.

subvertical portion. The dorsal part of the medial septum of the petrosquamosal fossa is formed by the parietal. The two crests (petrosal and parietal) do not contact. They are separated by a long and narrow slit posteriorly and by a wider opening anteriorly. The lateral wall of the petrosquamosal fossa is formed by the squamosal. The roof of the petrosquamosal fossa is pierced close to or at the squamosal-parietal suture by three large openings for the 


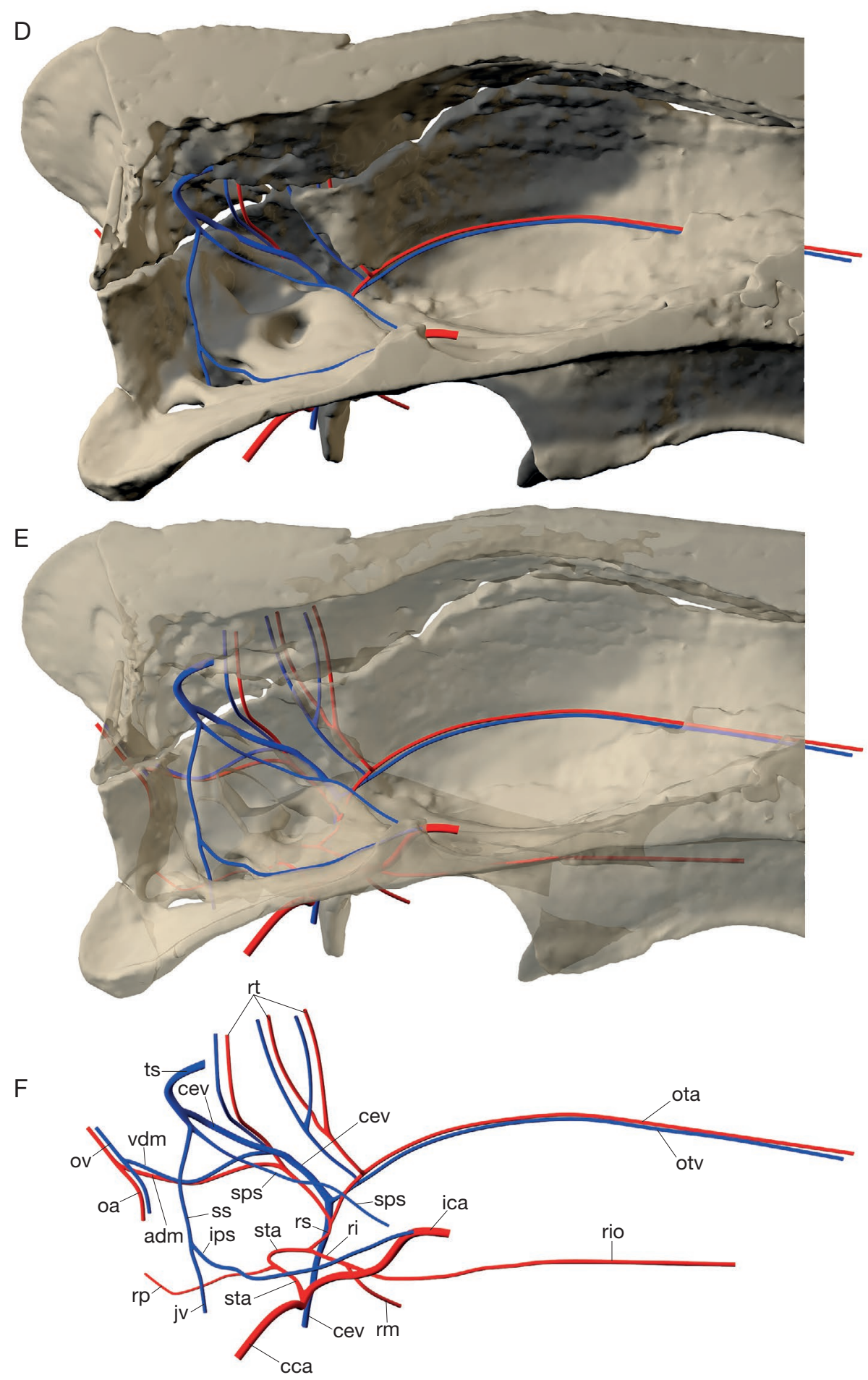

FIG. 47 (continuation). - Three-dimensional rendering of CT data illustrating the left side of the braincase of Alcidedorbignya inopinata (MHNC 8372) with vessels reconstruction. Vessels courses reconstruction has been performed directly on the slides of the image stack obtained by CT scanning of the skull: $\mathbf{D}$, medial view without transparency; $\mathbf{E}$, medial view with transparency; $\mathbf{F}$, vessels only in medial view. The 3D rendering of CT data is available in pdf format at the address http://sciencepress.mnhn.fr/en/periodiques/geodiversitas/37/4/alcidedorbignya-inopinata-additional-files.

temporal rami of the stapedial artery system, which are branches of the arteria diploëtica magna or orbitotemporal artery (Wible 1987; Rougier et al. 1992) (see above in the parietal section). The petrosquamosal fossa opens anterodorsally in the cerebral cavity at a level close to the internal triple junction petrosal, alisphenoid, and squamosal (see on MHNC 8372), close to the posterior starting point of the orbitotemporal sulcus. It also has an anteroventral outlet, visible on CT-images (Fig. 45), which leads outside of the skull through the postglenoid foramen.

According to the presence of the various sulci and foramina described above, the petrosquamosal fossa is the passageway 


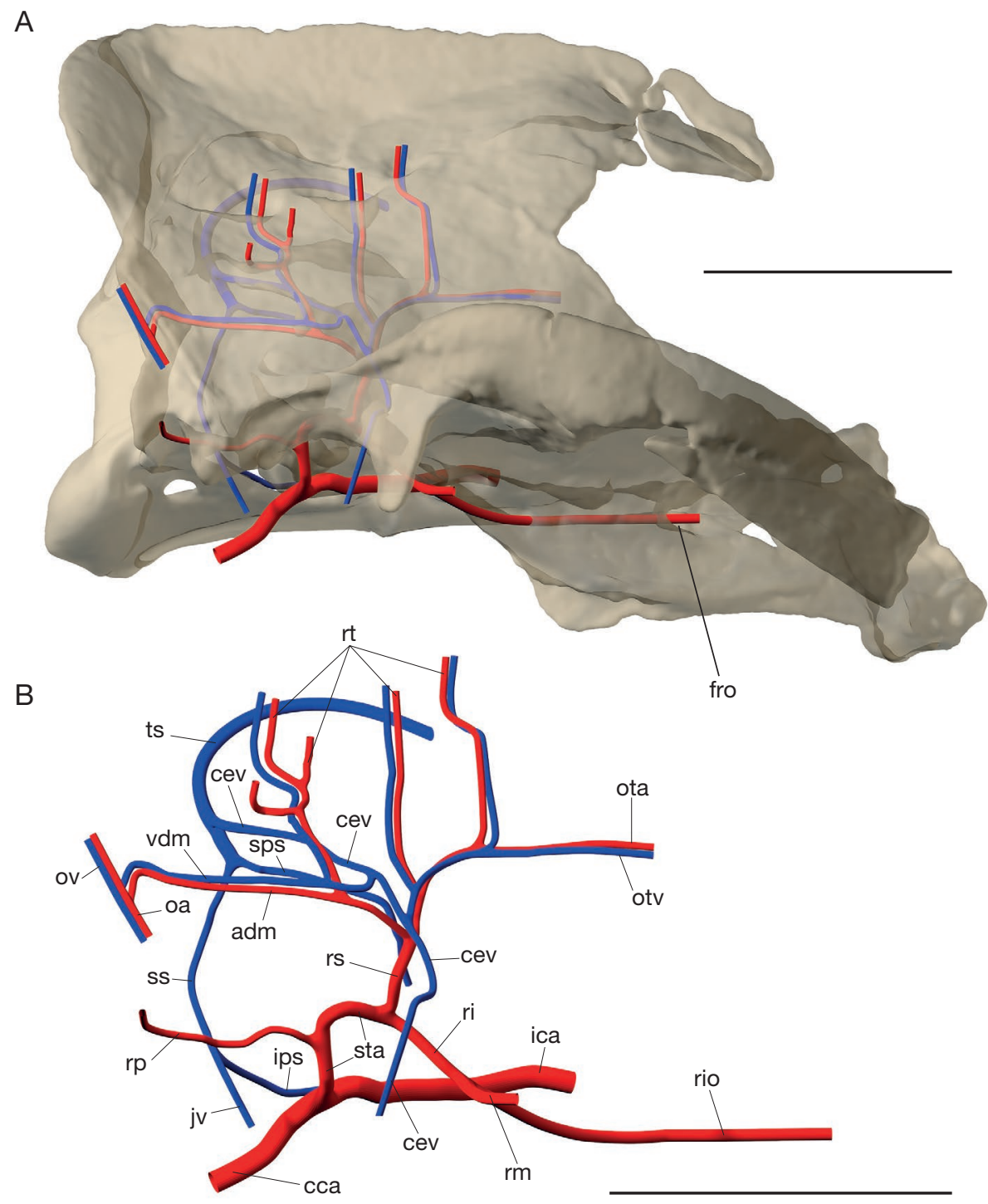

FIG. 48. - Three-dimensional rendering of CT data illustrating the right side of the braincase of Alcidedorbignya inopinata (MHNC 8399) with vessels reconstruction. Vessels courses reconstruction has been performed directly on the slides of the image stack obtained by CT scanning of the skull. A, lateral view with transparency; B, vessels only in lateral view. Abbreviations: adm, arteria diploëtica magna; cca, common carotid artery; cev, capsuloparietal emissary vein; fro, foramen rotundum; ica, internal carotid artery; ips, inferior petrosal sinus; jv, jugular vein; oa, occipital artery; ota, orbitotemporal artery, fro foramen rotundum; otv, orbitotemporal vein; ov, occipital vein; ri, ramus inferior; rio, ramus infrorbitalis; rm, ramus mandibularis; rp, ramus posterior; rs, ramus superior; rt, ramus temporalis; sps, superior petrosal sinus; ss, sigmoid sinus; sta, stapedial artery; ts, transverse sinus; vdm, vena diploëtica magna. Scale bars: $1 \mathrm{~cm}$. The 3D rendering of CT data is available in pdf format at the address http://sciencepress.mnhn.fr/en/periodiques/geodiversitas/37/4/alcidedorbignya-inopinata-additional-files.

for several major vessels (arteries as well as veins) of the skull (Figs 47, 48), Ventrally, the ramus superior of the stapedial artery entered the anteroventral part of the fossa via the anterior sulcus (mentioned above) of the dorsolateral side of the petrosal. There, it divided into two major vessels, the arteria diploëtica magna, posteriorly (see below in description of lateral face of isolated petrosals) and the orbitotemporal artery anteriorly (see above in orbitosphenoid and parietal sections). The arteria diploëtica magna ran posterodorsolaterally and penetrated the suture between the petrosal and the squamosal. It ran posteriorly in the posttemporal canal, and exits the skull via the posttemporal foramen (Wible 1987, 2008). Because of some transverse post-mortem compression of MHNC 8372, the entrance of the arteria diploëtica magna in the suture, on the lateral edge of the petrosquamosal fossa is not widely opened but is observable as an elongated slit (see below in the section on the lateral (squamosal) surface of the petrosal for the description of the posttemporal canal).

In eutherians the arteria diploëtica magna also supplies the rami temporales for vascularisation of the temporal muscles (Wible 1987). The temporal rami of Alcidedorbignya are described above in the parietal section. The anterior division of the ramus superior, the orbitotemporal artery, ran anteriorly in the orbitotemporal groove of the parietal and exited the skull in the orbit above the optic foramen (see above, parietal section).

The petrosquamosal fossa also corresponds to the passage for the capsuloparietal emissary vein, a ramification of the transverse sinus in eutherians (Wible 1993). In Alcidedorbignya, this vessel penetrated the petrosquamosal fossa passing between the 


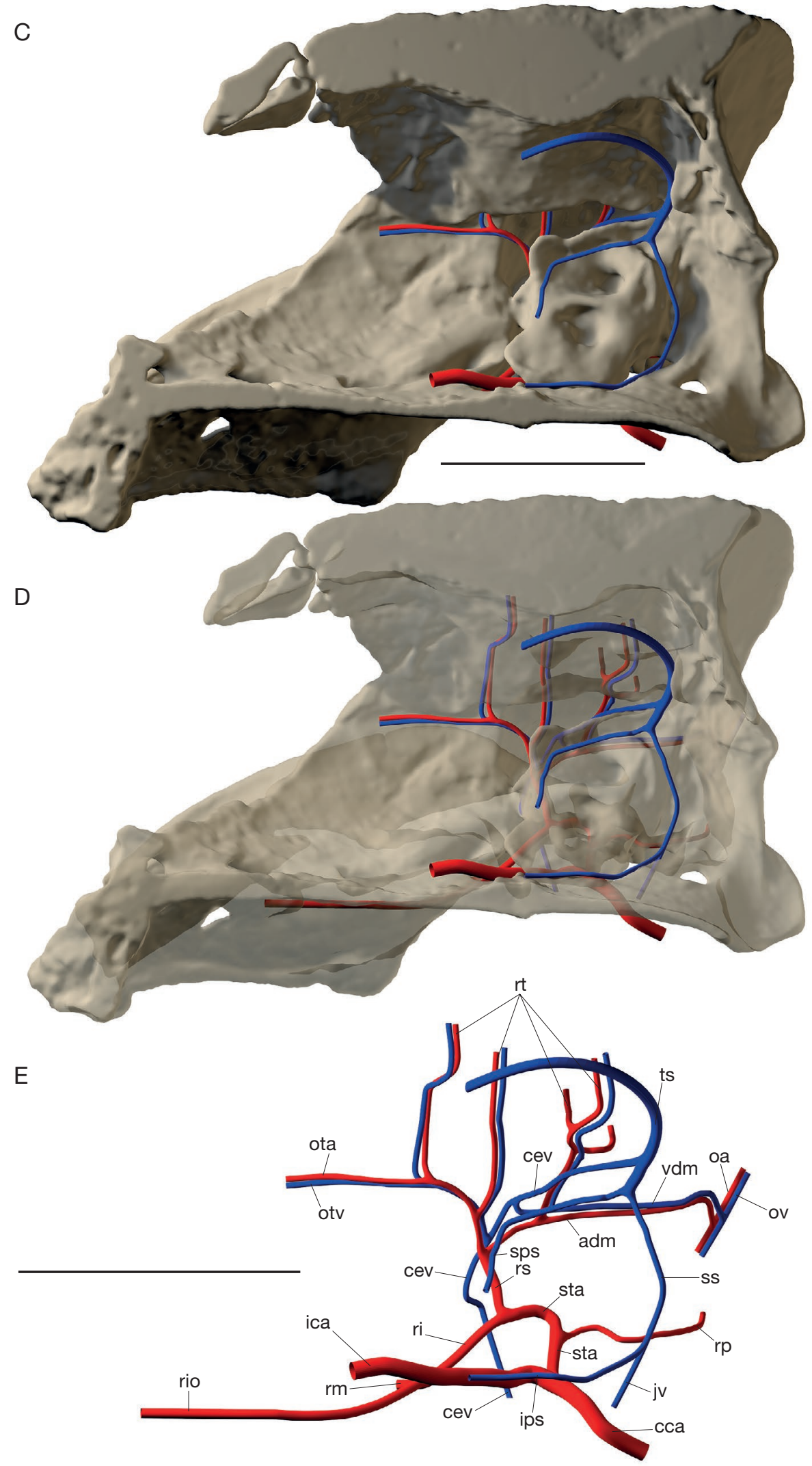

FIG. 48 (continuation). - Three-dimensional rendering of CT data illustrating the right side of the braincase of Alcidedorbignya inopinata (MHNC 8399) with vessels reconstruction. Vessels courses reconstruction has been performed directly on the slides of the image stack obtained by CT scanning of the skull. C, medial view without transparency; $\mathbf{D}$, medial view with transparency; $\mathbf{E}$, vessels only in medial view. Scale bars: $1 \mathrm{~cm}$. The 3D rendering of CT data is available in pdf format at the address http://sciencepress.mnhn.fr/en/periodiques/geodiversitas/37/4/alcidedorbignya-inopinata-additional-files. 
parietal and the petrosal portions of its medial septum. In the fossa it ran along a deep sulcus (the posterior of the two deep sulci mentioned above), which excavates the anterodorsolateral edge of the petrosal. It exited the fossa anteroventrally and left the skull through the postglenoid foramen as the postglenoid vein. In the petrosquamosal fossa, the capsuloparietal emissary vein sent off two venous branches: anteriorly the orbitotemporal vein (or cranio-orbital sinus), which accompanied the orbitotemporal artery, and posteriorly the vena diploëtica magna, the companion vein of the arteria diploëtica magna on the lateral face of the petrosal in its suture with the squamosal (Wible 1993). This posterior sulcus may have also conveyed the posterior temporal arterial and venous rami (see below).

In the posterolateral region of the braincase, dorsal to the petrosal, is a wide sulcus excavated in the supraoccipital that follows the curve of the lambdoid crest internally, descending from the sagittal region of the braincase. This groove conveyed the transverse sinus, which is connected dorsally to the median sagittal sinus (Wible 1984, 1990). In eutherians, the transverse sinus divides ventrally into three vessels, which spread over the petrosal: the sigmoid sinus posteromedially, the capsuloparietal emissary vein (or postglenoid vein) anterolaterally, and the superior petrosal sinus anteromedially (Wible 1984, 1990, 1993, 2011; Novacek 1986; Wible et al. 2009; Evans \& de Lahunta 2012). On the posterodorsalmost intracranial point of the petrosal is a marked large notch located just below the groove for the transverse sinus, immediately lateral to the dorsal part of the sulcus for the sigmoid sinus, and posteromedial to the petrosquamosal fossa. This notch is likely to have been the passageway of the superior petrosal sinus, which connects the transverse sinus to the cavernous sinus, which surrounds the dorsum sellae on the floor of the skull (Wible 1993; Evans \& de Lahunta 2012) (Figs 47, 48).

The internal acoustic meatus (IAM) is relatively wide and is approximately half the width of the petrosal at its level (Fig. 43). This size, however, is subject to some variation, as on MHNC 8360 the IAM is distinctly smaller than on the other specimens. Its anterior edge forms a gentle slope facing anteromedially. The anterolateral edge of the meatus is formed by the prefacial commissure, which floors the passage of the facial nerve, on its way to the ventral side of the petrosal. This bar of bone is a low and rounded area slightly expanded laterally; it does not protrude dorsally as in Leptictis (Novacek 1986). The posterolateral edge of the meatus is a narrow, wellindividualized transverse crest that separates the IAM from the fossa subarcuata dorsolaterally. The meatus and the fossa are therefore closely approximated. The foramina acustica inferius and superius are distinct, and are separated by a low septum that is located rather deeply in the recess (Fig. 43). The foramen acusticum superius (FAS) is anterodorsolateral to the foramen acusticum inferius (FAI). The FAS is made of two foramina: an anterolateral one for the passage of the facial nerve (facial foramen) exiting the braincase via the middle ear and a more medial fossa showing tiny perforations for the passage of bundles of the superior branch of the vestibular nerve to the superior vestibular area (Wible 2008, 2010). The FAI contains anteromedially the tractus spiralis foraminosus, a spiral cribriform tract, which transmits the fibers of the cochlear nerve (Meng \& Fox 1995), and more posteriorly the foramen singulare for the passage of inferior vestibular nerve bundles (see Wible 2008, 2010; Billet \& Muizon 2013) (see also inner ear description below).

The anterolateral edge of the pars cochlearis at the level of the prefacial commissure forms a relatively thin crest that extends from the posterolateral angle of the carotid foramen, anteriorly, to the anterior edge of the petrosquamosal fossa posteriorly. Ventral to this crest on MHNC 8372, the anterolateral side of the pars cochlearis is excavated by a distinct groove or elongated fossa. This groove is not so pronounced in MHNC 8399. The posterolateral part of this groove extends ventrolaterally in a small fossa excavated in the dorsomedial side of the medial wall of the foramen for the ramus superior of the stapedial artery. This fossa is part of the dorsal face of the tegmen tympani, which we regard as the vestigial portion of the posterior wall of the cavum epiptericum, the extradural endocranial space housing the trigeminal ganglion (Kuhn \& Zeller 1987; Wible 2008). This fossa therefore housed part of the trigeminal ganglion in Alcidedorbignya (see above) (Fig. 49). It is much smaller than in Leptictis and Prokennalestes because of the reduction of the tegmen tympani in Alcidedorbignya concomitant with the development of the pyriform fenestra. It constitutes a relict of the anterior lamina, a structure which is well developed in non-tribosphenic mammals and basal mammaliaformes (e.g., Vincelestes, multituberculates, triconodontids, monotremes, morganucodontids; Rougier et al. 1992; Hopson \& Rougier 1993; Kermack et al. 1981; Kermack 1963; Crompton \& Luo 1993; Wible \& Hopson 1993) and still present, but reduced, in some therians (Wible 1990; Marshall \& Muizon 1995, Ladevèze \& Muizon 2007; Billet \& Muizon 2013).

In the dog, Evans \& de Lahunta (2012: fig. 10-9) illustrate a common origin for the passage of the maxillary (CN V2) and ophthalmic (CN V1) branches of the trigeminal nerve. This common origin stems from the anteromedial edge of the trigeminal ganglion and passes medial to the foramen ovale towards the "foramen rotundum canal" and the sphenorbital fissure (see above and Fig. 30). The groove noted above in the lateral edge of the pars cochlearis is probably for this medial common origin of the CN V1 and CN V2.

Posteromedial to the fossa subarcuata, the cerebellar (intracranial) face of the petrosal presents on its large posterior rim a small transverse slit, the aperture of the vestibular aqueduct (Evans \& de Lahunta 2012), which transmits the endolymphatic duct (Fig. 43). In all specimens examined here, this aqueduct opens slightly anterior to the very posterior sharp edge of the petrosal. The slit morphology differs from that observed in Didelphodus and Leptictis, in which the aqueduct is more an elongated foramen than a narrow slit. Anteroventromedial to the vestibular aqueduct and dorsal to the sulcus for the internal jugular vein is a small foramen visible medially. This foramen is circular and located in a conical depression. It is the cochlear canaliculus (MacPhee 1981), which transmits the perilymphatic duct (Fig. 28 and below Figs 50, 51). This opening is located very close to the external aperture of the cochlear fossula laterally, especially when compared to Pantolambda. 

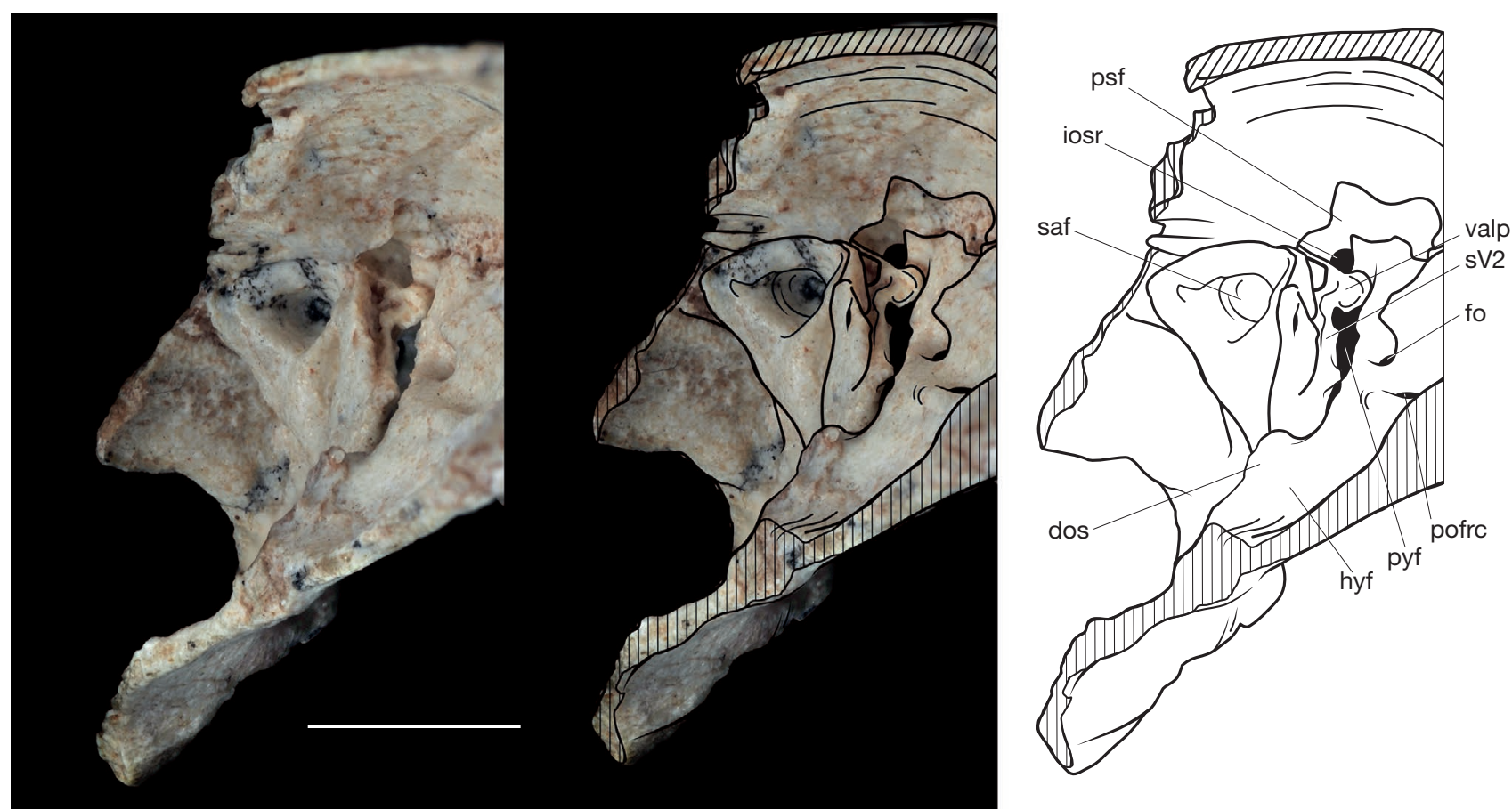

FIG. 49. - Anteromedial view of the endocranial aspect of the left auditory region of Alcidedorbignya inopinata (MHNC 8372) showing the vestigial anterior lamina of the petrosal, which bears the fossa for the trigeminal ganglion (photograph, schematic drawing on photo, and schematic drawing with captions). Abbreviations: dos, dorsum sellae; fo, foramen ovale; hyf, hypophyseal fossa; iosr, intracranial opening of the foramen for the superior ramus of the stapedial artery; pofrc, posterior opening of the foramen rotundum canal; psf, petrosquamosal fossa; pyf, pyriform fenestra; saf, subarcuate fossa; sV2, probable sulcus for the maxillary nerve (CN V2); valp, vestigial anterior lamina of the petrosal. Scale bar: $5 \mathrm{~mm}$.

Squamosal surface of the petrosal - Well-preserved isolated petrosals (e.g., MHNC 8359 and 8360) document the anatomy of the lateral or squamosal surface of the petrosal, otherwise unobservable because of its close approximation (suture) with the squamosal bone (Figs 40, 50). The suture with the squamosal occupies a triangular area that points dorsally. This sutural area lies just above and lateral to the crista parotica, and posterodorsal to the fossa incudis. It is bordered anterodorsolaterally by an oblique sulcus, which runs along the posteroventral edge of the elongated and bulbous posterodorsal root of the tegmen tympani process and reaches the dorsal edge of the posttemporal notch posteriorly. This sulcus is an articular structure, which is part of the squamosal suture and received a corresponding articular crest of the squamosal. This crest is a dorsal buttress of the squamosal portion of the epitympanic recess and fossa incudis. An approaching condition is observed in Tenrec. Dorsal to this articular sulcus and to the root of the tegmen tympani is a large vascular groove, the posttemporal sulcus (the petrosal portion of the posttemporal canal), for the arteria diploëtica magna. It runs subhorizontally along the squamosal surface of the petrosal and reaches the posttemporal notch of the petrosal posteriorly. On the two skulls, it is possible to follow the posttemporal canal to the posttemporal foramen on CT images (Figs 45 and 46), although this structure is, on some sections, partially obliterated because of slight distortion due to fossilisation. The posttemporal canal, which is marked by a sulcus on the isolated petrosals, is partially crushed and filled with ma- trix in both skulls. Undistorted and empty portions clearly show, however, that it corresponds to a large rounded canal that is tightly wedged between petrosal and squamosal. The arteria diploëtica magna is originally, in eutherians, a posterior anastomotic branch that connects the ramus superior of the stapedial artery to the occipital artery posteriorly (Wible 1987). In Alcidedorbignya, the sulcus of the arteria (and vena) diploëtica magna (the post-temporal groove) connects anteriorly with a vertically-oriented sulcus running from the foramen of the ramus superior ventrally. This marks the anterior connection of the arteria diploëtica magna with the ramus superior. This connection is located in the ventral half of the petrosquamosal fossa. The posttemporal sulcus also housed the vena diploëtica magna, which connects the capsuloparietal emissary and occipital veins in eutherians (Wible 1993; see above). In agreement with this configuration, the anterior end of the posttemporal groove in Alcidedorbignya also approximates the anteriormost part of the more medial sulcus that conveys the capsuloparietal emissary vein on the dorsal edge of the petrosal (see above). Posteriorly, the posttemporal sulcus extends back to the posttemporal notch, at which point the posttemporal foramen provided passageway to the diploëtic vessels out of the skull toward the occipital artery and vein. The large posttemporal foramen opens posteriorly, in the dorsolateral portion of the mastoid exposure of the petrosal within the suture between this bone and the squamosal as in most therians which have such a foramen (Wible 2008). The posttemporal notch also marks the connection between 

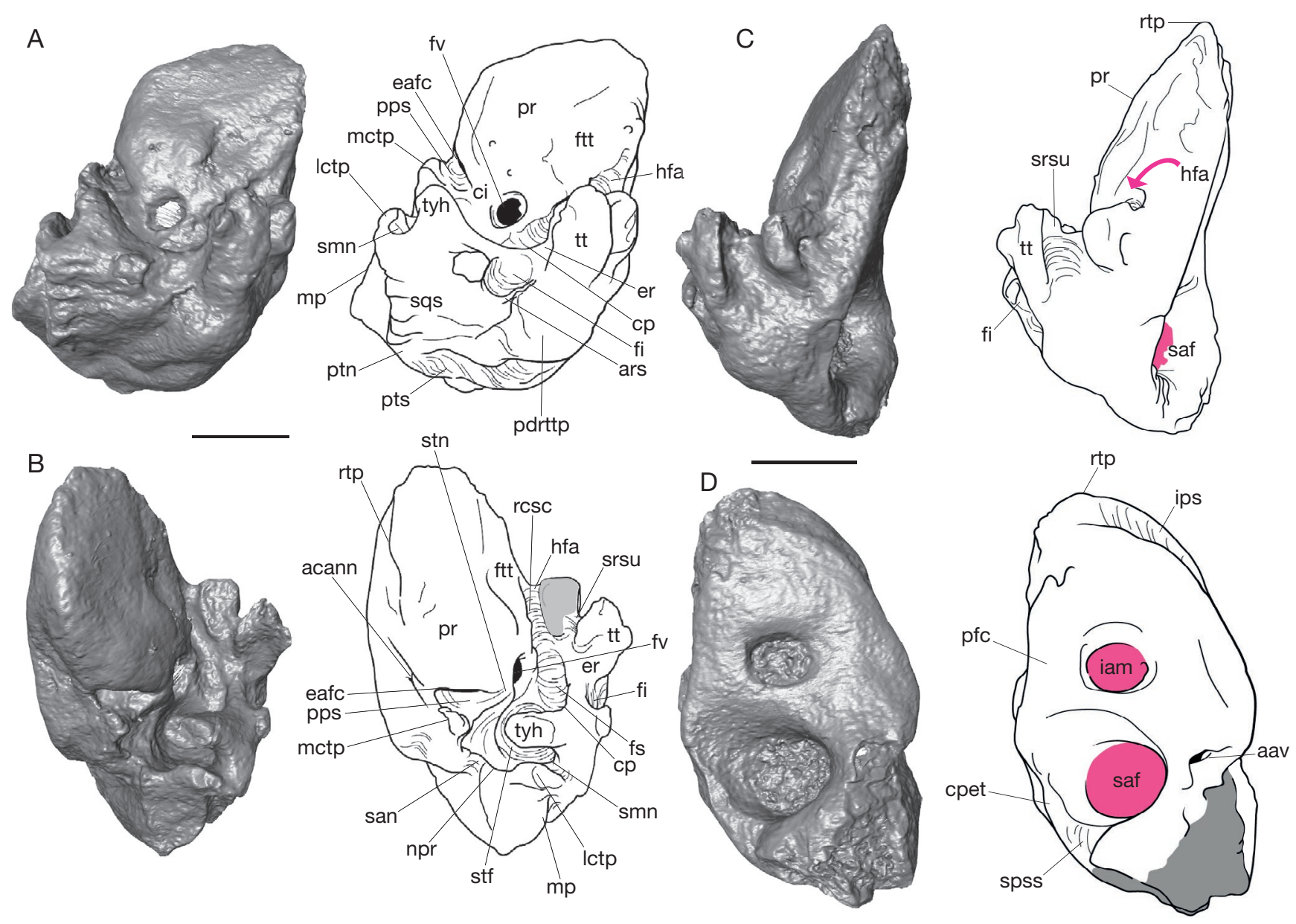

FIG. 50. - Surface rendering of CT data illustrating an isolated petrosal of Alcidedorbignya inopinata (MHNC 8360) in: A, lateral view; B, medioventral view; C, anterior view; D, cerebellar views. Abbreviations: aav, aperture of the vestibular aqueduct; acann, notch housing the external aperture of the cochlear canaliculus; ars, articular sulcus with the squamosal; $\mathbf{c p}$, crista parotica; cpet, crista petrosa; eafc, external aperture of the cochlear fossula; er, epitympanic recess; fi, fossa incudis; fs, facial sulcus; ftt, fossa for the tensor tympani; fv, fenestra vestibuli; hfa, hiatus Fallopii; iam, internal acoustic meatus; ips, sulcus of inferior petrosal sinus; Ictp, lateral caudal tympanic process; mctp, medial caudal tympanic process; mp, mastoid process; npr, notch for the posterior ramus of the stapedial artery; pdrttp, posterodorsal root of the tegmen tympani process; pfc, prefacial commissure; pps, postpromontorial sinus; pr, promontory; ptn, posttemporal notch; pts, posttemporal sulcus; rcsc, roof of the cavum supracochleare; rtp, rostral tympanic process; saf, subarcuate fossa; san, sulcus for the auricular branch of the CN X; smn, stylomastoid notch; spss, sulcus for the superior petrosal sinus; sqs, squamosal articulation surface of petrosal; srsu, sulcus for the ramus superior of the stapedial artery; stf, stapedial fossa; stn, sulcus for the tympanic nerve; tt, tegmen tympani; tyh, tympanohyal. Scale bars: 2 mm.

the posttemporal sulcus and a vertical occipital groove on the mastoid surface (possibly for the occipital artery). The course of the posttemporal sulcus is not strictly straight but rather slightly undulating. Our reconstructions of the course of the arteries and veins based on the examination of CT images of the skulls MHNC 8372 and 8399 confirm this course for the arteria and vena diploëtica magna. As discussed in the parietal section the arteria and vena diploëtica magna each send off a ramification before entering the post-temporal canal, which corresponds to the posterior temporal artery and vein (see below). No isolated petrosal specimen preserves entirely the posterodorsal tip/ apex of the squamosal surface of the petrosal.

Inner ear. The cavity of the inner ear (the bony labyrinth) of Alcidedorbignya has been reconstructed from CT scan data obtained from three specimens: MHNC 8399 (a petrosal in situ on a partial skull with associated upper and lower jaws),
MHNC 8360 (an isolated left petrosal), and MHNC 8419 (an isolated right petrosal, crushed dorsoventrally). The two latter might belong to juveniles or young adult individuals. The reconstruction of the inner ear of MHNC 8399 is presented in Figures 51 and 52. Measurements have been taken on MHNC 8399 and 8360, the two best-preserved specimens (Table 5).

Cochlear canal - The cochlear canal has 1.5 turns $\left(540^{\circ}\right)$ in the three specimens investigated (from its base, at the fenestra cochleae, to its apex). The cochlea is rather loosely coiled; that is, the successive turns do not contact much each other (Fig. 52). The bony laminae that provide support to the spiral organ of hearing in life (Fleischer 1973) have been observed on MHNC 8399 and 8360. The secondary bony lamina that runs on the external wall of the cochlear canal is distinct in the first $90^{\circ}$ of coiling. It is not strongly developed, and occupies less than one-third of the corresponding cochlear 


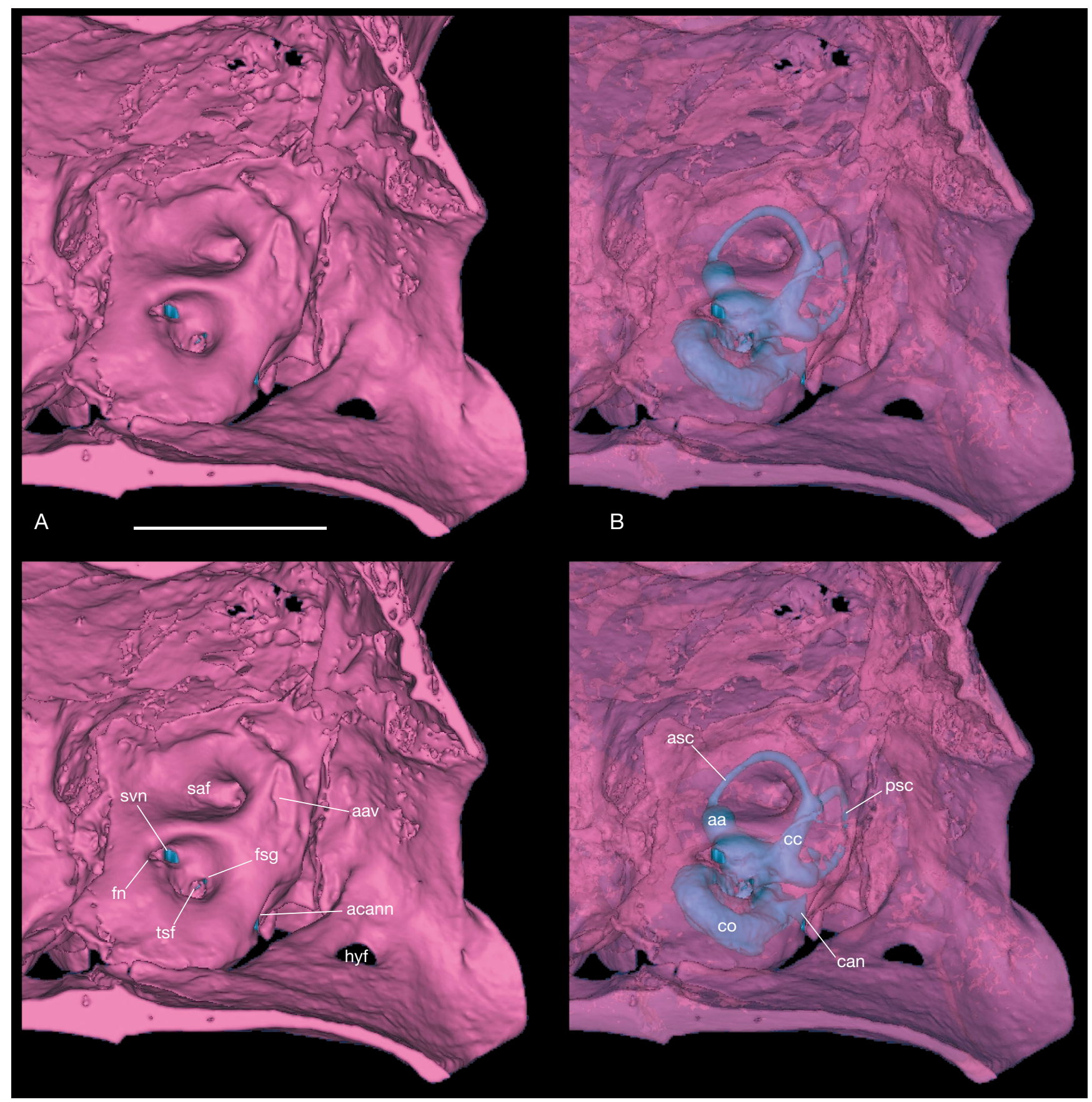

FIG. 51. Three-dimensional rendering of CT data illustrating the internal aspect of the posterior part of the braincase of Alcidedorbignya inopinata, in medial view (MHNC 8399): A, opaque view; B, view with bone transparency leaving the bony labyrinth of the inner ear apparent (in blue). Abbreviations: aa, anterior ampulla; acann, notch housing the external aperture of the cochlear canaliculus; aav, aperture of the vestibular aqueduct; asc, anterior semicircular canal; psc, posterior semicircular canal; can, cochlear canaliculus; cc, common crus; co, cochlear canal; saf, subarcuate fossa; fn, canal for the facial nerve; fsg, foramen singulare; tsf, tractus spiralis foraminosus (canal and cribriform tract for cochlear nerve); hyf, hypoglossal foramen. Scale bar: $5 \mathrm{~mm}$.

canal width. The primary bony lamina of the internal wall is present along most of the cochlear canal length where it contributes between one third and half of its width. Due to the rapid evanescence of the primary bony lamina, the laminar gap quickly increases towards the apex of the cochlea past the fenestra cochleae.

The modiolus (bony axis) of the cochlea houses the canal for the auditory branch of cranial nerve VIII (or cochlear nerve). A cribriform plate of moderate size is located between this latter canal and the cochlear canal. The canal for the ganglion of the spiral nerve has not been clearly identified as a single canal close to the primary bony lamina internal root, and may thus be somewhat confounded within the cribriform tract.

The maximum height of the cochlea is slightly less than half its maximal width (low-spired; Table 5). The kidney-shaped 
TABLE 5. - Measurements on reconstructed bony labyrinths of MHNC specimens referred to Alcidedorbignya inopinata (the unit for linear measurements is $\mathrm{mm}$ ) Abbreviations: ASC, anterior semicircular canal; $\mathbf{H}$, height; IEH, inner ear height; L, length; LSC, lateral semicircular canal; P, inner perimeter; PSC, posterio semicircular canal; W, width. Measurements follow Billet \& Muizon (2013), Billet et al. (2013) and Segall (1970).

\begin{tabular}{lcc}
\hline & MHNC 8399 & MHNC 8360 \\
\hline Cochlear canal length & 10.86 & 8.48 \\
Cochlea height & 1.29 & 1.19 \\
Cochlea width & 3.25 & 2.48 \\
Number of cochlear turns & 1.5 & 1.5 \\
Stapedial ratio & 1.8 & 1.7 \\
ASC L & 2.25 & 2.27 \\
ASC W & 2.26 & 2 \\
PSC L & 2.01 & - \\
PSC W & 2.03 & - \\
LSC L & 2.05 & 1.77 \\
LSC W & 2.06 & 1.79 \\
Radius ASC & 1.13 & 1.07 \\
Radius PSC & 1.01 & - \\
Radius LSC & 1.03 & 0.89 \\
ASC P & 7.42 & 7.21 \\
PSC P & 6.51 & - \\
LSC P & 6.8 & 5.81 \\
ASC L/W & 1.00 & 1.14 \\
PSC L/W & 0.99 & - \\
LSC L/W & 1.00 & 0.99 \\
Angle ASC/PSC & 90.06 & 69.39 \\
Angle ASC/LSC & 83.71 & 87.08 \\
Angle PSC/LSC & 90.59 & 90.33 \\
Angle LSC/cochlea & 35.86 & 23.11 \\
ASC P/PSC P & 1.14 & - \\
ASC P/LSC P & 1.09 & $\mathbf{4 . 3 3}$ \\
PSC P/LSC P & 0.96 & \\
\hline IEH & 4.78 & \\
\hline
\end{tabular}

fenestra cochleae, to which the secondary tympanic membrane attached in life (Wible et al. 2009; Wible 2010), covers an area that does not exceed twice the size of the more external and central to the external aperture of the cochlear fossula (defined in Wible 2008). The latter depression is shallow in Alcidedorbignya. The cochlear canaliculus is rather short; it is less than twice the size of the semicircular canal (SC) arcs diameters and projects from the posteroventral corner of the fenestra cochleae, directed towards the posterior direction.

The most basal/basal departure of the cochlear canal corresponds to a strong narrowing/constriction of the bony labyrinth when compared to the much larger portion of vestibule at the level of the utricule (see Luo et al. 2012) and SC ampullae. (Fig. 52) However, when observing at the cochlea only, the cochlear canal is the widest at its base; from there it narrows gradually and slightly toward its apex.

The cochlea contributes $c$. $45-50 \%$ of the total volume of the bony labyrinth (vestibular aqueduct and cochlear canaliculus excluded) in the two isolated petrosal specimens.

Vestibule and Semicircular Canals - From a lateral or medial perspective, the vestibule (including the region of the fenestra vestibuli) is elongate in the horizontal plane (approximately anteroposteriorly) and has in contrast a very low height. The spherical recess (for the membranous saccule) and elliptical recess (for the membranous utricule) are not easily discern- ible. A canal for nervus ampullaris (see Macrini et al. 2010) ventromedial to the root of the secondary common crus is poorly developed and opens into the foramen singulare of the IAM. The aqueductus vestibuli is closely approximated to the common crus. Its ventral origin is slightly anterior and medial to the common crus. The course of this aqueduct is mostly directed dorsally although it curves somewhat posteriorly along the common crus in its dorsalmost portion. It has a short course, with the external foramen of the vestibular aqueduct being much less dorsal than the dorsal tip of the common crus.

A short and wide canal is observed connecting the portion of the vestibule just ventromedial to the anterior ampulla (utricle?), to the foramen of the superior vestibule area (see Wible 2010) in the foramen acusticum superius (in the internal auditory meatus).

On MHNC 8399, the anterior semicircular canal (ASC) is the largest of the semicircular canals. The lateral one (LSC) is slightly larger than the posterior one (PSC). On the three specimens, the SC almost does not show any planar deviation. The SC arches exhibit a grossly semicircular shape, with an aspect ratio most often close to 1 (see Material and Methods, and Table 5). The SC bony ducts are moderately thick, i.e. they have a large diameter compared to the development of their arc (SC D/mean SC R). The dorsal extent of the ASC and PSC extend slightly beyond the dorsal tip of the common crus dorsally. All three specimens exhibit a secondary common crus between the PSC and LSC. The lateral and anterior ampullae are well individualised on the bony labyrinth, whereas the posterior ampulla rather presents as a posterior inflation of the secondary common crus. Of the three, the anterior ampulla is clearly the most inflated. The LSC and PSC show a similar lateral extent that goes slightly beyond the secondary crus commune's most distal (lateral) point.

The angles between the three SCs are $90^{\circ}$ for the ASC-PSC and PSC-LSC in MHNC 8399, and slightly more acute for the ASC-LSC. The angles in the two other specimens slightly differ from MHNC 8399, which might be due to postmortem deformation or some individual variation. The basal turn of the cochlear canal makes an angle of $35^{\circ}$ with the LSC in MHNC 8399, 21-23 in the two isolated petrosals (MHNC 8360 and 8419).

Ectotympanic, entotympanic, and ear ossicles. The ectotympanic is not preserved on MHNC 8372. However, the small facets described above on the anteromedial edge of the epitympanic recess and on the posterior face of the postglenoid eminence indicate that an ectotympanic was present in Alcidedorbignya. Furthermore, on the perinatal (either near-term foetus or neonate) specimen (MHNC 8373) preserved between the limbs of the articulated skeleton MHNC 8372, the ectotympanic is completely preserved. The ectotympanic is also preserved on MHNC 8423, a skull of a juvenile individual bearing complete deciduous dentition with erupting M1s and therefore probably slightly older than MHNC 8373. In the following description we follow the terminology of Wible (2008). However, some definitions of the major regions of the ectotympanic are neces- 

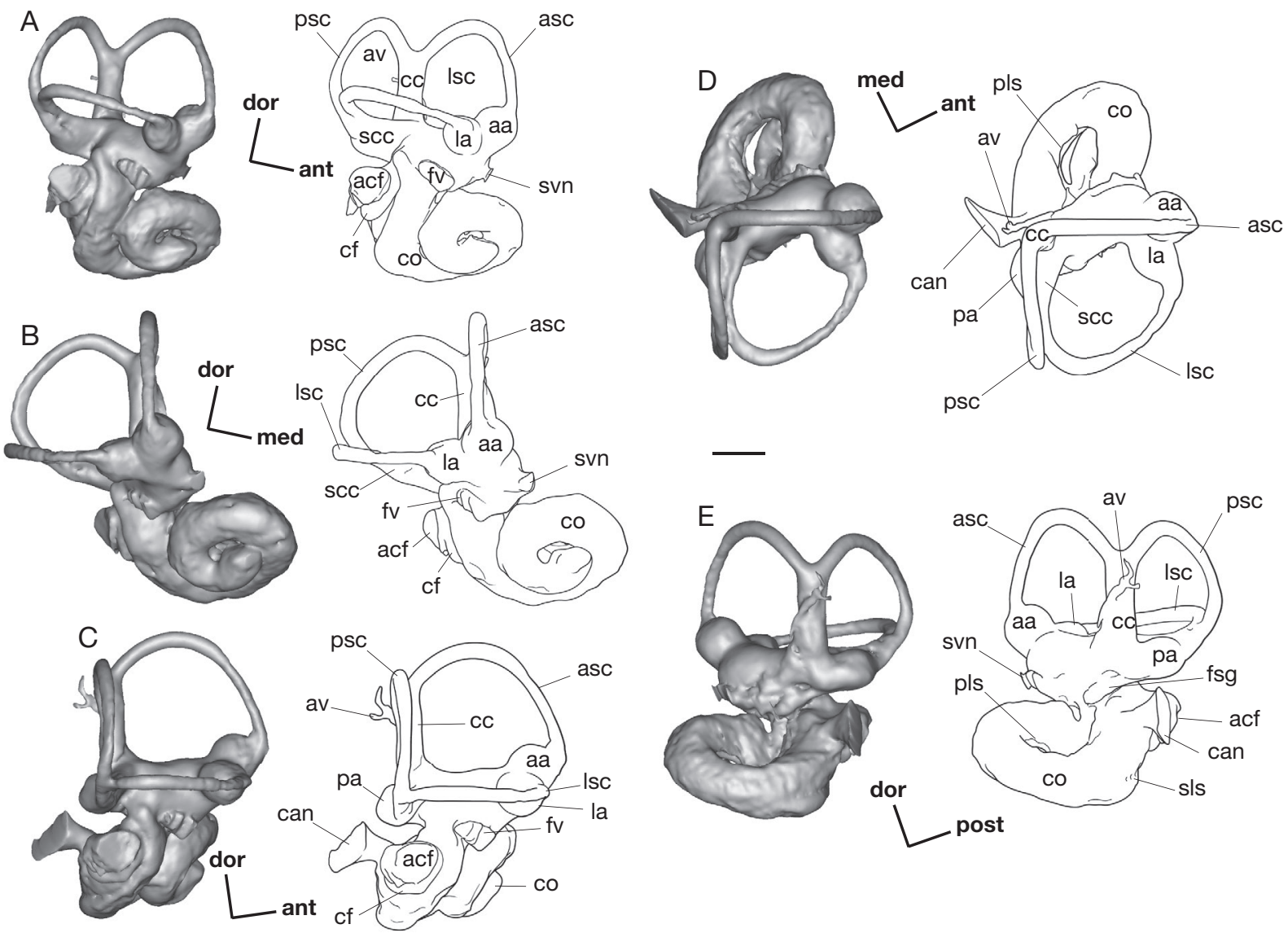

FIG. 52. - Virtual 3D reconstruction and outline drawing of the bony labyrinth of Alcidedorbignya inopinata (MHNC 8399): A, lateral view; B, anterior view; C, posterolateral view; D, dorsal view; E, medial view. Abbreviations: aa, anterior ampulla; acf, aperture of the cochlear fossula; ant, anterior; asc, anterior semicircular canal; av, vestibular aqueduct; can, cochlear canaliculus; cc, common crus; cf, cochlear fossula; co, cochlear canal; dor, dorsal; fsg, foramen singulare; fv, fenestra vestibuli; la, lateral ampulla; Isc, lateral semicircular canal; med, medial; pa, posterior ampulla; pls, primary bony lamina sulcus; post, posterior; psc, posterior semicircular canal; scc, secondary common crus; sls, secondary bony lamina sulcus; svn, tractus for the passage of the superior branch of the vestibular nerve. Scale bar: $1 \mathrm{~mm}$.

sary. The anterior crus is the anterior branch of the tympanic, which articulates with the postglenoid eminence and the petrosal (in the case of Alcidedorbignya) and the posterior crus is the posterior branch of the bone which articulates with the anterior edge of the tympanohyal (see above). The crura are restricted to the articular portions of the tympanic and have the same distal extent as the articular surface they bear. Between the distal limits of the articular surfaces and joining the crura is the arched portion of the ectotympanic, which we herein call the arc of the tympanic.

On the perinatal specimen (MHNC 8373) the bone is distinctly horseshoe-shaped and does not present the large medial expansion observed in Pantolambda (AMNH 16663) (see Appendix 3; Figs 125, 126). On the juvenile MHNC 8423, the ectotympanic is also horseshoe-shaped but the arc of the ectotympanic is slightly expanded distally (on its anteromedioventral edge) and bears a distinct triangular styliform process (sensu Wible 2008). However, the arc of the ectotympanic is not expanded medially in a large blade as is observed in Pantolambda (see below, Appendix 3; Figs 125, 126). In this respect, the general shape of the ectotympanic is grossly similar to that of Solenodon. As is observed in the latter genus, in lateral view, the ectotympanic is asymmetrical. The styliform process is in an anterior position and is located straight distal to and in line with the rectilinear anterior crus. The tympanic opening is roughly square and forms a conspicuous right angle, anteriorly, above the styliform process. Again, this condition closely resembles that of Solenodon (Wible 2008). Furthermore, this angle is distinctly notched on both ectotympanics of the specimen. The posterior crus is concave anteriorly and its rounded apex (slightly displaced medially on the fossil) probably articulated with the small cupular facet observed on the anterior face of the tympanohyal. The crura are not noticeably expanded transversely. Although the anterior crus is not as narrow as the posterior crus, it contrasts sharply with the condition with the condition observed in Pantolambda (see Appendix 3 and comparison section) and Solenodon. On its medial side, the ectotympanic bears a sulcus tympanicus which is deep, but which is apparently narrower (proportionally) than that of Pantolambda. As preserved the tympanic of MHNC 8423 is subvertical based on the position of the anterior crus which floors the fissura Glaseri. This condition is clearly observable on the CT scan of the specimen, on which it possible to observe 
that the posterior crus articulated with the tympanohyal and the anterior crus had a double articulation with the petrosal and the squamosal. On this scan it is possible to observe the fissura Glaseri, which is closed all along its length laterally by the squamosal-ectotympanic articulation and medially by the petrosal-ectotympanic articulation. Its roof is comprised of the squamosal and its floor is formed by the ectotympanic. The bone is slightly displaced from its anatomical position and the contact between the three bones is not tightly maintained all along the fissure. However, displacement is moderate and restoring the three bones to presumed anatomical position places the tympanic in a roughly anteroposterior position, and does not affect a subvertical orientation. Furthermore, the auditory ossicles are preserved and the malleus is articulated to the ectotympanic. The incus and the stapes are also only slightly displaced. These bones as well as the ectotympanic and skull will be described in a work in progress dealing with the juvenile specimens of Alcidedorbignya inopinata.

No entotympanic is preserved in MHNC 8372, 8399, or 8423 and there is no indication of an articulation or a contact with the preotic crest or the basioccipital, which could witness such an ossification. It is therefore probable that an entotympanic was absent in Alcidedorbignya. Furthermore, the basicranium of Alcidedorbignya bears no process of the basioccipital, basisphenoid, alisphenoid, tympanohyal, squamosal, or petrosal, which could indicate the presence of some kind of auditory bulla. It is therefore likely that the ectotympanic described above was the only bone flooring the middle ear of Alcidedorbignya.

Basioccipital, exoccipital, and supraoccipital. The basioccipital of MHNC 8372 is not completely fused to the basisphenoid but the suture with the exoccipital is totally obliterated. Surprisingly, however, on MHNC 8399, a presumed older individual, in which the most of the braincase sutures are more fused than in MHNC 8372, the lateral part of the basioccipitalexoccipital suture is still clearly visible on the left side of the specimen. On both specimens the exoccipital-supraoccipital sutures are not completely fused and are observable (grossly) horizontally on the occipital face, from the dorsal edge of the foramen magnum to the dorsomedial edge of the mastoid exposure. These bones will be described jointly below.

In ventral view, the basioccipital continuously broadens posteriorly. Its lateral edge runs parallel to the medial edge of the petrosal without contacting it. The space between the petrosal and the basioccipital is the basicochlear fissure described above in the petrosal section. The basioccipital has a regularly convex surface in the frontal plane but it is slightly concave ventrally in a sagittal or parasagittal plane. In lateral view it is distinctly oblique, its ventral side facing anteroventrally (i.e. the anteroventral edge of the foramen magnum is lower than the basioccipital-basisphenoid suture). The ventral surface of the basioccipital is smooth in MHNC 8372 while in MHNC 8399 it presents a short median crest in its middle third. This crest separates the two areas of insertion of the recti capitis ventralis (e.g., Evans \& de Lahunta 2012; Barone 2000). These areas are only slightly concave and are not deeply excavated as in Leptictis. The anterior and posterior thirds of the midline of the bone bear no crest and are flat. Posterolateral to the lateral edge of the basioccipital-exoccipital suture is the jugular foramen (or posterior lacerate foramen), the medial edge of which is formed by the lateral edge of the exoccipital. This edge is concavo-convex, being concave posteriorly and convex anteriorly. At the posteroventral region of the exoccipital-petrosal suture, the exoccipital has a salient paroccipital process (= paracondylar process of Wible et al. 2004; 2009), which projects posteroventrally. In MHNC 8372 this process is relatively low and rounded and does not project strongly ventrally. On the contrary, on MHNC 8399 the process is an elevated blade-like crest, which projects ventrally far from the exoccipital-petrosal suture. On MHNC 8399, the anterior part of the process, which is not so expanded ventrally, bears a small cupula, from which, in our view, the digastricus muscle took origin as inferred from condition in the dog (Evans \& de Lahunta 2012) and other domestic mammals (Barone 1997). The posterior part of the process (especially the ventral crest and the lateral side of the blade) likely received the remaining origin of this muscle. Such a crest-like paroccipital process is absent in Pantolambda and Leptictis. On the occiput, the posteromedial edge of the paroccipital process and the part of the exoccipital dorsal to the occipital condyle are deeply excavated. This fossa probably received mainly the insertion of the obliquus capitis cranialis (inferred from dissections in domestic mammals in Evans \& de Lahunta 2012 and Barone 2000). This fossa is limited dorsally by an obliquely-oriented rounded crest, which follows approximately the exoccipitalsupraoccipital suture. The bones are distinctly thickened on the suture. On the ventral face of the exoccipital, between the jugular foramen and the condyle, is a large, single hypoglossal foramen. It is slightly oval-shaped with the great axis having an anteromedial-posterolateral orientation. The foramen is located midway between the jugular foramen and the anterolateral edge of the atlantal facet of the exoccipital. It opens directly on a flat surface of the exoccipital bone, that is, not within a fossa. A single hypoglossal foramen is also present in Pantolambda and Leptictis. The hypoglossal foramen is relatively smaller and is located much closer to the jugular foramen in Pantolambda. Alcidedorbignya differs from Zalambdalestes and Maelestes, in which the foramen is multiple.

The foramen magnum faces posteriorly with a slight ventral component. In ventral view its ventral edge is deeply notched between the occipital condyles in ventral view, but it is almost straight in posterior view. Another narrow notch, smaller than the ventral notch, is present on the dorsal edge of the foramen. In MHNC 8399 this notch is narrower than in MHNC 8372. As a whole, the foramen magnum of MHNC 8399 is higher than wide, while it is roughly circular in MHNC 8372. This could be related to some distortion of the specimens, but none of them presents any obvious evidence of major post-mortem deformation. Therefore, this difference in the morphology of the foramen magnum is probably the result of some individual variation.

The occipital condyles strongly project posteriorly and ventrally. This feature is much more pronounced in Alcide- 
dorbignya than in Pantolambda and is absent in Leptictis, in which they are distinctly oriented ventrally. The articular facet of the condyles (the atlantal facet) is subdivided into two lobes, dorsal and ventral, separated by a transverse rounded ridge. In lateral view, this ridge is oriented anterodorsallyposteroventrally. In posterior view the dorsal lobe of the atlantal facet is oblique and elongate. Its ventral portion is distinctly convex but its dorsolateral portion is relatively flat to slightly concave. This part of the atlantal facet is located just below the fossa for insertion of the obliquus capitis cranialis. The ventral lobes of the condyles are anteroposteriorly narrow but wide transversely. They are widely separated and the articular surfaces do not contact medially on the ventral edge of the foramen magnum. However, the ventral edge of the foramen magnum between the medial side of the ventral lobe and the basioccipital-exoccipital suture on MHNC 8399 is distinctly thickened even though apparently it is not occupied by any articular surface. This condition differs from that in Pantolambda and Leptictis, in which the articular surfaces of the ventral lobes extend widely on the anteroventral edge of the foramen magnum. Therefore, the anterior border of the atlantal facet of Alcidedorbignya is markedly convex, but not sigmoid as in Leptictis. In ventral view, the lateral edge of the condyle is strongly salient posteriorly in Alcidedorbignya and there is a deep lateral notch between the condyle and the paroccipital process.

The supraoccipital is a deeply excavated plate of bone, which forms the posterior edge of the very large nuchal crest (Fig. 53). This crest is greatly developed and strongly protrudes posterodorsally. It is larger on MHNC 8399 than on 8372; however both specimens are regarded as female (see above; sexual dimorphism section). In dorsal view, the nuchal crest is bilobate and deeply $\mathrm{V}$-shaped medially. The point of junction of the sagittal and nuchal crest is thus well anterior to the lateral lobes, which form the posteriormost part of the skull, and is approximately dorsal to the dorsal edge of the foramen magnum. The nuchal crest of Alcidedorbignya strongly protrudes posteriorly and is distinctly visible when looking at the skull from a ventral perspective. The mediolateral extent of the nuchal crest is also narrow and only slightly wider than the greater width between the lateral edges of the condyles, while it is much wider in other pantodonts. The supraoccipital basin does not present a vertical median crest as in Leptictis, but is regularly concave. However, a robust nuchal tubercle is present on MHNC 8399 on the posterior aspect of the dorsomedial point of the crest. Because of the posterior projection of the nuchal crest, the supraoccipital basin is deep and presented a major area for insertion of the nuchal muscles (e.g., recti capitis dorsalis major and minor, semispinalis capitis) (inferred from dissections of domestic mammals in Evans \& de Lahunta 2012 and Barone 2000). The strong development of the nuchal crest and other structures of the occiput of Alcidedorbignya, indicate very powerful neck musculature, more so, relatively, than in the other pantodonts. Because the exoccipitals do not contact dorsally, the supraoccipital participates in the formation of the dorsal edge of the foramen magnum.
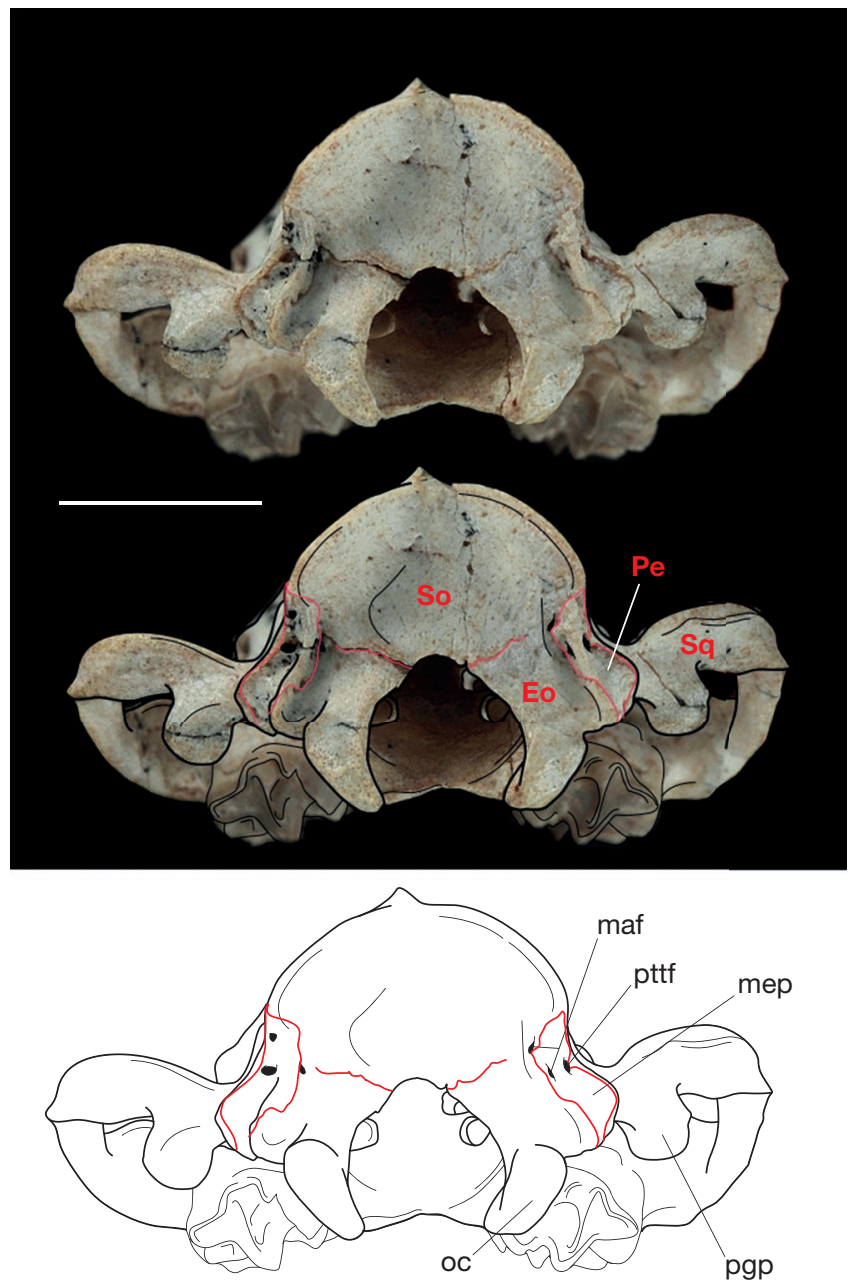

FIG. 53. - Posterior view of the skull of Alcidedorbignya inopinata (MHNC 8372) (photograph, schematic drawing with bone sutures on photo, and schematic drawing with captions). Abbreviations: Eo, exoccipital; maf, mastoid foramina; mep, mastoid exposure of the petrosal; oc, occipital condyle; Pe, petrosal; pgp, postglenoid process; pttf, posttemporal foramen; So, supraoccipital; Sq, squamosal. Scale bar: $1 \mathrm{~cm}$.

Braincase endocast (Figs 54-58). The braincase of MHNC 8372 has been entirely excavated of matrix and the digital rendering illustrated in Figures 54 and 57 represents the reversed volume of the braincase. The internal aspect of the walls of the braincase of mammals generally bears imprints of some boundaries of the brain. However, the absence of imprints on the braincase wall does not imply that the boundaries did not exist on the brain. In MHNC 8372 theses structures are extremely faint on the wall of the braincase; nonetheless, a few observations and interpretations can be made (see below). On this specimen there is no trace of an ossified falx cerebri, which is not surprising given the fact than MHNC 8372 is a young adult, i.e. not fully ossified. Furthermore, anteriorly, the cribriform plate was not preserved. However, the suture of the cribriform plate with the frontal dorsally and laterally and with the ethmoid ventrally is clearly visible (especially on the right side of the skull) and distinctly delimitates its periphery. This condition provides a good indication of the anterior end of the olfactory bulbs, although the anterior convexity of the bulbs has to be estimated. Measurements for the endocast are given in Table 7. 


\begin{tabular}{|c|c|c|}
\hline & MHNC 8372 & MHNC 8399 \\
\hline Anteroposterior length from alveolar border of $\mathrm{I} 1$ to posterior edge of condyle & 55.30 & 59.75 \\
\hline Anteroposterior length from alveolar border of 11 to posterior edge of nuchal crest & 57.75 & 62.56 \\
\hline Length of orbitotemporal fossa between ventral lacrimal foramen and anterior edge of glenoid fossa & 21.51 & 23.02 \\
\hline Length of palate from posterior edge of I1 to ventral edge of choanae & 27.28 & 29.67 \\
\hline Length of basicranium from posterior edge of palate to anterior ventral edge of foramen magnum & 23.52 & 25.62 \\
\hline Length of right tooth row (P1-M3) & 22.66 & 22.50 \\
\hline Bizygomatic width & 37.53 & $18.25 \times 2=36.50 \mathrm{e}$ \\
\hline Width between lacrimal tuberosities & 20.69 & 23.19 \\
\hline Width of rostrum at anterior opening of infraorbital canal & 17.01 & - \\
\hline Width of palate between Cs & 6.77 & - \\
\hline Width of palate between P2s & 6.77 & - \\
\hline Width of palate between M1s & 8.10 & - \\
\hline Width of palate between M3s & 8.91 & - \\
\hline Minimum width of interorbital bridge & 7.44 & 7.56 \\
\hline Maximum width of nasals posteriorly & 11.34 & - \\
\hline Maximum width of nasals anteriorly & 6.45 & - \\
\hline Minimum width of nasals & 4.81 & - \\
\hline Width of basicranium (in ventral view) between ventral ends of nuchal crests (just lateral to mastoid processes) & 20.20 & 19.71 \\
\hline Length of promontorium from ventral edge of external aperture of cochlear fossula to apex & 5.61 & 5.68 \\
\hline Width of basisphenoid between carotid notches & $4.91 \mathrm{e}$ & 4.75 \\
\hline Width of glenoid cavity & 6.55 & 7.21 \\
\hline Maximum length of glenoid cavity (in orthogonal projection) & 4.41 & 5.68 \\
\hline Width of skull between lateral edges of condyles & 14.2 & 13.76 \\
\hline Height of foramen magnum & 6.97 & 8.76 \\
\hline Width of foramen magnum & 7.57 & 8.36 \\
\hline Width of occipital face of skull at posttemporal foramina & 15.76 & 16.59 \\
\hline Maximum width of nuchal crest above posttemporal foramina & 14.74 & $16.71 \mathrm{e}$ \\
\hline
\end{tabular}

Overall morphology - The overall morphology of the endocast of Alcidedorbignya is elongate and narrow with large and cylindrical olfactory bulbs anteriorly. It is very similar to that of Pantolambda (AMNH 16663 and 3957) (Figs 54, 56, and personal observations) and also resembles in this respect the endocast of Leptolambda (Edinger 1956; Lucas 1984). It differs however from the proportionally shorter and more massive endocast of Coryphodon (Marsh 1876, 1877, 1884; Lucas 1984). When compared to other known endocasts of pantodonts, the endocast of Alcidedorbignya is much larger relative to the overall volume of the skull (41\%; see Table 7 and see Silcox et al. 2010, who provided an exhaustive dataset on endocranial volumes of fossil and extant mammals). In length, the braincase occupies slightly more than half of the of the skull length (57\%) and is distinctly longer than in the other pantodonts for which endocast is known. In Pantolambda the estimated length of the skull of AMNH 16663 is $157 \mathrm{~mm}$ (see above in the description of the general morphology of the skull) and the estimated length of the braincase (the anterior extent has been obtained with a CT scan image) is $57 \mathrm{~mm}$, which corresponds to approximately one third (36\%) of the skull length. Proportions of the braincase in Coryphodon (C. hamatus Marsh, 1876) are even smaller as the endocast occupies approximately a quarter of the skull length (28\%). The endocast of the brain of Alcidedorbignya extends farther anteriorly than in other pantodonts for which the endocast is known. Its anteriormost end approaches the orbit and is at the level of the anterior edge of M2, while in Pantolambda it reaches anteriorly the apex of the protocone of M3 and in Coryphodon it remains at the level of the posterior region of the orbitotemporal fossa.
Forebrain region - The olfactory bulb casts are the anteriormost structures of the forebrain cast and in life they contained the main olfactory bulbs and associated meninges. The casts of olfactory bulbs provide a good approximation of the size and shape of the corresponding olfactory bulbs in extant mammals (see for example Bauchot \& Stephan 1967; Macrini 2006). The olfactory bulbs of Alcidedorbignya are ovoid in shape and anteroposteriorly elongated (width/length ratio of 0.63 for the right bulb and 0.58 for the left bulb). However, their anterior boundary was not preserved because the cribriform plate is missing and the length measurements are based on its suture with the frontal. Alcidedorbignya has large olfactory bulbs, which represent the rostralmost $8.49 \%$ of the endocast length (Table 7). Among pantodonts, they are longer than in Pantolambda (5.26\%) and Coryphodon (two determinations; $6.45 \%$ and $4.44 \%$ ) but shorter than in Leptolambda (10.57\%). The olfactory bulbs of Alcidedorbignya are joined for most of their length as is observed in Pantolambda but contrary to the condition of Leptolambda and Coryphodon in which the olfactory bulbs diverge anteriorly. The olfactory bulbs of Alcidedorbignya are separated from the cerebral hemispheres by a distinct circular fissure. Concurrently, the cerebrum does not overlap onto the olfactory lobes, a condition, which resembles that observed in other pantodonts (e.g., Pantolambda, Leptolambda, Coryphodon). Immediately posterior to the circular fissure are cast of the two cerebral hemispheres. In dorsal view (Fig. 54A, B), they are ovoid in shape and anteroposteriorly elongate, with a width/length aspect ratio of 0.65 for both hemispheres. The surface of the cerebral hemisphere endocast is smooth with no gyri and sulci (except for the rhinal sulcus, 

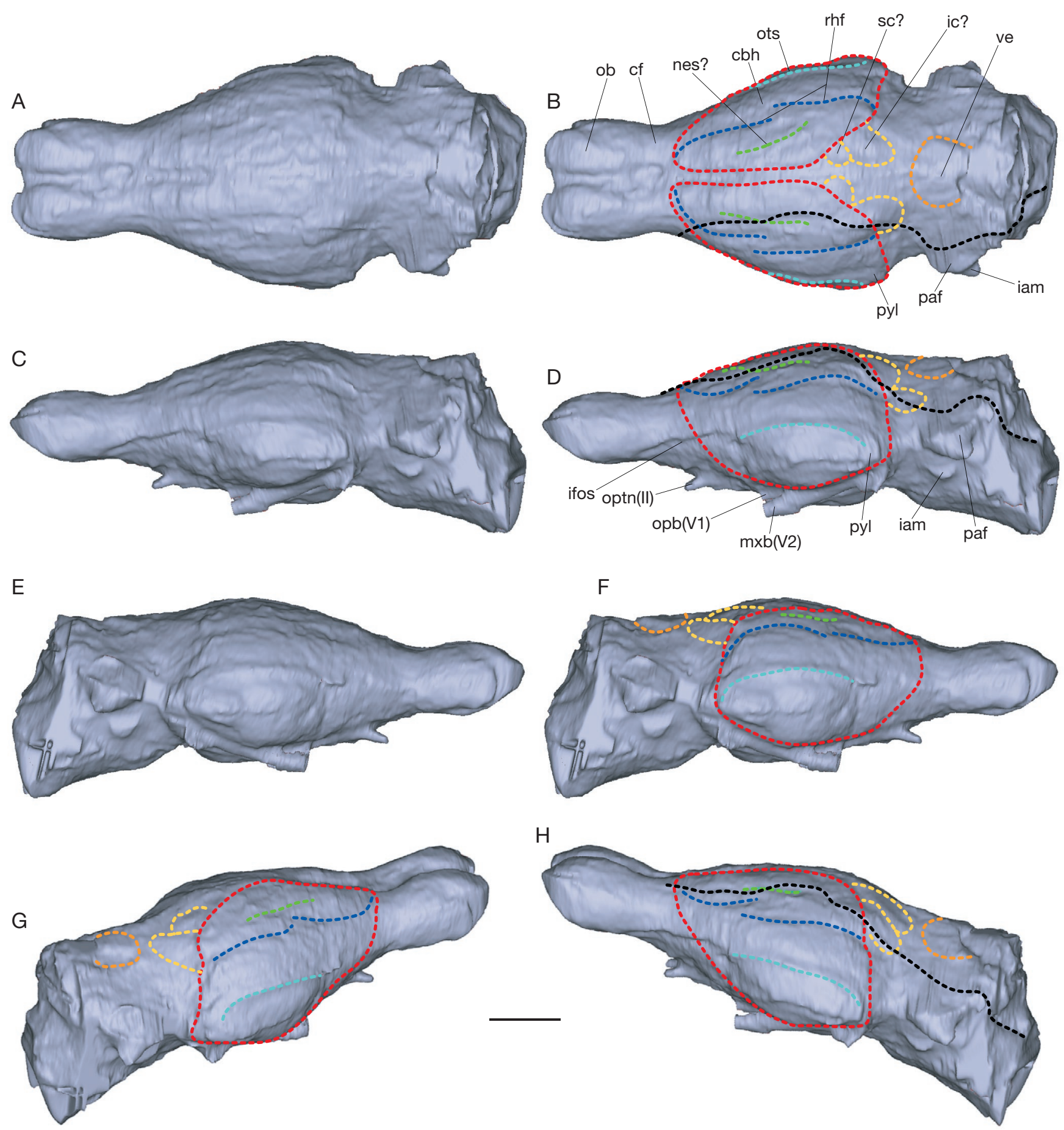

FIG. 54. - Digital rendering of the endocranial cavity of Alcidedorbignya inopinata (MHNC 8372) with hypothetical position of the various elements of the brain. These reconstructions are based on careful observation and movement of the 3D endocast model. A, dorsal view; B, dorsal view with reconstructions; C, right lateral view; D, right lateral view with reconstructions; $\mathbf{E}$, left lateral view; $\mathbf{F}$, left lateral view with reconstructions; $\mathbf{G}$, right posterodorsolatreral view; $\mathbf{H}$, left posterodorsolateral view. Abbreviations: cbh, cerebral hemispheres; cf, circular fissure; iam, internal acoustic meatus; ic?, possible imprint of the inferior colliculus; ifos, imprint of the frontal-orbitosphenoid suture; mxb(V2), maxillary branch of the trigeminal nerve (CN V2); nes?, possible imprint og the neocortical sulcus; ob, olfactory bulbs; opb(V1), ophtalmic branch of the trigeminal nerve (CN V1); optn(II), optic nerve; pf, parafloculus; ots, orbitotemporal sulcus; paf, parafloculus; pyl, pyriform lobe; rhf, rhinal fissure; sc?, possible imprint of the superior colliculus; ve, vermis. Dotted lines colors: red, cerebral hemispheres; blue, rhinal fissure; green, possible neocortical sulcus; light blue, orbitotemporal sulcus; yellow; midbrain; orange; cerebellar vermis; black, imprint of the breakage line, which separates the two parts of the skull. Scale bar: $5 \mathrm{~mm}$. The 3D rendering of CT data is available in pdf format at the address http://sciencepress. mnhn.fr/ en/periodiques/geodiversitas/37/4/alcidedorbignya-inopinata-additional-files.

see below) and the cerebral hemispheres were therefore lissencephalic. The median or sagittal sinus clearly separates the two cerebral hemispheres at their anterior dorsal end.
In fact, the groove, which separates the anterior part of the cerebral hemispheres and the olfactory bulbs, corresponds to the crista frontalis, a broad crest on the dorsal midline of 


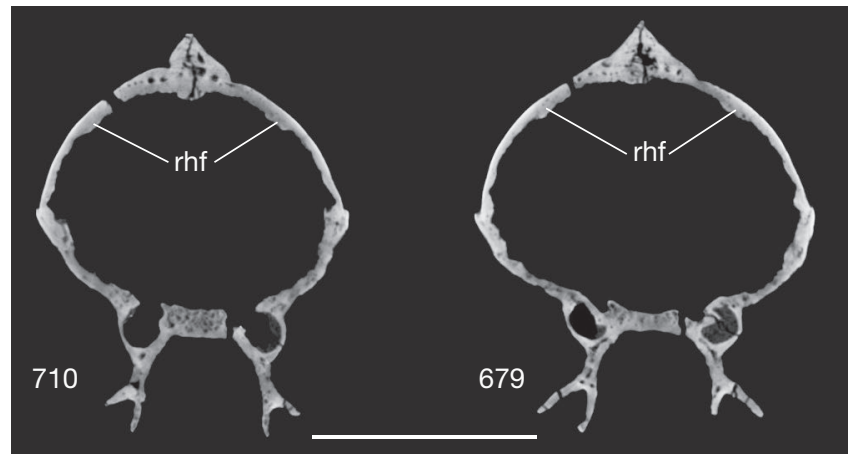

FIG. 55. - CT-slices through the skull of MHNC 8372. The numbers for each illustrated slice refer to the position of the slice within the sequence of 1812 images (resolution: on binned images, voxel size $=0.033 \mathrm{~mm}$ ). Top of the skull toward the top, right side of the skull toward the left. Sections of the zygomatic arches have been omitted. Abbreviations: rhf, ridge on the braincase wall, probably corresponding to the sulcus of the rhinal fissure on the brain. Scale bar: $1 \mathrm{~cm}$.

the braincase for the attachment of the falx cerebri. Posteriorly, the cerebral hemispheres are poorly separated medially and the roof of the braincase was apparently flat. At their posterior end, the two cerebral hemispheres diverge from each other at a point well anterior to the cast of the vermis, slightly anterior to the posterior third of the length of the hemispheres (c. $40 \%$ of the length). A similar condition is observed in Pantolambda, Leptolambda and Coryphodon. Therefore, in Alcidedorbignya, between the vermis of the cerebellum posteriorly and the point of divergence of the cerebral hemispheres anteriorly is a vast, roughly pentagonal to triangular area, which may correspond to a large exposure of the midbrain (see below). Hence, the cerebral hemispheres were well separated (anteroposteriorly) from the cerebellum and did not (or not completely) overlap the midbrain either. As a consequence, the brain of Alcidedorbignya was relatively rectilinear with a weak flexure $\left(21^{\circ}\right)$, a condition similar to that of Pantolambda.

In lateral view (Fig. 54C-F), the cerebral hemisphere are more elevated than the olfactory bulbs, and their ventral parts are expanded into piriform lobes. The piriform lobes are large but do not protrude strongly ventrally, resembling the condition observed in Pantolambda and Coryphodon.

The lateral boundary of the neocortex is often difficult to determine precisely. Although it can be fixed in histological slices, it is not always visible on the external surface of the brain because the lateral rhinal fissure, which marks the division between the paleocortex ('olfactory cortex' or piriform lobe of cerebrum) and neocortex (isocortex), can be incomplete or missing (Bauchot \& Stephan 1967). As a consequence, the cast of the rhinal fissure may be hardly visible or totally absent on endocranial casts. However, the absence of a distinct rhinal fissure on fossil endocranial casts does not imply the absence of neocortex. For instance, the rhinal fissure is not visible on any of the endocasts of Monodelphis domestica, but it is clearly visible on dissected brains (Macrini et al. 2007b). In Alcidedorbignya, on the internal side of the parietal and within the braincase of MHNC 8372 , dorsal and roughly parallel to the orbitotemporal sulcus is an irregular ridge, which is slightly closer to the sulcus than to the dorsal suture of the parietals. This ridge (a sulcus on the digital reconstruction of the cranial endocast) is here interpreted as a probable rhinal fissure (Fig. 55). If this interpretation is correct, then the rhinal fissure has a dorsoventral position relatively elevated on the brain, being clearly located on the dorsal half of the cerebral hemisphere. Therefore, the neocortex of Alcidedorbignya would be restricted to a small narrow dorsal region of the cerebral hemispheres. This condition strongly resembles that observed in other pantodonts such as Pantolambda (Fig. 56) and Leptolambda (Edinger 1956). On MHNC 8372, on the part of the endocast dorsal to the rhinal fissure (i.e. on the neocortex), no obvious neocortical sulci could be observed, although some faint structures on the neocortex could possibly be interpreted as such (Fig. 54). Neocortical sulci are possibly present in Pantolambda, as observed on AMNH 3957 and on the interpretation of Edinger (1956: fig. 18). However, we have not observed neocortical sulci on the CT scan of AMNH 16663, wherein the rhinal fissure is clearly visible in an elevated position on the cerebral hemispheres (Fig. 56). No sulci are apparently present in Leptolambda (fide Edinger 1956: fig. 18 and Lucas 1984: fig. 11).

Venous system - The pathways of the sinuses and veins draining blood from the brain are poorly marked on the endocranial cast surface of Alcidedorbignya. If dural blood vessels are mostly internal to the dura-mater, meningeal vessels (and more particularly sinuses) on the contrary lie in the intra-dural space (between the dura-mater and periostis), and are likely to leave an imprint on casts. Those sinuses are generally well visible on endocranial casts (for example in primates, Tenrec, Potamogale, Solenodon; see Bauchot \& Stephan 1967): in the inter-hemispheric fissure runs the sagittal sinus, from which originate the two transverse sinuses that run in the tentorial fold between the cerebrum and cerebellum.

On the dorsal surface of the neocortex of Alcidedorbignya, the superior sagittal sinus forms a median line, separating the two cerebral hemispheres by drawing a median line. At its posterior end is supposedly the division of the transverse sinus, between the cerebrum and cerebellum (Macrini et al. 2007c; Orliac et al. 2012a, 2014). The course of the transverse sinuses is not visible on the endocast. However, a shallow sulcus posterolateral to the vermis may represent a posteriormost course of the transverse sinus, just before it emitted the capsuloparietal emissary vein in the petrosquamosal fossa (for further details, see above in the petrosal section and Figs 47, 48). Such a posterior course of the transverse sinus is unusual, and in the absence of any undisputable imprints, we are unable to retrace the pathway of the transverse sinuses from their divergence from the median sagittal sinus to their emission of the capsuloparietal emissary veins and sigmoid sinuses. However, although no imprints are observable between the posterior end of the sagittal sinus and the shallow groove mentioned above, it is clear that the transverse sinus had to cross the midbrain. We hypothesized its course on Figure 58. 
A

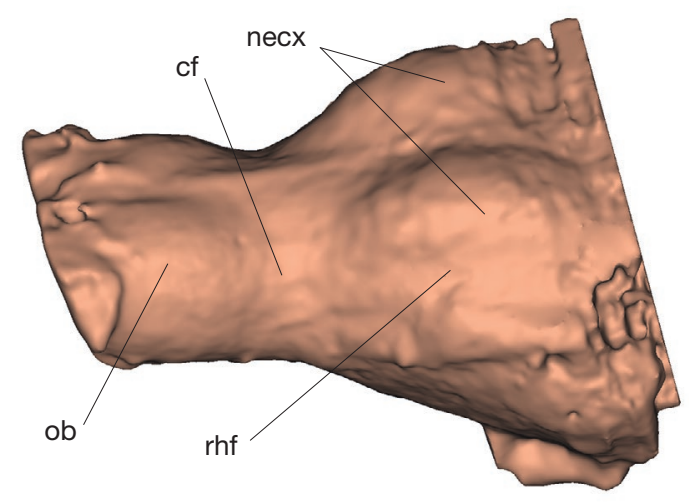

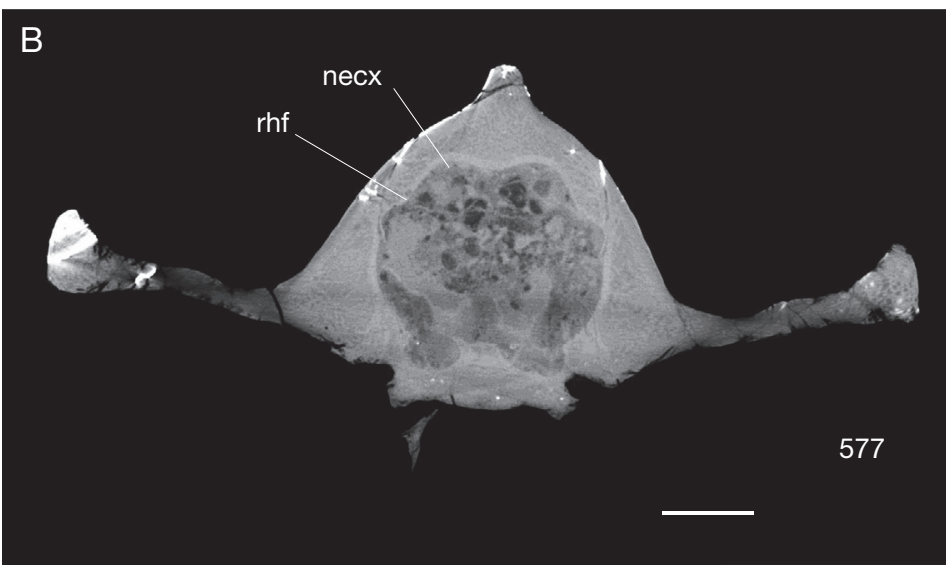

FIG. 56. - Pantolambda bathmodon (AMNH 16663): A, digital rendering of part of the endocranial cavity (it has not been possible to reconstruct the whole cavity because of the presence of iron oxide mineralisation in the central and posterior parts of the braincase). This left dorsolateral view clearly shows the small neocortical cap and the wide rhinal fissure; B, CT-slice through the skull of AMNH 16663 showing the neocortex and the rhinal fissure. The number of the illustrated slice refers to the position of the slice within the sequence of 1892 images (resolution binned images, voxel size=0.076 mm). Abbreviations: cf, circular fissure; necx, neocortex; ob, olfactory bulbs; rhf, rhinal fissure. Scale bar: $1 \mathrm{~cm}$.

Midbrain region - As exposed from above, the wide space observable between the point of divergence of the cerebral hemispheres and the vermis probably corresponds to the tectum of the midbrain. Wide dorsal exposure of the midbrain is a common condition in early mammals (see below). Midbrain exposure has been observed in all the other pantodonts for which an endocast is known. Lucas (1984: figs 10-12) illustrated the endocasts of Pantolambda bathmodon, Leptolambda schmidti, and Coryphodon "hamatus" and the three specimens clearly have a broadly exposed midbrain on the dorsal surface of the endocast as is observed in Alcidedorbignya.

As for the other structures of the brain, there are almost no imprints of the midbrain structures on the skull roof. Nevertheless, a faint elevation is observed on the endocast in the postero medial region of each cerebral hemisphere. If this structure actually corresponds to an imprint of the midbrain, it may represent either the colliculi superior or anterior or both (Fig. 54). Lucas (1984) did not observe any structural details on the midbrain of the endocasts of Coryphodon, Leptolambda, and Pantolambda. Transverse sinus imprints also might be expected to be in this region of the endocast, but none are visible. It is likely however that the transverse sinus followed the posterior edge of the cerebral hemispheres from their point of divergence (where they individualise from the sagittal sinus). At the posterolateral angle of the cerebral hemisphere, the transverse sinus had to turn posteriorly in order to reach the dorsalmost region of the petrosal where it would have divided into sigmoid sinus, superior petrosal sinus, and capsuloparietal emissary vein (Fig. 58). As a matter of fact, it is possible to follow on the skull roof (especially on the CT scan of MHNC 8399; Fig. 48) a probable passageway of the transverse sinus from the dorsal apex of the petrosal. From this point the sinus forms a drastic U-turn anteriorly and runs along a shallow but broad groove on the lateral wall of the skull (see above) in order to reach the posterior edge of the cerebral hemisphere anteriorly. At this point we hypothesize that the transverse sinus (Fig. 58) turns anteromedially, following the posterior edge of the cerebral hemisphere to reach the sagittal sinus medially.
Hindbrain region - The cast of the cerebellum of Alcidedorbignya is quite large (approximately 28\% of the endocast, 32\% of the cerebrum) and the casts of the vermis, cerebellar hemispheres, and paraflocculi are clearly visible, although the boundaries are subtle. As in most therian mammals (Macrini et al. 2007), the paramedian fissures separate the lateral lobes of the cerebellum from the vermis. The right paramedian fissure is best preserved on the 3D model (Fig. 54). The vermis of the cerebellum forms a wide bulge that extends anterior to the position of the cast for the paraflocculus. In lateral view, the dorsalmost point of the vermis is slightly lower than the dorsal surface of the cerebrum when the anteroposterior axis of the endocast is horizontal. This condition is also observed in Pantolambda, Leptolambda, and Coryphodon.

The paraflocculi lie on the ventrolateral part of the hindbrain; they are conical in shape and their apices point posteroventrolaterally. These conical lobes of the cerebellum are accommodated in the corresponding subarcuate fossae, which have been described above as conical (see above, petrosal section; and see discussion by Sánchez-Villagra 2002). Such a conical shape of the paraflocculi is also observed in Pantolambda and Leptolambda. It differs from the broadly spherical morphology observed in basal metatherians and basal eutherians (see Macrini et al. 2007c).

The caudal end of cerebellum is well separated from the brainstem. The medulla oblongata and the pons did not leave distinctive marks on the ventral surface of the endocast, as in many other mammal cranial endocasts (Macrini 2006). We can assume that they were likely obscured in ventral view by meninges and the pontine cistern, as in other mammals (Evans \& de Lahunta 2012).

Midventral surface of the endocast - The base of the cerebrum of Alcidedorbignya preserves casts of the foramina for the optic and trigeminal nerves (Fig. 57). The optic nerve casts are located anteriorly posterior to the posterior end of the olfactory lobes. The cast of the optic chiasma is visible on the endocast, posterior to the olfactory bulbs and anterior to 

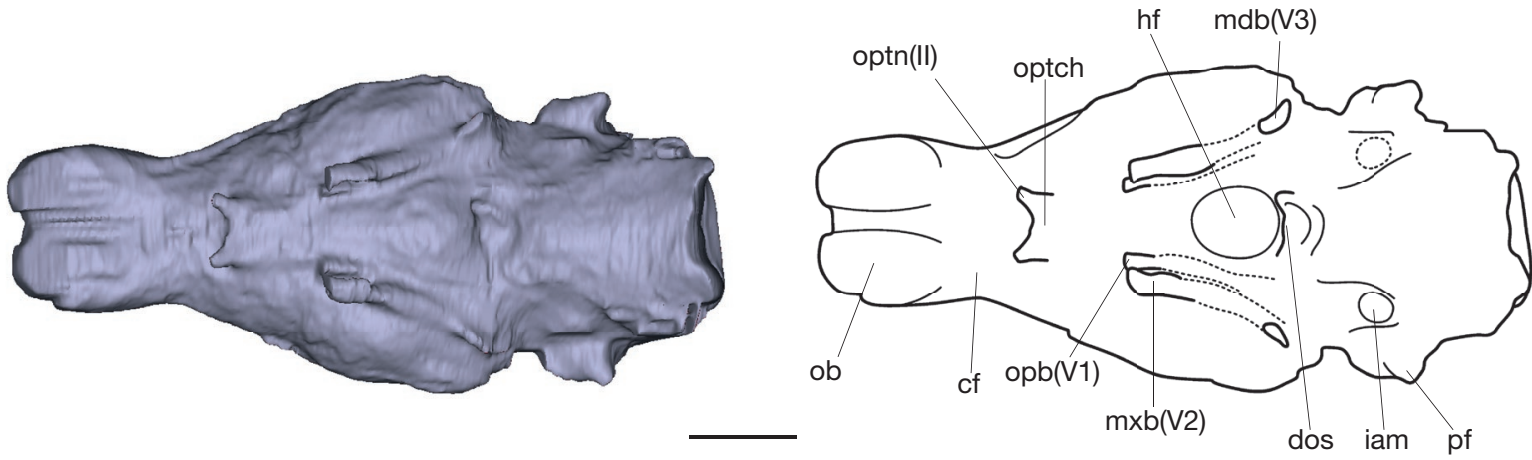

FIG. 57. - Digital rendering of the endocranial cavity of Alcidedorbignya inopinata (MHNC 8372) in ventral view with schematic interpretation. Abbreviations: cf, circular fissure; dos, dorsum sellae; hf, hypophyseal fossa; iam, internal acoustic meatus; ob, olfactory bulbs; optn (II), optic nerve; optch, optic chiasm; pf, parafloculus; mdb(V3), mandibular branch of the trigeminal nerve (CN V3); $\mathbf{m x b}(\mathbf{V} 2)$, maxillary branch of the trigeminal nerve (CN V2); opb(V1), ophtalmic branch of the trigeminal nerve (CN V1). Scale bar: $5 \mathrm{~mm}$. The 3D rendering of CT data is available in pdf format at the address http://sciencepress.mnhn.fr/en/ periodiques/geodiversitas/37/4/alcidedorbignya-inopinata-additional-files.

TABLE 7. - Endocast measurements for Alcidedorbignya inopinata (MHNC 8372).

\section{Endocast flexure}

Endocast anteroposterior length

Endocast maximum width

Endocast maximum height

Cerebrum anteroposterior length

Cerebrum maximal width

Olfactory bulbs cast anteroposterior length

Olfactory bulbs cast maximum width

Olfactory bulbs cast maximum height

Cerebellum anteroposterior length

Cerebellum maximal width

Cerebellum maximal height

Hypophyseal fossa cast anteroposterior length

Hypophyseal fossa cast maximum width

Hypophyseal fossa cast maximum height

Total endocast volume

Olfactory bulbs volume/endocast volume

Skull maximal length

Endocast length/skull length

Overall skull volume

Endocast volume/skull volume
Olfactory bulb volume $21^{\circ}$

$31.52 \mathrm{~mm}$

$14.15 \mathrm{~mm}$

$10.06 \mathrm{~mm}$

$13.96 \mathrm{~mm}$

$14.15 \mathrm{~mm}$

$8.07 \mathrm{~mm}$

$7.72 \mathrm{~mm}$

$4.80 \mathrm{~mm}$

$6.36 \mathrm{~mm}$

$9.85 \mathrm{~mm}$

$4.02 \mathrm{~mm}$

$4.37 \mathrm{~mm}$

$1.82 \mathrm{~mm}$

$2203.22 \mathrm{~mm}^{3}$

$187.06 \mathrm{~mm}^{3}$

$8.49 \%$

$55.3 \mathrm{~mm}$

$57 \%$

$5591 \mathrm{~mm}^{3}$

$39 \%$
$10.06 \mathrm{~mm}$

the hypophyseal fossa. The right and left optic canals, which transmitted cranial nerve II are confluent. More posteriorly are the casts of the exit of $\mathrm{CN} V 1$ from the foramen rotundum and of CN V2 from the sphenorbital fissure, which are distinctly separated according to what is observed on the skull (see above: Figs 31, 32), and the cast of the exit of CN V3 from the foramen ovale. The cast of the sphenorbital fissure is medial to that of the foramen rotundum and corresponds to the passage of the ophthalmic branch of the trigeminal nerve, as well as the oculomotor (CN III), trochlear (CN IV), and abducens (CN VI) nerves plus the ophthalmic artery and vein. The cast of the foramen rotundum corresponds to the passage of the maxillary branch of the trigeminal nerve (CN V2), which is generally much larger than the $\mathrm{CN} \mathrm{V1}$ (Forasiepi 2009; Evans \& de Lahunta 2012). Posterolaterally to the cast of the foramen rotundum and on the ventromedial edge of the piriform lobe is the cast of the foramen ovale, which corresponds to the passage of the mandibular branch of the trigeminal nerve (CN V3).
Between the foramina ovalia is the cast of the hypophyseal fossa, located in the center of the ventral surface of the endocast. It is a deep, roughly circular depression with a width/ length aspect ratio of approximately 1.09 , and a height/length ratio of 0.45 . The cast of the hypophyseal fossa composes approximately $1.5 \%$ of the endocranial cavity. Although the hypophyseal fossa may not be an accurate measure of the size of the pituitary gland, it is the only available bony proxy for the pituitary gland in fossils (Edinger 1942). Behind the cast of the hypophyseal fossa is a depression, which is the mold of the dorsum sellae. On the left side of the depression is a pit for the caudal clinoid process. The hypophyseal fossa is much more distant posteriorly from the optic chiasm than what is observed in Coryphodon and (to a lesser extent) in Leptolambda, a condition probably related to the greater massiveness and anteroposterior shortness of the cerebral hemispheres in these taxa.

Endocast volume and encephalization quotient- The endocranial cast of Alcidedorbignya (MHNC 8372), reconstructed with Mimics 16 (@Materialise), has a volume of $2203.22 \mathrm{~mm}^{3}$. As mentioned above, this volume is an approximation because of the absence of cribriform plate and because of some possible distortion of the skull. The encephalization quotient (EQ) is the ratio of actual to expected (based on correlation) brain sizes for a particular taxon (Jerison 1973: 61) relative to its group, with 1.0 representing an average. Expected brain size is calculated from regression equations relating brain mass or endocranial volume to body mass in a panel of related taxa (Jerison 1973). Although often criticized about their biological significance (e.g., Radinsky 1978, Deaner et al. 2007), EQs may be useful, for example, to infer relative brain sizes in extinct taxa, which can be correlated with functional features and behavior (e.g., Jerison 1973; Eisenberg \& Wilson 1981). The EQ of Alcidedorbignya was calculated using the equation of Eisenberg (1981), because it is based on a wide range of extant mammalian taxa: $\mathrm{EQ}=\mathrm{Ev} /(0.055[\mathrm{Bm}] 0.74)$ (Ev, endocast volume; Bm, Body mass). The body mass of Alcidedorbignya (MHNC 8372), calculated with the BODYMASS software of Gingerich (1990) on the basis of long bone length and diameter, is estimated at $579 \mathrm{~g}$. The mean of the 


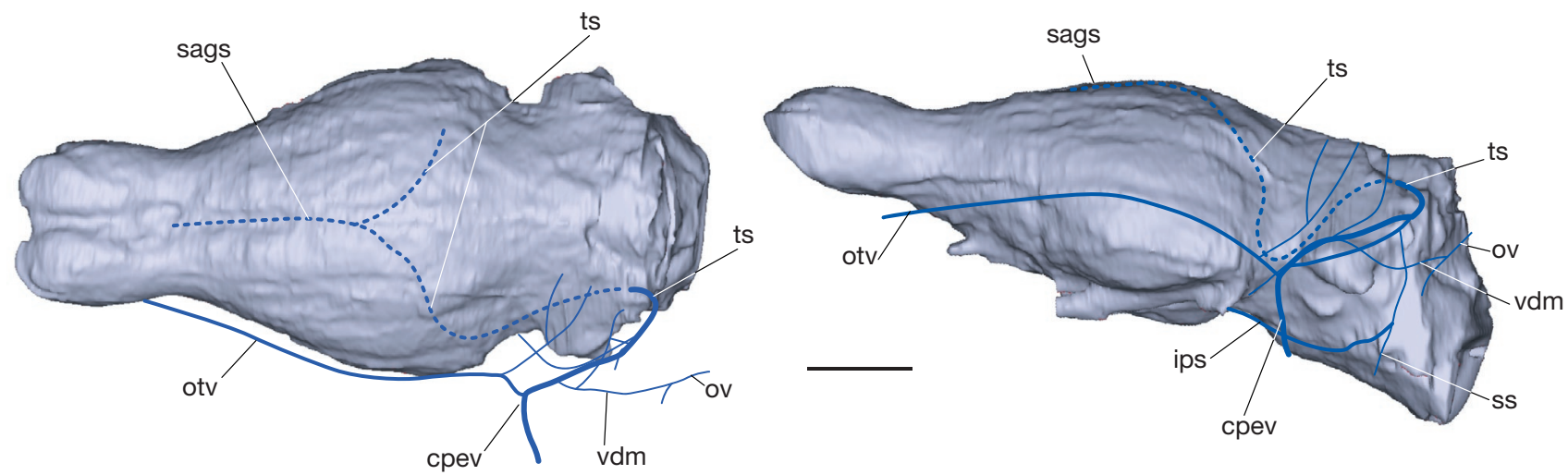

FIG. 58. - Digital rendering of the endocranial cavity of Alcidedorbignya inopinata (MHNC 8372) with reconstruction of the venous system and hypothetical course of the anterior portion of the transverse sinus and sagittal sinus (dotted line): A, dorsal view; B, lateral view. Abbreviations: cpev, capsuloparietal emissary vein; ips, inferior petrosal sinus; otv, orbitotemporal vein; ov, occipital vein; sags, sagittal sinus; ss, sigmoid sinus; ts, transverse sinus; vdm, vena diploëtica magna. Scale bar: $5 \mathrm{~mm}$. The 3D rendering of CT data is available in pdf format at the address http://sciencepress. mnhn.fr/en/periodiques/geodiversitas/37/4/ alcidedorbignya-inopinata-additional-files.

TABLE 8. - Body mass and encephalization quotient values for Alcidedorbignya inopinata (MHNC 8372).

\begin{tabular}{|c|c|c|c|c|c|}
\hline Source of body mass estimate & $\begin{array}{l}\text { Body mass } \\
(\mathrm{g})\end{array}$ & $\begin{array}{l}\text { Endocranial } \\
\text { volume }\left(\mathrm{mm}^{3}\right)\end{array}$ & $\begin{array}{c}E Q \\
\text { (Jerison, 1973) }\end{array}$ & $\begin{array}{c}E Q \\
\text { (Eisenberg, 1981) }\end{array}$ & $\begin{array}{c}\text { EQ } \\
\text { (Martin, 1990) }\end{array}$ \\
\hline $\begin{array}{l}\text { Cranial length insectivore equation } \\
\text { (Thewissen \& Gingerich 1989) }\end{array}$ & 383.03 & 2.20 & 0.34 & 0.49 & 0.41 \\
\hline $\begin{array}{l}\text { Cranial length generic primate } \\
\text { equation (Martin 1990) }\end{array}$ & 488.88 & 2.20 & 0.29 & 0.41 & 0.34 \\
\hline $\begin{array}{l}\text { Postcranial dimensions BodyMass } \\
\text { Software (Gingerich 1990) }\end{array}$ & 579.00 & 2.20 & 0.26 & 0.36 & 0.30 \\
\hline \multicolumn{3}{|c|}{ MEAN $=483.63$} & \multicolumn{2}{|r|}{ MEAN $=0.42$} & \\
\hline
\end{tabular}

values of bodymass calculated from cranial length equations (Thewissen \& Gingerich 1989; Martin 1990) and long bones (Gingerich 1990) provides a bodymass estimate of $483 \mathrm{~g}$. Therefore, with the equation of Eisenberg (1981), we obtained a mean EQ of 0.42. A similar EQ is calculated in Pantolambda, but the EQ is significantly smaller in Coryphodon (0.21) and Leptolambda (0.18) (see Table 8 and Silcox et al. 2010, who provide an exhaustive dataset on body masses and EQs for a wide range of fossil and extant mammals).

\section{Dentary (Figs 59, 60)}

The dentary is relatively short and stout (Figs 59, 60; Table 9). The corpus mandibularis (horizontal ramus) slightly increases in depth posteriorly to the level of $\mathrm{m} 2$, where its ventral border shows a distinct convexity. Posterior to this level its ventral margin is slightly concave below the anterior edge of the coronoid process and the masseteric fossa. Anteriorly, the symphyseal region is short and stout and the two dentaries are fused in the adult. The anteroventral edge of the symphysis forms a distinct angle $\left(c .120^{\circ}\right)$ with the ventral edge of the corpus mandibularis. In lateral view, this angulation, which marks the posteroventral end of the symphysis, is located approximately below $\mathrm{p} 1$ or $\mathrm{p} 2$. In ventral view the posteroventral end of the symphysis presents a small posteriorly-directed spine probably for the attachment of the geniohyoideus muscle (Barone 1997; Evans \& de Lahunta 2012). A relatively large anterior mental foramen is present below $\mathrm{p} 1$. The position of this foramen is apparently fairly constant as it is in the same position in the five specimens in which it is preserved. The position of the posterior mental foramen is more variable, either below p 3 or p 4 or between the two teeth.

The ramus mandibularis (vertical ramus) is anteroposteriorly short and slightly shifted anteriorly, i.e. its main axis is oriented anterodorsally-posteroventrally and is at an angle slightly less than $90^{\circ}$ with the corpus. This condition is more pronounced in MHNC 8399 than in MHNC 8372 (Fig. 60). The masseteric fossa is deep and limited anteriorly by a salient tuberosity located below the talonid of $\mathrm{m} 3$. This tuberosity extends posterodorsally in a massive and elevated coronoid crest along the anterior edge of the coronoid process. The masseteric fossa reaches the base of the coronoid process and extends posterodorsally to the anterior border of the condyloid process. The coronoid process is narrow and almost as wide at its base as at its apex. It is regularly curved posteriorly and its anterior edge is concave at the base and convex in its distal two-thirds. The angular process is short and relatively low when compared to that of Pantolambda, but distinctly hook-like. Although not as gracile as in Leptictis and Erinaceus, this hook-like morphology is clearly plesiomorphic for eutherians. The medial side of the angular process of MHNC 8372 bears a thick ridge, dorsally concave, extending from the apex posteriorly to slightly posterior to the level of the mandibular foramen (Fig. 59D). This ridge is probably for 


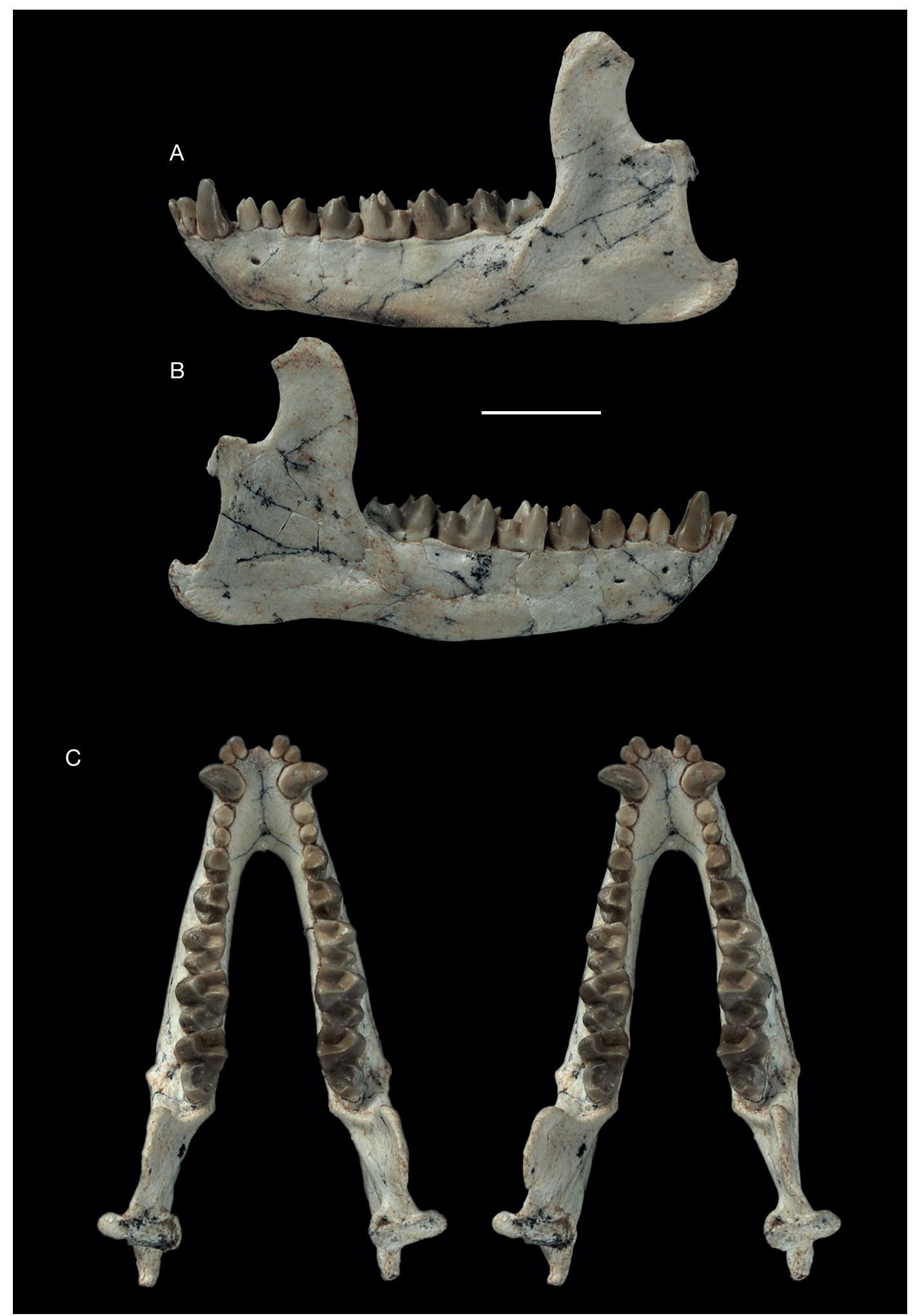

FIG. 59. - Mandible of Alcidedorbignya inopinata (MHNC 8372): A, left lateral view; B, right lateral view; C, stereophotographs of dorsal view. Scale bar: A-C, $1 \mathrm{~cm}$.

the attachment of the pterygoideus medialis muscle (Barone 1997; Evans \& de Lahunta 2012). This structure is apparently variable, as it is distinctly weaker in MHNC 8399 although the angular process of this specimen is larger (more extended dorsoventrally) than on the other. The condyloid process is transversely expanded and the articular surface is wider medially than laterally. The medial half of the articular surface extends posteroventrally, a condition that corresponds to the medially positioned postglenoid process. The condyle is located well above the occlusal plane of the molars. The pos- 

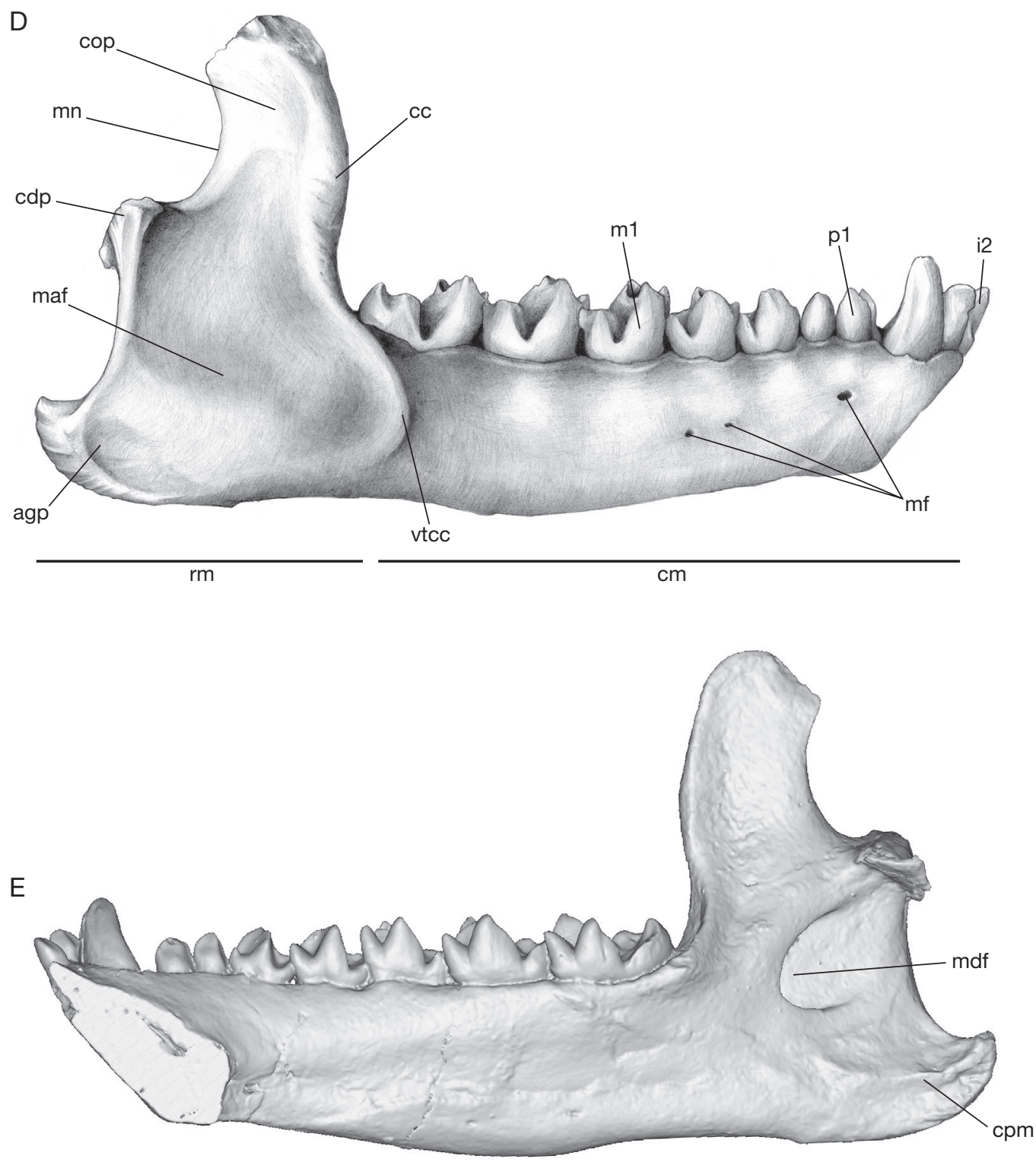

FIG. 59 (continuation). - Mandible of Alcidedorbignya inopinata (MHNC 8372): D, drawing of lateral view with captions; $\mathbf{E}$, digital rendering of the medial view of the left mandible (reversed). Abbreviations: agp, angular process; cc, coronoid crest; cdp, condyloid process; cm, corpus mandibularis; cop, coronoid process; cpm, crest for the pterygoideus medialis?; maf, masseteric fossa; mdf, mandibular foramen; mf, mandibular foramina; mn, mandibular notch; rm, ramus mandibularis; vtcc, ventral tubercle of the coronoid crest.

terior crest of the ramus mandibularis, between the condyle and the angular process, is relatively straight or very slightly concave on MHNC 8372. It is oriented slightly oblique, anterodorsally-posteroventrally. This condition is much more pronounced in Pantolambda because of the enlargement and posterior projection of the large angular process in this genus.

In dorsal view, the tooth row is not really aligned with the virtual line joining the coronoid process to the anterior tip of the mandible on MHNC 8372. The tooth row is rather slightly shifted medially in its posterior part. This is barely the case on MHNC 8399. This medial shift of the posterior part of the tooth row is perceptible in the shape of the dentary bordering the $\mathrm{m} 3$ posteromedially, which exhibits a medially protruding tuberosity. This tuberosity, also better marked on MHNC 8372 than on MHNC 8399, is connected postero- dorsolaterally with the medial crest of the anterior border of the ramus mandibularis.

The mandibular foramen is large, level with the tooth row dorsoventrally and slightly anterior to the midpoint of the ramus mandibularis (Fig. 59D).

\section{POSTCRANIAL SKELETON}

MHNC 8372 is a nearly complete skeleton, which preserves most of the postcranial skeleton (Fig. 2). No other skeleton (even partial) of Alcidedorbignya inopinata is known. However, some isolated vertebrae or limb bones have been referred to A. inopinata. The skeleton MHNC 8372 is that of a young adult, as all the epiphyses of the limb bones and vertebrae are unfused. Therefore, this skeleton is less completely ossified than would have been that of an old adult. As a consequence, 


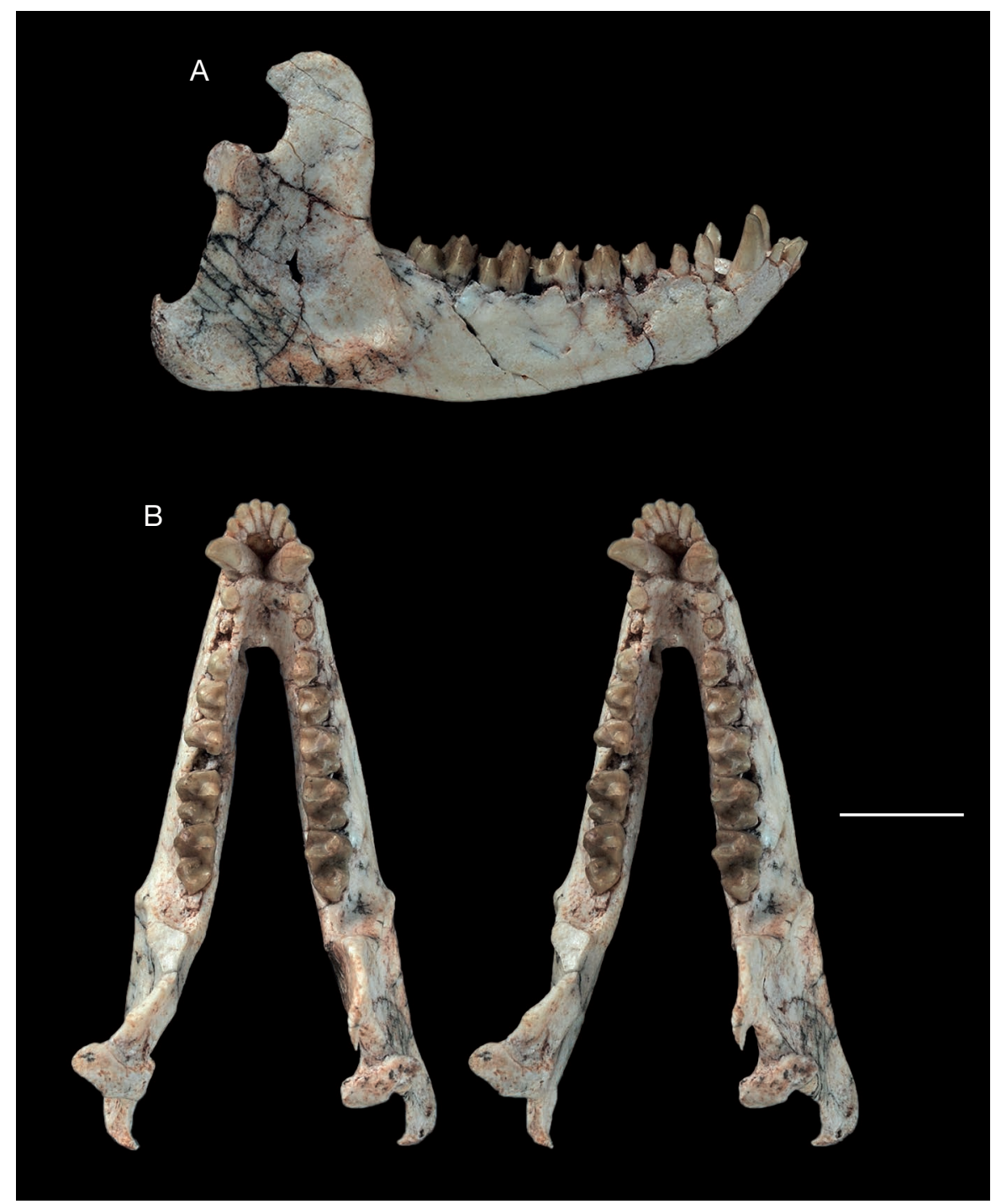

FIG. 60. - Mandible of Alcidedorbignya inopinata (MHNC 8399): A, lateral view; B, stereophotographs of dorsal view. Scale bar: $1 \mathrm{~cm}$.

TABLE 9. - Measurements of the dentary of Alcidedorbignya inopinata (in mm).

\begin{tabular}{lrr}
\hline & MHNC 8372 & MHNC 8399 \\
\hline Maximum length of dentary from alveolar border of i1 to posterior edge of angular process & 47.87 & 53.39 \\
Height of ramus & 24.61 & 27.84 \\
Length of tooth row (p1-m3) & 62.83 & 52.07 \\
Greatest length of symphysis & 11.76 & 12.94 \\
Greatest width of symphysis & 4.90 & 5.26 \\
Medial depth of body below anterior root of p3 & 6.43 & 7.69 \\
Medial depth of body below anterior root of m1 & 8.08 & 8.87 \\
Medial depth of body below anterior root of m2 & 9.25 & 10.04 \\
Medial depth of body below posterior root of m2 & 9.51 & 10.26 \\
Medial depth of body below posterior root of m3 & 8.93 & 11.38 \\
Width of the condyle & 6.74 & 7.02 \\
Maximum depth of condyle (on medial edge) & 3.67 & 3.86 \\
\hline
\end{tabular}

the bones are also less strong and less resistant to external pressure. Therefore, the bones (especially limb bones) have suffered some distortion and compression during fossilisation, which sometimes prevents an exact evaluation of the actual morphology of the bones. In some cases the compression is oriented and a distinct direction is observed in the distortion. This case is the worst as the actual morphology of the bone can be strongly modified and its original anatomy difficult to evaluate. In other cases, the compression could be defined as "surrounding". In other words, the compressive forces are 
approximately equivalent in all directions and are oriented toward the centre of the bone. Their action tends to squeeze the bone on itself. In such a case, the compression tends to exaggerate the superstructures of the bone: the crests are sharpened, and the cavity and fossae are deepened. However, in some cases this difficulty of observation related to postmortem distortion can be compensated for by the isolated bones referred to Alcidedorbignya inopinata, which are generally less distorted than those of the skeleton MHNC 8372.

The description below of the postcranial skeleton of $A$. inopinata is partly comparative. When possible, a comparison will be primarily with Pantolambda bathmodon, which is the closest taxon to $A$. inopinata for which the postcranial skeleton is known. The only reasonably complete skeleton of Pantolambda bathmodon is that associated with the skull referred to in the description of the skull (AMNH 16663). This specimen was described by Matthew (1937). Since Matthew's description, the skull has been further prepared and is partly described in Appendix 3. The block presented in Matthew (1937: pl. XLIII), shows part of the anterior postcranial skeleton, still unprepared, and most of the anterior vertebrae and ribs as well as both scapulae are still partly embedded in matrix. Of the anterior vertebrae, only a fragment of the atlas, the centrum and dens of the axis, and the third cervical were available for this study. Several posterior vertebrae were figured by Matthew (1937). They are: three posterior thoracics, five lumbars, and ten caudals. Most of the limb bones (except the scapulae and carpal bones) and the innominates are preserved and perfectly prepared. Therefore, it is not possible to compare most of the anterior vertebrae of Alcidedorbignya with those of Pantolambda bathmodon. Other comparisons will be, when possible, with the North American archaic ungulates Hyopsodus and Meniscotherium, which are similar in size to Alcidedorbignya and for which postcranial material is known. Among the Recent eutherians, we found some similarities with Solenodon and Tenrec. Comparison will also be with Mayulestes ferox, a borhyaenoid from Tiupampa similar in size to Alcidedorbignya, which has been interpreted as partially arboreal (Muizon 1998; Argot 2001, 2002, 2003).

\section{Axial Skeleton}

All the vertebrae of Alcidedorbignya inopinata (specimen MHNC 8372) are known, except, probably the caudal vertebra (Ca) 12 and the two or three most distal caudals (see below).

Atlas. (Fig. 61 and Table 10) In anterior view, the atlas presents a roof-like dorsal arch, with the two sides of the roof being relatively flat and forming an angle close to $120^{\circ}$. In dorsal view, the dorsal arch has the same anteroposterior length along all its width and the anterior and posterior borders are roughly straight. In its median region, it presents a robust dorsal tubercle that is triangular in dorsal view and that bears a strong scar for the rectus capitis dorsalis minor. On the lateral extremities of the anterior edge of the dorsal arch, above the articular facets for the occipital condyles, is a large laterally-opening notch. This structure represents the dorsal edge of a large atlantal foramen, which can be observed

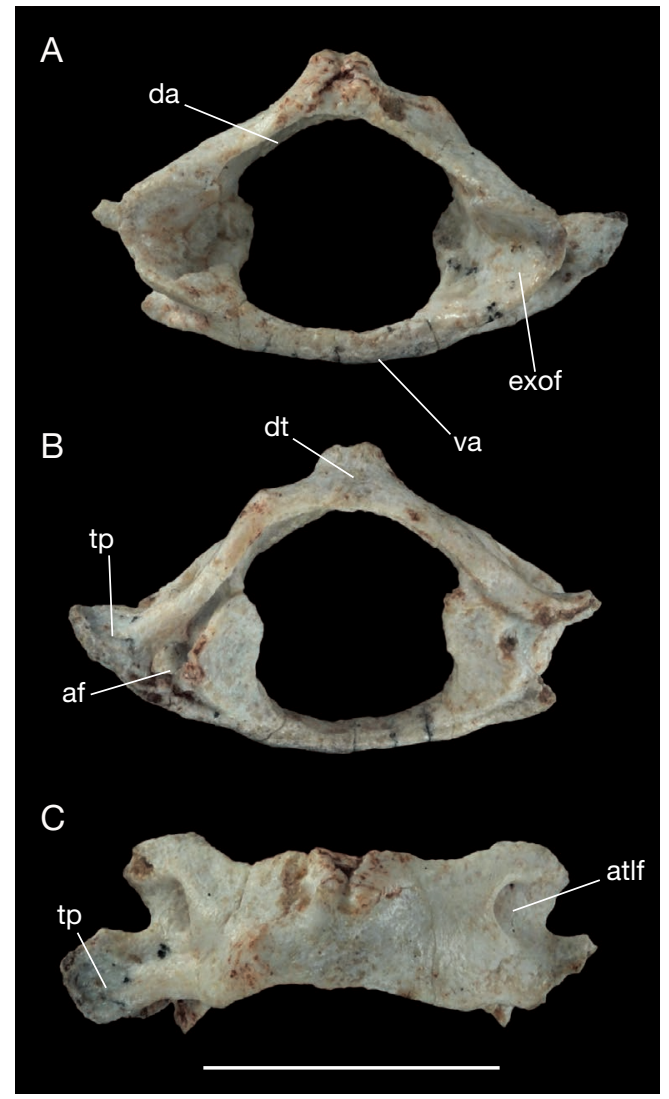

FIG. 61. - Atlas of Alcidedorbignya inopinata (MHNC 8372): A, anterior view; B, posterior view; C, dorsal view. Abbreviations: af, arterial foramen; atlf, atlantal foramen; da, dorsal arch; dt, dorsal tubercle; exof, exoccipital articular facet; tp, transverse process; va, ventral arch. Scale bar: $1 \mathrm{~cm}$.

in lateral view. This foramen opens above the deep notch that forms the anterior edge of the transverse process. A deep sulcus separates the posterior edge of the dorsal arch from the articular facets with the axis. The ventral arch is fused to the rest of the vertebra. It is smooth and gently concave dorsally; no ventral tubercle is present. It bears the articular facet for the dens of the axis. The neural canal is approximately as high as wide, being constricted at the level of the dorsal edge of the facet for the axis. The anterior facets for the occipital condyles open anteromedially and are strongly concave. The posterior articular facets for the axis are roughly triangular in outline and are only slightly concave. The transverse process (only the left one is preserved) is small and its dorsal side faces dorsoposteriorly. Posteroventrally, a large arterial foramen can be observed between the lateralmost edge of the axis facet and the posterior edge of the transverse process. The posterior apex of the process does not protrude beyond the posterior edge of the dorsal arch. In posterior view the transverse process is slightly deflected dorsally and forms a regular smooth curve with the ventral arch.

Axis. (Fig. 62 and Table 11) The axis of MHNC 8372 is preserved in articulation with $\mathrm{C} 3-\mathrm{C} 5$. In addition to this specimen, one isolated axis and another articulated to a partial C3 are referred to $A$. inopinata (MHNC 8424 and 8425 re- 


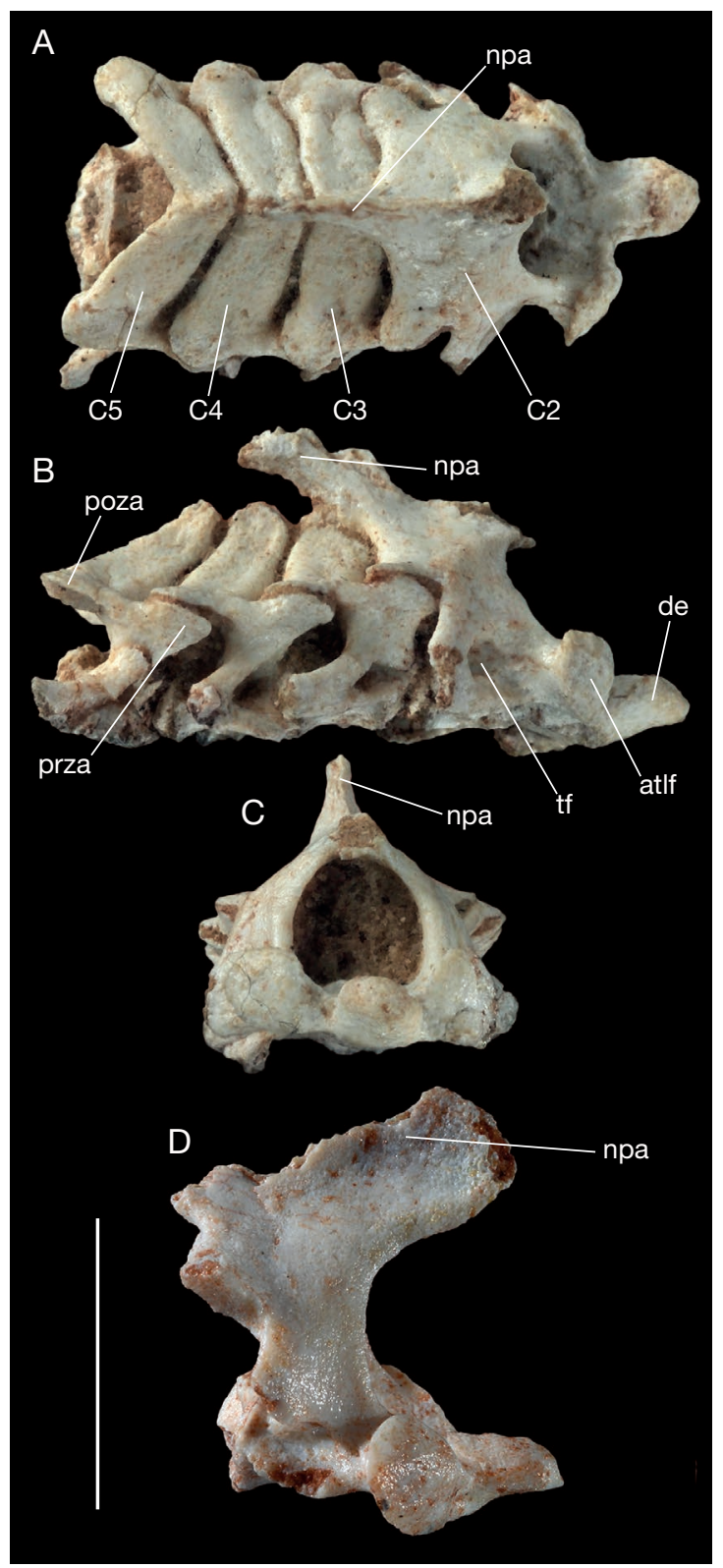

FIG. 62. - Cervical vertebrae of Alcidedorbignya inopinata: A, C2-C5 of MHNC 8372 in dorsal view; B, C2-C5 of MHNC 8372 in lateral view; $\mathbf{C}$, axis of MHNC 8372 in anterior view; D, axis of MHNC 8425 in lateral view. Abbreviations: atlf, atlantal factet; de, dens; npa, neural process of the axis; poza, postzygapophysis; prza, postzygapophysis; tf transverse foramen. Scale bar: $1 \mathrm{~cm}$.

spectively). None of the specimens has a completely preserved neural process. However, its anterior extremity is preserved on MHNC 8425 and its posterior part on MHNC 8372. Therefore, if the dorsoventral extension of the process can only be estimated, its anteroposterior length can be reconstructed. The neural process was very long anteroposteriorly. Its anterior extremity overhangs the anterior half of the odontoid process and its posterior extremity overhangs the dorsal arch of $\mathrm{C} 4$. However, the posterior apex of this process on MHNC 8372 is broken and clearly extended further posteriorly at least to a point above $\mathrm{C} 5$, perhaps more. This process was therefore conspicuously long and presented a condition quite similar
TABLE 10. - Measurements of the atlas of Alcidedorbignya inopinata (in mm).

Length of neural arch at sagittal plane

4.52

Length of intercentrum at sagittal plane

1.67

Height of atlas at sagittal plane

10.42

Maximum height of the neural canal

7.35

Minimum width of neural canal between medial

5.90

edges of facets for the axis

Maximum width between lateral edges of occipital

14.76

facets

Maximum height of left occipital facet

Maximum width of left occipital facet

Maximum height of left facet for the axis

Maximum width of left facet for the axis

3.66

4.20

4.60

3.31

TABLE 11. - Measurements of the axis of Alcidedorbignya inopinata (in mm).

\begin{tabular}{|c|c|c|c|}
\hline & $\begin{array}{c}\text { MHNC } \\
8372\end{array}$ & $\begin{array}{c}\text { MHNC } \\
8425\end{array}$ & $\begin{array}{c}\text { MHNC } \\
8424\end{array}$ \\
\hline \multicolumn{4}{|l|}{ Maximum ventral length (including dens) } \\
\hline at sagittal plane & 9.94 & 10.47 & 12.10 \\
\hline Length of dens & 3.82 & 3.66 & 3.90 \\
\hline \multicolumn{4}{|l|}{ Width between lateral edges of atlantal } \\
\hline facets & 9.67 & 10.39 & 10.84 \\
\hline Width of right atloidian facet & 3.88 & 4.64 & 5.14 \\
\hline Height of right atloidian facet & 3.00 & 3.66 & 3.77 \\
\hline Height of neural canal & 4.11 & 5.73 & 5.58 \\
\hline Width of neural canal & 4.53 & 5.47 & 5.10 \\
\hline Posterior maximum width of centrum & 6.15 & 6.11 & 6.03 \\
\hline $\begin{array}{l}\text { Width at lateral edges of } \\
\text { postzygapophyses }\end{array}$ & 9.40 & 8.95 & 9.46 \\
\hline
\end{tabular}

to that of Mayulestes ferox, carnivorous metatherian known from Tiupampa (Muizon 1998). It is clearly shorter in Tenrec or Solenodon, in which the posterior extent of the process is above C3.

The posteroventral edge of the neural process on the axis faces posteroventrally with the ventral component of the angle being much greater than the posterior one. It forms an angle of c. $20^{\circ}$ with the anteroposterior axis of the centrum. This angle is $c .30^{\circ}$ in Mayulestes, $c .65^{\circ}$ in Tenrec, and nearly vertical in Echinosorex. The posteroventral edge of the neural process is slightly concave in Alcidedorbignya, as it is in Tenrec, while it is straight in Mayulestes. The pedicles are anteroposteriorly longer in Alcidedorbignya than in Mayulestes. The postzygapophyses face ventrally on MHNC 8372 and 8425 but they face slightly ventroposteriorly in MHNC 8425 as they do in Mayulestes. The transverse process is triangular in anterolateral view and is perforated by a large transverse foramen as in Tenrec and Solenodon. Its distal extremity is pointed and extends posteriorly at the level of the pedicle of C3. It is more massive than those on the following vertebrae. The ventral side of the vertebral body exhibits a relatively elevated ventral keel. This keel is sharp on MHNC 8372 but is slightly rounded on MHNC 8424 and 8425 . Its posterior extremity does not end in a prominent tuber. In this respect, it resembles the condition observed in Tenrec contrasting with Mayulestes and Solenodon and especially Echinosorex. The ventral keel separates two fossae for the longus colli muscle. As in Tenrec, these fossae are 


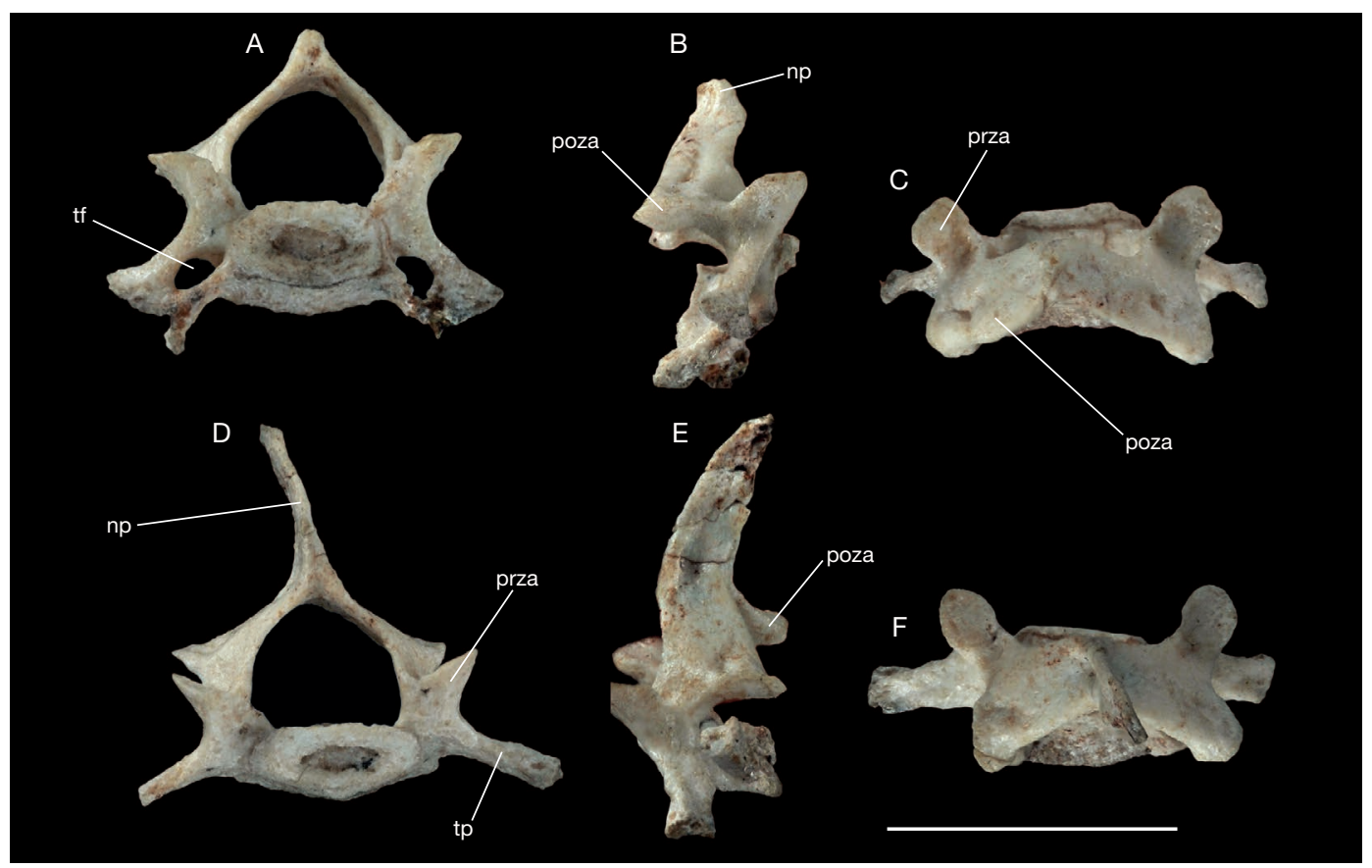

FIG. 63. - Cervical vertebrae (C6-C7) of Alcidedorbignya inopinata (MHNC 8372): A, C6 in anterior view; B, C6 in lateral view; C, C6 in dorsal view; D, C7 in anterior view; E, C7 in lateral view; F, C7 in dorsal view. Abbreviations: np, neural process; poza, postzygapophysis; prza, postzygapophysis; tf, transverse foramen; tp, transverse process. Scale bar: $1 \mathrm{~cm}$.

relatively shallow and differ from the deep ones observed in Mayulestes. In anterior view the atlantal facets are ovoid, and are more elevated laterally than medially. In this respect they resemble the condition observed in Mayulestes and Solenodon but differ from that of Tenrec, in which the atlantal facets are distinctly lower. The odontoid process is robust and has parallel edges as in Mayulestes but contrary to that of Tenrec, which is more conical. On its ventral side is an ovoid articular facet for the dorsal edge of the ventral arch of the atlas. This facet is distinctly separated from the pair of anterior atlantal facets.

The axis of Pantolambda bathmodon (AMNH 16663) is incomplete and only the centrum with the odontoid process is preserved. The latter is proportionally shorter and more massive than in Alcidedorbignya. Its lateral edges diverge posteriorly on the anterior two-thirds of its length, which results in a conical morphology that differs from the cylindrical morphology of this process in Alcidedorbignya. As in Alcidedorbignya, the dens facet is not continuous with the anterior atlantal facets. The centrum is anteroposteriorly shorter than in Alcidedorbignya and on its ventral face the fossae for the m. longi colli are as deep as in Alcidedorbignya. The median keel separating the fossae is well pronounced but much wider than in Alcidedorbignya. In fact, instead of a crest between the fossae, as in Alcidedorbignya, it is more an elevated plateau, which strongly widens posteriorly. At the level of the posterior epiphysis it is approximately twice as wide as anteriorly.

Posterior cervical vertebrae (C3-C7). (Figs 62, 63; Table 12) These vertebrae are anteroposteriorly short, as in Tenrec and Solenodon. There is no neural process on C3-C5 because of the posterior extension of the neural process of the axis. On
C6, a small process is present and the two lateral sides of the dorsal arch make an acute angle in anterior view. A long and thin neural process, inclined posteriorly, can be observed on C7. It is very similar to those of the anterior thoracic vertebrae except that in the latter the anterior edge is straight and not slightly convex as in C7. The prezygapophyses protrude anteriorly well beyond the narrow dorsal arch and their articular facet does not extend onto the arch itself. They are oriented dorsomedially, the dorsal component being distinctly more pronounced than the medial one. On C7 the medial component of their orientation is more important than on the anterior cervicals. The transverse processes of C3 are directed posterolaterally, whereas on C4-C5, they are blade-like owing to the presence of a ventral extension that protrudes also anteriorly. On C6 they are not complete but the right transverse process preserves the posterior protrusion of its ventral part. On C7 the transverse processes strongly protrude laterally with a small ventral component. A transverse foramen can be observed on all cervicals but C7. The anterior and posterior articular facets of the centra are strongly oblique relative to the long axis of the centrum. The anterior articulation therefore faces anteroventrally and the posterior one faces posterodorsally. This orientation suggests that the neutral position of the neck may have sloped anteriorly, with C1 higher than $\mathrm{C} 7$ and with the centra articulations vertically oriented. On the ventral side of C3, as on the axis, a distinct median ridge (ventral keel) separates the fossae for the longus colli. This ridge widens but becomes lower on $\mathrm{C} 4$, almost disappears on C5, and is absent on C6 and C7. An isolated cervical vertebra (MHNC 8426) has been referred to Alcidedorbignya inopinata. It is identified as a C4 or C5. 
TABLE 12. - Measurements of the posterior cervical vertebrae (C3-C7) of Alcidedorbignya inopinata (in $\mathrm{mm}$ ).

\begin{tabular}{|c|c|c|c|c|c|}
\hline MHNC 8372 & C3 & C4 & C5 & C6 & C7 \\
\hline Height of neural canal & - & - & 4.09 & 4.40 & 4.17 \\
\hline Width of neural canal & - & - & 5.24 & 5.19 & 4.95 \\
\hline $\begin{array}{l}\text { Ventral length of the centrum } \\
\text { at sagittal plane }\end{array}$ & 4.23 & 4.04 & $4.06 \mathrm{e}$ & $3.70 \mathrm{e}$ & $3.96 \mathrm{e}$ \\
\hline $\begin{array}{l}\text { Anterior maximum width } \\
\text { of centrum }\end{array}$ & 6.01 & 5.83 & 5.21 & 5.25 & 5.90 \\
\hline $\begin{array}{l}\text { Posterior maximum width } \\
\text { of centrum }\end{array}$ & 5.49 & 4.96 & - & 5.96 & 7.08 \\
\hline $\begin{array}{l}\text { Width at lateral edges } \\
\text { of prezygapophyses } \\
\text { Width at lateral edges }\end{array}$ & 9.32 & 10.96 & 10.88 & 10.55 & 10.25 \\
\hline of postzygapophyses & 10.89 & 10.76 & 10.32 & 9.79 & 9.35 \\
\hline Length of neural process & - & - & 1.50 & 1.82 & 6.97 \\
\hline
\end{tabular}

It is morphologically very similar to the cervical vertebrae of MHNC 8372 but notably larger. This size difference is probably related to sexual dimorphism, especially given the fact that MHNC 8372 appears to represent a small female.

A partial third cervical vertebra is known in the skeleton AMNH 16663 of Pantolambda bathmodon. Because the C3 of Alcidedorbignya is not isolated from C2 and C4 comparison is difficult with this specimen. The prezygapophyses are apparently more concave and do not project anteriorly as far as in Alcidedorbignya. The articular facet clearly extends posteriorly onto the neural arch, whereas this feature is absent in Alcidedorbignya. The transverse foramen of Pantolambda is totally enclosed in the transverse process, as in Alcidedorbignya, but is proportionally larger.

Thoracic vertebrae. (Fig. 64; Tables 13, 14) MHNC 8372 has 13 thoracic vertebrae articulated with ribs. The thoracic vertebrae count of Alcidedorbignya is less than in Tenrec (19), Solenodon (15), and Erinaceus (15) but equals that of Echinosorex (13). The thoracics of MHNC 8372 are distributed into four groups of three to five vertebrae still in articulation, the last block including also two lumbar vertebrae (T1-T3; T4-T6; T7-11 and T12-L2). When articulated, the thoracic area of the vertebral column is $7 \mathrm{~cm}$ long. Anteriorly, T2 is the transitional vertebra between the cervical and thoracic vertebral articular mode. The zygapophyses are widely separated on T1. The articular facets of the prezygapophyses of T1 and T2 are concave, strongly oblique, and face mediodorsally, and those of the postzygapophyses of T1 face ventrolaterally. This condition represents the cervical mode of vertebral articulation. On T2 the postzygapophyses are set closer to each other and their articular facets are roughly flat and face ventrally; a similar condition is observed on the prezygapophyses of T3 with the articular facets facing dorsally. This condition of the T2-T3 articulation represents the dorsal pattern of vertebral articulation. The neural process is not completely preserved on T1, T2, T4, and T6. The preserved base of the neural processes of T1 and T2 is much wider anteroposteriorly than the neural process of $\mathrm{T} 3$ to $\mathrm{T} 10$. The process is complete to almost complete on T3, T5, T7, T8 T9, and T10. It is long, slender and strongly reclined posteriorly back to T10. As preserved, the longest neural processes are located on T3-T7 $(10 \mathrm{~mm}$ on T3) and they only slightly decrease in length posteriorly until T7. Their length greatly reduces posteriorly from T8 to $\mathrm{T} 10$ (3.5 $\mathrm{mm}$ on T10). The neural process of T3 forms an acute angle of approximately $35^{\circ}$ with the horizontal plane. The apex of the neural process of T9 and T10 is anteroposteriorly expanded in comparison to the previous ones, this condition being more pronounced on T9. On T11 the neural process is strongly reduced and its anterior edge is concave whereas the posterior edge is slightly convex, and the main axis of the process is still slightly inclined posteriorly. In lateral view, the process appears strongly curved anterodorsally. On T12 and T13, the neural process is vertical, longer than high, short dorsoventrally and enlarged anteroposteriorly. On T13 in particular, the neural process is as long as the neural arch itself a point of departure from the condition seen among metatherians of "the quarry" at Tiupmapa. Therefore, it can be considered that the anticlinal vertebra is T12, which is also the diaphragmatic vertebra. The length, slenderness, and inclination of the neural process of the anterior thoracic vertebrae of Alcidedorbignya approache the condition observed in living "insectivores" such as Tenrec, Echinosorex, and, to some extent, Solenodon. In these genera, however, the processes are less inclined posteriorly than in Alcidedorbignya. The condition of Alcidedorbignya strongly differs from the very short processes of Erinaceus. Comparison with the thoracic vertebrae of Pantolambda is difficult because the anterior vertebrae of AMNH 16663 are not prepared. However, from what can be seen on the block including the scapulae and the anterior ribs and vertebrae, it seems than the anterior thoracics also had a long and slender neural process.

The transverse width (width of the dorsal arch between the lateral edges of the articular facets of the postzygapophyses) of the thoracic vertebrae of Alcidedorbignya decreases regularly posteriorly, from T1 $(7 \mathrm{~mm})$ to $\mathrm{T} 10(4 \mathrm{~mm})$. The length of the dorsal arch (length of the anteromedial angle of the prezygapophysis facet to the posteromedial angle of the postzygapophysis facet) increases posteriorly, from T1 $(3 \mathrm{~mm})$ to T13 $(5 \mathrm{~mm})$, and in such a way that the last space visible between the neural arches of two successive vertebrae is between T2 and T3. The first thoracic vertebra exhibits the most robust transverse processes of the series, where the first pair of ribs attaches. The articular facets of the prezygapophyses of T1 face dorsomedially and, as in Mayulestes, are in a lateral position, located more on the anteromedial edge of the transverse process than on the anterior edge of the dorsal arch. The pedicles of its dorsal arch are extremely short anteroposteriorly. The condition of T2 is similar to that of T1 especially in the orientation and position of the articular facet of its prezygapophyses. On the postzygapophyses, the posterior articular facets differ in their ventral orientation in correlation with the dorsally-facing anterior articular facets of T3. It is only until T5 that the prezygapophyses articular facets move medially from the medial edge of the transverse process to the anterior edge of the neural arch. The third thoracic vertebra exhibits a 


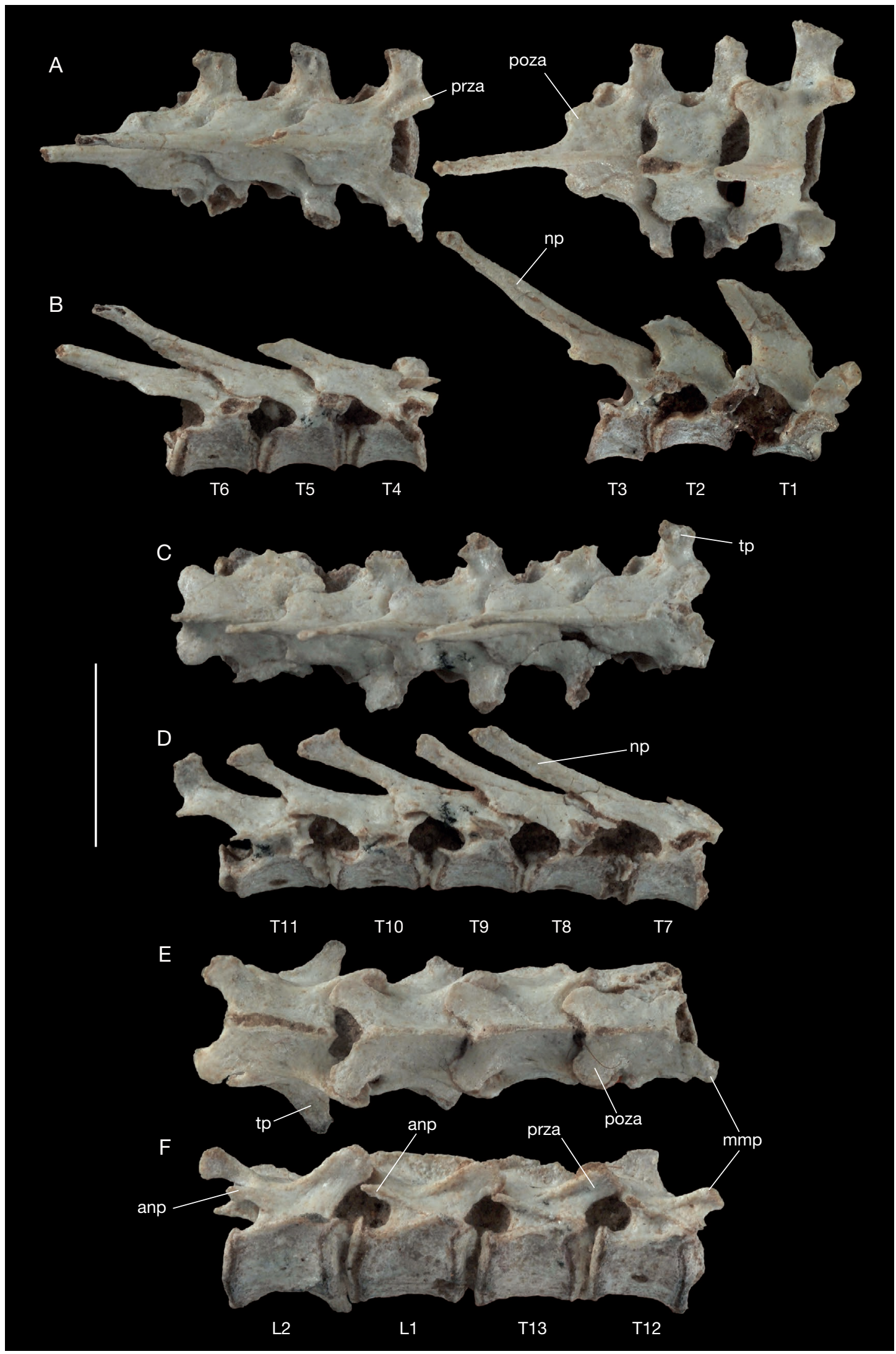

FIG. 64. - Thoracic and lumbar vertebrae of Alcidedorbignya inopinata (MHNC 8372): A, T1-T6 in dorsal view; B, T1-T6 in lateral view; C, T7-T11 in dorsal view; D, T7-T11 in lateral view; E, T12-T13, L1-L2 in dorsal view; F, T1-T13, L1-L2 in lateral view. Abbreviations: anp, anapophysis; mmp, mammillary process; np, neural process; poza, postzygapophysis; prza, postzygapophysis; tp, transverse process. Scale bar: $1 \mathrm{~cm}$. 
TABLE 13. - Measurements of the anterior thoracic vertebrae (T1 to T6) of Alcidedorbignya inopinata (in mm).

\begin{tabular}{|c|c|c|c|c|c|c|}
\hline MHNC 8372 & T1 & T2 & T3 & T4 & T5 & T6 \\
\hline Ventral length of the centrum at sagittal plane & 4.20 & 4.92 & 3.86 & $4.77 \mathrm{e}$ & 5.02 & 4.90 \\
\hline Anterior maximum width of centrum & 5.33 & 6.76 & 5.59 & 4.76 & 4.56 & 4.47 \\
\hline Posterior maximum width of centrum & - & 5.61 & 6.07 & 4.95 & 5.01 & 4.89 \\
\hline Height of neural canal & 3.75 & - & 3.28 & 3.11 & - & 3.27 \\
\hline Width of neural canal & 4.97 & - & 4.71 & 4.55 & - & 4.66 \\
\hline Width between transverse processes & 14.55 & - & - & - & - & - \\
\hline Width at lateral edges of prezygapophyses & 9.93 & 9.55 & 7.28 & 6.12 & 5.03 & 5.01 \\
\hline Width at lateral edges of postzygapophyses & 8.93 & 6.81 & 5.48 & 4.64 & 4.67 & 4.54 \\
\hline Proximodistal length of neural process & - & - & 9.91 & - & - & - \\
\hline Length of neural arch from anteromedian edge to posterior edge of postzygapophysis & 3.47 & 3.66 & 4.47 & 4.80 & - & - \\
\hline
\end{tabular}

TABLE 14. - Measurements of the posterior thoracic vertebrae (T7 to T13) of Alcidedorbignya inopinata (in mm).

\begin{tabular}{|c|c|c|c|c|c|c|c|}
\hline MHNC 8372 & T7 & T8 & T9 & T10 & T11 & T12 & T13 \\
\hline Ventral length of the centrum at sagittal plane & 5.34 & 5.10 & 5.11 & 5.33 & 5.98 & 5.78 & 5.94 \\
\hline Anterior maximum width of centrum & 5.4 .4 & 4.75 & 5.07 & 5.11 & 5.52 & 5.79 & 5.92 \\
\hline Posterior maximum width of centrum & 4.77 & 5.21 & 5.01 & 5.50 & 7.19 & 6.42 & 5.98 \\
\hline Height of neural canal & 3.26 & - & - & - & 3.25 & 3.46 & - \\
\hline Width of neural canal & 4.25 & - & - & - & 4.61 & 4.71 & - \\
\hline Width between transverse processes & - & - & 10.23 & 9.23 & - & 6.96 & 6.33 \\
\hline Width at lateral edges of prezygapophyses & 4.81 & _- & 5.18 & 5.67 & _ & - & 6.82 \\
\hline Width at lateral edges of postzygapophyses & 4.41 & 4.77 & 5.09 & 4.10 & 5.68 & 5.76 & 6.22 \\
\hline Proximodistal length of neural process & 7.86 & & 5.86 & 4.05 & 1.93 & 1.95 & 1.76 \\
\hline Anteroposterior proximal length of neural process & - & - & - & - & 2.61 & 4.01 & 5.60 \\
\hline \multicolumn{8}{|l|}{ Length of neural arch from anteromedian edge } \\
\hline to posteromedial edge of postzygapophysis & _- & _- & _- & _ & _ & 5.10 & 5.85 \\
\hline
\end{tabular}

robust ventral crest (more a plateau than a crest), which is somewhat present on T4 but less pronounced. The other thoracics have no ventral crest. The mammillary processes are present but small above the prezygapophyses of T7 and T9-13 (the transverse processes of T8 are incomplete). Anapophyses exist on T11-T13. They are sharp and small on T11 and prominent posteriorly on T13, where they reach the level of the prezygapophyseal pedicles.

Lumbar vertebrae. (Figs 64, 65; Tables 15, 16) Nine lumbars are known, L1 and L2 are still in articulation with the two last thoracics, L3-5 and L7-9 remain in articulated series, respectively and L6 is isolated. When articulated, the lumbar segment is $7 \mathrm{~cm}$ long, i.e. as long as the thoracic section of the vertebral column. The neural processes are anteroposteriorly long, as long as the dorsal arch. The length of the dorsal arch itself decreases posteriorly, which increases the space between two sequential vertebrae, as seen in dorsal view. The neural processes of the anterior lumbars are low (especially on L1) and stand upright. Their height increases posteriorly, from $1.5 \mathrm{~mm}$ on L1 to $5.5 \mathrm{~mm}$ on L9. From L1 to L6 the neural process is longer than high, a condition which reverses from L7 to L9. On L7-L8, the anterior edge of the neural process is concave whereas the posterior edge is slightly inclined anteriorly. It stands vertical on L9. The robustness and lack of inclination of the neural processes of the lumbar vertebrae contrast sharply with the morphology of the neural processes of the thoracics. This morphology also contrasts with that observed in Mayulestes, in which the fifth lumbar shows a high neural process, inclined anteri- orly. The condition of the neural processes of the lumbars of Alcidedorbignya (large and plate-like) resembles those of some Recent "insectivores" such as Solenodon, Echinosorex, and Tenrec (especially the two former).

The zygapophyses protrude anteriorly and posteriorly beyond the dorsal arch. The mammillary processes are not developed above the prezygapophyses. Anapophyses exist from L1 to L6 but they are weakly developed and hardly reach the level of the prezygapophysis pedicle of the following vertebra. On the ventral side of the vertebral bodies, a median crest can be observed, sharper on L3-L6 and almost absent on L9.

The transverse processes are reduced to a small triangular flat prominence on L1. They increase regularly in length posteriorly (until reaching $8 \mathrm{~mm}$ on L9) and extend anteroventrolaterally in a long and narrow bladelike process. On L4-6, on which the transverse processes are completely preserved, their apices have an anteriorly-projecting anterior angle, which gives them a hook-like morphology. Their anterior tips point anteroventrally: on L6 (Fig. 65C), seen in anterior view, the process is straight and not convex dorsally, and the apex almost reaches the ventral level of the centrum. In dorsal view, the transverse processes of the lumbar vertebrae do not reach anteriorly as far as the anterior extremity of the prezygapophyses (Fig. 65D). The transverse processes are more slender anteroposteriorly than the neural processes, their base occupying approximately half of the length of the vertebral body. On the last lumbar, the transverse processes are almost horizontal in anterior view and are much wider anteroposteriorly than on the preceding lumbars. Moreo- 


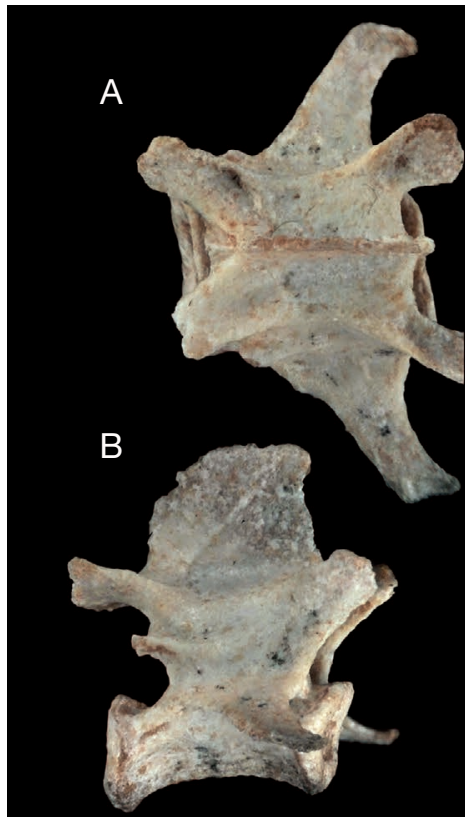

L6
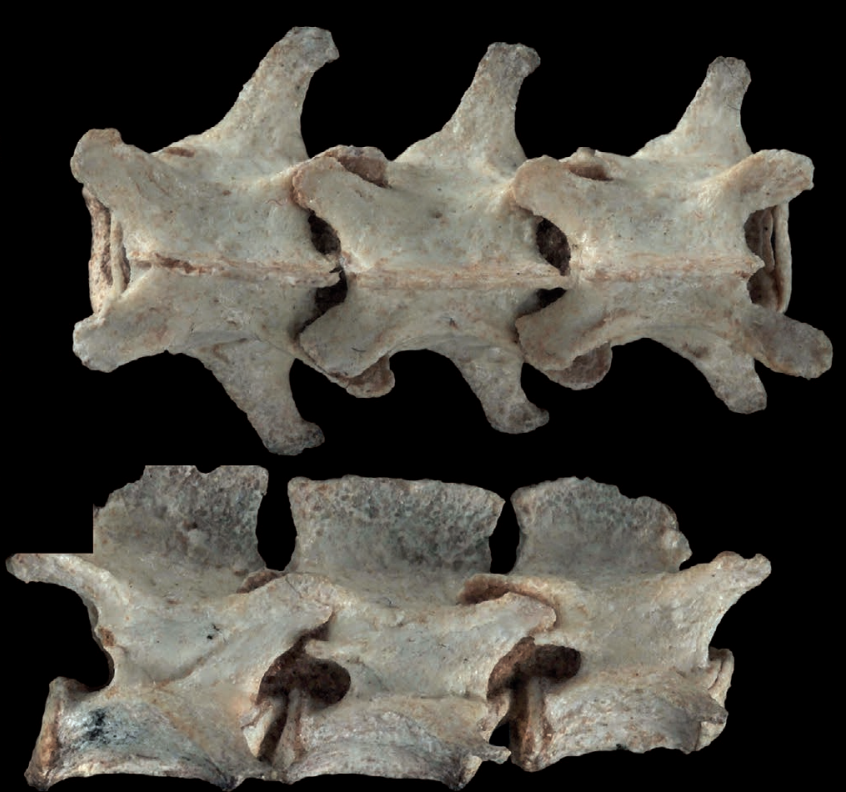

L5

L4

L3
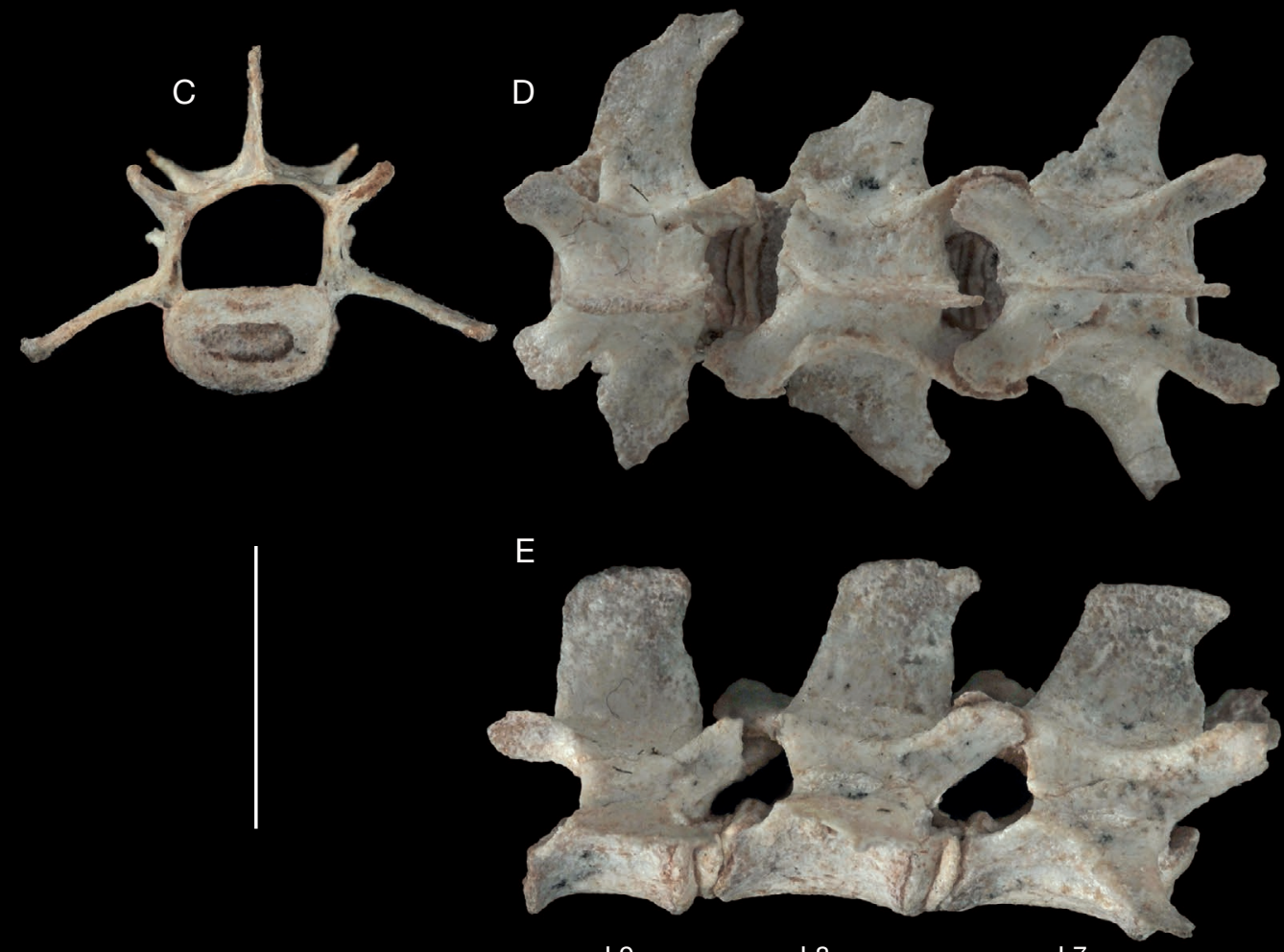

L9

L8

L7

FIG. 65. - Lumbar vertebrae of Alcidedorbignya inopinata (MHNC 8372): A, L3-L6 in dorsal view; B, L3-L6 in lateral view; C, L6 in anterior view; D, L7-L9 in dorsal view; E, L7- L9 in lateral view. Scale bar: $1 \mathrm{~cm}$.

ver, they are distinctly curved anteriorly with a concave anterior edge and a convex posterior one. In other words, their proximal portion attaches to the vertebral body and protrudes laterally, whereas the apex points anterolaterally. The transverse processes of Alcidedorbignya are much more developed than in Tenrec, which hardly bears transverse processes on the lumbars. Solenodon and Echinosorex have distinct transverse processes on their last three lumbar vertebrae, but in these genera the count of lumbar vertebrae is smaller than in Alcidedorbignya (five in Solenodon and six in Echinosorex) and the transverse processes are half the size of those in Alcidedorbignya. 
TABLE 15. - Measurements of the anterior lumbar vertebrae (L1 to L4) of Alcidedorbignya inopinata (in $\mathrm{mm}$ ).

\begin{tabular}{|c|c|c|c|c|}
\hline MHNC 8372 & L1 & L2 & L3 & L4 \\
\hline Ventral length of the centrum at sagittal plane & 6.06 & 5.70 & 7.09 & 7.07 \\
\hline Anterior maximum width of centrum & 5.87 & 5.78 & 5.90 & 6.22 \\
\hline Posterior maximum width of centrum & 6.26 & 6.20 & 6.29 & 6.39 \\
\hline Height of neural canal & - & 3.47 & 3.36 & - \\
\hline Width of neural canal & - & 4.81 & 4.66 & - \\
\hline Width between transverse processes & 8.91 & 11.27 & 12.52 & 13.72 \\
\hline Width at lateral edges of prezygapophyses & 7.39 & 8.17 & 7.92 & 8.20 \\
\hline Width at lateral edges of postzygapophyses & 7.13 & 7.35 & 7.38 & 7.67 \\
\hline Proximodistal length (= dorsoventral height) of neural process & 1.85 & - & 2.90 & 3.42 \\
\hline Anteroposterior proximal (= basal) length of neural process & 5.67 & - & 6.59 & 7.50 \\
\hline Length of neural arch from anteromedian edge to posteromedial edge of postzygapophysis & 5.77 & 6.34 & 5.59 & 7.50 \\
\hline
\end{tabular}

TABLE 16. - Measurements of the posterior lumbar vertebrae (L5 to L9) of Alcidedorbignya inopinata (in mm).

\begin{tabular}{|c|c|c|c|c|c|}
\hline MHNC 8372 & L5 & L6 & L7 & L8 & L9 \\
\hline Ventral length of the centrum at sagittal plane & 7.26 & 7.47 & 7.70 & 8.22 & $6.64 \mathrm{e}$ \\
\hline Anterior maximum width of centrum & 6.04 & 5.93 & 5.96 & 5.95 & 6.00 \\
\hline Posterior maximum width of centrum & 6.56 & 6.36 & 6.41 & 6.27 & 6.56 \\
\hline Height of neural canal & 4.12 & 3.51 & 3.61 & - & 2.60 \\
\hline Width of neural canal & 6.10 & 4.96 & 4.98 & - & 4.63 \\
\hline Width between transverse processes & 14.26 & 16.79 & - & - & - \\
\hline Width at lateral edges of prezygapophyses & 8.68 & - & 9.15 & 8.44 & $8.11 \mathrm{e}$ \\
\hline Width at lateral edges of postzygapophyses & 8.11 & 7.79 & 7.75 & - & 7.90 \\
\hline Proximodistal length (= dorsoventral height) of neural process & 3.98 & 4.45 & 5.04 & 5.62 & 5.77 \\
\hline Anteroposterior proximal (= basal) length of neural process & 6.44 & 6.14 & 5.35 & 5.55 & 5.37 \\
\hline $\begin{array}{l}\text { Length of neural arch from anteromedian edge } \\
\text { to posteromedial edge of postzygapophysis }\end{array}$ & 7.48 & 8.05 & 7.70 & 6.51 & 5.54 \\
\hline
\end{tabular}

TABLE 17. - Measurements of the sacral vertebrae of Alcidedorbignya inopinata (in $\mathrm{mm}$ ).

\begin{tabular}{|c|c|c|c|c|}
\hline MHNC 8372 & S1 & S2 & S3 & S4 \\
\hline Ventral length of the centrum at sagittal plane & 6.67 & 8.03 & $8.04 \mathrm{e}$ & 7.35 \\
\hline Anterior maximum width of centrum & 6.81 & 5.07 & 4.64 & 4.30 \\
\hline Posterior maximum width of centrum & 5.07 & 4.64 & $3.66 \mathrm{e}$ & 4.80 \\
\hline Height of neural canal & 2.18 & - & 1.45 & 1.47 \\
\hline Width of neural canal & 3.97 & - & 3.57 & 2.49 \\
\hline Width between transverse processes anteriorly & 16.34 & 11.93 & $8.84 e$ & 9.67 \\
\hline Width between transverse processes posteriorly & 13.89 & 8.67 & - & $13.50 \mathrm{e}$ \\
\hline Width at lateral edges of prezygapophyses & 8.25 & 5.14 & $4.36 \mathrm{e}$ & 5.71 \\
\hline Width at lateral edges of postzygapophyses & 5.14 & $4.36 \mathrm{e}$ & $4.50 \mathrm{e}$ & 5.24 \\
\hline Proximodistal length (= dorsoventral height) of neural process & \multicolumn{2}{|c|}{-} & 2.91 & 1.16 \\
\hline Anteroposterior proximal (= basal) length of neural process & \multicolumn{2}{|c|}{12.59} & 6.61 & 6.43 \\
\hline $\begin{array}{l}\text { Length of neural arch from anteromedian edge } \\
\text { to posteromedial edge of postzygapophysis }\end{array}$ & \multicolumn{2}{|c|}{13.16} & 6.99 & 7.09 \\
\hline
\end{tabular}

Sacrum. (Fig. 66; Table 17) The sacrum of MHNC 8372 is composed of four vertebrae, which were presumably fused in the adult. On MHNC 8372 the fourth sacral vertebra is not fused yet to predescessor probably because the specimen represents a young adult, in which all the long bones have unfused epiphyses. Although the centrum of S4 is not fused to that of of S3, it is clear that the anterior apex of its transverse processes had a cartilaginous contact with the posterior apex of the anterior transverse processes of S3, which indicates that S3 and S4 were totally fused in a fully grown adult. As a matter of fact, the transverse processes of S2 and S3 are not totally fused. They maintain a flat vertical contact, which corresponds to a hyaline cartilage joint, an articulation that ontogentically precedes synostosis (Grassé 1967). A similar condition is also present between $S 1$ and
S2, although less pronounced as in this case the synostosis is more advanced.

The total length of the sacrum (S1-S4) of Alcidedorbignya (MHNC 8372) is $33 \mathrm{~mm}$. Only the first vertebra articulates with the innominates (ossa coxae) and, in lateral view, the articular area (the auricular surface) is obliquely oriented (posteroventral-anterodorsal), the anterior part pointing proximodorsally. In dorsal view, the apices of the wings diverge anteriorly and the plane of the auricular surface faces dorsolaterally. This surface has the shape of an elongated right triangle: the hypotenuse is dorsal and sub-horizontal; the large side of the right angle faces anteroventrally and the small side, posteroventrally. The surface of the bone is rough, reflecting the attachment of the fibrocartilage of the sacroiliac articulation. At the anterodorsal border of the 


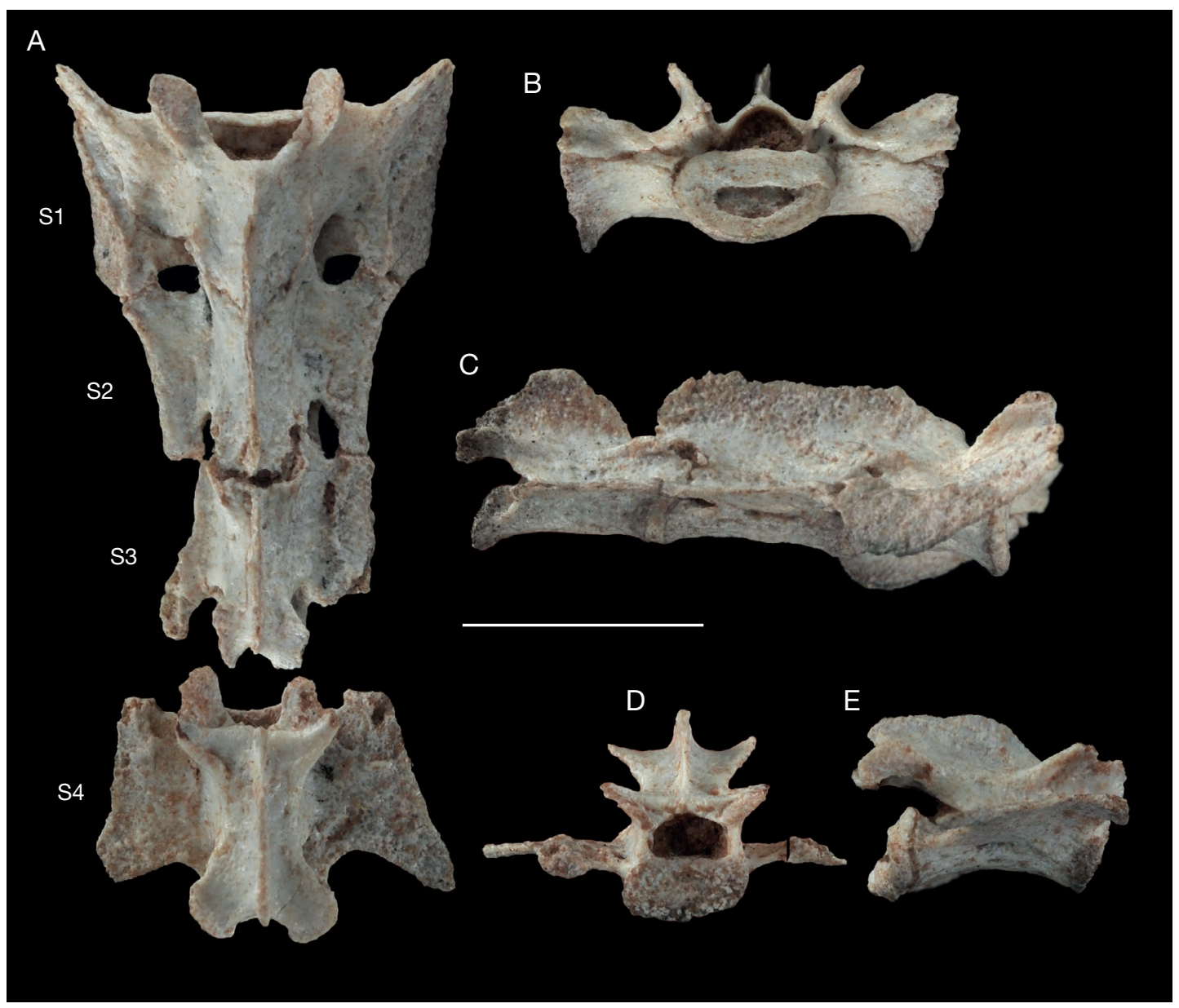

FIG. 66. - Sacrum of Alcidedorbignya inopinata (MHNC 8372): A, fused S1-S3 and free S4 in dorsal view; B, S1 in anterior view; C, fused S1-S3 in lateral view; D, S4 in anterior view; E, S4 in lateral view. Scale bar: $1 \mathrm{~cm}$.

auricular surface is an anteroposteriorly-elongated groove overhung by a robust ridge. These structures also probably received ligaments of the sacroiliac articulation. The sacrum tapers caudally, being $18 \mathrm{~mm}$ wide anteriorly versus $10 \mathrm{~mm}$ at the level of S3 (the posterior measurement is estimated, because the transverse processes of S3 is damaged). Posterior to $\mathrm{S} 3$, the sacrum widens again and reaches a width of $13.5 \mathrm{~mm}$ at the level of S4. The neural processes of the two anterior vertebrae (S1-S2) are anteroposteriorly wide and fused, forming a continuous wall. The anterior edge of the neural process of S1-2 almost reaches the anterior border of the neural arch. The dorsal edge of the neural process is incomplete but can be reconstructed on the basis of size of the neural processes of L9 and S3. The neural process of S1-2 was elevated anteriorly (as high as the process on L9) and decreased progressively in height posteriorly until the anterior height of the neural process of S3. It therefore formed a large and elevated blade for attachment of the tendons of the epaxial musculature. The neural processes of S3 and S4 are well separated. The anterior edge of the process of S3 is markedly convex and its dorsal edge is slightly thickened and straight in the preserved portion. The posterodorsal angle of the process is missing. The neural process of S3 is as high as that of S2. Its anterior edge reaches the anterior border of the neural arch. The neural process of S4 is dorsally incomplete but it is clear that, as on S3, it was as long as the neural arch. The transverse processes of the first three vertebrae are fused together except at the level of the large sacral foramina present at the junction of the processes. As mentioned above, the transverse processes of S4 are not fused to those of S3, but their surface of contact clearly indicates the presence of a hyaline cartilage joint. Therefore, in a full adult Alcidedorbignya the four sacral vertebrae were completely fused, as is usual in therian mammals.

When articulated to the pelvis, the sacrum of MHNC 8372 extends posteriorly beyond the posterior edge of the acetabulum. In lateral view, its posterior end stands above the dorsal edge of ischium and is sub-parallel to it. Posteriorly, it reaches the level of the ischiatic spine (see below, Fig. 118). On the whole, the sacrum of Alcidedorbignya resembles that of Solenodon paradoxus (MNHN-AC-14487), which has five sacral vertebrae, and that of Marmota monax (MNHN-AC-2691), which has four sacral vertebrae, and which, in both taxa, distinctly widens posteriorly as in Alcidedorbignya. 
TABLE 18. - Measurements of the anterior caudal vertebrae (Ca1 to Ca5) of Alcidedorbignya inopinata (in mm).

\begin{tabular}{|c|c|c|c|c|c|}
\hline MHNC 8372 & Ca1 & Ca2 & Ca3 & Ca4 & Ca5 \\
\hline Ventral length of the centrum in sagittal plane & 6.94 & 7.35 & 7.63 & 8.18 & 9.00 \\
\hline Anterior maximum width of centrum & 4.65 & 4.16 & 3.57 & 3.94 & 4.41 \\
\hline Posterior maximum width of centrum & 4.43 & 4.47 & 4.53 & 5.05 & 5.06 \\
\hline Height of neural canal & 1.09 & 1.10 & 0.26 & 0.21 & - \\
\hline Width of neural canal & 1.88 & 1.89 & 2.13 & 2.10 & 1.02 \\
\hline Width between transverse processes posteriorly & $14.20 \mathrm{e}$ & $13.10 \mathrm{e}$ & - & 11.13 & - \\
\hline Width at lateral edges of prezygapophyses & 6.45 & 6.36 & 5.71 & 5.39 & 3.95 \\
\hline Width at lateral edges of postzygapophyses & 4.93 & 4.56 & 4.10 & 0.97 & $1.43 \mathrm{e}$ \\
\hline Proximodistal length (= dorsoventral height) of neural process & 1.64 & 0.99 & 1.03 & 0.83 & 0.89 \\
\hline Anteroposterior proximal (= basal) length of neural process & 5.35 & 4.55 & 3.42 & 3.25 & 2.90 \\
\hline $\begin{array}{l}\text { Length of neural arch from anteromedian edge to posteromedial edge of } \\
\text { postzygapophysis }\end{array}$ & 6.94 & 6.29 & 5.52 & 5.82 & 3.63 \\
\hline
\end{tabular}

TABLE 19. - Measurements of the posterior caudal vertebrae (Ca6 to Ca11) of Alcidedorbignya inopinata (in mm).

\begin{tabular}{|c|c|c|c|c|c|c|}
\hline MHNC 8372 & Ca6 & Ca7 & Ca8 & Ca9 & Ca10 & Ca11 \\
\hline Ventral length of the centrum in sagittal plane & 9.60 & 10.39 & 10.45 & 11.28 & 11.61 & 11.47 \\
\hline Anterior maximum width of centrum & 4.84 & 4.24 & 4.02 & 4.08 & 3.43 & 3.43 \\
\hline Posterior maximum width of centrum & 5.57 & 4.55 & 4.47 & 4.22 & 3.97 & 3.42 \\
\hline Width between anterior transverse processes & $8.32 e$ & $7.22 \mathrm{e}$ & 6.40 & $5.91 e$ & 5.17 & 3.99 \\
\hline Width between posterior transverse processes & 8.75 & 6.53 & 6.16 & 5.27 & 4.98 & 3.91 \\
\hline Width at lateral edges of prezygapophyses & $4.53 e$ & $3.73 e$ & 3.54 & 3.68 & 3.01 & 2.71 \\
\hline
\end{tabular}

TABLE 20. - Measurements of the posterior caudal vertebrae (Ca13 to Ca16) of Alcidedorbignya inopinata (in mm).

\begin{tabular}{|c|c|c|c|c|}
\hline MHNC 8372 & Ca13 & Ca14 & Ca15 & Ca16 \\
\hline Ventral length of the centrum in sagittal plane & 9.57 & 9.06 & 7.97 & 7.36 \\
\hline Anterior maximum width of centrum & 3.00 & 2.30 & 1.74 & 1.67 \\
\hline Posterior maximum width of centrum & - & 1.85 & 1.67 & 1.30 \\
\hline
\end{tabular}

Caudal vertebrae. (Fig. 67; Tables 18-20) Fifteen caudal vertebrae of Alcidedorbignya are known in MHNC 8372 and some of them were found in articulation. The last one, although very thin, is $7 \mathrm{~mm}$ long, and it is likely that some posterior caudals are lacking (possibly no more than two or three). Because not all the caudal vertebrae were found in articulation their succession was reconstructed partially on the basis of their size. Because the vertebra just posterior to Ca11 seems too narrow to have articulated with it we suggest that Ca12 is missing and that the original series was characterized by smooth transition.

The first caudals of MHNC 8372 present a morphology similar to that of the last lumbars, although the vertebrae are more slender: the first caudal is slightly shorter than L9 between the apex of the pre- and postzygapophyses (L9: $12.5 \mathrm{~mm}$; Ca1: $10.5 \mathrm{~mm}$ ), and the median width of the dorsal arch is $5.5 \mathrm{~mm}$ on L9 vs $3 \mathrm{~mm}$ on Ca1. The foramen vertebrale is present, although reduced, until Ca5. It is somewhat triangular on $\mathrm{Ca} 1$ and $\mathrm{Ca} 2$ and abruptly flattens on $\mathrm{Ca} 3$ and $\mathrm{Ca} 4$. A well-developed neural process exists on $\mathrm{Ca} 1$ and $\mathrm{Ca} 2$, and contrary to the condition on S4 it does not reach the anterior edge of the neural arch anteriorly. It extends anteroposteriorly along the posterior three-quarters of the dorsal arch, with an anterior edge sloping backwards. On Cal the dorsal edge of the neural process is straight and sub-horizontal. On Ca2 the process is much lower and its dorsal edge is regularly convex. On Ca3-4, this process reduces to a small dorsal crest between the postzygapophyses. The transverse processes (especially that of $\mathrm{Ca} 1$ ) are different from those of lumbars. The transverse process of $\mathrm{Ca} 1$ still retains the large trapezoid shape observed on S4. On Ca2-4 they form wide triangular wings extended anteroposteriorly (their base is almost as long as the vertebral body), with the apex protruding postero-laterally. From $\mathrm{Ca} 2$ the anteroposterior length of these processes decreases, but they still form triangular wings protruding postero-laterally. The ventral side of the vertebral bodies does not exhibit a median crest but Ca2-4 preserve well-developed ventral chevrons (haemapophyses) bearing a ventral crest that protrudes posteriorly. On Ca1 the chevron is not preserved but the posterior end of the centrum (just anterior to the epiphysis) presents two distinct protuberances indicating the articulation of a hemapophysis. The appearance of such protuberances in the vertebral column of mammals marks the beginning of the caudal series. As a matter of fact no protuberances are observed on the vertebra anterior to Ca1, which is the last sacral vertebra. On Ca4 the postzygapophyses are not functional any more.

The change of the morphology between the anterior and posterior caudals occurs at the level of $\mathrm{Ca} 5-\mathrm{Ca}$. $\mathrm{Ca} 5$ is not well preserved, but the right transverse process is complete. It is as long at its base as the centrum and is perforated by a large median foramen, separating an anterior and a posterior part. 


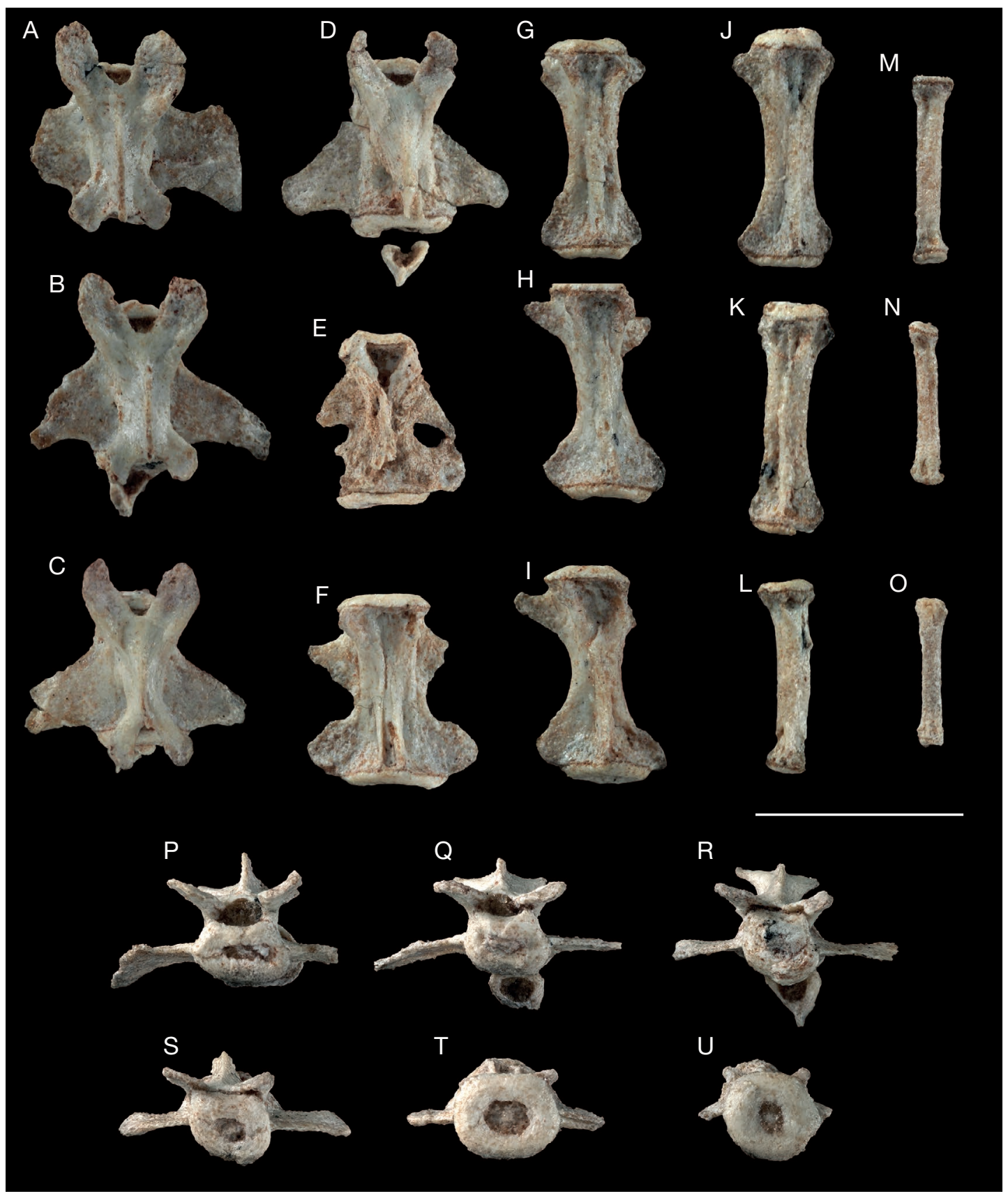

FIG. 67. - Caudal vertebrae of Alcidedorbignya inopinata (MHNC 8372) in dorsal view (A-O) and anterior view (P-U): A, Ca1; B, Ca2 (with haemal arch); C, Ca3 (with haemal arch); D, Ca4 (with haemal arch); E, Ca5; F, Ca6; G, Ca7; H, Ca8; I, Ca9; J, Ca10; K, Ca11; L, Ca13; M, Ca14; N, Ca15; O, Ca16, P, Ca1; Q, Ca2; R, Ca3; S, Ca4; T, Ca 6; U, Ca 10. Scale bar: $1 \mathrm{~cm}$.

On Ca5, prezygapophyses still exist but postzygapophyses are extremely reduced; neither are functional. On $\mathrm{Ca} 6$ the split of the transverse processes is completed, and the vertebra shows anterior and posterior lateral processes.

From $\mathrm{Ca} 7$ the typical rod-like morphology of distal caudals is achieved, and the length and robustness of the vertebrae regularly decrease posteriorly: the length is $10 \mathrm{~mm}$ on $\mathrm{Ca} 7$ and $7 \mathrm{~mm}$ on Ca16 (the last preserved caudal), with a mid-shaft diameter of $2 \mathrm{~mm}$ on Ca7 vs $0.8 \mathrm{~mm}$ on Ca16. Although in Mayulestes the most posterior caudal known (Ca?9) is much longer and more massive than the most anterior caudal vertebrae known, this is not the case in Alcidedorbignya.

Ribs and sternum. (Figs 68, 69; Tables 21, 22) Thirteen complete pairs of ribs are known in MHNC 8372 (Fig. 68). Only one point of articulation with the vertebrae remains after the ninth pair. As usual, the first one is the most robust and wide dorsoventrally. The relief of the posterior side is more emphasized on the left element than on the right one. The posterior pairs of ribs present the usual shape in mam- 


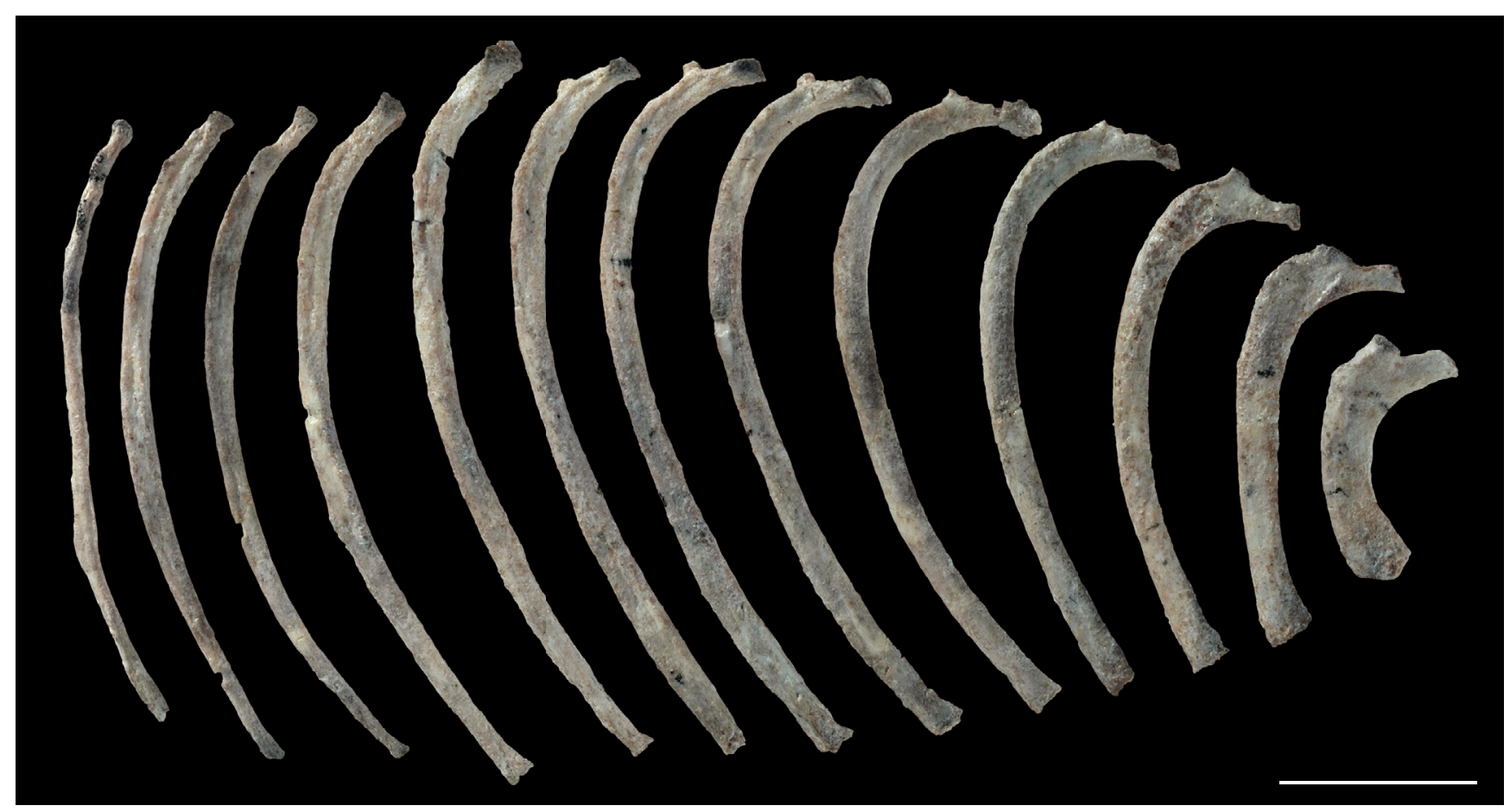

FIG. 68. - Right ribs of Alcidedorbignya inopinata (MHNC 8372) in anterior view. Scale bar: $2 \mathrm{~cm}$.

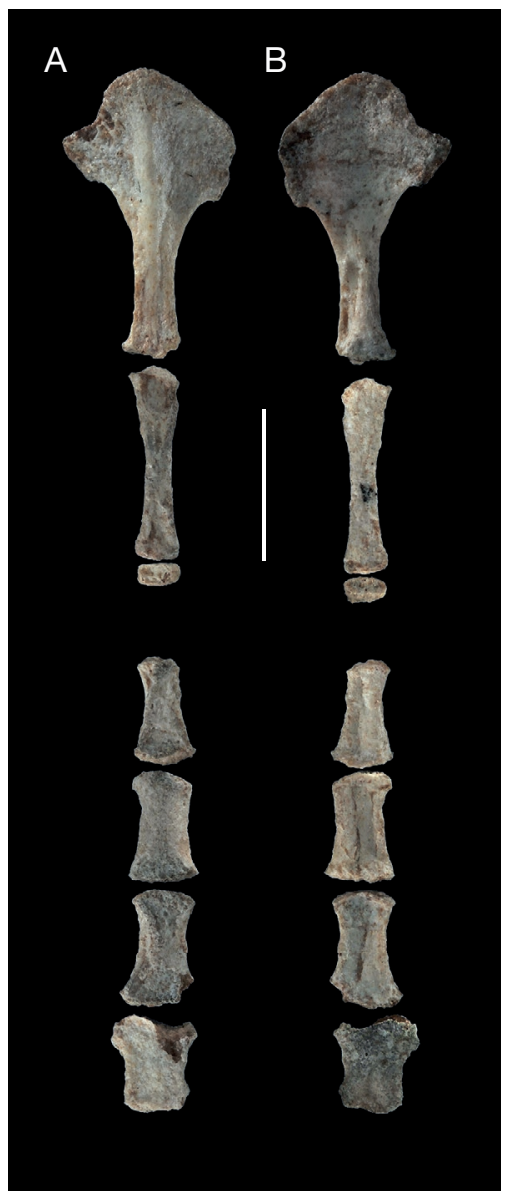

FIG. 69. - Sternum of Alcidedorbignya inopinata (MHNC 8372): A, ventral view; B, dorsal view. Scale bar: $1 \mathrm{~cm}$. mals, and do not exhibit particular crests. The longest are the pair 7 to 9, ribs 6-7 presenting the greatest curvature.

Additionally, the manubrium and five posterior sternebrae are known (Fig. 69). The manubrium presents a well-developed rhombic anterior portion and a rod-like posterior half. It is dorsoventrally compressed, concave dorsally, and bears a well-developed carina ventrally. In anterior view, it forms a widely opened roof. The anterior half of the manubrium bears two large laterally prominent wings-like processes, which articulate with the first pair of ribs, whereas the anterior apex relates to the clavicles (here without discernible articular facets). The manubrium of Pantolambda is quite similar to that of Alcidedorbignya, although in the former the rod-like posterior portion is longer than the anterior rhombic part. The second sternebra is anteroposteriorly long, about two-thirds the length of the manubrium, and narrow. The third sternebra, St 3 is fragmentary, and only its anterior extremity is preserved. It is as narrow as St2. St4 is half the length of St 2 and slightly wider. St 5 and St6 are as long as St 4 but conspicuously wider. St7 is the shortest and the widest anteriorly probably because it received several pairs of cartilaginous ribs, as is observed in Solenodon ( 4 to 5) and Erinaceus (5 to 6). From St4 to St6 the posterior extremity is clearly wider than the anterior while this condition is reversed on St7.

\section{Pectoral girdle and Forelimb}

Scapula and clavicle. (Figs 70-74; Tables 23, 24) Only four scapulae of Alcidedorbignya inopinata are known: the two of the skeleton (MHNC 8372) (Figs 70, 71); one isolated specimen, MHNC 8431, referred to this species on the basis of its size and morphology which are very similar to those of 


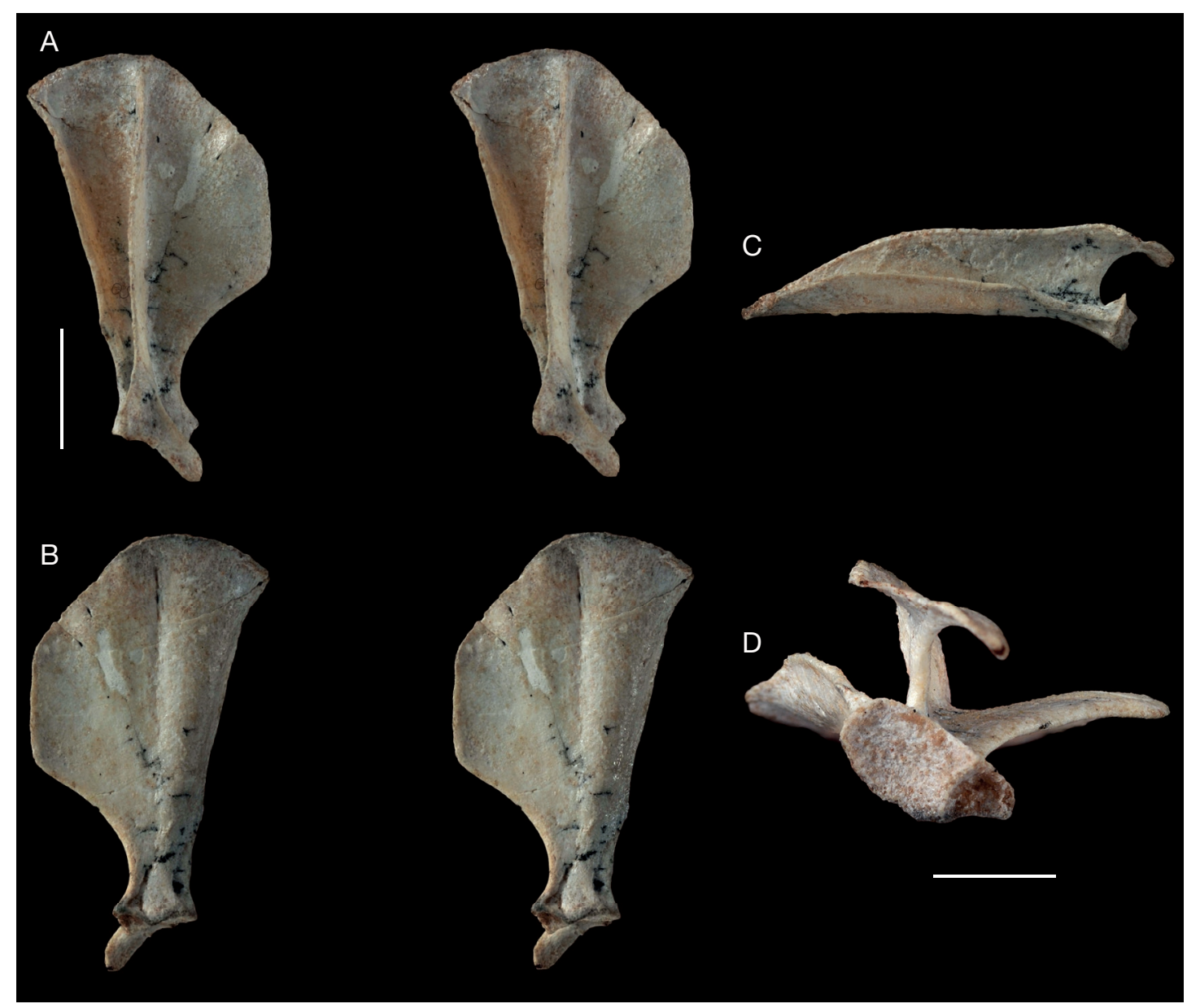

FIG. 70. - Right scapula of Alcidedorbignya inopinata (MHNC 8372): A, stereophotographs of lateral view; B, stereophotographs of medial view; C, posterior view; D, distal view. Scale bars: A-C, $1 \mathrm{~cm}$; D, $5 \mathrm{~mm}$.

TABLE 21. - Proximodistal length (PL) of the right ribs in right line from proximal articulation to distal extremity (in $\mathrm{mm}$ ).

\begin{tabular}{cccccccc}
\hline & $\mathbf{R 1}$ & $\mathbf{R 2}$ & $\mathbf{R 3}$ & $\mathbf{R 4}$ & $\mathbf{R 5}$ & $\mathbf{R 6}$ & $\mathbf{R 7}$ \\
$\mathrm{PL}$ & 11.62 & 19.15 & 22.77 & 27.35 & 29.41 & 32.28 & 34.16 \\
\hline & $\mathbf{R} 8$ & $\mathbf{R 9}$ & $\mathbf{R 1 0}$ & $\mathbf{R 1 1}$ & $\mathbf{R 1 2}$ & $\mathbf{R 1 3}$ & \\
$\mathrm{PL}$ & 34.76 & 35.90 & 34.89 & 33.34 & 33.18 & 30.97 & \\
\hline
\end{tabular}

TABLE 22. - Measurements of the sternebrae of Alcidedorbignya inopinata (in mm).

\begin{tabular}{lrrccccc}
\hline MHNC 8372 & St1 & St2 & St3 & St4 & St5 & St6 & St7 \\
\hline Length & 15.21 & 10.82 & - & 6.00 & 6.08 & 6.44 & 5.26 \\
Anterior width & 9.06 & 2.52 & 2.33 & 2.36 & 3.35 & 3.52 & 4.76 \\
Posterior width & 3.09 & 2.86 & - & 3.43 & 3.86 & - & 3.71 \\
\hline
\end{tabular}

the skeleton (Fig. 72); and a third partial scapula (MHNC 8432), which has been referred to $A$. inopinata in spite of it size, slightly larger than that of the other three. None of these specimens preserves the distal epiphysis of the bone, because the former three are young adults and have unfused epiphyses and the latter is broken in this region. However, two scapula fragments (anterior part of the articular angle, which bears the supraglenoid tuberosity and coracoid process) are referred to Alcidedorbignya (MHNC 8439 and 8440) (Fig. 73). Comparison of the scapula of Alcidedorbignya with that of Pantolambda bathmodon is difficult because the scapulae of the only specimen in which they are preserved (AMNH 16663) are incomplete and not fully prepared. Furthermore, the description and illustration in Matthew (1937: 177, pl. 43) do not provide a detailed anatomy of the bone. However, an acceptable reconstruction is provided by Simons (1960: fig. 2). Other pantodonts with a well-preserved scapula are Barylambda faberi (FMNH 14902; Patterson 1934), Haplolambda quinni (FMNH 15542; Patterson 1939), Leptolambda schmidti (PU 14990; Patterson \& Simons 1958), Bemalambda pachyoesteus (IVPP-V 4115; Zhou et al. 1977), and Bemalambda nanhsiungensis (IVPP-V 4157; Zhou et al. 1977). In other respects, interesting similarities exist with the scapula of the North American marmot (Marmota monax) and that of the Haitian solenodon (Solenodon paradoxus), as well as with the basal borhyaenoid, Mayulestes ferox. The scapula of Alcidedorbignya will be described below with the main axis of its spine in a vertical position.

The general shape of the scapula of Alcidedorbignya is roughly triangular, like those of Mayulestes and Marmota. It is, how- 


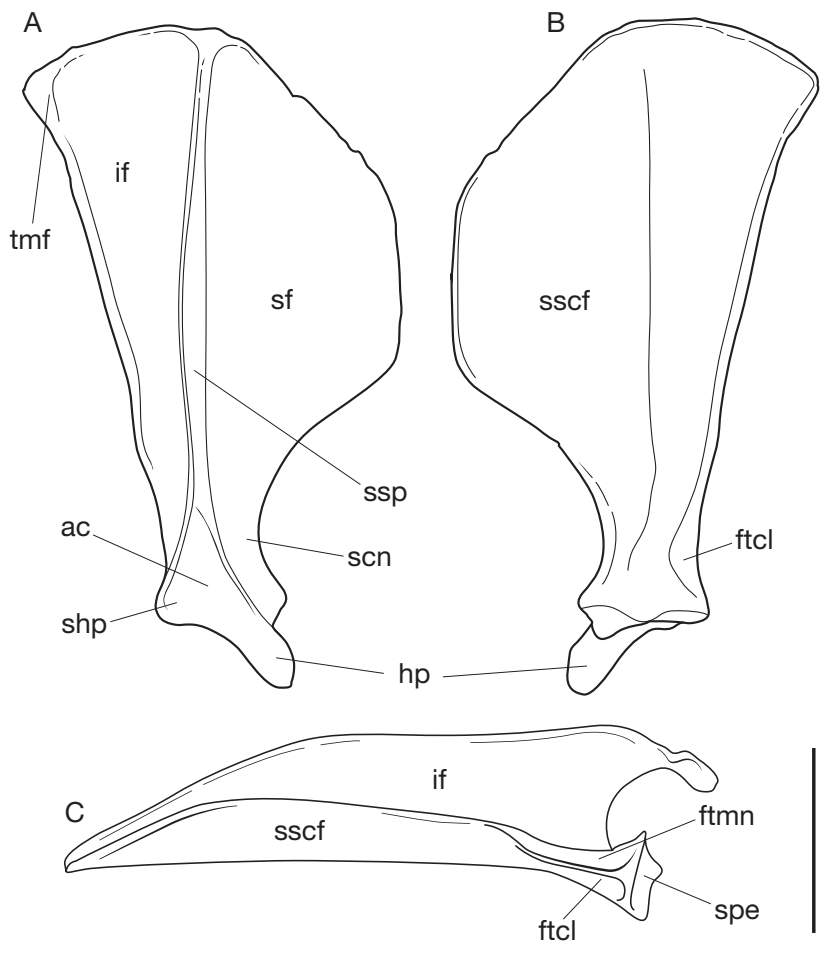

FIG. 71. - Right scapula of Alcidedorbignya inopinata (MHNC 8372): A, lateral view; B, medial view; C, posterior view. Abbreviations: ac, acromion; ftcl, fossa for the origin of the caput longum of the triceps; ftmn, fossa for the origin of the teres minor; $\mathbf{h p}$, hamatus process; if, infraspinatus fossa; scn, scapular notch; sf, supraspinatus fossa; shp, suprahamatus process; spe, suture for the dista epiphysis (missing on MHNC 8372); sscf, subscapular fossa; ssp, scapular spine; tmf, teres major fossa. Scale bar: $1 \mathrm{~cm}$.

ever, less triangular than that of the metatherian Mayulestes because of the morphology of its anterior and vertebral borders. First, its anterior edge presents two angulations rather than one as in Mayulestes: it is straight from the top of the spine and slopes anteroventrally at a point approximately one-third of the dorsoventral height of the bone. There, it turns ventrally at an angle of $c .140^{\circ}$ (on MHNC 8372). Further ventrally, it turns again posteroventrally at an angle of $c .125^{\circ}$ (on MHNC 8372). This angulation is responsible for a well-developed scapular notch, which forms the ventral third of the anterior edge of the bone. A similar condition is present on MHNC 8431. Because of these angulations the supraspinous fossa of the scapula of Alcidedorbignya is roughly trapezoid-shaped rather than triangular-shaped as in Mayulestes (Muizon 1998: fig. 16). Second, the vertebral border of the infraspinatus fossa forms a slightly acute angle with the scapular spine $\left(c .85^{\circ}\right)$, whereas this angle is obtuse in Mayulestes $\left(c .105^{\circ}\right)$. This means that when the spine is oriented vertically, the highest point of the scapula is located at the dorsal extremity of the spine (MHNC 8372) or in the middle of the dorsal edge of the infraspinatus fossa (MHNC 8431 and 8432), and not at the caudal angle of the scapula as in Mayulestes. It is noteworthy, however, that the proximocau$\mathrm{dal}$ angle of the scapula is only slightly lower than the dorsal extremity of the spine. The general shape of the scapula of Alcidedorbignya more closely resembles that of Pantolambda (based on the reconstruction provided by Simons 1960), but in this genus the anterior edge is more regularly convex and the posterior angle is lower relatively to the dorsal end of the spine than in Alcidedorbignya.

The supraspinatus fossa of Alcidedorbignya is wide and relatively flat. It is much broader than the deep and narrow infraspinatus fossa. This relative size of the scapular fossae of Alcidedorbignya clearly resembles the conditions of Mayulestes, Solenodon, and Marmota. It differs from the condition in other pantodonts, in which the infraspinatus fossa is much wider, either subequal to the supraspinatus fossa (e.g., Pantolambda, Leptolambda, Bemalambda), or slightly larger (e.g., Haplolambda). The infraspinatus fossa is very deeply excavated, a condition enhanced by the orientation of its caudal border, which is strongly deflected laterally. On the medial side of the scapula, the subscapularis fossa is flat anteriorly, below the supraspinatus fossa, concave below the spine, and strongly convex below the infraspinatus fossa. The scapular neck is narrow and well-marked. The scapular spine is extremely high, slightly deflected posteriorly in its mid-part. A similar size of the scapular spine is present in Pantolambda and most other pantodonts but apparently the posterior deflection is only observed in Alcidedorbignya.

The acromion is perfectly preserved on the right scapula of MHNC 8372 and almost complete in MHNC 8431. It is triangular in shape and bears a well-developed, anteriorly prominent hamatus process. It is quite similar to that observed in Mayulestes, and, as in this taxon, the apex of the hamatus process is bent medially in anterior view. In medial view, the acromion extends ventrally to the junction of the epiphysis and probably also extended ventral to the latter, as in Mayulestes. In proximal view (Fig. 70D), the hamatus process overhangs the area where the coracoid process (lost) attached. When articulated to the humerus, it overhangs a broad part of the greater tubercle. The anterodistal extension of the acromion seen in Alcidedorbignya is also present in Pantolambda (based on Simon's reconstruction). In other pantodonts the anterior projection is apparently less pronounced than in Alcidedorbignya. A small suprahamatus process is present on the posterior edge of the distal extremity of the spine of Alcidedorbignya. It is rounded and no more developed than in Mayulestes. A distinct suprahamatus process is not present in the other pantodonts, which instead present a convex and large posterior edge of the acromion. In this respect the acromion of Alcidedorbignya clearly differs from those of Solenodon and Marmota, each of which has a well-developed suprahamatus process. In Marmota, however, the hamatus process also presents a distinct anterior and distal projection, but a marked suprahamatus process is present and extends posteriorly. In Solenodon the projection is only distal.

As mentioned above, the scapulae of MHNC 8372 and 8431 have lost their distal epiphysis (which bears the glenoid cavity and coracoid process), which was not fused to the bone. In therians, the epiphysis of the glenoid cavity is generally a simple ring surrounding the glenoid cavity (personnal observations). Therefore, in spite of this lack in the available scapulae of Alcidedorbignya, the morphology of the cavity 


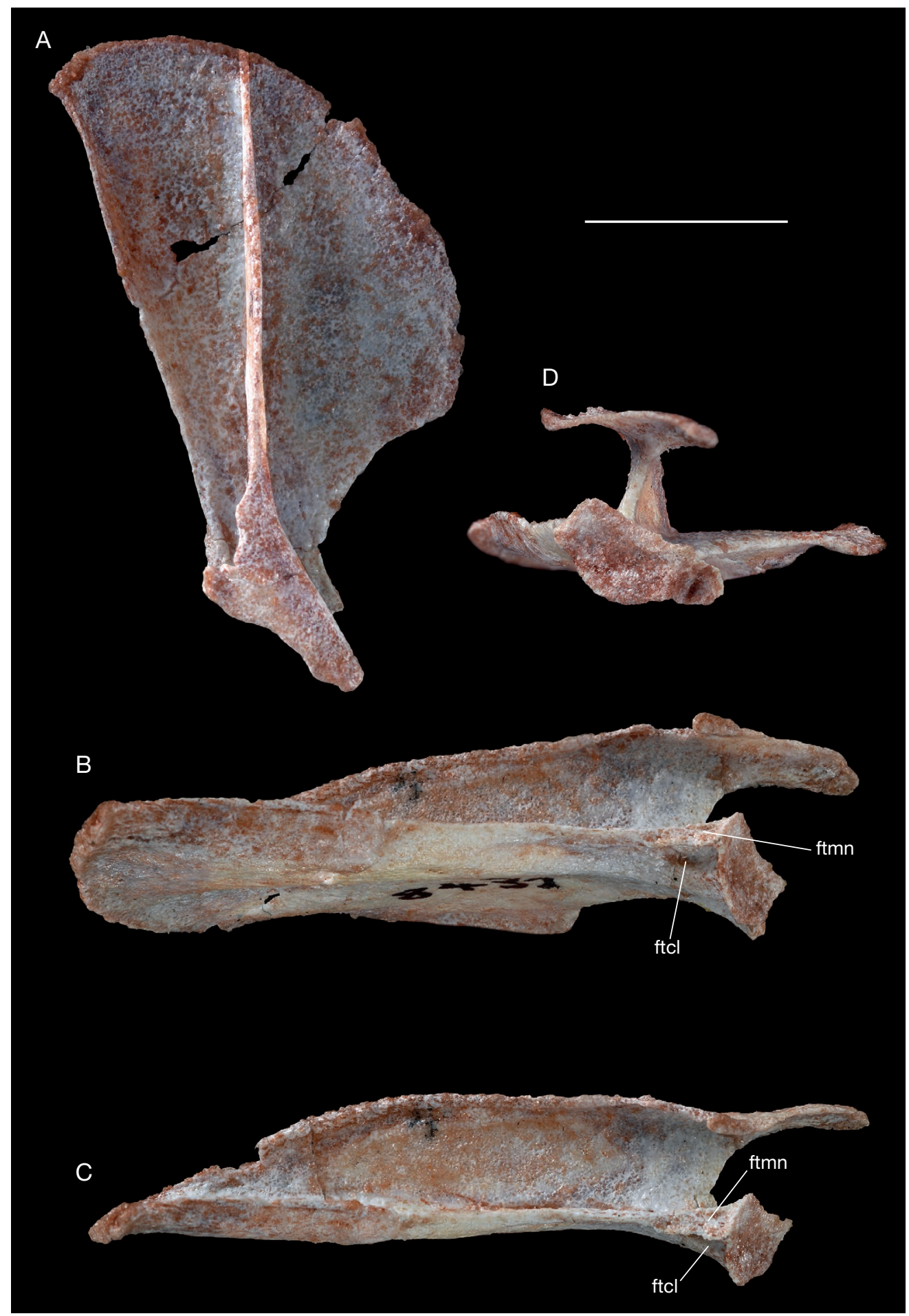

FIG. 72. - Right scapula of Alcidedorbignya inopinata: A, lateral view of MHNC 8431; B, posteromedial view; C, posterior view; D, distal view. Abbreviations: $\mathrm{ftcl}$, fossa for origin of the triceps caput longum; ftmn, origin of the teres minor. Scale bar: $1 \mathrm{~cm}$.

can be appreciated, although its anterior extremity (located on the coracoid) is missing. It is shallow, with a lateral border almost rectilinear in MHNC 8372 or even slightly concave in MHNC 8431 and a medial border distinctly convex. The preserved portion of the glenoid cavity is approximately twice as long as wide. In proximal view, with the plane of the spine in a vertical position, the main axis of the glenoid cavity is distinctly oblique, its anterior end (as well as the articular junction for the coracoid) being clearly ventral to the plane of the supraspinatus fossa. In other words, the distal end of the scapula, in distal view, is twisted clockwise about $45^{\circ}$ relative to the plane of the supraspinatus fossa. This condition is present in Pantolambda but it could not be observed in other pantodonts. In this respect, Alcidedorbignya contrasts with 

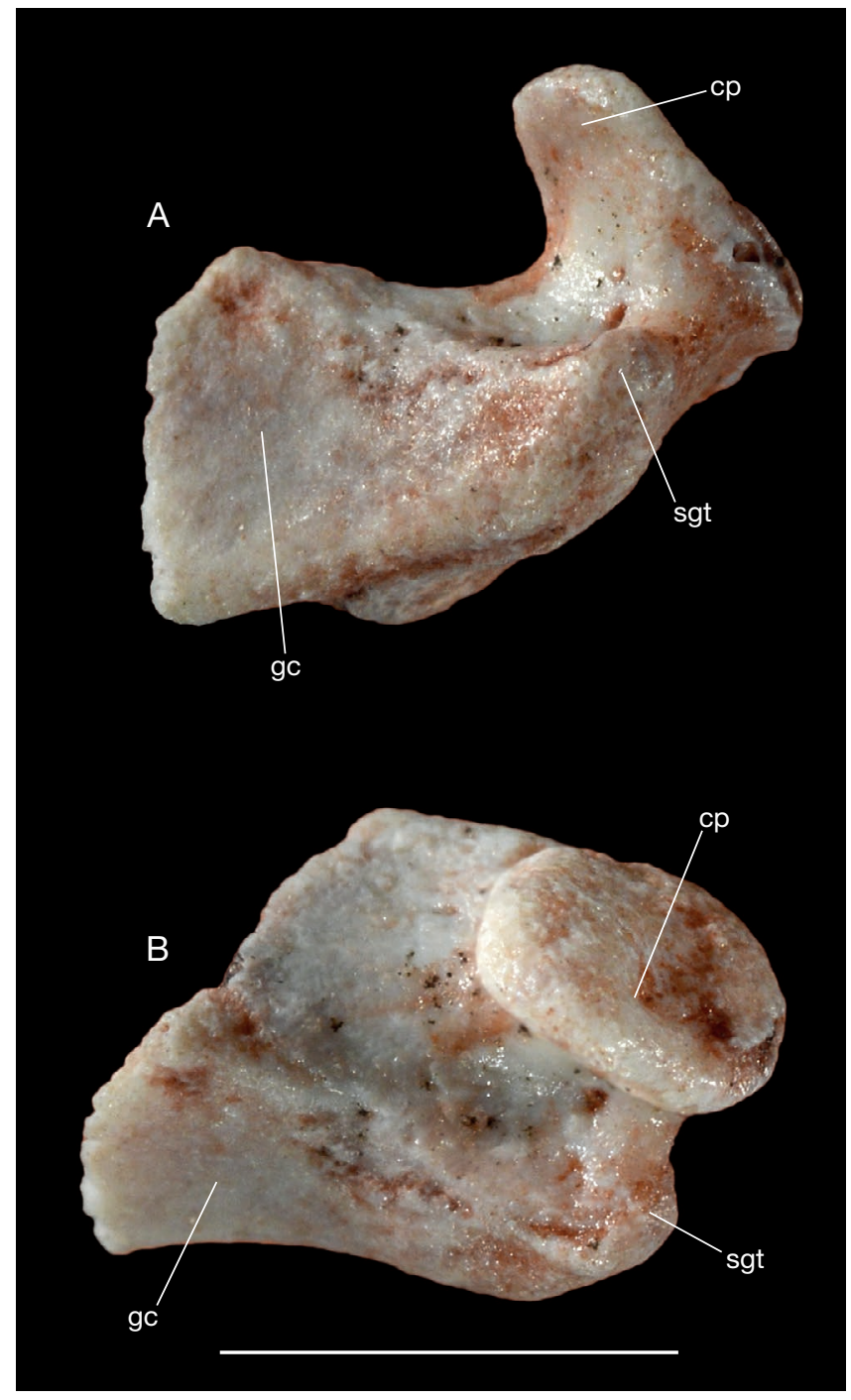

FIG. 73. - Anterodistal angle of left scapula of Alcidedorbignya inopinata (MHNC 8439): A, distal view; B, distomedial view. Abbreviations: $\mathbf{c p}$, coracoid process; gc, glenoid cavity; sgt, supraglenoid tubercle. Scale bar: $5 \mathrm{~mm}$.

Mayulestes, in which the long axis of the glenoid fossa is roughly parallel to the plane of the supraspinatus fossa. The condition in Alcidedorbignya is also absent in Solenodon and Marmota. The supraglenoid tubercle and coracoid process are lacking, as this part, still unfused to the blade, was lost postmortem. However, on the two fragments of the anterior part of the articular angle mentioned above, (MHNC 8439 and 8440) the two processes are preserved. The supraglenoid tubercle is relatively small, anteroposteriorly elongated, transversely narrow, and does not extend much proximally. The coracoid process is located on the medial side of the anterior region of the glenoid cavity. It is a small tongue-like blade that is posterodistally oriented. It does not extend distally further than the distal level of the supraglenoid tuberosity (Fig. 73). On its posterior edge, it is separated from the supraglenoid tuberosity by a deep groove and fossa, while on its anterior edge, the groove is distinctly shallower. At the apex of the supraglenoid process a deep notch separates it from the coracoid process.
At the posterodorsal angle of the scapula is a small, elongate and laterally facing surface, which extends the posterior crest of the infraspinatus fossa proximally. This surface is wider proximally than distally. It probably represents the attachment area for the origin of the teres major muscle. Such a well-developed muscle scar is also present in Solenodon and Marmota but faces posteriorly in the latter. It is also apparently well developed in Caenolambda and Haplolambda. It could not be observed in Pantolambda and is apparently not well developed in Barylambda.

On the posterior region of the neck, just posterior to the posterolateral extremity of the glenoid cavity, are two distinct fossae for muscle attachment (Fig. 72B, C). The deepest fossa faces almost totally medially and extends on the medial side of the posterior edge of the scapula on approximately $33 \%$ of its length. It is much deeper distally and becomes a slightly concave to almost flat area proximally. This fossa is likely to be for the origin of the caput longum of the triceps. The other fossa is located on the posterior edge of the scapula and faces posteriorly (not medially as the triceps fossa). It extends from the posterior edge of the epiphysis suture (the epiphysis is missing in the two specimens known). At this point is has its greatest width. The fossa becomes narrower proximally and extends on the posterior ridge of the infraspinatus fossa for approximately $25 \%$ of its length. We interpret this fossa as the area of origin of the teres minor muscle. It is possible that the origin of the triceps brachii caput longum also extended onto the posterior face of the scapula and attached, in part, jointly with that of the teres minor, as is observed in the dog (Evans \& De Lahunta 2012). These structures are well developed and suggest relatively powerful muscles, especially the triceps brachii caput longum.

In Alcidedorbignya, the hamatus process articulated with a well-developed clavicle (Fig. 74). The clavicle is long, slim, and ventrally curved. The left one is $21 \mathrm{~mm}$ long, which is approximately two-thirds as long as the scapula $(34 \mathrm{~mm}$ long). The right is only $17 \mathrm{~mm}$ because it suffered from a premortem fracture, which did not repair in anatomical position. As a consequence the bone is slightly shortened and presents a pathological angulation. The clavicle of Alcidedorbignya does not present the common sigmoid morphology generally observed in therians. It presents a single, dorsal curvature and is relatively flat in dorsal view. A similar condition is present in Pantolambda. This morphology is not common among mammals, but we have also observed it in Erinaceus. The lateral extremity of the bone, which articulates with the anterodorsal edge of the processus hamatus, is flattened and distinctly triangular-shaped. Its dorsal angle is conspicuously more extended than its ventral angle, which gives it a characteristic morphology also present in Pantolambda. The condition in other pantodonts could not be observed during this study. The medial extremity of the bone, which had a ligamentous attachment with the manubrium of the sternum, is roughly circular. In being more globular than the lateral extremity, it also resembles that of Pantolambda. 


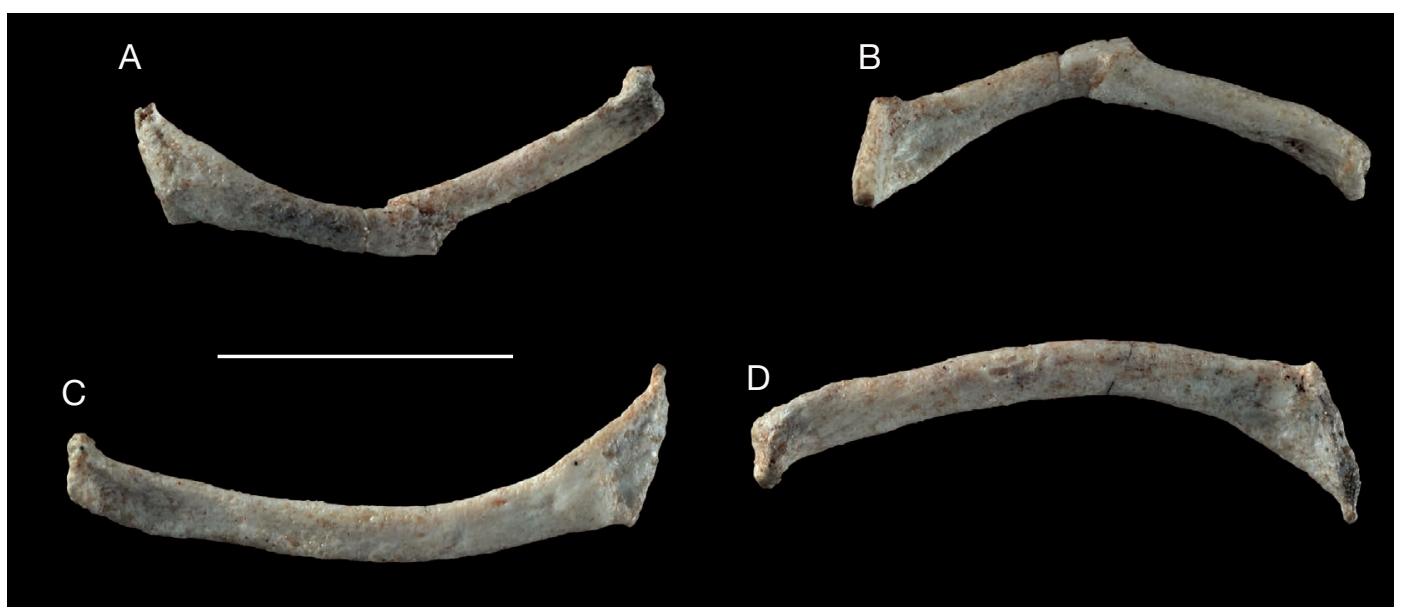

FIG. 74. - Clavicles of Alcidedorbignya inopinata (MHNC 8372): A, right clavicle in anterior view; B, right clavicle in posterior view; C, left clavicle in anterior view; D, left clavicle in posterior view. Scale bar: $1 \mathrm{~cm}$.

TABLE 23. - Measurements of the scapula of Alcidedorbignya inopinata (in mm).

Maximum length of infraspinatus fossa, parallel to spine

Maximum width of infraspinatus fossa, perpendicular to spine

Maximum width of supraspinatus fossa perpendicular to spine

Maximum length of spine

Maximum height of spine

Length of acromion along distal edge

Length of distal extremity of scapula (epiphyses of glenoid cavity and coracoid process are missing)

Width of distal extremity of scapula (epiphyses of glenoid cavity and coracoid process are missing)
MHNC 8372 (R)

31.6

9.35

10.42

28.92

5.99

9.78

8.23

4.17
MHNC 8431

28.11

9.45

10.63

24.84

5.12

9.60

7.70

3.85
TABLE 24. - Measurements of the left clavicle of Alcidedorbignya inopinata (in mm).

MHNC 8372

\begin{tabular}{lr}
\hline Length & 20.68 \\
Width of medial extremity & 4.40 \\
Width of lateral extremity & 2.67
\end{tabular}

Humerus. (Figs 75-78; Table 25) Both humeri of the skeleton MHNC 8372 are known (Fig. 75) but show a significant difference in length, the right one being shorter than the left one ( 42 vs $45 \mathrm{~mm}$ ). A similar discrepancy has been observed on the holotype of Mayulestes and is likely to be the result of postmortem distortion. Six isolated humeri have been referred to Alcidedorbignya inopinata. Three are almost complete and undistorted right humeri missing the proximal epiphysis MHNC 8433, 8434, 8435), one is an incomplete humerus missing the proximal epiphysis and part of the distal extremity of the diaphysis (MHNC 8437), another is a juvenile humerus missing both epiphyses (MHNC 8438), and the sixth is an undistorted left proximal epiphysis (MHNC 8436) (Fig. 77). The proximal epiphysis (the head) of both humeri of MHNC 8372 is anteroposteriorly compressed, a condition related to postmortem deformation. One of the major consequences of this distortion is the change in the original orientation of the head from an almost proximally (natural) to a posteroproximally facing position. Comparison with two other isolated specimens, missing the proximal epiphysis but preserving the proximal extremity of the diaphysis and undistorted (or little distorted), indicates that this artificial modification of the orientation in MHNC 8372 could approach $22^{\circ}$ to $35^{\circ}$. This rotation mainly affected the bone along a transverse axis (i.e. the head rotates posteriorly, i.e. counterclockwise in lateral view of the right humerus) (Fig. 78). This rotation is distinctly more pronounced on the left humerus of MHNC 8372 (Fig. 78A), than on the right bone (reversed on Fig. 78B). Apparently some rotation along an anteroposterior axis also occurred (i.e. the head rotates medially, i.e. counterclockwise in posterior view of the right humerus. Both rotations artificially placed the greater tubercle in a more elevated position when the proximodistal axis of the bone is vertical. As shown on Figure 78C, D, such distortion apparently did not occur on the other isolated humeri referred to Alcidedorbignya (MHNC 8435 and 8437) (Fig. 78C, D).

On the proximal epiphysis (MHNC 8436), the greater tubercle is massive (Fig. 77). In proximal view it appears longer than wide, and is obliquely oriented. It forms a thick rounded ridge that is concave medially. Its anterior end forms the lateral edge of the proximal extremity of the bicipital groove and its posterior end reaches the level of the centre of the articular area of the head. It is roughly as high as (possibly slightly lower) the head. On Fig. 78E, MHNC 8436 has been adapted on the diaphysis MHNC 8437 (although they were not discovered associated) in order to provide a 


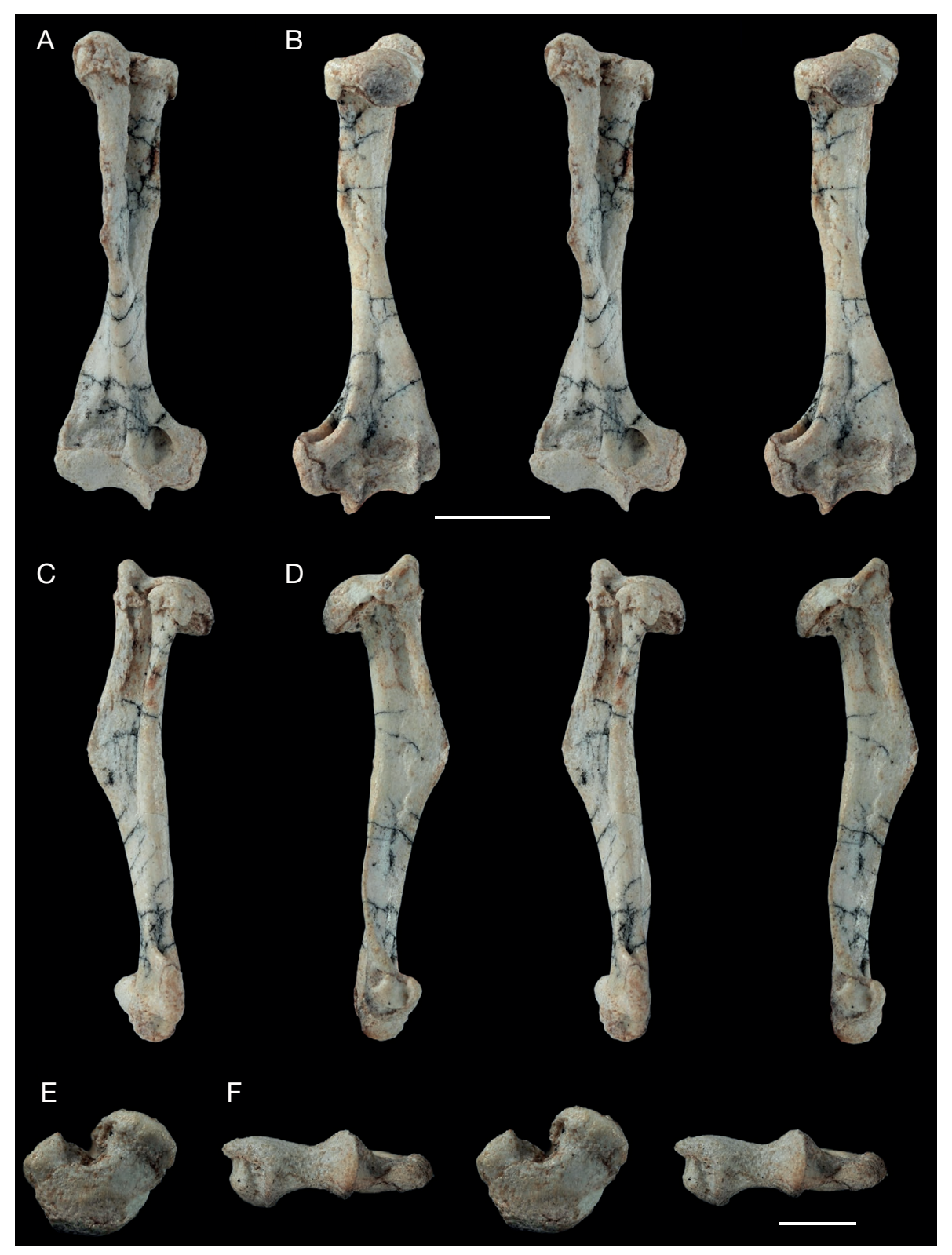

FIG. 75. - Right humerus of Alcidedorbignya inopinata (MHNC 8372): A, stereophotographs of anterior view; B, stereophotographs of posterior view; C, stereophotographs of medial view; D, stereophotographs of lateral view; E, stereophotographs of proximal view; $\mathbf{F}$, stereophotographs of distal view. Scale bars: $1 \mathrm{~cm}$.

better visualization of the actual orientation of the head and of the relative elevation of the greater tubercle and head. This montage confirms that the greater tubercle is not more elevated than the head. On MHNC 8372 the greater tubercle appears to be higher than the head, which contrasts with the condition of MHNC 8436. In fact, even if some individual variation is always possible, we rather interpret this difference as the result of the distortion of the specimen, which modified the orientation of the head (see above and Fig. 78). Because the epiphysis itself is apparently little deformed, the relative elevation of the greater tubercle (in lateral view) can be evaluated in placing the head alone in anatomical position (i.e. in lateral view, the concavo-convex suture being sub-horizontal, in a position similar to that of the epiphysis MHNC 8436 in Fig. 78E) In this position the elevation (proximal exten- sion) of the greater tubercle is similar to that observed on MHNC 8436. Therefore, the greater tubercle of the humeri of MHNC 8372 was at the most as high as the head but not higher (Figure 78). The greater tubercle is separated from the humeral head by a well-marked broad sulcus. On its posterolateral side, on approximately one-third of its length, the greater tubercle bears a sub-triangular scar for insertion of the infraspinatus. The lesser tubercle is small, rounded, appressed against the head. On the whole, the tubercles of Alcidedorbignya are more developed and more salient than in Pantolambda and somewhat resemble in their development those of Mayulestes. The proximal epiphysis is slightly wider (mediolaterally) than long (anteroposteriorly). On MHNC $8436, \mathrm{~L}=12.45 \mathrm{~mm}$ and $\mathrm{W}=13.50 \mathrm{~mm}$. The head is globular and sub-hemispherical. A similar condition is present in 

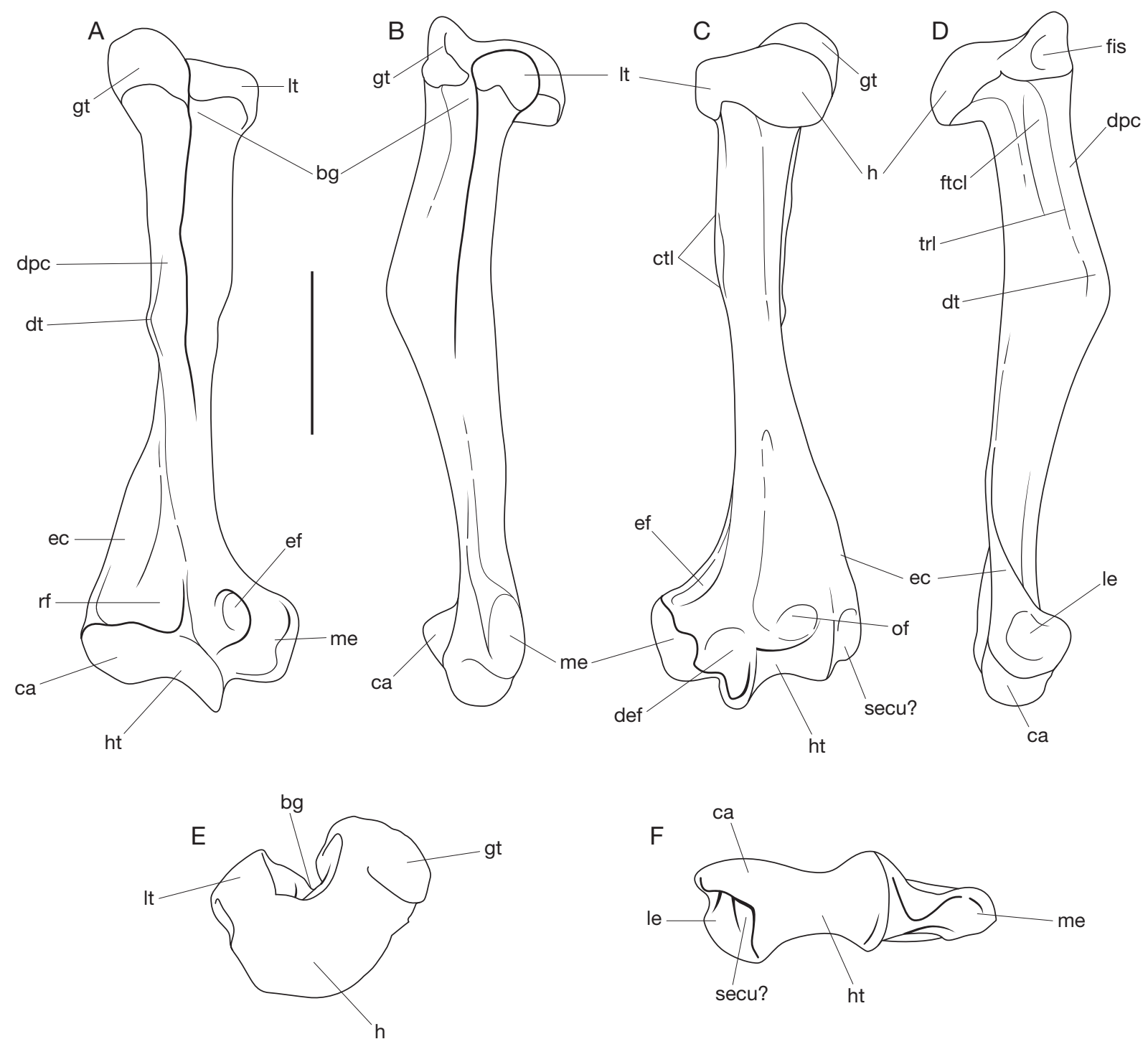

FIG. 76. - Right humerus of Alcidedorbignya inopinata (MHNC 8372). The position of the greater tubercle is artificially higher than the head because of distorsion of the proximal epiphysis of the specimen (see figure 78): A, anterior view; B, lateral view; C, posterior view; D, medial view; E, proximal view; F, distal view. Abbreviations: bg, bicipital groove; ca, capitulum; ctl, crest for insertion of the teres major and latissimus dorsi; def, dorsoepitrochelar fossa; dt, deltoid tuberosity; dpc, deltopectoral crest; ec, epicondylar crest; ef, entepicondylar foramen; ftcl, fossa for the caput longum of the triceps brachii; fis, fossa for insertion of the infraspinatus; gt, greater tubercle; h, head; ht, humeral trochlea; le, lateral epicondyle; It, lesser tubercle; me medial epicondyle; of, olecranon fossa; rf, radial fossa; secu?, sulcus for the extensor carpi ulnaris (possibly); trl, tricipital line. Scale bar: $1 \mathrm{~cm}$.

Mayulestes, Pantolambda, Marmota and Erinaceus. It differs from the condition in Solenodon, in which the articular area is distinctly narrower than long. The orientation of the head is difficult to evaluate on MHNC 8372 because of the distortion of the specimen. However, observation of the isolated specimens, which preserve the proximal extremity of the diaphysis with the neck (MHNC 8435 and 8437) (Fig. 78), clearly indicates that the distal epiphysis (and therefore the humeral head) was not as posteriorly oriented as in MHNC 8372 (see discussion above). In fact, it seems that the posterior component of this orientation (if any) was reduced and was probably close to that in Mayulestes and Pantolambda. The posterior edge of the proximal extremity of the diaphysis, just distal to the epiphysis (i.e. the neck) is strongly concave in such a way that at least one-third of the head overhangs the diaphysis posteriorly as is observed in Mayulestes and Pantolambda. There is no significant torsion of the proximal epiphysis in relation to the distal one. The bicipital groove (or intertubercular groove) is deep on MHNC 8372, but, again, this depth may have been enhanced by the distortion of the bone. On undistorted specimens the bicipital groove appears to be relatively wide but moderately deep. The deltopectoral crest and tricipital line surround the $\mathrm{V}$-shaped deltopectoral area, which faces anteriorly. These two crests end distally in a sharp deltoid tuberosity that protrudes anterolaterally, at about mid-shaft but remaining on the proximal half of the diaphysis. Because of the lateral component of the protrusion, the tricipital line appears slightly concave laterally in anterior view. The condition of Alcidedorbignya, with a laterally projecting tuberosity, is present, but to a much greater 


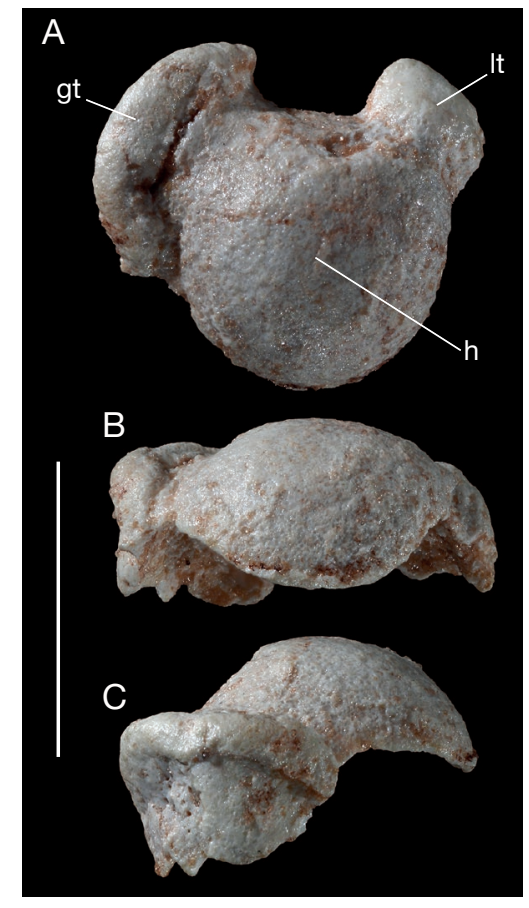

FIG. 77. - Proximal epiphysis of a left humerus of Alcidedorbignya inopinata (MHNC 8436): A, proximal view; B, posterior view; C, lateral view. Abbreviations: gt, greater tubercle; $\mathbf{h}$, head; It, lesser tubercle. Scale bar: $1 \mathrm{~cm}$.

extent, in Pantolambda. In this genus the deltoid tuberosity is long ( $25 \%$ the total length of the bone), thick and strongly projecting laterally and curved posteriorly, almost reaching a plane parallel to the transverse axis of the distal epiphysis. Furthermore, in Pantolambda the deltopectoral crest extends slightly onto the distal half of the diaphysis, contrary to the condition in Alcidedorbignya. As compared to Mayulestes, the deltopectoral crest of Alcidedorbignya is more prominent anteriorly but it is shorter, representing $43 \%$ of the diaphyseal length as opposed to $60 \%$ in Mayulestes. In Solenodon the deltopectoral crest extends onto the distal half of the diaphysis, while in Marmota it remains on the proximal half. On the medial ridge running from the lesser tubercle, on the proximal third of the diaphysis, is a short but conspicuous crest, which distinctly projects posteriorly. It is especially well-developed on MHNC 8433 (Fig. 79). This crest is for the insertion of the teres major and it probably received the insertion of the latissimus dorsi as well. This crest is also welldeveloped (but rather as a tuberosity) in Pantolambda, while in Solenodon and Marmota it is weaker than in Alcidedorbignya. On the lateral side of the diaphysis, below the greater tubercle, a fossa, probably emphasized by the anteroposterior compression, is related to the origin of the caput laterale of the triceps brachii and probably the brachialis (Fig. 76). This fossa is moderately developed in MHNC 8433 and 8434 , but more conspicuous in MHNC 8435, 8436, and 8437.

The distal epiphysis of the humerus is anteroposteriorly flattened as in the metatherians from Tiupampa and forms a distinct humeral plate. The lateral epicondylar crest extends along about $30 \%$ of the diaphysis. It is little expanded laterally and posteriorly in contrast to the condition in Pantolambda. In this genus the extreme posterior convexity of the lateral epicondylar crest gives the lateral view of the bone a strongly sigmoid appearance in lateral view, while it is sub-rectilinear in Alcidedorbignya. The proximal apex of the crest (in both posterior and anterior views) does not protrude laterally, in contrast to Mayulestes and, especially, Pucadelphys. Proximally, it does not reach the level of the deltoid tuberosity a condition also present in Pantolambda. The medial epicondyle is massive and much more developed proximo-distally in $\mathrm{Al}$ cidedorbignya than in Mayulestes. It is a rectangular structure with a long, sub-rectilinear, and vertical medial edge. The medial epicondyle extends medially with almost no distal component and appears sub-horizontal. In medial view its apex is elongated and oval-shaped. The proximal angle of this apex stands at the level of the proximal edge of the olecranon fossa, which means far above the aticular level of the elbow articular level. In its massiveness and general morphology, the medial epicondyle of Alcidedorbignya resembles, those of Pantolambda, Solenodon, and Marmota. An entepicondylar foramen can be observed on all the humeri of Alcidedorbignya as in Pantolambda and Solenodon. Above the capitulum, the radial fossa is reasonably developed in MHNC 8372, better defined on the right humerus. It presents some individual variation, as it is relatively deeper in MHNC 8433 than in the other specimens. Posteriorly, the olecranon fossa is shallow and as wide as the articular area.

The lateral epicondyle is much smaller than the medial. Together with the anterior side of the lateral epicondylar crest, it received the origin of the digit extensors. On the posterior aspect, between the lateral epicondyle and the lateral crest of the trochlea, is a small fossa (sometimes a deep sulcus as in MHNC 8433), which may have received the extensor carpi ulnaris (Figs 76C, F; 79A). On the posterior face of the distal epiphysis, medially, a deep and large dorsoepitrochlear fossa can be observed between the medial epicondyle and the medial border of the trochlea, probably for the attachment of the ulnar collateral ligament. This fossa is sometimes even as large as the olecranon fossa as is observed on MHNC 8433 (Fig. 79A). A well-developped dorsoepitrochlear fossa is common in primates (e.g., most new world monkeys, most adapoids, all Fayoum anthropoids, and Plesiadapis [Conroy 1976; Seiffert et al. 2000; personal observations]). In posterior view, the trochlea has sharp and asymmetrical crests, the lateral one placed more proximal than the medial one, which protrudes distally. In anterior view, the articular facet of the trochlea is very narrow in comparison to the capitulum. The capitulum is oval-shaped and more developed proximo-distally in Alcidedorbignya than in Mayulestes. It appears flattened antero-posteriorly on the left humerus, a condition related to post-mortem distortion. The lateral capitular tail is not well distinguished from the capitulum and is not developed as such. In fact, what corresponds to the capitular tail is simply a broad lateral extension of the capitulum, which is proximodistally wide and does not form the narrow extension observed in most primates. In anterior view, the medial lip of the trochlea protrudes more distally 


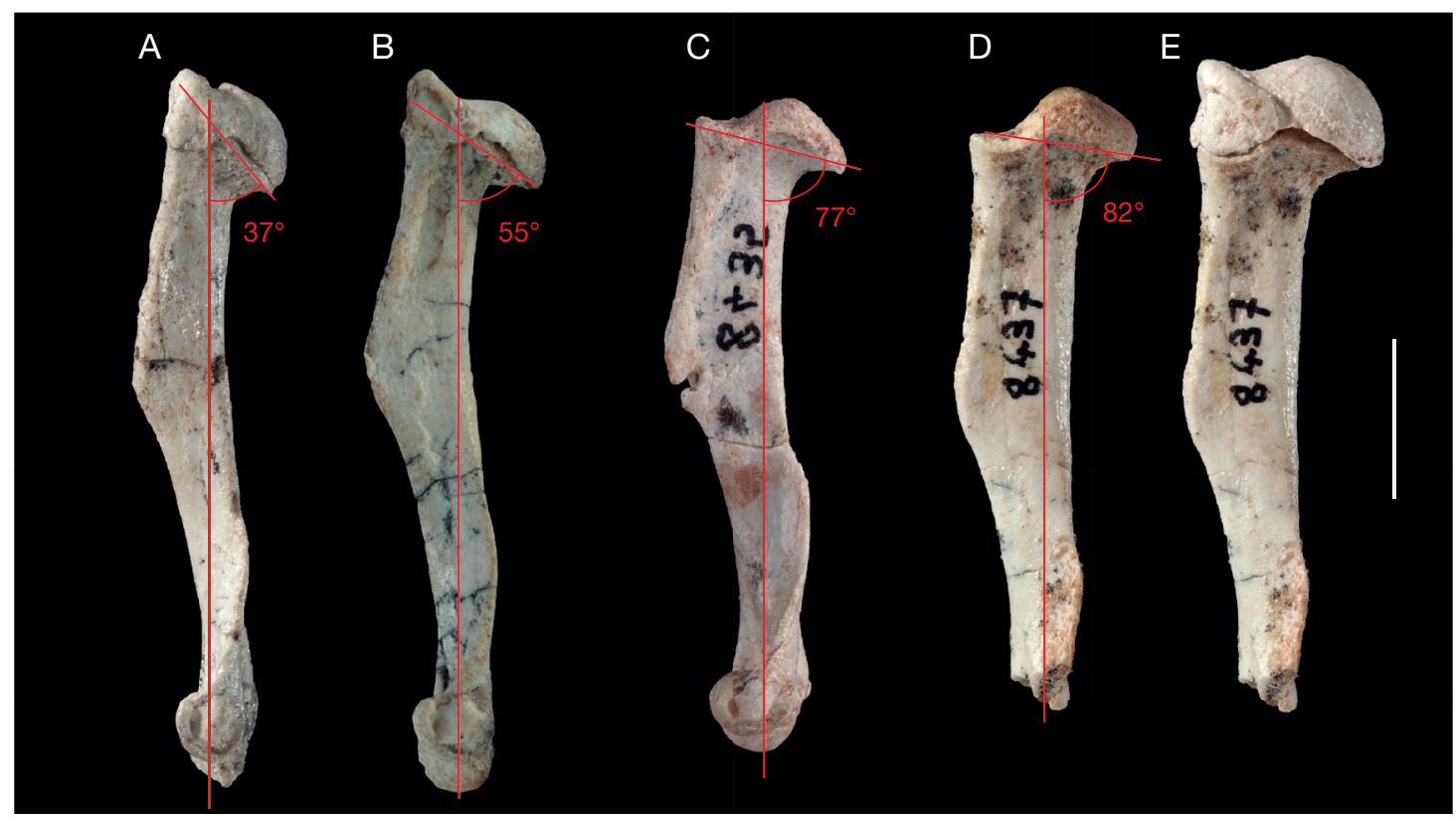

FIG. 78. - Lateral views of humeri of Alcidedorbignya inopinata showing the distorsion of the head, which has been rotated posteriorly in MHNC 8372 (on figure $\mathrm{A}$ and $\mathrm{B}$ the position of the greater tubercle is artificially higher than the head because of distorsion of the specimen): $\mathbf{A}$, lateral view of the left humerus (MHNC 8372), in which the posterior rotation of the head in greater than on the left bone; B, lateral view of the right humerus of the same (reversed); C, lateral view of an undistorted (or little distorted), immature right humerus lacking the proximal epiphysis but preserving the proximal epiphyseal suture (MHNC 8435), reversed; D, lateral view of an undistorted (or little distorted), immature left humerus lacking the epiphyses (MHNC 8437); $\mathbf{E}$, lateral view of the same on which the proximal epiphysis MHNC 8438 has been artificially placed, in order to provide a better idea of the elevation of the greater tubercle. In this position, which we regard as close to the natural position, the greater trochanter is as high as the head. The red lines materialize the proximodistal axis of the bone (vertical) and the rough plane of the epiphyseal suture (oblique). Scale bar: $1 \mathrm{~cm}$.

TABLE 25. - Measurements of the humerus of Alcidedorbignya inopinata (in mm).

MHNC 8372 (R) MHNC 8372 (L) MHNC 8433 MHNC 8434 MHNC 8435 MHNC 8438

\section{Length}

Anteroposterior length of proximal epiphysis

Width of the proximal epiphysis

Anteroposterior length of head

Width of head

Length of deltopectoral crest

(greater tubercle to deltoid tuberosity)

Width of distal extremity

Width of the trochlea in posterior view

Width of trochlea in distal view

Width of the capitulum

Height of capitulum in anterior view

Proximodistal length of medial epicondyle

$\begin{array}{rr}41.73 & 45.5 \\ 8.14 & 8.4 \\ 10.30 & 9.8 \\ 5.89 & 6.7 \\ 7.61 & 6.7 \\ 18.42 & 20.6 \\ 13.75 & 13.5 \\ 5.05 & 4.7 \\ 5.80 & 4.5 \\ 5.88 & 5.6 \\ 3.84 & 4.2 \\ 5.68 & 6.4\end{array}$

\begin{tabular}{rrrrc}
45.55 & - & - & - & - \\
8.45 & - & - & - & 12.45 \\
9.82 & - & - & - & 13.50 \\
6.73 & - & - & - & 9.30 \\
6.71 & - & - & - & 9.27 \\
20.60 & - & - & - & - \\
13.57 & 16.90 & 14.27 & 14.17 & - \\
4.76 & 4.91 & 4.89 & 5.63 & - \\
4.50 & 5.90 & 4.96 & 5.42 & - \\
5.61 & 6.70 & 5.46 & 5.70 & - \\
4.26 & 4.29 & 3.74 & 3.81 & - \\
6.44 & 6.60 & 6.45 & 5.59 & - \\
\hline
\end{tabular}

than the capitulum. Its prominence suggests asymmetrical flexion/extension of the elbow joint. The condition of the distal extremity of the humerus of Pantolambda is very similar to that of Alcidedorbignya. However, in Pantolambda the bone, besides being larger, is much more massive. The capitulum is slightly transversely narrower but especially the distal extremity of the bone (the humeral plate) is clearly less asymmetrical because of the lesser distal development of the medial trochlear crest, which is still, however, slightly more distally extended than the capitulum. The dorsoepitrochlear fossa forms a deep pit but is clearly less extended than in Alcidedorbignya. In contrast, the olecranon fossa is apparently deeper than in Alcidedorbignya. The radial fossa is not preserved on AMNH 16663.
Ulna. (Figs 80, 81; Table 26) Both ulnae of MHNC 8372 are preserved. The right ulna is complete but the left one has lost its distal epiphysis. The ulna of Alcidedorbignya is approximately as long as the humerus, as in Pantolambda but contrary to the condition of Mayulestes in which the ulna is slightly longer than the humerus. The bone is flattened medio-laterally but robust in lateral view. Its posterior border is convex approximately at the level of the radial notch. Because of this convexity the proximal part of the bone (olecranon and articular region) is bent anteriorly (in lateral view). This condition clearly differs from that of Pantolambda, in which the ulna is sub-rectilinear with a barely convex posterior edge. In turn, it resembles Mayulestes in the anterior bending of the bone, but the ulna of Alcidedorbignya is not as sigmoid as that of Mayulestes in 


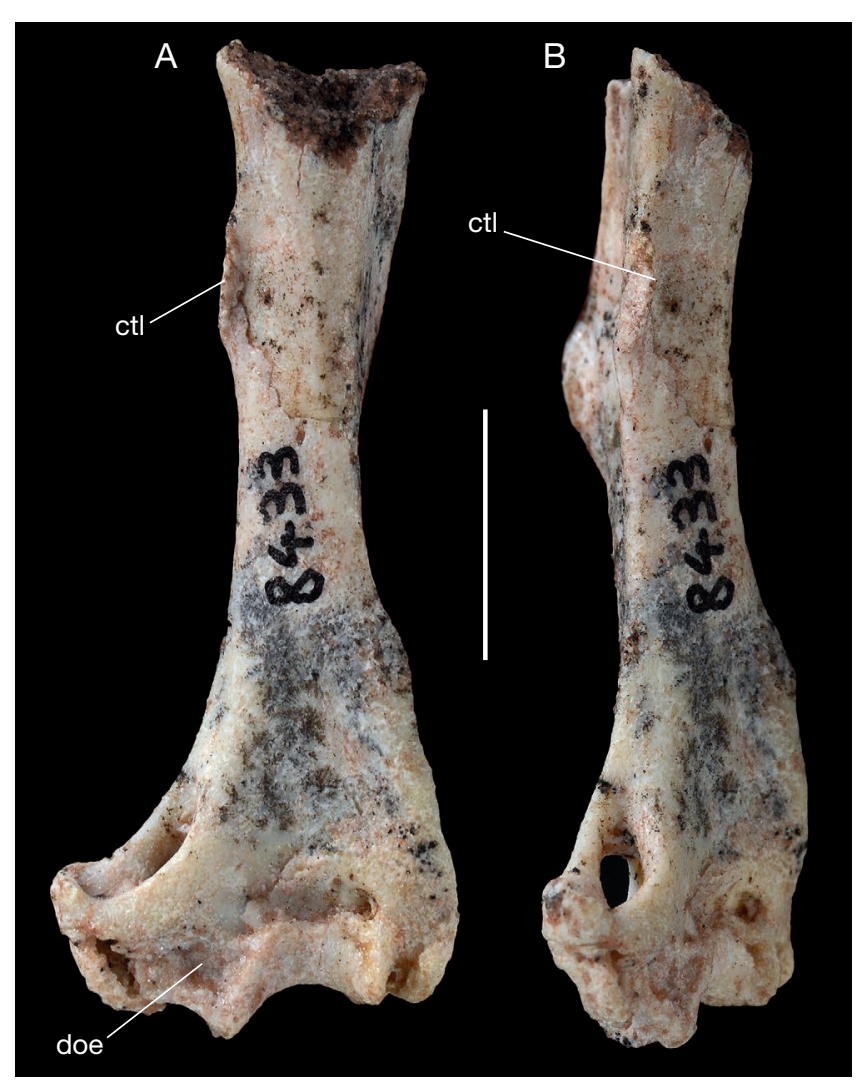

FIG. 79. - Right humerus of Alcidedorbignya inopinata (MHNC 8433): A, posterior view; B, posteromedial view. Abbreviation: ctl, crest for insertion of the teres major and latissimus dorsi; doe, dorsoepitrochlear fossa. Scale bar: $1 \mathrm{~cm}$.

lateral view. In Mayulestes the greatest posterior convexity of the bone is more distal (well distal to the radial facet) reaching the proximal third of the bone. In anterior view, the ulna of Alcidedorbignya is concave medially but less than in Mayulestes. It differs from that of Pantolambda, which is rectilinear. The olecranon is massive, wider anteroposteriorly than in Mayulestes, but shows a similarly an obtuse angle between its posterior and proximal edges. In Pantolambda the olecranon is sub-vertical and its posterior and proximal edges are approximately at a right angle. In lateral view, its functional length (measured from the tip to the centre of the trochlear notch) represents $20 \%$ of the total diaphysis length. The proximal edge of the olecranon of Alcidedorbignya, at the limit of the epiphysis, protrudes medially and, as a consequence, the medial edge of the olecranon bears a deep fossa for the flexor carpi ulnaris and the proximal origin of the flexor digitorum profundus. This fossa is wider and deeper than in Pantolambda but slightly shallower than in Mayulestes. It is much deeper than in Solenodon and Marmota. The lateral side of the olecranon is not excavated as is observed in Mayulestes but differs from the marked fossa present in Pantolambda. The proximal epiphysis is not fully fused to the diaphysis and not fully ossified. Its medial part is not completely preserved in either of the two ulnae of MHNC 8372. It shows a lateral notch seen in proximal view, where the anconeus may have inserted. From this notch and from the proximolateral angle of the epiphysis, on the lateral side of the olecranon, a salient oblique ridge extends distally
TABLE 26. - Measurements of the ulna of Alcidedorbignya inopinata (in mm).

MHNC 8372

Length

43.29

Length from apex of olecranon to anconeal process

7.74

Anteroposterior length of olecranon at level of most

proximal edge of humeral articular surface

Width of olecranon at apex

6.20

3.37

Proximodistal length of trochlear notch in medial view $\quad 7.97$

Width of distal extremity

Anteroposterior length of distal extremity

3.27

4.91

to the posterolateral edge of the diaphysis at the level of the junction between the radial and humeral facets. This ridge was also probably for the insertion of the anconeus. The beak of the olecranon (or anconeal process) is not prominent anteriorly, resembling Mayulestes, but contrary to the condition in Pantolambda, which bears a salient process. It is asymmetrical, the lateral lip being prominent laterally whereas the medial one extends proximally. The trochlear notch appears widely open in medial view, with the coronoid process facing more anteriorly than proximally. This condition strongly recalls that of Mayulestes but differs from Pantolambda in which the articular surface of the coronoid process faces antero-proximally with the two components being roughly similar. In lateral view the plane of the distal portion of the humeral facet on the coronoid process is approximately at an angle of $c .20^{\circ}$ with the diaphysis in Alcidedorbignya, while the angle is $c .55^{\circ}$ in Pantolambda. As a consequence, the coronoid process of Alcidedorbignya is much less salient anteriorly than in Pantolambda and the crest, wich separates the humeral and radial facets of the ulna is extremely low. Laterally, the radial notch is small and flat, in continuity with the diaphysis, and faces more laterally than anteriorly. It is smaller than the coronoid process. In anterodistal view, the distal edge of the articular surface for the radius on the radial notch forms an angle of approximately $120^{\circ}$ with the distal edge of the articular surface for the humerus on the coronoid process (Fig. 80D). This condition is strongly reminiscent of the angle observed in Mayulestes, but clearly differs from the right angle of Pantolambda. The articular facet for the radius is roughly triangular to sub-oval in outline. Distal to the radial notch, the lateral side of the diaphysis bears a long fossa for the abductor pollicis longus. Distal to the coronoid process, the fossa for the brachialis is deep, twice as long proximodistally as transversely, and faces anteriorly. Because of its depth and the relative weakness of the bicipital tuberosity on the radius, this fossa probably also received part of the insertion of the biceps brachii. It resembles that of Pantolambda but is more developed than in Mayulestes. On the medial side of the diaphysis, the fossa for the flexor digitorum profundus extends along the first two-thirds of the diaphysis. It is slightly shallower than in Mayulestes in its proximal half but slightly deeper in the median third of the diaphysis. The interosseous crest is sharp and, on the medial face of the distal extremity of the diaphysis, the pronator quadratus crest is salient as in Mayulestes but in contrast to the condition in Pantolambda. The styloid process of the ulna is very well-developed, conical, sharp, and prominent. 


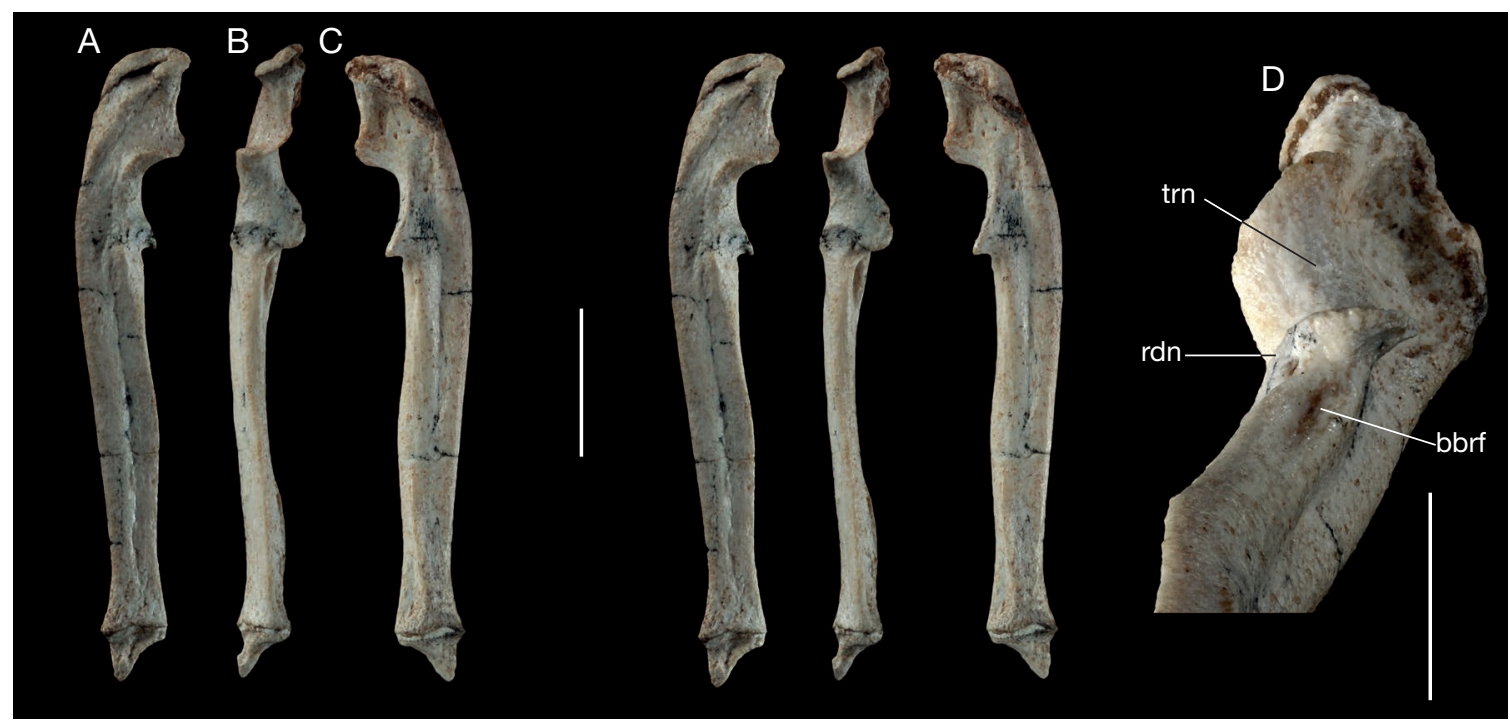

FIG. 80. - Right ulna of Alcidedorbignya inopinata (MHNC 8372): A, stereophotographs of lateral view; B, stereophotographs of anterior view; C, stereophotographs of medial view; D, anterodistalal view of the right ulna of MHNC 8372 showing the obtuse angle between the anterior edges of the trochlear and radial notches. Abbreviations: bbrf, biceps and brachialis fossa; rdn, radial notch; trn, trochlear notch. Scale bars: A, B, C, $1 \mathrm{~cm}$; D, $5 \mathrm{~mm}$.

Radius. (Figs 82-84; Table 27) The two radii of MHNC 8372 are preserved. The right is complete but the left one lacks the distal epiphysis. Two other specimens have been referred to Alcidedorbignya inopinata. One is a distal half of a large radius with a very strong pronator crest (MHNC 8441); the other is missing the distal epiphysis and the pronator crest (MHNC 8442).

The bone is more massive distally than proximally. The proximal third of its diaphysis is concave anteriorly, while the distal two-thirds are anteriorly convex. The anterior (pronator) crest is sharp distally. In proximal view the radial head is oval in outline $(\mathrm{L}=3.6 \mathrm{~mm} ; \mathrm{W}=5.7 \mathrm{~mm} ; \mathrm{L} / \mathrm{W}=0.63)$ and its width is approximately $50 \%$ longer than its anteroposterior length. However, these proportions vary, as on MHNC 8442 it is clearly longer in relation to its width than in MHNC 8372 $(\mathrm{L}=4.6 \mathrm{~mm} ; \mathrm{W}=6.3 \mathrm{~mm} ; \mathrm{L} / \mathrm{W}=0.73)$. In other words, the articular area of this specimen appears more ovoid and less elongate transversely. The articular surface for the capitulum is slightly concave. In anterior view the proximal epiphysis is oblique in MHNC 8372 and its lateral border protrudes proximally. This condition is less pronounced on MHNC 8442. The curvatures of the radius, and the morphology and orientation of the proximal epiphysis of the radius of Alcidedorbignya inopinata resembles the conditions observed in Pantolambda. In turn, the asymmetry of the proximal epiphysis is much more emphasized in Alcidedorbignya than in Mayulestes. On the anteromedial angle of the articular surface, the epiphysis presents a small proximal elevation, the capitular eminence, which gives to its anterior edge a distinct sigmoid profile. The capitular eminence is very weak in Mayulestes but it is well developed in Pantolambda. On the posterior edge of the epiphysis, the ulnar facet is gently convex posteriorly and narrow proximodistally. On the whole, the proximal end of the radius of Alcidedorbignya is very similar to that of Pantolambda. On the posterior edge of the proximal ex-

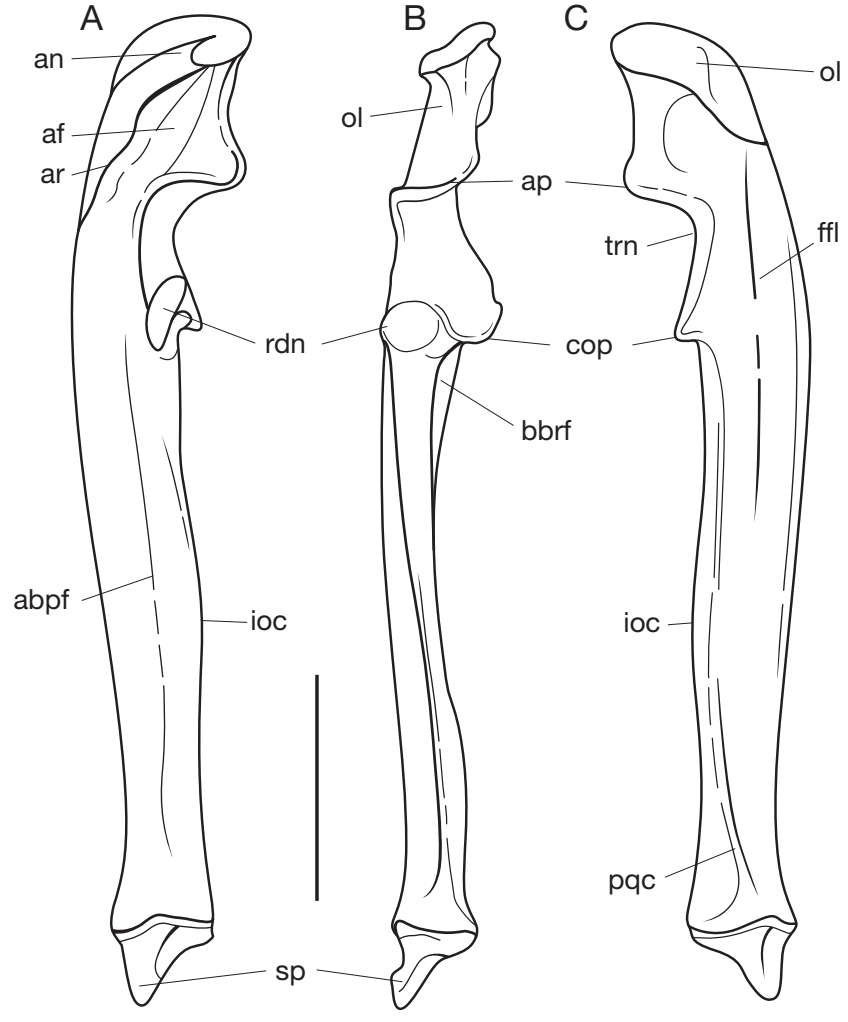

FIG. 81. - Right ulna of Alcidedorbignya inopinata (MHNC 8372): A, lateral view; B, anterior view; C, medial view. Abbreviations: abpf, abductor pollicis longus fossa; af, anconeal fossa; an, anconeal notch; ap, anconeal process (beak of the olecranon); ar, anconeal ridge; bbrf, biceps and brachialis fossa; cop, coracoid process; ffl, flexor fossa; ioc, interosseous crest; ol, olecranon; pqc, pronator quadratus crest; rdn, radial notch; sp, styloid process; trn, trochlear notch. Scal bar: $1 \mathrm{~cm}$.

tremity of the diaphysis the bicipital tuberosity is weak. It is proximodistally elongate, being approximately twice as long as wide. On the medial side of the tuberosity and just distal 


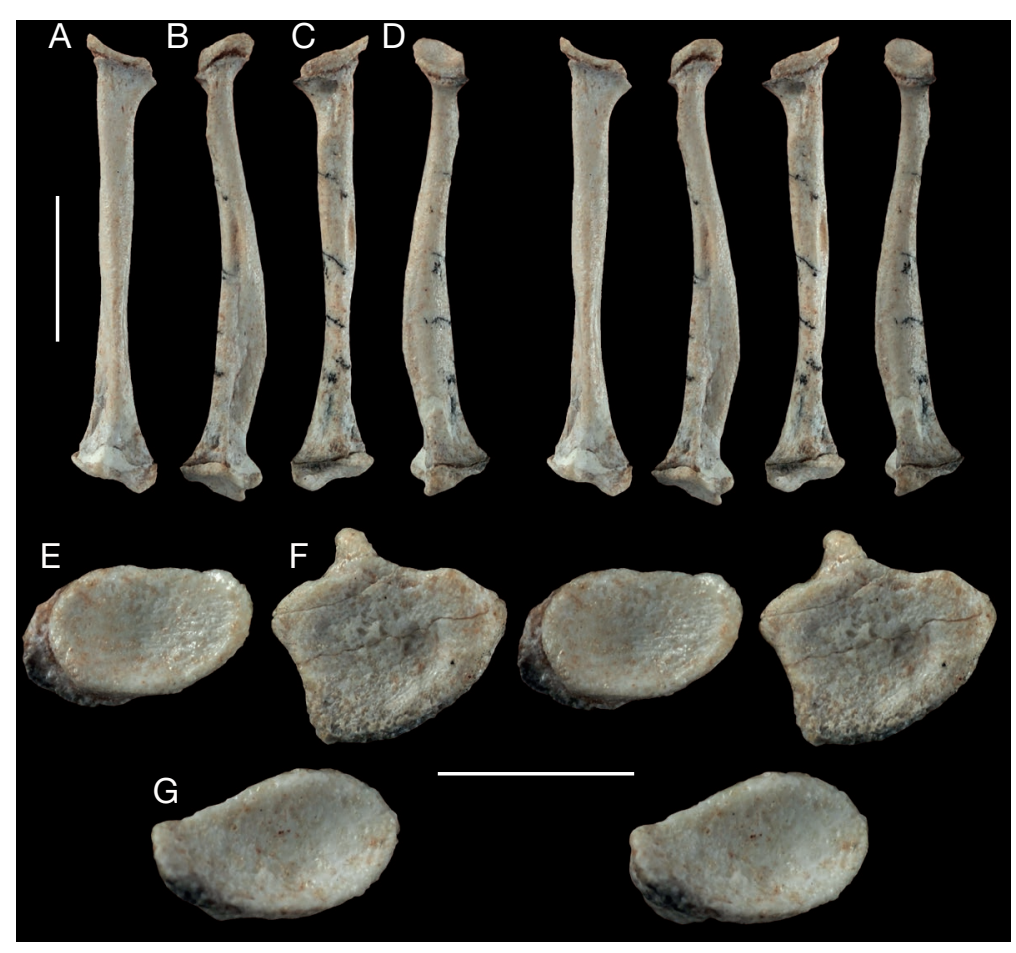

FIG. 82. - Right (A-F) and left (G) radius of Alcidedorbignya inopinata (MHNC 8372): A, stereophotographs of anterior view; B, stereophotographs of lateral view; C, stereophotographs of posterior view; D, stereophotographs of medial view; E, stereophotographs of proximal view; $\mathbf{F}$, stereophotographs of distal view; G, stereophotographs of proximal view. Scale bars: A-D, $1 \mathrm{~cm}$; E-G, $5 \mathrm{~mm}$.

to the epiphyseal line is a small but deep triangular fossa. We have no interpretation for this structure. Because there is no muscle likely to attach in this area, we suspect that it may receive a ligament of the humeroradial articulation. On the lateral side, at mid-shaft level, is an elongate fossa, which probably received the radial head of the abductor pollicis longus. On the anterior edge of the distal half of the diaphysis is a strong, anteriorly protruding, and rectilinear pronator crest. This crest is proportionally more developed on MHNC 8441, a specimen that is larger (probably a male) than MHNC 8372 (a small female). A similarly-developed pronator crest is present in Pantolambda, but it is weak in Mayulestes. Medially, along the sharpest part of the pronator crest, a scar indicates the insertion of the pronator teres, along the distal end of the diaphysis. The area where the pronator quadratus inserts is not salient as it is on the ulna. The pronator crest extends distally in a rounded ridge, which reaches the distal epiphysis at the level of a salient tubercle. This tubercle is located on the anterior edge of the epiphysis itself. It separates the passageway of the tendon of the extensor carpi radialis (medially) from that of the extensor digitorum communis (laterally). The passageways of these tendons are not deeply grooved and the lateral one is approximately twice as wide as the medial. The tubercle provided an area attachment for a carpal ligament, probably the radial collateral ligament. The distal epiphysis is mostly flat, and the styloid process, located medially, is much less prominent than that of the ulna. Seen in distal view and in articulation, the distal epiphysis of the radius is about twice broader than that of the ulna. On the lateral side of the distal epiphysis, the articulation with the ulna is reduced to a narrow triangular strip.

When the radius and ulna are articulated a conspicuous interosseous space is present. Its edges are sub-parallel on most of its length.

Carpal bones. (Figs 85, 86; Table 28) Three carpal bones are known for the right manus: the pisiform, the cuneiform (also called pyramidal), and the unciform. Four are known for the left hand: the pisiform, the unciform, the trapezoid, and the trapezium. They were found in the sediment close to the forelimb bones but were not articulated to each other or to the metacarpals.

Pisiform (Fig. 85) - The pisiform, known in both manus, is a small, rod-like bone, $5.5 \mathrm{~mm}$ long. The free extremity is massive with an expanded apex, whereas the articular end forms a sharpened triangle. It bears two facets: one is triangular, concave, and articulated with the lateral part of the styloid process of the ulna. The other facet, which contacts the cuneiform, is flat and trapezoidal in outline, elongated proximodistally, and oblique in anterior view when the bone is articulated with the ulna. The distal apex forms a slim hook and the ventral border of the pisiform is, therefore, concave.

Cuneiform (Fig. 86A-C) - This element is transversely elongate and flattened proximo-distally. It presents two processes, one protruding laterally (towards the outer side of the hand), while the other on the medial edge of the 


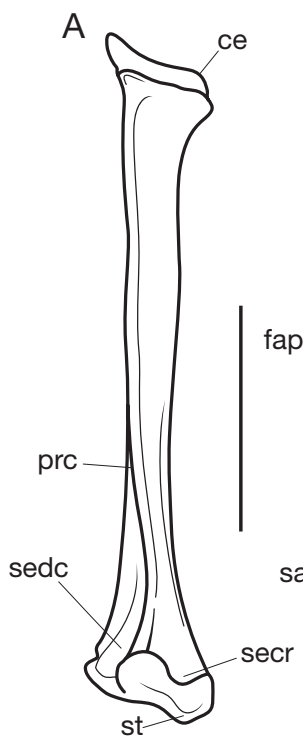

B

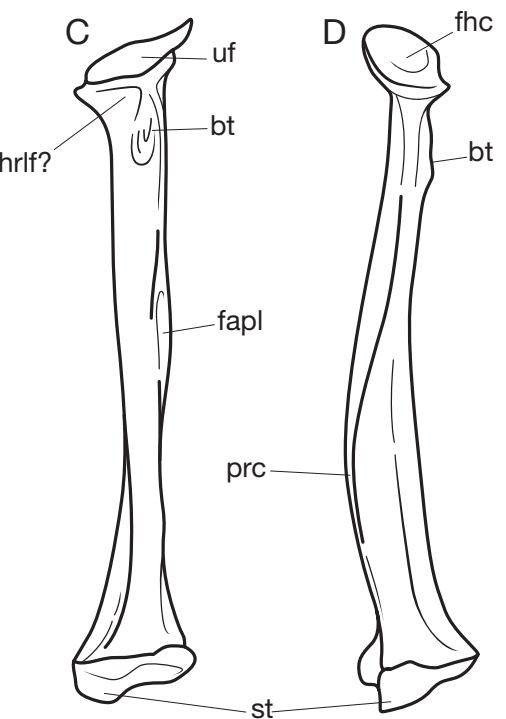

FIG. 83. - Right radius of Alcidedorbignya inopinata (MHNC 8372): A, anterior view; B, lateral view; C, posterior view; D, medial view. Abbreviations: bt, bicipital tuberosity; ce, capitular eminence; fapl, fossa for the abductor pollicis longus; fhc, facet for the capitulum of the humerus; hrlf?, possible fossa for the humeroradial ligament; prc, pronator crest; sapl, sulcus for the tendon of the abductor pollicis longus; secr, sulcus for the extensor carp radialis; sedc, sulcus for the extensor digitorum communis; st, styloid process; uf, ulnar facet. Scale bar: $1 \mathrm{~cm}$.

bone is smaller and protrudes posteriorly (palmarly). Additionally, the anterior (dorsal) side also presents a tuberosity on the proximo-medial angle of the bone. The proximal view of the bone forms a roughly right triangle and bears two transversely elongated articular facets separated by an elevated carina. The planes of the two facets form an angle of approximately $110^{\circ}$. The posterior (palmar) facet is for the pisiform and the anterior (dorsal) one is for the styloid process of the ulna. On the medial face of the bone is a relatively large facet for the lunate; this facet also extends onto the medial process mentioned above. The distal facet for articulation with the unciform is roughly oval-shaped and distinctly concavoconvex.

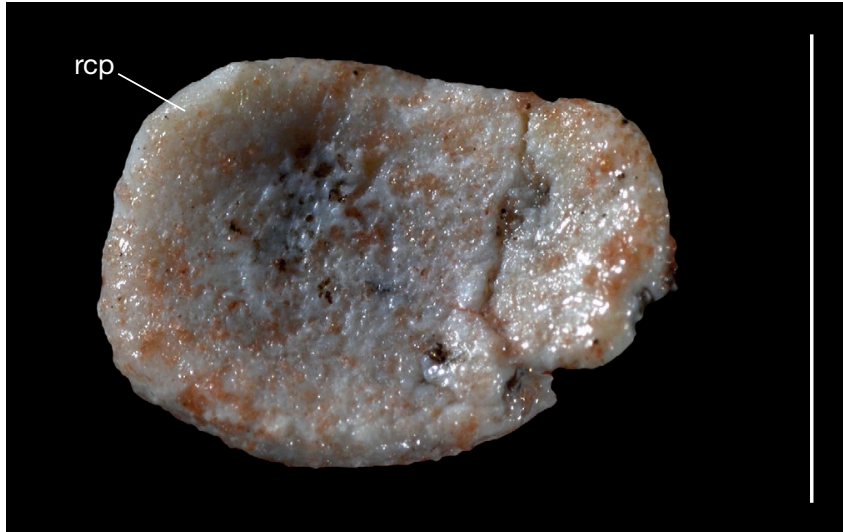

FIG. 84. - Right radius of Alcidedorbignya inopinata (MHNC 8442) in proximal view. Abbreviation: rcp, radial coronoid process. Scale bar: $5 \mathrm{~mm}$.

TABLE 27. - Measurements of the radius of Alcidedorbignya inopinata (in mm).

MHNC 8372

Length

31.70

Width of proximal epiphysis

5.34

Anteroposterior length of proximal epiphysis 3.52

Width of distal epiphysis

6.06

Anteroposterior length of distal epiphysis

5.61

Unciform (Fig. 86D-F) - Known from both manus, the unciform is relatively massive and exhibits the usual pyramidal shape in anterior view, the medial border, articulating with the magnum, being more elevated than the lateral one. The proximal facet for the cuneiform is slightly concavo-convex (concave laterally and convex medially), broadly rectangular in outline in proximal view, and slopes down laterally. Medially, the small facet articulating with the magnum is proximal. Posteriorly, a palmar tuberosity can be observed. Distally, the facet articulating with Mc IV and V is broad, concave anteroposteriorly, and transversely wider anteriorly than posteriorly.

Trapezoid (Fig. 86G, H) - The left trapezoid is the smallest bone of the known series. It is flattened proximodistally, with a proximal and a distal articular facet. The proximal one is for the centrale. It is triangular in outline, extended lateroanteriorly, and exhibits a quite rough surface. The articular facet on the opposite side is for the McII. It is more concave anteroposteriorly and is pentagonal in outline. Lateral or medial articular facets cannot be discerned but on the medial side of the bone a notch can be observed. When the trapezoid is articulated to the left Mc II, this notch is located just above another notch present on the medial side of the proximal epiphysis of this metacarpal, suggesting a ligamentous attachment between the two elements.

Trapezium (Fig. 86I, J) - The left trapezium is a robust bone, twice as big as the trapezoid but smaller than the unciform and cuneiform, high proximodistally and triangular in crosssection. The distal articular facet for the Mc I is triangular in outline and is transversely wider on its anterior edge than on 


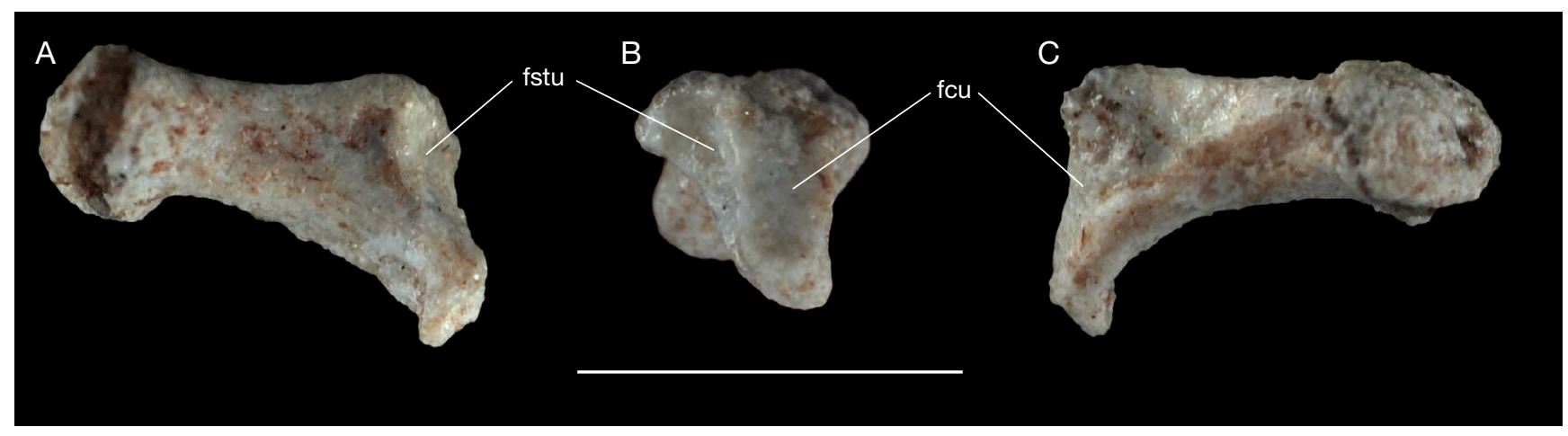

FIG. 85. - Left pisiform of Alcidedorbignya inopinata (MHNC 8372): A, dorsal view; B, distal view; C, ventral view. Abbreviations: fcu, facet for the cuneiform; fstu, facet for the styloid process of the ulna. Scale bar: $5 \mathrm{~mm}$.
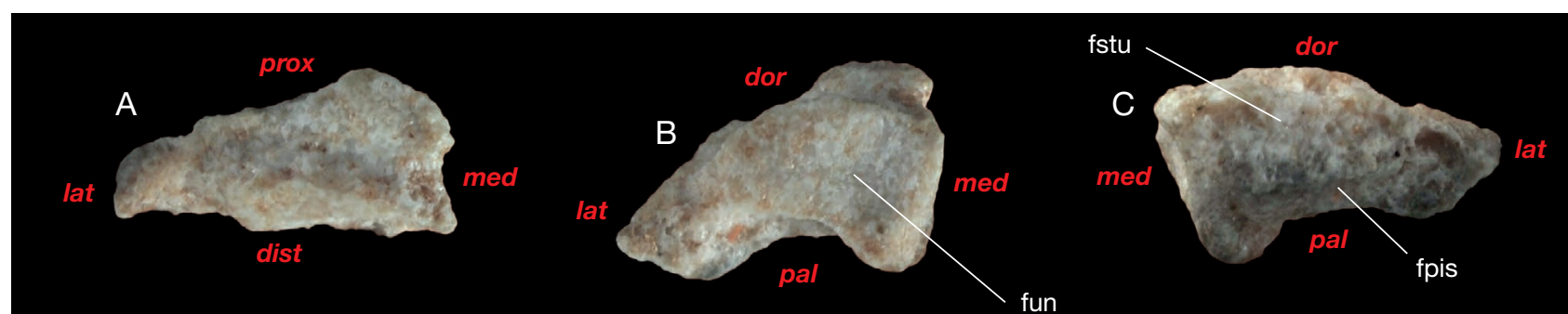

fun
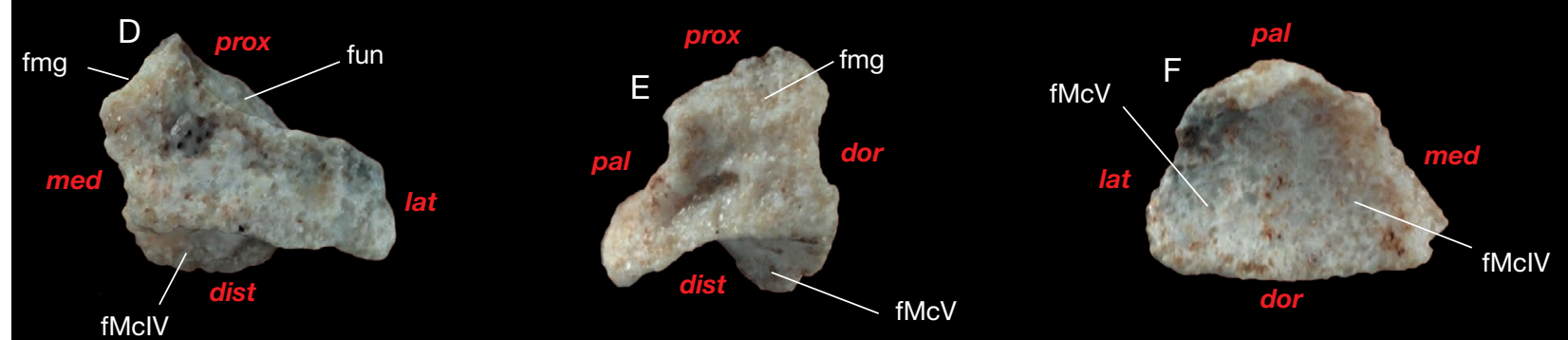

fMclV
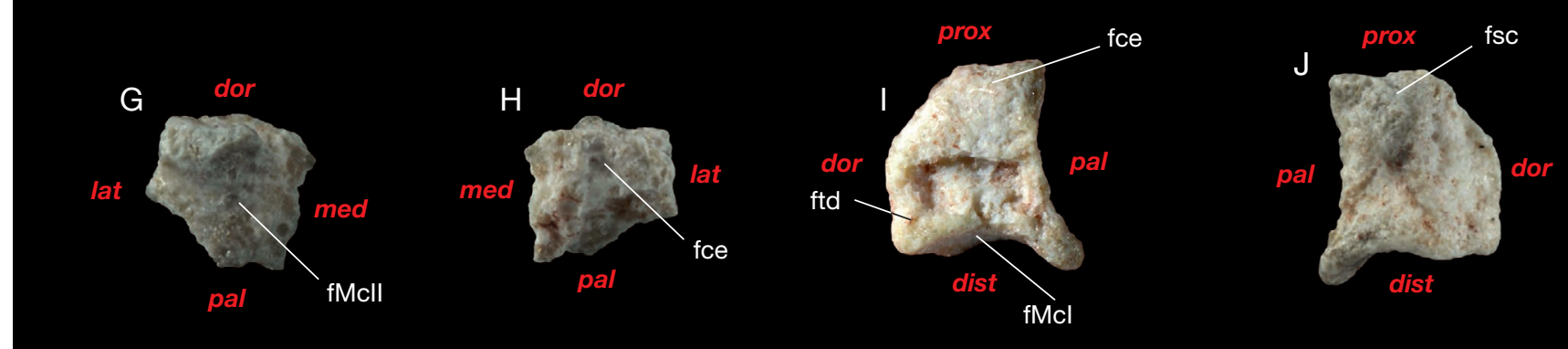

$\overline{-10}$

FIG. 86. - Carpals of Alcidedorbignya inopinata (MHNC 8372): A, right cuneiform in dorsal view; B, right cuneiform in distal view; C, right cuneiform in proximal view; D, left unciform in dorsal view; E, left unciform in medial view; $\mathbf{F}$, left unciform in distal view; G, left trapezoid in distal view; $\mathbf{H}$, left trapezoid in proximal view; I, left trapezium in lateral view; J, left trapezium in medial view. Abbreviations: dist, distal; dor, dorsal; fce, facet for the centrale; fMcl, facet for the proximal epiphysis of the Mcl; fMcll, facet for the proximal epiphysis of the Mcll; fMclV, facet for the proximal epiphysis of the MclV; fMcV, facet for the proximal epiphysis of the McV; fmg, facet for the magnum; fpis, facet for the pisiform; fsc, facet for the scaphoid; fstu, facet for the styloid process of the ulna; ftd, facet for the trapezoid; fun, facet for the unciform; lat, lateral; med, medial; pal, palmar; prox, proximal. Scale bar: $5 \mathrm{~mm}$.

TABLE 28. - Measurements of the carpal bones of Alcidedorbignya inopinata (in mm).

\begin{tabular}{lccccc}
\hline MHNC 8372 & Cuneiform (R) & Unciform (R) & Trapezoid (L) & Trapezium (L) & Pisiform (R) \\
\hline Proximodistal length & 2.13 & 2.87 & 1.47 & 3.24 & 5.78 \\
Width & 5.14 & 4.32 & 2.05 & 2.25 & - \\
Dorsopalmar depth & 2.75 & 3.23 & 2.09 & 2.38 & - \\
\hline
\end{tabular}




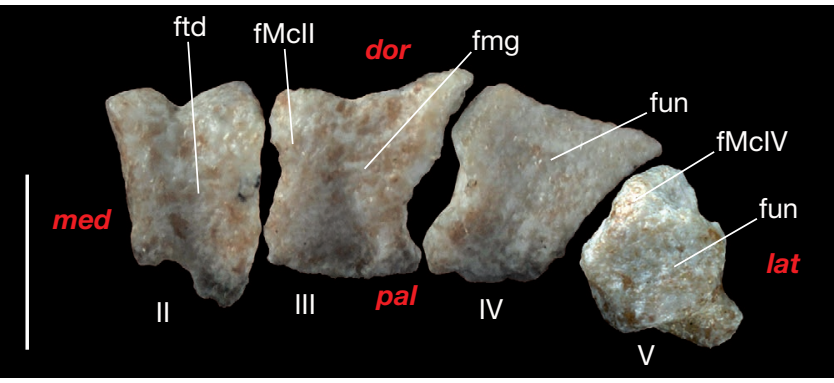

FIG. 87. - Proximal view of right metacarpals (Mc II to Mc V) of Alcidedorbignya inopinata (MHNC 8372). Mcl is not illustrated because the proximal epiphysis is missing on both metacarpals of MHNC 8372. Abbreviations: dor, dorsal; fMcll, facet for the Mcll; fMclV, facet for the McIV; fmg, facet for the magnum; ftd, facet for the trapezoid; fun, facet for the unciform; lat, lateral; med, medial; pal, palmar. Scale bar: $5 \mathrm{~mm}$.

the posterior one. It is deeply concave anteroposteriorly and slightly concave mediolaterally, suggesting a relatively mobile articulation with the Mc I. The anterior side of the bone is broadly triangular and the proximal apex exhibits a smoothly convex articular facet that slopes down medially (towards the inner side of the manus) and articulated with the distal articular facet of the scaphoid. The lateral side of the bone also exhibits a proximal articular facet, which apparently does not fit the trapezoid, which articulated with the distal portion of the lateral edge of the trapezium. Because the trapezoid is very flat proximodistally (see below) this articulation of the lateral edge of the trapezium is probably for the centrale, which would have compensated for the reduced proximodistal extension of the trapezoid. It is noteworthy that a centrale is also present in Pantolambda (Simons 1960).

Metacarpals and phalanges (Figs 87-90; Tables 29-35). The right manus is known with all the metacarpals and phalanges except the ungual phalanx of the fifth digit. It is composed of: five metacarpals, five proximal phalanges, four intermediate phalanges (on each of the lateral four digits) and four ungual phalanges. On the left manus the five metacarpals are also known, but only five phalanges, complete or partial are preserved: the distal extremity of a proximal phalanx, the proximal extremity of another one, two complete proximal phalanges and a complete intermediate phalanx.

Five isolated metacarpals have been referred to Alcidedorbignya inopinata: MHNC 8444 (a left Mc V); MHNC 8445 (a right Mc II); MHNC 8446 (proximal half of Mc III and IV); MHNC 8447 (a right Mc III); MHNC 8448 (a right Mc II); MHNC 8449 (a left Mc II).

On the whole the metacarpals of Alcidedorbignya are proportionally more slender but apparently not longer and than those of Pantolambda (Fig 88). The robustness can be evaluated with the ratio of width of distal extremity of Mc III/ length Mc III. In Alcidedorbignya (MHNC 8372) this ratio is: $3.96 / 13.96 \mathrm{~mm}=0.283$; in Pantolambda (AMNH 16663 ) the ratio is $13.46 / 37.98 \mathrm{~mm}=0.354$. Therefore, the metacarpals of Pantolambda are approximately $25 \%$ more massive than those of Alcidedorbignya. The relative length of the metacarpals can be evaluated with the ratio of: length of Mc

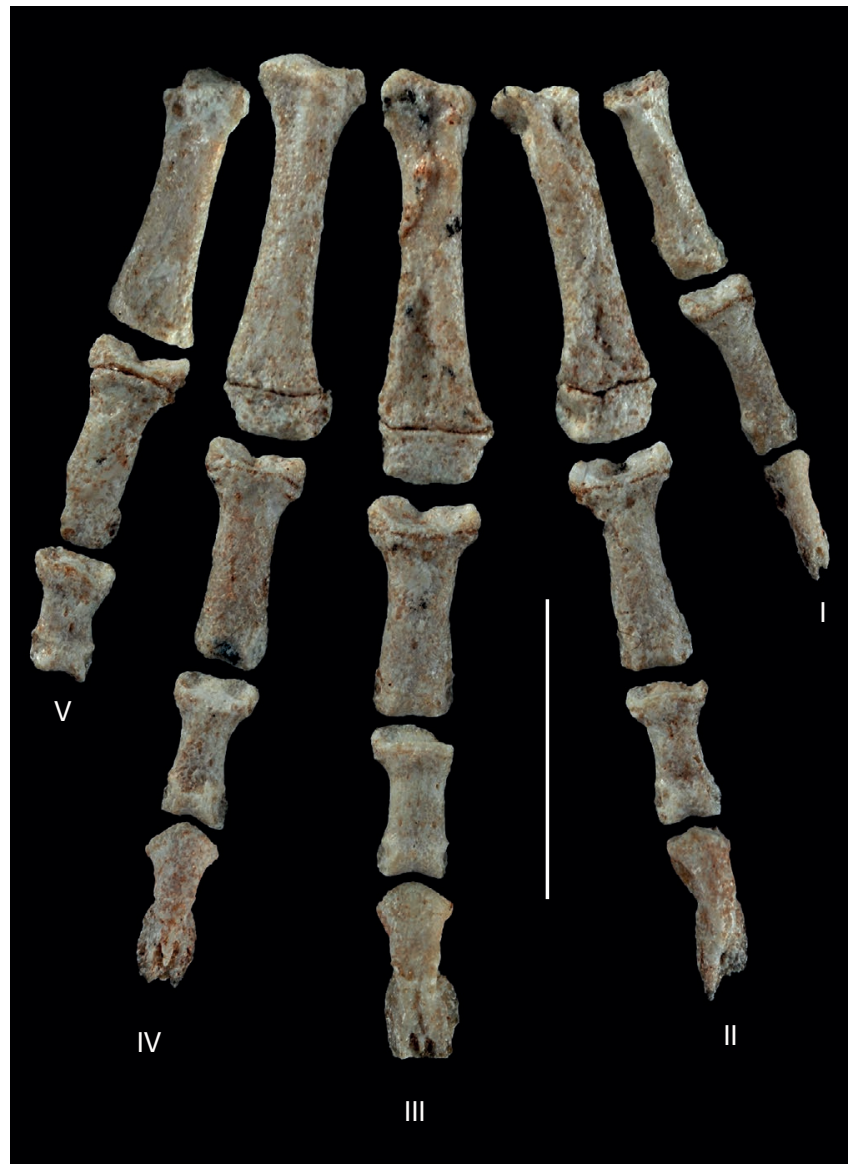

FIG. 88. - Right metacarpals and digits of Alcidedorbignya inopinata (MHNC 8372 ) in dorsal view. Scale bar: $1 \mathrm{~cm}$.

III/length of the humerus or ulna. In Alcidedorbignya (MHNC 8372) this ratio is: for the humerus, $13.96 / 43.99 \mathrm{~mm}=0.317$ and for the ulna 13.96/43.30 $\mathrm{mm}=0.322$. In Pantolambda this ratio is for the humerus: $37.98 / 122.14 \mathrm{~mm}=0.31$ and for the ulna $37.98 / 122.12 \mathrm{~mm}=0.31$. The ratios are very similar for the two bones in Alcidedorbignya and identical in Pantolambda. Therefore, the metacarpals of Alcidedorbignya are approximately $2.2 \%$ (compared with the humerus) and $3.7 \%$ (compared with the ulna) longer relative to the forelimb long bones than in Pantolambda. In the case of the robustness the difference observed between the two taxa is significant, but in the case of the relative length of the metacarpals the difference could very well be the result of individual variation. The relative length of the metacarpals of Alcidedorbignya approaches those of Solenodon and Erinaceus.

Both first metacarpals of MHNC 8372 lack the proximal epiphysis, and the distal one is damaged on the left Mc I. It is a short ( $7 \mathrm{~mm}$ long) and slender bone relative to the other metacarpals. It is apparently relatively smaller than in Pantolambda. The diaphysis is smooth and regularly convex. The distal epiphysis does not present well-separated condyles and is not asymmetrical. Not all the other metacarpals still preserve their distal epiphyses. It is missing on the right Mc IV and V and on the left Mc III. Comparison of the complete metacarpals on both hands clearly shows that the 
TABLE 29. - Comparison of robustness (Distal Width of McIII/Length of McIII) and relative length (Length McIII/Length Humerus, Length Mclll/Length Ulna) of the McIll in Alcidedorbignya inopinata and Pantolambda bathmodon (length of the humerus of Alcidedorbignya inopinata is the average of the lengths of the right and left humeri of MHNC 8372: $42.15 \mathrm{~mm}+45.84 \mathrm{~mm} / 2=43.99 \mathrm{~mm})$. Abbreviations: DW, distal width; $\mathbf{L}$, length.

\begin{tabular}{|c|c|c|c|c|c|c|c|}
\hline & DW McIII & L McIII & DW/L & L Hum & L Uln & LM/LH & LM/LU \\
\hline A. inopinata (MHNC 8372) & $3.96 \mathrm{~mm}$ & $13.96 \mathrm{~mm}$ & 0.283 & $43.99 \mathrm{~mm}$ & $43.30 \mathrm{~mm}$ & 0.317 & 0.322 \\
\hline P. bathmodon (AMHN 16663) & $13.46 \mathrm{~mm}$ & $37.98 \mathrm{~mm}$ & 0.354 & $122.14 \mathrm{~mm}$ & $122.12 \mathrm{~mm}$ & 0.31 & 0.31 \\
\hline
\end{tabular}

TABLE 30. - Measurements of the metacarpals of Alcidedorbignya inopinata (in mm).

\begin{tabular}{lcccc}
\hline MHNC 8372 & Mcl (R) proximal epiphysis missing & Mcll (R) & McIII (R) & McIV (R) \\
\hline Length & 7.14 (as preserved) & 12.16 & 13.46 & 12.89 \\
Proximal width & - & 2.46 & 2.88 & 2.83 \\
Distal width & 2.21 & 3.62 & 3.96 & 3.20 \\
\hline
\end{tabular}

TABLE 31. - Measurements of an isolated Mclll referred to Alcidedorbignya inopinata (MHNC 8445) (in mm).

\section{McIII (MHNC 8445)}

\begin{tabular}{lr}
\hline Length & 16.04 \\
Proximal width & 3.31 \\
Distal width & 4.43
\end{tabular}

TABLE 32. - Measurements of the proximal phalanges of Alcidedorbignya inopinata (in $\mathrm{mm}$ ).

\begin{tabular}{lccccc}
\hline MHNC 8372 & Digit I & Digit II & Digit III & Digit IV & Digit V \\
\hline Length & 6.00 & 7.34 & 7.43 & 7.35 & 7.05 \\
Proximal width & 2.50 & 3.59 & 3.86 & 3.45 & 3.46 \\
Distal width & 1.89 & 1.51 & 2.76 & 2.50 & - \\
\hline
\end{tabular}

TABLE 33. - Measurements of the intermediate phalanges of Alcidedorbignya inopinata (in $\mathrm{mm}$ ).

\begin{tabular}{lcccc}
\hline MHNC 8372 & Digit II & Digit III & Digit IV & Digit V \\
\hline Length & 4.84 & 4.91 & 5.03 & 4.12 \\
Proximal width & 2.78 & $3.1 \mathrm{e}$ & 2.83 & 2.77 \\
Distal width & 2.25 & 2.39 & 2.14 & 2.27
\end{tabular}

TABLE 34. - Measurements of the ungual phalanges of Alcidedorbignya inopinata (in $\mathrm{mm}$ ).

\begin{tabular}{lccccc}
\hline MHNC 8372 & Digit I & Digit II & Digit III & Digit IV & Digit V \\
\hline Length & 4.53 & 5.68 & $6.7 e$ & 5.74 & - \\
Proximal width & 1.79 & 2.18 & 2.61 & 2.51 & - \\
Proximal depth & 2.28 & 2.31 & 2.42 & 2.79 & - \\
Distal width & 1.42 & 1.99 & 2.49 & 1.99 & - \\
\hline
\end{tabular}

TABLE 35. - Measurements of an isolated ungual phalanx referred to Alcidedorbignya inopinata (MHNC 13846); (in mm).

\begin{tabular}{lc}
\hline MHNC $\mathbf{1 3 8 4 6}$ & Digit III ? \\
\hline Length & $8.09 \mathrm{e}$ \\
Proximal width & 2.87 \\
Proximal depth & 2.85 \\
Distal width & 2.73 \\
\hline
\end{tabular}

Mc III is the longest metacarpal of the hand $(13.5 \mathrm{~mm})$, whereas the Mc II equals Mc IV in length $(12.3 \mathrm{~mm})$. The $\mathrm{Mc} \mathrm{V}(10.3 \mathrm{~mm})$ is longer and more robust than Mc I, for which an estimated value is $8 \mathrm{~mm}$ (in Pantolambda Mc V is approximately as long and as robust as Mc I). The Mc II, III, and IV have a transversely compressed proximal epiphysis, but a dorsoventrally compressed distal epiphysis. They are robust bones providing for some space in between them. The diaphyses do not exhibit particular scars or tuberosities. The distal epiphyses present the usual shape with two condyles and a well-developed ridge in between.

They do not present marked asymmetry, even on Mc V. The proximal epiphysis of Mc II is trochlear shaped. It is trapezoidal in outline, with a median groove. The medial (internal) part of the trochlea is reduced in length and width in comparison to the lateral one. The proximal epiphysis of Mc III shows a similar shape, but is broader with a laterally prominent dorso-lateral corner. The proximal epiphysis of Mc IV is also trapezoidal in proximal view with a betterdeveloped lateral part, but without a median sulcus, and the lateral edge presents a notch. The left Mc V presents a smoothly convex proximal epiphysis, which slightly overhangs that of Mc IV. It also presents a posterolaterally prominent and sharp proximal process. The proximal epiphysis of $\mathrm{Mc} \mathrm{V}$ is slightly compressed dorsopalmarly. It is distinctly twisted (approximately $45^{\circ}$ ) relatively to the distal epiphysis in such a way that its palmar face is oriented medially.

Like the metacarpals, the manual phalanges of Alcidedorbignya are much more slender than those of Pantolambda (Fig 88). The ratio of the proximal width/length of the first phalanx of digit III is, again, relevant. In Alcidedorbignya the ratio is $4 / 7.3 \mathrm{~mm}=0.548$; in Pantolambda the ratio is $12.3 / 15.7 \mathrm{~mm}=0.78$. The phalanges of Alcidedorbignya are therefore approximately $43 \%$ more slender than those of Pantolambda, and the massiveness of the digits in Pantolambda is even more pronounced than that of the metapodials.

The proximal phalanges of Alcidedorbignya present an unspecialised morphology. The four lateral phalanges (digits II to $\mathrm{V}$ ) have the same length, $7 \mathrm{~mm}$, and approach the length of the Mc I as preserved (distal epiphysis missing). 


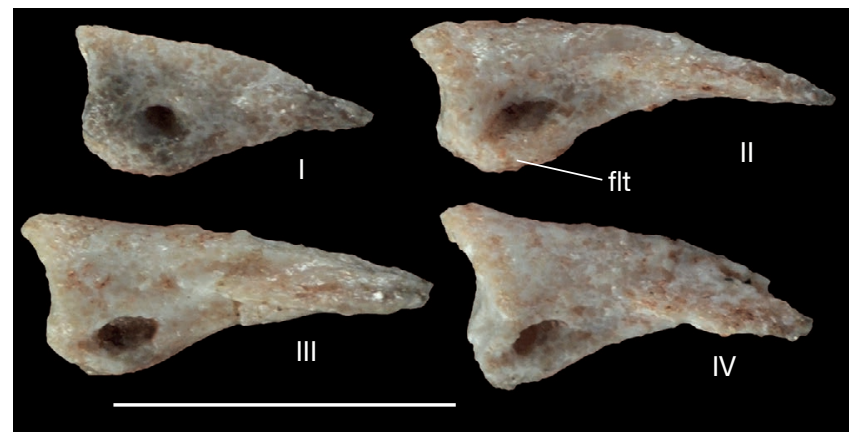

FIG. 89. - Lateral view of the ungual phalanges of digits I to IV of right manus of Alcidedorbignya inopinata (MHNC 8372). Abbreviation: flt, flexor tubercle. Scale bar: $5 \mathrm{~mm}$.

The proximal phalanx of digit III is more robust, having a mid-shaft transverse diameter of $2 \mathrm{~mm}$ vs 1.6 in the others. The proximal phalanx of the first digit is slender and shorter than the others, being $5.6 \mathrm{~mm}$ long. The diaphyses of these phalanges do not present tuberosities on their ventral side, and their epiphyses are not asymmetrical. On the whole the first phalanges of Alcidedorbignya are very similar to those of Solenodon and Marmota, but slightly longer than those of Erinaceus. They are distinctly more slender than those of Pantolambda.

The four intermediate phalanges are relatively slender as compared to Pantolambda, and are between 4 and $5 \mathrm{~mm}$ long.

The morphology of the four ungual phalanges known is of particular interest (Figs 88, 89). On MHNC 8372, they are between 5 and $5.5 \mathrm{~mm}$ long, except that of the first digit, which is shorter ( $4.5 \mathrm{~mm}$ long). This means that on the digits II-IV, the ungual phalanx is longer than the intermediate one. The ungual phalanges especially exhibit a flattened distal half, enlarged transversely. This distal half of the phalanx is oval-shaped in dorsal view. It presents two elongated and narrow lateral wings with thin and sharp edges, between which seem to penetrate the median region of the proximal half of the phalanx. At the apex of the bone the two lateral wings are well separated. At the posterior end of the wings in the median region of the phalanx, the bone is markedly constricted, resulting in a lanceolate morphology in dorsal view. The distal half of the phalanx with the elongated wings bore the nails or hoofs. In lateral view, the dorsopalmarly flattened distal half is slightly deflected palmarly but not strongly curved. Posterior to this widened apex on the plantar face of the phalanx, a robust flexor tubercle (for the flexor digitorum profundus muscle) is present overhung by a large foramen on each side (Figs 89, 90). On the dorsal side, the extensor tubercle is not particularly robust. The ungual phalanx of digit I presents the same morphology although the distal enlargement is much less developed than on the other digits. The morphology of the ungual phalanges of Alcidedorbignya is quite different from that of Pantolambda, in which they are shorter and more flattened, and with a lesser median constriction. The ungual phalanges of Alcidedorbignya clearly differ from those of

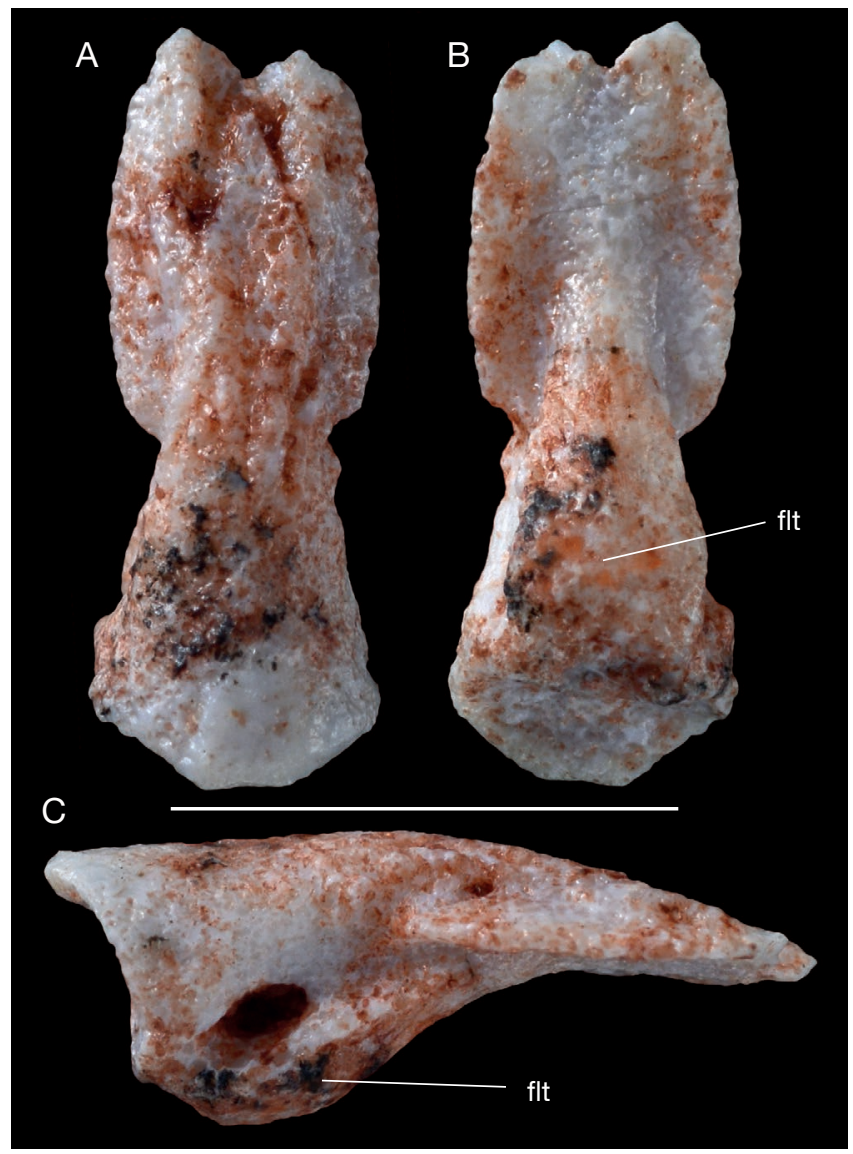

FIG. 90. - Isolated anterior ungual phalanx (digit III?) of Alcidedorbignya inopinata (MHNC 13846): A, dorsal view; B, palmar view; C, lateral or medial view. Abbreviation: flt, flexor tubercle. Scale bar: $5 \mathrm{~mm}$.

Solenodon and Erinaceus, which are transversely compressed and bear claws. They also differ from Hyopsodus, in which the hook-like ungual phalanges also probably bore claws (Gazin 1968). The ungual phalanges of Alcidedorbignya greatly resemble those of Meniscotherium in their general morphology (in dorsal view), with the difference that, in this genus, the hoofed part of the phalanx is not cleft and not deflected palmarly as in Alcidedorbignya. Like those of Meniscotherium (as suggested by Marsh 1892: 448; Gazin 1965: 64), the ungual phalanges of Alcidedorbignya did not bear true hooves (and certainly not claws), but elongated, primate-like nails. As a matter of fact, the ungual phalanges of Alcidedorbignya closely resemble, in dorsal view the scutiform morphology observed in the adapoid primates although the ungual phalanges of the latter are uncleft. This scutiform morphology has been regarded as related to the presence of nails (Franzen et al. 2009; Koenigswald et al. 2011). They differ, however, from the adapoid ungual phalanges in being slightly deflected palmarly.

When articulated, the digits II-IV are about $30-32 \mathrm{~mm}$ long, whereas the digit I is only $17 \mathrm{~mm}$ long (it does not reach the level of the distal extremity of the first phalanx of the second digit). The length of digit $\mathrm{V}$ can be estimated at $25-26 \mathrm{~mm}$. 


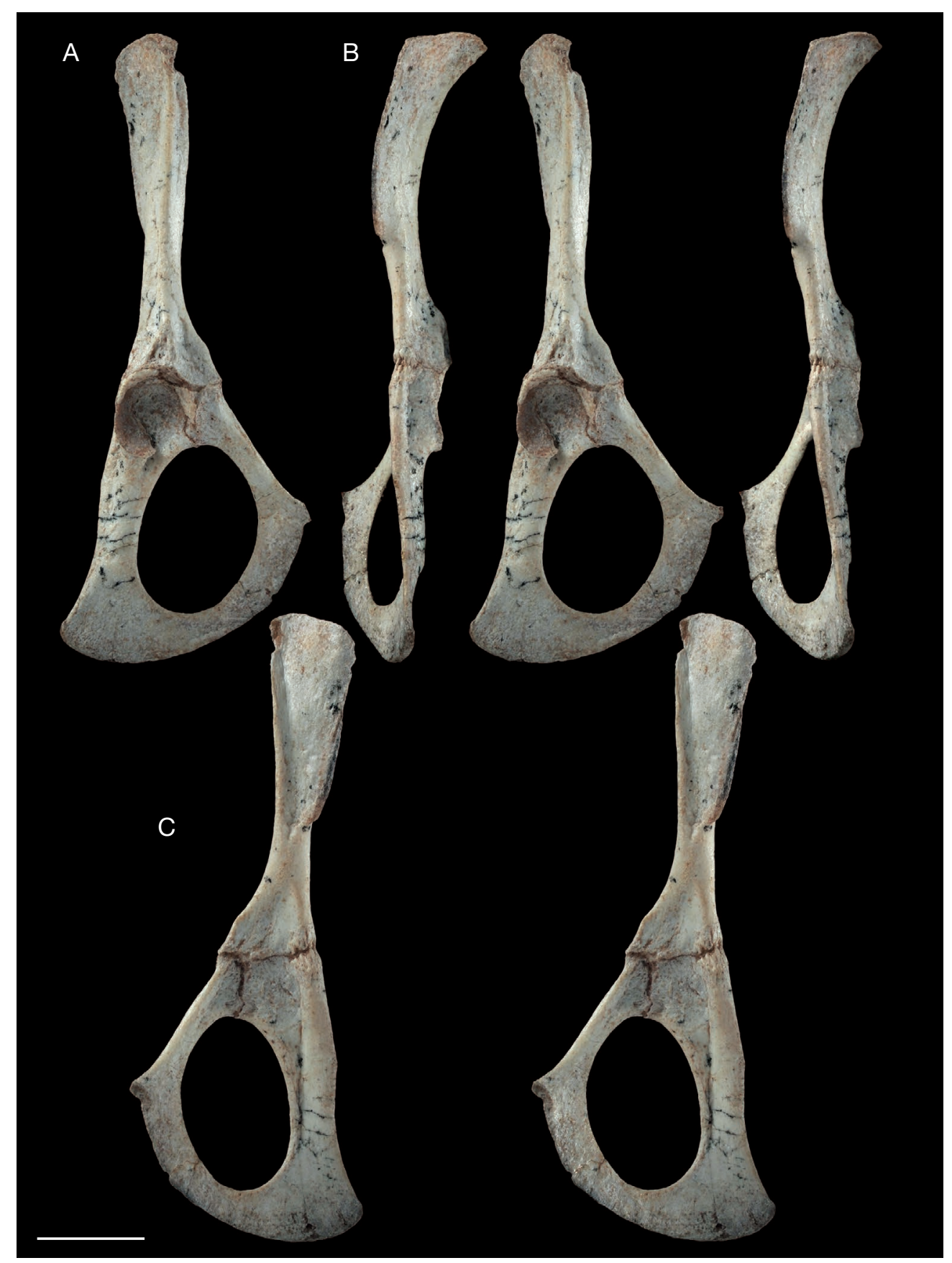

FIG. 91. - Right innominate of Alcidedorbignya inopinata (MHNC 8372): A, stereophotographs of lateral view; B, stereophotographs of dorsal view; C, stereophotographs of medial view. Scale bar: $1 \mathrm{~cm}$.

\section{Pelvic girdle and Hind limb}

Os Coxae. (Figs 91, 92; Table 36) The complete pelvis of MHNC 8372 is known in Alcidedorbignya by both complete innominates (ossa coxae). The ilium represents approximately $59 \%$ of the total length of the bone. On the wing of the ilium, the gluteal fossa is broad and oriented latero-dorsally whereas the iliac fossa is limited to a narrow, ventral strip. The two fossae are separated by a sharp acetabular border (sensu Grassé 1967). This condition is also present in Pantolambda, but it is absent in Solenodon, Marmota and Erinaceus in which the gluteal and iliac fossae are approximately the same size.
The gluteal fossa of Alcidedorbignya is more triangular than rectangular in outline, in relation to the dorsoventral development of the anterior part of the ilium and to the weakly developed posterodorsal iliac spine. Furthermore, this triangular morphology is emphasized by the great development of the anteroventral iliac spine, which is conspicuously deflected ventrolaterally. This condition is similar to that observed in Pantolambda although in this genus the anteroventral iliac spine is much larger and more deflected ventrally and the anterior edge of the ilium reaches an angle of $90^{\circ}$ with the dorsal edge of the bone. A ventrolateral inflection of the 

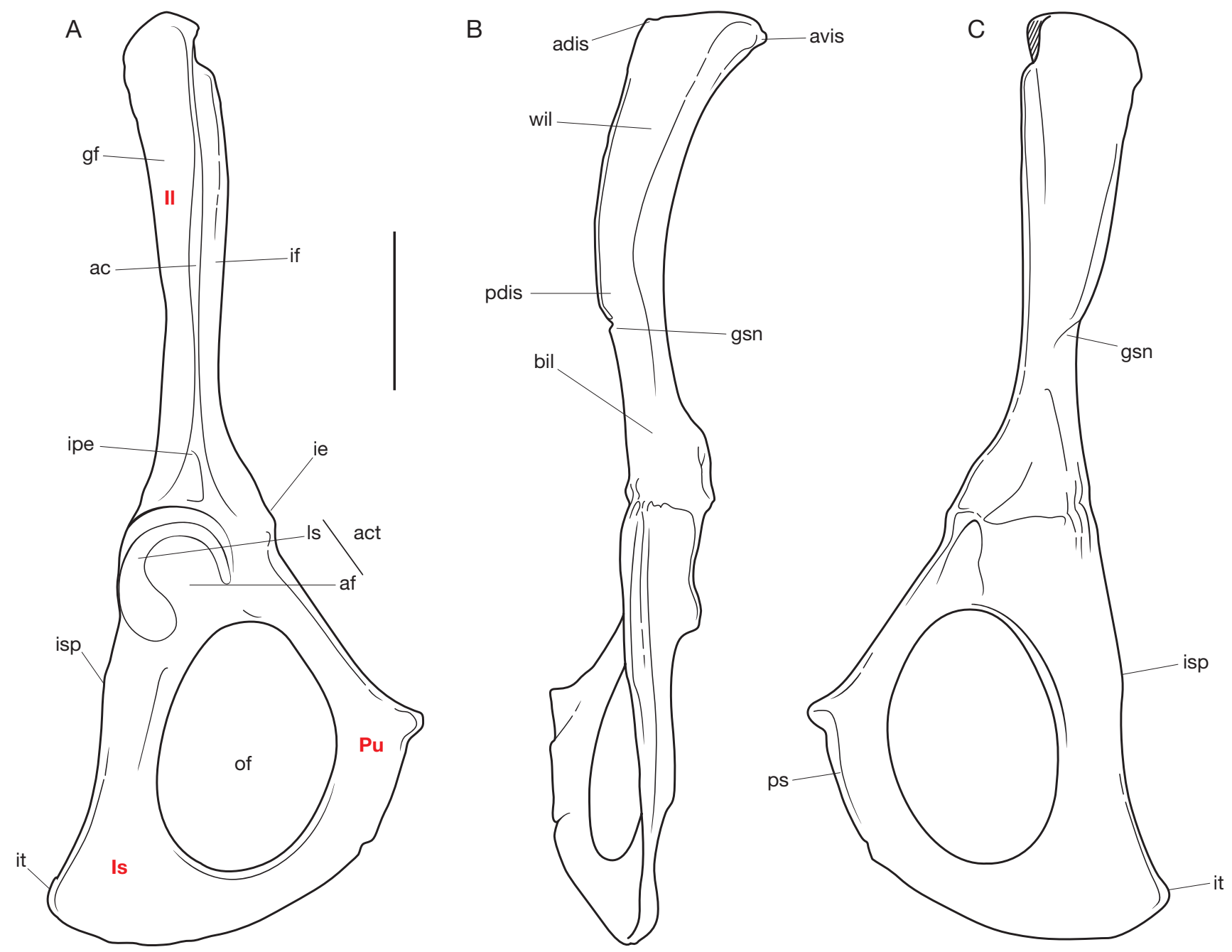

FIG. 92. - Right innominate of Alcidedorbignya inopinata (MHNC 8372): A, lateral view; B, dorsal view; C, medial view. Abbreviations: ac, acetabular crest; act, acetabulum; adis, anterodorsal iliac spine; af, acetabular fossa; avis, anteroventral iliac spine; bil, body of the ilium; gf, gluteal fossa; gsn, greater ischiatic notch; if, iliacus fossa; ie, iliopubic eminence; II, ilium; ipe, iliopectineal eminence; Is, ischium; isp, ischiatic spine; it, ischiatic tuberosity; Is, lunate surface; of, obturator foramen; pdis, posterodorsal iliac spine; ps, pubis symphysis; Pu, pubis; wil, wing of the ilium. Scale bar: $1 \mathrm{~cm}$.

anteroventral iliac spine is also present, although to a much lesser extent, in Mayulestes. It is absent in Solenodon, but in Marmota the relatively modest anteroventral iliac spine is strongly deflected laterally. The distance between the centre of the acetabulum and the posterior edge of the sacroiliac articulation (approximately the posterodorsal iliac spine) is long, representing approximately $25 \%$ of the total length of the innominate. In contrast to Mayulestes, there is no angulation between the ilium and the ischium in Alcidedorbignya. The ischium and pubis form a triangle surrounding a broad obturator foramen, and the pubic symphysis is short. The ischiatic tuberosity protrudes dorsally (more than in Mayulestes), whereas the ischiatic spine is weak. The acetabulum is widely open; the lunate surface, the articular area, extends along three-quarters of the circumference. It is composed of two lobes, the anterior one facing posterolaterally and the posterior one facing ventrolaterally. The posterior lobe is smaller and distinctly narrower than the anterior one. At the contact of the two lobes the lunate surface is strongly
TABLE 36. - Measurements of the right os coxae of Alcidedorbignya inopinata (in mm).

MHNC 8372

\section{Total length}

Length of ilium from anterior edge to centre of acetabulum

Length of ischium from centre of acetabulum to posterior edge

59.92

ength of ischium from dorsal edge of suture with ilium to posterior edge

Length of pubis from centre of acetabulum to anterior point of pubic symphysis

Length of pubis from ventral edge of suture with ilium to pubic symphysis

Maximum dorsoventral width of the ilium

Width of the ilium at the level of the greater ischiatic notch

Greatest width of iliac fossa

Greatest diameter of the obturator foramen

Lesser diameter of the obturator foramen

Anteroposterior diameter of the acetabulum

Dorsoventral diameter of the acetabulum

Length from posterior border of ischiatic spine to ischiatic tuberosity
35.46

24.85

27.47

18.36

15.98

10.66 


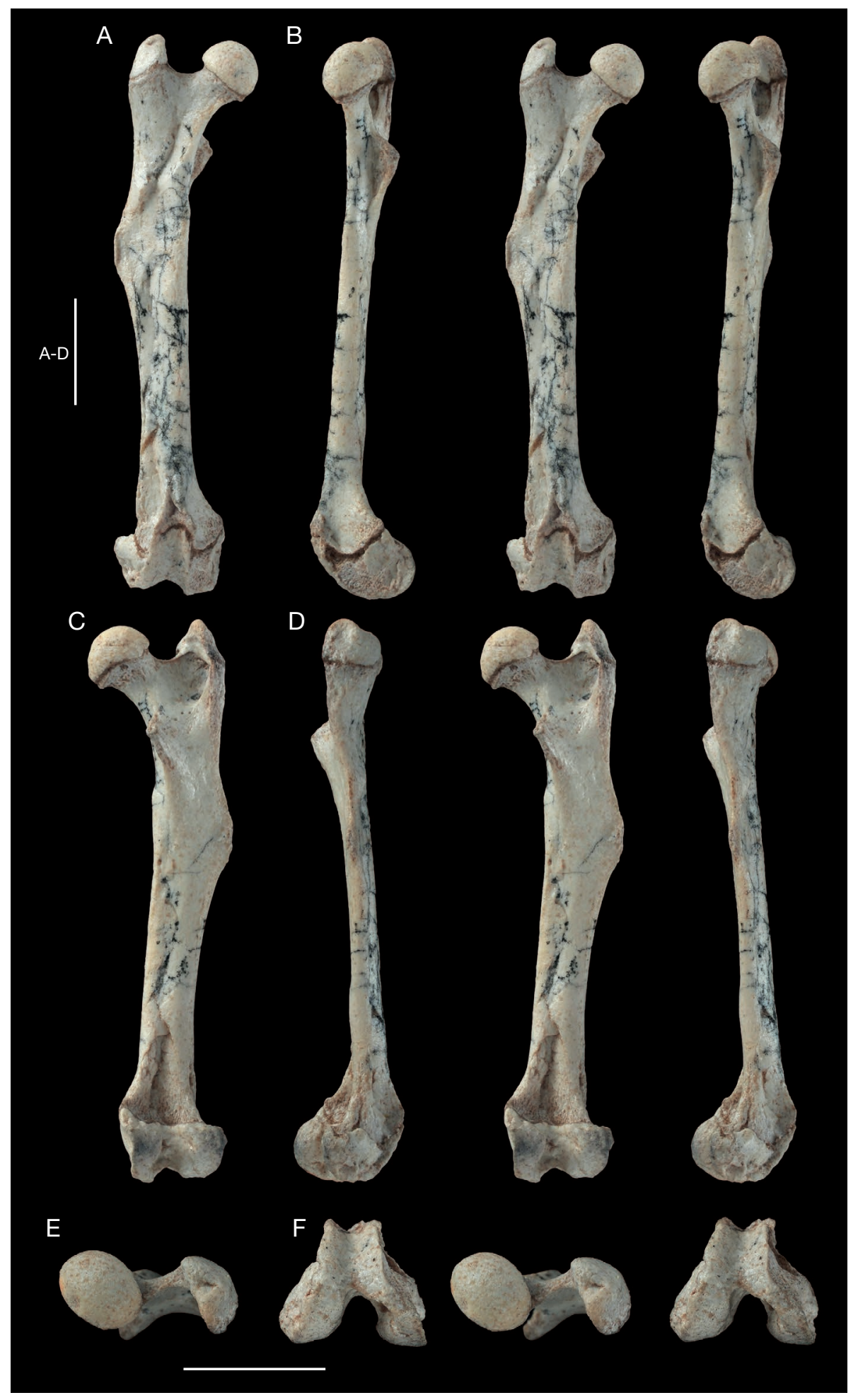

FIG. 93. - Right femur of Alcidedorbignya inopinata (MHNC 8372): A, stereophotographs of anterior view; B, stereophotographs of medial view; C, stereophotographs of posterior view; D, stereophotographs of lateral view; E, stereophotographs of proximal view; F, stereophotographs of distal view. Scale bars: $1 \mathrm{~cm}$.

constricted and the transition between the lobes is markedly pronounced. Just anterior to it is the ventrocaudal iliac spine, for the origin of the rectus femoris muscle. This eminence is in fact a small, elevated fossa, which denotes a relatively strong muscle attachment. Ventrally, anterior to the junc- tion of the ilium and pubis, the attachment area of the psoas minor is weak. The ischium is thicker than the pubis, and the origin of adductors is not emphasized. In posterior view, when the two innominates are articulated with the sacrum, the ischium faces more laterally than ventrally. 
A

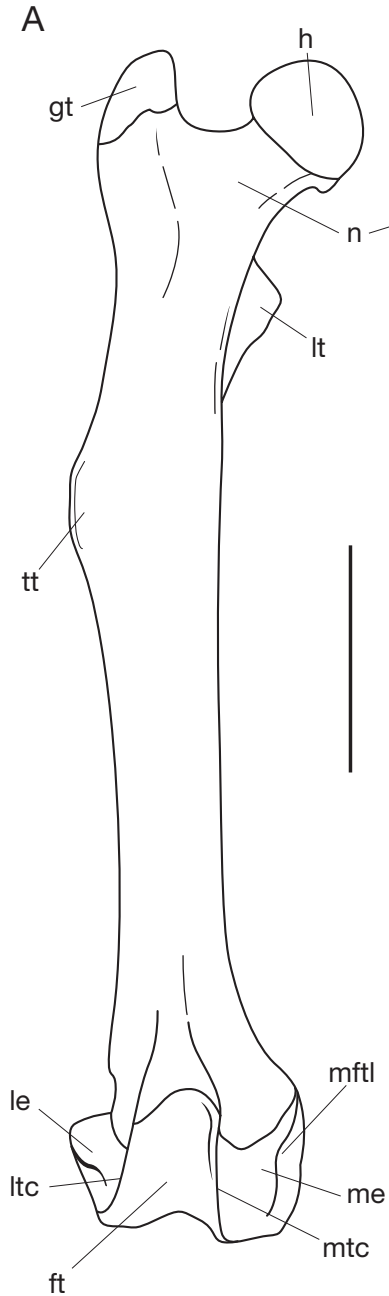

B

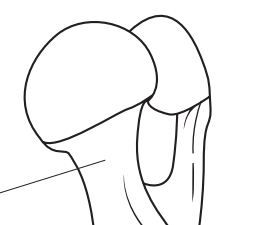

It
$\mathrm{C}$

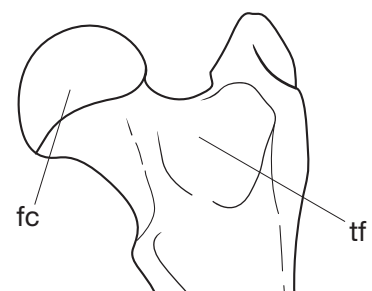

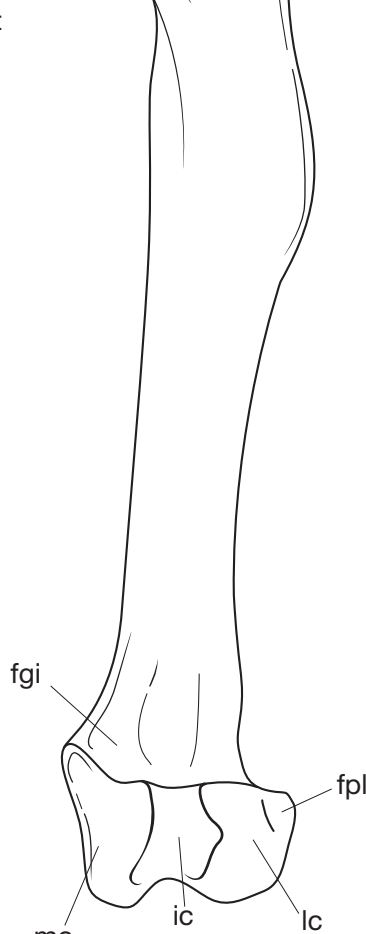
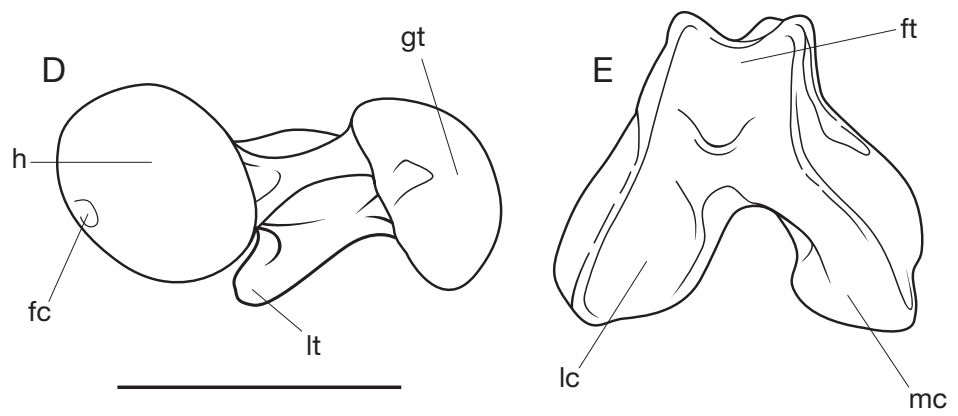

FIG. 94. - Right femur of Alcidedorbignya inopinata (MHNC 8372): A, anterior view; B, medial view; C, posterior view; D, proximal view; E, distal view. Abbreviations: fc, fovea capitis; fgi, fossa for the gastrocnemius internus; fpl, fossa for the popliteus muscle; ft, femoral trochlea; gt, greater trochanter, h, head; ic, interdondylar fossa; Ic lateral condyle; le, lateral epicondyle; It, lesser trochanter; Itc, lateral trochlear crest; mc, medial condyle; me, medial epicondyle; mftl, fossa for attachment of the medial femorotibial ligament; $\mathbf{m t c}$, medial trochlear crest; $\mathbf{n}$, neck; $\mathbf{t f}$, trochanteric fossa, $\mathbf{t t}$, third trochanter. Scale bars: $1 \mathrm{~cm}$.

Femur and Patella. (Figs 93-95; Tables 37, 38) The two femora of MHNC 8372 are known, and suffered some distortion during fossilization especially at their distal extremity (Figs 93, 94). Three isolated partial femora have been referred to Alcidedorbignya inopinata (MHNC 8451, 8452, 8453). They all lack the distal extremity of the bone and the proximal epiphyses of the head and greater trochanter. Three other specimens have also been referred to Alcidedorbignya inopinata (Fig. 95). MHNC 8454 is a distal extremity of a left femur, which probably suffered some surrounding compression (see above), MHNC 8455 is a distal epiphysis of a right femur and MHNC 8456 is a distal extremity of a left femur (lacking part of the lateral condyle) articulated to the proximal extremity of the tibia.

The femur is the longest limb bone in Alcidedorbignya and exhibits a slender diaphysis, straight in anterior and lateral view. In this respect it resemble the femur of Pantolambda but is distinctly more slender. In proximal view, the proximal 

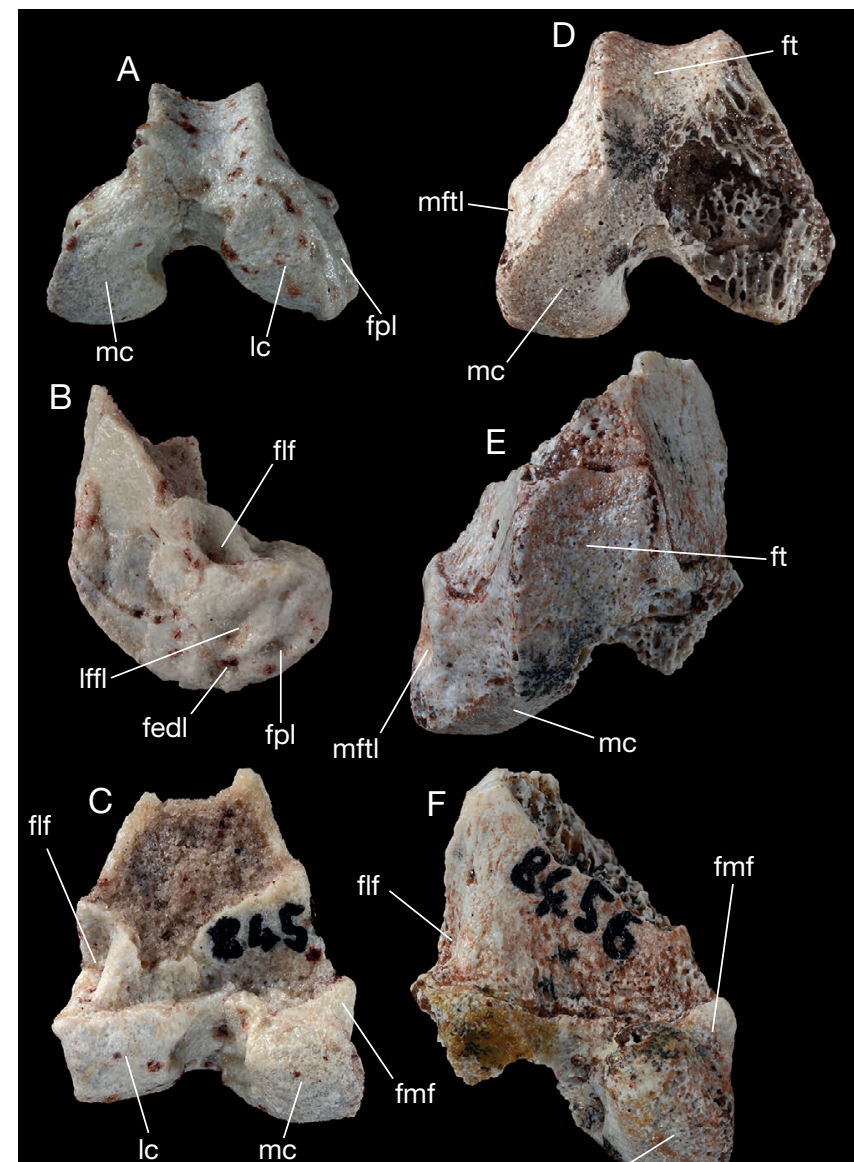

$\mathrm{m}$
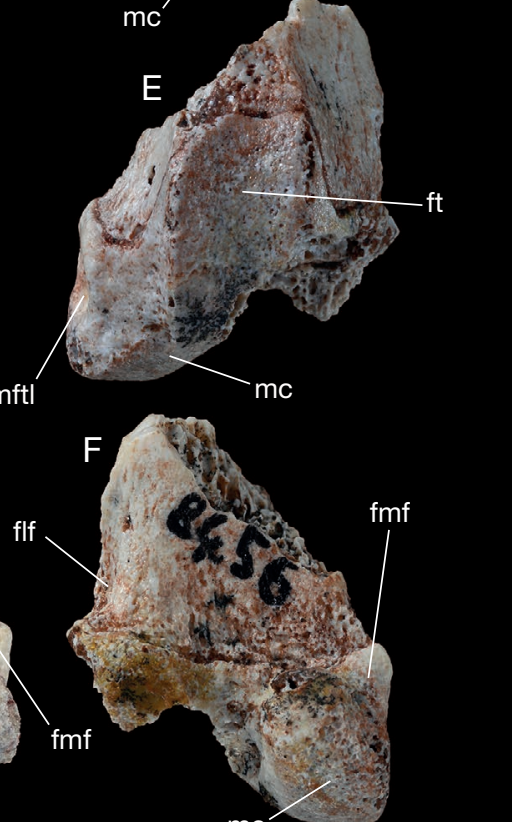

nc

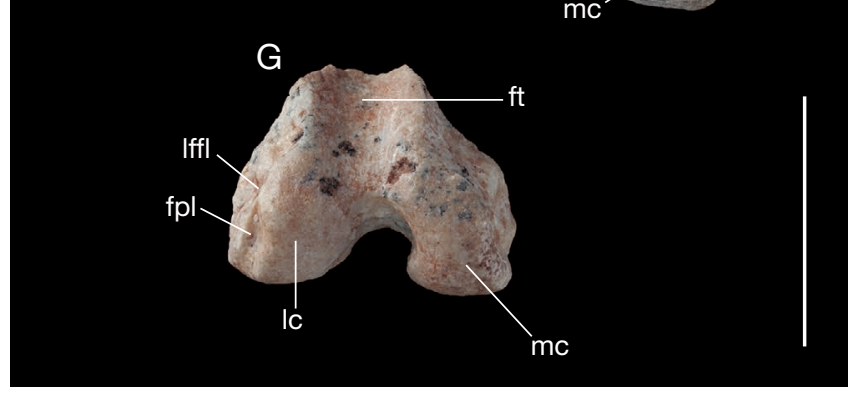

FIG. 95. - Distal extremity of femora of Alcidedorbignya inopinata: A, distal view of left femur (MHNC 8454); B, the same in lateral view; $\mathbf{C}$, the same in posterio view; D, distal view of left femur (MHNC 8456); E, the same in anterior view; F, the same in posterior view; $\mathbf{G}$, distal view of right femur (MHNC 8455). Abbreviations: fedl, fossa for the tendon of the extensor digitorum longus; flf, fossa for latera fabella; fmf, facet for medial fabella; fpl, fossa for the popliteus muscle; ft, femoral trochlea; Ic, lateral condyle; Iffl, fossa of attachment for the lateral femorofibular ligament; $\mathbf{m c}$, medial condyle; $\mathbf{m f t l}$, fossa for attachment of the medial femorotibial ligament. Scale bar: $1 \mathrm{~cm}$.

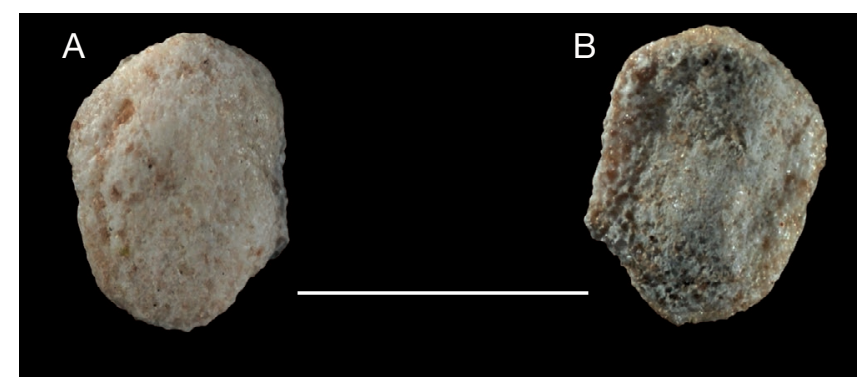

FIG. 96. - Right patella of Alcidedorbignya inopinata (MHNC 8372): A, anterior view; B, posterior view. Scale bar: $5 \mathrm{~mm}$.
TABLE 37. - Measurements of the right patella of Alcidedorbignya inopinata (in mm).

\begin{tabular}{lc}
\hline & MHNC 8372 \\
\hline Length & 5.03 \\
Width & 3.92 \\
\hline
\end{tabular}

epiphysis appears slightly twisted in relation to the distal one (transverse axis of the epicondyles), the femoral head protruding anteriorly. This anterior protrusion can also be observed in lateral view on the left femur. A twisting of the proximal epiphysis in relation to the axis of the epicondyles is hardly present in Pantolambda. It is absent in Solenodon, Erinaceus, and Marmota, but it is very pronounced in Procavia and especially in Dendrohyrax. The right femoral head has suffered anteroposterior compression during fossilisation and the left one is not distorted but is missing some of its posteromedial edge. The articular area of the head is almost perfectly hemispherical but slightly less convex than in Pantolambda. There is no posterior extension of this articular facet towards the femoral neck or trochanteric fossa. The fovea capitis is reduced and located posteromedially. It is surrounded by the articular surface and not located in a triangular notch that excavates the posteromedial edge of the articular surface of the head, as is observed in Procavia or Dendrohyrax. The condition of Alcidedorbignya is present in Solenodon, Erinaceus, Echinosorex, Marmota, and Pantolambda. In the latter genus, however the fovea capitis is much more developed than in Alcidedorbignya. In medial view, the head (as well as the whole proximal extremity of the bone) is only slightly bent anteriorly as is observed in Pantolambda, Echinosorex, and Marmota, but differing from the condition of Solenodon, Mayulestes and Recent didelphids in which the inflexion is strong. The femoral neck is long and slender. It is slightly longer than in Pantolambda and distinctly longer than in Solenodon, Tenrec, and Erinaceus. It approaches the morphology observed in Echinosorex. The greater trochanter is higher than the femoral head, especially the proximally prominent posterior part, where the gluteus medius inserted. This condition is similar to that in Echinosorex but differs from that of Pantolambda, in which the greater trochanter is as high as the head. Posteriorly, the trochanteric fossa is wide and deep as in Pantolambda. Laterally, on the distal extension of the lateral edge of the greater trochanter, a prominent third trochanter can be observed, located approximately on the proximal $2 / 5$ of the diaphysis. It differs from that of Pantolambda, which is more distal, located almost at mid-shaft. The third trochanter of Alcidedorbignya is more distally located than in Solenodon, Tenrec, Echinosorex, and Marmota. It is slightly more proximal than in Hyopsodus and Meniscotherium but less than in Pleuraspidotherium, in which it is located at the level of the lesser trochanter. Medially, the lesser trochanter is a triangular flat prominence, which protrudes posteromedially, located proximally below the femoral neck. In Pantolambda the lesser trochanter is similar to that of Alcidedorbignya, with the same posteromedial orientation, but slightly larger. Posteriorly, the insertions of the quadratus femoris and adductors cannot be discerned. 
TABLE 38. - Measurements of the femur of Alcidedorbignya inopinata (in mm).

\begin{tabular}{|c|c|c|c|c|}
\hline Length & 51.70 & - & - & - \\
\hline Proximal transverse width & 12.81 & - & - & - \\
\hline Anteroposterior diameter of head & 6.69 & - & - & - \\
\hline Transverse diameter of head & 6.57 & - & - & - \\
\hline Length from tip of greater trochanter to distal edge of third trochanter & 21.70 & - & - & - \\
\hline Length from tip of greater trochanter to distal end of trochanteric fossa & 8.34 & - & - & - \\
\hline Length from proximal edge of head to distal end of lesser trochanter & 15.68 & - & - & - \\
\hline Anteroposterior diameter at mid-length of the shaft & 3.36 & - & - & - \\
\hline Transverse width of distal epiphysis & 11.50 & 12.65 & 11.72 & 13.36 \\
\hline Anteroposterior length of distal epiphysis & 9.17 & 10.62 & 9.39 & 12.61 \\
\hline Width of lateral condyle & 4.70 & 4.41 & 4.27 & 5.11 \\
\hline Width of medial condyle & 4.23 & 4.74 & 4.35 & - \\
\hline Width of femoral trochlea & 3.93 & 3.88 & 4.71 & 5.08 \\
\hline Length of the femoral trochlea & 6.77 & 8.03 & - & 10.62 \\
\hline
\end{tabular}

The distal epiphysis is slightly wider than antero-posteriorly long on the right femur of MHNC 8372 (W=10 mm vs $\mathrm{L}=8.5 \mathrm{~mm}$ ), on MHNC 8456 (W $=13.3 \mathrm{~mm}$ vs $\mathrm{L}=$ $11.4 \mathrm{~mm}$ ), and on MHNC 8454 (W = $12.4 \mathrm{~mm}$ vs $\mathrm{L}=$ $10.2 \mathrm{~mm})$. A similar condition is present in Pantolambda (W $=41.2 \mathrm{~mm}$ vs $\mathrm{L}=38.5 \mathrm{~mm}$ ), but in this taxon the difference is much less pronounced than in Alcidedorbignya. The groove of the trochlea is very deep on the two femora of MHNC 8372. However, it is not as deep in the three other isolated femora in which it is preserved (MHNC 8454,8455 , and 8456). In fact, the depth of the trochlea in MHNC 8372 is likely to be the result of post-mortem "surrounding compression" (see above) during fossilisation. Similarly MHNC 8454 seems also to have suffered some "surrounding compression" but to a much lesser extent than MHNC 8372. This slight distortion probably increased the sharpness of the trochlear crests and the depth of the trochlea but apparently not the proportions of the epiphysis. Taking these taphonomic considerations into account, the femoral trochlea of Alcidedorbignya was probably not as narrow as suggested by the distorted femora of MHNC 8372. It was moderately deep, and bordered by sharp but not extremely elevated trochlear crests (as observed on MHNC 8455 and 8456, which are not distorted) (Fig. 95). The width and depth of the trochlea as well as the development of the crests greatly resembles the condition of Pantolambda. They also resemble the condition in Marmota and Procavia (although slightly narrower than in the latter genus). It is also very similar to that of Mayulestes but proximodistaly longer and not asymmetrical as in this metatherian. The trochlea of Alcidedorbignya is narrower, deeper and has sharper crests than in Solenodon, Tenrec, and Erinaceus. It is wider, shallower, and has slightly lower crests than in Echinosorex. The trochlea of Alcidedorbignya also resembles that of Hyopsodus, although in the latter genus the trochlea is slightly deeper, the crests slightly more elevated and especially the trochlea is asymmetrical in distal view with the medial crest more developed than the lateral one. It is not as narrow or as deep as that of Meniscotherium.

The two ossified patellae were found associated with the specimen (Fig. 96). The fact that they do not perfectly fit in the grooves of their respective trochlea is another indication that the narrowness of the trochleae of MHNC 8372 is an artefact and that they have suffered some post-mortem transverse compression. The patellae are ovoid and bear a longitudinal, median saddle-shaped ridge on their articular surface. This ridge is smooth but distinctly developed. It is bordered medially and laterally by two longitudinal shallow fossae. This condition is also present on the patella of Pantol$a m b d a$, but less emphasized. It is also possible that the patella of MHNC 8372 has suffered some surrounding compression (as is observed on the distal extremity of the femur), and that the morphology of the ridge and fossae has been emphasized by this taphonomic effect.

In lateral view, the femoral trochlea of the distal epiphysis of Alcidedorbignya does not extend further proximally than the level of the proximal edge of the condyles (but see below). It is therefore relatively short and differs from the elongated trochlea observed in some saltatorial mammals as Lepus, Dasyprocta, or Rhynchocyon or cursorial mammals as Tragulus or the fossils Diacodexis or Meniscotherium.

However, the distal extremity of the femoral shaft, on its anterior side and proximal to the trochlea, bears a fossa in both femora of MHNC 8372. This fossa could also be an artefact of fossilisation, but in MHNC 8454 and 8456 (visible only on lateral side of this latter specimen) the trochlear groove and crests clearly extend proximally on the distal end of the diaphysis possibly corresponding to the suprapatellar bursa, on which the patella could have slid more proximally. Therefore, the condition observed on the femora of MHNC 8372 , although enhanced by compression of the bone, actually reveals that the patella could have slid slightly more proximally on the diaphysis. This condition has not been observed in Pantolambda.

The medial epicondyle exhibits a deep fossa for the attachment of the medial (femorotibial) collateral ligament (Fig. 95B). On the lateral epicondyle three distinct fossae are observable, arranged in a triangle. The most proximal fossa is located roughly in the centre of the epicondyle and is probably for the lateral (femorofibular) collateral ligament. Slightly distal and posterior to this ligament fossa, on the posterolateral edge of the lateral condyle, is another 
TABLE 39. - Measurements of the left tibia of Alcidedorbignya inopinata (in mm)

\begin{tabular}{lc}
\hline & MHNC 8372 \\
\hline Length & 46.07 \\
Transverse width of proximal epiphysis & 9.68 \\
Anteroposterior length of proximal epiphysis & 9.31 \\
Width of lateral condyle & 4.41 \\
Width of medial condyle & 4.31 \\
Length of lateral condyle & 5.61 \\
Length of medial condyle & 7.68 \\
Length from proximal epiphysis to tibial tuberosity & 22.12 \\
Transverse width of distal epiphysis & 7.25 \\
Anteroposterior length of distal epiphysis & 4.70 \\
Width of the malleolus & 2.56 \\
Length of the malleolus & 3.15 \\
\hline
\end{tabular}

depression, which excavates the lateral edge of the lateral condyle. This fossa, which borders the articular surface is for the origin of the tendon of the popliteus muscle. Anterior to the popliteus fossa, also on the edge of the condyle articular surface and located approximately at the limit between the condyle and the trochlea is a smaller fossa for the tendon of the extensor digitorum longus. In Pantolambda the popliteus fossa is two to three times larger than in Alcidedorbignya. On the posterior side of the distal extremity of the diaphysis of Alcidedorbignya, laterally, and just proximal to the lateral epicondyle and lateral edge of the lateral condyle is a deep fossula (this feature is especially visible on MHNC 8454 and 8456) (Fig. 95B, C). It probably received the lateral fabella embedded in the tendon of the gastrocnemius externus, which had its origin in this area just proximal to the lateral condyle. In posterior view, on the medial side of the diaphysis no distinct fossula is observed. However, just proximal to the medial condyle, a wide and shallow depression bordered by a sharp crest medially, is present. This depression probably received the origin of the gastrocnemius internus. In posteromedial view of the medial condyle, proximal to the fossa for the medial femorotibial ligament, is a small slightly concavoconvex facet, on which probably rested the medial fabella (Fig. 95C, F). It is present on all four specimens, which still preserve this region of the bone. Because this facet is smaller than the lateral fossula for the lateral fabella, it is therefore possible that the medial fabella was smaller than the lateral, as is generally the case in mammals (Lessertisseur \& Saban 1967). Fabellar fossae are apparently absent in Pantolambda. Fabellae are well developed in Procavia, Marmota, and Solenodon. Small fabellae are also present in Echinosorex and Erinaceus.

In distal view, the lateral condyle appears slightly wider and distinctly more massive than the medial one on MHNC 8372 and 8455 . However, this is not true in MHNC 8454. The medial condyle slightly projects medially but not posteriorly, which gives a slightly asymmetrical outline to the distal epiphysis. In Pantolambda, the medial condyle does not project medially but extends strongly posteriorly, well posterior to the level of the posterior edge of the lateral condyle. A medially projecting medial condyle is also present in
Erinaceus and Echinosorex. The medial condyle has a deep concavity on its anterolateral (internal) edge. Because of this morphology, in distal view, it is much wider posteriorly than anteriorly. This concavity is much less pronounced in Pantolambda but, as in Alcidedorbignya, the medial condyle is distinctly narrower anteriorly than posteriorly. The width of the lateral condyle of Alcidedorbignya is more constant anteroposteriorly. Because of the median concavity of the medial condyle, the intercondylar fossa is broad and deep.

Tibia. (Figs 97, 98; Table 39) The two tibiae of MHNC 8372 are preserved and almost complete. Two other isolated specimens are referred to Alcidedorbignya inopinata. They are: MHNC 8456 (a partial proximal epiphysis missing the lateral condyle and associated with a distal extremity of the femur); and MHNC 8457 (a diaphysis of a left tibia). The tibiae of the skeleton MHNC 8372 have suffered some "surrounding compression" but the isolated diaphysis and the proximal epiphysis are apparently undistorted. The bone is slender. In MHNC 8372 it is straight in anterior view and slightly bowed anteriorly in lateral view. The diaphysis MHNC 8457 is slightly bowed medially in anterior view. In MHNC 8372 the tibia is $88 \%$ of the total femur length. The ratio in Pantolambda (85\%) is almost the same as that of Alcidedorbignya. The proximal half is transversely compressed below the proximal epiphysis because of postmortem compression and the depth of its fossae and the sharpness of its crests are probably emphasized. In MHNC 8457 the section of the proximal extremity of the diaphysis is very roughly triangular (slightly longer than wide) and clearly differs from the condition in Pantolambda, in which the diaphysis is two to three times longer than wide on most of its proximal half. The anterior tibial crest is wide and rounded. It extends to mid-shaft, and its distal apex forms an elongated protruding tuberosity, which is distinctly deflected laterally. It bears scars for insertion of the gracilis and semitendinosus. This morphology is also present in Pantolambda, but in this genus the tibial crest extends further distally, protrudes more anteriorly and the distal tubercle is more developed and more deflected laterally. The morphology of the tibia of Alcidedorbignya is strikingly different from that of Mayulestes. On the proximal end of the bone, on its anterior side, the tibial tuberosity is low and does not reach the level of the condyles proximally. It is not very salient anteriorly, which gives to the proximal epiphysis the shape of an equilateral triangle with greatly rounded angles. This condition is even more pronounced in Pantolambda, in which the tibial tuberosity is almost flat and the proximal extremity of the tibia is roughly ovoid to semicircular. In proximal view, this proximal epiphysis of the tibia of Alcidedorbignya is slightly wider than long. The condyles are sub-equal in size on the right tibia, whereas the medial condyle appears longer anteroposteriorly and extends posteriorly more than the lateral one on the left tibia. This corresponds to the asymmetry observed also on the condyles of the left femur, which, therefore is probably not related only to post-mortem deformation. The medial tibial condyle is concave whereas the lateral one is flat. In Pantolambda the 


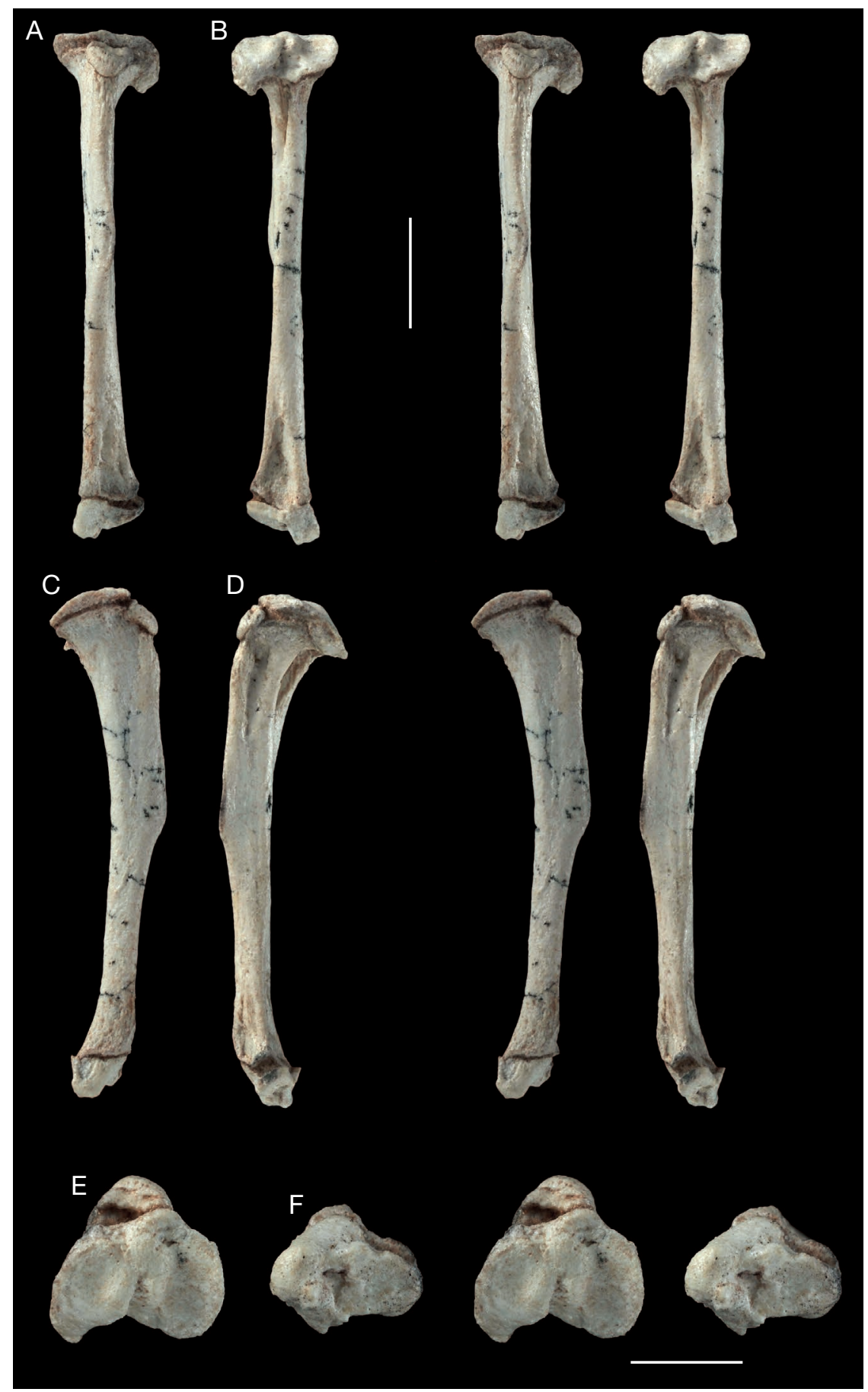

FIG. 97. - Left tibia of Alcidedorbignya inopinata (MHNC 8372): A, stereophotographs of anterior view; B, stereophotographs of posterior view; C, stereophotographs of medial view; D, stereophotographs of lateral view; E, stereophotographs of proximal view; $\mathbf{F}$, stereophotographs of distal view. Scale bars: A-D, $1 \mathrm{~cm}$; E, F, $5 \mathrm{~mm}$.

lateral condyle is distinctly smaller than the medial one and, relatively, much smaller than in Alcidedorbignya. On the posterolateral angle of the proximal epiphysis a posteriorly protruding process bears on its distal side the articular facet for the fibula (see especially the left tibia of MHNC 8372; Fig. 97). The articular surface of the lateral condyle does not extend onto this process. On the posterolateral angle of the epiphysis, distal to the process, the fibular facet is well formed and faces ventrally. The presence of a distinct proximal tibiofibular facet is evidence that the bones articulated at a synovial joint. The intercondyloid eminence is low and much less developed than in Pantolambda. Laterally, the proximal fossa, where the tibialis anterior originated, is deep but narrow as in Pantolambda. Posteromedially to this fossa, on the posterior edge of the proximal diaphysis, is a deep posterior fossa. The two fossae are separated by a salient ridge, which is located just distally to the tibiofibular articulation on the epiphysis. In Pantolambda, this ridge is much lower than in 

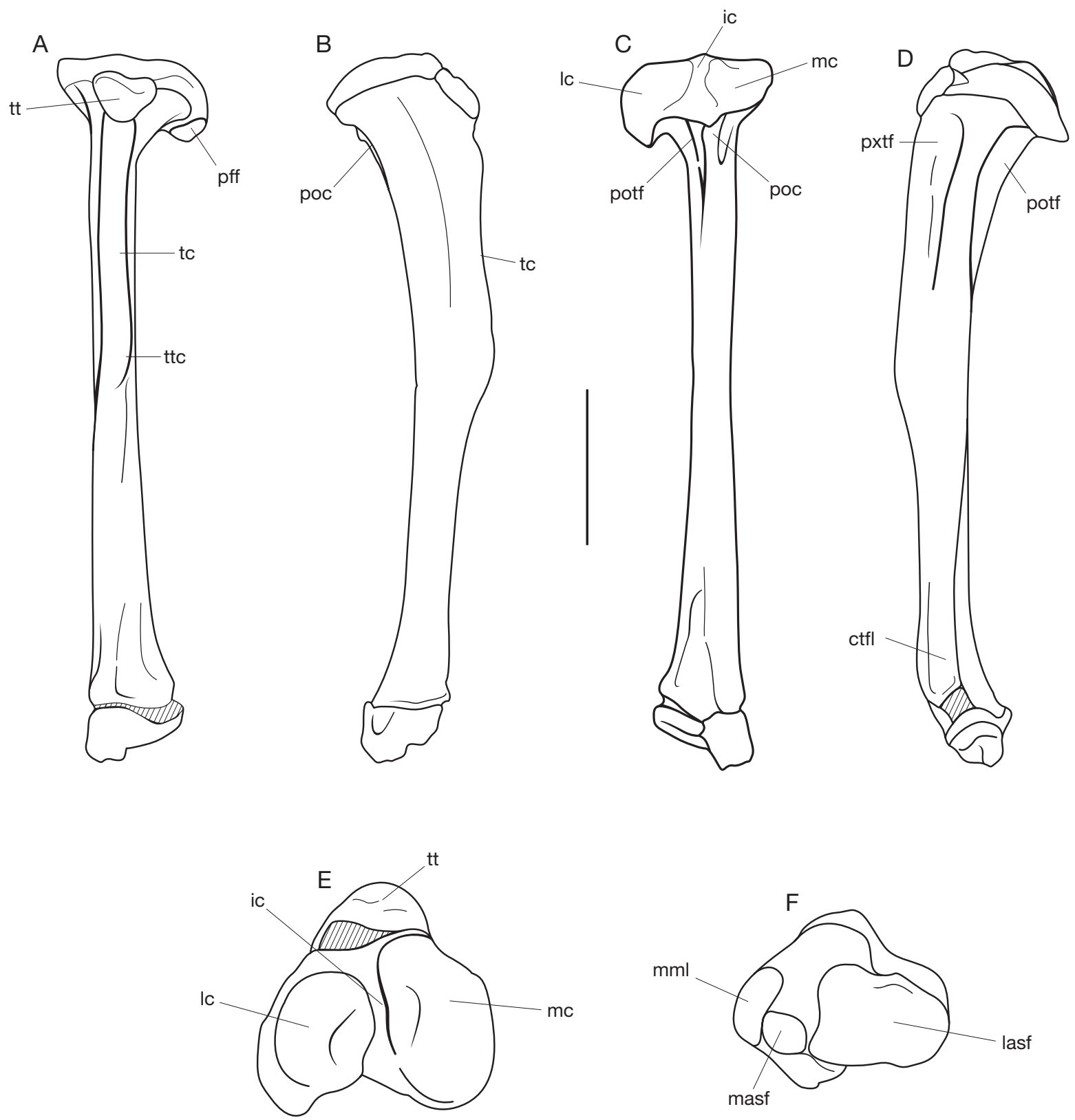

FIG. 98. - Left tibia of Alcidedorbignya inopinata (MHNC 8372): A, anterior view; B, medial view; C, posterior view; D, lateral view; E, proximal view; F, distal view. Abbreviations: ctfl, crest for the tibiofibular ligament; ic, intercondyloid eminence; lasf, lateral astragalar facet; Ic, lateral condyle, masf, medial astragalar facet; mc, medial condyle; mml, medial maleolus; pff, proximal fibular facet; poc, popliteus crest; potf; posterior tibial fossa; pxtf proximal tibial fossa; tc, tibial crest; tt, tibial tuberosity; ttc, tuberosity of the tibial crest. Scale bar: A-D, $1 \mathrm{~cm}$; E, F, $5 \mathrm{~mm}$.

Alcidedorbignya. The posterior tibial fossa is bordered medially by a salient crest, on the medial side of which probably inserted the popliteus (Fig. 98C). Muscle scars indicate that this insertion is likely to have extended further distally on the diaphysis of the tibia (approximately on the proximal third of the diaphysis), as it does in the dog (Evans \& de Lahunta 2012). In Pantolambda, the popliteus crest is well developed and the muscle scar appears to be larger than in Alcidedorbignya, in correlation with the large size of the origin of the popliteus on the femur. On the proximal extremity of the popliteus crest, facing posteromedially, is an excavated muscular scar, which could be for the insertion of the semi-membranosus muscle. However, because the insertion of the semimembranosus is generally located more medially to anteromedially (Davis 1964; Barone 2000; Evans and de Lahunta 2012) while in Alcidedorbignya this scar is located and faces posteromedially, it could also be for the proximal part of the insertion of the popliteus. On the distal extremity of the diaphysis, laterally, is a salient crest for the attachment of a tibiofibular ligament. On the distal epiphysis, the malleolus is massive and prominent and forms an obtuse angle with the lateral part of the articular surface. On the lateral edge of the malleolus 


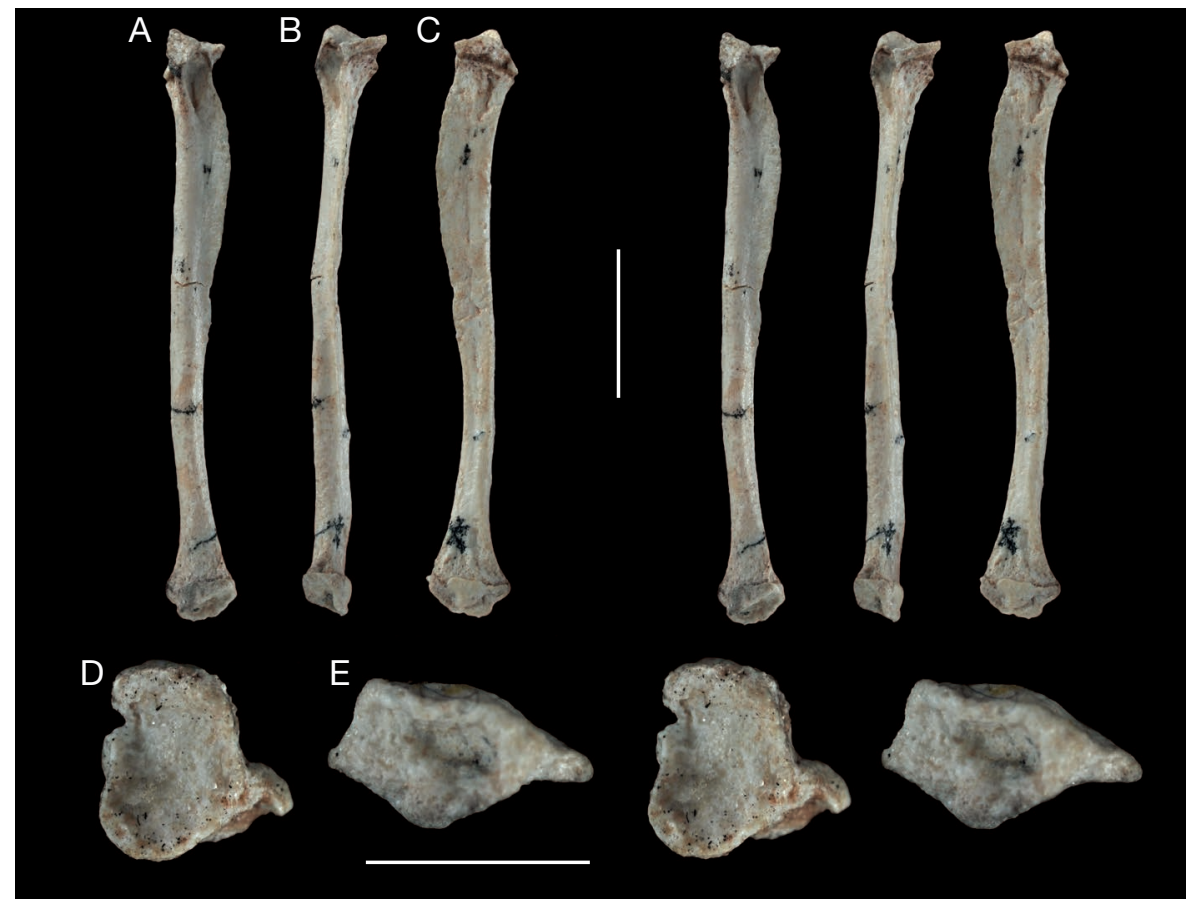

FIG. 99. - Left fibula of Alcidedorbignya inopinata (MHNC 8372): A, stereophotographs of posterior view; B, stereophotographs of medial view; C, stereophotographs of anterior view; D, stereophotographs of proximal view; E, stereophotographs of distal view. Scale bars: A-C, $1 \mathrm{~cm}$; D, E, $5 \mathrm{~mm}$.

is a small and ovoid medial articular facet for the astragalus (malleolar facet). The lateral articular facet for the astragalus is slightly concave (almost flat) and shorter antero-posteriorly than wide. The medial and lateral facets are widely separated anteriorly by a conspicuous groove. This groove does not extend posteriorly and the two articular surfaces are probably in contact posteriorly.

Fibula. (Figs 99, 100; Table 40) The two fibulae of MHNC 8372 are known and are long and slender bones. In anterior view, the medial edge of the fibula is markedly sigmoid because of the presence of a sharp and thin interosseous crest extending towards the tibia. It is markedly convex medially on most of its length. This crest is located on the proximal half of the bone but disappears along the distal half of the diaphysis. Despite the extension of the crest, the two bones of the leg remain widely separated when articulated, especially distally. On the posterior edge of the proximal portion of the diaphysis is a deep elongated fossa limited posteromedially by the interosseous crest. Laterally, the fossa is limited by a short and rounded ridge of the diaphysis. This fossa, as well as the posterior edge of the interosseous crest, probably housed the origin of the flexor hallucis longus (Fig. 100).

An interosseous crest is present in Pantolambda but it differs from that of Alcidedorbignya in being concave medially, narrower, and much shorter as it extends only on the proximal quarter of the diaphysis. Furthermore, the flexor hallucis fossa is very shallow in this genus. A well-developed interosseous crest is present on the fibulae of Ectoconus (Matthew 1937: pl. 31, fig. 2) and Periptychus (Matthew 1937: pl. 21, fig. 2a), and is very similar to that of Alcidedorbignya, although the

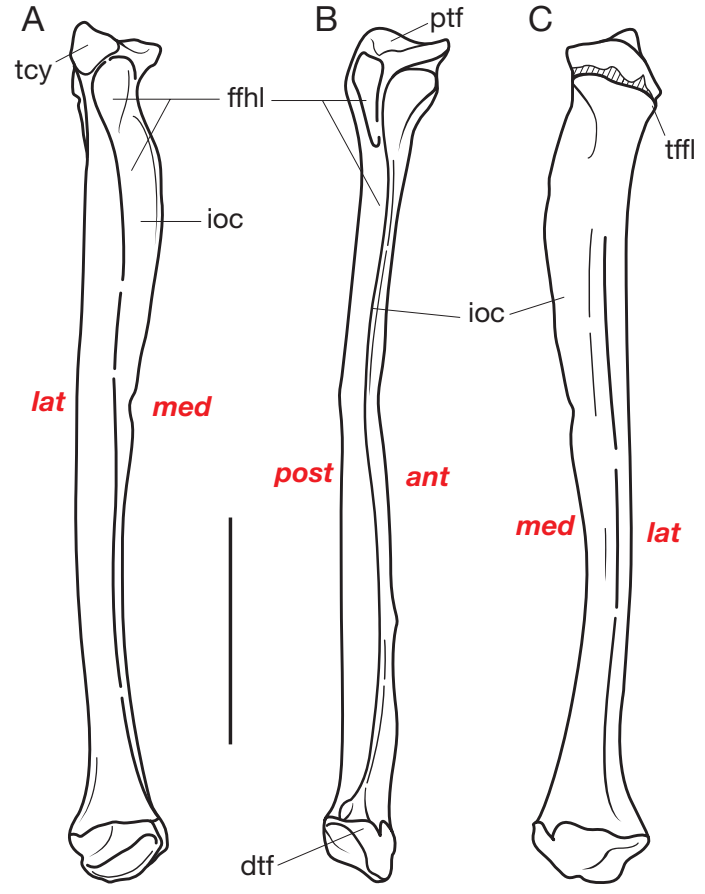

FIG. 100. - Left fibula of Alcidedorbignya inopinata (MHNC 8372): A, posterior view; B, medial view; C, anterior view. Abbreviations: ant, anterior; dtf, distal tibial facet; ffhl, fossa for the flexor hallucis longus; ioc, interosseous crest; lat, lateral; med, medial; post, posterior; tcy, tubercle for attachment of the cyamella ligament; ptf, proximal tibial facet; tffl, tubercle for the femorofibular ligament. Scale bar: $1 \mathrm{~cm}$.

crest is slightly smaller in Periptychus. It is also present but much smaller in Solenodon and Marmota. It is absent in Tenrec, Erinaceus, and Echinosorex. 
TABLE 40. - Measurements of the right fibula of Alcidedorbignya inopinata (in mm)

\begin{tabular}{lc}
\hline & MHNC 8372 \\
\hline Length & 41.33 \\
Width of tibial facet & 3.00 \\
Length of tibial facet & 3.96 \\
Width of diaphysis at level of greatest extension & \\
of interosseous crest & 4.23 \\
Width of distal epiphysis & 3.87 \\
Length of distal epiphysis & 5.23 \\
\hline
\end{tabular}

The proximal epiphysis is reduced to a concave proximal facet articulating with the tibia. On the anterolateral edge of the epiphysis, a tuberosity buttressed by a short lateral crest of the diaphysis provided attachment for the lateral femorofibular collateral ligament, which had its origin on the lateral epicondyle of the femur. A posterolateral tuberosity of the epiphysis, buttressed by the lateral crest of the flexor hallucis longus fossa, was probably for a cyamella, a sesamoid bone embedded in the articular ligament. The cyamella probably also articulated with the protruding posterior angle of the lateral condyle of the tibia and, possibly, with the lateral condyle of the femur. This interpretation is based on observations made on an articulated skeleton of Solenodon (MNHN-A-144887), which presents a well-developed cyamella articulating with the tibia, fibula, and femur. A small, wedge-like sesamoid that was discovered in the vicinity of the right knee of MHNC 8372 could represent a cyamella. These two tuberosities of the proximal epiphysis of the fibula of Alcidedorbignya are apparently present and well developed in Hyopsodus and Meniscotherium (Gazin 1965; 1968).

The distal epiphysis is better preserved on the left fibula and apparently not or little distorted. It is massive with a weaklydeveloped malleolus (contrasting with that of the tibia). The articular facet for the astragalus is triangular and slightly convex. It is bordered anterolaterally by a small V-shaped wall, which bears an elongated articulation with the calcaneus.

Tarsal bones. In MHNC 8372, the seven tarsal bones of the left pes are known, whereas only five are known for the right pes (all but the navicular and mesocuneiform). However, the tarsal bones of this specimen have suffered significant compression and distortion (especially the calcaneus and astragalus), such as they do not provide good basis for detailed reliable description. Therefore, the description below will also incorporate observations based on isolated specimens referred to Alcidedorbignya inopinata. Two of them (MHNC 8311 and 8315) were described by Muizon et al. (1998) and referred to either Alcidedorbignya inopinata or Andinodus boliviensis (two taxa similar in size) with a preference for the first alternative. Here we confirm this interpretation on the basis of the morphology observed on MHNC 8372. We add that A. inopinata is (with Pucadelphys andinus) the most abundant mammalian taxon of Tiupampa (known by hundreds of specimens), whereas $A$. boliviensis is a very rare taxon known from two specimens only (Muizon 1992). The other referred isolated tarsal bones are: MHNC 8459 (left astragalus), MHNC 8460 (right calcaneus), MHNC 8461 (partial right astragalus), and MHNC 8462 (partial right calcaneus).
Astragalus (Figs 101-103; Table 41) - Both astragali of MHNC 8372 are known but present an important variation in preservation, the left astragalus being strongly distorted transversely. The right astragalus is less modified but probably suffered some proximodistal compression (Fig. 101). Therefore, the following description is also based on MHNC 8311 and 8459 (Fig. 103), two undistorted specimens. The bone is slightly longer than wide, a condition that is reversed in Pantolambda. The astragalus of Alcidedorbignya is remarkably compressed dorsoplantarly and possesses a relatively flat tibial trochlea. The lateral tibial articular facet (i.e. the trochlea) is transversely wide with a very shallow relief. It is weakly convex anteroposteriorly, and slightly concavoconvex transversely. It is not distinctly separated from the medial tibial facet, which represents its medial edge. This facet (also the malleolar facet) articulates with the facet on the lateral edge of the tibial malleolus. It is relatively large, concave anteriorly, and convex posteriorly. It broadens anteriorly and gently slopes laterally. On its posteromedial edge is an oblique groove, probably for the astragalotibial ligament. The medial tibial facet is bordered anteromedially by a small obliquely-oriented groove, which excavates the posteromedial angle of the neck. Opposite to the groove, the lateral tibial facet does not really extend anteriorly on the medial region of the astragalar neck, although a slight extension can be observed on the medial side of the neck of MHNC 8311. The transition between the lateral and medial tibial articular facets is very smooth and forms a low and rounded dome. The two articular surfaces form an obtuse angle of approximately $120^{\circ}$ to each other. The posterior edge of the lateral tibial facet is deeply notched, corresponding to the presence of a large posterior foramen (astragalar canal).

Laterally, the fibular articular facet is separated from the lateral tibial facet by a sharp and elevated ridge, the two facets forming an angle of approximately $100^{\circ}$. The fibular facet is roughly triangular and extends onto the lateral process of the bone. This facet is slightly concave dorsally and strongly broadens anteriorly. On its posterolateral edge, just dorsal to the posterior border of the ectal facet is a deep fossa for the astragalofibular ligament.

On the ventral side, the ectal articular facet extends obliquely along the posterior edge, laterally. It is narrow and deeply concave. At the posteromedial edge of the ectal facet, the plantar tuberosity is a small and convex prominence. The astragalar neck is relatively short and transversely expanded but well individualised. The head is also extremely broad and is more elevated dorsoplantarly on its lateral than on its medial side. The plantar face of the neck bears a roughly circular and convex sustentacular facet, which does not really connect to the articular surface of the head, although it is close to it. The plane of the sustentacular facet faces roughly plantarly and, in distal view of the head, the plane of the sustentacular facet is sub-parallel to the transverse axis of head. On the plantolateral region of the articular surface of the head, facing the distolateral border of the sustentacular facet, is a small, elongated supplementary astragalocalcaneal 


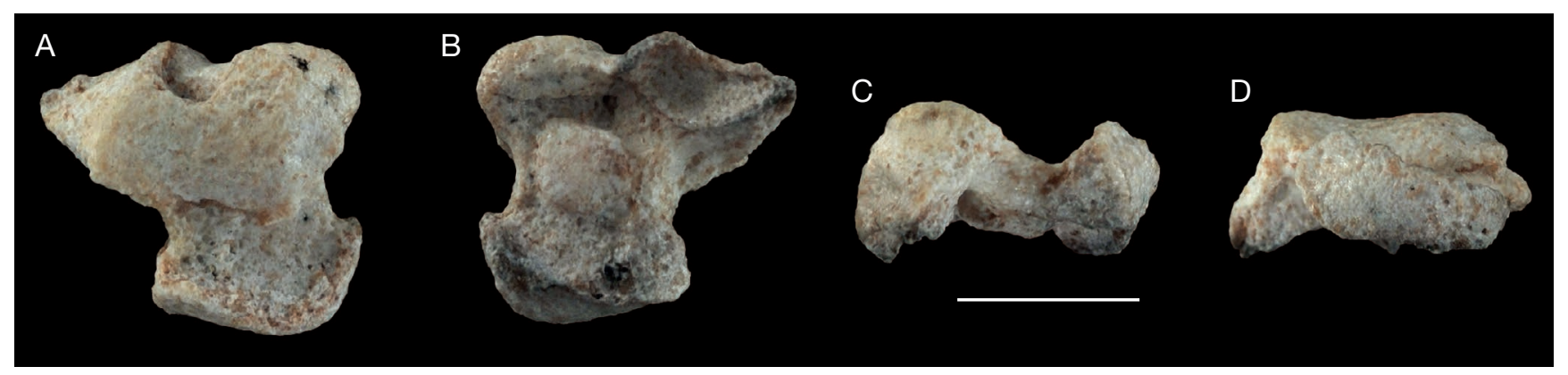

FIG. 101. - Right astragalus of Alcidedorbignya inopinata (MHNC 8372): A, dorsal view; B, plantar view; C, lateral view; D, distal view. Scale bar: $5 \mathrm{~mm}$.
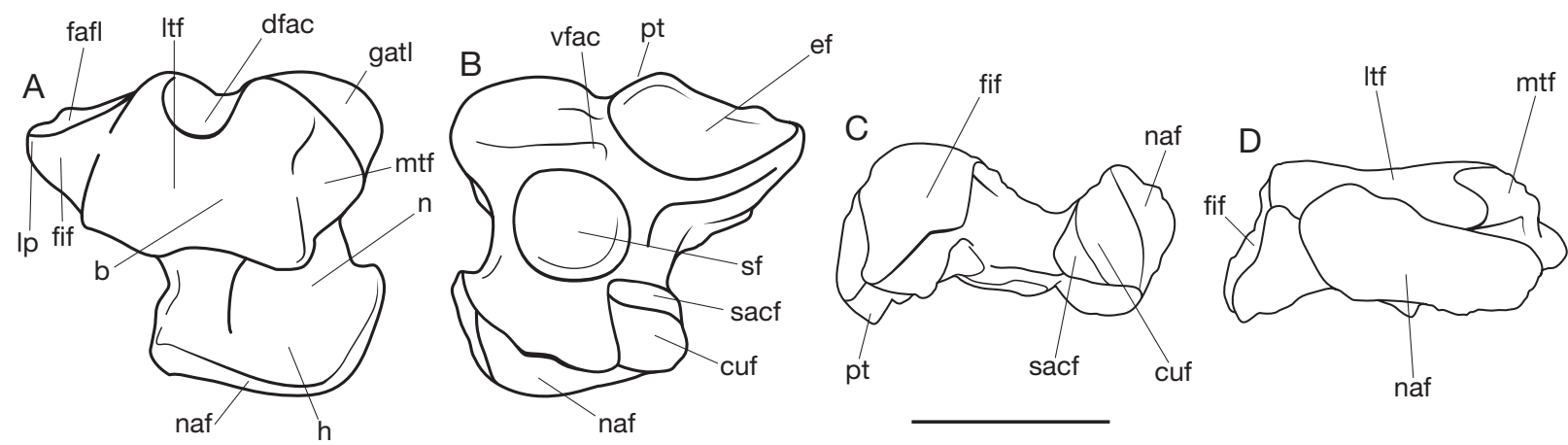

FIG. 102. - Right astragalus of Alcidedorbignya inopinata (MHNC 8372): A, dorsal view; B, plantar view; C, lateral view; D, distal view. Abbreviations: b, body; cuf, cuboid facet; dfac, dorsal foramen of the astragalar canal; ef, ectal facet; fafl, fossa for the astragalofibular ligament; fif, fibular facet; gatl, groove for the astragalotibial ligament; h, head; Ip, lateral process; Itf, lateral tibial facet; mtf, medial tibial facet; n, neck; naf, navicular facet; pt, plantar tuberosity; sacf, supplementary astragolocalcaneal facet; sf, sustentacular facet; vfac, ventral foramen of the astragalar canal. Scale bar: $5 \mathrm{~mm}$.

TABLE 41. - Measurements of the astragalus of Alcidedorbignya inopinata (in mm).

\begin{tabular}{|c|c|c|c|c|}
\hline & MHNC 8372 (R) & MHNC 8311 & MHNC 8459 & MHNC 13843 \\
\hline Anteroposterior length & 8.17 & 10.83 & 9.50 & - \\
\hline Width of body & 8.96 & 10.25 & 8.52 & - \\
\hline Width of articular tibial facet & 6.58 & 7.50 & 6.75 & - \\
\hline Maximum length of articular tibial facet & 4.90 & 6.70 & 5.58 & - \\
\hline Width of articular peroneal facet & 2.53 & 2.73 & 2.82 & - \\
\hline Maximum length of articular peroneal facet & 3.60 & 4.53 & 5.16 & - \\
\hline Width of neck & 4.63 & 4.86 & 4.71 & 4.35 \\
\hline Width of head & 6.16 & $6.54 \mathrm{e}$ & 6.07 & 5.86 \\
\hline Dorsoplantar height of head & 2.76 & 3.67 & 3.93 & 2.89 \\
\hline Maximum length of ectal facet & 4.27 & 5.70 & 4.86 & - \\
\hline Maximum length of sustentacular facet & 3.19 & 4.08 & 3.39 & 3.81 \\
\hline
\end{tabular}

TABLE 42. - Measurements of the calcaneus of Alcidedorbignya inopinata (in mm).

\begin{tabular}{lccccc}
\hline & MHNC 8372 (R) & MHNC 8315 & MHNC 8460 & MHNC 8462 & MHNC 8293 \\
\hline Length & 16.39 & 20.50 & - & - & 17.26 \\
Width of tuber at apex & 4.32 & 6.35 & - & - & - \\
Height of tuber at apex & 4.95 & 5.60 & - & - & 4.81 \\
Height of tuber on posterior edge of ectal facet & 4.50 & 6.79 & - & - & 6.39 \\
Minimum width of tuber & 2.29 & 4.18 & 2.93 & - & 3.09 \\
Length of ectal facet & 3.91 & 6.10 & 5.49 & - & 5.26 \\
Length of sustentacular facet & 3.33 & 4.36 & 3.46 & - & 4.72 \\
Dorsoplantar height of cuboid facet & 3.82 & 5.11 & 4.68 & 5.28 & 5.40 \\
Transverse width of cuboid facet & 4.51 & 5.42 & 4.75 & & 5.72 \\
\hline
\end{tabular}

facet (Figs 102, 103). As stated by Muizon et al. (1998) these facets were probably functionally contiguous. Dorsal to this facet is an elongated obliquely oriented (proximodorsolateraldistoplantaromedial) facet for the cuboid. On its medial edge a distinct ridge separates the cuboid facet from the large and strongly convex navicular facet, which represents most of the rest of the articular surface of the astragalar head. It indicates an extensive contact with the navicular. 


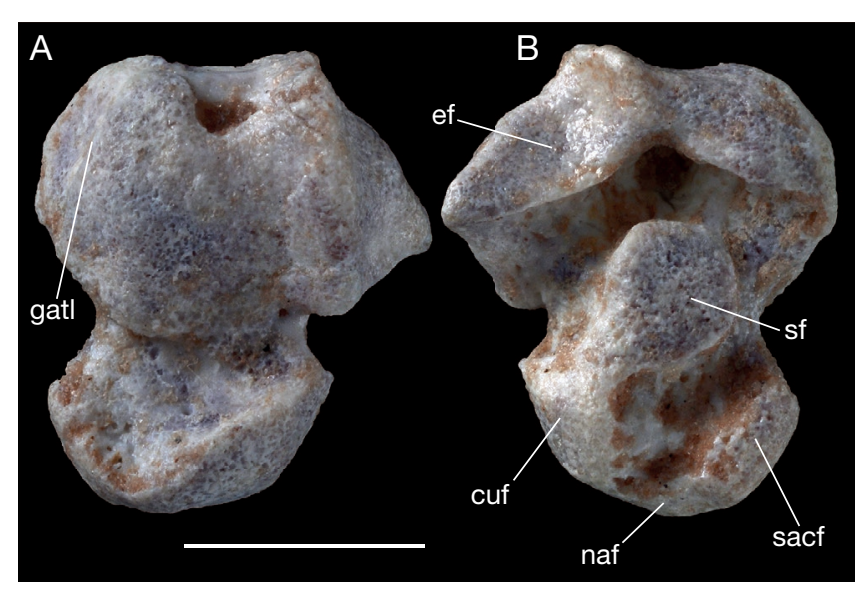

FIG. 103. - Left astragalus of Alcidedorbignya inopinata (MHNC 8459): A, dorsal view; B, plantar view. Abbreviations: cuf, cuboid facet; ef, ectal facet; gatl, groove for the astragalotibial ligament; naf, navicular facet; sacf, supplementary astragolocalcaneal facet; sf, sustentacular facet. Scale bar: $5 \mathrm{~mm}$.

The astragalus of Alcidedorbignya is markedly different from that of Pantolambda. The major difference is in the reduction of the astragalar neck of Pantolambda, which has almost disappeared laterally and which is invaded by the medial tibial facet medially. Plantarly the sustentacular facet is continuous with the cuboid facet of the head and the supplementary astragalocalcaneal facet is assimilated into the cuboido-sustentacular facet. In dorsal view the head is strongly shifted medially. The tibial trochlea of Pantolambda is even shallower than in Alcidedorbignya. The lateral tibial facet of the astragalar trochlea is almost flat transversely. The medial facet is larger than in Alcidedorbignya because of its distal extension toward the head and is markedly concave. Laterally the fibular facet is also separated from the tibial facet by a sharp ridge but the two surfaces form a smaller angle $\left(\right.$ c. $\left.90^{\circ}\right)$. Because of this morphology, the lateral process of the astragalus is smaller and less salient laterally.

Calcaneus (Figs 104-107; Table 42) - The right calcaneus of MHNC 8372 is slightly longer than the left one, $16 \mathrm{~mm}$ instead of 14.5 , this difference being probably the result of some distortion of the bones. The tuber calcanei of Alcidedorbignya represents more than half the length of the bone (approximately 60\%). Because of this length the astragalocalcaneal facets are located in a remarkably anterior proximodistal position. The tuber is relatively massive on $\mathrm{MHNC}$ 8315, but it is more slender on MHNC 8460 and MHNC 8372. The apex is robust and presents a groove that is not located at the same place on the two bones of MHNC 8372 (it is medio-posterior on the right calcaneus, but extended between the dorso-medial and the latero-plantar corners on the left one). On MHNC 8315 the groove is oblique as on the left calcaneus of MHNC 8372. The ectal and sustentacular facets are separated by a deep groove (the sulcus calcanei) and both face anterodorsally with a strong anterior component. The ectal facet is slightly convex and is antero-posteriorly short. Its posterior extremity turns medially; there, it tapers and faces more medially than anteriorly. The facet is weakly crescent-shaped and bears, on its posterolateral edge, a narrow fibular facet. The sustentacular facet is ovoid to subcircular and concave. It faces anterodorsally and is located in the same transverse plane as the ectal facet. It does not reach the cuboid facet distally (contra Muizon et al. 1998). Behind (posteroventral to) the sustentacular and cuboid facets a massive plantar tuberosity can be observed. The peroneal process is blade-like and, on MHNC 8372, presents no groove for the tendon of the fibularis longus. However, this condition may be the result of taphonomical bias as a much thicker peroneal process with a distinct groove is clearly present in MHNC 8462 (Fig. 107B). The peroneal process is in a distal position and reaches the lateral edge of the cuboid facet. The latter is broadly quadrangular in outline and mostly flat. In dorsal view, it is strongly oblique and faces mesiodistally. Its dorsomedial edge is bordered by and elongated supplementary astragalocalcaneal facet (Figs 103, 107D), which articulated with the corresponding facet on the posterolateral side of the head of the astragalus (see above and Muizon et al. 1998).

The general morphology of the calcaneus of Alcidedorbignya is roughly similar to that of Pantolambda, although the latter is, on the whole, much more massive. The bone is broader and shorter with proportionally larger astragalar facets and a more oblique cuboid facet. The peroneal process protrudes less laterally but is more extended proximodistally, on approximately half of the length of the bone. In distal view the bone is flatter dorsopalmarly with proportionally smaller cuboid facet and plantar tubercle. The dorsoplantar flattening of the bone is also clearly visible in medial view.

Cuboid (Figs 108A-C, 109; Table 43) - Both cuboids of MHNC 8372 are known, but again have suffered some post-mortem distortion. Several isolated undistorted (or little distorted) bones are herein referred to $A$. inopinata (MHNC 13837, 13838, 13939 and 13845) and the following description also refers to these specimens (Fig. 109).

The cuboid is a robust bone, mostly triangular in crosssection and proximodistally longer than transversely wide. The proximal articular calcaneal facet is extended dorsoplantarly along its medial side, but tapers laterally. In dorsal view the facet slopes laterally. On MHNC 8372 a distinct articular facet for the astragalus is observed. However, its orientation is almost totally medial while on the other specimens it is proximomedial. This unusual orientation is probably the result of some post-mortem distortion, causing the astragalar facet to be placed in a proximodistal orientation on the medial side of the bone in apparent continuity with the articular facets for the navicular and ectocuneiform. On the referred specimens, the medial edge of the calcaneal facet is bordered by a smaller, dorsoplantarly elongated, concave facet sloping medially (in dorsal view) (Fig. 109). This facet articulated with the elongated cuboid facet of the astragalar head. On the medial side of the bone, distal to the astragalar facet, two articular facets exist. The more proximal, of the two is slightly concave and dorsoplantarly elongate, and it articulates with the navicular. The more 


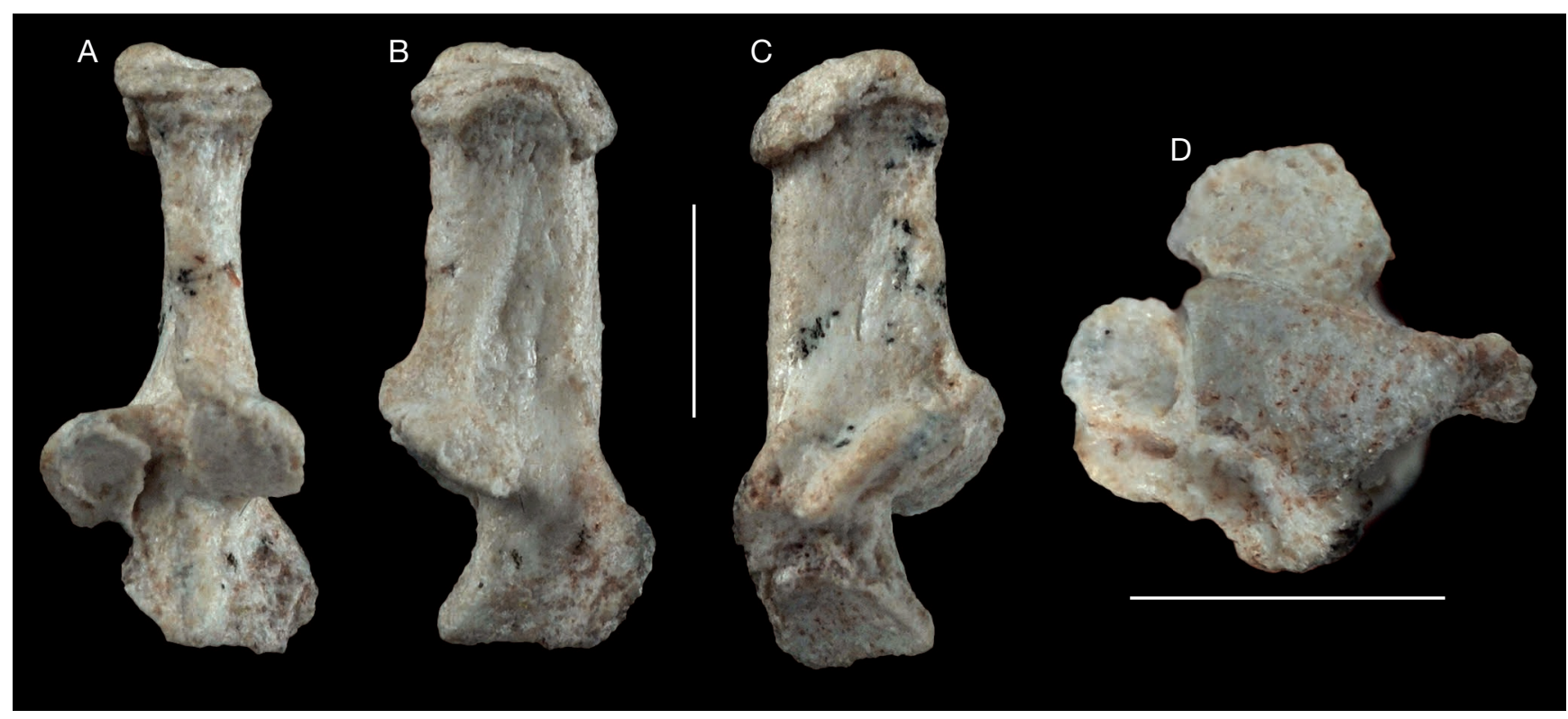

FIG. 104. - Left calcaneus of Alcidedorbignya inopinata (MHNC 8372): A, dorsal view; B, lateral view; C, medial view; D, distal view. Scale bars: 5 mm.

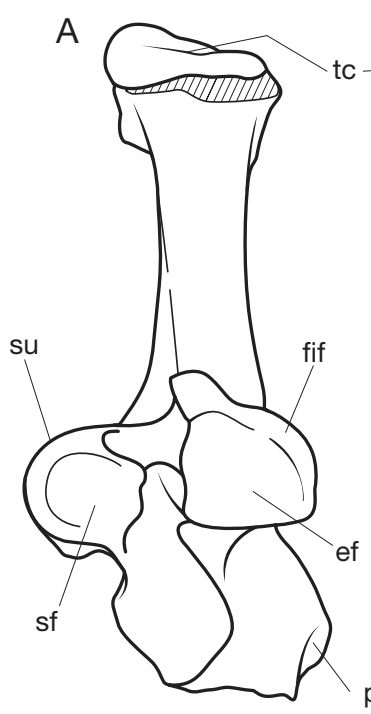

B
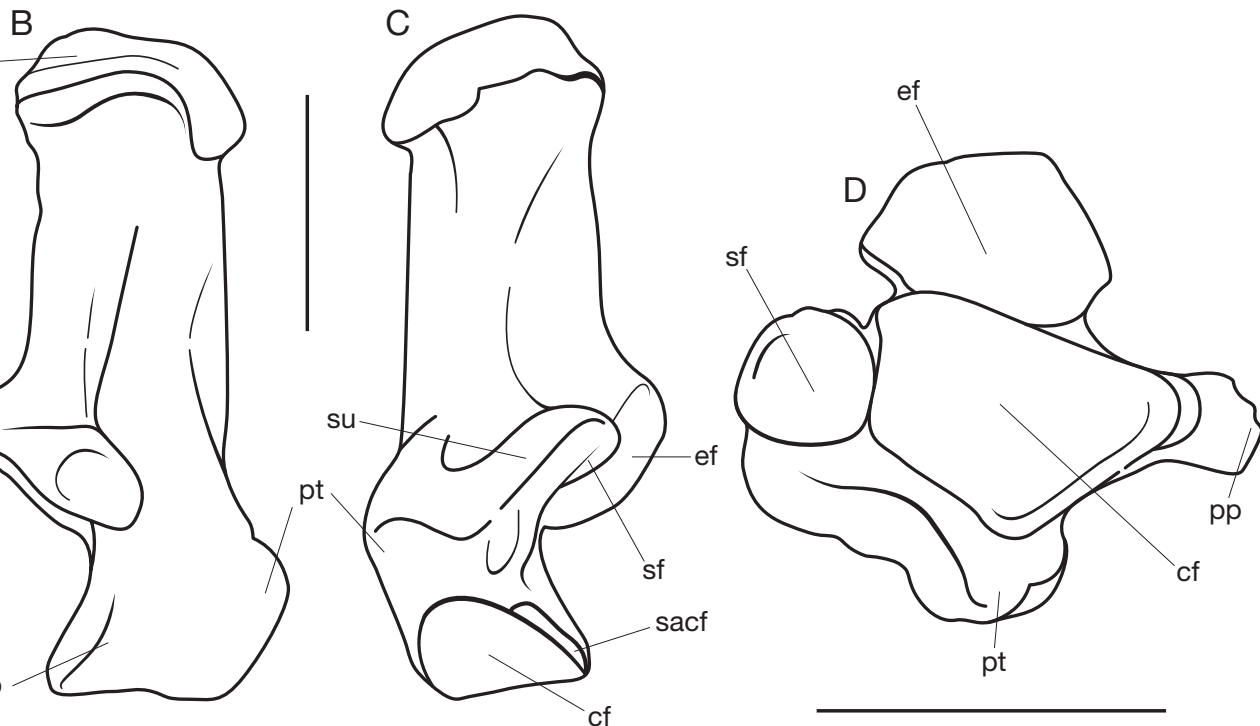

pt

FIG. 105. - Left calcaneus of Alcidedorbignya inopinata (MHNC 8372): A, dorsal view; B, lateral view; C, medial view; D, distal view. Abbreviations: cf, cuboid facet; ef, ectal facet; fif, fibular facet; pp peroneal process; pt, plantar tubercle; sacf, supplementary astragolocalcaneal facet; sf, sustentacular facet; su, sustentaculum; tc, tuber calcanei. Scale bars: $5 \mathrm{~mm}$.

distal, adjacent to the navicular facet, is also elongated and articulates with the ectocuneiform. These two facets are not discernible from each other on the right cuboid of MHNC 8372 but they are distinct on the referred specimens (e.g., MHNC 13837), the ectocuneiform facet being generally slightly larger than that for the navicular (Fig. 109 C). On the plantar side of the bone is a massive plantar tubercle, which overhangs a narrow and deep sulcus for the tendon of the fibularis longus. The distal articular facet is broad, concave, triangular in outline (more developed along the dorsal edge than the plantar one).

The cuboid of Pantolambda differs from that of Alcidedorbignya in being conspicuously shorter proximodistally (i.e. wider than long). The astragalar facet is much larger than in Alcidedorbignya. On the medial side of the bone, the articular facet for the navicular is a proximodistally narrow strip, much smaller than in Alcidedorbignya. . The ectocuneiform facet is, on the contrary, relatively large compared to that of Alcidedorbignya and forms a distinct angle with the navicular facet.

Navicular (Fig. 108D-F; Table 44) - The left navicular of MHNC 8372 is the only navicular known of Alcidedorbignya. It is proximodistally short and cradle-shape, as usual in basal eutherians, with a concave proximal articular facet for the astragalar head. It bears a small plantar tubercle. The 


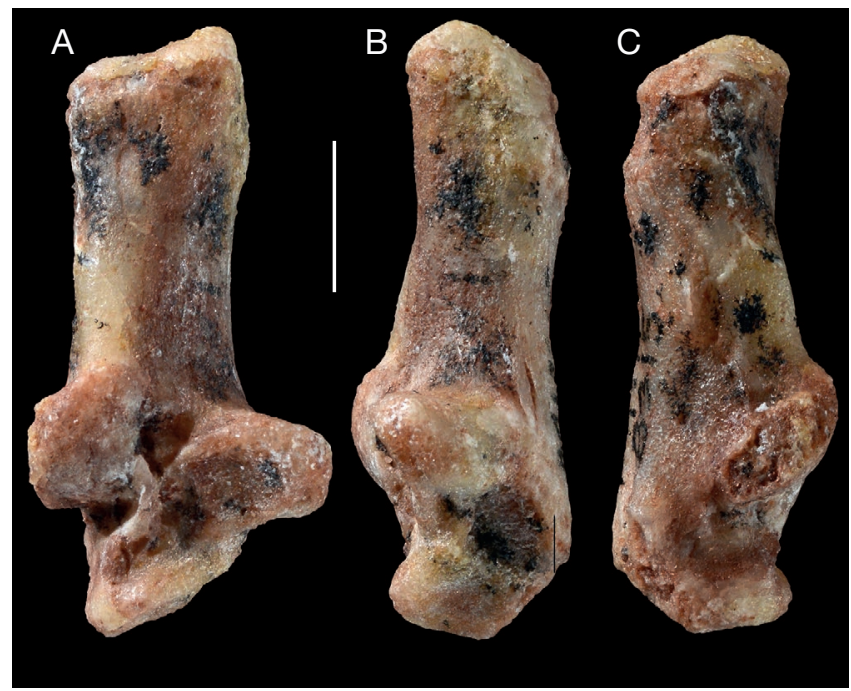

FIG. 106. - Right calcaneus of Alcidedorbignya inopinata (MHNC 8315): A, dorsal view; B, medial view; C, lateral view. Scale bar: $5 \mathrm{~mm}$.

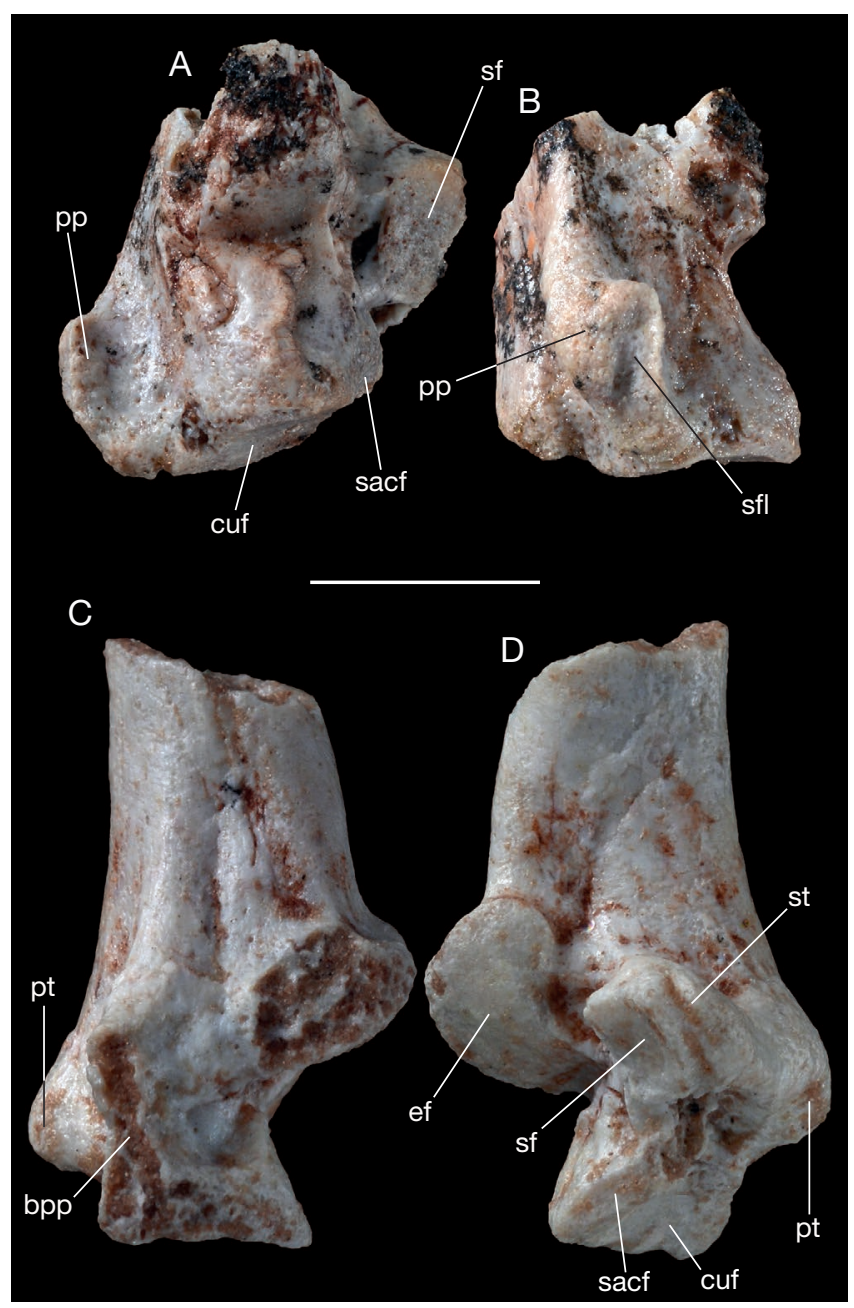

FIG. 107. - Right calcanei of Alcidedorbignya inopinata: A, MHNC 8462 distal portion of the calcaneus (body) in dorsal view; B, MHNC 8462 in lateral view; C, MHNC 8460, calcaneus missing the apex of the tuber, in lateral view; D, MHNC 8460 in medial view. Abbreviations: bpp, broken peroneal process; cuf, cuboid facet; ef, ectal facet; pp, peroneal process; pt, plantar tubercle; sacf, supplementary astragalocalcaneal facet; sf, sustentacular facet; sfl, sulcus for the tendon of the fibularis longus; st sustentaculum. Scale bar: $5 \mathrm{~mm}$.
TABLE 43. - Measurements of the cuboid of Alcidedorbignya inopinata (in mm).

\begin{tabular}{lcccc}
\hline & MHNC & MHNC & MHNC & MHNC \\
& $\mathbf{8 3 7 2}$ (L) & $\mathbf{1 3 8 3 8}$ & $\mathbf{1 3 8 3 9}$ & $\mathbf{1 3 8 4 5}$ \\
\hline Length & 4.97 & - & 8.19 & 5.84 \\
Width & 4.64 & 6.29 & 6.79 & 4.88 \\
Depth (dorsoplantar) & 4.36 & 5.65 & 5.89 & 4.06 \\
\hline
\end{tabular}

TABLE 44. - Measurements of the navicular and cuneiforms of Alcidedorbignya inopinata (in $\mathrm{mm}$ ).

\begin{tabular}{lcccc}
\hline MHNC 8372 & Navicular & $\begin{array}{c}\text { Ecto- } \\
\text { cuneiform }\end{array}$ & $\begin{array}{c}\text { Meso- } \\
\text { cuneiform }\end{array}$ & $\begin{array}{c}\text { Ento- } \\
\text { cuneiform }\end{array}$ \\
\hline Length & 2.50 & 4.14 & 2.60 & 4.70 \\
Width & 6.62 & 3.13 & 1.65 & 1.40 \\
$\begin{array}{l}\text { Depth } \\
\text { (dorsoplantar) }\end{array}$ & 4.04 & 4.92 & 3.60 & 3.07 \\
\hline
\end{tabular}

proximal facet presents a medial expansion that is upturned proximally, following the curvature of the astragalar head. Distally there are the three facets that articulated with the cuneiforms. Laterally, the facet for the ectocuneiform is the largest and is oriented dorsolaterally-plantomedially. The mesocuneiform facet is only slightly smaller, while that for the entocuneiform, the smallest, is a narrow strip on the anteromedial edge of the bone. The navicular of Pantolambda differs from that of Alcidedorbignya in being proximodistally thinner and more circular rather than transversely elongated.

Ectocuneiform (Fig. 109A-C; Table 44) - This element is the largest of the three cuneiforms. It presents a typical morphology, being quadrangular in lateral view and elongate dorso-plantarly but compressed transversely. A robust plantar tubercle is developed proximal to a shallow sulcus for the fibularis longus. The proximal facet articulating with the navicular is small and quadrangular in outline. The distal articular facet (contacting Mt III) is broader and pear-shaped, transversely wider on its dorsal than on its plantar edge. The lateral and medial faces exhibit small proximal articular facets, the lateral one, articulating with the cuboid, being broader. The medial one is triangular in outline, the anterior part extending distally.

Mesocuneiform (Fig. 110D-F; Table 44) - The mesocuneiform is by far the smallest of the tarsal bones. It is also extended dorsoplantarly but compressed transversely and proximodistally. The shape of the proximal and distal facets is congruent, elongated dorsoplantarly and compressed transversely. There is no discernible medial facet or plantar process. A lateral, distal facet, extended dorsoplantarly, can be observed.

Entocuneiform (Fig. 110G-I; Table 44) - The entocuneiform, known on both sides, is transversely narrow and elongated dorso-plantarly and proximo-distally: it is proxi- 


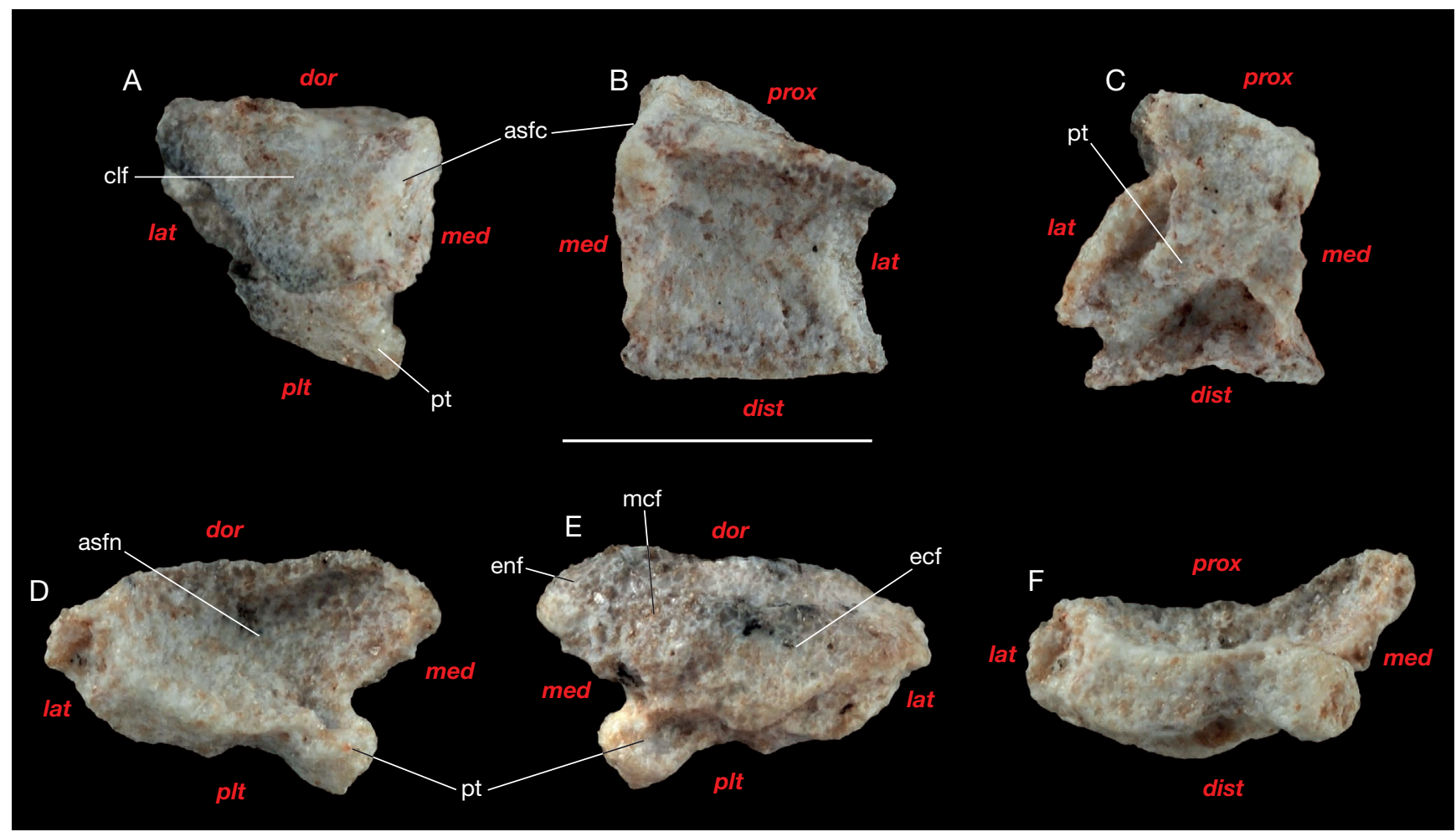

FIG. 108. - Left cuboid and navicular of Alcidedorbignya inopinata (MHNC 8372): A, left cuboid in proximal view; B, left cuboid in dorsal view; C, left cuboid in plantar view; D, left navicular in proximal view; E, left navicular in distal view; F, left navicular in plantar view. Abbreviations: asfc, astragalar facet of the cuboid; asfn, astragalar facet of the navicular; clf, calcanear facet; dor, dorsal; dist, distal; ecf, ectocuneiform facet; enf entocuneiform facet; lat, lateral; med, medial; mcf, mesocuneiform facet; plt, plantar; prox, proximal; pt, plantar tubercle. Scale bar: $5 \mathrm{~mm}$.

modistally twice as high as the adjacent mesocuneiform. As on the mesocuneiform, the proximal and distal facets are elongated dorso-plantarly and compressed transversely. The distal facet presents an anterior (dorsal) apex that protrudes distally, forming a small hook (especially on the left entocuneiform). Its transverse narrowness precludes a contact with Mt II and no lateral articular facet can be observed. The medial side of the bone exhibits a sharp crest running proximo-distally, better emphasized on the right entocuneiform.

Metatarsals and phalanges. (Figs 111-113; Tables 4551) In MHNC 8372 the left pes is known with the five metatarsals and some phalanges, including three proximal phalanges, the four intermediate phalanges, and two ungual phalanges. On the right pes the four lateral metatarsals are known, but that of the hallux is missing. Four proximal phalanges are known, as well as an intermediate and ungual phalanges associated with digit III. The left Mt V lacks the distal epiphysis and the right Mt II was broken and healed during the life of the animal.

Several other isolated specimens have been referred to A. inopinata. They are: one left Mt II (MHNC 13830); one left Mt IV (MHNC 13834); three right Mt III (MHNC 13831, 13832, 13833); and two right Mt V (MHNC $13835,13836)$.

Like the metacarpals, the metatarsals of Alcidedorbignya are relatively slender bones (Figs 111, 112). The distal

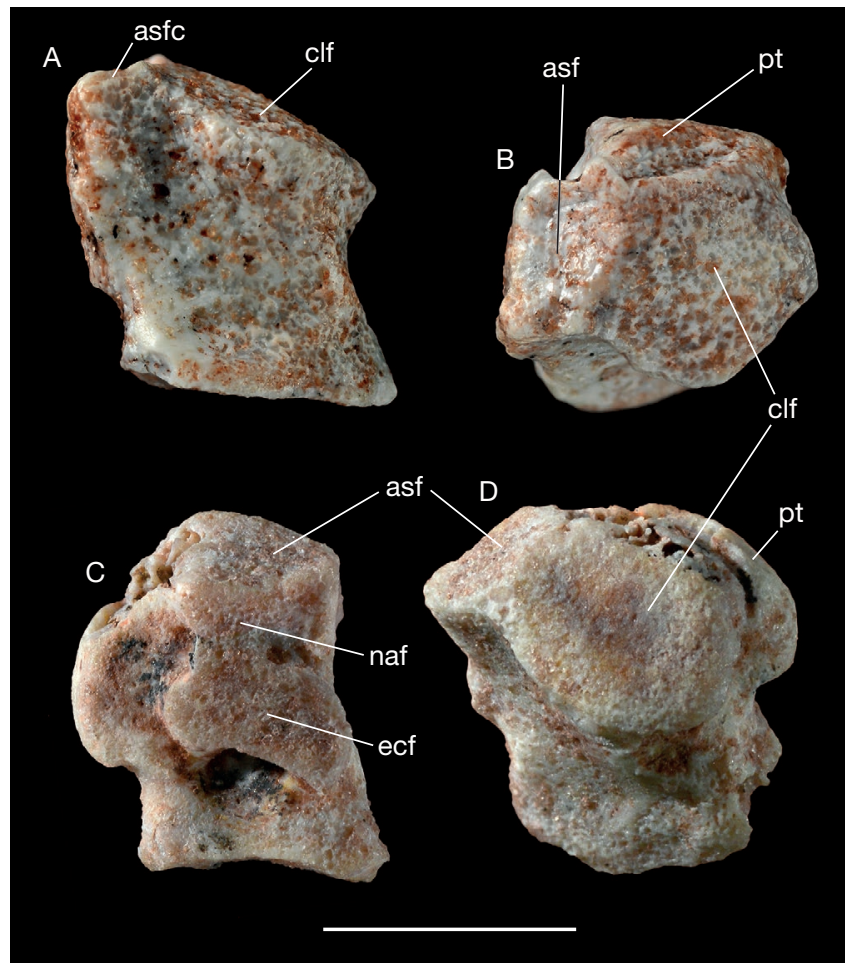

FIG. 109. - Left cuboids of Alcidedorbignya inopinata: A, dorsal view of (MHNC 13838); B, proximal view of (MHNC 13838); C, medial view of (MHNC 13837); D, proximodorsolateral view of (MHNC 13837). Abbreviations: asf, astragalar facet; asfc, astragalar facet of the cuboid; clf, calcaneal facet; ecf, ectocuneiform facet; naf, navicular facet; pt, plantar tubercle. Scale bar: $5 \mathrm{~mm}$. 


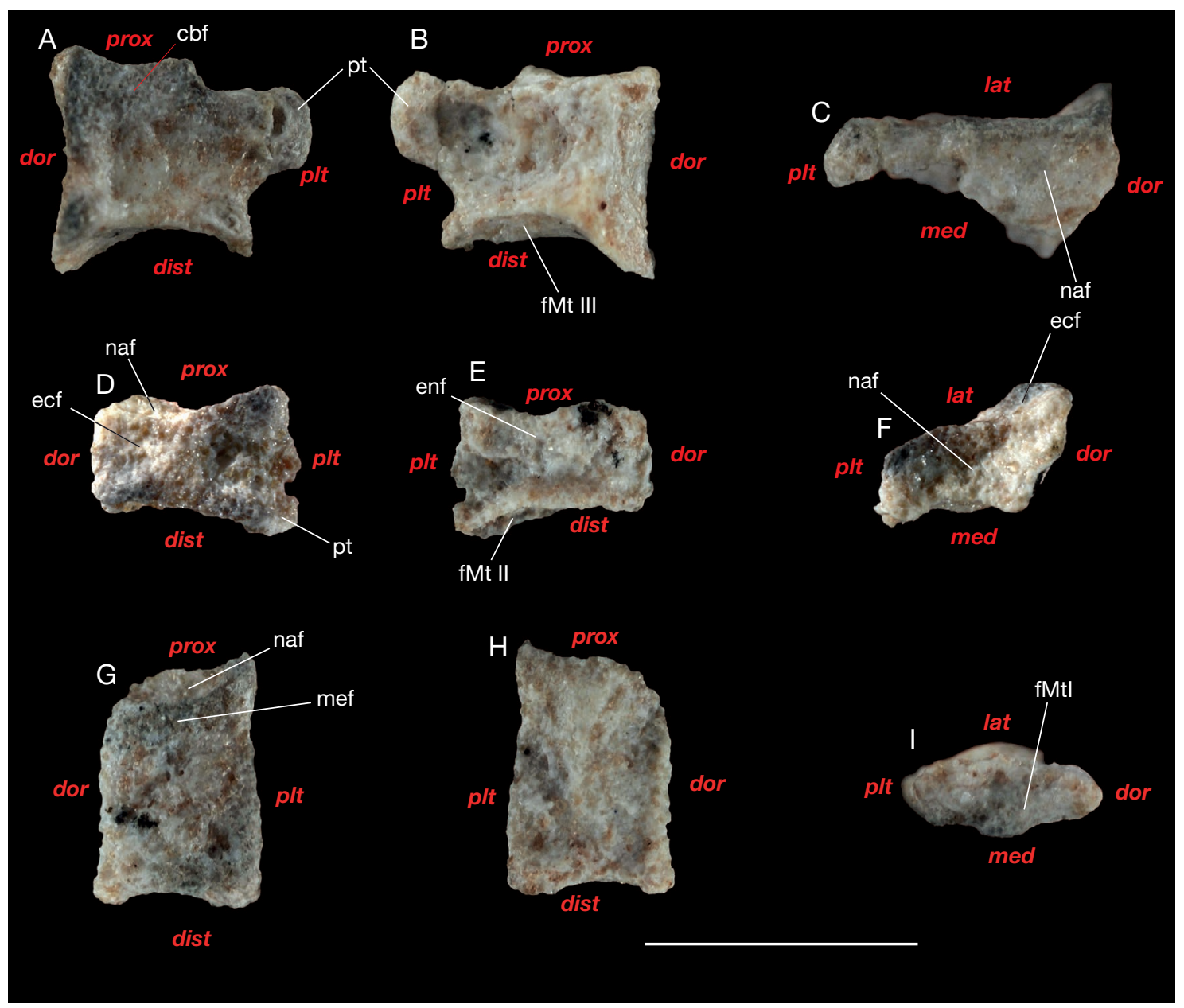

FIG. 110. - Left posterior cuneiforms of Alcidedorbignya inopinata (MHNC 8372): A, ectocuneiform in lateral view; B, ectocuneiform in medial view; C, ectocuneiform in proximal view; D, mesocuneiform in lateral view; E, mesocuneiform in medial view; $\mathbf{F}$, mesocuneiform in proximal view; $\mathbf{G}$, entocuneiform in lateral view; H, entocuneiform in medial view; I, entocuneiform in distal view. Abbreviations: cbf, cuboid facet; dist, distal; dor, dorsal; ecf, facet for the ectocuneiform; enf, facet for the entocuneiform; fMtl, facet for the Mtl; fMtll, facet for the Mtll; fMtIII, facet for the MtIII; lat, lateral; med, medial; mef, facet for the mesocuneiform; naf, navicular facet; plt, plantar; pt, plantar tubercle, prox, proximal. Scale bar: $5 \mathrm{~mm}$.

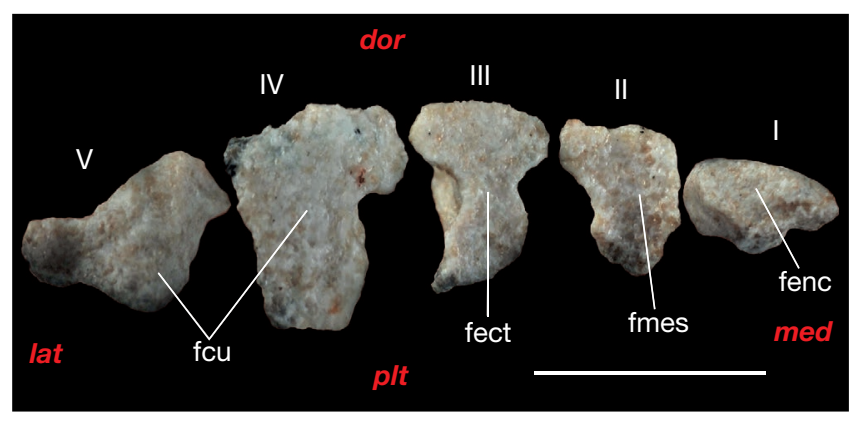

FIG. 111. - Proximal views of the left metatarsals of Alcidedorbignya inopinata (MHNC 8372). Abbreviations: dor, dorsal; fcu, facet for the cuboid; fect, facet for the ectocuneiform; fenc, facet for the entocuneiform; fmes, facet for the mesocuneiform; lat, lateral; med, medial; plt, plantar. Scale bar: $5 \mathrm{~mm}$.

width/length ratio of MtIII is 0.229 , this being the average of the ratios of the four specimens (three isolated and one preserved as part of the skeleton). In Pantolambda the ratio is 0.330 (AMNH 16663), which indicates that the meta- tarsal of Pantolambda is c. 44\% more massive than those of Alcidedorbignya. This difference is therefore significantly greater than that observed in the metacarpals (c. 25\%).

The relative length of the metatarsal can be evaluated by comparison with the length of the femur (length Mt III/ length femur). In this case only MHNC 8372 is relevant because it is the only specimen that preserves both the $\mathrm{Mt}$ III and the femur. The ratio is 0.322 in Alcidedorbignya and 0.256 in Pantolambda. Therefore the metatarsals of Pantolambda are c. 20\% shorter than those of Alcidedorbignya compared to the length of the femur. This difference is much greater than that observed in the case of the metacarpals (2.2\% and 3.7\% - see above) and shows that the metatarsals of Alcidedorbignya are proportionally longer (when compared to the metacarpals) than in Pantolambda.

A direct comparison of the McIII and MtIII of $A$. inopinata and $P$. bathmodon clearly shows a notable size difference between the two species (Table 46). The mean MtIII length of $A$. inopinata is $34 \%$ longer than that of McIII, while the difference is $5 \%$ in $P$. bathmodon. 


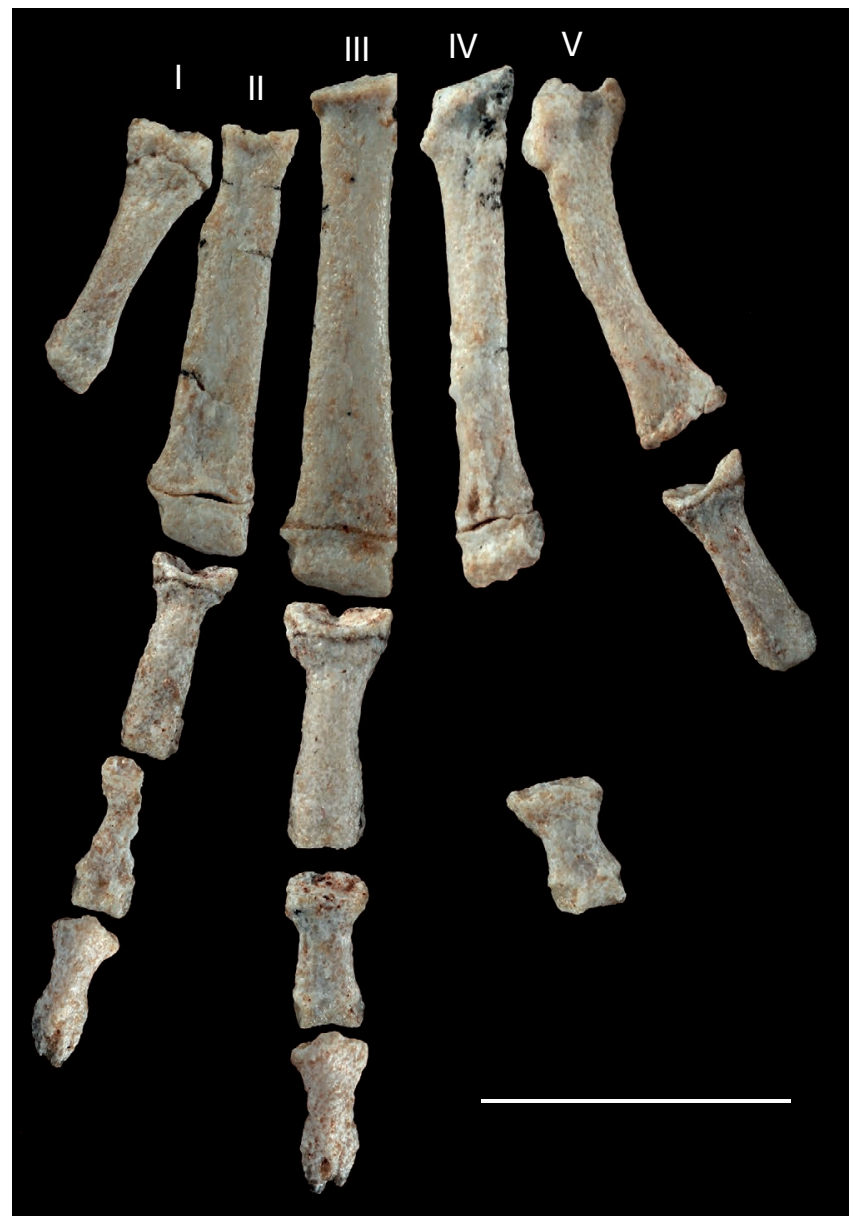

FIG. 112. - Left metatarsals and digits of Alcidedorbignya inopinata (MHNC 8372 ) in dorsal view. Scale bar: $1 \mathrm{~cm}$.

In other words, in Pantolambda the metacarpals and metatarsals are closer in size than in Alcidedorbignya. In generalised mammals metatarsals are generally longer than metacarpals. This length difference tends to be less in some specialised mammals such as cursorial taxa (e.g., Equus, Cervus). It is lacking in some large herbivorous mammals (Coryphodon, Coelodonta), or even reversed in graviportal mammals (Elephas, Uintatherium).

The left Mt I of MHNC 8372 is, like the Mc I, a short and slender bone; it is slightly longer than the Mc I $(9.5 \mathrm{~mm})$. It is compressed dorsoventrally but all the left metatarsals of MHNC 8372 show this unnatural, post-mortem compression. On the right pes, the Mt II is $14.6 \mathrm{~mm}$ long, the Mt III is $17.6 \mathrm{~mm}$ long, and the Mt IV is $17.4 \mathrm{~mm}$ long. On the left pes the three metatarsals are slightly shorter, (Mt II, $4.1 \mathrm{~mm}$; Mt III, $17.1 \mathrm{~mm}$; Mt IV, $17 \mathrm{~mm}$ ). These differences in MHNC 8372 are probably the result of postmortem deformation. Therefore, as in the manus, the Mt III is the longest metapodial of the pes. However, the Mt III $(17.6 \mathrm{~mm})$ is distinctly longer than the Mc III $(13.5 \mathrm{~mm})$. Furthermore, the Mt IV is significantly longer than the Mt II and is almost as long as the Mt III. In the hand Mc II and Mc IV are approximately of the same size. Although

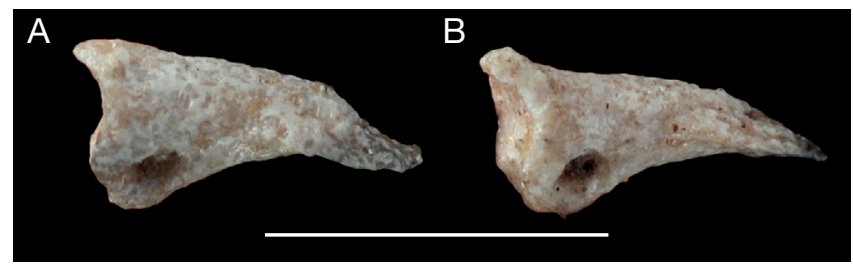

FIG. 113. - Medial view of left posterior ungual phalanges of Alcidedorbignya inopinata (MHNC 8372). A, digit II; B, digit III. Scale bar: $5 \mathrm{~mm}$.

incomplete, it is clear that the left $\mathrm{Mt} \mathrm{V}$ was also longer than Mt I. The right Mt I is missing on MHNC 8372 but the right $\mathrm{Mt} \mathrm{V}$ is complete and is $13.7 \mathrm{~mm}$ long, i.e. distinctly longer than $\mathrm{MtI}$. The metatarsals are transversely compressed proximally, and dorso-ventrally compressed distally, like the metacarpals. If this characteristic is somewhat altered on the metatarsals of MHNC 8372 because of post-mortem deformation, it is conspicuous on the isolated metatarsals mentioned above, which are not deformed.

The proximal epiphysis of Mt I is wider than long, a condition possibly related to post-mortem compression. In proximal view, it bears an oblique crest (dorsolateralmedioplantar orientation), which separates the articular facet for the entocuneiform medially and the facet for the $\mathrm{Mt}$ II laterally. This facet presents on its distal edge a smooth tuberosity formed by the proximal part of the diaphysis and the distal part of the epiphysis. A small tuberosity is also present medially and a well-developed plantar tubercle is observed in the median region of the epiphysis. The proximal epiphysis of Mt II is triangular to trapezoidal in proximal outline (wider dorsally than ventrally), with a dorsoplantar median sulcus and a notched medial edge. The proximal epiphysis of Mt III is trapezoidal in proximal outline. Its edges are notched, and the lateral border more deeply than the medial one. The proximal articular facet slopes dorsomedially. The proximal epiphysis of Mc IV is also trapezoidal in proximal view. Its medial edge is deeply notched (corresponding to the deep notch of the lateral edge of the Mt III) and its lateral edge is only regularly concave and deeply excavated on its lateral edge for the articulation with the MT V. Its dorsomedial angle slopes distomedially. It extends on the medial edge of the diaphysis and articulates with the adjacent Mt II. The Mt $\mathrm{V}$ presents a triangular proximal epiphysis that is smoothly convex, with its medial part being deflected medially, where it articulates with the adjacent Mt IV. Like the Mc V, it presents a laterally prominent proximal process and also a ventral one, prominent proximally. The lateral process is separated from the articular facet by a deep sulcus.

The distal epiphysis of Mt I does not present well-separated condyles and is more asymmetrical than on Mc I, the medial part positioned more proximally than the lateral one. The distal epiphyses of Mt II to V each present two condyles separated by an elevated median crest. Their asymmetry is difficult to evaluate because it may have been exagerated by the post mortem distortion, which affected MHNC 
TABLE 45. - Comparison of robustness (Distal Width of MtIII/Length of MtIII) and relative length (Length MtIII/Length Femur) of the MtIII in A. inopinata and P. bathmodon

\begin{tabular}{lrlccc}
\hline & Dist W MtIII & L MtIII & DWMtIII/LMtIII & L Fem & LMtIII/LFem \\
\hline $\begin{array}{l}\text { Alcidedorbignya inopinata } \\
\quad \text { (Mean of MHNC 8372, 13831, 13832, 13833) }\end{array}$ & $4.29 \mathrm{~mm}$ & $18.68 \mathrm{~mm}$ & 0.22 & & \\
$\begin{array}{l}\text { Alcidedorbignya inopinata (MHNC 8372) } \\
\text { Pantolambda bathmodon (AMHN 16663) }\end{array}$ & $3.99 \mathrm{~mm}$ & 17.07 & 0.23 & 52.93 & 0.32 \\
\hline
\end{tabular}

TABLE 46. - Comparison of the relative lengths of the Mclll and MtIII in Alcidedorbignya inopinata and Pantolambda bathmodon.

\begin{tabular}{lccc}
\hline & $\begin{array}{c}\text { Length } \\
\text { McIII }\end{array}$ & $\begin{array}{c}\text { Length } \\
\text { MtIII }\end{array}$ & $\begin{array}{c}\text { Length McIII/ } \\
\text { Length MtIII }\end{array}$ \\
\hline $\begin{array}{c}\text { Alcidedorbignya inopinata } \\
\text { (MHNC 8372) }\end{array}$ & $13.96 \mathrm{~mm}$ & $18.68 \mathrm{~mm}$ & 0.747 \\
$\begin{array}{c}\text { Pantolambda bathmodon } \\
\text { (AMNH 16663) }\end{array}$ & $37.98 \mathrm{~mm}$ & $39.85 \mathrm{~mm}$ & 0.953 \\
\hline
\end{tabular}

TABLE 47. - Measurements of the metatarsals belonging to the skeleton (MHNC 8372) of Alcidedorbignya inopinata (in $\mathrm{mm}$ ).

\begin{tabular}{lccccc}
\hline MHNC 8372 & Mt I (L) & Mt II (R) & MT III (R) & Mt IV (R) & Mt V (R) \\
\hline Length & 9.38 & 14.6 & 17.63 & 17.46 & 13.69 \\
Proximal width & 3.27 & 2.26 & 3.49 & 3.65 & 3.26 \\
Distal width & 1.90 & 3.14 & 4.15 & 3.34 & 3.03
\end{tabular}

TABLE 48. - Measurements of isolated Mt Ills referred to Alcidedorbignya inopinata (in $\mathrm{mm}$ ).

\begin{tabular}{lccc}
\hline & MHNC 13831 & MHNC 13832 & MHNC 13833 \\
\hline Length & 19.91 & 19.23 & 18.69 \\
Proximal width & 4.10 & 3.25 & 3.81 \\
Distal width & 4.34 & 4.51 & 4.41 \\
\hline
\end{tabular}

8372. On this specimen the left Mt II presents an asymmetry that is in agreement with its position in the foot, i.e. the medial condyle is proximodistally slightly shorter than the lateral one. On the right bone the epiphysis is relatively symmetrical, which could be due to distortion. The distal epiphysis of the Mt III is relatively symmetrical, although some variation exists. For instance, of the three isolated right Mt III, two are perfectly symmetrical but one (MHNC 13831) presents a slight asymmetry, i.e. the medial condyle is proximodistally shorter than the lateral. On the Mt IV the asymmetry is reversed and on both sides of MHNC 8372 the lateral condyle is proximodistally shorter than the medial one.

The proximal phalanges present the typical morphology and they are between 7 and $8 \mathrm{~mm}$ long, i.e. they are barely longer than those of the manus (Fig. 112). The proximal phalanx associated with digit III is also the longest. The proximal phalanx of the first digit, known on the right pes, is extremely slender but incomplete. Like the manual proximal phalanges, the diaphyses of the proximal pedal phalanges do not present tuberosities on their ventral side, nor are their epiphyses are not asymmetrical. The four intermediate phalanges show the same robustness and are between 4 and
TABLE 49. - Measurements of the proximal phalanges of Alcidedorbignya inopinata (in mm).

\begin{tabular}{lccccc}
\hline MHNC 8372 & $\begin{array}{c}\text { Digit I } \\
(\mathbf{R})\end{array}$ & $\begin{array}{c}\text { Digit II } \\
(\mathbf{L})\end{array}$ & $\begin{array}{c}\text { Digit III } \\
(\mathbf{L})\end{array}$ & $\begin{array}{c}\text { Digit IV } \\
\text { (R) }\end{array}$ & $\begin{array}{c}\text { Digit V } \\
\text { (L) }\end{array}$ \\
\hline Length & - & 8.25 & 8.40 & 8.02 & 7.19 \\
Proximal width & - & 3.48 & 3.60 & - & 3.04 \\
Distal width & 1.68 & 2.42 & 2.64 & 2.70 & 2.21 \\
\hline
\end{tabular}

TABLE 50. - Measurements of the intermediate phalanges of Alcidedorbignya inopinata (in $\mathrm{mm}$ ).

\begin{tabular}{lcccc}
\hline MHNC 8372 & Digit II (L) & Digit III (L) & Digit IV & Digit V (R) \\
\hline Length & 5.31 & 5.10 & - & 4.41 \\
Proximal width & - & 2.71 & - & 2.65 \\
Distal width & 2.09 & 2.39 & - & 2.03 \\
\hline
\end{tabular}

TABLE 51. - Measurements of the ungual phalanges of Alcidedorbignya inopinata (in $\mathrm{mm}$ ).

\begin{tabular}{lccccc}
\hline MHNC 8372 & Digit I & Digit II (L) & Digit III (L) & Digit IV & Digit V \\
\hline Length & - & 5.13 & 5.19 & - & - \\
Proximal width & - & 2.32 & 2.34 & - & - \\
Proximal depth & - & 2.51 & 2.54 & - & - \\
Distal width & - & 1.71 & 1.83 & - & - \\
\hline
\end{tabular}

$5 \mathrm{~mm}$ long, as in the manus. The morphology of the ungual phalanges known is similar to that described in the manus section, with a flattened distal extremity, cleft, relatively wide transversely, and slightly deflected plantarly (Figs 112, 113). However, they are slightly shorter and more slender (with a distal enlargement less developed) on the pes: they are between 4 and $5 \mathrm{~mm}$ long, i.e. they are as long as the intermediate phalanges, whereas on the manus, the ungual phalanges of digits II-IV are longer than the intermediate ones. When articulated, digit III (metatarsal included) is $35 \mathrm{~mm}$ long vs $31 \mathrm{~mm}$ for digit II, which is hardly longer than on the hand. The metatarsals being longer than the metacarpals, this means that the phalanges present approximately the same length on the manus and pes.

\section{DISCUSSION}

The discussion below has separate emphases for the cranial or the postcranial anatomy. The cranial discussion focuses on the character analysis, with a comparison and an evaluation of the possible state of the characters in Alcidedorbingya that is to say, discussion of the skull has an obvious phylogenetic 
orientation. In the postcranial discussion, the characters will be functionally considered in order to provide a hypothesis on the locomotory adaptations and behaviour of Alcidedorbignya and to approach some aspects of its palaeobiology.

\section{COMPARISON AND ANALYSIS OF CRANIAL CHARACTERS}

In the following comparison, we survey the major cranial anatomical features of Alcidedorbignya and analyse their distribution among various eutherians and metatherians as well as some non-therian mammalian outgroups, mentioned in the Material and Methods section. This comparison section considers some characters, and many taxa not included in the data matrix below. A goal of this comparison is to provide some interpretation on character polarity of Alcidedorbignya within Pantodonta and/or Placentalia and Eutheria. However, in some cases, the great intraspecific or interspecific variation and the broad and irregular distribution of the characters makes interpretation problematic. This also occurs in the case of highly homoplastic characters. In some of these cases, the cladistic analysis performed below provided an interpretation of the unresolved polarities.

\section{Teeth}

Comparison of the cheek teeth has been detailed in Muizon \& Marshall (1992) and will not be considered here. In the following section we only discuss aspects of the incisors and molars relative size.

Incisors. The upper incisors of Alcidedorbignya are slightly procumbent, and, in this respect, they resemble those of Bemalambda, which appear to be anteroventrally oriented based on illustrations of Zhou et al. (1977: pl. 10 [fig. 1] and pl. 11 [fig. 3]). They differ from the condition of most of the other pantodonts, in which the upper incisors are generally subvertical. It is noteworthy, however, that the incisors of the terminal taxon Coryphodon are also procumbent, although to a much greater extent than in Alcidedorbignya (they are also distinctly splayed anteriorly, a condition absent in other pantodonts). Upper incisors are not procumbent in the early Palaeocene metatherians Pucadelphys and Andinodelphys (although the I1 is enlarged in the latter genus) nor in the Late Cretaceous eutherians Asioryctes and Kennalestes. It is therefore possible that the condition of Alcidedorbignya and Bemalambda is derived within Eutheria. It is difficult to determine whether or not this condition is plesiomorphic for pantodonts. Few Palaeocene placentals document this character; however, it is noteworthy that a slight procumbency of upper incisors is present in Arctocyon and Arctocyonides (Russell 1964: figs 22 and 46).

As in all pantodonts (in which the anterior region of the premaxillae is preserved) contralateral I1 are well separated and do not contact each other. Although the anterior tips of the premaxillae are not preserved in the two skull of Alcidedorbignya, this region can be reconstructed (Figs 11, 14). This can be done either by duplicating the right premaxilla and incisors of MHNC 8372 and placing on the left side a reversed copy, or by joining the original and a reversed copy of the right complete premaxilla + maxilla of MHNC 8399. Fig. 14 provides sufficient documentation to demonstrate that the I1s of Alcidedorbignya had a conspicuous space between them. A similar condition is present in tillodonts (Trogosus, Tillodon), in which the anterior upper incisors (I2s according to Gazin 1953, and Gingerich \& Gunnell 1979) are widely separated. This condition is also present in some groups of Recent mammals such as erinaceomorphs (e.g., Erinaceus) or tenrecoid afrotheres (e.g., Tenrec, Setifer). It is absent in early metatherians and eutherians (e.g., Pucadelphys, Andinodelphys, Asioryctes, Kennalestes) and in many extant and fossil placentals that preserve the first pair of upper incisors. It is likely to represent a synapomorphy of pantodonts and possibly of both pantodonts and tillodonts.

Molars relative size. The relative size of the molars in Alcidedorbignya (see Muizon \& Marshall 1992) can be compared to that of other eutherians and to the Inhibitory Cascade model of Kavanagh et al. (2007). Lower molar areas for determination of molar ratios (in relation to IC model of Kavanagh et al. 2007) can be calculated as the product of the mean length and width of the considered molar based on data from Muizon \& Marshall (1992: table 1). Alcidedorbignya exhibits the following mean ratio values of molars areas: $\mathrm{m} 2 / \mathrm{m} 1=1.41$ and $\mathrm{m} 3 / \mathrm{m} 1=1.47$. With these values, Alcidedorbignya falls on the lower right boundary of the Pantodonta polygon cluster within the range of Pantodonta as studied by Halliday \& Goswami (2013: fig. 3). It barely falls in the area predicted by the IC model (Polly 2007; Kavanagh et al. 2007), and not within the center of the morphospace that includes many "basal" mammalian groups (australosphenidans, stem therians and stem placentals) - those in which molar size is equal or subequal along the tooth row (Halliday \& Goswami 2013). Alcidedorbignya is distinct from these taxa in having both $\mathrm{m} 2$ and $\mathrm{m} 3$ much larger than $\mathrm{m} 1$ (ratios > 1.4). In this respect, it resembles some artiodactyls, perissodactyls, later diverging pantodonts (e.g., Asiocoryphodon, Titanoides) and a few condylarths (Halliday \& Goswami 2013); this also resembles a less extreme version of the condition of xenungulates, pyrotheres and astrapotheres among South American ungulates (see Wilson et al. 2012).

\section{Bony skull}

General morphology of the skull. Alcidedorbignya differs from the other pantodonts except Pantolambda bathmodon in having a regularly domed dorsal profile of the skull, this character being especially pronounced on the snout (Figs 10, $11,14)$. The other pantodonts generally have relatively flat nasals in lateral view and the dorsal sagittal profile is either subrectilinear (e.g., Haplolambda, Titanoides, Barylambda, Coryphodon) or presents a distinct angulation at the contact between the rostrum and the braincase (e.g., Caenolambda, Bemalambda). The condition of Alcidedorbignya inopinata and Pantolambda bathmodon corresponds to a lower rostrum and therefore a smaller and lower narial opening 
than in the other pantodonts, in which the nasal cavity is strongly elevated dorsoventrally (at least in the North American pantodonts). Muizon \& Marshall (1992) have regarded Bemalambda as an early-diverging pantodont, which would be an indication that a domed lateral view of the skull could be a derived feature of Alcidedorbignya and Pantolambda bathmodon. However, this character state is not present in Pantolambda cavirictus, a species slightly younger than $P$. bathmodon, and the distribution of this character state is therefore probably more complex. Furthermore, this character is difficult to observe in other early-diverging eutherians because the available specimens are generally heavily distorted. A domed dorsal profile of the skull (especially the rostrum) seems, however, to be present in Maiorana, an early Palaeocene "condylarth" (personal observation), although the only known skull of this genus (YPM-PU 14111) is also slightly compressed dorsoventrally. Apparently a domed cranium and rostrum vault is also present in the early-diverging eutherians such as Maelestes, Ukchudukodon, Kennalestes, Asioryctes, Barunlestes, and possibly Kulbeckia (Kielan-Jaworowska 1975a; McKenna et al. 2000; Archibald \& Averianov 2003; Wible et al. 2009). It is absent in the highly specialized skull of Zalambdalestes. Therefore, because this condition is present in several Late Cretaceous eutherians (and many extant placentals), it may represent a plesiomorphic condition for the broader group. Alcidedorbignya and Pantolambda would therefore exhibit the plesiomorphic condition within pantodonts. This character is also present in many extant placentals.

Another general feature of the skull of Alcidedorbignya is the stoutness and apparent shortness of its snout. However, as mentioned above, the rostrum of Alcidedorbignya is proportionally longer than that of Pantolambda. In fact, as observed in Table 3 the two genera are the most extreme in the order in terms of relative rostrum length, the longest being that of Alcidedorbignya and the shortest, those of Barylambda, Harpyodus, and Pantolambda. Among pantodonts Coryphodon and Haplolambda have relatively long rostra, which approach that of Alcidedorbignya.

Billet (2010) defined a character (ch. 68 of Billet 2010) on the relative lengths of the rostrum and the braincase: rostrum length/ braincase length $<0.5$ (braincase more than twice as long as the rostrum). This character can be formulated slightly differently: rostrum long (longer than one- third of the total length of the skull from the occipital condyle to anterior alveolar border of the premaxilla); rostrum short (shorter than one-third of skull length). According to this character, the pantodonts that have a relatively short rostrum are Barylambda and Pantolambda, with a rostrum slightly shorter than one-third of the length of the skull. The short rostrum in the large-sized taxon Barylambda suggests that pantodonts do not strictly follow the allometric constraint evidenced for several placental lineages, i.e. that larger taxa have relatively longer faces (Cardini \& Polly 2013). The relative rostrum length of the tillodont Azygonyx falls within the pantodont range, while that of the early Palaeocene "condylarth" Maiorana is 5\% larger than in Alcidedorbignya.
Premaxilla. The posterodorsal ascending process of the premaxilla has a large contact with the nasal in Alcidedorbignya, Pantolambda, Caenolambda, and Bemalambda. There is no contact in the other North American pantodonts probably because of the elevation of the rostrum and nasal cavity. The condition of Alcidedorbignya clearly represents the plesiomorphic character state, which is present in all other early mammals. The absence of contact of the premaxilla with the frontal is also a plesiomorphic character state for eutherians (Novacek 1986; Wible et al. 2009).

The posterodorsal ascending process of the premaxilla of Alcidedorbignya extends dorsally but not posteriorly and remains dorsal to the anterior edge of the canine. This condition is present in Pantolambda and other North American pantodonts. In Bemalambda, Caenolambda, and Barylambda the apex of the ascending process apparently extends posteriorly slightly beyond the canine and is above P1 (Simons 1960; Zhou et al. 1977). A short posterodorsal ascending process of the premaxilla is present in the basal metatherians Pucadelphys and Andinodelphys but not in Mayulestes in which the process extends beyond the canine, above the posterior edge of P1 (as in other borhyaenoids). A short posterodorsal ascending process of the premaxilla is present in most basal eutherians for which this character can be observed such as Maelestes, Kennalestes, Asioryctes, Ukhaatherium, and Zalambdalestes. A short posterodorsal ascending process, which does not extend posteriorly beyond the canine is therefore likely to be the plesiomorphic condition for therians (Luo \& Wible 2005).

The palatal process of the premaxilla of Alcidedorbignya extends posteriorly and nearly contacts the alveolus of the canine. A similar condition is present in Pantolambda. It is also present in many metatherians (including basal metatherians such as Pucadelphys, Andinodelphys, and Mayulestes), in Erinaceus, Solenodon, Potamogale, and Tenrec; and in the archaic ungulates Maiorana, Hyopsodus, Meniscotherium, Pleuraspidotherium, Cynodictis and Phenacodus. This condition is absent in Mongolian asioryctitheres (Asioryctes, Kennalestes, Barunlestes, Zalambdalestes), in Kulbeckia, and in the archaic ungulates Arctocyon and Arctocyonides. A palatal process of the premaxilla contacting (or almost contacting) the alveolus of the canine has been regarded by Rougier et al. (1998) and Wible et al. (2009) as a synapomorphy of metatherians and of some eutherians (including placentals) (node Q in Wible et al. 2009). However, the condition of Alcidedorbignya and other eutherians indicates that this character state presents some degree of homoplasy or may represent a plesiomorphic condition for therians.

Nasal. The great width of the posterior portion of the nasals of Alcidedorbignya is a plesiomorphic character state that is present in most early mammals (e.g., triconodonts, morganucodonts, multituberculates, Vincelestes, Pucadelphys, Andinodelphys, Mayulestes, Kennalestes, Zalambdalestes, and the tillodonts Trogosus and Tillodon). It is also present in Bemalambda and very slightly developed in Pantolambda and Titanoides. It is absent in Haplolambda, Caenolambda, Barylambda, and Coryphodon. Therefore, within pantodonts, Alcidedorbignya 
presents the plesiomorphic condition of a posteriorly expanded nasal (Wible et al. 2009). This character is responsible for a narrow contact of the maxilla with the frontal in the facial region, which represents a plesiomorphic condition (Novacek 1986). Pantodonts, however, always present a frontal-maxilla contact, a derived condition among Theria. The plesiomorphic condition is a nasal-lacrimal contact, which is present in e.g., triconodonts, morganucodonts, multituberculates, Deltatheridium, borhyaenoid metatherians, Zalambdalestes, and Kennalestes. It is noteworthy that a narrow contact of the maxilla with the frontal in the facial region may also be the result of an expansion of the lacrimal on the face as is observed in notoungulates.

In dorsal view the nasals of Alcidedorbignya appear transversely expanded at their apex, although to a much lesser extent than their posterior end. This condition, which is due to the medial inflection of the maxilla-nasal suture in the median region of the nasals, is also present in Bemalambda, while other pantodonts have nasals with subparallel lateral edges. Expanded apices of the nasals are absent in early metatherians (e.g., Pucadelphys, Andinodelphys) and eutherians (Asioryctes, Kennalestes, Zalambdalestes, Leptictis), in which they could represent the plesiomorphic condition. Alcidedorbignya and Bemalambda would be derived for this character state within eutherians. However, given the fact that the two genera are the earliest-diverging pantodonts, for which the skull (with well-preserved nasals) is known, their condition may represent the plesiomorphic character state for pantodonts. It is noteworthy that apically expanded nasals are also present in Aphronorus, a late Palaeocene pantolestid (Gingerich et al. 1983) and in Erinaceus.

The more anterior location of the anterior tips of the nasals relative to the most anterior point of the premaxillary-nasal suture (i.e. nasals overhanging nasal aperture), which is present in Alcidedorbignya, appears to be a widespread and possibly plesiomorphic feature for eutherians and placentals (Wible et al. 2009; O'Leary et al. 2013). However, the alternative state, a more posterior location of the nasals, is however present in various placentals like primates (Adapis, Magnadapis), carnivorans (Cynodictis and Amphicynodon), afrotherians (Procavia [contra Wible et al. 2009], Tenrec, Potamogale and Orycteropus [Wible et al. 2009]) and eulipotyphlans (Erinaceus, Solenodon, Talpa). This character state is also present in the archaic ungulates, Meniscotherium, Arctocyonides, and possible Hyopsodus.

In Alcidedorbignya, the posteriormost extent of the nasofrontal suture is level with the anterior edge of the orbit (MHNC 8372) or slightly more posterior (MHNC 8399). Such a condition is present in basal eutherians, such as Zalambdalestes, Asioryctes, Kulbeckia, and Leptictis. It is also present in Pantolambda but in Haplolambda it is distinctly posterior. The suture is level with the anterior edge of the orbit in Azygonyx but well posterior to it in Trogosus. The condition of Alcidedorbignya is present in Recent Solenodon and in tenrecoids and lipotyphlans. Conversely, a posterior end of the nasofrontal suture well anterior to the anterior edge of the orbit occurs in some afrotherians (Procavia), some notoungulates (Proty- potherium, Archaeohyrax), some litopterns (Diadiaphorus), some scandentians (Tupaia), some bats (Pteropus) and some xenarthrans (Dasypus). A suture roughly level with or slightly posterior to the anterior edge of the orbit, as observed in Alcidedorbignya, seems to represent the plesiomorphic condition, while the derived condition is a suture well anterior to it. The posteriorly-located suture observed in Haplolambda and Trogosus may also be a derived condition.

Maxilla. The maxilla of Alcidedorbignya is conspicuously inflated laterally (in dorsal view) between the anterior root of the zygomatic arch and the canine. This condition is absent in other pantodonts and in early tribosphenidans. It probably represents a derived condition in Alcidedorbignya indicating that, although similar to Pantolambda, it is probably not directly ancestral to the North American genus. This absence of a direct ancestor-descendant relationship has already been noted by Muizon \& Marshall (1992) on the basis of the presence of single rooted P2 and p2 in Alcidedorbignya, a derived condition absent in Pantolambda. A laterally-inflated maxilla is also present in the tillodont Azygonyx. Conversely, many placentals instead exhibit a concavity on this region of the rostrum.

A characteristic feature of Alcidedorbignya and Pantolambda is the strong posterior convex projection of the maxilla in the orbitotemporal fossa, which forms the floor of the orbit and the consequently strongly concave (notched anteriorly) anterior edge of the orbitotemporal fossa (in ventral view). This condition is especially pronounced in MHNC 8372 (a young adult) but is less developed in MHNC 8399 and 8400, thus indicating some individual variation of this character (possibly related to ontogenetic age). This condition is present in other pantodonts although to a lesser extent. A posteriorly convex extension of the maxilla with a concave (anteriorly) anterior edge of the orbitotemporal fossa is also present in the early Eocene esthonychid tillodont Azygonyx and to a lesser extent in Trogosus as well as in many "condylarths" (e.g., $H y$ opsodus, Meniscotherium, Pleuraspidotherium, Arctocyonides), some notoungulates (Archaeohyrax; variable in Periphragnis), astrapotherians (Trigonostylops) and basal artiodactyls (Dichobune). In the oldest "condylarths" skulls known (Maiorana, Baioconodon) the anterior edge of the temporal fossa is slightly concave (anteriorly) to straight. It is noteworthy that a slightly concave (anteriorly) anterior edge of the orbitotemporal fossa is also present in some palaeoryctids (Eoryctes). The anterior edge of the orbitotemporal fossa is distinctly to slightly convex (anteriorly) and the maxilla does not project posteriorly in Vincelestes, in early metatherians (e.g., Pucadelphys, Andinodelphys, Mayulestes) and eutherians (e.g., Maelestes, Asioryctes, Kennalestes, Zalambdalestes), which is likely to represent the plesiomorphic condition. A posterior projection of the maxilla is also absent in Leptictis, Tenrec, Solenodon, Erinaceus, Potamogale, carnivorans Cynodictis, Amphicynodon, primates (Adapis, Magnadapis), litopterns, etc. The fact that Bemalambda and Harpyodus, the earliest-diverging pantodonts for which the skull is known, and Lofochaius, the earliest known tillodont do not present a posterior projection of the maxilla flooring 
the orbit and have a straight to convex (anteriorly) anterior edge of the orbitotemporal fossa is also an indication that this morphology could represent the plesiomorphic condition for these groups. It is noteworthy that in the case of a maxilla which does not project posteriorly in the orbit, the convex (anteriorly) anterior edge of the orbitotemporal fossa is generally anterior and lateral to the last molars (e.g., Bemalambda, Harpyodus); in the other (probably derived) condition (e.g., Alcidedorbignya) the last molar "abuts" the anterior edge of the orbitotemporal fossa.

The roots of the last molars (M2-3) of Alcidedorbignya pierce the maxilla dorsally and outcrop on the floor of the orbit. This condition is present in Pantolambda but is apparently absent in Haplolambda and Coryphodon. It is also absent in tillodonts (Azygonyx, Trogosus). It is present in the archaic ungulates Maiorana, Baioconodon, and Arctocyonides, but is absent in Pleuraspidotherium. It is scored by Wible et al. (2009) as absent in Hyopsodus, Meniscotherium and Phenacodus. It is present in early-diverging metatherians [Pucadelphys, Mayulestes (contra Wible et al. 2009) and Andinodelphys], in Maelestes, Kennalestes, Asioryctes, Zalambdalestes, Tenrec, Solenodon, Erinaceus, Tupaia, and Dichobune but it is absent in Leptictis. Therefore, because this condition appears to be present in most early-diverging metatherian and eutherian taxa it probably represents a plesiomorphic condition.

The maxilla of Alcidedorbignya and Pantolambda has no orbital ascending process in the orbit and has neither contact with the frontal nor participation in the orbital wall. This condition is the plesiomorphic character state for eutherians (Novacek 1986; Wible et al. 2009). There is no orbital ascending process of the maxilla in early metatherians (e.g., Pucadelphys, Andinodelphys) and eutherians (e.g., Maelestes, Asioryctes, Kennalestes, Zalambdalestes, Leptictis, Maiorana, Arctocyonides, Phenacodus, Pleuraspidotherium, Dichobune, Acotherulum, Archaeohyrax, Protypotherium, Trigonostylops, Dasypus, Tupaia, Ptilocercus, Cynodictis). A large ascending process of the maxilla in the orbit is present in e.g., Tenrec, Solenodon, Erinaceus, Meniscotherium. A moderately-sized orbital ascending process of the maxilla is present in Procavia and Magnadapis.

The bifurcated maxilla-jugal suture of Alcidedorbignya, Pantolambda, Bemalambda, and probably Harpyodus is an apomorphic character state within eutherians (Novacek 1986; Wible et al. 2004; Wible et al. 2009). Because in pantodonts the maxilla-jugal suture is rapidly fused in ontogeny, it is not possible to observe the condition in other pantodonts. However, the presence of a bifurcated suture in the oldest and earliest-diverging taxa of the group (Muizon \& Marshall 1992) indicates that this character state is probably plesiomorphic within pantodonts. A bifurcated jugal-maxilla suture is absent in metatherians. However, this feature is also absent in Vincelestes, and in some early-diverging eutherians (e.g., Prokennalestes, Kennalestes, Zalambdalestes, Maelestes). It is also absent in Recent erinaceomorphs (e.g., Erinaceus, Echinosorex). A bifurcated maxilla-jugal suture is also present in the tillodonts Azygonyx and Trogosus, in the archaic ungulates Baioconodon, Maiorana (possibly), in Leptictis and in the carnivorans Cynodictis and Vulpavus (Wible et al. 2009). It is absent in Hyopsodus, Meniscotherium, Archaeohyrax, Protypotherium, Macrauchenia, Procavia, Tupaia, and Acotherulum. Both conditions are present in Phenacodus and Adapis (fide Wible et al. 2009).

The single infra-orbital foramen of Alcidedorbignya is located above the posterior upper premolars. An infra-orbital foramen located above the premolars rather than above the molars is also found in most early-diverging eutherians (including Maiorana, Baioconodon) and might represent the plesiomorphic condition for Placentalia (Wible et al. 2009 character 165).

Turbinals and nasal cavity. Each nasal fossa of Alcidedorbignya possesses eight major turbinals, including one maxilloturbinal, one nasoturbinal, two frontoturbinals, and four ethmoturbinals, as observed in Leptictis (Novacek 1986), Carcinella (Koenigswald et al. 2009), Solenodon (Wible 2008), and Ptilocercus (Wible 2011). In this respect, the nasal cavity of Alcidedorbignya exhibits the condition that is presumed to be primitive for eutherians (Smith \& Rossie 2008; Van Valkenburgh et al. 2014).

In Alcidedorbignya, the primary laminae of the maxilloturbinal are clearly observable on the posterior floor of the nasal cavity but not on the medial wall of the maxilla and premaxilla, a condition that differs from that observed in e.g., didelphids, tupaiids, carnivores, primates, Echinosorex, Setifer, Potamogale, in which a primary lamina of the maxilloturbinal also originates on the medial wall of the maxilla and premaxilla. However, Tenrec exhibits a condition that is apparently similar to that of Alcidedorbignya, in which the primary lamina of the maxilloturbinal only originates from the posterior floor of the nasal cavity (from the maxilla).

Alcidedorbignya presents two frontoturbinals (ectoturbinals) a condition also present in didelphids (Caluromys [Macrini 2012, 2014] and Monodelphis [Rowe et al. 2005]), Leptictis, tenrecoids, erinaceomorphs, and soricomorphs. Two frontoturbinals probably represent the plesiomorphic condition for eutherians (Novacek 1986, 1993).

Alcidedorbignya has four ethmoturbinals (endoturbinals), a condition present in lipotyphlans, chiropterans, hyracoids some primates, many rodents and many carnivorans, which apparently represent the plesiomorphic condition for eutherians (Gregory 1910; Novacek 1993). Five ethmoturbinals are present in didelphids, but there are seven in Tachyglossus and three in Ornithorhynchus.

In terms of paranasal recesses, the primitive eutherian condition includes a lateral recess composed of both frontal (upper) and maxillary (lower) recesses, separated by a horizontal lamina (frontomaxillary septum; Maier 2000), which is the condition observed in Alcidedorbignya. Among the paranasal sinuses, the maxillary sinus is widely present in eutherians and considered as a plesiomorphic character (Novacek 1993). Alcidedorbignya would therefore, exhibit the plesiomorphic state for this character. It is noteworthy, however, that the maxillary sinus of Alcidedorbignya is much more inflated than in other pantodonts. Additional paranasal sinuses (i.e. sphenoidal and frontal) occur in a large number 
of mammals (Novacek 1993). However, in Alcidedorbignya, there is no clear evidence of a frontal sinus or a sphenoidal sinus. In this respect, it resembles the condition observed in the apatemyid Carcinella (Koenigswald et al. 2009).

Palatine. The palatines of Alcidedorbignya are very broad and almost contact M2 and M3 on the posterolateral edge of the palate. This condition is less pronounced in Pantolambda, where the palatine-maxilla suture is slightly more medial. In this genus the Pal-Mx suture is located 2 to $3 \mathrm{~mm}$ medial to M2 and M3. In the tillodont Azygonyx (A. ancylion, UM68511) the palatines are very close to (but not contacting) the posterior molars (M2-3) and in Trogosus the palatine contacts the M3s only (Gazin 1953). Very broad palatines are present in the archaic ungulates Arctocyon and Arctocyonides, where these bones are in contact with the M3s but not with the M2s. In Meniscotherium the palatines contact the M2 (posteromedial angle) and M3 laterally. Very wide palatines contacting the alveolar border of M2 and/or M3 are not present in the archaic ungulates, Pleuraspidotherium and Phenacodus (condition in Maiorana and Baioconodon are unclear on the available specimens) but in these genera the lateral edge of the palatine is close to the alveolar border as in Azygonyx. This latter condition is also present in Maelestes, Leptictis, tupaiids, Solenodon and litopterns. In the early-diverging metatherians Pucadelphys and Andinodelphys the lateral edge of the palatine is more medial than in the latter taxa and iswell-separated from the medial edge of the molars. Palatines well separated from the medial edges of the molars are also present in e.g., Zalambdalestes, Asioryctes, Kennalestes, Aphronorus, Erinaceus, Tenrec, and Archaeohyrax.

The major palatine foramina of Alcidedorbignya are located in the maxillopalatine suture at the limit between the two bones. This condition is also present in early-diverging metatherians (Pucadelphys, Andinodelphys and Mayulestes) and eutherians (Maelestes). It is also present in the archaic ungulates Arctocyon and Pleuraspidotherium, in the lipotyphla Solenodon (contra Wible et al. 2009), in the artiodactyl Dichobune, in some notoungulates (Periphragnis), pyrotheres (Pyrotherium) and astrapotheres (Trigonostylops). The condition in other condylarths (Maiorana, Baioconodon, Hyopsodus, Meniscotherium) is unclear or highly variable (Wible et al. 2009). According to these authors the plesiomorphic condition is a major palatine foramen within the palatine. This plesiomorphic character state is present in Vincelestes, Kennalestes (fide Wible et al. 2009) and Leptictis but it is also present in the artiodactyl Acotherulum. In some taxa the condition of the major palatine foramina cannot be observed because it is obliterated by the presence of palatine fenestrae such as in many didelphids or in Erinaceus. The major palatine foraminae may also perforate the maxillae well ahead of the maxillopalatine suture. This is a derived condition (Wible et al. 2009), which is present in some early-diverging eutherians (Barunlestes, Zalambdalestes) but also in many Recent eutherians and in some notoungulates (Archaeohyrax, Protypotherium (variable intra-individually). A particular condition is found in some litopterns with the major palatine foramen opening in a lateral location, very close to the lingual edges of molars and close (anterolateral to) or within the maxillary-palatine suture (Diadiaphorus, Macrauchenia). Therefore the distribution of this character seems to be extremely variable, both taxonomically and stratigraphically.

The minor palatine foramen (or canal) of Alcidedorbignya is opened posteroventrally and-limited to a deep notch in the posterolateral angle of the palate. This condition is present in all other pantodonts, in tillodonts (Azygonyx, Trogosus), and "condylarths". A fully enclosed minor palatine canal is present in early-diverging metatherians (e.g., Pucadelphys, Andinodelphys, Mayulestes) and eutherians (e.g., Maelestes, Asioryctes, Kennalestes, Zalambdalestes, Uchkudukodon), in leptictids, in Erinaceus, in some Scandentia, soricoids, tenrecoids, rodents, tubulidentates, euprimates, and a variety of early mammals (Novacek 1986; Wible \& Rougier 2000; Wible et al. 2004, 2009). Because it is almost constantly present in early-diverging metatherians and eutherians, it likely represents the plesiomorphic character state for therians. Pantodonts, therefore, present the apomorphic character state for eutherians.

The presence of an accessory palatine foramen (= middle palatine foramen) in Alcidedorbignya and Pantolambda is a plesiomorphic character state for eutherians (Novacek 1986).

The posterior edge of the palatines of Alcidedorbignya is almost straight to incipiently double notched with a small postpalatine spine. The double notch is present but more pronounced in Pantolambda, Caenolambda, and Haplolambda. In Bemalambda the posterior edge of the palate is almost straight. A straight posterior edge or slightly curved (but not double arched) of the palate likely represents the plesiomorphic condition within therians (Novacek 1986), which is present in early-diverging metatherians (e.g., Pucadelphys, Andinodelphys, Mayulestes) and eutherians (e.g., Asioryctes, Kennalestes, Zalambdalestes, Maelestes, Uchkudukodon). Therefore, the American pantodonts mentioned above present the apomorphic character state and Alcidedorbignya is more primitive than the North American taxa for this character. The presence of a postpalatine spine (or posterior nasal spine), probably partly correlated with the preceding feature, is widespread within placentals and observed in Plesiadapis, Amphicynodon, Cynodictis, Procavia, Dichobune, Leptictis, Erinaceus, Notostylops, Trigonostylops, Tenrec, Solenodon, Tupaia, Sciurus, and Arctocyon. It is, however, absent in the early-diverging eutherians Maelestes and Zalambdalestes and in some placentals like Dasypus and Pteropus. The development of the postpalatine spine varies from weak, as in Alcidedorbignya, to prominent as seen in Erinaceus and Procavia, and even to a massive and salient process as in Trigonostylops. The presence of a strong post palatine torus has been regarded as a derived character state within eutherians by Novacek (1986). However, a well-developed torus is present, among others, in early-diverging metatherians (e.g., Pucadelphys, Andinodelphys, Mayulestes, Asiatherium) and eutherians (e.g., Asioryctes, Kennalestes, Ukhaatherium, Zalambdalestes, Barunlestes, Maelestes, Uchkudukodon, Cimolestes). Therefore, its presence in most early-diverging therians (in which this feature is known) casts doubt on the polarity proposed by Novacek. A strong post-palatine torus is also present in a variety of placentals (e.g., Leptictis, Trigonostylops,Notostylops, 
Maiorana?, Arctocyonides, Pleuraspidotherium, Plesiadapis, Magnadapis, Azygonyx, Dichobune, Acotherulum, Diacodexis, Paramys, Hyopsodus, Erinaceus, Tenrec, Solenodon, Tupaia, Aphronorus [pers. obs. and Wible et al. 2009]). It is strictly absent (not weak) in xenarthrans (Wible et al. 2009), in many (if not all) carnivorans (Amphicynodon, Cynodictis, Miacis [Wible et al. 2009], Lynx, Felis, Genetta, Ursus, Hyaena, etc.), in many perissodactyls (e.g., Tapirus, Equus, Palaeotherium, Ceratherium) and chiropterans (Simons \& Conway 2003). The presence of a postpalatine torus in Alcidedorbignya may therefore be regarded as a plesiomorphic character state, which is also present in Pantolambda and apparently in Bemalambda (Zhou et al. 1977).

In Alcidedorbignya, the sphenopalatine foramen is distinctly separated from the caudal palatine foramen (= dorsal palatine foramen), but both foramina open in a common recess. According to Novacek (1986), this condition, which is present in Leptictis, is a derived character state within eutherians. The condition is apparently present in Pantolambda but is difficult to observe because of the crushing of the bones of the orbit in AMNH 16663. The condition in Bemalambda and Harpyodus is unknown because specimens of these genera were not available during this study. It is absent in early metatherian (e.g., Pucadelphys, Andinodelphys). This character could not be checked in Mongolian asioryctitheres. In the "condylarths" Arctocyonides, Baioconodon and Maiorana, (and possibly Pleuraspidotherium) these two foramina are more closely approximated to each other than in Alcidedorbignya. The same is true for the early-diverging eutherian Zalambdalestes (Wible et al. 2004), the litoptern Diadiaphorus, the artiodactyls Dichobune and Acotherulum (merged in the latter), the tenrecoid Potamogale, the carnivoran Amphicynodon, various notoungulates (Plesiotypotherium, Paedotherium; foramina merged) and the primate Plesiadapis. Therefore, the approximate location of these two foramina in a recess is a widely-distributed feature within eutherians. The sphenopalatine foramen opens at the level of the anteroposterior mid-length of the orbital floor in Alcidedorbignya. It opens relatively more posterior (at the posteromedial corner of the orbital floor) in many eutherians, such as Plesiadapis, Maiorana, Baioconodon, Amphicynodon, Cynodictis, Dichobune, Arctocyonides, Pleuraspidotherium, Tenrec, Solenodon, Notostylops, and Trigonostylops. A more anterior position is less common and is found in Diadiaphorus, Acotherulum, interatheriid notoungulates and Tupaia.

A large ascending (orbital) process of the palatine, which articulates with the frontal and lacrimal in the orbit, is a plesiomorphic character state for eutherians (Novacek 1986; Wible et al. 2009). Alcidedorbignya and Pantolambda, which present such an ascending process of the palatine, are therefore plesiomorphic for this character state. This character could not be observed in the other pantodonts, mostly because the orbit is generally poorly preserved or crushed, or because of the fusion of the bones. The plesiomorphic condition is present in early metatherians (e.g., Pucadelphys, Andinodelphys), in the asioryctitheres eutherians from Mongolia (e.g., Kennalestes, Asioryctes, and Barunlestes), in the archaic ungulates Hyopsodus, Arctocyonides, Maiorana, Pleuraspidotherium, Di- chobune; in Leptictis, Potamogale, Tupaia, in notoungulates (but moderate; Protypotherium, Plesiotypotherium, Puelia), and in the litoptern Diadiaphorus. An ascending process of the palatine in the orbit is absent in Zalambdalestes, Moeritherium, Erinaceus, Tenrec, and Solenodon (Wible et al. 2009) as well as in Trigonostylops (Simpson 1967).

The posterior margin of the palate is even with the ultimate molar in Alcidedorbignya, Bemalambda, Pantolambda, and Haplolambda. This condition of the palatal extension with regard to the ultimate molar, has been regarded as the plesiomorphic character state within eutherians by Rougier et al. (1998) and Wible et al. (2009) and probably represents a general condition within pantodonts. This plesiomorphic character state is also present in tillodonts, (Azygonyx and Trogosus), in some archaic ungulates (Arctocyonides, Arctocyon, Pleuraspidotherium, Hyopsodus), in the astrapothere Trigonostylops and in some early eutherians (e.g., Zalambdalestes, Kennalestes, Asioryctes, Barunlestes). It is absent in some early metatherians (e.g., Pucadelphys, Andinodelphys, Mayulestes), in which the palatine projects posteriorly well behind the last molars (a derived condition fide Wible et al. 2009) but is present in Deltatheridium, Asiatherium and in the Gurlin Tsav skull (Szalay \& Trofimov 1996 and personal observations). In the basal ungulates Maiorana and Baioconodon it is slightly posterior to the last molar. Another derived condition (posterior margin of the palate anterior to last molar or even to its anterior border) is present in the early-diverging eutherian Maelestes, in the pantodont Harpyodus, in the archaic ungulate Meniscotherium, in the litopterns Diadiaphorus and Proterotherium; in Palaeotherium (and many perissodactyls), in the artiodactyls Dichobune and Acotherulum and in the palaeoryctid Eoryctes. The broad taxonomic distribution of this character state (except for rodents; see Wible et al. 2009) is an indication of a strong degree of homoplasy. The notoungulates are a good illustration of the large taxonomic variation of this character, as all states are represented within this order.

In general the palatine of Alcidedorbignya is relatively primitive (when compared to other pantodonts) in the weakness and straightness of the torus and in the small size of the postpalatine spine, and in the large size of the ascending orbital process contacting the lacrimal and the frontal (Novacek 1986). It is derived in the presence of a minor palatine notch and in lacking a minor palatine canal totally enclosed by the palatine or maxilla. The posterior border of the palate, which is anterior to the posterior edge of the last molar, is probably a derived character state in pantodonts.

Lacrimal. Alcidedorbignya bears a rather large facial process of the lacrimal at the anterior edge of the orbit, which represents a plesiomorphic character state (Gregory 1920; Salomon 1930; Novacek 1986; O’Leary 2013), but an apomorphic character state according to the results of the analysis of Wible et al. (2009). In Pantolambda, the facial wing, if any, is very reduced and the condition of Harpyodus decorus and Bemalambda is unclear. A facial process is present in the tillodonts Azygonyx and Trogosus (Gazin 1953), but is apparently smaller than in Alcidedorbignya. A large facial process is present in Vince- 
lestes, Deltatheridium, Asiatherium (probably), and in the early-diverging borhyaenoid metatherians (e.g., Mayulestes, Sallacyon, Callistoe), but a facial process reduced to a rim is present in Pucadelphys, Andinodelphys and in Recent South American metatherians. A large facial wing of the lacrimal is present in many basal eutherians (Eomaia, Zalambdalestes, Maelestes, Uchkudukodon, Kulbeckia), in some "condylarths" (Maiorana -probably-, Baioconodon -probably-, Hyopsodus, Meniscotherium, Phenacodus, Arctocyon, Arctocyonides, Pleuraspidotherium), in many artiodactyls including Dichobune (especially large in this genus) and Acotherulum, in the litoptern Proterotherium and in tupaiids (Tupaia, Ptilocercus). A much reduced facial rim is present in the basal eutherians Asioryctes, Ukhaatherium, and Kennalestes, which probably represents a derived condition (Novacek 1986). It is also small in Eoryctes (Thewissen \& Gingerich 1989), in the primate Adapis, in many notoungulates (Billet 2011), in Procavia, in many carnivorans, in Solenodon (Wible 2008) and in Leptictis. This character is not applicable in several genera, which have lost the lacrimal (e.g., Erinaceus, Potamogale). In the astrapothere Trigonostylops, the facial process of the lacrimal is somewhat intermediate in size between those of Alcidedorbignya and Leptictis. In Tenrec, the lacrimal is exclusively facial and has no intraorbital process.

A distinct lacrimal tubercle is present in Alcidedorbignya, Pantolambda, and Bemalambda. This feature is also present in tillodonts (Azygonyx, Trogosus), in archaic ungulates (e.g., Baioconodon, Maiorana [possibly, but weak if present], Arctocyonides, Phenacodus, Meniscotherium, Hyopsodus [fide Wible et al. 2009], Pleuraspidotherium), in early artiodactyls (Dichobune, Acotherulum, Diacodexis), in typotherian notoungulates, in litopterns, in the primate Magnadapis, in Procavia (prominent), in Tupaia, Leptictis, carnivorans (e.g., Cynodictis, Amphicynodon), in perissodactyls (Equus, Ceratherium, Tapirus), in some xenarthrans (Tamandua, Hapalops; fide O'Leary et al. 2013), in the rodent Sciurus, as well as in the basal eutherian Zalambdalestes (but weak as noted by Wible et al. 2009, if any), and in the pantolestid Aphronorus. A lacrimal tubercle is said to be present in Vincelestes, in the early metatherians Deltatheridium and Asiatherium, although observation of this feature is unconvincing on the published illustrations of these taxa (Bonaparte \& Rougier 1987; Szalay \& Trofimov 1996; Rougier et al. 1998). Furthermore, we did not observe a lacrimal tubercle on a cast of the Deltatheridium specimen (ZPAL MgM-I/102) described by Kielan-Jaworowska (1975b). As a matter of fact, this author does not mention a lacrimal tubercle in this specimen either. It is clearly absent in the basal metatherians Pucadelphys, Andinodelphys, and Mayulestes (contra Rougier et al. 1998 and Wible et al. 2009) as well as in the Gurlin Tsav skull (observation from a cast of the specimen). A lacrimal tubercle is also absent in several early-diverging eutherians (e.g., Asioryctes, Kennalestes, Uchkudukodon, Ukhaatherium) and in many placentals, e.g., Tenrec, Solenodon, Pteropus, Vulpavus, Ptilocercus, Plesiadapis, Magnadapis, Bradypus, Dasypus (Wible et al. 2009 and personal observations). The presence of a lacrimal tubercle has been regarded as a derived character state within eutherians by
Novacek (1986). The absence of a lacrimal tubercle in several basal metatherians and eutherians apparently seems to favour this interpretation. However, because of its extremely variable distribution among eutherians at any level, this character is probably strongly homoplastic and no consistent pattern for distribution of this character emerges from this comparison.

Alcidedorbignya has two lacrimal foramina, which likely represents the plesiomorphic condition for therians. Two lacrimal foramina are present in Vincelestes, in early metatherians (e.g., Deltatheridium, Pucadelphys, Andinodelphys, Mayulestes), in most South American metatherians (with the exception of some borhyaenoids), and in some early eutherians (Acristatherium, Zalambdalestes, Maelestes). A single lacrimal foramen is present in many basal eutherians (Uchkudukodon, Kennalestes, Asioryctes, Barunlestes, Leptictis), "condylarths" (Baioconodon, Maiorana, Pleuraspidotherium, Arctocyonides, Arctocyon, Hyopsodus, Meniscotherium, Phenacodus). Pantolambda apparently has a single lacrimal foramen. The condition of other basal pantodonts such as Harpyodus and Bemalambda is unknown. Many placentals present a single lacrimal foramen but two such foramina are found in some taxa (including Recent placentals), among which are some perissodactyls (Tapirus), some notoungulates (Plesiotypotherium) and some artiodactyls (Dichobune, Capreolus).

A small jugal-lacrimal contact is present in Alcidedorbignya. This is a plesiomorphic character state, which is present in Vincelestes, early metatherians, early-diverging Mongolian eutherians, "condylarths" (e.g., Maiorana, - probably - Baioconodon, - probably-Arctocyonides, Pleuraspidotherium, Hyopsodus, Meniscotherium, etc.) and most placentals. The lacrimal and jugal have no contact (for the taxa that preserve both bones) in tupaiids and interatheriid notoungulates.

Frontal. The postorbital process of the frontal is hardly present in Alcidedorbignya and Pantolambda. It is more a blunt tuberosity than a proper process. The condition of Alcidedorbignya greatly resembles the morphology observed in Leptictis or Erinaceus. A well-developed postorbital process is present in Vincelestes, Zalambdalestes, and possibly Maelestes. A distinct postorbital process is also present in some "condylarths" (e.g., Arctocyonides - in which it is present but weak -, Pleuraspidotherium, Meniscotherium), early artiodactyls (Dichobune, Acotherulum), notoungulates, astrapotheres, the primate Plesiadapis, the bat Pteropus, the carnivorans Cynodictis and Amphicynodon, and the perissodactyl Palaeotherium. In some groups, the strong development of the postorbital process of the frontal, together with the formation of a postorbital process of the jugal closes or almost closes the orbit posterolaterally: this is the case in the sparassodont Thylacosmilus, equids, many ruminant artiodactyls, many primates, some felids, and tupaiids (Tupaia, Ptilocercus). In hyraxes (Procavia), this is instead a process of the parietal (more than the frontal) that forms this postorbital process and almost closes the orbit posterolaterally. A postorbital process, small but distinct, is present in early metatherians (e.g., Pucadelphys, Andinodelphys, Mayulestes) and in some early eutherian (Cimolestes, Kulbeckia, fide Wible et al. 2009). No true postorbital process (rather a postorbital 
tuberosity) is present in early eutherians such as Mongolian asioryctitheres (e.g., Kennalestes, Asioryctes, Ukhaatherium), in the tillodont Azygonyx, in the pantolestid Aphronorus, in Leptictis, Erinaceus, Solenodon, Dasypus, Tamandua, Manis, Tenrec, and Potamogale. It is noteworthy, however, that relatively large postorbital process and crest are present in the primitive pantodonts Harpyodus decorus and Bemalambda. In other respects a reasonably well-developed (somewhat crest-like) postorbital process is present in the derived pantodonts Haplolambda, Titanoides, and Coryphodon. Similarly, the most primitive tillodonts (Azygonyx and Lofochaius) have no postorbital process, but the derived tillodont Trogosus has a pronounced process. Therefore, the distribution and the polarity of this character are confusing. It is noetworthy, however, that in the analysis of Wible et al. (2009) the absence of a postorbital process of the frontal is a synapomorphy of their node "Q" (Wible et al. 2009: fig. 29), which mainly includes the Placentalia. The presence of a well-developed postorbital process would therefore be a plesiomorphic trait within eutherians, whereas most basal eutherians have a process that is small or lacking.

The frontals of Alcidedorbignya have a broad median anterior process wedged between the nasals, which represents an apomorphic condition within Placentalia and close relatives (node Q of Wible et al. 2009). Such a process is present in Pantolambda, Haplolambda, and Bemalambda. An anterior process of the frontal wedged between the nasals is present in some early metatherians (e.g., Deltatheridium, Pucadelphys, Mayulestes; it is absent in Andinodelphys) and in some early eutherians (Asioryctes, Kennalestes, Zalambdalestes). Several early ungulates (e.g., Maiorana, Arctocyonides, Meniscotherium - but variable -, Hyopsodus) do not have an anterior process of the frontals, but a small process is apparently present in Baioconodon. The condition of the tillodont Azygonyx is not observable on the only skull known but it is clearly absent in Trogosus (Gazin 1953). It is absent in tupaiids, in many lipotyphlans (e.g., Solenodon, Tenrec, Potamogale), primates and afrotherians (Wible et al. 2009), in at least some litopterns (Diadiaphorus, Proterotherium), in the artiodactyls Dichobune and Acotherulum, in many carnivorans (e.g., Vulpavus, Miacis [see Wible et al. 2009], Cynodictis), in the bat Pteropus, and in pantolestids (Aphronorus, Pantolestes). The process is present in most notoungulates. Both conditions are notably present in perissodactyls (e.g., Equus, Tapirus, Palaeotherium) and xenarthrans (e.g., Dasypus, Chaetophractus, Tamandua). Therefore, both states of this character have a broad distribution within placentals. It seems, however, that pantodonts retain the plesiomorphic condition for this character.

In the orbit, the orbital process (wing) of the frontal is well developed in Alcidedorbignya, in which it reaches the recess of the sphenopalatine foramen. The process separates the palatine anteriorly and the orbitosphenoid posteriorly. A large orbital process of the frontal (as in Alcidedorbignya) is a plesiomorphic character state for eutherians according to Novacek (1986). A large orbital wing of the frontal is present in early metatherians (e.g., Pucadelphys, Andinodelphys). This condition could not be observed in the Mongolian asioryctitheres. It is apparently present in early ungulates, but difficult to observe on the specimens available during this study (e.g., Maiorana, Baioconodon, Arctocyonides, Pleuraspidotherium), in basal artiodactyls (Dichobune, Acotherulum), in tupaiids, in Leptictis, in notoungulates and many other placentals (Novacek 1986). A large orbital process of the frontal is absent in Erinaceus, Solenodon, Potamogale and the litoptern Proterotherium.

The ethmoidal foramen of Alcidedorbignya is in the orbital process of the frontal. This foramen is located at the limit of the frontal and the orbitosphenoid in Vincelestes, in most metatherians, especially in such basal taxa as Pucadelphys and Andinodelphys, in some basal eutherians (Maelestes, Asioryctes, Kennalestes, Uchkudukodon), in Leptictis, and in Eoryctes. This latter condition is also present in several Recent eutherians (e.g., Erinaceus [variable in this genus], Echinosorex, Solenodon, Tenrec, Procavia, Felis). A foramen in the frontal (as in Alcidedorbignya) is present in Zalambdalestes, in some "condylarths" (Arctocyonides, Pleuraspidotherium, Phenacodus, Hyopsodus), in basal artiodactyls (Dichobune, Acotherulum), in tupaiids, in Potamogale, in Euphractus (Wible \& Gaudin 2004) and in the notoungulate Pachyrukhos. Given the occurrence of an ethmoid foramen located at the limit of the frontal and the orbitosphenoid in Vincelestes and many early-diverging therians (metatherians as well as eutherians) this condition may represent a plesiomorphic character state. Therefore, the condition of Alcidedorbignya probably represents the derived character state.

The frontal of Alcidedorbignya has a small contact with the anterior angle of the dorsal plate of the alisphenoid, posterodorsal to the orbitosphenoid, which is relatively expanded. The condition in other pantodonts and tillodonts could not be observed either because of the fusion or crushing of the bones or because the specimens were not available for this study. The condition observed in Alcidedorbignya is present in Vincelestes (fide Wible et al. 2009), Erinaceus, Solenodon, Tenrec, basal artiodactyls (Dichobune, Acotherulum), Magnadapis, notoungulates (e.g., Billet et al. 2009: fig. 20), and pilosan xenarthrans (Wible et al. 2009). A large contact of the dorsal border of the alisphenoid (approximately half of the border) with the frontal is regarded by Novacek (1986: $84)$ as the primitive eutherian condition. As a matter of fact, a large contact of the two bones is present in basal metatherians, (Pucadelphys, Andinodelphys), in Zalambdalestes, in Leptictis, in Hyopsodus, in the astrapothere Trigonostylops (according to Simpson 1967: fig. 43) and in many armadillos (e.g., Chaetophractus, Dasypus, Euphractus [Wible \& Gaudin 2004], Kuntinaru [Billet et al. 2011]). The absence of contact between alisphenoid and frontal is another character state (Wible et al. 2009), that is notably present in some archaic ungulates (Phenacodus, Meniscotherium), tupaiids and Procavia. Therefore, because of the variable distribution of the states of this character its polarity is difficult to establish securely although the occurrence of a large contact in early metatherians would favour an interpretation of this state as the plesiomorphic condition for therians.

In Alcidedorbignya, the frontal has no intraorbital contact with the maxilla, from which it is separated by the palatine. This condition is also present in Pantolambda. This condition 
is often difficult to observe because of the preservation of the orbitotemporal fossa (crushed or missing bone fragments in this area) or because the bones are often fused (e.g., in pantodonts, tillodonts, or archaic ungulates). Absence of contact between maxilla and frontal in the orbit is present in, for example, Vincelestes, basal metatherians (e.g., Deltatheridium, Pucadelphys, Andinodelphys), basal eutherians (e.g., Asioryctes, Kennalestes, Maelestes), archaic ungulates (e.g., Baioconodon, Maiorana, Arctocyonides, Hyopsodus, Meniscotherium, Pleuraspidotherium, Phenacodus), archaic artiodactyls (Acotherulum, Dichobune), Leptictis, Eoryctes, Potamogale, and tupaiids (e.g., Ptilocercus, Tupaia). A large contact between the frontal and maxilla is present in for example the basal eutherian Zalambdalestes, in erinaceomorphs (e.g., Erinaceus, Echinosorex), in Solenodon, Tenrec, and Procavia. Because the absence of contact is commonly observed in a large number of basal therians as well as in Vincelestes, it probably represents the plesiomorphic condition. Alcidedorbignya and Pantolambda would therefore retain the plesiomorphic character state.

In Alcidedorbignya, the frontal has no contact with the squamosal and a large contact between the parietal and the alisphenoid is present. This condition is also present in Vincelestes, in multituberculates, and in the basal metatherians Pucadelphys, Andinodelphys, Mayulestes and Allkoqirus (unpublished data). It is the most common condition in Recent metatherians although variation is present among didelphids (Metachirus, for example presents a conspicuous squamosalfrontal contact). This parietal-alisphenoid contact is present is the early-diverging eutherian Zalambdalestes, and Asioryctes, A parietal-alisphenoid contact is also present in Pleuraspidotherium, Arctocyon, Tenrec, Pteropus, Leptictis, Procavia, Erinaceus, Potamogale, Solenodon, tupaiids (Wible 2011), the primates Magnadapis and Nycticebus, the artiodactyls Acotherulum and Dichobune, most notoungulates (e.g., Puelia; see Billet et al. 2009: fig. 20 for typotherians), the litopterns Diadiaphorus and Proterotherium, many carnivorans (many felids, viverrids, mustelids, canids) and also the rodent Sciurus. A large squamosal-frontal contact is present in armadillos (Dasypus, Kuntinaru, Euphractus, Chaetophractus) and Choloepus but not in Tamandua and Bradypus. A squamosal-frontal contact is also notably present in the astrapothere Trigonostylops (Simpson 1967: fig. 43), the lagomorph Oryctolagus, the perissodactyl Tapirus and the suid Sus. To conclude, given the distribution of a parietal-alisphenoid contact, almost omnipresent in earlydiverging mammals, it is highly probable that it represents the plesiomorphic condition, which is therefore retained in Alcidedorbignya.

The presence in Alcidedorbignya of supraorbital foramina (Barone 1997) on the medial surface of the skull roof, on the frontal but close to the nasals (paired on the right side of MHNC 8372), is comparable to the condition observed in the basal artiodactyls Dichobune, Acotherulum, and Diacodexis (Russell et al. 1983), in suids, in notoungulates (e.g., Pachyrukhos, Archaeohyrax, Puelia, Plesiotypotherium), in litopterns (Diadiaphrous, Proterotherium), and in Maiorana and Baioconodon. These foramina are located more laterally on the dorsal edge of the orbit in Tupaia, in many late-diverging artiodactyls (e.g., Bos, Capreolus), in Equus, in some rodents (e.g., Aguti, Dolichotis; with a sulcus on all the lateral face of muzzle) and in the bat Pteropus. These dorsal supraorbital foramina are absent in primates (e.g., Magnadapis, Plesiadapis, Nycticebus), in xenarthrans (e.g., Dasypus, Kuntinaru, Euphractus, Bradypus, Tamandua, Choloepus), in carnivorans (e.g., Felis, Cynodictis, Amphicynodon, Canis), in Tapirus, in many rodents (e.g., Cavia, Sciurus, Rattus), in Erinaceus, Solenodon, Tenrec, and Procavia. They are apparently absent too in Pleuraspidotherium and Arctocyonides. This feature actually deserves further study (CT scan), notably on its exact course and network that are sometimes not well understood, such as in litopterns or Alcidedorbignya (i.e. possible relation to orbital foramina?), as well as its comparability between taxa (e.g., Giannini et al. 2006).

Two frontal diploic foramina are present above the orbit of Alcidedorbignya. A frontal diploic foramen is absent in Vincelestes, Mayulestes, Phenacodus and Zalambdalestes, but present in Pucadelphys, Maelestes, Kulbeckia, Leptictis (two foramina in this genus, Novacek 1986) and Ptilocercus (Wible et al. 2009). It is also present in Pleuraspidotherium, in Tupaia and Talpa (Thewissen 1989), Potamogale, Solenodon, and probably Erinaceus (contra Wible et al. 2009), variably present in Procavia (Wible et al. 2009), absent in Tenrec, present in xenarthrans (e.g., Dasypus, Kuntinaru, Chaetophractus, Bradypus, Tamandua, Choloepus), in carnivorans (Miacis [Wible et al. 2009], Amphicynodon, Cynodictis, Felis, Canis, Hyaena, Herpestes), in the primates Magnadapis and Nycticebus, variably present in artiodactyls (sometimes possibly confounded with the orbital opening of the supraorbital canal; but present in Diacodexis fide Wible et al. 2009), possibly present in notoungulates (but requires additional CT scan investigation), variably present in rodents (present in Cavia, absent in Sciurus), present in Pteropus (Giannini et al. 2006), in the perissodactyls Tapirus and Equus, and apparently in the litoptern Macrauchenia. A tiny foramen anterior to the postorbital process and on the dorsal edge of the orbit in Arctocyonides (in a position similar to that of Alcidedorbignya) may represent a frontal diploic foramen. Therefore, the apparently extremely variable distribution of this character does not provide decisive arguments for its polarisation.

Pterygoid. In Alcidedorbignya the posterior opening of the pterygoid canal is located on the medial side of the pterygoid in the dorsolateral angle of the basipharyngeal canal at the level of the anterior fourth of the length of the basisphenoid. In eutherians, the pterygoid canal is generally located within the suture between the pterygoid and the basisphenoid (De Beer 1937; MacPhee 1981) even if the entrance of the canal is sometimes in the pterygoid (but close to the pterygoidbasisphenoid suture) as in Alcidedorbignya (specimens MHNC 8399 and 8423). In Pantolambda (AMNH 16663) the basipharyngeal canal is not as perfectly preserved as in the specimens of Alcidedorbignya and the pterygoids are slightly displaced from their anatomical position. However, several observations can be made. Several foramina pierce the basisphenoid in the bottom of the carotid notch. Three foramina 
are present on the left side of AMNH 16663 and a single very large slit-like foramen on the right. These foramina open in a similar position as the foramen described by Wible (2008) in Solenodon as the posterior opening of the pterygoid canal and could therefore had a similar function in Pantolambda. However, a CT scan of the skull reveals that these foramina extend in the bone in a short canal, which quickly disappears anteriorly as is observed in Alcidedorbignya (MHNC 8399). Therefore, as with the foramina on the basisphenoid of this specimen (MHNC 8399), we also interpret these structures as nutrient foramina. In other respect, on the dorsolateral angles of the basipharyngeal canal of Pantolambda (AMNH 16663), along the basisphenoid-pterygoid suture, delicate but distinct grooves run anteriorly from the carotid notch (observable $c .5 \mathrm{~mm}$ anterior to the bottom of the notch), extending on approximately $15 \mathrm{~mm}$ before penetrating the pterygoid-basisphenoid suture (observable on the right side of the specimen). We interpret these grooves as the passageway of the nerve of the pterygoid canal, which runs some distance in the basipharyngeal canal before entering the pterygoid canal anteriorly. The condition of Alcidedorbignya is very similar to that of Pantolambda. In the two genera the posterior foramen of the canal opens well anterior to the carotid notch, at the level of the anterior region of the ectopterygoid fossa close to the level of the basisphenoid-presphenoid suture. In both genera, from its exit from the tympanic cavity posteriorly, the nerve runs in the dorsolateral sulcus of basipharyngeal canal to the posterior foramen of the pterygoid canal anteriorly.

We have observed a foramen of the pterygoid canal distinctly penetrating the pterygoid-basisphenoid suture (or close to it) in many Recent carnivores and primates (e.g., Canis, Felis, Hyaena, Potos, Nasua, Ursus, Martes, Mustela, Nycticebus, Hapalemur), in Ptilocercus (Wible 2008), in Solenodon (Wible 2008), in Pteropus (Giannini et al. 2006), in fossil carnivores (e.g., Cynodictis, Amphicynodon), and in some basal ungulates (e.g., Arctocyon, Arctocyonides, Pleuraspidotherium). It is also present in the basal artiodactyls Acotherulum and Dichobune and in extant artiodactyls (e.g., Capreolus). In many lipotyphlans and tenrecoids, which possess a tympanic process of the basisphenoid, the posterior opening of the pterygoid canal is in the tympanic cavity (e.g., Tenrec, Setifer, Hemicentetes, Erinaceus, Echinosorex). In Leptictis a large entotympanic is present and the posterior opening of the pterygoid canal is also located in the tympanic cavity (Novacek 1986). It is noteworthy, however that the posterior opening of the pterygoid canal of Alcidedorbignya and Pantolambda is located significantly more anteriorly than in the non-pantodont taxa mentioned above.

Squamosal, orbitosphenoid, and alisphenoid. The squamosal of Alcidedorbignya is very similar to that of Pantolambda. The postglenoid process is high and narrow (at least as high as wide) and does not border posteriorly the lateral side of the dentosquamosal articulation (i.e. the process is located on the medial edge of the fossa). As a matter of fact on the dentary the dentosquamosal articular facet greatly extends ventrally on the medial half of the condyle to articulate with the postglenoid process. However, the condition of MHNC
8399 is not identical to that of MHNC 8372 as the postglenoid process is in a more median position with respect to the glenoid fossa. The condition of Alcidedorbignya resembles that of Haplolambda. The postglenoid process of Pantolambda is incompletely preserved on AMNH 16663 (the only specimen in which the basicranium is well preserved). However, the base of the process clearly indicates that it was narrower than the total width of the articulation for the mandibular condyles and that it only bordered the medial part of the posterior edge of the dentosquamosal articulation. It is noteworthy however, that the archaic pantodont Bemalambda differs from Alcidedorbignya and Pantolambda in having a wide and low postglenoid process, which borders posteriorly the whole width of the articular facet for the dentary. The postglenoid process is high and narrow in the tillodonts Azygonyx and Trogosus and in the archaic ungulates Maiorana and Baioconodon. It is also the case in Leptictis, in the early-diverging notoungulate Simpsonotus and in Deltatherium (AMNH 16610). In some other archaic ungulates, the postglenoid process is wider and lower and borders posteriorly most of the dentosquamosal articulation (Meniscotherium, Pleuraspidotherium, Arctocyon, Arctocyonides, Hyopsodus). The basal eutherian Maelestes also has a relatively high and narrow postglenoid process (Wible et al. 2009). This process however is extremely variable within therians. It varies from totally absent (Tamandua) or very reduced (Bradypus, Dasypus, Kuntinaru) to hypertrophied in mysticetes. When moderately developed it can be located on the medial edge of the glenoid fossa (with its apex shifted medially) and facing anteriorly as in most Carnivora. In Erinaceus, tupaiids (e.g., Ptilocercus, Tupaia), it is small to greatly reduced and rather located on the lateral edge of the glenoid fossa. It is well developed in the primates Magnadapis and Nycticebus (grossly as high as wide). In Tenrec, Potamogale, and Nesophontes it is probably reduced and fused to the entoglenoid process of the squamosal therefore located in a medial position (see below). In Solenodon and other "soricomorph", the postglenoid process is considered to be absent and only an entoglenoid process is recognized (McDowell 1958; MacPhee 1981; Wible 2008). A similar condition is observed in Zalambdalestes in which the postglenoid process is continuous with the entoglenoid process (see below). In early metatherians (Pucadelphys, Andinodelphys, Mayulestes) it is elevated, located posterior to the glenoid fossa and extends transversely along the whole width of the fossa, its apex being roughly median. Because of the great diversity of the morphology and position of the glenoid process in mammals it is difficult to establish an obvious pattern of evolution of this structure. Nevertheless, it is clear that the postglenoid process of Alcidedorbignya is very similar to that of other pantodonts (except Bemalambda), tillodonts and basal ungulates in its elevation and narrowness.

The anterolateral border of the middle ear (posterolateral to the foramen ovale) of Alcidedorbignya is limited by a distinct crest made of the squamosal posteriorly (entoglenoid process of the squamosal) and the alisphenoid anteriorly (tympanic process of the alisphenoid): the preotic crest (Novacek 1986). We interpret the groove on the medial side of the crest (in 
the alisphenoid) in Alcidedorbignya as the passageway for the ramus inferior of the stapedial artery. Laterally, between the crest and the glenoid cavity + postglenoid foramen is a wide groove, which has been identified above as the Glaserian fissure for the passage of the chorda tympani. A similar condition is present in Pantolambda, Bemalambda (apparently; see Zhou et al. 1977), and in the tillodont Azygonyx. A preotic crest with a conspicuous (but shallow) groove for the ramus inferior is also present in the archaic ungulates Baioconodon, and Maiorana. In these genera a shallow but distinct groove for the Glaserian fissure is present. In Arctocyonides the preotic crest is extremely massive, the groove of the Glaserian fissure is conspicuous but the groove for the inferior ramus is tenuous on the medial side of the crest. In Pleuraspidotherium, Meniscotherium, Hyopsodus and Phenacodus the three structures (crest and grooves) are well-developed and resemble the condition of Alcidedorbignya. Although slightly modified, a comparable structure is observable in the basal artiodactyls, Acotherulum and Dichobune when the tympanic bulla is removed. In Leptictis a preotic crest is also present but the Glaserian fissure is narrower and shorter than in Alcidedorbignya. Furthermore, on the medial edge of the entoglenoid process of the preotic crest, a distinct groove (not mentioned by Novacek 1986) runs anteroventrally, slightly medial to the Glaserian fissure. This groove is for the ramus inferior, which apparently was not conveyed by the Glaserian fissure as hypothesised by Novacek (1986: fig. 26). On the only partial skull known of Didelphodus (specimen USNM 18369), in spite of its poor preservation, the tympanic process of the alisphenoid is well preserved and is excavated by a conspicuous groove for the inferior ramus. In the basal eutherian, Maelestes, the anterolateral border of the middle ear is more a thickening than a crest at the level of the alisphenoid-squamosal suture (Wible et al. 2009: fig. 15 and personal observations of PSS-MAE 607). In this taxon, lateral to the preotic crest is a groove, the Glaserian fissure, which separates it from the postglenoid foramen. On the medial side of the preotic crest is a faint depression in the alisphenoid, unlikely for the passage of the ramus inferior of the stapedial artery, which probably entered the skull through the pyriform fenestra (Wible et al. 2009: 47, fig. 19) because Maelestes does not have an alisphenoid canal. This depression could possibly have conveyed the ramus mandibularis. Therefore, the condition of Maelestes, which lacks a patent entoglenoid process, differs from that of Alcidedorbignya. The condition of Zalambdalestes is even more divergent. In this taxon, the preotic crest bears a large entoglenoid process, which is continuous with the postglenoid process laterally. Another peculiarity of Zalambdalestes is the position of the postglenoid foramen, which is anterior to the entoglenoid + postglenoid process instead of being posterior or medial to the postglenoid process. This condition raises the problem of the Glaserian fissure, the passageway of the chorda tympani, which exits the middle ear at the anterior region of the epitympanic recess (MacPhee 1981). The chorda tympani (not reconstructed in Wible et al. 2004) probably passed on the medial edge of the preotic crest medial to the entoglenoid process or on the posterior edge of the common crest and between postglenoid and entoglenoid processes, as is observed in Solenodon (Wible 2008: figs 25, 26; see below). A similar condition is apparently also present in Asioryctes, Kennalestes, and Barunlestes (Kielan-Jaworowska \& Trofimov 1980; Kielan-Jaworowska 1981; Wible et al. 2009). A condition very similar to that of Zalambdalestes is present in Tenrec, although in this genus the postglenoid process is absent and the postglenoid foramen is located posterior to the posterior edge of the articular facet of the glenoid cavity approximately midway between its lateral and medial edges. In Tenrec the medial edge of the entoglenoid process bears a shallow (sometimes very faint) but distinct oblique sulcus that originates at the anterior border of the epitympanic recess and that extends to the apex of the entoglenoid process (i.e. in a perfect position for conveying the chorda tympani). Therefore, there is no Glaserian fissure sensu stricto as a deep notch observed slightly medial to the postglenoid foramen. In Erinaceus and Echinosorex, the preotic crest is excavated by a deep groove for the inferior ramus on the tympanic process of the alisphenoid in the former and on the alisphenoid-squamosal suture in the latter. In Echinosorex the inferior ramus is even sometimes totally enclosed in the alisphenoid. In Echinosorex, the entoglenoid process of the squamosal is separated from the small postglenoid process by a distinct Glaserian fissure. In Erinaceus, there is no deep fissure for the chorda tympani but a faint notch is present just lateral to the ramus inferior groove and anteromedial to the anterior border of the epitympanic recess. As a matter of fact, the ectotympanic presents a well-marked groove, which perfectly matches the faint notch mentioned above and closes ventrally what we interpret as the passageway for the chorda tympani. In the erinaceid Parechinus, the preotic crest is better materialised than in Erinaceus and the passageway of the chorda tympani is clearly a small slit between the ectotympanic and the preotic crest, which are closely appressed one against the other. The condition of Solenodon is relatively similar to that of Tenrec and not fundamentally different from that of Zalambdalestes. Solenodon bears a large process present on the posteromedial edge of the squamosaldentary articulation, which Wible (2008) identified as the entoglenoid process following McDowell (1958). However, on the posterior edge of the process (as mentioned above) is a groove (sometimes very deep, as in MNHN-AC-1902-156) for the chorda tympani, which therefore corresponds to the Glaserian fissure. In Solenodon also the ectotympanic is appressed against the entoglenoid process and closes the entoglenoid groove for the chorda tympani ventrally. As a mater of fact, Wible et al. (2004: fig. 37B) observed a facet for the ectotympanic on the posteromedial face of the entoglenoid process of Zalambdalestes, which therefore seems to present a condition remarkably similar to those of Tenrec, Solenodon, Erinaceus, Parechinus, and Echinosorex.

Now, the Glaserian fissure (the passageway for the chorda tympani) is generally located between the postglenoid and entoglenoid processes of the squamosal (e.g., Alcidedorbignya, Leptictis). In fact, apparently the passageway of the chorda tympani between the two processes and medial to the postglenoid foramen may represent, in some cases, a good criterion to 
identify the postglenoid process (lateral) and the entoglenoid process (medial), because as stated by Wible (2008: 355), "the chorda tympani nerve lies medial or dorsomedial to a true postglenoid process" (see also McDowell 1958 and MacPhee 1981). It is therefore tempting to interpret the process of Solenodon (as well as that of Tenrec) as the coalescence of the two processes, postglenoid and entoglenoid, processes, which are also fused in Zalambdalestes. The condition of Nesophontes is very similar to that of Solenodon (McDowell 1958). On the medialmost edge of the entoglenoid process of Solenodon is a deep groove for the exit of the inferior ramus of the stapedial artery on its way toward the posterior opening of the alisphenoid canal (Wible 2008: fig. 26).

In basal metatherians (e.g., Mayulestes, Andinodelphys, Pucadelphys), the postglenoid process is large and distinctly lateral to the postglenoid foramen. There is no preotic crest as in Alcidedorbignya or Leptictis, but the squamosal presents a conspicuous medial process (Muizon 1994; Muizon et al. 1997; Muizon 1998), which could be homologous to the entoglenoid process of eutherians. As a matter of fact, the passageway of the chorda tympani is on the medial edge of the postglenoid process and foramen and on the lateral side of the medial process (possibly homologous to entoglenoid process). In Vincelestes there is neither a postglenoid nor entoglenoid process, which is likely to represent the plesiomorphic condition. Therefore, the presence of an entoglenoid process could apparently represent a synapomorphy of therians and a plesiomorphy within therians. The presence of well-separated postglenoid and entoglenoid processes is a plesiomorphic condition for eutherians when compared to coalescent processes, which represent a derived condition as suggested by Wible et al. (2004: 130).

In Alcidedorbignya, the foramen rotundum is adjacent to but not confluent with the sphenorbital fissure on the medial wall of the orbitotemporal fossa. Novacek (1986) regarded the confluence of the two foramina as a plesiomorphic character state, which is present in Leptictis. In basal metatherians (Pucadelphys, Andinodelphys, Mayulestes), the foramen rotundum is well separated from and well posterior to the sphenorbital fissure. In the basal eutherian Zalambdalestes the foramen rotundum is located in a position similar to that of early metatherians, i.e. separated and posterior to the sphenorbital fissure (Wible et al. 2004). In the archaic ungulates Baioconodon, Maiorana, Arctocyonides, Pleuraspidotherium, Meniscotherium, and Phenacodus, the foramen rotundum is adjacent and slightly posterior to the sphenorbital fissure. The condition in these genera differs from that of Alcidedorbignya in the fact that the two foramina do not open in a common anteriorly facing opening and, as a consequence, the foramen rotundum is distinctly visible in lateral view of the orbitotemporal fossa. Well-separated foramina are present in many carnivores (e.g., Hyaena, Martes, Meles, Lutra, Canis, Potos, Felis, Amphicynodon), except for Cynodictis in our sample. It is also present in Procavia and in pilosan xenarthrans (Gaudin 2004), but apparently absent in cingulate xenarthrans (Dasypus, Euphractus [Wible \& Gaudin 2004], Kuntinaru). The foramina are coalescent in the early artiodactyls Dichobune and Acotherulum, in Solenodon, Tenrec, Potamogale, Erinaceus, Echinosorex, in many xenarthrans, notoungulates (e.g., Puelia, Notostylops, Plesiotypotherium, Hegetotherium; see also Gabbert 2004), in the astrapothere Trigonostylops (Simpson 1967) and possibly in the litopterns (Diadiaphorus, Proterotherium). Within tupaiids, a separated foramen rotundum is absent in Ptilocercus but present in Tupaia (Wible 2011). It is also absent in the strepsirhine primates Nycticebus, Hapalemur, Lepilemur (but present in Magnadapis). It is absent in Plesiadapis. Because early eutherians and metatherians present well-separated foramen rotundum and sphenorbital fissure, with the former being posterolateral to the latter this condition is likely to be plesiomorphic. Therefore, Alcidedorbignya presents an intermediate condition between this plesiomorphic condition and the derived condition, in which the two foramina are coalescent.

A suprameatal foramen has been regarded as lacking in Alcidedorbignya. We regard the tiny foramen observed on MHNC 8372 (but absent in MHNC 8399) as related to the temporalis system (or foramina for rami temporales of Wible \& Gaudin 2004). The absence of a suprameatal foramen has been considered as a derived character (Butler 1956; Novacek 1986; Wible et al. 2001). Among therians, a suprameatal foramen is present in basal metatherians (e.g., Pucadelphys, Andinodelphys, Mayulestes), in all well-known Late Cretaceous basal eutherians (e.g., Asioryctes, Kennalestes, Maelestes, Zalambdalestes, Barunlestes), in Leptictis, in Didelphodus, in Eoryctes, in Scandentia (e.g., Tupaia, Ptilocercus), in Plesiadapis (Wible et al. 2009), and in many (but not all) notoungulates (Gabbert 2004; Billet et al. 2008, 2009). A very large suprameatal foramen is also present in the basal archaic ungulates Baioconodon and Maiorana (c. $2 / 3$ of the size of the postglenoid foramen) and in Dasypus, and Kuntinaru (c. the same size of the postglenoid foramen). A smaller foramen is present in later archaic ungulates (e.g., Pleuraspidotherium, Hyopsodus, and Phenacodus) and in the early-diverging artiodactyl Acotherulum. It is small to tiny and inconstantly present in e.g., Potamogale, Hemicentetes, Erinaceus, Echinosorex, Solenodon, and in the perissodactyl Tapirus. It is absent in, for example, Vincelestes; in the archaic ungulates, Arctocyonides, and Meniscotherium; in Pantolambda, and Azygonyx; in Tenrec, Setifer, Oryzoryctes, and Suncus; in Procavia; in most carnivorans (e.g., Protictis, Cynodictis, Martes, Canis, Thalarctos, Hyaena, and Felis); in euprimates (e.g., Magnadapis, Microcebus, and Hapalemur) in Bradypus; in Rhyncocyon, in Orycteropus; in the glires Paramys, Rhombomylus, and Gomphos (fide Wible et al. 2009); in the bat Pteropus (Giannini et al. 2006); and apparently in the astrapothere Trigonostylops. Therefore, the suprameatal foramen is fairly constantly present in basal metatherians and eutherians. The fact that it is present and large in the earliest known skulls of "condylarths" and missing in most others, together with its occurrence in Plesiadapis and its absence in other primates, is an indication that it tends to disappear in some lineages of therians. Therefore, its absence in Alcidedorbignya is a derived character state that is likely to characterize the whole order Pantodonta. 
The alisphenoid canal of Alcidedorbignya is small and its anterior end opens in the lateral edge of the foramen rotundum canal, posterior to the foramen rotundum. The absence or indistinct aspect of the alisphenoid canal with an anterior opening confluent with the sphenorbital fissure has been considered as a plesiomorphic character state for eutherians (Novacek 1986), and its presence as a synapomorphy of a clade including placentals and their closest relatives (node Q in Wible et al. 2009). In Pantolambda, the alisphenoid canal is distinctly larger than in Alcidedorbignya, which represents a derived condition relatively to the Bolivian genus. An alisphenoid canal is absent in metatherians, in basal eutherians (e.g., Zalambdalestes, Asioryctes, Kennalestes), in Erinaceus, in some archaic ungulates (e.g., Arctocyonides, Pleuraspidotherium), in basal artiodactyls (e.g., Diacodexis [Wible et al. 2009], Dichobune, Acotherulum), in tupaiids, in the bat Pteropus (where it is interpreted as coalescent with foramen ovale; Giannini et al. 2006), in Orycteropus (Wible 2012), in many primates (e.g., Plesiadapis, Notharctus, Adapis [Wible et al. 2009], Archaeolemur), in xenarthrans (e.g., Wible \& Gaudin 2004), in notoungulates, in astrapotheres (Trigonostylops, Astrapotherium and apparently Astraponotus (Kramarz et al. 2011) and in litopterns (Diadiaphorus, Proterotherium). An alisphenoid canal is present in the early tillodont $A z y g$ onyx, in some archaic ungulates (e.g., Maiorana, Baioconodon, Hyopsodus, Meniscotherium, Phenacodus), in Eoryctes, in Echinosorex, in Tenrec, in Solenodon, in Potamogale, in Procavia, in the perissodactyls Equus, Tapirus and Ceratherium, and in early-diverging carnivorans (e.g., Miacis, Vulpavus [Wible et al. 2009], Amphicynodon, Cynodictis). It is variably present in rodents (e.g., Wible et al. 2007, 2009; MacPhee 2011). An alisphenoid canal is also present in Didelphodus (USNM 18369). Therefore, given the broad distribution of the alisphenoid canal and given the fact that it may be present or absent in different taxa of the same group it is likely that this highly homoplastic character evolved independently several times in many lineages.

A large ectopterygoid process of the alisphenoid is present in Alcidedorbignya, a feature that is regarded as a derived character state within eutherians by Novacek (1986). It is present in Pantolambda, apparently absent in Bemalambda and possibly present in Harpyodus decorus. No ectopterygoid process has been observed in Haplolambda, Caenolambda, Titanoides, and Coryphodon. However, this condition could be the result of either the poor preservation of the specimens available during this study, or a reversal. A distinct process (separated from the entopterygoid process of the pterygoid) is present in basal eutherians (e.g., Kennalestes, Asioryctes, Zalambdalestes), in early ungulates (e.g., Maiorana, Arctocyonides, Pleuraspidotherium, Phenacodus), in the artiodactyls Diacodexis (Wible et al. 2009), in the tillodont Esthonyx, in the pantolestid Aphronorus, in the Paleocene viverravid Protictis schaff, in leptictids, Procavia, in at least some notoungulates (see Billet et al. 2008), and in fossil primates and rodents (in which it approaches the ear region; Wible et al. 2009). Among recent mammals, it is present in, erinaceomorphs, rodents, lagomorphs, macroscelideans, dermopterans, chiropterans, and primates. It is absent in Tenrec, Solenodon, Potamogale, and xenarthrans; and is small (presumably reduced) in many carnivorans [Amphicynodon, Cynodictis, Miacis (Wible et al. 2009)]. It is also not individualized from the entopterygoid process in astrapotheres (Trigonostylops, Astraponotus, Astrapotherium) and also possibly in litopterns (Diadiaphorus). Therefore, the broad distribution of the ectopterygoid process and its almost constant occurrence in basal eutherians would rather indicate that it represents a plesiomorphic condition for eutherians. However, its absence in Vincelestes and metatherians suggests that its absence is a plesiomorphic condition for therians.

The large anterodorsolateral ascending process of the alisphenoid of Alcidedorbignya is a feature that is regarded as a derived character state by Novacek (1986). This condition is generally difficult to observe on fossil taxa because of the poor preservation and crushing of that region of the skull and/or because of the fusion of the bones. Therefore, this condition is unknown in other pantodonts, in Azygonyx, in all archaic ungulates available during this study, in Aphronorus and in Eoryctes. A large ascending process that approximately reaches mid-height of the lateral view of the skull is present in Vincelestes, in basal metatherians (Pucadelphys, Andinodelphys), in Zalambdalestes, in Leptictis, in notoungulates (Puelia also see Billet et al. 2009: fig: 20), in the litoptern Proterotherium, and in carnivorans (e.g., Cynodictis, canids, ursids, hyaenids, felids, viverrids). The ascending process is small to moderately developed in erinaceomorphs, in Tenrec, in Potamogale, in Solenodon, in the basal artiodactyl Acotherulum, and in the astrapothere Trignonostylops (Simpson 1967). Both states of the character are present within xenarthrans. The occurrence of a large ascending process of the alisphenoid in Vincelestes and basal metatherians is an indication that this feature is plesiomorphic (rather than apomorphic) within therians. However, it is noteworthy that this character is difficult to evaluate as the transition from a large process to a small process is continuous and the intermediate conditions are not easy to qualify. Be that as it may, Alcidedorbignya exhibits the plesiomorphic state of this character.

The shape of the zygomatic arches in dorsal view are greatly expanded laterally relative to other parts of the skull laterally and forms an elbow posteriorly in Alcidedorbignya and Pantolambda. This is similar to the condition in many carnivorans (when the arch is not rounded), Arctocyonides and Arctocyon. This condition is however absent in many other eutherians (because either the arches are subparallel or rounded, and/or do not show a posterior elbow): e.g., Maelestes, Asioryctes, Kennalestes, Zalambdalestes (Wible et al. 2004), Dasypus, Procavia, Pteropus, Pleuraspidotherium, Erinaceus, most Notoungulata, Xenarthra, many artiodactyls and perissodactyls, many rodents (e.g., Wible et al. 2007), Azygonyx, and Leptictis.

Parietal. Alcidedorbignya presents three large temporal rami that were supplied by the orbitotemporal artery (anterior and median temporal rami) and by the arteria diploëtica magna (posterior temporal ramus) - but see above in the description for variation. This condition differs from that generally observed in eutherians, in which the arteria diploëtica magna 
supplies the rami temporalis for vascularisation of the temporal muscles (Wible 1987). Foramina for the temporal rami in therians are generally in the squamosal or between squamosal and parietal, a condition, which is widely distributed within therians (Wible 1987, 1993; Wible et al. 2001; Wible \& Gaudin 2004; Giannini et al. 2006). Alcidedorbignya differs from Pantolambda in which the temporal foramina are in the squamosal only and are smaller. The condition of Azygonyx is unknown because of the condition of preservation of the only skull known for this genus of tillodont. In Trogosus and Tillodon several large foramina (three to five) are present and are in the squamosal and in the parietal only but they apparently are not in or near the suture (Gazin 1953). In Vincelestes a single temporal ramus was emitted by the orbitotemporal artery and two others were emitted by the ramus superior (Rougier et al. 1992) and the three foramina are in the anterior lamina of the periotic. In Kryptobaatar (Wible \& Rougier 2000) the three rami temporales were emitted by the ramus superior and also exited the skull via the anterior lamina of the periotic. Asioryctes and Zalambdalestes present two rami emitted by the arteria diploëtica magna and which exited the skull via the suprameatal and "subsquamosal foramina" (= foramen for rami temporales, Wible 2008) of the squamosal (Rougier et al. 1992; Wible et al. 2004). Therefore, these early-diverging eutherians have rami temporales exiting the skull through the squamosal (at least externally) and not mainly within the suture between parietal and squamosal as in Alcidedorbignya. The condition of Alcidedorbignya (large foramina for rami temporales within pa-sq suture) is present in tenrecoids (e.g., Tenrec, Potamogale, Setifer, Hemicentetes) and in lipotyphlans (e.g., Echinosorex, Erinaceus), although in these genera only one temporal foramen (not three) is generally present. Some variation occurs, however, as in Erinaceus the foramen is sometimes in the squamosal only and in some specimens of Echinosorex we have observed two foramina. On our specimens of Solenodon, the number and size of the main foramina also varies more significantly. There are generally two or three foramina (sometimes one only) and not always the same number on each side of one specimen; they generally exit the skull at or near the squamosal-parietal suture but may also exit more dorsally through the parietal or, occasionally, more ventrally, through the squamosal. Wible (2008) (and see also MacPhee 1994) counted three to six foramina on various specimens of Solenodon, thereby also considering the "subsquamosal foramen" within this cluster. In Scandentia (e.g., Tupaia and Ptilocercus) we have observed one to three foramina generally one in the parietal-squamosal suture and the others, when present, in the parietal. Wible (2011: 215) mentions three to seven foramina and a great variability in Tupaia. In the archaic ungulates Baioconodon and Maiorana apparently a single, large temporal foramen is present at or near the parietal-squamosal suture and a large "subsquamosal foramen" (foramen for rami temporales in the squamosal) is present just above the posterior root of the zygomatic arch. In Baioconodon one smaller foramen perforates the squamosal posterodorsal to the large one. There are two in Maiorana. In Pleuraspidotherium and Arc- tocyonides, apparently (the specimens available for this study, MNHN.F.CR146, CR733, are partly crushed) a single large foramen is present at the parietal-squamosal suture but, in the former, one or two smaller foramina also perforate the squamosal externally; there are two in Hyopsodus, generally one in the suture and another either in the squamosal or in the parietal; in Meniscotherium an extremely large number of foramina (generally close to ten) are present in the squamosal and/or the parietal. In the basal artiodactyl Acotherulum (the specimen available for this study, MNHN.F.QU16366) there are four foramina in the parietal, one in the suture and another smaller one next to it in the squamosal, while Dichobune (MNHN.F.QU16586) exhibits one large foramen in the suture and another smaller one in the squamosal. In the carnivorans Cynodictis and Amphicynodon a single, mediumsized temporal foramen generally pierces the parietal in the dorsal region of the temporal fossa. Foramina for the rami temporales within the parietal, the suture and/or squamosal are also present in notoungulates (Billet \& Muizon 2013), in astrapotheres (Astrapotherium, Trigonostylops), in litopterns (Proterotherium), in fossil primates (Notharctus (fide Wible et al. 2009), Magnadapis, Plesiadapis), in fossil glires (Wible et al. 2007 2009), in xenarthrans (Dasypus, Kuntinaru, Euphractus, Bradypus, Tamandua [Wible \& Gaudin 2004]), in the bat Pteropus (Giannini et al. 2006) and in perissodactyls (Palaeotherium, Tapirus, Equus). The temporal foramina are absent in a number of Recent taxa: e.g., Procavia, Rhynchocyon (Wible et al. 2009), the rhinoceros Ceratherium, Recent Carnivora and many modern primates. In basal metatherians (e.g., Pucadelphys, Andinodelphys, Mayulestes), there are one to three temporal foramina in the parietal exclusively, on the top of the braincase close to the sagittal crest. Therefore, the position and number of temporal foramina show important intra-individual (asymmetry), intraspecific, and interspecific variation. In non-therian taxa such as Vincelestes or multituberculates they perforate the anterior lamina of the petrosal (Rougier et al. 1992; Rougier \& Wible 2000). In basal therians (e.g., Pucadelphys, Andinodelphys, Mayulestes, Prokennalestes -possibly-, Asioryctes, Kennalestes, Ukhaatherium, Zalambdalestes) it perforates the squamosal and/or the parietal (possibly the petrosal in Prokennalestes) and in many therians (eutherians or metatherians) temporal foramina are simply absent. Therefore, the distribution, position on the skull, and number of foramina for the temporal rami are extremely variable and an obvious evolutionary pattern does not appear easily, except that their presence is a plesiomorphic state, certainly within therians.

Petrosal. The promontorium of Alcidedorbignya is isolated from the surrounding bones (alisphenoid, basisphenoid and presphenoid) by the pyriform fenestra anterolaterally, the carotid foramen (or notch) anteromedially and the basicochlear fissure medially. A similar condition is present in Pantolambda, Haplolambda and Caenolambda. The condition of Bemalambda is peculiar. In this genus the pyriform fenestra is relatively narrow as in Alcidedorbignya, while the basicochlear fissure is greatly widened; the carotid foramen is 
also distinctly opened. The condition in Harpyodus decorus is unclear on the photographs available during this study. A petrosal isolated from the surrounding bones is also apparently present in the tillodont Trogosus but the condition of Azygonyx could not be elucidated because of the crushing of the single skull known for this taxon. A promontorium isolated from the surrounding bones is present in the basal ungulates Maiorana, Baioconodon, Meniscotherium, Hyopsodus (Cifelli 1982), and Phenacodus (Thewissen 1990). An opposite condition is observed in Arctocyonides and Pleuraspidotherium, and in the basal artiodactyl Dichobune, in which the carotid foramen is distinctly closed and there is neither pyriform fenestra nor continuous basicochlear fissure. A pyriform fenestra was possibly present in the basal artiodactyl Acotherulum. The condition of Alcidedorbignya is present in the pantolestid Aphronorus, in litopterns (Diadiaphorus, Proterotherium, Macrauchenia), in astrapotheres (Astrapotherium, Astraponotus [Kramarz et al. 2011]), in the bat Pteropus (Giannini et al. 2006), in perissodactyls (Tapirus, Palaeotherium, Equus, Ceratherium) and in Procavia (observable in this genus when the tympanic is removed). There is no pyriform fenestra and basicochlear fissure in Eoryctes, Erinaceus, Echinosorex, Tenrec, and in many notoungulates (but some large-sized ones like Periphragnis do show this feature when the tympanic is removed). A pyriform fenestra is present in Solenodon (Wible 2008), in Nesophontes (McDowell 1958), and Microgale. A basicochlear fissure is present in Solenodon (variably) and in Nesophontes. An incomplete basicochlear fissure is present in some tenrecoids (e.g., Tenrec, Setifer) although it is partly obliterated in these genera by the development of the tympanic process of the basisphenoid. A pyriform fenestra is present in the basal eutherians Maelestes (Wible et al. 2009) and Zalambdalestes (Wible et al. 2004) but they apparently lack a basicochlear fissure.

As mentioned by MacPhee (1981), the pyriform fenestra is a large opening in the basicranium anterior to the auditory capsule and it is present in all foetal mammals and some adults. Similarly the basicochlear fissure is a "space included between the ventromedial edge of the auditory capsule and the lateral edge of the parachordal cartilage (basal plate)" (De Beer 1937: 399 and MacPhee 1981:48-49). According to MacPhee (1981) the pyriform fenestra and the basicochlear fissure are almost always obliterated in adults (of Recent mammals). A pyriform fenestra and a basicochlear fissure are, however, retained in some adult soricomorphs (see above). Although the presence of a pyriform fenestra is rare among adult Recent mammals, it commonly occurs in early eutherians and in the most archaic ungulates. This is an indication that it probably represents a plesiomorphic character within eutherians.

There is no pyriform fenestra and basicochlear fissure in adult metatherians. In Vincelestes, Rougier et al. (1992) describe a large vacuity between the anterior end of the petrosal and the posterior edge of the alisphenoid and lateral to the carotid foramen. These authors called this vacuity the ventral opening of the cavum epiptericum. It is noteworthy however that this opening is in the same position as the pyriform fenestra of eutherians. If this opening can actually be regarded as homologous to the pyriform fenestra of Eutherians, it strengthens the interpretation that the presence of this structure in adults is a plesiomorphic condition within eutherians. Alcidedorbignya therefore retains the plesiomorphic condition for this character.

In Alcidedorbignya, the carotid foramen notches the basisphenoid but is opened posterolaterally and communicates with the pyriform fenestra posterolaterally and the basicochlear fissure posteromedially. However, this carotid notch is constricted by a lateral projection of the posterolateral angle of the basisphenoid. A carotid foramen totally enclosed in the basisphenoid is present in Vincelestes. In metatherians (e.g., Pucadelphys, Andinodelphys, Mayulestes, Didelphis) the carotid foramen perforates the lateral edge of the basisphenoid almost on the suture with the alisphenoid. Because the suture between these two bones obliterates early in the ontogeny of these taxa we have not been able to observe if the alisphenoid actually participates in the foramen. In basal eutherians the carotid foramen is totally enclosed in the basisphenoid (e.g., Zalambdalestes, Barunlestes, Maelestes, Asioryctes, Kennalestes). In these taxa the carotid foramen is not obviously included in the middle ear cavity. The carotid foramen is a notch in the posterolateral angle of the basisphenoid (and bordered posteriorly by the petrosal) in pantodonts, tillodonts, in some basal archaic ungulates (e.g., Baioconodon, Maiorana, Phenacodus, Arctocyonides), in some lipotyphlans (e.g., Solenodon (Wible 2008), Nesophontes), in Dasypus (Wible 2010: fig. 10), in Orycteropus (Wible 2012), in some archaic carnivores (e.g., Protictis), possibly in litopterns (but the notch is not well delimitated), in the bat Pteropus (with entotympanic participation; Giannini et al. 2006) and in perissodactyls (e.g., Tapirus, Equus, Palaeotherium). In some taxa the development of a tympanic bulla closes the notch posteroventrally and removal of the bulla is necessary to observe the character state (e.g., Procavia, Tupaia, Ptilocercus, Cynodictis, Canis, Nasua, Martes, Lutra, Meles, Felis). In many lipotyphlans and tenrecoids, which do not develop a totally closed tympanic bulla, but in which the pyriform fenestra is totally or partially obliterated by a posterodorsolateral wing of the basisphenoid, the internal carotid artery is totally enclosed in a foramen located between the petrosal posteriorly and a posterior extension of this lateral wing of the basisphenoid anteriorly. In these taxa the carotid foramen may also be variably enclosed in the posterodorsolateral wing of the basisphenoid but within the middle ear cavity and not external to it in the posterolateral angle of the body of this bone, which differs from the plesiomorphic condition of the basal eutherians cited above. This is the case in, for example, Leptictis, Eoryctes, Erinaceus, Echinosorex, Suncus, Tenrec, Potamogale, Microgale, Setifer, Hemicentetes, and Oryzorictes. This condition is clearly derived when compared to that of Alcidedorbignya and basal archaic ungulates. Therefore, Alcidedorbignya is derived when compared to the Late Cretaceous basal eutherians, but presents a plesiomorphic pattern within the Tertiary eutherian radiation.

The apex of the promontorium is truncated in Alcidedorbignya and Pantolambda, this condition being more pronounced in the latter. As a consequence, the opening formed by the coalescent carotid foramen and pyriform fenestra, which separates the promontorium from the alisphenoid and the basisphenoid is 
increased in size. This vacuity is larger in Pantolambda than in Alcidedorbignya. This character is apparently absent in Bemalambda, Haplolambda, and Caenolambda, in which the apex of the promontorium is not truncated. A blunt apex of the promontorium is also present in the tillodont Azygonyx, although not as truncated as in Pantolambda and Alcidedorbignya. In this genus the apex of the promontorium is exactly at the level of the anterior edge of the tegmen tympani and the step, which exists between these two structures in some pantodonts (large in Alcidedorbignya, small in Pantolambda), is absent. In this genus the morphology of the carotid foramen and pyriform fenestra cannot be observed on the single skull known, which is dorsoventrally crushed. To conclude, the square apex of the promontorium and the consecutive enlargement of the carotid+pyriform fenestra vacuity appears to be characteristic of Alcidedorbignya and Pantolambda, although it must be noted that no observation of a well-preserved auditory region could be made on other pantodonts. It is noteworthy that a truncated apex of the promontorium and large confluent pyriform fenestra and carotid foramen (and basicochlear fissure) like in these pantodonts is also observed in some perissodactyls (Palaeotherium, Tapirus, Equus). A large confluent carotid foramen and pyriform fenestra is also present in some early ungulates such as Meniscotherium, Hyopsodus, and Phenacodus. However, in these genera the apex of the promontorium is pointed and relatively narrow, thus differing from the condition of Alcidedorbignya and Pantolambda.

The promontorium of Alcidedorbignya is grooved by a shallow and wide sulcus for the transpromontorial branch of the internal carotid. The transpromontorial sulcus is very shallow in Pantolambda and apparently hardly discernible, if present, in other pantodonts. The wide and shallow groove of Alcidedorbignya clearly differs from the deep and narrow sulcus observed (with variation in depth) in e.g., Vincelestes, Prokennalestes, Uchkudukodon, Maelestes, leptictids, some erinaceomorphs, Eoryctes, Potamogale, and some early ungulates (e.g., Protungulatum, Pleuraspidotherium, Orthaspidotherium, Arctocyonides, Hyopsodus, Meniscotherium, Acotherulum, Dichobune [contra Orliac \& O'Leary (2014) based on the same specimen MNHN.F.QU16586]. In Baioconodon, a transpromontorial groove is present but very shallow as in Alcidedorbignya. In some basal eutherians this sulcus is not transpromontorial but excavates the medial edge of the promontorium as in, e.g., Zalambdalestes, Barunlestes, Asioryctes (Kielan-Jaworowska \& Trofimov 1980; Kielan-Jaworowska 1981; Wible et al. 2004). This condition is also present in some Recent eutherians (e.g., Procavia, xenarthrans, etc.). The fact that Vincelestes, Prokennalestes, Maelestes, and Uchkudukodon have a transpromontorial groove is an indication that its presence is likely to be a plesiomorphic character state among eutherians (see also Wible 1986; Wible et al. 2009).

Furthermore, in Alcidedorbignya, a distinct notch for the stapedial artery excavates the medial edge of the fenestra vestibuli. Pantolambda differs from Alcidedorbignya in lacking a notch for the stapedial artery on the medial edge of the fenestra vestibuli. The orientation of the stapedial groove differs in Alcidedorbignya and Leptictis in being oriented anteromedially- posterolaterally while it is anterolaterally-posteromedially oriented in Leptictis. In this genus the bifurcation between the stapedial and carotid artery is just ventral to the ventral edge of the fenestra cochleae. In Alcidedorbignya, it probably occurred in a more anterolateral position, on the promontorium (more or less central). Grooves marking the bifurcation between the stapedial and carotid arteries ventral to the promontorium are present in e.g., Orthaspidotherium (Ladevèze et al. 2011), Pleuraspidotherium, Arctocyonides, Acotherulum, Dichobune (contra Orliac \& O'Leary [2014] based on the same specimen MNHN.F.QU16586), Maelestes (Wible et al. 2009), Leptictis (Novacek 1986), Solenodon (Wible 2008), in tenrecoids (e.g., Tenrec, Setifer, Microgale, Potamogale), in Orycteropus (Wible 2012), in litopterns (Proterotherium), Deltatherium (AMNH 16610), Protungulatum (O'Leary 2010), and in some early Cenozoic artiodactyls but not the extant ones, which lack a groove for the stapedial artery (O'Leary 2010; Orliac \& O'Leary 2014). This is supposed to represent a condition present in a hypothetical ancestral eutherian (Wible 1987: fig. 1). It differs from that in Zalambdalestes, in which the dichotomy is below the exoccipital (Wible et al. 2004: fig. 37D). A distinct stapedial groove or notch is present in for example, Vincelestes, Prokennalestes, Mongolian asioryctitheres, (Maelestes, Zalambdalestes, Barunlestes), Azygonyx, Protungulatum, archaic ungulates (e.g., Baioconodon, Maiorana, Arctocyonides, Pleuraspidotherium, Hyopsodus, Meniscotherium, Dichobune, Acotherulum), tupaiids, erinaceomorphs, soricomorphs (e.g., Tenrec, Setifer, Microgale, Solenodon, Potamogale), litopterns, Deltatherium, and early Cenozoic artiodactyls (O'Leary 2010; Orliac \& O'Leary 2014). It very probably represents a plesiomorphic condition.

In Alcidedorbignya, the foramen for the superior ramus of the stapedial artery is almost totally enclosed by the petrosal (totally on some specimens). The plesiomorphic condition for eutherians (Wible et al. 2001) is a foramen totally enclosed in the petrosal. It is present in Vincelestes, Prokennalestes, and Leptictis (contra Wible et al. 2001, 2009 who code this character as present between the petrosal and the squamosal). In Leptictis the presence of a foramen for the superior ramus totally enclosed in the petrosal is clearly observed in AMNH 80213. The foramen is also completely enclosed in the petrosal in several archaic ungulates (e.g., Baioconodon, Pleuraspidotherium, Meniscotherium, Arctocyonides, Arctocyon), in some basal artiodactyls (e.g., Acotherulum, Dichobune; but absent in most others [O'Leary 2010]), in possible early-diverging notoungulates (but reduced; see Billet \& Muizon 2013) and litopterns (Diadiaphorus). It is absent in Hyopsodus, and Phenacodus. A foramen for the superior ramus totally enclosed in the petrosal is present in the basal tillodont Azygonyx (probably), and in erinaceomorphs (e.g., Erinaceus, Echinosorex). The derived character state is a foramen (or simply a space) in the petrosal-squamosal suture, which is present in Pantolambda. Therefore, the derived condition of Pantolambda is incipiently developed in Alcidedorbignya because in this genus the foramen in the petrosal is generally slightly opened anteriorly. The derived condition of a foramen in the suture is also present in Zalambdalestes, in Maelestes (possibly, as Wible et al. 
2009 mention that it may also be absent), in tenrecids (e.g., Tenrec, Setifer, Microgale, Potamogale), in Solenodon, and in tupaiids (Wible 2009: fig. 12b). A foramen for the superior ramus is absent e.g., in adult metatherians, in Procavia, and in xenarthrans.

The lateral and medial caudal tympanic processes of Alcidedorbignya are located posterior to the external aperture of the cochlear fossula. The medial caudal tympanic process is located on the posterior edge of the postpromontorial tympanic sinus (in the sense of Wible et al. (2009) and Billet \& Muizon [2013]) just posterior to the cochlear fossula and medial to the posterior opening of the middle ear sulcus. The lateral caudal tympanic process is located at the posterior end of the middle ear sulcus posterior to the stapedial fossa. As noted in the description, its size and presence are variable and the process may even be absent. Its presence is responsible for the $\omega$-shaped morphology of the posterior opening of the middle ear sulcus (see above, petrosal section of the description). As mentioned above, the lateral caudal tympanic process is a structure, that probably separates the passageways of the facial nerve laterally and that of the posterior ramus of the stapedial artery medially (see Fig. 39).

In Vincelestes, there is no patent medial caudal tympanic process nor postpromontorial tympanic sinus [since the original definition of Wible 1990 (i.e. continuity of the cochlear fossula and fossa for the stapedius muscle = synapomorphy of Vincelestes and therians) has been revised by Wible et al. 2009 and Billet \& Muizon 2013] and the paroccipital process (sensu Rougier et al. 1992) is interpreted here as the lateral caudal tympanic process (see Appendix 5). It is a well-developed tubercle that we regard as possibly homologous to the lateral caudal tympanic process of eutherians. We believe that the crest-like caudal tympanic process identified by Rougier et al. (1992: fig. 2) is a different structure from the caudal tympanic process, lateral or medial (see Appendix 5). In basal metatherians, such as Pucadelphys, Andinodelphys, and Mayulestes there is no medial caudal tympanic process nor postpromontorial tympanic sinus (the posterior extension of the cochlear fossula is reduced and does not form a distinct fossa) and the caudal tympanic process (probably homologous to the lateral caudal tympanic process of eutherians, see Ladevèze \& Muizon 2007) is a small crest-like protuberance, just medial to the stylomastoid notch and mastoid process and bordering the posteroventral edge of the stapedial fossa. In Prokennalestes, the medial caudal tympanic process is apparently absent and the caudal tympanic process mentioned by Wible et al. (2001), is in the same position (on the posteroventral edge of the stapedius fossa) as what we regard as the lateral caudal tympanic process of Vincelestes, metatherians and Alcidedorbignya. In Late Cretaceous basal eutherians (e.g., asioryctitheres, zalambdalestids, Maelestes) the lateral caudal tympanic process is either absent or faintly developed (Kielan-Jaworowska 1981; Wible et al. 2001, 2004, 2009; Ekdale et al. 2004), but a medial caudal tympanic process is well-developed (="tympanic process" of Kielan-Jaworowska 1981; Wible et al. 2004, 2009) and located on the posterior edge of the postpromontorial tympanic sinus. The lateral caudal tympanic process is absent in some early-diverging ungulates (Maiorana, Baioconodon, Arctocyonides, Arctocyon), but it is weakly developed in others (e.g., Meniscotherium and Hyopsodus). It is absent in other possible early-diverging placentals like Leptictis and Didelphodus.

A small lateral caudal tympanic process is present in the basal pantodonts Alcidedorbignya (very weak in some specimens) and Pantolambda, (the condition of Bemalambda could not be elucidated from illustrations of Zhou et al. 1977), in the tillodont Azygonyx, and in the archaic ungulates Pleuraspidotherium and Phenacodus. It is also variably present in several lipotyphlans (e.g., Erinaceus, Echinosorex) and tenrecoids (e.g., Tenrec, Setifer, Microgale, Potamogale). The lateral caudal tympanic process varies in size in these genera: it can range from a relatively large crest, which gives the posterior opening of the middle ear sulcus the $\omega$-shaped morphology mentioned in the description, to a tiny hump, which hardly alters the circular morphology of the opening or it may be totally absent. The lateral caudal tympanic process in these genera is generally a small peg, which differs from the large tubercle observed in Vincelestes (according to our interpretation) and the crest-like structure of early metatherians. However, given their position on the posteroventral edge of the stapedial fossa, just medial to the passageway of the facial nerve (stylomastoid notch) they are likely to represent the same structure.

Given its absence in Vincelestes and basal metatherians, the medial caudal tympanic process probably represents a synapomorphy of eutherians, while the lateral caudal tympanic process is likely to be a plesiomorphy within therians.

In the above description we have provided an interpretation of the passageways of the tympanic and auricular nerve in Alcidedorbignya. The former passed below the postpromontorial tympanic sinus and anterior to the medial caudal tympanic process while the latter passed posterior to the process. A similar condition is also probably present in Erinaceus, which bears deep notches in the medial and lateral walls of the postpromontorial tympanic sinus for the passage of the tympanic nerve and a groove on the posterior edge of medial caudal tympanic process for the passage of the auricular branch (cf. MacPhee 1981:205). In Echinosorex the condition is similar but the passages are generally closed anterior and posterior to the medial caudal tympanic process. In Tenrec it is probable that the two nerves (tympanic and auricular) pass anterior to the medial process, the tympanic passing clearly below the postpromontorial tympanic sinus and the auricular between the sinus and the process. In Potamogale, a large tympanic process of the basisphenoid forms most of the tympanic bulla and contacts the medial process and the enlarged tympanohyal posteriorly. However, the condition of the tympanic and auricular nerves is basically similar to that of Alcidedorbignya: a groove on the posterior edge of the medial process indicates the passage of the auricular branch and notches in the wall of the postpromontorial tympanic sinus indicates that the tympanic nerve ran below the sinus and passed through a canal, which also conveyed the internal carotid artery. A condition similar to that of Potamogale is apparently present in Setifer and Hemicentetes. 
In Solenodon the two nerves have the same passageways, the tympanic nerve below the postpromontorial tympanic sinus and anterior to the medial caudal process; and the auricular branch behind the process (for the latter, see Wible 2008: fig. 27B). The peculiarity of Solenodon is that the rostral process of the tympanic extends posteriorly and contacts the medial caudal tympanic process, therefore flooring the postpromontorial tympanic sinus. It further develops posterior to the medial caudal tympanic process and contacts a lip of the mastoid, which is a ventral extension of the posterior ridge of the groove for the auricular branch, on the posterior edge of the medial caudal process. This posterior extension closes ventrally the passageway of the auricular nerve, and forms the mastoid canaliculus (NAV 1994; Wible 2008).

In Tupaia a distinct groove is present on the posteromedial edge of the bulla for the auricular branch, as observed by MacPhee (1981: 168) and Wible (2009: 230). This nerve therefore passes posterior to the medial caudal tympanic process. In Ptilocercus its passageway is within a canal on the posterior edge of the medial caudal tympanic process. In both genera, the auricular nerve connects to the facial nerve outside the tympanic cavity (e.g., Wible 2009: fig.11). The tympanic nerve runs along the posterior aspect of the external aperture of the cochlear fossula in Ptilocercus and Tupaia (MacPhee 1981; Wible 2009). Therefore, the pattern of these nerves in Scandentia is basically similar to that hypothesised in Alcidedorbignya.

In Dasypus, Wible (2010: fig. 3) illustrates passageways of the tympanic and auricular branch, which are exactly in the same position as those hypothesised for Alcidedorbignya. In fact, the morphology of the posterior opening of the middle ear sulcus of Dasypus is also very similar to that of Alcidedorbignya and is probably not so different from the basal eutherian pattern (except for the fact that Dasypus has no stapedial artery, and no posterior ramus).

A condition similar to that of Alcidedorbignya and Pantolambda (see Appendix 3) is present in the tillodont Azygonyx and in the archaic ungulate Arctocyonides, in which the grooves for the tympanic nerve and auricular branch are distinctly identifiable. In Baioconodon and Maiorana the morphology of the posterior opening of the middle ear presents the same pattern as in Alcidedorbignya except for the absence of a lateral caudal tympanic process. It is therefore probable that the pattern of passageways of the tympanic nerve and auricular branch was the same as that suggested in Alcidedorbignya. In Pleuraspidotherium the pattern seems to be as in Alcidedorbignya with grooves variably present on the anterior and posterior edge of the medial tympanic process. However, in some specimens one or two small foramina open at the medial base of the medial caudal process (MNHN.F.BR12534, BR17498, CR964, CR1128). These foramina could be the medial opening of an auricular canaliculus (not of a tympanic canaliculus) because in most specimens a notch is present on the medial edge of the postpromontorial tympanic sinus, probably for the passage of the tympanic nerve. However, we have not observed the medial opening of this supposed auricular canaliculus in any of the available specimen. Therefore, these foramina instead probably represent nutrient foramina, which are not uncommon on the periotic of Pleuraspidotherium. In the early-diverging litoptern Miguelsoria, a notch or foramen for the auricular branch are present, and the interpreted pattern for both tympanic and auricular branch is similar to that in Alcidedorbignya (Billet et al. 2015). In Leptictis, the groove for the auricular branch is conspicuous on the posterior edge of the medial caudal process, and a notch on the medial wall of the postpromontorial tympanic sinus possibly indicates a passageway for the tympanic nerve similar to that of Alcidedorbignya. In Zalambdalestes, Maelestes, Kennalestes, and Asioryctes based on figures by Kielan-Jaworowska (1981), Wible et al. (2004, 2009), there are apparently no grooves for the passages of the tympanic and auricular nerves. However, the morphology and position of the medial tympanic process and of the posterior opening of the middle ear are quite similar to that of Alcidedorbignya and similar passageways for the tympanic and auricular nerves are quite plausible. It is noteworthy, however, that this interpretation differs from the "standard eutherian pattern" hypothesized by MacPhee (1981: 44), who instead suggests a course of the tympanic nerve posterior to the medial caudal tympanic process (see above, description section).

In the Notoungulata, a foramen that has been previously regarded as a possible postcarotid foramen in this group (Patterson 1936; Billet \& Muizon 2013) may instead be a tympanic canaliculus for the transmission of the tympanic nerve to the tympanic cavity (MacPhee 1981, 2014). This foramen pierces the bulla or between the bulla and the petrosal and opens posteromedially within the tympanic cavity. In Posnanskytherium (MNHN.F.AYO191), this foramen opens just posterior to the internal opening of the stylomastoid foramen, and therefore likely posterior to a medial caudal tympanic process. Whether it may also convey the auricular branch of the vagus nerve in notoungulates also remains to be clarified.

In the Litopterna, the course of these nerves might resemble that of Alcidedorbignya, as Proterotherium exhibits a groove on the postpromontorial tympanic sinus that may have conveyed the tympanic nerve and a possible passageway for the auricular branch posterior to the medial caudal tympanic process.

To conclude, because the pattern of passageways of the tympanic nerve and auricular branch hypothesised for $\mathrm{Al}$ cidedorbignya is probably present in most basal eutherians and in many Recent taxa with a relatively unspecialised (or little specialised) middle ear, this condition, given the available fossil data, is likely to be plesiomorphic for eutherians and to approach the plesiomorphic condition for eutherians.

In the description above, the notch posterior to the medial caudal tympanic process of Alcidedorbignya has been interpreted as a passageway also for a posterior branch of the stapedial artery. A posterior ramus of the stapedial artery has been identified in Solenodon and Nesophontes (McDowell 1958) and in tenrecoids (MacPhee 1981). This latter author identified a large (one-half to two-thirds the size of the parent vessel) posterior ramus in Hemicentetes, Setifer, Microgale, and Solenodon. He also mentions that this ramus passes to the rear 
of the skull in Hemicentetes and enters the posterior part of the auricle in Solenodon. Asher (2001) also identified a posterior branch of the stapedial artery in several tenrecids (Echinops, Potamogale, Micropotamogale, Geogale, Microgale). However, this author reports the absence of a ramus posterior in Tenrec. In Erinaceus MacPhee (1981: 203) mentions that a small posterior ramus is present in the three youngest specimens he sectioned; this vessel is replaced by a network of arterioles in the young infant and disappears in the adult. Asher (2001) also observed an unambiguous posterior ramus passing into the occiput on a 31-mm Erinaceus embryo but not on 37$\mathrm{mm}$ embryo or on a neo/postnatal specimen, which seems to corroborate the fact that the ramus posterior of the stapedial artery disappears in adults. A ramus posterior is present in the scandentian Ptilocercus and exits the tympanic cavity as the stylomastoid artery, which joins the caudal auricular artery (Wible 2009: 218). The posterior ramus of Ptilocercus, however, may not be homologous to that of tenrecids and Solenodon, as it bifurcates from the stapedial artery lateral to the stapes (not medial as in tenrecids and Solenodon). Neither MacPhee (1981) nor Wible (2009) describe a posterior ramus in Tupaia.

Therefore, the observations of MacPhee and Asher strengthen our interpretation that a posterior ramus of the stapedial artery was present in adult Alcidedorbignya and extended to the rear of the skull and/or auricle via the path hypothesised in Figure 39. Furthermore, our reconstruction also reinforces Asher's hypothesis (2001) that the presence of a large ramus posterior of the stapedial artery is part of the plesiomorphic pattern of cranial arterial supply of eutherians. This interpretation is also in agreement with Wible $(1987,1993)$, who regards the ramus posterior as part of the hypothetical primitive pattern of eutherian basicranial circulation.

The mastoid process in Vincelestes (sensu this paper; see Appendix 5) and early metatherians is a round thickening of the pars mastoidea posterior or posteromedial to the stylomastoid notch. In the basal eutherian Prokennalestes, the mastoid process (= paroccipital process of Wible et al. 2001) is in a similar position. In Late Cretaceous eutherians such as Asioryctes and Zalambdalestes, the mastoid process is slightly displaced posterolaterally and does not form the posteromedial wall of the stylomastoid notch. There is great variation of the morphology of the mastoid process in mammals but, when distinctly identified, it is located on the posterolateral angle of the auditory region, posterior to the external auditory meatus. The condition in Alcidedorbignya, lateral to posterolateral to the stylomastoid notch, is similar to that in Pantolambda. It is also present in many archaic ungulates (Baioconodon, Maiorana, Pleuraspidotherium, Meniscotherium, Phenacodus, Arctocyonides, Arctocyon, Hyopsodus), in the tillodont Azygonyx, and in the pantolestid Aphronorus. In Leptictis no well-differentiated mastoid process is visible but a distinct muscular attachment for the depressor of the head is present on the lateral part of the pars mastoidea, on the petrosal-squamosal suture, posterior to the posttympanic process of the squamosal. As in Alcidedorbignya, the muscular attachment also extends slightly onto the posttympanic process.
On the endocranial side of the petrosal of Alcidedorbignya, the subarcuate fossa is not very deep and voluminous, and its widest diameter is located at its aperture (i.e. the fossa is vaguely conical). A similar condition is observed in Pantol$a m b d a$, in which the subarcuate fossa is conical and shallow. This condition has been observed on the isolated petrosal of AMNH 15934 and on the CT scan of AMNH 16663. A shallow, conical subarcuate fossa is also present in the tillodont Azygonyx (from CT scan data of UM 68511), in the early ungulates Hyopsodus (Cifelli 1982 and personal observations), Meniscotherium, and some specimens of Pleuraspidotherium (MNHN.F.R1128), in notoungulates (Billet \& Muizon 2013), in litopterns, in many xenarthrans (Chaetophractus, Tolypeutes [Babot et al. 2012], Dasypus [Wible 2010], Bradypus, Choloepus, Megatherium, but not in Tamandua), in the astrapothere Astrapotherium, in the perissodactyls Palaeotherium, Tapirus and Equus, in many artiodactyls (but not in some early-diverging ones; O’Leary 2010; Orliac \& O’Leary 2014) and in Procavia.

A very deep (i.e. deeper than its widest diameter) fossa with an aperture diameter smaller than its widest diameter ("constricted fossa subarcuata" in Wible et al. 2009) (i.e. the fossa is roughly spherical) is present in early-diverging metatherians (e.g., Pucadelphys, Andinodelphys, Mayulestes), in Prokennalestes (probably - see Wible et al. 2001), Kulbeckia, in the "zhelestid" URBAC 99-41 of Ekdale et al. (2004), Kennalestes (Kielan-Jaworowska 1981), Maelestes (see Wible et al. 2009), Leptictis (AMHN 80213), Maiorana (PU 16520), Protungulatum (O'Leary 2010), Arctocyon (Russell 1964), and Arctocyonides (CT scan of MNHN.F.R733). A large and spherical fossa subarcuata with a constricted opening is also present in erinaceomorphs (e.g., Erinaceus, Echinosorex), in Solenodon, in some tenrecids e.g., Potamogale, Microgale), in tupaiids (Tupaia), in the bat Pteropus (Giannini et al. 2006), and in at least some rodents (e.g., Sciurus, Myocastor). In Didelphodus (USNM 18369), in some specimens of Pleuraspidotherium (MNHN.F.R963), in Baioconodon (probably), and in Tenrec an intermediate condition (between conical and spherical) is present; the aperture of the fossa subarcuata is not distinctly constricted and the fossa is rather cylindrical, with a roughly constant diameter. A shallow and conical subarcuate fossa is also present in Vincelestes, which could suggest that it represents the plesiomorphic condition. However, it is noteworthy that a large spherical subarcuate fossa is present in multituberculates (Kielan-Jaworowska et al. 1986; Wible \& Rougier 2000), in basal metatherians, in Prokennalestes (probably), and in some other early-diverging eutherians. This is an indication that this condition may instead represent the plesiomorphic condition within therians. Furthermore, according to Macrini et al. (2007), a broad and rounded subarcuate fossa (spherical condition mentionned here) is the condition that would be observed in the most recent common ancestor of therians. Alcidedorbignya would therefore be derived for this character.

A foramen for the petromastoid canal, which transmits the subarcuate artery and vein from the subarcuate fossa to the mastoid cells (Gannon et al. 1988; Krombach et al. 
2002; O'Leary 2010) is present in Alcidedorbignya as well as in Pantolambda. Such a canal is variably present in mammals. It is best visible when the bottom of the subarcuate fossa is not very deep and notably occurs in several archaic ungulates such as Pleuraspidotherium, Hyopsodus, and $\mathrm{Me}$ niscotherium, in primates (Gannon et al. 1988; Krombach et al. 2002), in many ungulates (e.g., most suids, and hippopotamids, mesonychids [O’Leary 2010]), in earlydiverging notoungulates (Billet \& Muizon 2013), and in Tenrec and Procavia. It probably represents a plesiomorphic character state.

On the lateral edge of the endocranial side of the petrosal of Alcidedorbignya, the prefacial commissure is a low and rounded area that is slightly expanded laterally. A low prefacial commissure is present in some archaic ungulates (e.g., Baioconodon, Pleuraspidotherium, Orthaspidotherium, the early artiodactyls Diacodexis and Dichobune [Orliac \& O'Leary 2014]), in Deltatherium, and in Dasypus. The condition of Alcidedorbignya differs from that observed in Didelphodus (USNM 18369), Leptictis (Novacek 1986), and Prokennalestes (Wible et al. 2001), in which the prefacial commissure is an elevated crest rounded in the former, sharp in the latter two genera. An elevated prefacial commissure is also present in Tenrec, Potamogale, Microgale, Hyopsodus, Acotherulum, Procavia, in some litopterns (Diadiaphorus, Proterotherium), and in Pteropus (Giannini et al. 2006; Wible 2010). An intermediate condition is present in earlydiverging notoungulates (Billet \& Muizon 2013).

A vestigial anterior lamina of the petrosal is present in Alcidedorbignya. This lamina bears a small fossa on its endocranial side, which received part of the trigeminal ganglion. This fossa is the dorsal face of the tegmen tympani. A reduced anterior lamina is also present in early metatherians such as Pucadelphys, and Andinodelphys (Marshall \& Muizon 1995; Ladevèze \& Muizon 2007). Among eutherians, when present, it is smaller than in these basal metatherians. It has been observed by Wible et al. (2001) in Prokennalestes and we observed it in the Eocene eutherian Didelphodus; it is also seen in early-diverging notoungulates (Billet \& Muizon 2013). It is absent in most Recent eutherians, although we observed that a tiny little hint of a vestigial anterior lamina is present in, e.g., Potamogale, and Myocastor. Its occurrence in Alcidedorbignya is clearly a plesiomorphic character.

In Alcidedorbignya, the most basal part of the cochlear canal of the inner ear exhibits a strong constriction relative to the (more dorsal) portion of the vestibule at the level of the ampullae. This constriction is smaller in the specimen MHNC 8399 than in the two isolated petrosals with reconstructed inner ears (MHNC 8360 and 8419). This means that postmortem deformation may have accentuated this constriction in the isolated petrosals, which show overall more deformation than the perfectly 3D-preserved basicranium of MHNC 8399. This being said, the constriction observed in each of these specimens remains strong when compared to many placentals. It is indeed clearly lesser in notoungulates, litopterns, xenarthrans, Didelphodus, Erinaceus, Talpa, Martes, Myocastor, Sciurus, Procavia, and Leptictis. This constriction is just slightly weaker in Pleuraspidotherium, Baioconodon, Astrapotherium and Deltatherium than it is in Alcidedorbignya. The condition of the early-diverging eutherians described by Ekdale \& Rowe (2011) are difficult to observe because the available views are not ideal for this feature. Furthermore, no data on other pantodonts are known that could permit to know if this could be an exclusive feature of the group.

Ectotympanic. The ectotympanic of Alcidedorbignya is horseshoe-shaped and, because we did not find evidence for the presence of an entotympanic, it is arguably widely exposed ventrally and not covered by any bone (i.e. "phaneric") as in Pantolambda. It is narrow and not expanded medially in a large blade, as is observed in Pantolambda (see Appendix 3; Figs 125, 126). The ectotympanic of Alcidedorbignya, however, presents a small distally-expanded triangular styliform process similar to that of Solenodon, Potamogale, Hemicentetes, Asioryctes, and Kennalestes, which have a pronounced styliform process (Kielan-Jaworowska 1981; Wible 2008). A distinct styliform process is absent in e.g., Leptictis, Echinosorex, Suncus, and Tupaia. It differs from Zalambdalestes, in which the arc of the bone is apparently not expanded at all (Wible et al. 2004: fig. 37c). In other respect, the anterior and posterior crura of the ectotympanic of Alcidedorbignya are not strongly expanded transversely, which differs from the expanded anterior crus of Pantolambda, Zalambdalestes, Solenodon, and Orycteropus (Wible et al. 2004; Wible 2008, 2012). The ectotympanic of Alcidedorbignya probably had a sub vertical orientation a condition, also present in Pantolambda. A similar condition has been observed in Zalambdalestes and Ukhaatherium by Wible et al. (2004). On the basis of their observation Wible et al. (2004) regarded the almost vertical orientation of the ectotympanic of the Asioryctes skull ZPAL MgM-I/98 as the original position of this bone, rather than the oblique position observed on ZPAL MgM-I/56 (contra Kielan-Jaworowska 1981). This condition differs from that of most tenrecoids (e.g., Tenrec, Setifer, Hemicentetes), and lipotyphlans (e.g., Erinaceus, Solenodon, Suncus), in which the ectotympanic is either at $45^{\circ}$ or sub-horizontal. The ectotympanic is also strongly oblique in tupaiids. No comparison is possible with tillodonts and archaic ungulates as we have not observed specimens with articulated ectotympanic bones. The basal artiodactyle Acotherulum has a large bulla flooring the middle ear, apparently mostly made of the ectotympanic, which is therefore strongly expanded medially. Because of its development into a bulla, comparison of the orientation of the ectotympanic is inapplicable.

Therefore, the condition of Alcidedorbignya, with a subvertical, and horseshoe-shaped ectotympanic and which lacks any kind of auditory bulla, is highly plesiomorphic. In contrast, the development of a styliform process, which increases the width of the arc in its distal portion, is derived when compared to the ectotympanics of e.g., Leptictis, Suncus, Echinosorex, and Tupaia, which have a constant diameter between the crura. 
Basisphenoid. In Alcidedorbignya the hypophyseal fossa on the dorsal face of the basisphenoid is located anterior to the level of the foramen ovale. This condition radically differs from that of Leptictis, Eoryctes, many lipotyphlans [e.g., Erinaceus, Echinosorex, Suncus, Solenodon (Wible 2008: fig. 22)], and tenrecoids (e.g., Tenrec, Oryzorictes, Potamogale), in which the fossa is totally posterior to the foramen. A hypophyseal fossa posterior to the foramen ovale is also present in the archaic ungulate Pleuraspidotherium. An anterior one is notably present in Ptilocercus (Wible 2011: fig. 24), Hapalemur, and in Martes. Because the hypophyseal fossa is on the dorsal face of the basisphenoid and just anterior to the basioccipitalbasisphenoid suture, the ventral aspect of the latter provides a good approximation of the position of the fossa (of its posterior edge). On the basis of the observation of the suture, a hypophyseal fossa with a posterior edge approximately at the level of the foramen ovale (like Alcidedorbignya) is probably present in Zalambdalestes, in the archaic ungulate Arctocyonides, in Procavia, in Tupaia, in the early-diverging artiodactyls Dichobune and Acotherulum, and Dasypus. A hypophyseal fossa with a posterior edge (based on the suture) well posterior to the level of the foramen ovale is present in the archaic ungulates Maiorana, Hyopsodus, Meniscotherium, and Phenacodus; and in the litoptern Diadiaphorus. The condition in Maelestes is uncertain because the foramen ovale is not observable on the single specimen known. However, the position of the basioccipital-basisphenoid suture relatively to the position of the anterior edge of the pyriform fenestra indicates that, if separated from the piriform fenestra, the foramen ovale was closer to the level of the anterior edge of the hypophyseal fossa, therefore a condition similar to that observed in lipotyphlans. The condition of Asioryctes and Kennalestes, on the basis of the illustrations of Kielan-Jaworowska (1981), is probably similar to that of Alcidedorbignya. A hypophyseal fossa anterior to the level of the foramen ovale is present in the basal metatherians (e.g., Pucadelphys, Andinodelphys, and Mayulestes), which is an indication that this character state could represent a plesiomorphic condition within eutherians, although its distribution among basal eutherians is variable.

Basioccipital, exoccipital. The basioccipital of Alcidedorbignya is proportionally larger and more flattened than in Pantolambda. In this genus it is strongly convex ventrally and narrow anteriorly. The two genera share an absence of contact of the basioccipital and petrosal all along their length. This condition is also present in Bemalambda and probably in Haplolambda. The basioccipital does not contact the petrosal in some "condylarth" or archaic ungulates (Baioconodon, Maiorana, Phenacodus), in some artiodactyls (Diacodexis, Homacodon, etc.), in Solenodon, and in Procavia. It articulates with the petrosal in Maelestes, asioryctitheres, zalambdalestids, Leptictis, and Aphronorus (probably). The condition in early tillodonts such as Esthonyx of Azygonyx is unknown.

A single hypoglossal foramen is present in the exoccipital of Alcidedorbignya. This condition is also present in Pantolambda and Haplolambda but it is apparently absent in Bemalambda, in which two hypoglossal foramina are present on the left side of IVPP V4116. A single hypoglossal foramen is present in Azygonyx, Trogosus and Tillodon but also in some basal eutherians (Asioryctes, Kennalestes), in the archaic ungulates Baioconodon, Maiorana, Arctocyonides, Hyopsodus, Meniscotherium; the early-diverging artiodactyl Acotherulum, and in Leptictis, Potamogale, Microgale, Suncus, Hemicentetes, Tenrec, Setifer, and Erinaceus. In the last three genera, although the most common condition is a single hypoglossal foramen, a double foramen may occasionally occur. A single hypoglossal foramen is also present in Tupaiids (Tupaia, Ptilocercus), in Procavia, and Dasypus. A multiple hypoglossal foramen is present in Vincelestes, in basal metatherians (e.g., Pucadelphys, Andinodelphys, Mayulestes), in the basal eutherians Zalambdalestes, Barunlestes, Maelestes, and Eoryctes; in the Recent lipotyphlans, Solenodon and Echinosorex; and in the archaic ungulate Phenacodus. Therefore, there seem to exist a great deal of variation in the distribution of this character, as well as some individual variation. However, the fact that it is present is basal taxa (Vincelestes, basal metatherians and some basal eutherians) tends to suggest that Alcidedorbignya apparently bears the apomorphic state for this character.

The hypoglossal foramen of Alcidedorbignya opens as a large foramen on a flat ventral surface of the exoccipital bone in front of the occipital condyles. This foramen thus faces more ventral than anterior or lateral, and is not accompanied by any crest or fossa. The same condition is present in Pantolambda, but also in the xenungulate Carodnia (in which it faces anteroventrally), the condylarths Baioconodon, Arctocyon and Maiorana; and Potamogale; possibly also in Azygonyx. Many other eutherians do not show this condition but rather exhibit a hypoglossal foramen within a fossa or bordered by a crest: e.g., Erinaceus, Echinosorex, Solenodon, Cynodictis, Amphicynodon, Nycticebus, Magnadapis, Dichobune, Acotherulum, Xenarthra, Leptictis, Procavia, Astrapotheria, Tenrec, Setifer, Tapirus, Equus, Tupaia, Zalambdalestes (Wible et al. 2004), Maelestes (Wible et al. 2009), Notoungulata, Litopterna, Pleuraspidotherium, and Arctocyonides (bordered by a crest, but ventral and large).

Supraoccipital. The nuchal crest of Alcidedorbignya differs from those of Pantolambda and Haplolambda in its strong posterior projection, which extends further posteriorly than the occipital condyles. It is also much narrower than in the other American pantodonts, in which the crest almost as wide as the total width of the skull. In this respect, Alcidedorbignya resembles the Asiatic pantodonts Harpyodus decorus and Bemalambda nanhsiungensis and its condition is likely to be plesiomorphic.

The nuchal crest of Alcidedorbignya bears a deep median notch in dorsal view and differs, in this respect, from those of Pantolambda and Haplolambda, which are almost semicircular in dorsal and occipital views. In Alcidedorbignya it is hardly as wide as the condylar width while it is much wider in Pantolambda and Haplolambda. Like that of Alcidedorbignya, the nuchal crest of Bemalambda strongly projects posteriorly and is also notched at the junction point between sagittal and nuchal crests, although to a much lesser extent 
than in Alcidedorbignya (Zhou et al. 1977). The condition of Harpyodus decorus is also apparently similar to that of $\mathrm{Al}$ cidedorbignya (Wang 1979). A similar condition is present in the tillodont Azygonyx, in which the nuchal crest greatly projects posteriorly and is deeply notched in its median region. The nuchal crest is notched and projects posteriorly in Pleuraspidotherium, Meniscotherium, and Deltatherium. This condition is variably present in Solenodon and Potamogale. It is notched in some armadillos (e.g., Kuntinaru), but it does not project posteriorly (more or less similar in Leptictis; note that in these taxa the temporal lines do not meet to form a sagittal crest). In Maiorana and Baioconodon the nuchal crest is apparently not notched in its median region and is relatively straight, but it strongly projects posteriorly. A similar condition is observed in Arctocyon and Arctocyonides, which have no nuchal notch and in which the point of junction between the sagittal and nuchal crests projects posteriorly and forms the posteriormost point of the skull (Russell 1964). Among pantodonts, the condition of Alcidedorbignya is only present in early divergent Asiatic taxa (e.g., Bemalambda, Harpyodus) (Muizon \& Marshall 1992) and probably represents a plesiomorphic character state. In fact, a nuchal crest deeply notched in its median region and posteriorly projecting in its lateral portions (as is observed in Alcidedorbignya) is common among aquatic mammals (sea otters, pinnipeds, archaeocetes) and could be related to lateral movements of the head or in any case to a greater mobility of the head in all directions. In other respects, the condition of Arctocyon and Arctocyonides is common in large terrestrial carnivores (felids, canids); it is also seen in the primate Magnadapis, in astrapotheres, and in some litopterns (Proterotherium) and is probably related to strong dorsoventral movements rather than lateral or multidirectional. The nuchal crest is rather straight and transverse in many placentals, e.g., Tupaia, Bradypus, Tenrec, Erinaceus, Procavia (not a continuous crest), in most notoungulates, and in early-diverging artiodactyls.

\section{Brain endocast}

Olfactory bulbs, circular fissure. The olfactory bulbs of $\mathrm{Al}$ cidedorbignya are large (8.49\% of the total endocast volume), although smaller than in the theriiform Vincelestes $(10.80 \%)$ and the basal metatherian Pucadelphys (11.68\%). Among eutherians, they are smaller than in the basal taxa Zalambdalestes (9.24\%), Kennalestes (10.03\%), Asioryctes (10.35\%); and much smaller than in Leptictis (16.66\%). Prominent olfactory bulbs, larger than or subequal to those of Alcidedorbignya, are observed in some pantodonts (Leptolambda), in Uintatherium, in some "condylarths" (Arctocyon, Arctocyonides, Phenacodus) (see Silcox et al. 2010 who provided an exhaustive dataset on endocranial volumes of fossil and extant mammals). The olfactory bulbs of Alcidedorbignya are distinctly larger than those of some other pantodonts (Pantolambda Coryphodon), the unitathere Tetheopsis, and some "condylarths" (e.g., $\mathrm{Me}$ niscotherium, Pleuraspidotherium, Hyopsodus). Among Recent taxa, the olfactory bulbs of Alcidedorbignya are notably smaller (relative to the cerebrum) than those of tenrecids (except for Potamogale and Limnogale), Solenodon, Rhynchocyon, and
Erinaceus, but are comparable in size to those of soricids (see the dataset provided by Silcox et al. 2010). The presence of large olfactory bulbs in basal theriiform, metatherian, and eutherian mammals as well as in Leptictis is likely an indication that this condition represents a plesiomorphic character state among therians and mammals (Macrini et al. 2007a). It is noteworthy, however, that the very large olfactory bulbs of some extant mammals (e.g., Tenrec, Solenodon, Rhynchocyon, Erinaceus) could be interpreted as a derived trait correlated with highly developed olfactory abilities (Novacek 1986).

A marked circular fissure separates the olfactory bulbs of Alcidedorbignya from the cerebral hemispheres posteriorly, which is an indication that the cerebrum does not overlap onto the olfactory bulbs. A similar condition is observed in other pantodonts, condylarths, Leptictis; the Cretaceous eutherians Asioryctes, Kennalestes, Zalambdalestes, the metatherian Pucadelphys; and the early-diverging theriiform Vincelestes. This condition, which is also present in extant tenrecids (e.g., Tenrec), and eulipotyphlans (e.g., Solenodon, Rhynchocyon, Erinaceus), is probably an ancestral feature of therians (Macrini et al. 2007a) and most likely of mammals (Rowe et al. 2011).

Lissencephalic brain. Small lissencephalic brains usually imprint lissencephalic endocasts, although some mammals with large gyrencephalic brains can have lissencephalic endocasts (i.e. hominids, proboscideans, cetaceans; see Edinger 1955; Tobias 1971; Holloway et al. 2004; Racicot \& Colbert 2013). As a consequence, only fossil mammals with small endocranial cavities and lissencephalic endocasts are supposed to have had lissencephalic brains. The endocranial cavity of Alcidedorbignya is not as small relative to the skull size, as compared to other pantodonts or to Uintatherium, which are much larger animals with very small endocranial cavities. If we assume that brain tissue has an average density of $1.0 \mathrm{~g} / \mathrm{cm}^{3}$ (following Jerison 1973), then Alcidedorbignya had a brain of approximately $2.2 \mathrm{~g}$. Such a low brain weight is consistent with the hypothesis of a lissencephalic brain, since most extant mammals with brain weights less than $5 \mathrm{~g}$ have relatively smooth brains (see Macrini et al. 2007a).

Macrini et al. (2007c) stated that the presence of lissencephalic cerebral hemisphere casts is the likely ancestral condition of therians, based on endocasts of the eutherians Kennalestes, Barunlestes, and Zalambdalestes (Kielan-Jaworowska 1984, 1986); basal metatherians (e.g., Pucadelphys; Macrini et al. 2007c); and Vincelestes (Macrini et al. 2007a).

Fissuration: rhinal fissure and neocortical sulcus. The supposed position of the rhinal fissure in Alcidedorbignya delimitates a narrow and elevated neocortex (not overlapping the piriform lobes laterally), which approaches the condition observed in Erinaceus, Solenodon, and Tenrec (Bauchot \& Stephan 1967; Kaas 2009). This condition is also observed in the other pantodonts (Edinger 1956; Lucas 1984). This would indicate, in Alcidedorbignya, a moderate development of the neocortex. A similar condition is also observed in the "condylarth" Phenacodus (Simpson 1933; Edinger 1956) in which the rhinal fissure is in an elevated position on the hemisphere, does not 
extend laterally and does not overlap the piriform lobe in dorsal view. The neocortex of Phenacodus appears as two "ovoid caps", especially distinct on the MCZ specimen (MCZ 4440) illustrated by Edinger (1956: fig. 15). On the two specimens of Phenacodus (MCZ 4440 and AMNH 4369), however, no obvious cast of the orbitotemporal sulcus has been observed. The condition of Periptychus is similar to those of Alcidedorbignya and Phenacodus in the narrow, ovoid-shaped neocortical caps (although shorter than in the two latter genera [Edinger 1929, 1956]). In Arctocyon, and in Pleuraspidotherium, the condition is similar to that in Alcidedorbignya, with an elevated and narrow neocortex and a rhinal fissure dorsal to and well separated from the orbitotemporal sulcus. In dorsal view of the endocast, the rhinal fissure is clearly medial to the orbitotemporal sulcus and the piriform lobe is clearly lateral to the rhinal fissure (Russell \& Sigogneau 1965).

In the slightly younger "condylarths" Hyopsodus (Orliac et al. 2014) and Meniscotherium (Gazin 1965) from the Eocene, the dorsal surface of the endocasts show cast of the rhinal fissure and orbitotemporal sulcus. In both taxa the rhinal fissure is in a more ventrolateral position than in Alcidedorbignya in such a way that the rhinal fissure is hardly visible in dorsal view. In lateral view, however, it is distinctly separated from the orbitotemporal sulcus (Gazin 1965: fig. 2; Orliac et al. 2014: fig. 3A, B). This condition denotes a greater development of the neocortex than in Alcidedorbignya and therefore a more derived condition. It is noteworthy that Kielan-Jaworowska (1984) observed, in Asioryctes, a possible rhinal fissure, which delimitates an even smaller neocortex than in Alcidedorbignya or the "condylarths" Periptychus, Arctocyon or Phenacodus. An orbitotemporal sulcus is clearly present in Asioryctes, well ventral to the rhinal fissure. In turn, the structure on the ventrolateral side of the endocast of Zalambdalestes interpreted by Kielan-Jaworowska as the rhinal fissure is likely to be the orbitotemporal sulcus. In Leptictis, Novacek (1986) interpreted the course of the rhinal fissure as following the ventral edge of the orbitotemporal groove. According to this interpretation, the neocortex of Leptictis would be larger than that of Erinaceus (Moodie 1922). However, it is noteworthy that Bauchot \& Stephan (1967: 581) stated that the orbitotemporal sulcus (their "canal sinusaire") does not correspond to the rhinal fissure, even if they may be approximated. They further compared the position of the orbitotemporal sulcus in Solenodon and in Loris. In both taxa the orbitotemporal sulcus is in the same lateral position relatively to the cerebral hemisphere, but in the former it is located at the level of the pririform lobe and in the latter at the level of the neocortex, which therefore extends much more ventrally in Loris than in Solenodon. A condition similar to that of Solenodon is present in Tenrec and Erinaceus. Therefore, the rhinal fissure of Leptictis may not follow the orbitotemporal groove but may correspond to the sulcus identified as neocortical sulcus by Novacek $(1982,1986)$. As a matter of fact, the sulcus identified as a neocortical sulcus by Novacek extends on the entire length of the hemisphere, while, when present in the "condylarths" considered in this section (e.g., Phenacodus, Arctocyon, Arctocyonides), the sulcus is restricted to an anterior depression. A neocortical sulcus is probably present in Alcidedorbignya. It has not been described in the other pantodonts by Lucas (1984), but Edinger (1956: fig. 18) illustrated a structure that could be interpreted as such in Pantolambda. However, we have not observed the structure in the CT scan of AMNH 16663 (Fig. 56A). A neocortical sulcus ("sillon latéral” of Russell \& Sigogneau 1965) has been identified in Arctocyon, Arctocyonides and Phenacodus, in a similar position to that possibly present in Alcidedorbignya.

However, it is worth remembering to remind that the identification of cerebral sulci in endocranial casts of extinct animals should be taken cautiously because the rhinal fissure, for instance, is not always printed in endocranial casts, which does not mean that it was absent on the actual brain (see above, comments on statement by Bauchot \& Stephan 1967: 581 and Macrini et al. 2007b). According to Macrini et al. (2007a), the ancestral condition for therians is lack of a rhinal fissure on endocasts, which is likely to be the consequence of the small size of their brains. As a matter of fact, modern mammals with brain masses lower than 5 g generally lack neocortical sulci and have smooth cerebral hemispheres (see Macrini et al. 2007a).

Alcidedorbignya presents a very anterior point of posterior divergence of the cerebral hemispheres approximately at the posterior third of the length of the cerebrum. This condition resembles that observed in Mongolian basal eutherians (e.g., Kennalestes, Asioryctes, Zalambdalestes), although in these genera the condition is more pronounced and the hemispheres diverge almost at half the length of the cerebrum. Cerebral hemispheres also diverge posteriorly in other pantodonts (e.g., Pantolambda, Leptolambda and Coryphodon). Among Recent mammals, posteriorly-diverging hemispheres are observed in Tenrec, Potamogale and Rhynchocyon, although to a lesser extent than in Alcidedorbignya. Given the distribution of this character state, an anteriorly placed point of posterior divergence of the cerebral hemisphere, which leaves a large area of exposure of the colliculi, is therefore likely to be plesiomorphic, at least for eutherians.

The evolution of the mammalian cerebrum is understood as following three related trends (see Sanides 1970; Novacek 1982): increase in size of the neocortex, downward migration of the rhinal fissure and increased gyrification. The lissencephalic endocranial cast and the dorsal position of the rhinal fissure of Alcidedorbignya represent plesiomorphic conditions, but, if our interpretation is correct, the presence of a neocortical sulcus represents a derived beginning of gyrification.

Midbrain exposure. The midbrain is widely exposed in $\mathrm{Al}$ cidedorbignya and faint swellings on the posterior edge of the cerebral hemisphere can be interpreted as imprints of the colliculi (Fig. 54). Exposure of the midbrain has also been observed in other pantodonts (Lucas 1984); in the basal eutherians Asioryctes, Zalambdalestes, Kennalestes, and Barunlestes (Kielan-Jaworowska 1984); in the the "condylarths" Pleuraspidotherium, Arctocyon, Arctocyonides, Hyopsodus, Meniscotherium; and in many living mammals (Edinger 1964; Gazin 1965; Russell \& Sigogneau 1965; Jerison 1973; Orliac et al. 
2012a). Although midbrain exposure of highly-specialized mammals such as bats (Edinger 1964) or flying lemurs (Gingerich \& Gunnel 2005) is interpreted as a secondary sensory specialization, it can also be regarded as a primitive feature of mammals, resulting from a weak neocortical expansion (Edinger 1964) or from a weak expansion of the posterior cerebral hemisphere, as argued by Orliac et al. (2014) for the plesiadapiform Plesiadapis tricuspidens. While describing the endocast of Alcidedorbignya, we assumed that the distance between the neocortex and the hindbrain correlates with the exposure of the midbrain, which is apparently corroborated by the presence of possible colliculi in this area of the endocast (Fig. 54). It is noteworthy that some extant mammals exhibit exposed colliculi on the dorsal surface of their brains but not on the corresponding endocasts (for instance in $D i$ delphis virginiana, Dom et al. 1970; Monodelphis domestica, Macrini et al. 2007b; Tenrec ecaudatus, Bauchot \& Stephan 1967). Exposure of colliculi has been documented in few fossil eutherians (Asioryctes, Kennalestes, Zalambdalestes, Barunlestes, Coryphodon, Leptictis) and few extant placentals (Tenrec, Solenodon, Microgale, Rhynchocyon, Erinaceus, Gymnura, Crocidura, Talpa, Chrysochloris, Le Gros Clark 1932; Bauchot \& Stephan 1967; Stephen \& Andy 1982). Macrini et al. (2007a) argued that the ancestral condition of therians was most probably the lack of dorsal exposure of the midbrain on endocasts. However, the fact the dorsal exposure of the midbrain is widely present in basal eutherians is an indication that this condition is probably plesiomorphic within eutherians.

Hindbrain - cerebellum. The cerebellum of Alcidedorbignya is slightly lower in lateral view than the cerebrum. According to the figures of Lucas (1984) the cerebella of Leptolambda and Coryphodon resemble the condition observed in Alcidedorbignya in being slightly lower than the cerebral hemispheres. Apparently a similar condition is also present in Pantolambda.

A cerebellum that is lower or nearly as high as the cerebrum has also been observed in some "condylarths" (Hyopsodus, Meniscotherium, Arctocyon), Diacodexis, Erinaceus, Tenrec, and Solenodon. In other condylarths (Pleuraspidotherium, Phenacodus), in Leptictis, and in basal eutherians from Mongolia (e.g., Asioryctes, Kennalestes, Zalambdalestes), the top of the cerebellum is slightly more elevated than the top of the cerebrum. Cerebellar height is characterized by an elevated vermis with respect to the cerebrum height and is associated with a weakly expanded neocortex (Novacek 1982, 1986). However, this statement is contradicted by the condition observed in pantodonts, which have a poorly developed neocortex and a cerebellum slightly lower than the cerebral hemispheres.

The paraflocculi of Alcidedorbignya are conical, like those of other pantodonts (e.g., Pantolambda, Leptolambda and Coryphodon). This morphology of the paraflocculi resembles that observed in the theriiform Vincelestes, and in some "condylarths" (e.g., Hyopsodus, Pleuraspidotherium), but clearly differs from the spherical condition observed in basal metatherians (e.g., Pucadelphys, Andinodelphys, Mayulestes), in basal eutherians (e.g., Asioryctes, Kennalestes, Zalambdalestes, Leptictis) or in Recent Lipotyphla (e.g., Solenodon, Erinaceus, Echinosorex), or tenrecoiids (e.g., Tenrec, Potamogale, Hemicentetes). In fact, the presence of well-developed parafloccular lobes is considered as a plesiomorphic feature of therians (Macrini 2006). Therefore, the condition of Alcidedorbignya probably represents a derived condition within eutherians.

\section{Dentary}

As a whole the dentary of Alcidedorbignya is more gracile and slender than that of Pantolambda. The corpus mandibularis is slightly shallower than in Pantolambda. However, the dentaries of the two genera are remarkably similar except for the angular process. The small and hook-like angular process of Alcidedorbignya differs from that of Pantolambda, which is much larger and wider and strongly projects posteriorly. A very wide and large angular process is present in all other pantodonts (e.g., Haplolambda, Caenolambda, Titanoides, Barylambda, Bemalambda). Within Pantodonta, the hooklike morphology of the angular process of Alcidedorbignya is apparently unique. Among tillodonts the angular process is unknown in Esthonyx and Azygonyx, but that of Trogosus is wide and rounded in lateral view.

The posterior part of the dentary is not commonly preserved in early ungulates. A small and hook-like angular process is present in the archaic ungulates Arctocyon, Arctocyonides, Simoclaenus, Molinodus, and Protungulatum (Sloan \& Van Valen 1965). A large and broad (in lateral view) process is present in Pleuraspidotherium, Meniscotherium, Hyopsodus, Ectocion, Carodnia, notoungulates, and Trigonostylops. Among other early mammals, a hook-like angular process is common in Cretaceous eutherians (e.g., asioryctitheres - sensu Archibald \& Averianov 2006 -, Zalambdalestes, Montanalestes, Zhelestes). This feature is also present in Leptictis, several Lipotyphla (e.g., Erinaceus, Nesophontes, soricoids, talpids), Potamogale, and some Archonta (e.g., Tupaia, saxonellid and carpolestid Plesiadapiforms). In the earliest known eutherians, Juramaia and Eomaia, the angular process is short and narrow but it is not distinctly hook-like as in the taxa cited above (Ji et al. 2002; Luo et al. 2011). A hook-like angular process in $\mathrm{Al}$ cidedorbignya clearly represents a plesiomorphic condition absent in the other pantodonts.

As in Pantolambda, the coronoid process of Alcidedorbignya is anteroposteriorly narrow, approximately constant in width all along its proximodistal length, and distinctly bent posteriorly. This morphology is present in most other North American pantodonts (Caenolambda, Haplolambda, Titanoides, Barylambda, and Coryphodon). In the Asiatic genus Bemalambda, however, the coronoid process is triangular and distinctly wider at its base than at its apex.

A coronoid process that is narrow and strongly bent posteriorly is notably present in Zalambdalestes, Protungulatum (Sloan \& Van Valen 1965), many rodents (e.g., Rattus, Castor, Ictidomys [Morphobank], Cocomys [Wible et al. 2005]), Leptictis, xenarthrans (e.g., Dasypus, Bradypus), erinaceids and tenrecoids. However, many early eutherians have a triangular coronoid process with a straight posterior edge (e.g., Eomaia, Uchkudukodon, Asioryctes, Kennalestes). In archaic ungulates the coronoid process is bent posteriorly (i.e. distinctly concave 
posteriorly); e.g., Arctocyon, Loxolophus, Ectoconus, Phenacodus, Meniscotherium, and Hyopsodus. The tillodont Trogosus has a posteriorly bent coronoid process. Therefore, because several basal eutherians have a triangular coronoid process (i.e. not concave posteriorly), the narrow and anteriorly bent coronoid process of Alcidedorbignya may represent a derived character state within eutherians present in most pantodonts (except Bemalambda).

In posterior view the transverse axis of the mandibular condyle slopes medially in Alcidedorbignya and Pantolambda, but this feature is clearly more pronounced in the latter.

As noted in the description, the ramus mandibularis (vertical ramus) of Alcidedorbignya is anteroposteriorly short and its coronoid process is slightly tilted anteriorly, i.e. its main axis is oriented anterodorsally-posteroventrally and is at an angle slightly less than $90^{\circ}$ with the corpus. This condition is found in the xenungulate Carodnia, in Tapirus, Glyptodon, Procavia and astrapotheres (Trigonostylops, Astrapotherium, Astraponotus). It is absent (main axis shifted posteriorly) in many eutherians, among which are Erinaceus, Dasypus, Bradypus, Arctocyon, lagomorphs (Oryctolagus, Prolagus [Wible et al. 2009]), Leptictis, Maelestes, Zalambdalestes, the rodent Cocomys (illustration in Wible et al. 2009), Barunlestes, Asioryctes, Kennalestes and Daulestes (illustrations in Wible et al. 2009), Potamogale, Tenrec, Pteropus, many carnivorans and artiodactyls, and the perissodactyl Equus. A somewhat intermediate condition, i.e. the main axis vertical, is present in Solenodon, Protungulatum (Sloan \& Van Valen 1965), Pleuraspidotherium, notoungulates (e.g., Simpsonotus, Notostylops, Posnanskytherium, Plesiotypotherium) and in litopterns (Proterotherium). This character partly overlaps with character 135 of Wible et al. (2009), which illustrates the relatively rare occurrence, within eutherians, of an anterior border of the coronoid process tilted anteriorly, as is the case in Alcidedorbignya. Therefore, given its absence in most basal eutherians, the condition in Alcidedorbignya is likely to be derived.

In Alcidedorbignya, the coronoid crest extends ventrally onto the corpus mandibularis and forms a salient elongated tubercle below $\mathrm{m} 3$. This condition is present in the pantodonts Pantolambda, Bemalambda, and Barylambda; in the tillodonts Azygonyx and Trogosus; and in the xenungulates Etayoa and Carodnia. Because it is absent in the early eutherians (for which the dentary is known) from the Jurassic and Cretaceous (e.g., Juramaia, Eomaia, Prokennalestes, Asioryctes, Kennalestes, Maelestes, Zalambdalestes), the condition in Alcidedorbignya is likely to represent an apomorphic character state.

\section{DISCUSSION OF POSTCRANIAL CHARACTERS}

The discussion on the postcranial skeleton of Alcidedorbignya will be focused on its functional interpretation rather than on its phylogenetic signal in order to interpret the locomotory behaviour of this basal pantodont. Nevertheless, postcranial characters will be included in the parsimony analysis on the basis of those used by Wible et al. (2009). Therefore, in the following discussion, comparison will be made with taxa that do not necessarily exhibit close phylogenetic affinities with Alcidedorbignya but that bear similar morphological traits, which can be interpreted as related to a comparable function (or which can allow a better understanding of our fossil taxon), given their biology in the case of Recent taxa (e.g., Solenodon, Tenrec, Sciurus, Ailurus, Marmota, Dendrohyrax, Caluromys, Metachirus). In the discussion on possible scansorial habits of Alcidedorbignya, comparison with the arboreal Dendrohyrax may appear surprizing given the fact that the postcranial anatomy of this genus shows many traits suggesting a long cursorial ancestry (Fischer 1986). Nevertheless, we regard this comparison as highly relevant since tree hyraxes climb very well without claws (also absent in Alcidedorbignya) and without the great capacity of prehension of arboreal primates (also absent in Alcidedorbignya). Their ability to climb is related to the association of digital friction pads associated to unusual carpal mobility that allows wrist (instead of elbow) supination, two features that will be discussed below. Therefore, we regard Dendrohyrax as a good potential functional analogue of Alcidedorbignya on some aspects.

\section{Axial skeleton}

Cervical vertebrae. The extensive neural process of the axis vertebra in Alcidedorbignya suggests strong neck musculature, especially the development of long fibers of the obliquus capitis caudalis (on the lateral side of the process) and semispinalis capitis (on the top of the neural process - through the nuchal ligament - and partly on its lateral sides), powerful rotator and extensor of the head. However, the transverse processes of the atlas, which receive the insertion of the obliquus capitis caudalis, are poorly developed and do not protrude posteriorly. A similar condition is found in Tenrec, in which the neural process of the axis is well developed and prominent posteriorly, but the transverse processes of the atlas are not, and the neural processes of the posterior cervical vertebrae are low. However, Tenrec differs from Alcidedorbignya in lacking the anterior extension of the neural process.

In Alcidedorbignya, the sagittal and occipital crests of the skull are prominent, especially the nuchal crest, which protrudes posteriorly. This condition suggests powerful splenius and semispinalis capitis muscles, which elevate the head but also bend the head and neck laterally in unilateral action. Therefore, the great development of the nuchal crest corroborates the capacities of powerful extension of the head hypothesized from the morphology of the axis but also indicates possibility of powerful lateral movements of the head and neck. In this respect, Alcidedorbignya resembles Tenrec, which also has a well-developed nuchal crest concomitant with a large neural process of the axis. Solenodon also has a very large, posteriorly extended, and dorsally strongly convex neural process of the axis. In this genus, the nuchal crest is strongly extended posteriorly and sometimes bilobate, as in Alcidedorbignya, suggesting powerful dorsoventral and lateral movements of the head and neck. However, the three genera mentioned above have moderately developed transverse processes of the atlas, which suggests, in contrast, that dorsoventral movements of the head were probably not exceptionally powerful. A strongly developed neural process of the axis is present in hypercarnivorous borhyaenoids (e.g., Mayulestes, Prothylaci- 
nus, Cladosictis, Borhyaena), and the transverse processes of the atlas of these genera are not much more developed than in Alcidedorbignya. Be that as it may, the great development of the neural process of the axis and of the nuchal crest are indicators of powerful movements (dorsoventral as well as lateral) of the neck and head and of development of the three muscles (splenius, semispinalis capitis and obliquus capitis caudalis), which are likely involved in this mobility. In this context, it is noteworthy that the three genera (Alcidedorbignya, Tenrec and Solenodon) have either well-developed upper canines or canine-like upper I1s (for Solenodon). Powerful movements of the head in these genera could be related to the function of the canines or canine-like incisors in the case of Solenodon.

Thoracolumbar vertebrae. Alcidedorbignya exhibits 22 thoracolumbar vertebrae, with the usual 13 pairs of ribs and 9 lumbar vertebrae (the thoracolumbar length of MHNC $8372-14 \mathrm{~cm}-$ is approximately $1.5 \mathrm{~cm}$ longer than Tenrec ecaudatus, specimen MNHN-AC-1261, and $2 \mathrm{~cm}$ longer than Solenodon paradoxus, specimen MNHN-AC-14487). The first 11 thoracolumbar vertebrae of Alcidedorbignya show a typical thoracic morphology with a posteriorly inclined neural process, whereas the 10 most posterior vertebrae show a radial articular mode (lumbar-like) and a quadrangular neural process. The $12^{\text {th }}$ vertebra of the series is intermediate and is the diaphragmatic vertebra. Because the neural processes of the lumbar vertebrae and last two thoracics are oriented vertically and not anteriorly, there is no reversion (from posterior to anterior) in the orientation of the neural processes and therefore, strictly speaking, no anticlinal vertebra can be observed in Alcidedorbignya.

In comparison, Tenrec exhibits 24 thoracolumbar vertebrae, 19 with a pair of ribs and 5 without. The 14 anterior vertebrae present an elongate and posteriorly-inclined neural process, whereas the 9 most posterior vertebrae show a quadrangular neural process. In between, the $15^{\text {th }}$ vertebra, being intermediate between the thoracic and the lumbar articular mode, is the diaphragmatic vertebra. As in Alcidedorbignya, the neural processes of the anterior lumbars and posterior thoracics are quadrangular and vertical. Therefore, there is no anticlinal vertebra. There is, then, a similar number of posterior vertebrae with a lumbar morphology in the two taxa (10 vs 9). There is also an important posterior inclination of the neural processes of the thoracics in Tenrec, the longest being T4-T6. Posteriorly, the length of the neural processes decreases but can be observed until T14.

The condition in Solenodon slightly differs from that of $\mathrm{Al}$ cidedorbignya. Solenodon has 20 thoracolumbar vertebrae, 15 with a pair of ribs and 5 without. The 12 anterior are thoraciclike and have a long and posteriorly-inclined neural process, while the 7 posterior are lumbar-like with a quadrangular neural process. The $13^{\text {th }}$ vertebra is intermediate between the anterior and posterior. Its neural process is subvertical, not strongly elongated anteroposteriorly, and only slightly longer than high. This vertebra bears ribs and is the diaphragmatic vertebra. There is no anticlinal vertebra. Therefore, in Solenodon the number of lumbar-like vertebrae is smaller (7) than in Alcidedorbignya (10) and Tenrec (9). The diaphragmatic vertebra is the penultimate thoracic in Alcidedorbignya. In Tenrec it is located more anteriorly in the thoracic section as it is followed by four rib-bearing vertebrae (one in Alcidedorbignya), whereas in Solenodon, being the antepenultimate thoracic, it is followed by two rib-bearing vertebrae.

The thoracic section of Alcidedorbignya is approximately as long as the lumbar section $(c .7 \mathrm{~cm}$ each). In Tenrec and Solenodon the thoracic section is distinctly longer than the lumbar section, respectively 9 vs $4.2 \mathrm{~cm}$ and 8 vs $3.9 \mathrm{~cm}$. This condition is the consequence of the greater number of thoracic vertebrae and the smaller number of lumbar vertebrae in Tenrec and Solenodon compared to Alcidedorbignya.

The thoracic vertebrae of Alcidedorbignya are not wider than in Tenrec, but the dorsal arch is longer anteroposteriorly. The lumbar vertebrae are also larger and longer in Alcidedorbignya than in Tenrec. This is why, despite a lower number of vertebrae, the thoracolumbar segment is slightly longer in Alcidedorbignya than in Tenrec. The thoracic vertebrae of Alcidedorbignya are roughly of the same proportions as those of Solenodon but its lumbar vertebrae are more slender than those of Solenodon. Because Solenodon has two thoracolumbar vertebrae less than in Alcidedorbignya, its thoracolumbar segment is slightly shorter than that of Alcidedorbignya, in spite of its larger lumbar vertebrae.

The slenderness, great length, and posterior inclination of the neural processes of the anterior thoracics of Alcidedorbignya are also observed in Tenrec and Solenodon, and hyracoids. However, the processes are clearly longer and more inclined posteriorly in Alcidedorbignya than in Tenrec and Solenodon. The three genera also share anteroposteriorly long, quadrate, and robust neural processes on the lumbar vertebrae, but they are proportionally higher in Alcidedorbignya than in the other two genera. A similar morphology is also observed in hyracoids and Marmota. Table 52 compares the heights of the neural process to the total height of the penultimate lumbar vertebra in Alcidedorbignya, and in Tenrec, Solenodon, selected squirrels and arboreal carnivores, hyracoids, and in the curso-saltatorial didelphid, Metachirus. The process of Alcidedorbignya is distinctly closer in size to those of Procavia and Sciurus than to that of the other taxa considered. The neural processes of the lumbars are also remarkably similar (being anteroposteriorly long and quadrate) to those of Marmota, an agile fossorial rodent, which demonstrates a great ability to stand bipedally, and to the hyracoids Dendrohyrax and Procavia. In contrast, the height of the neural process of the penultimate lumbar vertebra of Alcidedorbignya departs from those of Solenodon and Tenrec, which are relatively lower, and from those of Metachirus and Nasua, which are higher.

In Tenrec the neural processes of the lumbar vertebrae stand vertically on the anterior lumbars and show a slight posterior inclination on the posterior lumbars. In Solenodon the processes are vertical but the last lumbar has a slightly anteriorly inclined neural process. In hyracoids all the neural processes are inclined slightly anteriorly. In Alcidedorbignya they are all subvertical as in Marmota. Moreover, in Alcidedorbignya, the space between the neural processes of the posterior lumbar 
vertebrae (L5-L9) varies, from half the anteroposterior length of the neural process between L5 and L6 to one neural process length between L8 and L9. Alcidedorbignya thus differs from Tenrec and Solenodon in which the neural processes are almost contiguous or, at the most, separated by half the length of the neural process as between L5 and L6 in Solenodon. In Marmota, the space between the neural processes of the lumbar vertebrae is half the length of the processes to slightly less. Therefore, the condition of Alcidedorbignya is an indication of better capacities of dorsiflexion of the vertebral column in the posterior lumbar region, and departs from the more rigid lumbar segment of Tenrec and especially Solenodon. The condition in Marmota indicates a slightly more flexible vertebral column than in Tenrec and Solenodon, but distinctly less than in Alcidedorbignya. The flexibility of the vertebral column of Alcidedorbignya is corroborated by the morphology of the zygapophyseal articular facets, which are almost flat in Alcidedorbignya while they are markedly concavoconvex and tend to be somewhat cylindrical in Solenodon.

The transverse processes of the lumbar vertebrae of Alcidedorbignya are well developed but not as much as in cursosaltatorial mammals (e.g., Oryctolagus, Metachirus). They are oriented slightly ventrally and anteriorly. They clearly increase in dorsoventral height, anteroposterior length, and curvature from L1 to L9. On the last lumbar vertebra (L9), the process has the characteristic falciform morphology observed in squirrels or arboreal carnivores. Furthermore, the transverse process of the posterior lumbar vertebrae is as wide (anteroposteriorly) at its base as the length of the vertebral canal. This condition resembles that observed in some very agile arboreal mammals such as squirrels. On L9 the proximodistal length is at least $7.6 \mathrm{~mm}$ (because the apex of the process is not preserved it is estimated that the process could have reached $8 \mathrm{~mm}$ or slightly more), which is almost $60 \%$ longer than the minimum width of the neural arch between the zygapophyses $(4.8 \mathrm{~mm})$. The transverse processes of Alcidedorbignya are more developed than in Solenodon, in which the length of the longest transverse process (on the penultimate lumbar vertebra) is roughly similar to the neural arch width. Furthermore, in Solenodon the transverse processes are oriented more anteriorly and less laterally than in Alcidedorbignya, providing less transversal width for attachment of the hypaxial musculature. The two genera differ from the condition of Tenrec, in which the transverse processes are almost absent. It is noteworthy that the transverse processes of the lumbar vertebrae of Alcidedorbignya are not drastically smaller than in some sciurids (e.g., Callosciurus flavimanus). The transverse processes of Alcidedorbignya also resemble those of some arboreal carnivores (Ailurus, Nasua) but are less robust than those of hyracoids.

The long and posteriorly-inclined neural processes of the thoracic vertebrae reflect the development of the extensor musculature (multifidus thoracis and semispinalis thoracis) attaching on their apices. This condition, in Alcidedorbignya, agrees with the capacities of dorsiflexion of the posterior lumbar vertebrae noted above. In other respects, the transverse processes of the lumbar vertebrae, which are inflected only slightly anteriorly and ventrally, would suggest a moderate
TABLE 52. - Comparative sizes of the neural processes relative to the maximum height (in sagittal plane) of the penultimate lumbar vertebra in several placentals.

\begin{tabular}{|c|c|c|c|}
\hline & $\begin{array}{c}\text { Ht } \\
\text { Penult L }\end{array}$ & $\begin{array}{l}\text { Ht Neurl } \\
\text { Proc }\end{array}$ & HNP/HPL \\
\hline $\begin{array}{l}\text { Alcidedorbignya inopinata } \\
\text { (MHNC 8372) }\end{array}$ & 12.42 & 5.65 & 0.455 \\
\hline $\begin{array}{c}\text { Solenodon paradoxus } \\
\text { (MNHN-AC-14487) }\end{array}$ & 16.93 & 6.10 & 0.360 \\
\hline $\begin{array}{l}\text { Tenrec ecaudatus } \\
\quad(\mathrm{MNHN}-\mathrm{AC}-6997)\end{array}$ & 12.82 & 4.87 & 0.379 \\
\hline $\begin{array}{l}\text { Sciurus igniventris } \\
\text { (Coll RH uncat. specimen) }\end{array}$ & 15.55 & 7.10 & 0.458 \\
\hline $\begin{array}{l}\text { Sciurus carolinensis } \\
\text { (MNHN-AC-2719) }\end{array}$ & 16.34 & 6.87 & 0.420 \\
\hline Sciurus niger (MNHN-AC-2760) & 12.73 & 6.33 & 0.497 \\
\hline $\begin{array}{l}\text { Callosciurus flavimanus } \\
\text { (MNHN-AC-2758) }\end{array}$ & 10.36 & 4.25 & 0.410 \\
\hline $\begin{array}{l}\text { Ailurus fulgens } \\
\quad \text { (MNHN-AC-1870-92) }\end{array}$ & 22.48 & 9.42 & 0.419 \\
\hline Nasua rufa (MNHN-AC-3497) & 28.98 & 14.7 & 0.507 \\
\hline $\begin{array}{l}\text { Metachirus nudicaudatus } \\
\quad \text { (Coll RH uncat. specimen) }\end{array}$ & 16.30 & 10.7 & 0.656 \\
\hline $\begin{array}{l}\text { Dendrohyrax arboreus } \\
\quad(\mathrm{MNHN}-\mathrm{AC}-1961-47)\end{array}$ & 19.96 & 9.27 & 0.464 \\
\hline $\begin{array}{l}\text { Procavia capensis } \\
\quad(\text { MNHN-AC-972-CG 429) }\end{array}$ & 20.18 & 9.23 & 0.457 \\
\hline $\begin{array}{l}\text { Marmota monax } \\
\quad(\text { MNHN-AC-2691) }\end{array}$ & 21.69 & 9.71 & 0.447 \\
\hline
\end{tabular}

development of the flexors of the posterior vertebral column (quadratus lumborum and psoas minor). However, they are clearly more developed than in Solenodon and Tenrec and, because of their relative width, they accommodated a relatively large space for the hypaxial musculature, as noted above, suggesting that these muscles were not so weak in Alcidedorbignya (see hind limb section below). Very agile small- to mediumsized cursorial or curso-saltatorial mammals, such as canids, felids, leporids, and Metachirus, have long transverse processes of the lumbar region, which are strongly inflected anteriorly and ventrally. In contrast, other mammals known to be less agile, such as hystricids, castorids, and ursids, have transverse processes of the lumbar vertebrae that are subhorizontal and subtransverse (they are inflected very slightly anteriorly and ventrally). Therefore, the condition of Alcidedorbignya, intermediate between these two morphotypes, suggests neither an extremely agile animal nor a slow animal. It is noteworthy that the very agile squirrels have processes of the lumbar vertebrae that are not much more developed than in Alcidedorbignya.

The anapophyses are distinctly present from T12 to L6 in Alcidedorbignya but weakly developed. The mammillary processes are hardly present. This feature suggests reduced traction by the epaxial musculature (longissimus dorsi lumborum in particular), which could be related to reduced lateral and rotatory movements and to moderately powerful extension of the lumbar portion of the vertebral column. In Tenrec and Solenodon the anapophyses are even more reduced than in Alcidedorbignya (almost absent in Solenodon). In contrast, the mammillary processes are clearly more developed in the two Recent genera, (especially in Solenodon) and the prezygapophyses of the lumbars are oriented much more dorsally than 
in Alcidedorbignya. This condition suggests stronger action of the longissimus dorsi lumborum and more powerful extensions of the posterior part of the thoracolumbar segment in these genera. Therefore, the morphology of the mammillary processes suggests an opposite interpretation than that based on the neural and transverse processes of Alcidedorbignya, which are larger than in Solenodon and Tenrec.

To conclude, some aspects of the thoracolumbar anatomy of Alcidedorbignya suggest that it was not very agile (absence or very small size of the mammillary processes and orientation of the prezygapophyses of the lumbar vertebrae). However, other features suggest that it was not so sluggish and slow either (neural processes of the anterior thoracolumbar vertebrae, neural and transverse processes of the lumbar vertebrae). In fact, in many aspects of their morphology the thoracolumbar vertebrae of Alcidedorbignya resemble those of Solenodon (except for the lack of mammillary processes) but with more developed neural and transverse processes. Little is published on the locomotion of Solenodon. However, Nowak (1999: 201) mentions that "they can run surprisingly fast, but can easily be caught by people. They seem incapable of jumping..." This indication suggests that Solenodon is a moderately agile animal but not a fast cursor or active saltator. This could probably apply to Alcidedorbignya as far as the thoracolumbar vertebrae are concerned. However, because of the larger size of the neural and transverse processes, the epaxial and hypaxial musculature of the lumbar vertebrae was probably more powerful than in Solenodon. It is therefore possible that Alcidedorbignya was at least as agile as (possibly slightly more agile than) Solenodon. It is noteworthy that the lack (or poor development) of mammillary processes of Alcidedorbignya, which seems to contradict this interpretation, also occurs in the very agile squirrels.

Sacrum. The long sacrum of Alcidedorbignya is indicative of a well-stabilized base for the epaxial musculature whose tendons reach the strongly inclined neural processes of thoracic vertebrae. The elevated and fused neural processes of $S 1$ and $S 2$ also suggest a strong attachment for the epaxial musculature. The length of the sacrum is probably more related to the epaxial musculature than to the length of the tail, considering that Marmota also exhibits a long sacrum with four vertebrae but has a reduced tail. The long and robust sacrum of Marmota denotes its strong epaxial musculature related (at least in part) to its frequent use of an erect posture.

The sacrum of Alcidedorbignya is also very similar to that of Solenodon, which distinctly widens posteriorly. It differs in having four vertebrae, whereas Solenodon has five. The sacrum of Tenrec also has four vertebrae but in this genus because of the extreme reduction of the tail, the sacrum strongly tapers posteriorly.

Caudal vertebrae. The tail of Alcidedorbignya is long and thin. The caudal vertebrae are rod-like and without zygapophyseal articulation from $\mathrm{Ca} 5$. The first caudal is reduced in comparison to the last lumbar and almost of the same size as the fourth sacral (unfused to the more anterior part of the sacrum in MHNC 8372). In Alcidedorbignya the foramen vertebrale and zygapophyseal articulation are lacking after $\mathrm{Ca} 5$; transverse and neural processes are well developed only on Ca1-2. In spite of the length of the tail, the thinness of the last caudals indicates that it was not prehensile. Furthermore, the tail of Alcidedorbignya also lacks several other features present in the prehensile tail of arboreal mammals, such as the large size of the neural canal, the number of caudal vertebrae that exhibit a neural canal (indicative of a complex innervation), and the development of the neural and transverses processes of the anterior caudals. These three characters confirm that the tail of Alcidedorbignya was not prehensile. No comparison is possible with Tenrec, in which the tail is vestigial. In contrast, the tail of Alcidedorbignya is very similar to that of Solenodon (MNHN-AC-14487) but slightly shorter. Fifteen vertebrae are preserved and estimating that three vertebrae may be missing $\left(12^{\text {th }}, 17^{\text {th }}\right.$, and $\left.18^{\text {th }}\right)$, the total count of the tail was probably 18 vertebrae. An estimation of the actual length of the tail of Alcidedorbignya, taking into account the intervertebral disc, could have approached $170 \mathrm{~mm}$. The tail of Solenodon is $229 \mathrm{~mm}$ with 22 vertebrae. In both genera, therefore, the average length of a caudal vertebra approximates $1 \mathrm{~cm}$. One major difference between the tails of the two genera is the shape of the hemal arches, which are small and V-shaped in Alcidedorbignya and strongly expanded at the apex, from Ca2 to Ca13 in Solenodon. This condition is related to a more powerful caudal musculature (essentially the sacrococcygeus ventralis medialis) in Solenodon. Functional comparison is difficult because the biology of Solenodon is poorly known and we have not been able to obtain information on the function of its tail.

Nevertheless, some observations can be made on the relative length of the tail of Alcidedorbignya. In MHNC 8372, it is approximately $70 \%$ of the head and body length $(170 / 241 \mathrm{~mm}$ $=0.705)$, from posterior end of the sacrum to the anterior end of the skull (measurement of head and body length is taken from Fig. 118B). The length of the tail greatly varies amongst mammals. It is generally long to medium-sized in arboreal mammals but several exceptions are observed (e.g., Dendrohyrax, Hylobates, Pongo, Cacajao, Ursus, Bradypus, Phascolarctos, Nycticebus). The tail is generally very short to absent in fossorial mammals. However, the tail of Oryzorictes, a semi-fossorial tenrecid, and that of Orycteropus are longer than in most other fossorial mammals. But, in these cases, the tail approximates $30 \%$ of the rest of the body in the former and $40 \%$ in the latter (Nowak 1999), which is roughly twice less than in Alcidedorbignya. In this context, it is noteworthy that the tail of arboreal sciurids (e.g., Sciurus, Callosciurus, Ratufa) is at least twice longer than that of terrestrial/fossorial ones (e.g., Cynomys, Spermophilus). The length of the tail in terrestrial mammals varies more considerably than in arboreal and fossorial ones. It can be almost totally absent (e.g., Phascolomys, Pan, Mandrillus, Hyaena, Cavia, Kerodon, Dolichotis...) or relatively long (Macropus, dipodids, Lagidium, Rhynchocyon - generally related to jumping) with all size-intermediates. Therefore, the tail of Alcidedorbignya is distinctly longer than that observed in fossorial mammals 
and is compatible with terrestrial and arboreal (or scansorial) habits. As mentioned above, the tail of Alcidedorbignya was not prehensile as commonly observed in arboreal mammals. However, many arboreal or scansorial mammals have a nonprehensil tail, especially carnivores (e.g., Cryptoprocta, Nasua, Paradoxurus, Genetta, Ailurus). In this case, the tail (which approaches the head and body length) acts as a balance organ, a function that is more efficient with a long tail (Dor 1937; Kiley-Worthington 1976; Hickman 1979). Though we hypothesize from this qualitative comparison that the tail of Alcidedorbignya was well compatible with scansorial habits, quantitative comparative analyses considering phylogenetic effects are certainly needed to examine further these links between the tail morphology and the locomotory habits of mammals.

\section{Pectoral girdle and Forelimb}

Scapula. The grossly triangular morphology of the scapula of Alcidedorbignya strongly differs from that of Tenrec, which is proximodistally elongated with sub-parallel anterior and posterior edges and subequal infra- and supraspinatus fossae. The scapula of Solenodon more resembles that of Alcidedorbignya. Both bear a narrow infraspinatus fossa with a laterally deflected posterior edge, and a triangular supraspinatus fossa (but see below). In both genera the spine is very elevated which suggests powerful shoulder musculature. But, as expressed above, the closest similarities are with Mayulestes, a borhyaenoid metatherian with arboreal habits from Tiupampa.. The acromion of Alcidedorbignya is almost identical to that of Mayulestes, although slightly less projecting distally and anteriorly, two features related to arboreality (Muizon 1998). In this respect Alcidedorbignya strongly differs from the strictly terrestrial Tenrec. The acromion of Solenodon is less projected anteriorly than in Alcidedorbignya but projects distally well below the glenoid cavity, a condition present in many arboreal mammals. As a matter of fact, Solenodon is said to be an occasional climber even on vertical surfaces (MacDonald 1995; Nowak 1999).

An interesting comparison can be made with the scapula of Recent didelphids. The family Didelphidae includes a series of genera, which present a gradient of locomotory adaptations from strictly arboreal (Caluromys) to strictly terrestrial (Metachirus). Comparison with these genera was exemplified by Muizon (1998) to evaluate the arboreal abilities of Mayulestes. In Caluromys several characteristics of the scapula have been related to arboreality: 1) the posteroproximal extension of the caudal angle of the scapula, which is the most proximal point of the bone (when the spine is vertical); 2) the large size of the acromion; 3 ) the anterior and distal projection of the acromion (especially the hamatus process), which hides, in lateral view, the glenoid cavity and the supraglenoid tubercle; 4) the triangular morphology of the supraspinatus fossa with the most anterior point of the fossa being approximately at mid-height of the spine or more proximal; 5) the elevation of the spine, which denotes a greater muscular mass of the spinati muscles than in a scapula with a lower spine; and 6) the posterior edge of the infraspinatus fossa strongly deflected laterally (Muizon 1998). This list is obviously not exhaustive but the conjunction of those features is always related to arboreal (or scansorial) habits in Recent mammals (rodents, carnivores, primates, marsupials, etc). Of course the development of these characters is subject to variation and not all of them are always fully developed in all arboreal mammals. Furthermore, some of these features may also be related to other functions such as digging (it is noteworthy that several of these features are also present in Marmota but see p. 578 for a discussion on possible fossorial habits in Alcidedorbignya).

These features are absent to weakly developed in Metachirus, a strictly terrestrial didelphid. The postero-proximal angle is not extended posteroproximally, the acromion is much smaller than in Caluromys, the acromion does not project anteriorly and distally, the supraspinatus fossa is not really triangular and its more anterior point is well below mid-height of the spine, the spine is much lower than in Caluromys, and the posterior edge of the infraspinatus fossa is not deflected laterally. A Caluromys-like scapula is present in Mayulestes; this condition is less emphasized in Andinodelphys and even less in Pucadelphys (two didelphimorphs from Tiupampa). These observations, among others, led Muizon (1998) and Muizon \& Argot (2003) to the conclusion that Mayulestes was mainly arboreal, Andinodelphys was intermediate and Pucadelphys was mainly terrestrial (although clearly capable of climbing).

In Alcidedorbignya, when compared to Metachirus and Caluromys, the most elevated (proximal) point of the scapula is either above the spine (MHNC 8372) or at mid-length of the dorsal edge of the infraspinatus fossa (MHNC 8431, 8432). It is noteworthy that the condition of MHNC 8372 may be related to the fact that the epiphysis of the posterior angle of the scapula is missing because the specimen is that of a not fully ossified young adult (see description). The acromion of Alcidedorbignya is larger than in Metachirus for a scapula that is approximately $50 \%$ smaller in surface area of the blade. The acromion is also clearly shifted anteriorly and extends distally (although to a lesser extent than in Caluromys). This development of the acromion is in relation to the presence of well-developed clavicles. The supraspinatus fossa is distinctly triangular and the anteriormost point of the triangle is roughly at mid-height of the spine, as in Caluromys but differing from Metachirus in which this point is distal to mid-height of the spine. The spine is as elevated as in Caluromys and much more so than in Metachirus. The posterior edge of the infraspinatus fossa is strongly deflected laterally as in Caluromys but in contrast to the condition in Metachirus. It is therefore clear that the scapula of Alcidedorbignya is more reminiscent of that of Caluromys than that of Metachirus even if some features are less pronounced than in Caluromys. The only feature that does not closely resemble Caluromys and somewhat recalls Metachirus is the morphology of the caudal angle of MHNC 8372, which is not strongly extended postero-proximally. However, this statement is true for MHNC 8372 only but not for the other two specimens referred to Alcidedorbignya (MHNC 8431 and 8432), which are intermediate between Caluromys and Metachirus (Figs 70A, 72A). It is also noteworthy that in some specimens of Caluromys the most elevated point of the 
scapula is located at mid-length between the proximalmost point of the spine and the caudal angle of the bone (similar to the condition observed in MHNC 8431). The generally triangular morphology of the scapula of Alcidedorbignya and the morphology of the spine are also indicative of good capacity of rotation of the scapula and anterior projection of the shoulder as shown by Jenkins \& Weijs (1979), Larson (1993) and Muizon (1998: fig. 51). Such a protraction of the shoulder involves rotator muscles (anterior and posterior portion of the trapezius, serratus ventralis thoracis, and atlantoacromialis) and is frequently used by arboreal mammals.

Furthermore, a possibility of abduction exists based on the development of the supraspinatus, the deltoideus pars acromialis as reflected by the acromion, and probably the deltoideus pars spinalis, as indicated by the posterior inclination of the scapular spine. Moreover, some abduction of the humerus at rest is also suggested by the rotation of the glenoid cavity relative to the supraspinous plane of the scapula (Figs 70D; 72D).

All these features of the scapula of Alcidedorbignya indicate some arboreality, some ability to climb or some active use of the forelimb like prehension. Because of the absence of claws (the almost exclusive organ employed for digging), fossorial habits are unlikely, although forearm adaptations of semifossorial mammals (e.g., Cynomys, Marmota) are very similar to those of arboreal or scansorial mammals (e.g., Caluromys, Mayulestes, Sciurus, Ratufa). As a matter of fact the scapulae of the arboreal Sciurus and Ratufa closely resemble those of the fossorial Cynomys and Marmota (but see p. 578 for a discussion on possible fossorial habits in Alcidedorbignya).

The scapula of Alcidedorbignya is clearly more similar to those of the arboreal Caluromys and Sciurus than to that of the terrestrial Metachirus and Tenrec. It is also very similar to that of Ailurus in its general outline and in specific characters such as the anterior and distal projection of the acromion.

The scapula of Solenodon differs from that of Alcidedorbignya in that the acromion is not placed anteriorly, and in the anteriormost point of the supraspinatus fossa, which is clearly proximal to mid-height of the spine. The scapulae of the two genera resemble each other in the posteroproximal angle of the scapula, which is intermediate between those of Caluromys and Metachirus, in the elevation of the spine, and in the laterally deflected posterior edge of the infraspinatus fossa.

The scapula of Alcidedorbignya also differs from those of the archaic ungulates Hyopsodus and Meniscotherium. The spine and acromion of these genera are clearly placed posteriorly, the acromion does not extend distally and anteriorly, and the caudal angle of the bone does not feature any posteroproximal extension. Furthermore, in these genera the spine is strongly inclined posteriorly, especially in Hyopsodus, which appears to be very specialized in this respect. Therefore, although the spine is relatively elevated in both genera (Gazin 1965, 1968), given the morphology of their scapula, they do not seem to have arboreal affinities.

Humerus. The humerus of Alcidedorbignya is similar in size to that of Tenrec but slightly smaller than that of Solenodon although the thoracolumbar segment of the vertebral column (taken as a proxy for body size) is slightly longer. It is noteworthy that the humeri of MHNC 8372 are those of a small individual and that they have been slightly distorted. Therefore, the isolated humeri referred to Alcidedorbignya, even if incomplete, are probably more reliable. On MHNC 8438, an isolated proximal epiphysis, the head is sub-hemispherical as in Caluromys, Mayulestes, and Sciurus, although slightly less convex than in these genera. It is not transversely compressed as in Metachirus. Transverse compression is present in Solenodon and Tenrec but to a much lesser extent than in Metachirus. Furthermore, in proximal view, the posterior border of the epiphysis is regularly convex from the lateral to the medial edge and semicircular, a condition observed in Caluromys, Mayulestes, and Sciurus. In the terrestrial Metachirus the posterior edge of the proximal epiphysis is clearly compressed transversely and is parabolic (almost v-shaped). This condition is also present in Solenodon and Tenrec but less pronounced than in Metachirus; however, it is by no mean semicircular. Such a condition (as observed in Metachirus) is present in Hyopsodus.

The humeral tubercles of Alcidedorbignya are well developed, on MHNC 8372, especially the greater tubercle, which is anteroposteriorly elongated and crestlike. On the undistorted epiphysis MHNC 8438 it is slightly lower than the head (fig. 78 demonstrates that the condition of MHNC 8372 is the result of post-mortem deformation), although to a lesser extent than in Sciurus. It is distinctly lower than in Tenrec (in which it projects above the head) and resembles Solenodon in this respect. It strongly resembles that of Mayulestes in its length and elevation. It differs from those of Hyopsodus and Meniscotherium, in which the greater tubercle is distinctly higher than the head (especially in Meniscotherium). The sharp, narrow, and prominent deltopectoral crest of Alcidedorbignya resembles that of Caluromys, and Sciurus, but strongly differs from those of Tenrec, Solenodon and Hyopsodus. It also differs from the robust and transversely thick deltopectoral crests of Marmota and Cynomys.

Therefore the proximal end of the humerus of Alcidedorbignya indicates a good mobility of the shoulder, although to a lesser extent than in arboreal taxa as Caluromys and Sciurus because of its less convex head and larger and slightly higher greater tubercle. Furthermore, the posterior concavity of the humeral neck and the posterior protrusion of the humeral head, are distinct from arboreal taxa like Caluromys, in which the humeral head protrudes little posteriorly beyond the humeral diaphysis. However, this condition in Alcidedorbignya is similar to that observed in the arboreal borhyaenoid, Mayulestes (Muizon 1998; Muizon \& Argot 2003). It is noteworthy that in the highly arboreal hyracoid Dendrohyrax the greater tubercle is at least twice as high as in Alcidedorbignya and the head overhangs the diaphysis posteriorly to a greater extent than in Alcidedorbignya.

On the medial face of the proximal region of the diaphysis of Alcidedorbignya, the well-developed tuberosity for the teres major (a very sharp and salient crest on MHNC 8433, 8434, 8435) (Fig. 78) suggests strength in the adduction and internal rotations of the arm. The teres major being also a flexor of 
the shoulder its inferred strength in Alcidedorbignya would also favor powerful flexion of the shoulder. A well-developed teres tuberosity is also present in Tenrec and Solenodon, but clearly not to the extent observed in Alcidedorbignya. A crestlike teres tuberosity is also often present in Caluromys, which also denotes a powerful teres major. A stout and large crestlike teres tuberosity is also present, among others, in arboreal sciurids (Sciurus, Ratufa), in adapiforms (Adapis, Notharctus), in Plesiadapis (especially developed in this taxon) and in semifossorial sciurids (Marmota and Cynomys).

The deltopectoral crest of Alcidedorbignya is short, as it remains on the proximal half of the diaphysis, and is prominent anteriorly, especially distally where a salient distal deltoid tuberosity forms the most anterior point of the humerus in mediolateral view. It is even more prominent in Pantolambda (see Matthew 1937: pl. 45), suggesting in this taxon (as well as in Alcidedorbignya) strong traction of the deltoid (pars spinalis), probably in relation to grasping (or possibly digging, but see discussion p.000) with a good mobility of the shoulder (low greater tuberosity and spherical head). Because of the lack of claws in these two genera (see Matthew 1937: 46) fossorial habits are unlikely (see description and discussion p. 578). In Solenodon the deltopectoral area is more developed and the crest is longer than in Alcidedorbignya. In the case of Solenodon, which is a good digger (Nowak 1999), this morphology could be related to fossorial habits because this genus bears robust claws. A short but strong deltopectoral crest with a protruding deltoid tuberosity is also present in Caluromys (arboreal) as well as in Marmota (fossorial with strong claws). A short but relatively weak deltopectoral crest is present in Sciurus and Dendrohyrax (both arboreal). If the deltopectoral crest of Alcidedorbignya is more robust than in the latter two genera, it is clearly thinner transversely and sharper than in the semi-fossorial Marmota and Cynomys and is not significantly different in this respect from that of Caluromys. The deltopectoral crest of Alcidedorbignya greatly resembles, in its moderate massiveness and thickness, the condition observed in Paramys, which has been regarded by (Rose \& Chinnery 2004) as equally adapted to terrestrial and arboreal locomotion (although it extends more distally than in Paramys). Therefore, the development of the deltopectoral crest appears to be related to several functions and its development cannot be retained as a decisive (positive) argument for one given locomotory habit but rather as a "non-negative" argument. In the case of Alcidedorbignya, the deltopectoral crest seems less compatible with fossorial taxa but more reflects terrestrial and/or scansorial habits.

On the distal extremity of the diaphysis, the radial and olecranon fossae are not very deep but are more developed than in Caluromys. They resemble in this respect the condition observed in Mayulestes and in Sciurus. This condition of Alcidedorbignya, as well as the widely open trochlear notch on the ulna (see below), indicates a poorly or moderately stabilized elbow joint.

On the distal epiphysis of the humerus of Alcidedorbignya, the trochlea is markedly asymmetrical. It is deep with salient and well-developed crests, especially the medial, which protrudes distally. This condition indicates a forelimb usually slightly abducted, a position that requires an elevated medial crest in order to preserve the stability of the articulation. It resembles the morphology of Solenodon but differs from the condition of Mayulestes and Caluromys, in which the crest are almost absent. However, in Sciurus and Ratufa the medial trochlear crest, is less developed than in Alcidedorbignya but as developed as in the fossorial Cynomys and Marmota. In didelphids the trochlear crest of terrestrial (Metachirus) and scansorial (Didelphis, Lutreolina) forms are clearly more developed than in the strictly arboreal Caluromys. Therefore, it seems that the well-developed trochlear crests of Alcidedorbignya are more indicative of terrestrial habits than arboreal of fossorial. It is noteworthy, however, that the asymmetry of the humeral trochlea of Alcidedorbignya and the development of its crests strongly resembles the condition observed in the Eocene paramyids, which have been regarded as equally terrestrial and arboreal (Rose \& Chinnery 2004). An elevated and distally projecting trochlear crest is also present in the scansorial Nasua (Gebo \& Rose 1993). Therefore, if elevated trochlear crests are more typical of terrestrial mammals they are not incompatible with climbing abilities.

One major feature of the distal epiphysis of the humerus of Alcidedorbignya is the size of the entepicondyle. It is large, massive, proximodistally elongate, and strongly protrudes medially. It resembles those of Tenrec, Solenodon, and Marmota but also those of Caluromys, Sciurus, and Paramys. It also resembles that of Mayulestes but in this genus the entepicondyle projects distomedially. On the entepicondyle is the origin of the flexor muscles of the manus and digits and these muscles were, therefore, well-developed in Alcidedorbignya. A robust entepicondyle (and therefore powerful flexors of the hand and forefingers) is common in arboreal Recent mammals. It is also frequently observed in fossorial mammals with robust claws (e.g., Dasypus, Marmota). A strong medial epicondyle is also present in Hyopsodus and Meniscotherium, which do not exhibit decisive features indicating arboreality. The lateral epicondylar crest of Alcidedorbignya is well developed and sharp, as in Solenodon Caluromys, Mayulestes, and Sciurus. It differs from the small and rounded crest observed in Tenrec. This crest provides origin for the extensors of the manus and digits, as well as the supinator of the forearm, which were also powerful in Alcidedorbignya. Therefore, the morphology of the distal extremity of the humerus of Alcidedorbignya is consistent with powerful extension and especially flexion, of the manus and digits, which could be related to some kind of grasping (digging is unlikely because of the absence of claws; see p. 578 comments on the digging abilities).

Furthermore, on the posterior face of the entepicondyle, the dorsoepitrochlear fossa is remarkably deep and large. This fossa received the origin of the medial ulnar collateral ligament, which is inferred to have been very strong in Alcidedorbignya. This condition indicates a well-stabilized articulation related to strong tension exerted on the elbow. A well-developped dorsoepitrochlear fossa is present in many arboreal and scansorial mammals (e.g., most new world monkeys, most adapoids, all Fayoum anthropoids, arboreal sciurids, arboreal carnivores 

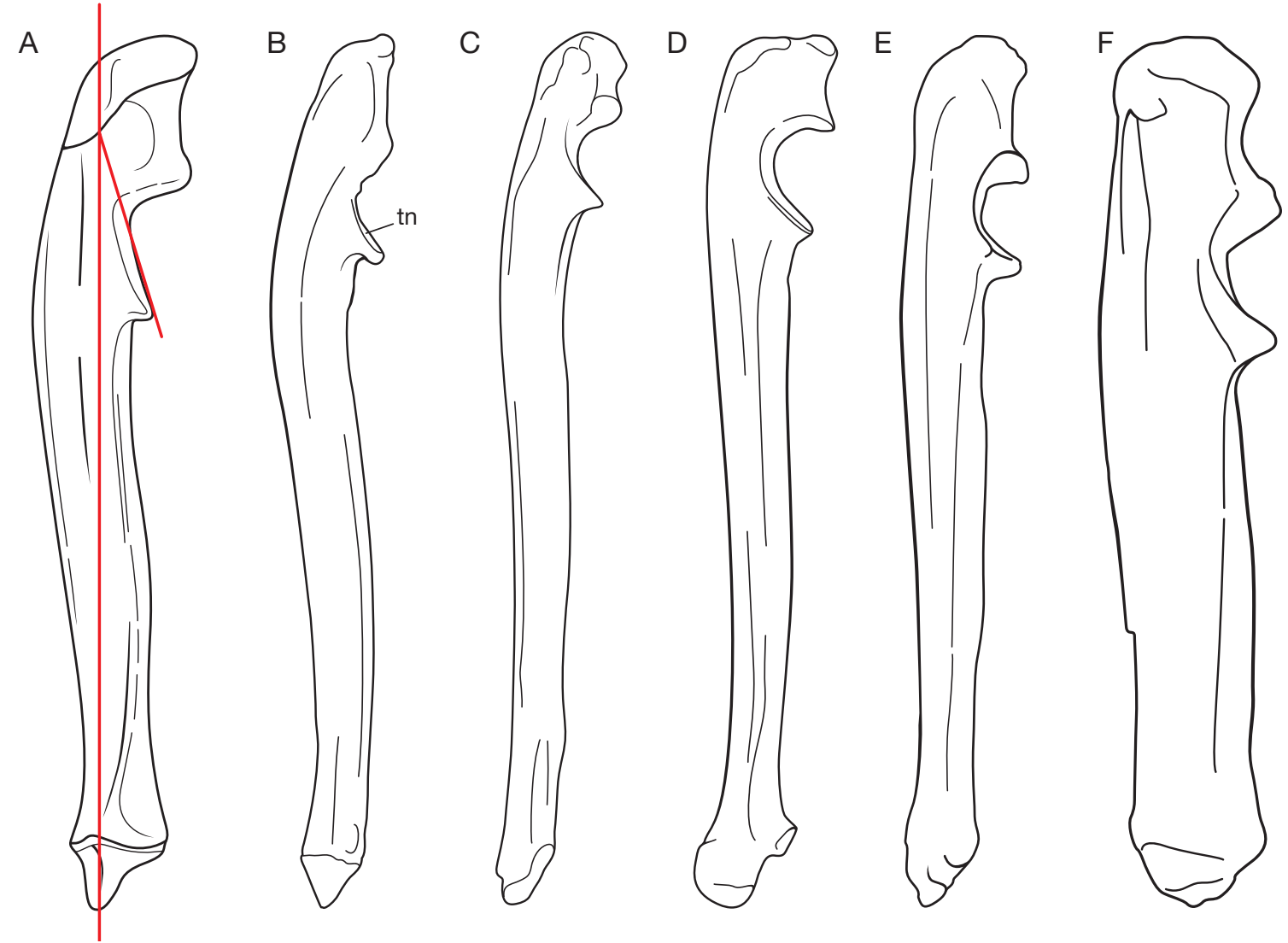

FIG. 114. - Medial view of the left ulna in selected fossil and extant mammals showing the distal orientation of the coronoid process. The red lines materialise the angle between the plane of the humeral articular surface of the process and the proximodistal axis of the bone: A, Alcidedorbignya inopinata (MHNC 8372, reversed), angle $=$ C. $20^{\circ} ; \mathbf{B}$, Mayulestes ferox (MHNC 1249), angle $=25^{\circ} ; \mathbf{C}$, Sciurus igniventris (coll RH uncatalogued), angle $=40^{\circ} ; \mathbf{D}$, Martes foina (uncatalogued), angle $=50^{\circ}$; E, Marmota, marmota, (MNHN-AC-1996-2438), angle $=60^{\circ} ; \mathbf{F}$, Pantolambda bathmodon (AMNH 16663), angle $=55^{\circ}$. Abbreviations: rdn, radial notch; trn, trochlear notch. Not to scale.

(Conroy 1976; Seiffert et al. 2000; personal observations). The dorsoepitrochlear fossa is generally less developed in fully terrestrial or fossorial mammals.

Ulna. On the ulna of Alcidedorbignya, the olecranon is distinctly shorter than in Tenrec and Solenodon, a condition that indicates faster but less powerful extension of the forearm, because of the smaller lever arm. It is distinctly bent anteriorly and medially, which suggests a powerful triceps brachii, especially the caput longum (Muizon \& Argot 2003: fig. 6). On the medial side of the bone the trochlear notch is wideopen and the coronoid process distinctly extends proximally (the apex of the process is displaced proximally), rather than protrudes anteriorly. In other words, the part of the humeral facet on the coronoid process faces anteroproximally (with an anterior component greater than the proximal one) rather than almost totally proximally. In lateral view, the angle between the plane of the humeral facet of the coronoid process and the length of the ulna allows quantification of the opening to the trochlear notch (Fig. 114). This angle is c. $20^{\circ}$ in Alcidedorbignya, $25^{\circ}$ in Mayulestes, $26^{\circ}$ in Paramys copei (measurement taken from Figure 3C in Rose \& Chinnery 2004), $30^{\circ}$ in Sciurus and Ratufa, $40^{\circ}$ in Cynomys, $55^{\circ}$ in Pantolambda and $60^{\circ}$ in Marmota. This proximal extension of the coronoid process and the opening of the trochlear notch are more pro- nounced in Alcidedorbignya than in the other taxa mentioned above and clearly more resemble the arboreal and scansorial taxa than the fossorial ones. They reflect a greater mobility of the humeroulnar joint. Interestingly, the angle between the distal edges of the radial and trochlear notches is wide open (distinctly obtuse) in Alcidedorbignya as it is in Mayulestes, Sciurus, Ratufa, and apparently (from Rose \& Chinnery 2004: fig. 3C) in Paramys. It is either a right angle or generally much less in terrestrial mammals, whether generalised, fossorial or cursorial (Fig. 115).

On the ulna, four features have been regarded as related to arboreality by Muizon (1998) and Muizon \& Argot (2003). They are: 1) the anterior bending of the bone in lateral or medial view; 2) the broadly open trochlear notch in medial view; 3) the obtuse angle between the distal edges of the radial and trochlear notches in distal view; in other words the medial contact between the radial and humeral facet is a low ridge rather than a sharp crest; and 4) the medial bending of the proximal end of the bone in anterior view with a deep flexor fossa on the medial side of the olecranon and which may extend distally on the diaphysis. These four features are present, for example, in Caluromys, Mayulestes, and Paramys copei. In the terrestrial Metachirus, the ulna is almost straight in lateral view, the trochlear notch is very constricted, the angle between the humeral and radial facets is approximately $90^{\circ}$, 

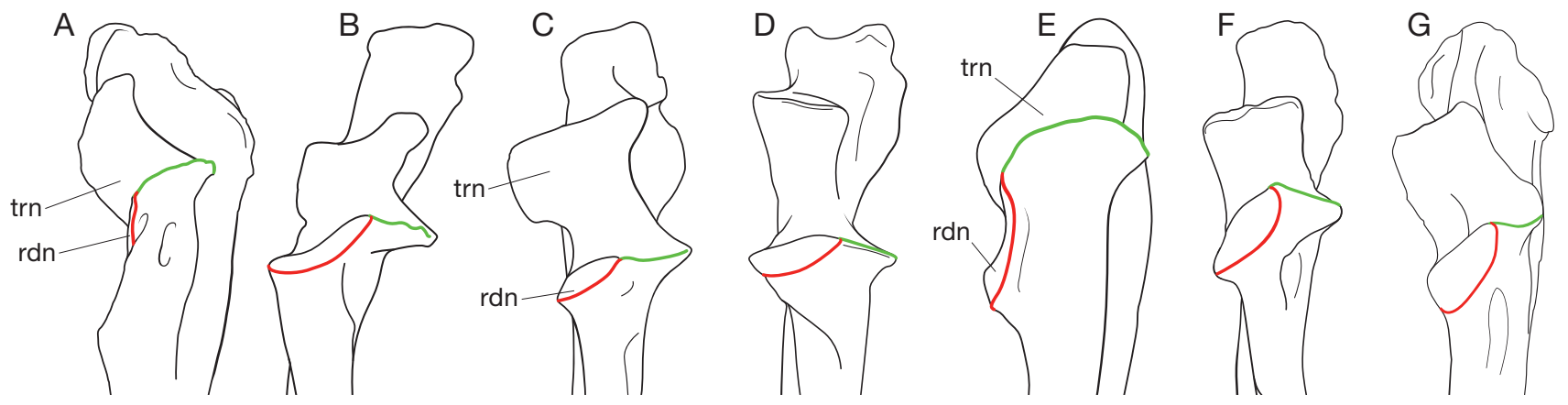

FIG. 115. - Anterodistal view of the right ulna in selected fossil and extant mammals showing the angle between the proximal edges of the radial and trochlear notches. A, Alcidedorbignya inopinata (MHNC 8372); B, Mayulestes ferox (MHNC 1249 reversed); C, Sciurus igniventris (coll RH uncatalogued); D, Martes foina (uncatalogued); E, Dendrolagus sp (uncatalogued); F, Marmota, marmota, (MNHN-AC-1996-2438) G, Pantolambda bathmodon (AMNH 16663 reversed). Abbreviations: rdn, radial notch; trn, trochlear notch. Colors: red, anterior edge of the radial notch; green, anterior edge of the trochlear notch. Not to scale.

the bone is almost straight in anterior view, and the flexor fossa is short and not very deep. In Alcidedorbignya the ulna in bent anteriorly and medially, the flexor fossa is very deep, the trochlear notch is widely open, and the angle between the radial and humeral facets is markedly obtuse with no elevated crest between the facets. However, the anterior bending of the ulna is much less pronounced than in highly arboreal taxa. The condition of Alcidedorbignya is also somewhat present in Solenodon, in which the anterior and medial bending of the ulna are present but weak and the trochlear notch is relatively open, although less than in Alcidedorbignya. However, in Solenodon the two radial and humeral facets are separated medially by a sharp crest and the angle between the facets in distal view is approximately $90^{\circ}$. The condition in Tenrec is that of a strictly terrestrial mammal. In the archaic ungulate Hyopsodus the four arboreal features are apparently present. In Paramys the four features are apparently present but the medial bending of the bone is weak.

In Alcidedorbignya, because of the wide-open trochlear notch, the elbow joint was probably not well stabilized, a condition that was probably compensated by the depth of the humeral trochlea and the elevation of its medial crest and the strength of the medial ulnar collateral ligament. In Hyopsodus the condition is almost identical, with an elevated medial crest of the deep trochlea. However, in this genus the articulation was probably better stabilized because of the greater mediolateral convexity of the capitulum and the perforated olecranon fossa (Gazin 1968). Furthermore, the posterior border of the ulnar diaphysis and the proximal border of the olecranon form an obtuse angle in Alcidedorbignya. This condition can be observed in mammals with more flexed than erected postures at rest, as is observed in some arboreal mammals such as sciurids and some tupaiids. It is present in Mayulestes, Sciurus, Ratufa, Marmota, and Solenodon but it is not conspicuous in Caluromys and variably present in Dendrohyrax.

The distal extremity of the ulna of Alcidedorbignya is relatively straight, thus differing from the more concave (posteriorly) morphology observed in Solenodon, Hyopsodus, and (to a lesser extent) Tenrec. In fact the ulna of Hyopsodus is more sigmoid in lateral view than in Alcidedorbignya, in relation to the strong posterior convexity at the level of the radioulnar articulation.
An interosseous space is clearly present in Alcidedorbignya, as in Tenrec, differing from the condition in Solenodon and Hyopsodus, in which the two bones are almost in contact all along their length. Furthermore, in Alcidedorbignya, the sharp interosseous crest and the prominent distal crest for the pronator quadratus suggest that the ulna and radius were better stabilized distally than proximally.

Radius. On the proximal end of the radius, the humeral facet is distinctly oval-shaped as is the capitulum on the humerus. This condition precludes a significant range of pronation-supination movements, although some rotation was certainly possible. A similar condition has been observed in the arboreal borhyaenoid Mayulestes. In the arboreal hyrax Dendrohyrax the proximal articular facet of the radius features two fossae (one in Alcidedorbignya and Mayulestes) and the radius-ulna articulation is distinctly ankylosed (reminiscent of its terrestrial ancestry), which demonstrates a total absence of pronation-supination movements [It is noteworthy however that this ankylose of the radioulnar articulation is compensated for by some carpal pronationsupination ability (Fischer 1986)]. The bicipital tuberosity of Alcidedorbignya is relatively weak, as in Tenrec and Solenodon, and this feature, together with the massiveness of the olecranon, suggests a triceps that was better developed, and exerted more powerful traction, than the biceps brachii. The curvature of the radius and the robustness of the distal epiphysis are comparable in the three taxa.

The long bones of the forelimb in Alcidedorbignya are slightly shorter than in Tenrec and much shorter than in Solenodon, while the thoracolumbar segment is approximately $10 \%$ longer. The ulna of Alcidedorbignya is slightly longer than the humerus, but its forearm is shorter than in Tenrec and especially Solenodon, relative to the rest of the limb and especially to the length of the manus (similar in the three taxa). This shorter forelimb suggests a body (at rest) more crouched anteriorly in Alcidedorbignya. In Dendrohyrax the forearm is relatively even shorter, as the ulna is shorter than the humerus. As a matter of fact, hyracoids have a distinctly crouched posture at rest that could approach that of Alcidedorbignya (Fischer 1994). 
Manus. On the manus of Alcidedorbignya, some gliding movements were possible between the trapezium and scaphoid, and between the trapezium and Mc I although the dorsoventrally concave distal facet of the trapezium suggests more flexionextension than abduction-adduction. Mc I is slender and short: when articulated, the extremity of the pollex does not reach the level of the distal extremity of the first phalanx of the second digit. Mc I is clearly much smaller than $\mathrm{McV}$, contrary to the condition observed in Pantolambda, in which the two metacarpals are approximately of the same size. This reduction of Mc I, as well as the anteroposteriorly oriented trapezium-McI articulation, would indicate a non-abductable pollex and, a priori, does not favor pronounced grasping capacities. This condition departs from that observed in some arboreal primates or marsupials. However, grasping ability is not strictly dependent on abduction of the pollex. The highly arboreal woolly monkey, Lagothrix has a reduced Mc I and digit I and Dendrohyrax, in which digit I is reduced to a vestigial McI, is highly arboreal and grasps small branches with no difficulty.

As a whole the metacarpals of Alcidedorbignya are relatively short but approach the length observed in Solenodon, Erinaceus, and Tenrec. As noted in the description they are proportionally slightly longer and more slender than in Pantolambda. The digits of Alcidedorbignya are not very long in relation to the palm of the hand, in contrast to what is observed in some arboreal taxa. However, they have proportions close (but slightly longer and more slender) to those observed in Dendrohyrax. The proximal phalanges of Alcidedorbignya do not exhibit the two salient crest-like tuberosities on the distal end of the diaphysis (for attachment of the middle annular ligament), whose presence is observed in extant taxa with powerful traction by the flexor digitorum profundus, such as Caluromys, Sciurus, and Potos. The small crests observed in Alcidedorbignya clearly differ from the salient tubercles observed, for instance, in Sciurus. This condition does not indicate powerful flexors of the digits and could argue against efficient grasping abilities in Alcidedorbignya. However, crestlike tuberosities for the medial annular ligament are also absent in Dendrohyrax. In spite of this absence and of its metacarpals and digits proportionally shorter and stouter than in Alcidedorbignya, Dendrohyrax has enough grasping ability to be a very agile arboreal mammal (see discussion below). It is noteworthy that developed tuberosities for the medial annular ligament are not strictly related to grasping but can be related to digging or simply to the active use of claws. Furthermore, in Alcidedorbignya the presence of a very strong ventral tubercle (which forms almost half of the length of the phalanx) on the proximal extremity of the ungual phalanges clearly indicates powerful flexors. This tubercle is even more developed than in Solenodon, which is an active digger (but not regarded as fossorial) with powerful claws and approaches the relative size of the fossorial Dasypus. This does not mean that Alcidedorbignya was fossorial (which is unlikely here because of the lack of claws, see p. 578) but it confirms the strength of the flexors of the digits observed on the humerus and the ulna and favors good grasping abilities.
In Tenrec and Solenodon the hands have five well-developed clawed digits. The digits II-III-IV are subequal in length (Mc III being the longest Mc as in Alcidedorbignya). The pollex is not opposable. The metacarpal and phalangeal length is comparable (in absolute size) in Alcidedorbignya and Tenrec, which means that Alcidedorbignya had a relatively longer hand in comparison to the forelimb length than in Tenrec. The length of the metacarpals and phalanges of Alcidedorbignya is clearly shorter than in Solenodon. The difference between the pollex and fifth digit is less emphasized than in the pes, and these two digits are comparable to those of Tenrec and Solenodon. The most obvious difference concerns the shape of the ungual phalanges, sharp and narrow for bearing claws in Tenrec and Solenodon, transversely enlarged for large and robust nails (not true hooves) in Alcidedorbignya. The relative size of the metacarpals of Alcidedorbignya appears to be similar to those of Hyopsodus and Meniscotherium. The ungual phalanges of Alcidedorbignya resemble those of Meniscotherium, which are transversely enlarged to bear small nail-like hooves (Williamson \& Lucas 1992). However, the ungual phalanges of Alcidedorbignya are curved palmarly and differ from those of Meniscotherium, which are flat in lateral view. In Hyopso$d u s$ the ungual phalanges of the forefoot are sharp, palmarly recurved, and transversely compressed and probably bore claws (Gazin 1968: pl. 10, fig. 12). The ungual phalanges of Dendrohyrax are much shorter than those of Alcidedorbignya but are splayed at their apex, and the ligament of the flexor muscles does not insert on a tubercle, as in Alcidedorbignya, but in a deep fossa, which indicates the strength of the flexor muscles. As mentioned above, the ungual phalanges of $\mathrm{Al}$ cidedorbignya also resemble those of adapoid primates in the splayed and scutiform morphology of their distal extremity, which has been regarded as related to nail-bearing condition (Fanzen et al. 2009; Koenigswald et al. 2011). They differ from the uncleft ungual phalanges of adapoids in being distinctly cleft. The palmar curvature observed in Alcidedorbignya is also present in some adapoid primates (Koenigswald et al. 2012)

Conclusion on the forelimb. Alcidedorbignya differs from Tenrec and Solenodon mostly in the length and general outline of the scapula, in the relative length of the forelimb long bones and of the clavicles (longer in Tenrec). However, the most striking characteristic of Alcidedorbignya is the conjunction of several features of the forelimb that are present in arboreal mammals and which could, therefore, be related to some climbing ability, in other words to scansoriality. On the scapula these features are mainly the posterodorsal extension of the posteroproximal angle of the bone, the elevation of the spine, the anteriormost point of the bone located at mid height (proximodistally) of the spine and especially the anterior shift and distal extension of the acromion. On the humerus the hemispherical morphology of the head, the large and deep dorsoepitrochlear fossa (strong ulnar collateral ligament, which stabilizes the elbow), and the medially salient robust entepicondyle could plausibly be related to climbing abilities. On the ulna the anterior and medial bending of the proximal part of the bone and the wide-open trochlear notch, 
are features generally present in arboreal mammals. The obtuse angle between the medial contact of the humeral and radial facets is observed only in arboreal or scansorial mammals.

However, some features of Alcidedorbignya are not obviously congruent with arboreality (scansoriality). They are: The relative shortness of the limb, the relatively developed greater tubercle (although not higher than the head), the relatively short deltopectoral crest, the oval-shaped capitulum of the humerus and corresponding proximal articular facet of the radius, the reduction of $\mathrm{McI}$, the non-abductable digit $\mathrm{I}$, the short digits, the lack of developed crest-like tuberosity for the medial annular ligament on the first phalanges. In this respect an interesting comparison can be made with the tree hyrax Dendrohyrax as compared with the rock hyrax Procavia. Of course, the anatomy of hyraxes is extremely derived when compared to that of Alcidedorbignya mainly because of their terrestrial ancestry. Among others, the morphology of the elbow markedly differs from Alcidedorbignya in having an ankylosed forearm, the radius being closely appressed against the ulna but not fused to it. Therefore, the characters mentioned above on the trochlear notch and the angle between the radial and humeral facets are not applicable in this case. However, some other features should be considered. The humeral head of Dendrohyrax is much more globose, convex, and hemispherical than in Procavia, in which it is slightly compressed transversely. The olecranon of the ulna is well developed and long in Procavia and in Dendrohyrax the olecranon is hypertrophied. It presents an enormous medial process, which accommodates a deep medial fossa for the flexor muscles of the hand and fingers (e.g., flexor carpi ulnaris, flexor digitorum communis). Furthermore, on the humerus, the medial epicondyle, which receives the humeral heads of the flexor muscles attached on the medial face of the olecranon, is clearly more developed in Dendrohyrax than in Procavia. We have mentioned above that the large size of the medial crest of the humeral trochlea in Alcidedorbignya could be related to a slightly abducted position of the forelimb. Interestingly the humeral trochlea of Dendrohyrax is markedly asymmetrical in contrast to that of Procavia, which is symmetrical; the medial crest of the trochlea of Dendrobyrax is much more elevated than the lateral in contrast to the condition in Procavia, where the two crests are more or less of the same size. It is noteworthy that, contrary to many arboreal mammals (e.g., marsupials, carnivores, rodents) and contrary to Solenodon, Alcidedorbignya has no claws, which obviously are of significant advantage in climbing, but had small hooves (or nail-like hooves). Hyraxes, have small hooves and short fingers and lack a thumb. Because this condition does not prevent Dendrohyrax from being extremely efficient climber (see video at: http://www.arkive.org/southern-treehyrax/dendrohyrax-arboreus/video-00.html) it is reasonable to hypothesize the same for Alcidedorbignya. The same reasoning could be applied to the size of the greater tubercle of the humerus, which is extremely developed in Dendrohyrax and which is also prominent in Alcidedorbignya (although clearly smaller than in Dendrohyrax and slightly lower than the head). A greater tubercle distinctly lower than the head of the humerus is generally present in arboreal taxa. However, in Dendrohyrax the greater tubercle is high and large (which is apparently contradictory with arboreality) but the head is salient and hemispherical, a condition present in arboreal taxa. Furthermore neither Dendrohyrax nor Alcidedorbignya have long fingers (which provides good grasping abilities extremely useful in climbing) as observed in most arboreal primates. However, again, the short fingers of Dendrohyrax do not prevent it from being an excellent climber, a condition that could also apply to Alcidedorbignya.

Therefore, the hemispherical humeral head of Alcidedorbignya, combined with the large olecranon, the deep medial (flexor) fossa, the robust and medially salient entepicondyle of the humerus, the large and deep dorsoepitrochlear fossa, and the elevated medial crest of the humeral trochlea, could indicate (along with the other features of the elbow and of the scapula mentioned above) some climbing abilities for Alcidedorbignya (see below discussion on the hind limb).

Several features of the forelimb of Alcidedorbignya, interpreted here as related to some climbing abilities could be related to digging habits. However, the hemispherical humeral head, the extremely open trochlear notch and the distinctly obtuse angle between the trochlear and radial notches are never present in fossorial mammals. Furthermore, Alcidedorbignya had no claws but nail-like hooves, which were probably inappropriate for digging. Therefore, because no clawless fossorial mammal exists so far, this character of Alcidedorbignya is regarded here as likely to preclude fossorial habits for this taxon. Finally, it is noteworthy that, although the morphology of the forelimb of Alcidedorbignya as exposed and discussed above reveals some climbing abilty, it is also perfectly compatible with a moderately agile terrestrial locomotion, which was probably frequent (see discussion below) in this taxon.

\section{Pelvic girdle and hind limb}

Innominate. One characteristic of the innominate of Alcidedorbignya is the relative length of the ilium and ischium (32.1 vs $26.8 \mathrm{~mm}$; ratio = 0.83). Although the ilium of Alcidedorbignya is slightly longer than the ischium, the difference is much smaller than that observed in Tenrec (30.6 vs $16.4 \mathrm{~mm}$; ratio $=0.53$ ), Solenodon (39.3 vs $22.5 \mathrm{~mm}$; ratio $=0.57$ ), Procavia (56.6 vs $30.1 \mathrm{~mm}$; ratio $=0.53)$, and Dendrohyrax (60.7 vs $32.4 \mathrm{~mm}$; ratio $=0.53)$. Therefore, the ilium of Alcidedorbignya is relatively shorter than in these taxa. In Sciurus (26.4 vs $18.7 \mathrm{~mm}$; ratio $=0.7$ ) and in Mayulestes (24.8 vs $18 \mathrm{~mm}$; ratio $=0.72$ ) the ratio is closer to that of Alcidedorbignya. The problem of the relative length of the ilium has been discussed by Muizon (1998) and, given the lack of complete skeletons of early mammals, it appears difficult to assess polarity of this character. In Pantolambda the ilium is slightly longer relatively to the ischium than in Alcidedorbignya (106.8 vs $79.8 \mathrm{~mm}$; ratio = 0.74), and, in Bemalambda (measurements taken from Zhou et al. 1977: fig. 19), it is even longer (c. 146 vs c. $95 \mathrm{~mm}=$ 0,65). In Barylambda (measurements taken from Patterson 1939: fig. 104) it is intermediate between Pantolambda and Bemalambda (c. 355 vs c. $250 \mathrm{~mm}=0.7$ ). Furthermore, Patterson (1939) mentions that the ilium of Barylambda is shorter 
anteroposteriorly than in Titanoides (= Spartacolambda) and Coryphodon. Because Alcidedorbignya is the oldest pantodont and Coryphodon and Titanoides among the youngest ones these ratios suggest that, in pantodonts, the ilium tends to lengthen in younger taxa.

The orientation of the gluteal fossa in Alcidedorbignya is dorsolateral, while in Tenrec, Solenodon, Procavia and Dendrohyrax it is dorsal to almost dorsal. In Sciurus and Mayulestes (dorsolateral orientation) the condition is closer to that of Alcidedorbignya. In Pantolambda, the ilium tends to have a more dorsal orientation, as is the case in the geologically younger pantodont Barylambda (Patterson 1934, 1935, 1939).

Another character of the innominate of Alcidedorbignya is the eversion of the ilium, which resembles the condition in Mayulestes. This condition differs from the relatively straight ilia of Tenrec, Procavia and Dendrohyrax, or the little-everted ilium of Solenodon. As mentioned by Muizon (1998), an everted ilium is present in arboreal tree shrews, squirrels, lemurs, indri, and spider monkey, which are all capable of powerful leaping. However, an everted ilium is also present in less agile mammals (e.g., koala, phalanger, wombat, small and giant panda, bears, and anteater). On the anteromedial side of the ilium are inserted the erector spinae muscles (iliocostalis and longissimus), powerful extensors of the vertebral column. The origin of this muscle is on the dorsal side of the thoracic and lumbar vertebrae and ribs. When the sacrum articulates with the posterior end of the medial face of the iliac wing, as is the case in Alcidedorbignya, an everted ilium leaves more space for insertion of the posterior portion of the erector spinae at the angle between the vertebral column and the pelvis. Such a condition indicates that this muscle was powerful in Alcidedorbignya. Furthermore, on the lateral side of the ilium are the origins of the gluteus profundus and medius, which are inserted on the greater trochanter and that of the gluteus superficialis, which is inserted on the third trochanter. An everted ilium allows for more space for the glutei and therefore a larger muscular mass. Other muscles originating on the anteroventral region of the ilium are the sartorius and the tensor fasciae latae. The sartorius inserts on the medial side of the proximal tibia. The tensor fasciae latae is distally continuous with the fascia lata, a ligament, which inserts on the patella. These two muscles are flexors of the hip and/or extensors of the leg. An everted ilium therefore suggests a powerful traction of these muscles. It also indicates, in Alcidedorbignya, a powerful musculature of the posterior part of the vertebral column and of the coxofemoral articulation (especially the gluteus medius - see femur section). However, this observation must be moderated in the light of the morphology of the postdiaphragmatic vertebrae (see above), in which the neural processes and the transverse processes are not extremely developed (although clearly more than in Tenrec and Solenodon) and which do not bear robust mammillary processes, three structures which receive the epaxial musculature. In Alcidedorbignya, these structures are less developed than in some squirrels (Sciurus igniventris), but it is noteworthy that others (Callosciurus flavimanus) have structures that are as weakly developed as those of Alcide- dorbignya. Furthermore, the woolly monkey, Lagothrix, also has moderately-developed neural and transverse processes of the lumbar vertebrae, lacks mammillary processes, and has an everted ilium as in Alcidedorbignya. The very agile Tupaia has relatively small anteriorly-oriented neural processes, moderately-developed transverse processes, and very reduced mammillary processes and bears an everted ilium (although to a lesser extent than in Alcidedorbignya).

As noted by Muizon (1998), the epaxial musculature contributes to a propulsive stroke of the hind limb with vertical movements of the vertebral column (in the case of terrestrial mammals). In the case of a potentially arboreal ecotype it could be related to some degree of leaping or leaping run and in any case to a good agility. It is noteworthy that living didelphids which generally (when unstressed) do not move very fast, which walk on the branches with the help of their grasping manus and pes, and, which are not jumpers, do not have an everted ilium. The only living didelphid that has a slightly more everted ilium than the others is Metachirus. This condition is probably related to the terrascansorial (Szalay 1994) mode of terrestrial locomotion of this taxon, which is extremely agile on the ground and whose locomotion is a sort of leaping run. However, Metachirus has lumbar vertebrae with neural and transverse processes much larger than those of Alcidedorbignya. Moreover, it is noteworthy that an everted ilium is not indispensable to agility, as Dendrohyrax, which is very agile in trees, does not have an everted ilium. However, in this genus, the medial face of the ilium, anterior to the sacroiliac articulation, bears a conspicuous fossa for the erector spinae, whereas in Procavia this region is flat to slightly convex. In other respects, an everted ilium is also a character related to bipedalism. This is true of course for higher primates, but also for bears. All bears, which are occasional bipeds, have a distinctly everted ilium to an extent never observed in the other carnivores. Squirrels also commonly adopt a bipedal posture. This does not mean that Alcidedorbignya was bipedal but, in light of the iliac anatomy and locomotor abilities in ursids and sciurids, it is possible to hypothesize that the eversion of the ilium in Alcidedorbignya could be related to some temporary need for the body to be carried or supported for a short time by the hind limbs alone. Such a condition could occur when the animal is in a tree and when its forelimbs are searching for grips in order to carry on its progression. This situation does not imply that the animal remained in a bipedal stance, but that it probably used this posture for very short episodes (even not fully erect) in order to facilitate an arboreal progression. A bipedal posture is also commonly used by some terrestrial mammals to watch out the surroundings. This interpretation of the eversion of the ilium in arboreal locomotion could also apply to Mayulestes. As mentioned by Argot (2002) the cursorial or curso-saltatorial abilities of Mayulestes suggested by Muizon (1998), are unlikely given the shallow acetabulum (even shallower than in Alcidedorbignya), which indicates an unstabilized hip articulation. In fact, agile locomotion and erect stance involve the same muscles (epaxial musculature among others) and may exist jointly. A good example is that of squirrels, which are extremely agile and frequently adopt 
an erect posture. Alcidedorbignya however, was certainly not as agile as a squirrel (see below, pes section). Furthermore, concerning Mayulestes, some agility is to be expected because of the predaceous habits of this taxon, which is probably not the case of the herbivorous-omnivorous Alcidedorbignya.

The ilium of Pantolambda is also strongly everted. In this genus a large space for the erector spinae is present at the anterior region of the pelvis (Matthew 1937: pl. 47). It differs however from Alcidedorbignya in being less recurved laterally, and in the gluteal fossa, which is almost flat, while it is distinctly concave in Alcidedorbignya. The morphology observed in Matthew's illustration suggests very strong epaxial musculature with gluteal musculature relatively less developed than in Alcidedorbignya and, therefore, possibly a less agile animal. This condition is in agreement with the larger size of Pantol$a m b d a$, and especially with the greater stoutness of its limbs, which are intermediate between an ancestral Alcidedorbignya morphotype and the sub-graviportal morphotype observed in later pantodonts like Barylambda, Titanoides, and Coryphodon. This is especially true concerning the astragalus, which has a well-developed head and neck in Alcidedorbignya, a much reduced head with no neck in Pantolambda and no head in the later pantodonts, but only a planar articular facet for the navicular on the ventral face of the astragalus. The robustness of the epaxial musculature of Pantolambda is unlikely to be related to saltatorial or curso-saltatorial locomotion (e.g., because of its plantigrady), but could be related to some ability to stand in a bipedal position (see below in the tibia discussion). It is noteworthy that a distinctly everted ilium is also present in Bemalambda, which has a strongly inflected anteroventral iliac spine as in Pantolambda and Alcidedorbignya.

Anterior to the anterior edge of the acetabulum is a salient tuberosity (with a small and deep triangular fossa) for the origin of the rectus femoris muscle, which we regard as well developed in Alcidedorbignya. The rectus femoris is an element of the quadriceps femoris, and inserts on the patella and patellar ligament. The three other elements of the quadriceps femoris (the vasti lateralis, intermedius, and medialis) have their origin on the femur and insert on the patella. The rectus femoris, which originates on the ilium, is, therefore, an extensor of the crus, when the hip articulation is immobilized or a flexor of the vertebral column on the thigh, when the knee is immobilized. This tuberosity (or ventrocaudal iliac spine) is also especially robust in ursids. In the case of taxa with flared ilia, a powerful rectus femoris could be related to the need to resist to flexion (which involves the extensor muscles and therefore corresponds to extension) of the knee, when the animal is in a bipedal erect posture, with all the weight of the body being borne by the hind limbs only, instead of the four limbs. Because the rectus femoris also acts as a flexor of the hip articulation, the extension of the knee must be compensated for by resistance to the flexion (equivalent to extension in terms of the muscles involved) of the femur on the pelvis. In this case the gluteus medius, which originates on most of the gluteal fossa of the ilium and inserts on the apex of the greater trochanter, is involved. Its function is extension of the hip articulation. As noted above, the glutei of Alcidedorbignya are relatively well developed because of the everted ilium with a concave gluteal fossa, but also because of the elevated and robust greater trochanter of the femur. Furthermore, two other muscles have the same action as the rectus femoris. They are the sartorius and the tensor fasciae latae, which originate on (or around) the anteroventral iliac spine and insert on the patella or patellar ligament. Like the rectus femoris, they flex the hip joint when the knee is immobilized and extend the knee when the hip articulation is stable. Therefore, both the eversion of the ilium and the deflection of the anteroventral iliac spine in Alcidedorbignya could be related to some need for a bipedal stance. It is noteworthy that in the case of a bipedal posture, the muscles that provide the main resistance to flexion of the knee are the vasti (vastus medialis, lateralis, and intermedius) and the rectus femoris. However, these muscles originate on the diaphysis of the femur and insert on the patella and therefore have no connection with the innominate. Nevertheless, the diaphysis of the femur of Alcidedorbignya is transversely wide with a laterally expanded third trochanter, on which inserts the gluteus superficialis. This condition provides for a large origin of the vasti muscles, which were possibly powerful in Alcidedorbignya. It is noteworthy that ability to stand bipedally has been hypothesized in many ground sloths on the basis (among others) of their transversely wide femora (Casinos 1996).

The acetabulum is widely open in Alcidedorbignya, a condition that allows greater mobility of the articulation, and which is often present in arboreal taxa. Such a condition is present in Mayulestes and arboreal didelphids. It is absent in the terrestrial Pantolambda and Metachirus, which have a much deeper acetabulum than in Alcidedorbignya.

The open acetabulum also suggests that the hip joint of Alcidedorbignya was not very stabilized. A non-stabilized articulation suggests that Alcidedorbignya was moderately agile. However, the acetabulum of Tupaia is also relatively open (although less than in Alcidedorbignya) and the agility of tree shrew is astonishing. Furthermore, in the tree hyrax, Dendrohyrax, the acetabulum is larger and more open than in the rock hyrax Procavia, and Dendrohyrax is also an extremely agile climber.

The wide-open acetabulum, combined with the laterally facing ischium (rather than ventrally), indicates good ability of femoral abduction. The limited articular facet of the femoral head (which does not present a posterior extension towards the femoral neck) could indicate a contradictory interpretation. However, the maximum angle of abduction of the femur to the vertical plane has been measured with the innominate articulated with the sacrum (in order to have a vertical reference). The remarkably high value of this angle, which approximates $75^{\circ}$, clearly demonstrates the increased capacities of femoral abduction of Alcidedorbignya. This angle was also measured in Sciurus, where it approximates $70^{\circ}$, while in the terrestrial Metachirus it is not higher than $25^{\circ}$.

On the ischium, the ischiatic tuberosity is elevated and salient posterodorsally but not very robust. However, the ischium is complete in a single specimen of Alcidedorbignya, MHNC 8372 , a young adult, which is not fully ossified. Therefore, it 
is possible that the ischiatic tuberosity may have been more robust on a fully-grown adult than on the available specimen. From the ischiatic spine originates (among others) the ischiocaudalis, and from the anapophyses of the lumbars originates the longissimus caudae. These two structures are moderately developed in Alcidedorbignya. Therefore, these two tail muscles were probably not very powerful in Alcidedorbignya, thus indicating a tail less robust and muscular than in arboreal taxa with a prehensile tail.

Femur. The femur of Alcidedorbignya is proportionally longer than those of Solenodon and Tenrec. Because of its length it appears more slender than that of Solenodon, however the width of the proximal half of the diaphysis is clearly greater in Alcidedorbignya, a feature even more developed on the referred specimens MHNC 8451 and 8453. When compared to Tenrec the femur of Alcidedorbignya has a wider and more robust diaphysis. The greater trochanter of Alcidedorbignya projects slightly above the head. It is much higher than that of Tenrec (which is distinctly lower than the head). It is roughly as high as that of Solenodon but more massive. The lesser trochanter is located at the same proximodistal level in the three genera, but the third trochanter is more distal and more prominent in Alcidedorbignya. The femoral head protrudes more proximally in Hyopsodus than in Alcidedorbignya and the third trochanter is still more distal.

The robust and blade-like lesser trochanter of Alcidedorbignya seems to indicate a good development of the iliopsoas (iliacus + psoas major). However, the iliacus fossa on the ventral face of the wing of the ilium is long and narrow and the origin of the psoas major on the ventral side of the transverse processes of the lumbar vertebrae, are moderately developed. As mentioned above, we consider the condition in Alcidedorbignya to be intermediate. Development is certainly greater than in Tenrec and even Solenodon. However, more than the strength of the iliopsoas, it is the orientation of the lesser trochanter that matters. The ilio-psoas is a flexor of the thigh on the trunk but also an efficient rotator of the femur laterally, especially when the thigh is semiflexed. Such a rotation turns the pes laterally in such a way that the sole faces somewhat medially (if the pes is extended). This position of the pes is critical in the case of an arboreal mammal walking on a branch, which it clasps between the soles of its feet. This position of feet (as well as hands) is observed, e.g., in arboreal didelphids, in Sciurus, in Dendrohyrax (see video of Dendrohyrax at: http://www.arkive.org/southerntree-hyrax/dendrohyrax-arboreus/video-00.html). Having in mind this possible locomotor behaviour of Alcidedorbignya, the posteromedial orientation (rather than medial) of the lesser trochanter clearly increases the angle of rotation of the femur. It is noteworthy that in Dendrohyrax the posterior orientation of the lesser trochanter is even more pronounced than in Alcidedorbignya, while in Sciurus, which uses its claws to grasp the branches (rather than clasp them between its feet) and does not need great capacities of external rotation of the femur to grasp the branches, the lesser trochanter is oriented medially only.
It is noteworthy, however, that the interpretation presented above may be specific to Alcidedorbignya or simply inapropriate because a posteromedially projecting lesser trochanter is more typical of generalized terrestrial and cursorial mammals (e.g., carnivores, Gebo \& Rose 1993; Heinrich \& Rose 1997; rodents, Rose \& Chinnery 2004), whereas arboreal and scansorial forms tend to have a medially directed lesser trochanter as observed in sciurids (Emry \& Thorington 1982). In fact, the orientation of the lesser trochanter may alternatively be regarded as related to terrestrial locomotion, which is likely to have been frequent in Alcidedorbignya. It is noteworthy, in this respect, that a posteriorly oriented lesser trochanter of the femur is observed in Paramys, an Eocene rodent that is regarded as having both terrestrial and scansorial locomotory habits (Rose \& Chinnery 2004). Therefore, the posterior orientation of the lesser trochanter may not be incompatible with the presence of both terrestrial and scansorial habits in Alcidedorbignya.

The slight torsion of the proximal epiphysis of the femur in relation to the distal one recalls the twist of the glenoid cavity of the scapula in relation to the main plane of the blade. It similarly suggests a slight abduction of the thigh at rest. This twist is still more pronounced in Procavia, which often maintains crouched posture.

The distal end of the femur of Alcidedorbignya is weakly asymmetrical. The medial condyle slightly projects medially and the lateral condyle is slightly wider than the medial. However, as mentioned above this asymmetry is subtle, and it may have been altered by some distortion suffered by the femora of MHNC 8372. Asymmetry of the distal epiphysis of the femur is apparently not strictly related to arboreal habits (Kappelman 1988). Arboreal Tupaia and Sciurus, as well as the terrestrial Procavia, have a symmetrical epiphysis, whereas the arboreal Dendrohyrax shows a conspicuous asymmetry (lateral crest of the trochlear notch higher than the medial and medial condyle much larger and longer than the lateral). The distal extremity of the femur of Alcidedorbignya also resembles those of Solenodon (terrestrial but which climbs well) and Tenrec (strictly terrestrial). The distal epiphysis of the femur of Alcidedorbignya is slightly wider than long anteroposteriorly as observed on the two undistorted specimens (Fig. 95D-G). The femoral trochlea is not flat but not deeply grooved and narrow either (Fig. 95D, E, G), as is observed in cursorial mammals. It is clearly shallower than in Meniscotherium but deeper than in Solenodon. It is also proximodistally short, as in lateral view its proximal edge does not extend proximally further than the proximal edge of the femoral condyles. In Solenodon, and especially in Meniscotherium and Diacodexis the proximal extension of the trochlea passes the level of the condyles proximally. Among recent mammals this condition is present in e.g., Lepus, Oryctolagus, Tragulus, Rhynchocyon, Dipus. This condition extends the length of the trochlea and is generally present in saltatorial and cursorial mammals, but is less conspicuous in the latter. Therefore, the distal extremity of the femur of Alcidedorbignya is not indicative of highly active specialized habits but rather denotes a more generalised terrestrial condition. Among Recent mammals, it 
is not fundamentally different from the conditions observed in Sciurus or Martes although patellar groove is slightly narrower and deeper. Because Sciurus and Martes are extremely agile, the condition of the distal extremity of the femur of Alcidedorbignya does not obligatorily preclude a potential for some agility of the knee. The distal extremity of the femur of Alcidedorbignya is certainly more similar to that of these scansorial/arboreal taxa than to those of cursorial taxa (Fig. 116). In fact, its divergence (although moderate) from the morphology observed in arboreal taxa (deeper and narrower patellar groove) indicates more terrestrial adaptations and could reflect in Alcidedorbignya the presence of both terrestrial and scansorial locomotor habits. However, the patellar groove of Alcidedorbignya also differs form the condition generally observed in semifossorial terrestrial mammals, in which it is wider and shallower. Therefore, the morphology of the distal extremity of the femur of Alcidedorbignya does not closely resemble one of the three ecotypes considered above (cursorial, arboreal, and fossorial) and could approach that of a generalised terrestrial mammal. This condition suggests that the terrestrial gait of Alcidedorbignya probably more resembled scampering than active running.

Tibia. The tibia of Alcidedorbignya is proportionally shorter and more massive than that of Tenrec and especially than that of Solenodon, which is $40 \%$ longer than the femur. As a whole the tibia of Alcidedorbignya more resembles that of Tenrec, which is roughly straight in lateral view whereas that of Solenodon is distinctly sigmoidal with its distal third closely appressed against the fibula. Close approximation and/or fusion of the distal extremity of the tibia and fibula reinforce the crus in saltatorial mammals (e.g., rabbits, macroscelidids, saltatorial rodents such as pedetids, dasyproctids, ect) but it is also present in erinaceids, which are terrestrial with no cursorial or saltatorial habits. The tibial crest of Alcidedorbignya is clearly more developed than in Tenrec and Solenodon. At its distal end (mid-shaft of the bone) is a salient elongated tubercle and the crest is distinctly bent laterally. This condition is even more pronounced in Pantolambda but is apparently absent (or poorly developed) in Bemalambda, judging from the illustrations in Zhou et al. (1977). A similar condition is present in Ectoconus (Matthew 1937) and in Hyopsodus (Gazin 1968: pl. 12-5). A laterally inflected tibial crest is also present in Sciurus and Tupaia. However, in these genera, the crest remains on the proximal third of the bone, the distal tubercle is less salient anteriorly, and the lateral inflection is less pronounced than in Alcidedorbignya. The tibial crest receives the insertion of the biceps femoris on its lateral side (Barone 1997, 2000; Evans \& de Lahunta 2012). On its medial side are the insertions of the semitendinosus and gracilis. In the $\operatorname{dog}$ (Barone 1997, 2000; Evans \& de Lahunta 2012) the insertion of the semitendinosus is at the distal extremity of the tibial crest, on the tibial tubercle, and immediately proximal to it on the crest is the insertion of the gracilis. The biceps femoris and the semitendinosus originate on the ischiatic tuberosity and were apparently well developed in Alcidedorbignya. These muscles are extensors of the thigh and may
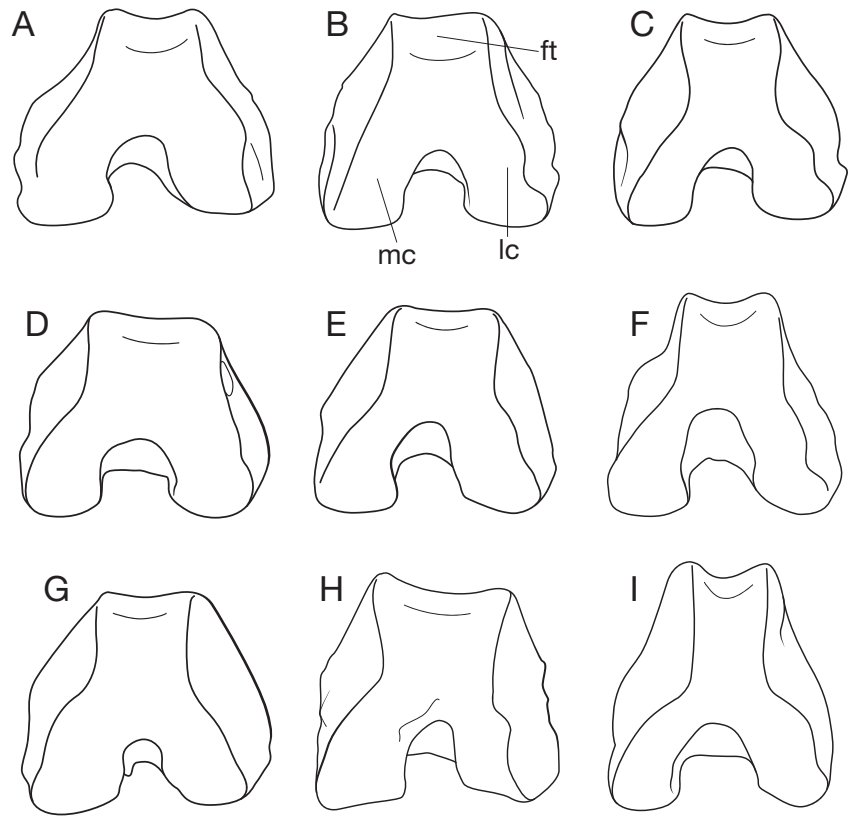

FIG. 116. - Distal view of the left femur in selected fossil and extant mammals: A, Alcidedorbignya inopinata (reconstructed on the basis of the undistorted specimens MHNC 8355 and 8456); B, Sciurus igniventris (coll RH uncatalogued); C, Sciurus vulgaris (uncatalogued); D, Ratufa bicolor (MNHN-AC-13435); E, Martes foina (uncatalogued); F, Felis silvestris (uncatalogued); G, Cynomys ludovicinanus (MNHN-AC-1895-4); H, Marmota marmota (MNHN-AC-1996-2438); I, Vulpes lagopus (MNHN-AC-1880.1313). Abbreviations: ft, femoral trochlea; Ic, lateral condyle; mc, medial condyle. Not to scale.

have been involved, in the case of a need for bipedal posture, to maintain the anterior part of the body erect without the help of the forelimbs. The condition of Pantolambda, more pronounced than in Alcidedorbignya, favours the hypothesis of a temporary need for bipedal posture in this genus. The gracilis, which inserted on the tibial crest, is inferred to have been also relatively powerful in Alcidedorbignya, because of the robustness of the crest; this muscle is an adductor of the leg, which could have been involved when clasping a branch on which Alcidedorbignya was walking.

On the posterior face of the tibia is a deep fossa for the tibialis caudalis (an extensor of the tarsus and an evertor of the foot) and the flexor digitorum longus. This fossa also probably received the origin of the flexor hallucis longus, which is likely to have also originated on the salient interosseous crest present on the posteromedial edge of the fibula in its proximal half. The development of this crest is an indication of a powerful muscle. The posterior tibial fossa and the interosseous crest of Alcidedorbignya are more developed than in Solenodon and especially than in Tenrec. A similar fossa is present in Dendrohyrax and Sciurus, but the large interosseous crest of the fibula of Alcidedorbignya is absent in these genera. Although it is also related to terrestrial locomotion, the development of the flexors of the digits could indicate scansorial habits. It reinforces the hypothesis of a possible locomotion on trees with the hind limbs slightly everted, the feet with the soles against the lateral sides of the branch they were clasping, possibly with the hallux remaining dorsal and the other fingers being more lateral (see discussion on the pes, below). 
Fibula. The fibula of Alcidedorbignya is more robust than that of Tenrec, with a much more prominent interosseous crest and a more massive distal extremity. However, the fibular head is less developed anteroposteriorly than in Hyopsodus. The tibia and fibula of Alcidedorbignya are separated by a wide space, which allowed for an important development of the distal musculature of the hind limb. A similar space is present in Tenrec but it is more reduced in Solenodon because of the close approximation of the bones in the distal third of the crus. Although the proximal head of the fibula is not extremely expanded (as in the case of the fan-shape observed in didelphids) and did not articulate with the femur, it is nevertheless significantly developed, with a large interosseous crest. This condition suggests that the peronei muscles are likely to have been well developed, especially the fibularis longus (see pes section below).

Pes. On the pes, the relatively flat astragalar trochlea of Alcidedorbignya denotes an obvious plantigrady. The tibial facet is very flat with no crest at all between the lateral and medial tibial facets. The only high crest is that between the tibial and fibular facet. This morphology is not that of a cursorial or fossorial mammals since it does not provide for a high astragalo-tibial stability or a wide range of flexionextension. Equally, the articulation does not suggest a very agile animal either. Highly plantigrade mammals (e.g., bears, badgers, apes) are well known not to be fast runners but are best regarded as scampering runners. This morphology of the astragalar trochlea clearly differs from that observed in the agile climbers like Sciurus or Nasua, which have a deep trochlea with sharp crests. It however resembles the shallow trochlea of slow climbers such as Potos, in which the medial crest is smooth and low and the lateral one is elevated and sharp, a condition similar to that of Alcidedorbignya. Furthermore, the condition of the astragalar trochlea observed in Plesiadapis rex (Boyer et al. 2010: fig. 3) and in Plesiadapis tricuspidens (personal observations of Cernay specimens) is distinctly reminiscent of that of Alcidedorbignya (although slightly thicker dorsoplantarly), with a very low and smooth medial crest (between the lateral and medial tibial facets) and a very elevated and sharp lateral crest (between the lateral tibial and fibular facets). As a matter of fact, Plesiadapis has been regarded as arboreal or scansorial (Gunnell \& Gingerich 1987; Gunnell 1989; Gingerich \& Gunnell 1992; Bloch \& Boyer 2007; Kirk et al. 2008). This astragalar articulation of Alcidedorbignya also differs from those of Tenrec and Solenodon. In these genera, the lateral and medial tibial facets are separated by an elevated crest, especially in Solenodon, in which the trochlea is very deep (clearly V-shaped in crosssection), which indicates a more stable articulation than in Alcidedorbignya. However, the well-developed medial tibial malleolus of Alcidedorbignya, combined with the sharp lateral crest between the tibial and fibular facet laterally, could have compensated for the absence of an elevated crest between the medial and lateral tibial facets, thus providing a reasonably stable articulation. Therefore, the morphology of the astragalotibial articulation of Alcidedorbignya, which, at first sight, suggests a relatively poor agility, is probably not that of a chronically slow animal. Furthermore, the plantigrady indicated by the astragalar trochlea, is probably not related only to terrestrial locomotion but could also be compatible with climbing ability.

The extensive and strongly convex astragalo-navicular contact suggests some mobility of this articulation. Because of the presence of the cuboid lateral to the navicular, the movement was dorsoplantar and increased the mobility of the foot. Such a movement could have improved the capacities of clasping branches between the feet during arboreal locomotion. However, it is also apparently a plesiomorphic character present in many early mammals (e.g., the Tiupampa condylarths, Hyopsodus, Meniscotherium, Promioclaenus, Periptychus, Ectoconus, Arctocyon). An astragalocuboid contact as observed in Alcidedorbignya usually permits less flexibility and, therefore, might not be advantageous to a climber. It is noteworthy, however, that an astragalocuboid articulation is also present in the arboreal Plesiadapis and we have observed a small astragalocuboid facet in Sciurus and in Potos.

On the calcaneus, the peroneal process is salient and large and bears a distinct groove for the tendon of the fibularis longus as is observed on MHNC 8462 (Fig. 107A, B). It is not as developed as in Mayulestes ferox. When compared to Tenrec, it does not protrude as laterally as in this genus, but it is proximodistally longer, extends further distally, and bears a deeper groove. The peroneal process of Solenodon is distinctly smaller than in Alcidedorbignya. The tendon of the fibularis longus muscle extended past in the groove of the peroneal process, passed obliquely in a groove on the plantar aspect of the cuboid and cuneiforms and, running medially, inserted on the proximal epiphysis of the $\mathrm{Mt} \mathrm{I}$. This muscle essentially rotates the pes so that the plantar surface faces laterally and is a flexor of the hallux. The lateral development of the peroneal process increases the lever arm and efficiency of the muscle. In Alcidedorbignya the lever arm was probably not as efficient as in Mayulestes, but the size of the process indicates that the action of the muscle was still relatively effective. In Tupaia and Sciurus the peroneal process is much smaller than in Alcidedorbignya and is in a more proximal position, with no contact with the cuboid facet distally. The peroneal process is totally absent in Procavia and Dendrohyrax. In the case of the arboreal locomotory hypothesis made for Alcidedorbignya, a well-developed fibularis longus would be congruent with a clasping action if the feet are on both sides of a branch, posteriorly oriented, (rotation of the foot) and with a grasping action of the hallux on top of the branch (flexion of the hallux). However, a distal peroneal process as is observed in Alcidedorbignya is usually more typical of terrestrial mammals, while a more proximal process is commonly observed in arboreal mammals (Gebo \& Rose 1993; Rose \& Chinnery 2004). Therefore the condition of the peroneal process of Alcidedorbignya would also indicate terrestrial habits.

The calcaneo-fibular contact was reduced in Alcidedorbignya, which suggests that the calcaneus and astragalus were located more one above the other than side by side. This condition is congruent with the orientation of the ectal and sustentacular 
facets and with the development of the plantar tuberosity, which is much more massive on the calcaneus than on the astragalus. The massive plantar tuberosity behind the sustentacular facet (found also on the cuboid and ectocuneiform, slightly weaker on the navicular) suggests a more common plantigrade than digitigrade posture, congruent with the general shape of the pes, with five well-developed digits slightly longer than on the forefoot, providing for a wide plantar sole.

As for the trapezium on the manus, the entocuneiform does not suggest opposability of the hallux. However, the articulation between Mt I and Mt II appears to be relatively mobile, with an opening angle between the two metapodials of approximately $30^{\circ}$. The mobility between the other metapodials is clearly more reduced. Although this mobility did not allow full abduction and opposability of the hallux, as is observed in many arboreal mammals, it certainly helped an arboreal locomotion with the hallux dorsal to the branch used and the other fingers in a more lateral position. However, because of the moderate capacity of abduction of the hallux, Alcidedorbignya was probably not capable to grasp branches of large diameter. Furthermore, the Mt I, although short and slender, is longer than the Mc I and presents a slight asymmetry of the distal epiphysis. Considering that the distal epiphysis of Mt II also presents this asymmetry (and Mt IV, an opposite asymmetry), it could be related to the position of these metatarsals in the foot. The Mt III and IV are the longest, whereas in the forelimb Mc II and IV are of similar length and shorter than Mc III, which suggests a slight lateralisation of the main axis of the pes.

In Alcidedorbignya the length of the pes is slightly longer than that of the manus mainly because the metatarsals are c. $34 \%$ longer than the metacarpals. However, the size difference, which is conspicuous at the level of the metapodials, is attenuated at the level of the digits because the length of the phalanges is similar in the manus and pes. This condition resembles that of Tenrec but differs from that of Solenodon, in which the metatarsals are proportionally clearly longer than in Alcidedorbignya. Therefore, the pes of Alcidedorbignya is relatively shorter than in Solenodon. The hallux is not opposable in any of the three genera. Mt I of Alcidedorbignya is less robust than $\mathrm{Mt} \mathrm{V}$, and conspicuously shorter, whereas they have roughly the same length in Tenrec. In Solenodon, Mt I is only slightly shorter than Mt V but it is stouter, in contrast to the condition in Alcidedorbignya. Mt III of Alcidedorbignya is the longest, as in the hand, a condition similar to that of Tenrec and Solenodon. Alcidedorbignya is also characterized by a Mt III that is slightly longer than in Tenrec and especially more robust.

The pedal digits of Alcidedorbignya are hardly longer than those of the manus. The ungual phalanges of Alcidedorbignya did not bear claws as they do in Tenrec and Solenodon, but had robust, elongated primate-like nails (not true hooves). Based on the ungual phalanges, they were smaller and less massive than on the manus, which suggests that they played a less active role in behaviours like foraging or grasping. However, as on the manus the unguals bear a very robust basal tubercle, which suggests powerful flexor muscles even though the attachment of the medial annular ligaments on the proximal phalanges are not strongly developed. The ungual phalanges of Alcidedorbignya resemble primate ungual phalanges in dorsal view in being scutiform and transversely flaring distally. However, they are cleft and deflected plantarly, in contrast to the condition generally observed in primates. Nevertheless, it is noteworthy that the ungual phalanges of Alcidedorbignya are very similar to the ungual of Notharctus venticolis figured by Koenigswald et al. (2012: fig. 12b) in being distinctly deflected plantarly and flared distally.

Conclusions on the hind limb. The hind limb of Alcidedorbignya exhibits little significant characters that could be related to possible arboreal habits. As in the case of Mayulestes such features have been mainly observed on the forelimb and have been discussed above. However, the hind limb bears several characteristics that can be interpreted and understood in the light of the arboreal (scansorial) habits suggested by the forelimb anatomy.

The major anatomical structure of the hind limb of $\mathrm{Al}$ cidedorbignya, which lends support to the hypothesis of arboreal habits, is the morphology of the hip articulation. The wide-open acetabulum of Alcidedorbignya indicates a great mobility of the hip and a great capacity of abduction of the femur, which are close to those of Sciurus (see above). This mobility allows for a wide range of movements and is generally regarded as related to arboreality (Jenkins \& Camazine 1977; Heinrich \& Rose 1997; Argot 2002).

The strong eversion of the ilia, which reflects powerful erector spinae muscles, is plausibly related to a need for periodic, presumably short-term bipedal posture. This character is very pronounced in Pantolambda, for which some bipedal stance has been hypothesized (Savage \& Long 1986). During bipedal stance, the hind limb is strongly involved as the hip and knee articulations must resist flexion and therefore involve the extensor muscles of the leg. In this context, the relatively powerful sartorius and tensor fascia latae (origin on the anteroventral iliac spine; insertion on the patella and/or tibial tuberosity), rectus femoris (origin on the ventrocaudal iliac spine; insertion on the patella), gluteus medius (origin on the gluteal fossa of the ilium; insertion on the apex of the greater trochanter), vasti (origin on the anterior, lateral and medial faces of the femur; insertion on the patella), biceps femoris and semitendinosus (origin on the ischiatic spine; insertion on the tibial crest and tibial tubercle), gracilis (origin on the ventral edge of the pubis; insertion on the tibial crest - an extensor of the hip joint), can be interpreted as supporting this hypothesis.

The lesser trochanter, on which inserts the illiopsoas, (a rotator of the leg and therefore of the pes) is large and posteriorly oriented. This posterior (instead of medial) orientation combined to the great mobility of the hip could have allowed significant capacity of external rotation of the limb. This condition could be an indication that Alcidedorbignya moved with the soles of the feet oriented medially in order to clasp the branch on which it was walking, in a 


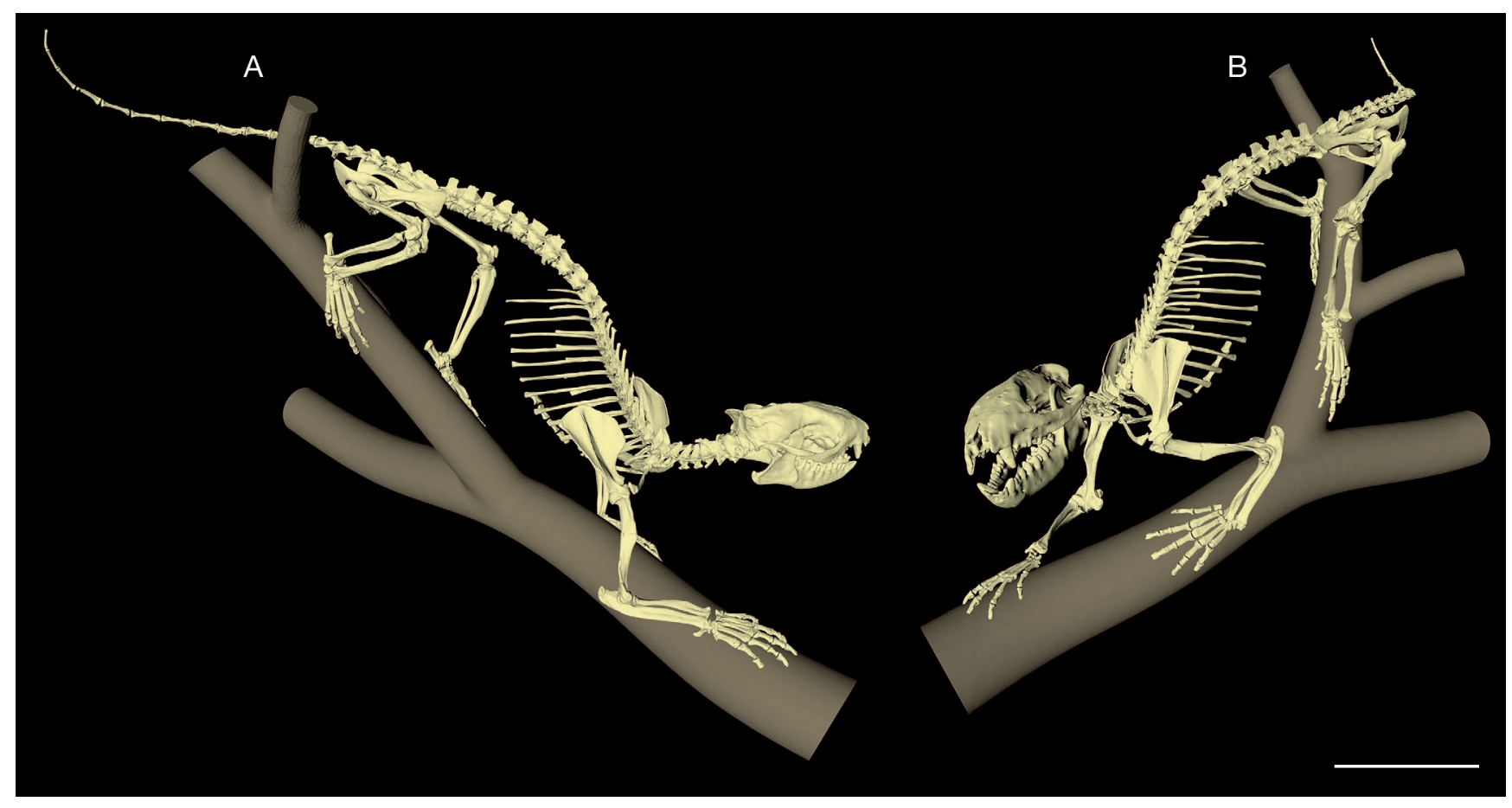

FIG. 117. - Mounted skeleton of Alcidedorbignya inopinata. Bones have been modeled using Materialise Mimics Innovation Suite 16.0 from the CT scan images of the skeleton MHNC 8372, then assembled, articulated and posed using Maxon Cinema 4D R16. The position is that hypothesized during arboreal locomotion, with the branches clasped between the manus and pedes: A, right lateral view; $\mathbf{B}$, left lateral view. The 3D rendering of CT data is available in pdf format at the address http://sciencepress.mnhn.fr/en/periodiques/geodiversitas/37/4/alcidedorbignya-inopinata-additional-files. Scale bar: $5 \mathrm{~cm}$.

pattern similar to that observed in Dendrohyrax (Fig. 117). During such locomotion, the role of the extensors of the pes and of the flexors of the digits is preponderant. In this context, the possibly powerful muscles: gracilis (origin on the ventral edge of the pubis; insertion on the tibial crest - an adductor of the limb), tibialis caudalis (origin on the posterior aspect of the proximal extremity of the tibia; insertion on the medial ligamentous mass of the tarsus - an extensor of the pes), flexor digitorum profundus (origin on the posterior tibial fossa; insertion on the plantar aspect of the phalanges), and flexor hallucis longus (origin on the proximal region of the posterolateral surface of the tibia and the posterior surface of the fibula; insertion on the plantar aspect of the ungual phalanx of digit I) could also favour this interpretation. The potential mobility of the astragalonavicular articulation and the potential opening between Mt I and Mt II (although moderate) are also possibly related to this pattern of arboreal locomotion. In other respect, the shallow acetabulum, as well as the flat astragalotibial articulation (which favours plantigrady), indicate a moderate agility. It is noteworthy, however, that many features of the hind limb, which have been interpreted as compatible with climbing ability are also characteristic of terrestrial locomotion, which may indicate that Alcidedorbignya was probably both terrestrial and scansorial. This condition is not surprizing in a generalised early placental as Alcidedorbignya. A similar interpretation has been suggested by Rose \& Chinnery (2004) concerning the basal Eocene rodent Paramys.
General conclusions on the postcranial skeleton

The general anatomy of the postcranial skeleton of Alcidedorbignya indicates that it was in part a generalised terrestrial mammal. Because it was plantigrade and because it has a relatively wide and not strongly excavated femoral trochlea, Alcidedorbignya was not cursorial but probably practiced a scampering locomotion, not extremely agile but not sluggish either (Figs 118, 119). It is difficult to evaluate the actual degree of agility of Alcidedorbignya, but considering its pedal and knee anatomy we suggest that appropriate living models of moderate agility might be the living lesser panda, Ailurus fulgens or Solenodon paradoxus. Furthermore, the size of the neural apophyses of its dorsal vertebrae seems to indicate that it could even be somehow more agile than Solenodon, as stated above. In other respects, the forelimb of Alcidedorbignya presents, on the scapula, on the humerus, and on the ulna, some features, which are clearly related to climbing ability although not as pronounced as in extremely arboreal mammals such as Caluromys or Sciurus. These features are, among others, the anterior and distal projection of the acromion, the globular and hemispherical shape of the head of the humerus with tubercles more distal than the head, the robust and medially salient entepicondyle of the humerus, the elevated medial crest of the humeral trochlea, the anterior and medial bending of the ulna, with a deep flexor fossa on the medial face of the proximal half of the bone, the widely opened trochlear notch, and the obtuse angle between the trochlear and radial notches in distal view. All these features converge to indicate, in Alcidedorbignya, some ability to climb 


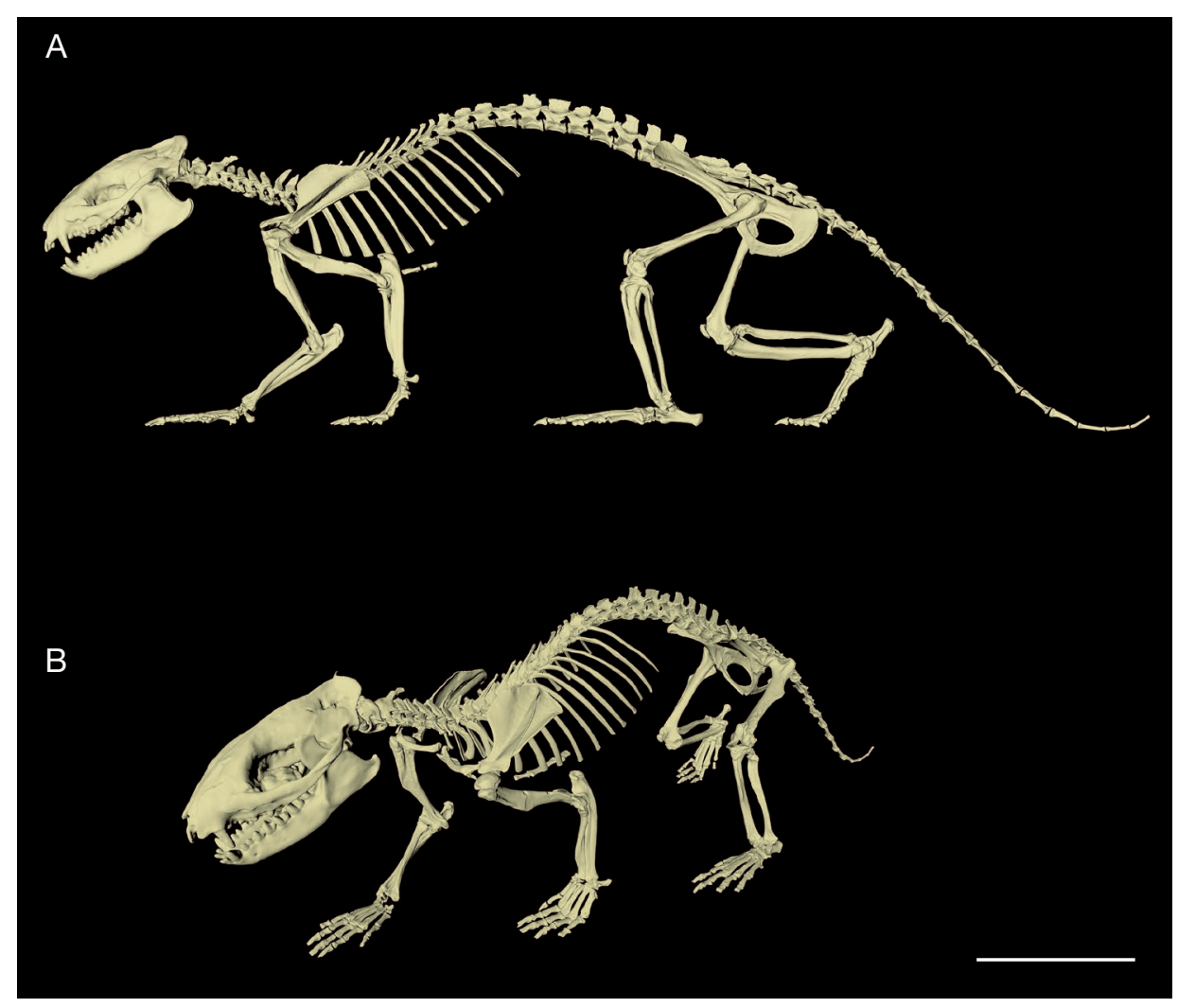

FIG. 118. - Mounted skeleton of Alcidedorbignya inopinata. Bones have been modeled using Materialise Mimics Innovation Suite 16.0 from the CT Scan images of the skeleton MHNC 8372, then assembled, articulated and posed using Maxon Cinema 4D R16. The position is that of the animal walking on the ground: A, left lateral view; B, left anterodorsolateral view. The 3D rendering of CT data is available in pdf format at the address http://sciencepress.mnhn.fr/en/periodiques/ geodiversitas/37/4/alcidedorbignya-inopinata-additional-files. Scale bar: $5 \mathrm{~cm}$.

and some inclination to scansoriality (but see p. 578 for a discussion on possible digging abilities in Alcidedorbignya). However, some contradictions exist, the most important being the radio-humeral articulation, which is not circular as generally observed in arboreal mammal but oval-shaped. This morphology therefore greatly reduces (but does not totally prevent) the capacity of pronation-supination movements of the forearm, which are abundantly involved in arboreal locomotion of many mammals. It is noteworthy that highly arboreal tree hyraxes (Dendrohyrax), which have an ankylosed radio-ulnar articulation have evolved a supinatory ability at the wrist (Fischer 1986). Some other features such as the shortness of the digits and the reduced abductability of the digit I, do not favour arboreality but seem more related to terrestriality. Nevertheless, they do not prevent from climbing since they are also present in tree hyraxes (short digits and lack of digit I). In spite of these contradictions and on the basis of comparison with tree hyraxes, we conclude that Alcidedorbignya was capable of climbing and was possibly partly scansorial but not fully arboreal (Figs 117, 119).

The interpretation of the locomotory adaptations of the hind limb is sometimes more difficult than that of the forelimb at least concerning potential arboreal adaptations (e.g., Muizon 1998). This is the case for the hind limb of Alcidedorbignya. However, the wide-open acetabulum and the great capacity of abduction of the femur of Alcidedorbignya (as mentioned above) indicate a great mobility of the hip joint that is generally related to climbing ability. The remaining features of the hind limb rather indicate a terrestrial scampering locomotion (as mentioned above) and do not present obvious arboreal adaptations, which could support the interpretations resulting from the study of the forelimb.

Therefore, as mentioned above, concerning some of its anatomical features, we have intented to provide an interpretation at least compatible with the hypothesis that Alcidedorbignya was scansorial. This interpretation only means that one given anatomical feature could possibly be interpreted as related to a posture or a movement related to arboreality even if a terrestrial interpretation is equally plausible. We are conscious that different interpretations could be made in the current state of our knowledge and, even if we provide an interpretation congruent with that made of the forelimb, we admit that both habits, terrestrial and scansorial probably existed in Alcidedorbignya (see below).

The eversion of the ilium, the anteroventrally projecting anteroventral iliac spine, the supposed strength of the rectus femoris, and the well-developed tibial crest (in part) suggest ability for an erected standing position on the hind limbs only. Such a posture is frequent in many arboreal or semi-arboreal mammals (e.g., bears, lesser panda, martens, sciurids), but also in terrestrial and semi-fossorial one (e.g., Marmota, Cynomys, Spermophilus, Suricata). A bipedal posture could be related to 
several behaviours: grasping food, which is not at the ground level (e.g., Ailurus), observation of the surroundings (e.g., Suricata, Martes, Sciurus) fight or defensive attitude (bears, squirrels). It could also be a useful ability when climbing trees, to free the forelimb in order to extend grasping range.

Several features have been tentatively related to a need for a medial orientation of the sole of the pes in order to clasp branches during arboreal locomotion. This mode of locomotion has been suggested because Alcidedorbignya had no claws to enable grasping in trees. Our interpretation is also based on the fact that tree hyraxes do not have claws either and apparently use this technique (see below coment on friction pads). The features that we regard as possibly related to this behaviour are: the posterior orientation of the lesser trochanter of the femur, the well-developed tibial crest (in part), and the development of the peroneal process of the calcaneus. It is noteworthy that because all these features are also present in terrestrial mammals that do not climb, they may indicate that Alcidedorbignya was both scansorial and terrestrial. In this context the function of the ungual phalanges is critical as they are one of the most important organ in arboreal locomotion. The morphology of the ungual phalanges of Alcidedorbignya (scutiform in dorsal view but ventrally deflected in lateral view) is unique and therefore difficult to elucidate. They certainly more resemble nail-bearing ungual phalanges than any other because of their scutiform morphology. They did not bear true hooves neither claws and therefore do not suggest respectively cursorial or fossorial habits. Claws are present in all fossorial mammals or in all mammals with digging abilities. Claws are also present in all arboreal mammals lacking abducted and opposable hallux and/or pollex (e.g., carnivores, rodents). For instance arboreal primates do not bear claws but have strong prehension ability (abducted and opposable hallux and/or pollex). The only arboreal mammals lacking boths, claws and abducted and opposable hallux and/or pollex are the tree hyraxes, which however grasp small branches well with flexion of the digits. In Alcidedorbignya the slightly ventrally deflected ungual phalanges may indicate some grasping abilities also with flexion of the digit because hallux and pollex were not opposable. Congruent with grasping ability is also the remarkable development of the flexor tubercle on the ventral face of the ungual phalanges even if such structure is present in diggers as well as in climbers. Strong flexor tubercles are also present on the palmar and plantar aspects of the carpus and tarsus of Alcidedorbignya. As noted above, Alcidedorbignya had no claws or prehensile tail to insure security when climbing. However, friction pads may have been present to increase the grip against substrate during arboreal locomotion. Because there is no indication of presence of pads on the carpus and tarsus, metapodials and digits of manus and pes, they have not been mentioned in the discussion above. However, such friction pads are commonly observed in most arboreal mammals such as marsupials, primates, carnivores, hyracoids, or rodents (Davis 1964; Cartmill 1974; personal observations) and in some cases may be more efficient than claws when both are present (Davis 1964). In didelphids the ungual phalanges bear claws, are distinctly recurved palmarly or plantarly, and bear well-developed friction pads, especially in arboreal taxa like Caluromys (Argot 2001, 2002). Pads are especially large on the metapodials. Therefore, it seems plausible that such structures may have been present in Alcidedorbignya, on the palmar and plantar surface of the ungual phalanges as well as on the rest of the metapodial and carpus and tarsus.

\section{Climbing vs digging or both?}

Because many features of the forelimb are generally similar in scansorial and semi-fossorial mammals, the anatomy of the postcranial skeleton of Alcidedorbignya could be partially related to fossorial rather than to scansorial habits. These characters are essentially observed on the forelimb (shoulder and elbow). In Alcidedorbignya, they are: 1) the posteroproximal extension of the caudal angle of the scapula, which is roughly the most proximal point of the bone (when the spine is vertical); 2) the large size of the acromion; 3) the anterior and distal projection of the acromion (especially the hamatus process), which hides, in lateral view, the glenoid cavity and the supraglenoid tubercle; 4) the triangular morphology of the supraspinatus fossa with the most anterior point of the fossa being approximately at mid-height of the spine or more proximal; 5) the elevation of the spine, which indicates a greater muscular mass of the spinati muscles than in a scapula with a lower spine; 6) the posterior edge of the infraspinatus fossa strongly deflected laterally; 7) the robust deltopectoral crest and tuberosity; 8) the strong teres major tuberosity (or crest) on the medial edge of the humerus; 9) the medially protruding entepicondyle; 10) the salient lateral epicondylar crest; 11) the olecranon bent anteriorly (bowed posteriorly) and medially (bowed laterally); and 12) the relatively open trochlear notch. Furthermore, the relative shortness of the limb bones of Alcidedorbignya, as well as the palmarly or plantarly deflected distal half of the ungual phalanges (in lateral view) are more indicative of fossorial than scansorial habits.

However, some features observed in Alcidedorbignya are generally more developed in arboreal than in fossorial mammals such as the anterior bending of the olecranon (posterior edge of the distal ulna distinctly convex) and the opening of the trochlear notch, which is generally shallower. Therefore, the widely open trochlear notch of Alcidedorbignya would indicate climbing rather than digging ability. Moreover, some features are more restricted to arboreal or scansorial mammals such as the hemispherical proximally facing humeral head, and, especially, the obtuse angle between the distal edges of the trochlear and radial notches (in anterodistal view of the ulna). This latter feature is exclusively present in arboreal and scansorial mammals (although it is not conspicuous, or even absent, in several scansorial carnivores such as viverrids or procyonids). Both features occur in Alcidedorbignya. Furthermore, as discussed above, the scutiform morphology of the ungual phalanges indicates that they bore nails (primate-like nails) or nail-like hooves, which are hardly compatible with digging. Moreover, the tail is generally short to very reduced in fossorial mammals, whereas it is long to very long in arboreal ones. The maximum length that we observed (see above) in semi-fossorial or fossorial mammals approximates $40 \%$ of 


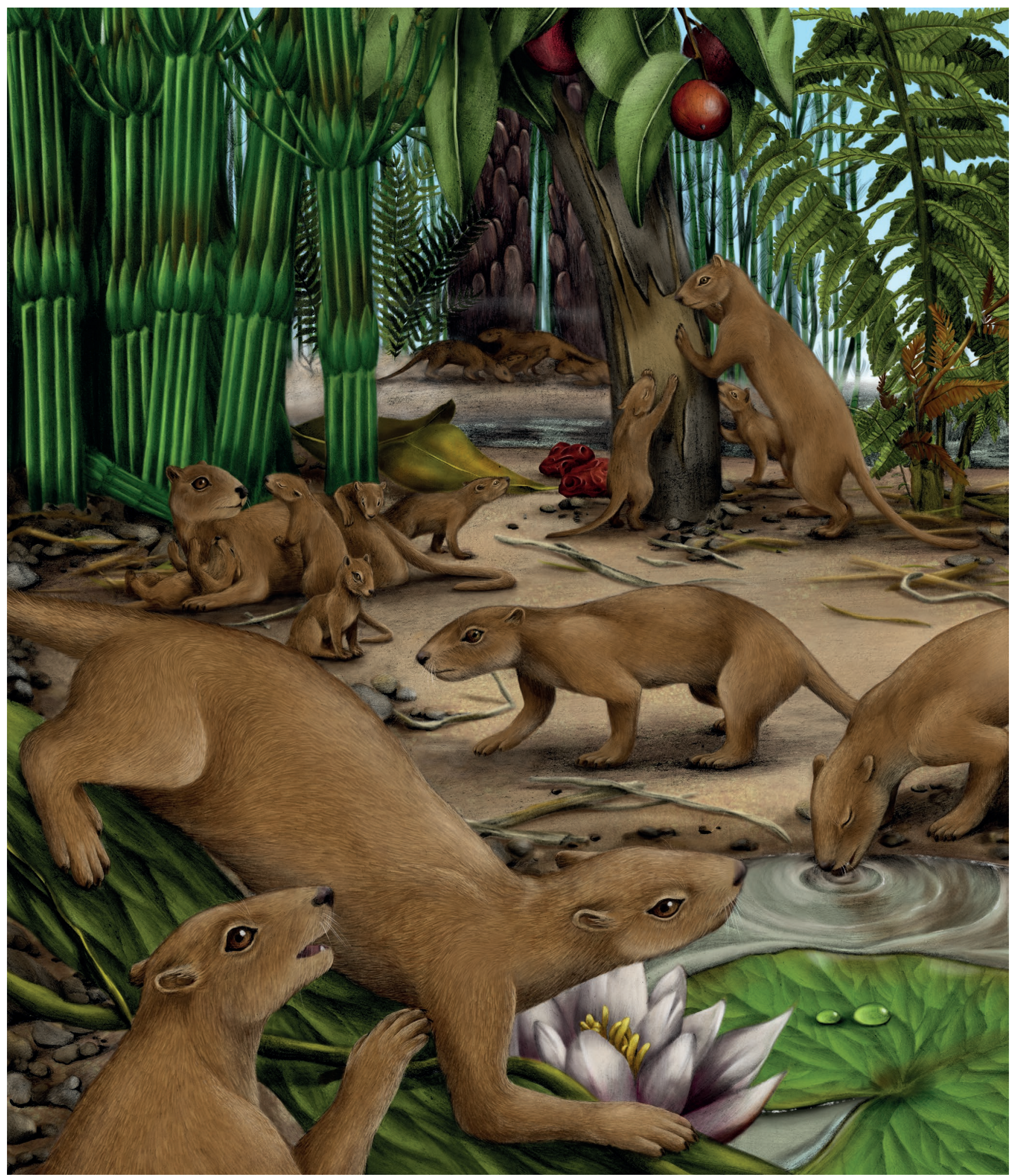

FIG. 119. - Life reconstruction of some individuals of Alcidedorbignya inopinata (c. $\times 0.5$ ).

the head and body length (it is almost always much shorter), whereas the length of the tail of arboreal mammals is generally (but see above for exceptions) between 50\% and 100\% of the head and body length. In Alcidedorbignya the tail is approximately $70 \%$ of the head and body length, which is more compatible with the condition we observed in arboreal and scansorial mammals. Therefore, because of the features exposed in this section and in the description and comparison of the postcranial skeleton above, we suggest that Alcidedorbignya was terrestrial with climbing rather than digging ability. 
Nevertheless, we admit that the palmarly or plantarly deflected ungual phalanges of Alcidedorbignya suggest some digging ability. However, true semi-fossorial habits (as in Marmota or Cynomys) would require that Alcidedorbignya bore extremely robust claw-like nails, which would be suprizing given the width and the scutiform morphology of the distal portion of the unguals. We therefore believe that, if some digging could have been performed with these ungual phalanges, it was not in a harder ground than the soft sand of banks of the river, where Alcidedorbignya was living.

To conclude, Alcidedorbignya was a moderately agile generalised, mainly terrestrial mammal with good climbing ability and occasionally capable of standing in a bipedal posture. Alcidedorbignya was not a strictly arboreal mammal (such as the marsupials Caluromys or Phascolarctos, the rodent Coendou or most cebids monkeys) but, since it was probably capable of efficient climbing, it should be therefore regarded as scansorial. Because of the absence of claws (among others), fossorial habits are here regarded as unlikely or extremely limited (Figs 117-119).

\section{PHYLOGENETIC ANALYSIS}

\section{Results}

Unconstrained analysis. The parsimony analysis of the matrix with some of the characters with multiple states treated as ordered (see Material and Methods) resulted in 7 most parsimonious trees (MPTs) of 3392 steps $(\mathrm{CI}=0.18, \mathrm{RI}=0.504)$. The strict consensus of these trees shows a large basal polytomy for placentals $(\mathrm{CI}=0.166, \mathrm{RI}=0.453 ; 3676$ steps) (Fig. 120). Among traditional extant clades, it supports the monophyly of Xenarthra, Euarchontoglires, Carnivora and Paenungulata. The Afrotheria, Laurasiatheria, Artiodactyla and Lipotyphla are not supported. The xenungulate Carodnia is nested within Pantodonta, and is the sister taxon of Coryphodon. The sister taxon to the clade including Pantodonta and Carodnia is the cimolestid Didelphodus. The South American ungulates Astrapotheria, Notoungulata and Pyrotheria are clustered with the Paenungulata.

The Bremer values supporting the clades that include $\mathrm{Al}$ cidedorbignya are rather weak, below the average Bremer value (Fig. 116; average Bremer value $=4.87$ ). A list of the synapomorphies is provided in the supplementary data. The inclusion of Carodnia within the Pantodonta is mostly supported by characters of the dentition and of the mandible (e.g., spatulate shape of the incisors [character 12,13], deep mandibular body [ch. 139] and strong coronoid crest [ch. 144], long mandibular symphysis [ch. 159]).

Within Pantodonta, the analysis does not group Carodnia with the South American pantodont Alcidedorbignya, though several derived character states are shared by the two genera (e.g., angle between anterior border of coronoid process and horizontal alveolar border of cheek teeth slightly obtuse or right [ch. 143], anterior extension of masseteric fossa on the body of the dentary, below the last molars [ch. 146]) and not by other pantodonts. Instead of Alcidedorbignya, the analysis groups Carodnia with the later diverging and larger-sized North American pantodont Coryphodon. Synapomorphies supporting the latter clade are especially found in the teeth and tarsus (e.g., incisors procumbent [ch. 14, 20, 24], absence of astragalar neck [ch. 410] and overlap between calcaneal ectal and sustentacular facets [ch. 417]) (see supplementary data).

Finally, the characters supporting unambiguously the clade Pantodonta in this analysis are: basined protocone of the penultimate upper premolar (ch. 39), double V-shaped penultimate and ultimate upper premolars (paracone and protoconeVshaped) (ch. 51), vestigial or absent paraconid of penultimate lower premolar (ch. 57), metaconid and paraconid distinctly separated on penultimate lower premolar (ch. 58), m1 much smaller $(80 \%<$ ) than $\mathrm{m} 2$ (ch. 69), crest-like molar cusps (ch. $71)$, stylar cusp A distinct but smaller than B on upper molars (ch. 78), metaconule weak or absent on upper molars (ch. 99), deep mandibular body (ch. 139) and strong coronoid crest on the mandible (ch. 144).

Analysis with backbone constraint. The analysis performed with a backbone constraint (see Material and Methods, and Figures 121 and 122, showing the molecular scaffold used) and with an additive treatment of several multistate characters (as in the unconstrained analysis above) yielded 11 MPTs with 3445 steps ( 53 steps more than the unconstrained analysis) $(\mathrm{CI}=0.177$; $\mathrm{RI}=0.494$; HI 0.506). The same internal relationships as in the unconstrained analysis are found in all 11 constrained MPTs (CMPTs) within the clade Pantodonta except that Carodnia is no longer included in this order, but is grouped with Pyrotherium in another distinct clade with other South American ungulates. In all CMPTs, Didelphodus is the sister taxon of Pantodonta. The sister taxon to the clade Didelphodus-Pantodonta, however, varies among the CMPTs: a clade uniting the Tillodontia, arctocyonids, Pleuraspidotherium, Baioconodon and Maiorana, or the Tillodontia alone. In all cases, the Pantodonta are nested in a fairly apical position within the clade Laurasiatheria. Two of the preferred CMPTs are illustrated which present two of the most differing topologies (figs 117, 118). The other South American taxa - Litopterna, Notoungulata, Astrapotheria and Pyrotheria - are all nested within Laurasiatheria as well, in another clade closely related to the one that contains the Pantodonta. However, in none of the CMPTs is there an exclusive relationship between the South American ungulates and the Pantodonta. An Adams consensus tree (Fig. 120), which preserves the shared phylogenetic relationships, highlights the conflicting position of Eoryctes (sister taxon to tenrecids or lipotyphlans), and of xenarthrans and afroinsectiphilians (sister-groups or nested to within other placentals). The contentious relationships of Protungulatum, Diacodexis, and carnivores is noteworthy, since these three taxa are either stem to a clade including artiodactyls, litopterns, astrapotheres, notoungulates, pantodonts, tillodonts, and "condylarths"; or placed more apically within this clade (Protungulatum is sister taxon to the clade including pantodonts and tillodonts; Diacodexis is sister taxon to the clade including artiodactyls and allies).

A two-tailed Templeton test (Templeton 1983) on the MPTs of the unconstrained analysis and on the CMPTs shows that the latter are significantly different from the former and would be rejected as an implausible hypothesis based on the morphological datamatrix $(\mathrm{P}=0.0192)$. 


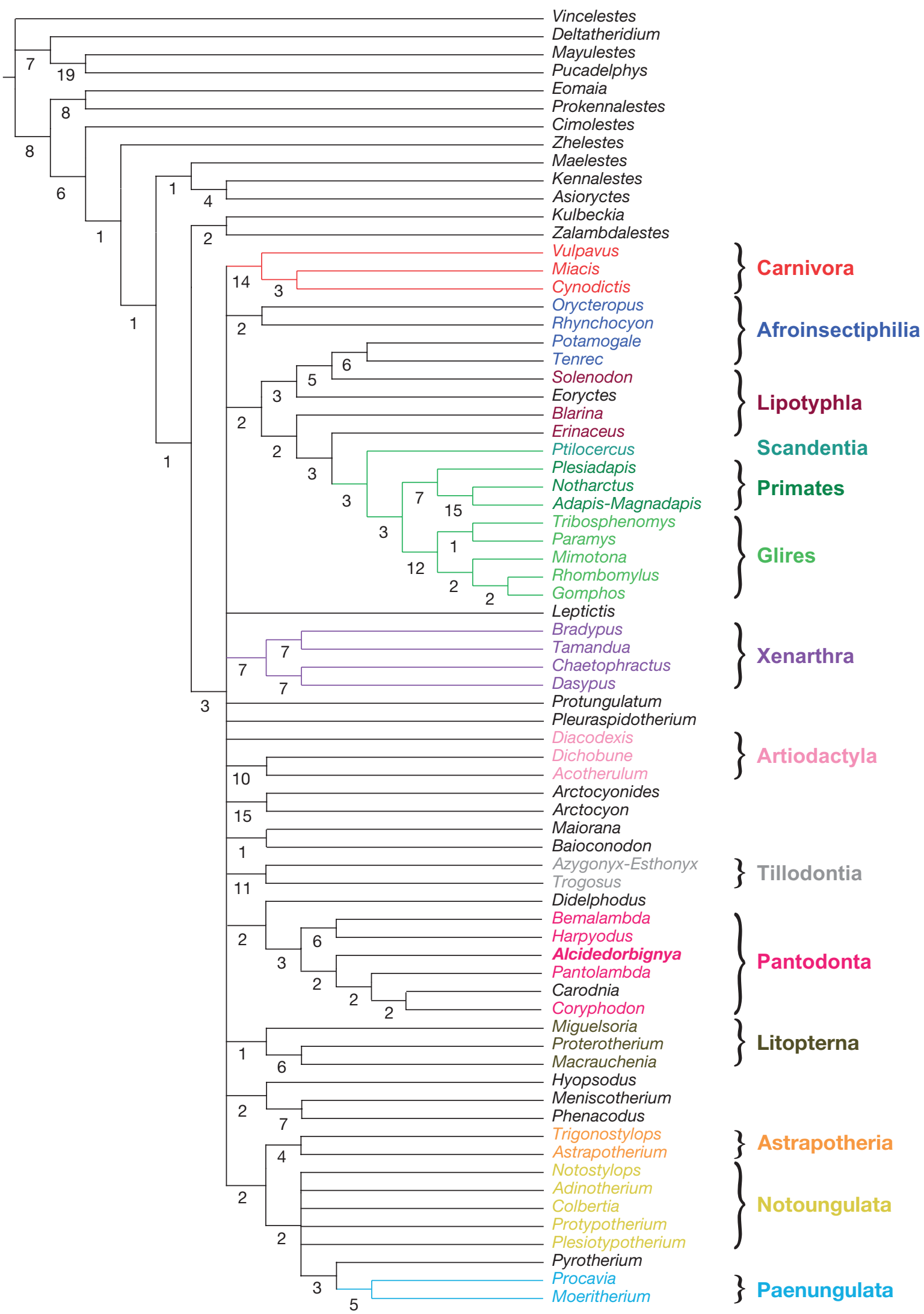

FIG. 120. - Strict consensus cladogram (consistency index $=0.166$, retention index $=0.453$, homoplasy index $=0.547 ; 3676$ steps) of the unconstrained analysis of a data matrix of 70 taxa and 426 characters. Analysis was performed employing the program TNT (Goloboff et al. 2008) (see above in material and methods). Numbers at nodes are Bremer values. Given the high homoplasy index, it is clear that the very unstable trees resulting from this analysis must be taken with extreme caution, since their topology may be drastically modified with introduction or withdrawal of taxa or characters. 


\section{Discussion}

Morphological analyses of placental phylogeny. As explained in the Material and Methods section, the main goal of our analysis was to propose hypotheses for the relationships of Alcidedorbignya and Pantodonta within placentals (in particular the five orders of "South American ungulates") and the matrix was built with this objective in mind. Therefore, the matrix is not best adapted to resolve internal relationships within Pantodonta, because not all pantodonts were sampled. Similarly, because some extant orders are missing, our taxonomic sample is certainly not best adapted to resolve all phylogenetic relationships at the base of the placental tree, but the large diversity of Paleogene fossils included in our datamatrix still confers to it some pertinence on this topic.

The strict consensus cladogram of our unconstrained analysis does not provide much resolution for the root of the placental tree. In agreement with most recent molecular and morphological studies (e.g., Wible et al. 2007, 2009; Prasad et al. 2008; Nishihara et al. 2009; Meredith et al. 2011; O’Leary et al. 2013; Romiguier et al. 2013), it supports two of the four main extant clades of placentals, the Euarchontoglires and the Xenarthra. The Laurasiatheria and Afrotheria are not supported however. At a lower taxonomic level, the Carnivora, the Glires, the Primates and the Paenungulata are supported. Other generally accepted extant ordinal or supraordinal taxa, such as Artiodactyla, Lipotyphla and Afroinsectiphilia (Asher et al. 2009), are not recovered. Among extinct orders, the monophyly of Litopterna, Astrapotheria and Tillodontia is supported. Moreover, to be monophyletic on the unconstrained strict consensus cladogram, the Pantodonta should include Carodnia (see below), and the Notoungulata should include Pyrotherium (see Billet 2010) and the Paenungulata.

One of the main results of the unconstrained analysis is the high level of homoplasy detected (RI close to 0.5 ; low CI below 0.2 ). The low value of the retention index RI, close to 0.5 , indeed means that only half of the similarities present in the matrix are interpreted as homologies (synapomorphies) on the cladogram (Farris 1989a, b). In other words, homoplasy accounts for half of the observed similarities. The case of the basal diversification of placental mammals would certainly deserve further treatment and extensive comparative analyses of homoplasy levels (e.g., see Williams 2007) to determine if the morphological characters issued from this radiation have been overwhelmed by an exceptionally high homoplasy in comparison with other major evolutionary events. Nevertheless, this high value of RI undoubtedly indicates that homoplasy is a non-negligible, if not dominating, explanation for the observed morphological similarities across our matrix of early-diverging placentals. This result is congruent with past studies that also obtained low RI values, close to or below 0.5, for placentals (e.g, Wible et al. 2007; O'Leary et al. 2013). The presence of pervasive convergence has previously been pointed out as the main reason why some morphological studies obtained very different results from molecular analyses (Lee \& Camens 2009) and might instead favour ecomorphological clusters (Springer et al. 2013). Our unconstrained analysis is not as divergent from molecular data as was the "phenomic" analysis of O'Leary et al. (2013: fig. S2), but it still contains major incongruences with the reconstructed molecular patterns, such as the absence of unambiguous support for Afrotheria and Laurasiatheria. Actually, the existence of conflicts between molecular and morphological analyses is not limited to placentals (e.g., Losos et al. 2012), and this situation emphasizes the need for a better mutual understanding between these two approaches in order to build more robust phylogenetic hypotheses with more explanatory power.

Though molecular and morphological partitions provide different results when analyzed separately, it has been demonstrated that the addition of morphological data to molecular datasets increases the support of most placental clades, thereby possibly corroborating several molecular-based hypotheses (Lee \& Camens 2009). Based on these considerations, we have also run an analysis with a backbone constraint (molecular scaffold) in order to; 1) explore if the pattern supported by molecules may also be a plausible hypothesis for our morphological datamatrix; and 2) determine the position of extinct orders on such a topology. The backbone constraint was set on the monophyly of the four major extant clades of Placentalia (two of which were already in the unconstrained analysis) (Figs 121, 122).

The Templeton tests (see Results) indicate that the most parsimonious trees of the constrained analysis (CMPTs) are significantly different from the MPTs of the unconstrained analysis and thus that the monophyly of the Afrotheria, Xenarthra, Euarchontoglires and Laurasiatheria could not be considered as a plausible hypothesis based on our morphological datamatrix. However, it is noteworthy that all CMPTs show an internal pattern of relationship within Xenarthra, Euarchontoglires and Laurasiatheria that is exactly or closely congruent with molecular analyses, e.g., (Lipotyphla (Carnivora, (Artiodactyla (Litopterna, Notoungulata))), even though we did not set a constraint for these internal relationships (Figs 117, 118) (e.g., Prasad et al. 2008; Meredith et al. 2011; Romiguier et al. 2013; Welker et al. 2015). This is not the case within Afrotheria, for which the Afroinsectiphillia clade (Tenrecoidea + Macroscelididae; see Asher et al. 2009) is not supported. Furthermore, concerning the position of several extinct orders, the constrained analysis provides more precise hypotheses than in the unconstrained analysis. The Pantodonta and Tillodontia are nested within Laurasiatheria. Moreover, the five orders of South American "ungulates", Litopterna, Notoungulata, Xenungulata, Pyrotheria, and Astrapotheria, are also included within the Laurasiatheria. The inclusion of Litopterna and Notoungulata was constrained based on the molecular scaffold (see Material and Methods; Welker et al. 2015), but that of the three other orders is a novel result seemingly contradictory with other recent hypotheses that allocate them, together with Notoungulata, to Afrotheria (e.g., Agnolin \& Chimento 2011 [but see also Billet \& Martin 2011]; O'Leary et al. 2013). The presence of North American "condylarths" as sister group of the meridiungulates also agrees well with a modified version of the Panameriungulata hypothesis (Muizon \& Cifelli 2000) that would encompass all members of Meridiungulata. 


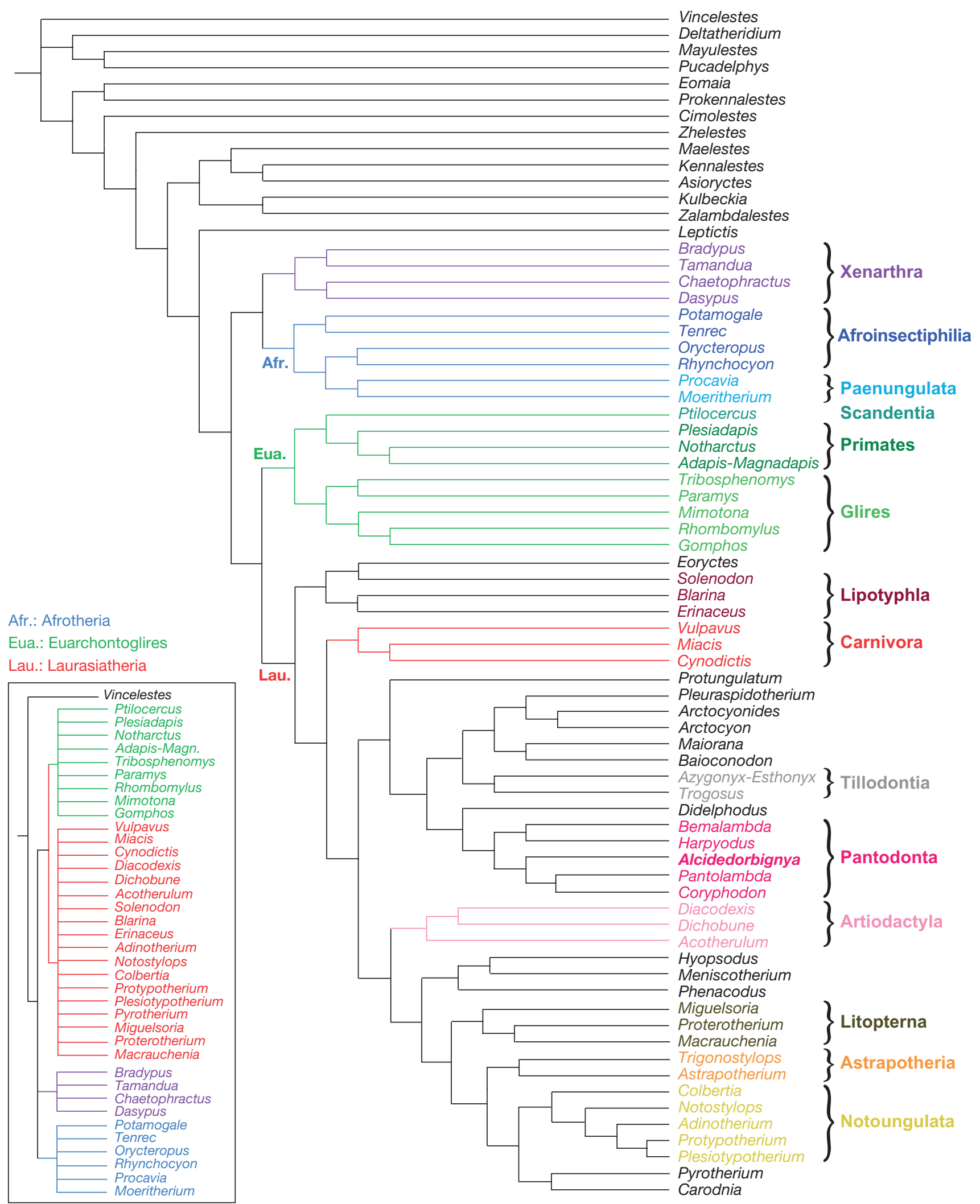

FIG. 121. - One of the 11 most parsimonious trees (consistency index $=0.177$; retention index $=0.494$; homoplasy index 0.506 ; 3445 steps) resulting from the constrained analysis of a data matrix of 70 taxa and 426 characters. Analysis was performed employing the program TNT (Goloboff et al. 2008) (see above in Material and methods). Given the high homoplasy index, it is clear that the very unstable trees resulting from this analysis must be taken with extreme caution, since their topology may be drastically modified with introduction or withdrawal of taxa or characters. The pattern of the molecular scaffold used for the constraint is shown in the lower left corner of the figure. 
Alcidedorbignya, Pantodonta and Xenungulata relationships. To our knowledge, there was until now no phylogenetic analysis that included pantodont taxa together with a large and diverse sample of placental orders. As summarized by Rose (2006), past studies first grouped Pantodonta with various ungulates (Simpson 1945; Van Valen 1988) before they were considered to be closer allies of tillodonts and possibly derived from cimolestids (Muizon \& Marshall 1992; Lucas 1993; McKenna \& Bell 1997). We have obtained results close to the latter hypotheses, as the cimolestid Didelphodus is the sister taxon of Pantodonta (including or not Carodnia) in both analyses, and the Tillodontia is the sister taxon of the clade Didelphodus-Pantodonta in 6 of the 11 CMPTs (see Figs 116-118).

Furthermore, the unconstrained analysis locates the xenungulate Carodnia in an apical position within Pantodonta, as the sister taxa of the genus Coryphodon. This relationship is not strongly supported: the values of Bremer supports for the pantodont nodes including Carodnia are either 2 or 3, which is lower than the average Bremer support in this analysis $(=4.87)$. The unambiguous synapomorphies of the pantodont clades that are observable in Carodnia come mostly from dental and mandibular anatomy, and the synapomorphies of the apical clade Carodnia-Coryphodon from the teeth and tarsus (see above). Many aspects of the cranial anatomy of Carodnia are currently unknown, a fact that prevents exploring if further support for this new relationship may come from other anatomical regions. However, one character of the basicranium observable in Carodnia may also provide support for a relationship of the latter with pantodonts: the ventral opening of the hypoglossal foramen on a flat to convex bony surface is indeed interpreted as a synapomorphy of the Pantodonta (including Carodnia) or the clade Didelphodus-Pantodonta (including Carodnia) in our analysis (ch. 333; see supplementary data). Nevertheless, the constrained analysis does not support the inclusion of Carodnia within the Pantodonta and groups it instead with the South American ungulate Pyrotherium, a relationship that is especially supported by dental and postcranial characters. Like Carodnia, Pyrotherium is a large-sized bilophodont taxon (Billet 2010) and therefore shares with it several dental characters related to the presence of bilophodonty. In addition, it is noteworthy that, as is the case with Coryphodon in the unconstrained analysis, several specializations of the tarsus are found among the synapomorphies that Pyrotherium shares with Carodnia on the CMPTs. Among them, the absence of the astragalar neck characterizes all these three animals, a character that may be related to their heavy weight, since most (but not all, e.g., Paraceratherium) graviportal animals have dorsoplantarly compressed astragali (Shockey \& Anaya 2004). Pending better evaluation of the exact influence body mass can have on other aspects of postcranial morphology, our results may suggest that the phylogenetic placement of the heavy taxon Carodnia was partly guided by several bodymass-related postcranial characters, since this taxon has been alternatively clustered with the two other heaviest taxa in our analyses. The cranial anatomy of Carodnia being almost unknown (see below) leaves relatively more power to other anatomical partitions of the datamatrix, like the postcranial one, for the resolution of the phylogenetic relationships of this taxon.

As is the case in our analyses, past studies did not recover strong support for any extra-ordinal relationship of Xenungulata, which shows how difficult it is to propose a wellcorroborated hypothesis for these poorly-known taxa. Because of the bilophodont nature of their two first upper molars, Xenungulata have long been compared and variably allied to pyrotheres, while they have been also allied to uintatheres because of the structure of their third upper molar (PaulaCouto 1952; McKenna 1981; Lucas 1993). However, the anatomy exhibited by etayoid xenungulates and early-diverging pyrotheres have later cast doubts on these hypotheses (Villarroel 1987; Cifelli 1993; Gelfo et al. 2008). Detailed studies of tarsal elements of pyrotheres and xenungulates have also challenged a relationship between these two groups (Cifelli 1983a, 1993; Shockey \& Anaya 2004). However, the result of our constrained analysis would tend to bring back the hypothesis of pyrotherian affinities for xenungulates, but caution should be used when considering this relationship. The unconstrained analysis indeed provides very different results for this taxon in comparison to the constrained analysis. In addition, the impact of body mass may be a non-negligible factor influencing phylogenetic hypotheses for this taxon.

Contrary to the case of pyrotherian affinities, a relationship of xenungulates with pantodonts, as is supported by our unconstrained analysis, has never been mentioned before (to our knowledge). Though not supported by the MPTs of this analysis, the possibility that xenungulates may be closer to Alcidedorbignya rather than to Coryphodon within pantodonts should also be explored. An exclusive relationship between these two South American taxa would indeed make much sense in terms of past biogeography. The inclusion of early-diverging and well-documented xenungulate taxa could certainly be decisive on that matter, but no such material exists yet. Apart from Carodnia, which belongs to the monogeneric and possibly late-diverging family Carodniidae (Antoine et al. 2015), fossil material of Xenungulata is very scarce and currently limited to mandibular remains (Villarroel 1987; Gelfo et al. 2008). The most complete of these fossils, attributed to the family Etayoidae, is that of the holotype of Etayoa batacensis from the earliest Eocene of northern Colombia (Morón et al. 2013), which consists of an incomplete left mandible (Villarroel 1987). Interestingly, like Carodnia and Alcidedorbignya, Etayoa also shows an anterior extension of the masseteric fossa onto the body of the dentary, below the last molar (ch. 146), and a mandibular symphysis extending up to $\mathrm{p} 3$ posteriorly (ch. 159). It also shows a coronoid crest that is hypertrophied and laterally flaring with a very deep masseteric fossa posterior to it (ch. 144), which is a synapomorphy of Pantodonta and Carodnia in our unconstrained analysis (see supplementary data). The anatomy of other xenungulates like Etayoa may therefore provide some additional support for a relationship between Xenungulata and pantodonts, but the current fossil record of 


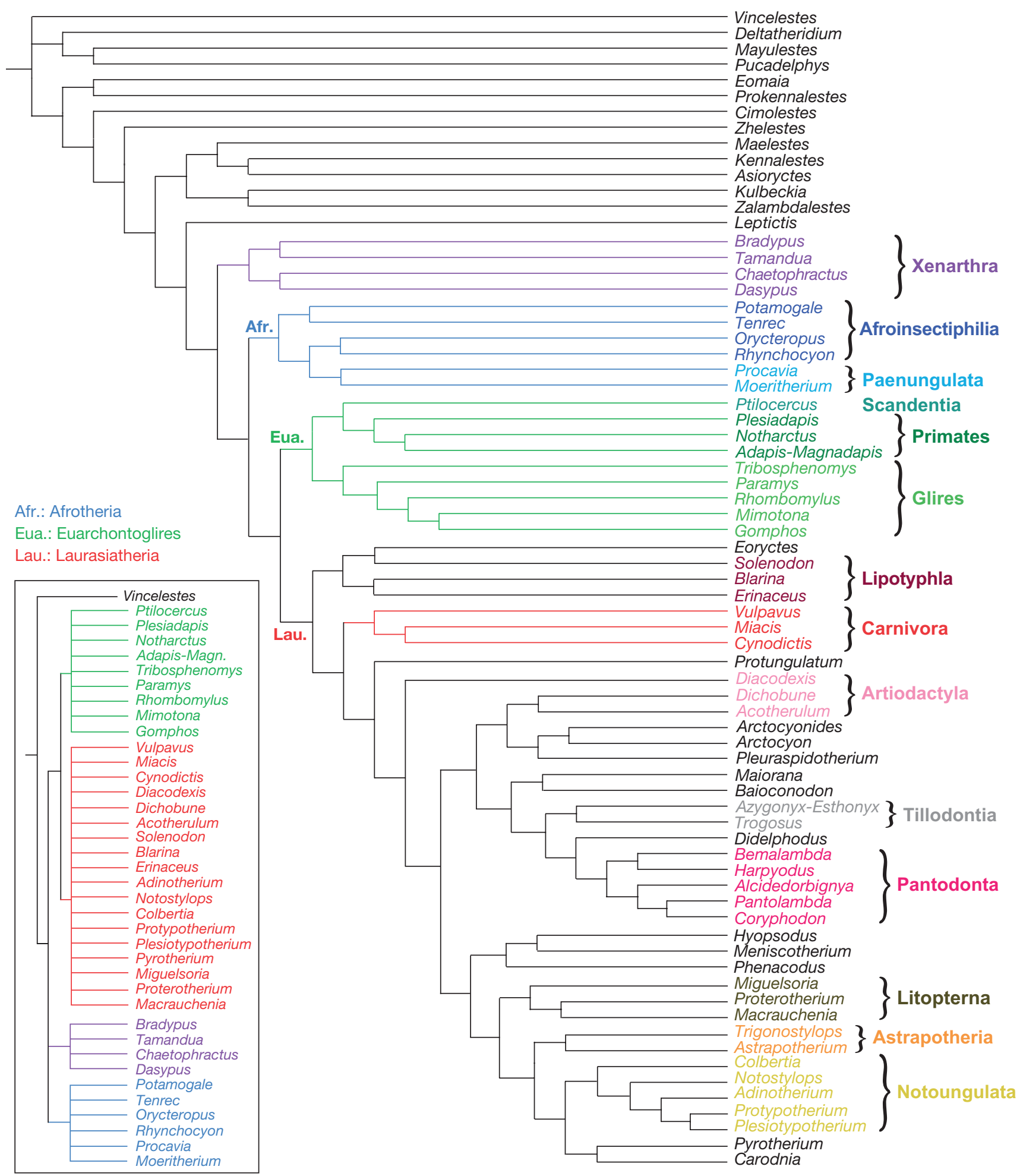

FIG. 122. - One of the 11 most parsimonious trees resulting from the constrained analysis of a data matrix of 70 taxa and 426 characters. Analysis was performed employing the program TNT (Goloboff et al. 2008) (see above in Material and methods). Given the high homoplasy index, it is clear that the very unstable trees resulting from this analysis must be taken with extreme caution, since their topology may be drastically modified with introduction or withdrawal of taxa or characters. The pattern of the molecular scaffold used for the constraint is shown in the lower left corner of the Figure.

the former group is too incomplete to provide robust tests of such hypotheses. In addition, homology hypotheses between the bilophodont upper molars of Carodnia and the more dilambdodont teeth of pantodonts such as Alcidedorbignya are difficult to establish so far, and derivation of the former dental type from the latter in that case seems a priori hardly conceivable. The absence of etayoid upper cheek teeth hinders progress on these questions for now. 
In addition to the hypotheses discussed above, other workers have regarded Xenungulata as taxa possibly derived from arctocyonid condylarths (Van Valen 1988), but this position has not been endorsed by later studies. Eventually, similarities have been noted between etayoid xenungulates and earlydiverging astrapotheres (Villarroel 1987; Cifelli 1993), but xenungulate-astrapothere relationships are not supported by our analyses. All these contrasting hypotheses and results demonstrate that much progress remains to be achieved, and in particular more complete early xenungulate fossils are needed in order to obtain more robust hypotheses of relationship for Xenungulata.

Potential paleobiogeographic and temporal implications on the history of Pantodonta. Independent of their potential relation with Xenungulata, the Pantodonta are otherwise mostly composed of northern taxa and/or nested in northern clades. In the unconstrained analysis, the genus Didelphodus from North America is the sister taxon of pantodonts, all of which, except Alcidedorbignya, originated on northern continents. In the constrained analysis, the pantodont clade is included within Laurasiatheria, which constitutes a novel and important result. Within Laurasiatheria, the Pantodonta are nested in different clades among the $11 \mathrm{CMPTs}$, but these are always composed of fossil taxa of exclusive northern origins (Eurasia or North America). All these patterns are congruent with a northern origin for pantodonts.

Both analyses support a clade of American pantodonts, with Pantolambda and Coryphodon being northern representatives and Alcidedorbignya (and possibly Carodnia) a southern one. In both analyses, Alcidedorbignya is the sister taxon of the clade that unites Pantolambda and Coryphodon. Parsimonious reconstruction of monomorphic ancestral areas (Springer $\mathrm{et} \mathrm{al}$. 2011) with Mesquite (Maddison \& Maddison 2009) argues for a North American origin for the clade Pantodonta on both the strict consensus of the unconstrained analysis and the CMPTs of the constrained analysis. Based on these arguments, it may be hypothesized that Alcidedorbignya issued from a dispersal of an early-diverging pantodont offshoot from North America into South America, which could have taken place by the earliest Palaeocene or Late Cretaceous before the record of Alcidedorbignya in Tiupampa. Indeed episodic connections between North and South America via island chains hypothesized to have existed during the Maastrichtian (Wilf et al. 2013) would have permitted such active dispersion of pantodonts in South America. Though the phylogenetic pattern of relationships within Pantodonta remains to be tested with a larger number of pantodont taxa, additional arguments exist that could support a North American origin for the offshoot that gave rise to the South American Alcidedorbignya. W. A. Clemens (personal communication) has brought to our knowledge an isolated upper molar (M1 or M2) that was discovered a few years ago in early Paleocene (Puerco 3) deposits of the Tullock Formation in Montana (from the Biscuit Springs locality in the Garbani Channel). This tooth is the basis for the citation as "Pantodont sp. A" by Wilson (2014). This molar closely resembles, in size and morphology, the upper molars of Alcidedorbignya although it clearly belongs to a different taxon. The Tiupampa fauna is assigned here an age slightly younger than Puerco 3, since it is now regarded as an equivalent of the base of Torrejonian 1 in North America (c. $65 \mathrm{Ma}$; see above, in the geological setting and age section). Therefore, if the Garbani channel tooth can actually be referred to an Alcidedorbignya-like pantodont, it may shed some light on the geographical origin of Alcidedorbignya. Because the dental anatomy of the upper molars of Alcidedorbignya clearly more closely resembles the Garbani Channel tooth than that of the oldest Chinese Pantodonta (e.g., Harpyodus, Bemalambda), it would be an indication that Alcidedorbignya is an early offshoot of the North American pantodont radiation rather than of the oldest Asiatic pantodonts. The age of the earliest Chinese pantodonts (Harpyodus and Bemalambda) is supposed to be late early Palaeocene (Torrejonian equivalent) (Qui \& Li 1977; Qui et al. 1977). If this estimated age is correct, then these Chinese pantodonts would be younger than the Garbani Channel tooth, which is likely to be the oldest known pantodont. Alcidedorbignya is clearly younger than the Garbani Channel tooth but possibly older than Harpyodus and Bemalambda. Finally, the fact that an Alcidedorbignya-like taxon is present in the Puerco 3 (c. $65.5 \mathrm{Ma}$; Sprain et al. 2014), i.e. c. 500 ka before Alcidedorbignya (but only c. $500 \mathrm{ka}$ after the K-Pg boundary; see Sprain et al. 2014) is a strong argument in favor of an occurrence of pantodonts in North America as soon as the very beginning of the Cenozoic, or that they were immigrants from Asia to North America. Therefore, it is possible that the origin of the order may have to be sought in the Late Cretaceous faunas of North America (as indicated by the parsimonious reconstruction of ancestral areas above) or Asia, if we consider the possibility of immigrants from Asia to North America.

Finally, the goal of our analyses was to test the existence of relationships between Alcidedorbignya and groups of endemic South American placentals, in order to explore the possibility that pantodonts may have had a longer and until now unrecognized descent in South America. Except for possible relationships with the xenungulates (see above), the analysis failed to recover any link to other South American ungulate groups or to Xenarthra. In the case of the constrained analysis, it is noteworthy that the CMPTs support a monophyletic Meridiungulata [as has been previously proposed by McKenna (1975) but based on paleobiogeography (Cifelli 1993; Rose 2006) rather than morphology] that is not related to Alcidedorbignya and Pantodonta, though both are found within Laurasiatheria.

Finally, concerning the much-debated divergence time estimations in placentals (e.g., Bininda-Emonds et al. 2012; Beck \& Lee 2014), the apical position of Alcidedorbignya in the unconstrained analysis, and even more in the constrained analysis, may be considered as an argument for a pre-K-Pg placental diversification, as this taxon, which diverges apically on our phylogeny, is dated at $65 \mathrm{Ma}$, shortly after the end of the Cretaceous (see above: geological setting and age section) (Figs 120-122). Furthermore, the fact that a possible pantodont is present in strata of the Puercan 3, just after the K-Pg boundary, would also favor the hypothesis that placental diversification took place in the Late Cretaceous. 


\section{GENERAL CONCLUSIONS}

Alcidedorbignya inopinata (Pantodonta, Placentalia, Eutheria) is the third species from the early Palaeocene mammal fauna of Tiupampa (Bolivia) to be thoroughly described on the basis of sub-complete skulls and skeleton. The other two are the metatherians Pucadelphys andinus (Marshall et al. 1995) and Mayulestes ferox (Muizon 1998). It is the first and only eutherian species of the Tiupampa fauna represented by skulls and skeleton discovered so far. The Tiupampa fauna is composed of at least 25 taxa and is the oldest mammalian assemblage of the Cenozoic of South America. It is the reference on which has been created the oldest Cenozoic South American Land Mammal age (SALMA), the Tiupampan (Ortiz-Jaureguizar \& Pascual 1989), which is regarded here as an equivalent of the base of the Torrejonian NALMA (Torrejonian 1) and assigned an age of approximately $65 \mathrm{Ma}$ (see above in geological setting and age section). Alcidedorbignya inopinata is the only South American representative of the Pantodonta (unless xenungulates are pantodonts, see above - phylogenetic analysis section - and below), an order of placentals otherwise known in the Palaeocene and Eocene of Laurasian continents. The oldest undisputed and wellknown North American pantodont is Pantolambda bathmodon from Torrejonian 2 and 3 (Lofgren et al. 2004). However, it is noteworthy that a single upper molar from deposits of the Tullock Formation in Montana referred to Puercan 3, exhibits morphological similarities with Alcidedorbignya ("Pantodont sp. A" of Wilson [2014]; W. A. Clemens, pers. comm.) and likely represents the oldest known pantodont so far. The earliest Asiatic pantodonts are Harpyodus spp. and Bemalambda spp., which are supposed to be late early Palaeocene (Torrejonian) in age (Qui \& Li 1977; Qui et al. 1977; Zhou et al. 1977). Therefore, according to the assessed ages of the earliest northern pantodonts, Alcidedorbignya is older than Pantolambda and therefore one of the earliest known pantodonts. Furthermore, considering the completeness and/or the quality of preservation of the two specimens described in this paper, Alcidedorbignya is undoubtedly also the best-known pantodont in terms of, both, cranial and postcranial anatomy. For that matter, given the scarcity of sub-complete skulls and skeletons from the basal Palaeocene (Puercan), the specimens of Alcidedorbignya here described are probably the most complete and best-preserved therian mammal remains from this critical interval of the early Cenozoic, which corresponds to a major diversification of the placentals. Although placentals are likely to have started their diversification well before the beginning of the Cenozoic (Bininda-Emonds et al. 2012; Dos Reis et al. 2012), no well-preserved placental skulls and skeletons have been recognized in the fossil record predating the K-Pg boundary. In this context the extraordinarily complete remains of Alcidedorbignya described here represent a welcome glimpse not only into early evolutionary history of pantodont, but also into the anatomy of early placentals. Indeed, several aspects of the basicranium of Alcidedorbignya (e.g., the basicranial circulation pattern) are similar to that of afroinsectiphile tenrecids (e.g., Tenrec, Potamogale), although both taxa have no close relationships within placentals. Therefore, these similarities are probably the result of common sharing of plesiomorphic characters, and may represent an indication that Alcidedorbignya may approach a relatively plesiomorphic morphology within placentals. In this connection, basicranial circulation of tenrecids retains several plesiomorphic features (Asher 2001), and that of Alcidedorbignya may be interpreted as extremely plesiomorphic when compared to the primitive eutherian pattern of basicranial circulation hypothesised by Wible (1993). If our interpretations are correct, Alcidedorbignya retains many plesiomorphic features of the basicranial circulation: superior, inferior, and posterior rami of the stapedial artery; mandibular ramus possibly originating from the ramus inferior of the stapedial artery; entry of the internal carotid artery in the braincase medial to the apex of the cochlea; ramus superior of the stapedial artery emitting anteriorly an orbitotemporal artery and posteriorly an arteria diploëtica magna; rami temporalis being emitted by the latter two arteries; transverse sinus supplying three major veins, the capsuloparietal emissary vein, the sigmoid sinus, and the superior petrosal sinus; capsuloparietal emissary vein emitting anteriorly an orbitotemporal vein and posteriorly a vena diploëtica magna (Wible 1993, Asher 2001, Wible et al. 2004, 2009). However, some features of the basicranial circulation of Alcidedorbignya are also likely to be derived for eutherians: opening of the carotid foramen in a distinct notch of the basisphenoid at the posterolateral angle of the bone, not in a foramen piercing the bone; ramus inferior of the stapedial artery entering the skull via the alisphenoid canal and not through the pyriform fenestra (endocranial course) (Kielan-Jaworowska 1981, Rougier et al. 1992; Wible \& Rougier 2000; Wible et al. 2004, 2009) or not entering the skull (extracranial course) (Wible 1993; Asher 2001; Wible et al. 2004). Therefore, and keeping in mind its derived traits, it appears that the impact of the cranial anatomy of Alcidedorbignya extends well beyond the order Pantodonta and presents essential elements for understanding the early pattern of placental cranial anatomy. As such, it widely corroborates the hypothetical reconstruction of the ancestral eutherian basicranial circulation as proposed by Wible (1987, 1993; see also MacPhee 1981; Wible et al. 2004, 2009).

The comparison of the endocast of Alcidedorbignya to that of tenrecids (e.g., Tenrec, Potamogale) also reveals many similarities, and the brain morphology of Alcidedorbignya as hypothesized from its endocast, indicates that these genera clearly share a plesiomorphic pattern [as hypothesised by Kaas (2009)]. Among others, the large size of the olfactory bulbs not overlapped by the cerebral hemispheres, the reduced neocortex with a high rhinal fissure, the rectilinear brain lacking a flexure, and the midbrain and colliculi widely visible in dorsal view of the brain with no overlap by the cerebral hemispheres, are clues for the plesiomorphic condition of the brain of Alcidedorbignya. This condition reinforces the idea that the cranial anatomy of Alcidedorbignya may represent a model that can enlighten the understanding of cranial anatomy in the ancestral placental. 
The postcranial skeleton of Alcidedorbignya is relatively generalised with complete manus and pes and a plantigrade morphology, which indicates a moderately agile animal. However, some features of the scapula, humerus, and ulna are suggestive of potential arboreality or at least ability to climb. This condition raises the problem of the plesiomorphy of arboreality as hypothesised by Matthew (1904). Arboreality (or at least characters compatible with climbing, i.e. scansorial) is commonly encountered in early mammals (e.g., Haines 1958; Jenkins \& Parrington 1976; Rose 1987; Krebbs 1991; Muizon 1998; Ji et al. 2002; Muizon \& Argot 2003; Luo et al. 2003, 2011; Goswami et al. 2011; Argot 2013). However, several Late Cretaceous mammals (multituberculates, metatherians, and eutherians), from Mongolia have been regarded as fully terrestrial, which is probably related to the very arid environment in which they lived (Kielan-Jaworowska 1977, 1978; Kielan-Jaworowska \& Gambaryan 1994; Szalay \& Trofimov 1996). Furthermore, discoveries during the last two decades, especially in China, have provided evidence that Mesozoic mammals have experimented with very diverse types of ecological behaviour (including locomotion) as synthesised by Luo (2007). But it is also noteworthy that, concerning mammals from the Late Cretaceous or Palaeocene, very few taxa are represented by sufficiently well-preserved specimens with reasonably complete postcranial remains to evaluate potential scansorial habits or even simple climbing abilities. Interestingly, Alcidedorbignya, one of the oldest pantodonts, was probably partly arboreal, while the younger Pantolambda and Bemalambda were not; and the later taxa such as Titanoides, Barylambda and Coryphodon, because of their much larger size, were likely confined to the ground. Furthermore, Goswami et al. (2011) in a study of late Cretaceous mammals from India, suggested that scansoriality and arboreality were prevalent early in eutherian evolution. A similar conclusion is also reached by Luo et al. (2011). Moreover, as a result of their phylogenetic analysis of placentals, O'Leary et al. (2013) concluded that the common ancestor of placentals was scansorial. Therefore, these independent results provide credit to Matthew's hypothesis and to our interpretation of scansorial habits in Alcidedorbignya.

Another interesting aspect of Alcidedorbignya is its geographical location. It is the only South American pantodont and, given the known distribution of the representatives of the order, in the northern continents, its presence in South America seems incongruous, especially considering that it is likely to be the second oldest known pantodont (taking into account the undetermined pantodont molar from the Puerco 3 of Montana). The locality of Tiupampa has yielded several other taxa of eutherians, notably a number of "condylarths" belonging to the same radiation: the Kollpaniinae, a clade probably related to the North American Mioclaenidae (Muizon \& Cifelli 2000). This interpretation was not accepted by Williamson \& Carr (2007), who did not suggest, however, an alternative interpretation. Whether or not the Kollpaniinae have close affinities with the Mioclaenidae, they nonetheless exhibit remarkably similar morphologies, especially with the mioclaenids Promioclaenus and Bomburia (probably including Platymastus). Furthermore, the specimens of Bomburia (including Platymastus) illustrated by Williamson \& Carr (2007: figs 1, 2) are so similar to the Tiupampa "condylarths" that, if discovered at Tiupampa, they would have easily been included in a new kollpaniine genus. Therefore, the new data provided by Williamson \& Carr (2007) reinforce the similarities existing between the Kollpaniinae and the North American Mioclaenidae, similarities that are greater than with any other group of North American "condylarths", and emphasize the idea of a North American origin of these South American "condylarths". Furthermore, recent analyses of ancient protein sequencing in Pleistocene litopterns and notoungulates show relationships with the clade Perissodactyla (Welker et al. 2015), which is exclusively known on northern continents during the Paleogene. Because kollpaniines have been allied to litopterns based on their dental morphology (Muizon \& Cifelli 2000), the proteomics finding is thus also in good agreement with a relationship of kollpaniines with northern "condylarths". In this context, it is likely that both Tiupampa's pantodont and kollpaniines issued from migrations of North American relatives to South America during ephemeral land connections through the Antillean volcanic arc system, which existed between the Americas during the latest Cretaceous or earliest Palaeocene (IturraldeVinent 2006; Wilf et al. 2013).

Relationships of the kollpaniines with the didolodontids and litopterns have been suggested by Muizon \& Cifelli (2000). Because so far the origin of the other four groups of South American "ungulates" remains unclear (at least on the morphological point of view concerning notoungulates), it was logical to investigate the phylogenetic proximity of Alcidedorbignya to groups of endemic South American eutherians. The parsimony analyses performed, either unconstrained or constrained, were disappointing, since they highlighted a high level of homoplasy in the dataset. However, the results, although weakly conclusive and to be treated carefully, were nevertheless of interest. First of all, it is noteworthy that half of the morphological similarities present in the datamatrix are subject to homoplasy. Significantly no close relationship was found between Alcidedorbignya and other South American mammals, although a potential affinity was evidenced with the Xenungulata as indicated by the unconstrained analysis. This result was not obtained with the constrained analysis, but it is noteworthy that cranial remains of xenungulates are limited mainly to teeth, and the analyses rest only on dental, mandibular and postcranial characters. This result of course may be drastically modified with the discovery of well-preserved cranial remains of early xenungulates such as Etayoa. With regard to pantodont affinities within Eutheria, the two analyses placed them in the Placentalia, and the constrained analysis indicates a close relationship with the tillodonts within Laurasiatheria, which was not the case in the unconstrained analysis. Another interesting potential result of the constrained analysis (Fig. 120) is the place- 
ment in the same clade of the five groups of South American "ungulates" with some North American "condylarths" and artiodactyls (see slightly different results in Fig. 121). This clade, in turn, is the sister group of another clade including pantodonts, Didelphodus, tillodonts, and some North American and European "condylarths". In turn, this interpretation provides a new life to the Meridiungulata of McKenna (1975), which unites in the same clade the five orders of South American ungulates, although this result should be considered cautiously in light of the high degree of homoplasy present in the analysis. Moreover, these results question the level of reliability of analyses of morphological matrices focused on basal and distant radiations with a high taxonomic diversity, which generally result in a high level of homoplasy ( $\mathrm{HI} \geq 0.5)$, as observed here. It is clear that the very unstable trees resulting from analyses of such datasets, where homoplasy is strongly present, must be taken with extreme caution since their topology may be drastically modified with introduction or withdrawal of taxa or characters and would require further investigation and comparison with molecular patterns.

\section{Author contributions}

$\mathrm{CM}$ organized the field expeditions during which the specimens described here were discovered (except MHNC 8399, which was discovered by R. Cespedes-Paz). CM prepared all the specimens and designed and started the research. CM and GB wrote the description of most of the skull anatomy - except for the bony labyrinth of the inner ear (GB and $\mathrm{CM})$, the endocast and nasal cavity (SL and $\mathrm{CM})-, \mathrm{CM}$ and $\mathrm{CA}$ wrote the description of the postcranial skeleton and associated functional morphology, $\mathrm{CM}, \mathrm{GB}, \mathrm{SL}$ wrote the anatomical comparison section, GB, CM and SL performed, discussed, and wrote the phylogenetic analysis section. FG and $\mathrm{CM}$ worked on $3 \mathrm{D}$ reconstructions of the skeleton and skull provided in this paper.

\section{Acknowledgements}

The Tiupampa locality was discovered during a field expedition funded by the National Geographic Society (grant 2467/82). Field expeditions during which the specimens mentioned in this paper were recovered (including the skeleton MHNC 8372), were made possible by five other grants of the NGS (2908/84, 3381/86, 6296/98, 7109/01, $9394 / 13)$. Funds for fieldwork were also provided by the Institut français d'Études andines (Lima, Peru) and by the Muséum national d'Histoire naturelle (Paris, France). Fossil collecting was carried out under the auspices of research agreements between the Museo de Historia Natural Alcide d'Orbigny of Cochabamba (Bolivia) and the Muséum national d'Histoire naturelle (France). All the specimens collected are the property of the MHNC and were provided on loan to the MNHN for curation and publication. Field expeditions have benefited of logistical support from the IDR (Institut de Recherche pour le Dévelopement) in Bolivia.
We thank our Bolivian colleagues (Ricardo Céspedes-Paz, Ramiro Suarez-Sorruco, Mario Suarez-Riglos) for their collaboration and logistic support during all the field seasons. Special thanks are due to Michael Novacek, Jin Meng, Ruth O'Leary, Judith Galkin, and Suzanne Goldberg (American Museum of Natural History), Sara Lyons (National Museum of Natural History, Smithsonian Institution), Daniel Brinkman and Christopher Norris (Yale Peabody Museum) Philip Gingerich (Museum of Paleontology, University of Michigan), Richard Cifelli (Oklahoma Museum of Natural History), for access to the specimens under their care and for their understanding in the loans of specimens. Céline Bens, Géraldine Véron, Jacques Cuisin, Christine Lefèvre, Luc Vives (MNHN) provided access to MNHN specimens under their care. We are indebted to Spencer Lucas (New Mexico Museum of Natural History) for providing photos of the holotype of Harpyodus decorus and copies of chapters of his unpublished dissertation on the genus Coryphodon. Our warmest and most gratefull thanks are due to R. L. Cifelli and K. D. Rose, who accepted the monstrous task of reviewing such a long manuscript. Their unvaluable comments and suggestions have allowed to greatly improve our manuscript. This paper has also benefited from fruitful discussions on various aspects of mammalian evolution with Eli Amson, Robert Asher, William Clemens, Fabrice Fack, Emmanuel Gheerbrant, Maeva Orliac (who provided CT scans of several fossil artidactils), and Gregory Wilson. Photographs were made by Philippe Loubry and Lilian Cazes and illustrations by Charlène Letenneur and Justine Jacquot-Haméon. Thanks are due to Jean Dejax, who provided advice on the paleoflora for the life reconstruction of Alcidedorbignya by Justine Jacquot-Haméon. CT scanning of the specimens was performed by Miguel Garcia at the AST-RX technical platform of scientific access to RX tomography (UMS 2700 "outils et méthodes de la systématique intégrative”, CNRS-MNHN, Paris) and by Peter Göddertz at the Steinmann Institut für Geologie, Mineralogie und Paläontologie, Universität Bonn, Germany. Data processing was undertaken at the $3 \mathrm{D}$ imaging facilities Lab of the UMR 7207 CR2P (MNHN CNRS UPMC-Paris6). Special thanks are due to Gaétan Langlois and Fabrice Escalier (3D WEAVE) and Frenchcinema4D online community for their invaluable advice on the Cinema4D reconstructions. GB also acknowledges past financial support from the Alexander von Humboldt Foundation for some scans used in this research with further thanks due to Thomas Martin at Universität Bonn, Germany.

\section{ADDENDUM}

When this monograph was almost at the end of the editorial process, two of us (GB and CM) discovered a third partial skull (Fig. 123) and skeleton of Alcidedorbignya inopinata in the context of a field expedition (October-November 2015) funded by the National Geographic Society (Grant no. 9394/13). The specimen is from the Santa Lucia For- 
mation beds in the Vila Vila outcrop but does not come from the site called "the quarry" at Tiupampa, which has yielded most of the specimens of the Tiupampa fauna.

The new pantodont specimen comes from another site (called Criadero de Loro) located approximately $1 \mathrm{~km}$ southward as the crow flies of the "the quarry" at Tiupampa. Direct observations on the field evidences that both fossil-bearing sites are found within a similar layer of pink sandstones located below a hardened reference layer containing a wealth of large fragmentary fossil specimens mostly belonging to crocodiles and turtles. In addition, aerial satellite images of the area also permit to follow the outcrops of geological layers between the two sites thanks to the different colorations each of them has. The continuity observed for the pink sandstone outcrop confirms that both sites are located in the same stratigraphic layer and, except for some possible lateral variation of layer thickness, they most probably come from a similar level within it.

The specimen (MHNC 13931) was lying on its right side and most of the left half of the skull, the occipital condyles, and the left mandible have been weathered off. Numerous elements of the postcranial skeleton were associated to the skull. The latter was immediately prepared by one of us (CM) at the "Museo de Historia Natural de Cochabamba" in order to include this important specimen in this monograph. The skull of MHNC 13931 does not significantly differ from the other two skulls (MHNC 8372 and 8399) described above except in its much larger size and a few other features discussed below.

The length of the skull of MHNC 13931 from the tip of the premaxilla to posterior end of the nuchal crest is $83.7 \mathrm{~mm}$, while in the other skulls this measurement is $57.75 \mathrm{~mm}$ in MHNC 8372 and $66.56 \mathrm{~mm}$ in MHNC 8399. MHNC 13931 is thus $31 \%$ longer than MHNC 8372 and $20 \%$ longer than MHNC 8399. Such a significant size difference could be explained by the fact than MNHC 13931 could belong to a new species of Alcidedorbignya. However, because the three specimens where discovered roughly in the same stratigraphic horizon and probably belong to the same faunal assemblage, it is unlikely that two species so similar morphologically and ecologically (dental morphology) could have been coeval.

This size difference could instead be explained by sexual dimorphism, MHNC 13931, being a male, larger than MHNC 8372 and 8399, which have been regarded above as females. The main feature, which has been related above to sexual dimorphism is the height of the maxilla from the anterolabial angle of M1 to anterior end of jugal. This measurement varies from 7 to $8.92 \mathrm{~mm}$ in a sample of eight specimens regarded as females and from 10.1 to $11.6 \mathrm{~mm}$ in a sample of 6 specimens referred to males. The measurement in MHNC 13931 is $10.09 \mathrm{~mm}$, which corresponds to that observed in the inferred males. Two other features have been related above to sexual dimorphism; these are the mesiodistal length vs the labiolingual width of the section of the upper canine at the alveolar border and the height vs the labiolingual width of the upper canine. The measurement of the section of the upper canine of MHNC 13931 are: $\mathrm{L}=4.9 \mathrm{~mm}$ and $\mathrm{W}=3.26 \mathrm{~mm}$ and the height of the canine is $10.12 \mathrm{~mm}$. These measurements, when placed on the scatter plots of Figure $7 \mathrm{C}, \mathrm{D}$, provide a position of MHNC 13931 distinctly closer to the specimens interpreted here as males than to those regarded as females. Furthermore, it is noteworthy that the canine of MHNC 13931 bears a large wear facet on its anterior edge and apex and, therefore, the actual height of the unworn canine was probably greater than that measured. Therefore, in spite of the small sample on which this dimorphism is hypothesized, the morphology of the upper canine of MHNC 13931 is also compatible with a referral of this specimen to a male. Therefore, MHNC 13931 is regarded here as the first known skull of a male of Alcidedorbignya inopinata. Given this interpretation, the size of the skull is also regarded here as a dimorphic character, being c. 20\% larger in males. The greater difference observed with MHNC $8372(31 \%)$ is probably related to the fact that this specimen is that of a young adult, the rostrum of which may not have reached its full-adult length yet.

Two other features of MHNC 13931 are also interpreted here as probably related to sexual dimorphism in Alcidedorbignya inopinata. They are, the greater elevation of the sagittal crest and the greater posterior projection of the nuchal crest as well as the larger size of the angular process of the dentary, as compared to MHNC 8372 and 8399, which are regarded as female individuals. Among Recent mammals, the sagittal crest and nuchal crests, when present, are generally more developed in males than in females. This is true for instance in most carnivores, carnivorous marsupials, and primates. As a matter of fact in these mammals the upper canine is also a sexually dimorphic character. The use of a large canine is related to the masticatory muscles (e.g., temporalis muscle, which attaches in part on the lateral edge of the sagittal crest and on the anterodorsal face of the nuchal crest) and to the neck muscles, which attach on the posteroventral side of the nuchal crest. On the angular process also attach the pterygoideus medialis (medial side) and the masseter superficialis (lateral side). These muscles are masticatory muscles and are probably also related to the size of the upper canine. Therefore, it is possible that, besides the larger size of the skull, a set of functionally related characters of Alcidedorbignya inopinata was sexually dimorphic, including the size and robustness of the canine, the size of the sagittal and nuchal crests, and the size of the angular process of the condyle (though allometric processes may be involved as well). The discovery of the new specimen (MHNC 13931) thus provides further supports to the presence of a distinct sexual dimorphism in Alcidedorbignya inopinata. The fact that a sexual dimorphism has also been observed in a population of the metatherian Pucadelphys andinus (Ladevèze et al. 2011) from Tiupampa, given the age of the locality (basal Palaeocene $-c .65 \mathrm{Ma}$ ), could be an indication that sexual dimorphism was a widespread condition for early diverging eutherians and metatherians. 


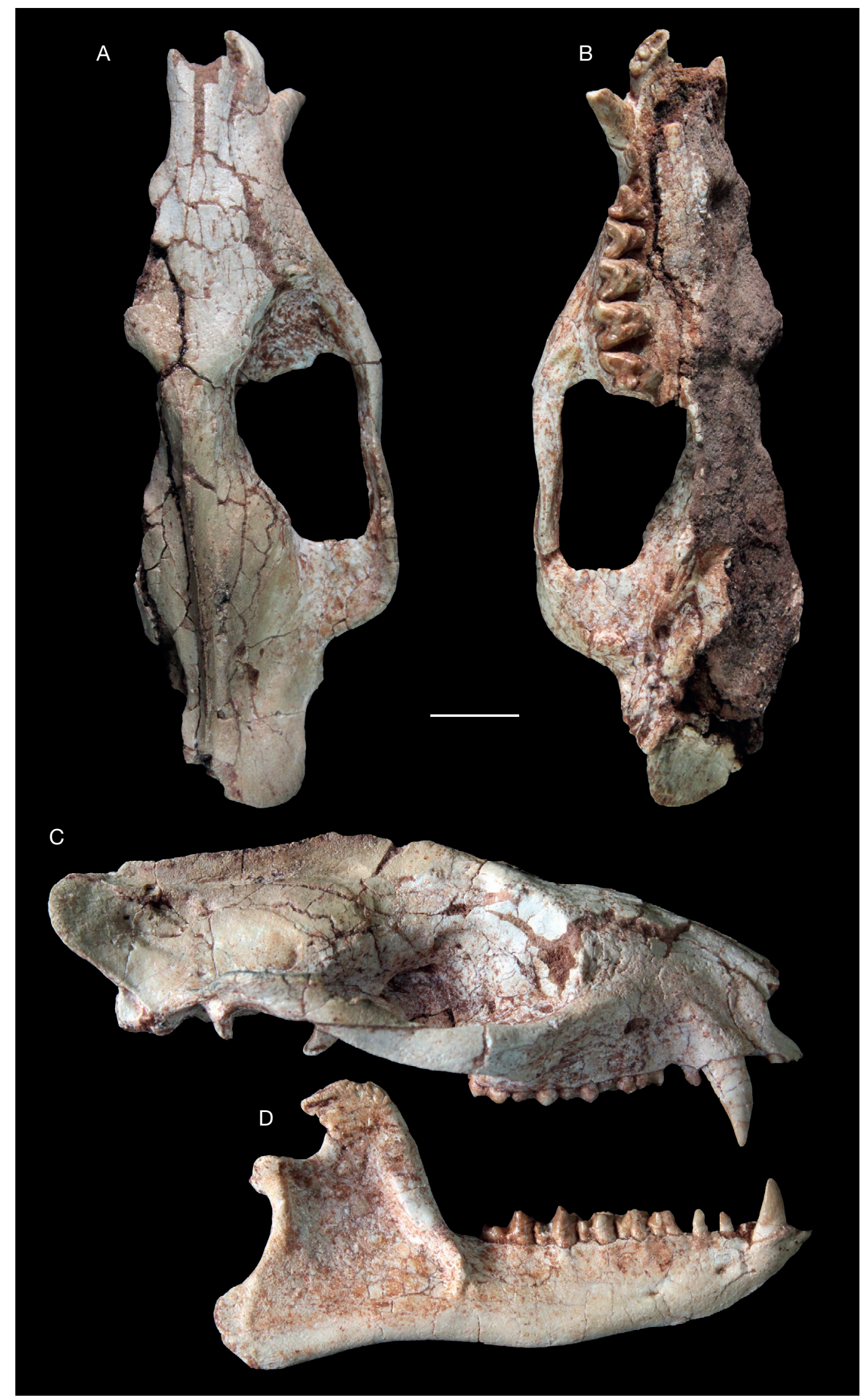

FIG. 123. - Partial skull of Alcidedorbignya inopinata (MHNC 13931): A, dorsal view; B, ventral view; C, right lateral view; $\mathbf{D}$, right lateral view of the mandible. Scale bar: $1 \mathrm{~cm}$. 


\section{REFERENCES}

Agnolín F. L. \& Chimento N. R. 2011. - Afrotherian affinities for endemic South American "ungulates". Mammalian Biology 76: 101-108.

ANdreWs C. W. 1906. - A Descriptive Catalogue of the Tertiary Vertebrata of the Fayum, Egypt. British Museum of Natural History, London, $324 \mathrm{p}$.

Antoine P.-O., Billet G., Salas-Gismondi R., Lara J. T., Baby P., Brusset S. \& Espurt N. 2015. - A new Carodnia Simpson, 1935 (Mammalia, Xenungulata) from the early Eocene of Northwestern Peru and a phylogeny of xenungulates at species level. Journal of Mammalian Evolution 22 (2): 129-140.

Archibald J. D. \& Averianov A. O. 2003. - The Late Cretaceous placental mammal Kulbeckia. Journal of Vertebrate Paleontology 23: 404-419.

ArChibald J. D. \& Averianov A. O. 2005. - Mammalian faunal succession in the Cretaceous of the Kyzlkum Desert. Journal of Mammalian Evolution 12: 9-22.

ArChibald J. D. \& Averianov A. O. 2006. — Late Cretaceous asioryctitherian eutherian mammals from Uzbekistan and phylogenetic analysis of Asioryctitheria. Acta Palaeontologica Polonica 51 (2): 351-376.

Archibald J. D., Averianov A. O. \& Ekdale E. G. $2001-$ Late Cretaceous relatives of rabbits, rodents, and other extant eutherian mammals. Nature 414: 62-65.

ARGOT C. 2001. - Functional-adaptive anatomy of the forelimb in the Didelphidae, and the paleobiology of the Paleocene marsupials Mayulestes ferox and Pucadelphys andinus. Journal of Morphology 247: 51-79.

ARGOT C. 2002. - Functional-adaptive anatomy of the hindlimb of extant marsupials, and the paleobiology of the Paleocene marsupials Mayulestes ferox and Pucadelphys andinus. Journal of Morphology 253: 76-108.

ARGOT C. 2003. - Functional-adaptive anatomy of the axial skeleton of some extant marsupials, and the paleobiology of the Paleocene marsupials Mayulestes ferox and Pucadelphys andinus. Journal of Morphology 255: 279-300.

ARGOT C. 2013. - Postcranial analysis of a carnivoran-like archaic ungulate: the case of Arctocyon primaevus (Arctocyonidae, Mammalia) from the late Paleocene of France. Journal of Mammalian Evolution 20 (2): 83-114.

AsHer R. J. 2001. - Cranial anatomy in tenrecids insectivorans: character evolution across competing phylogenies. American Museum Novitates 3352: 1-54.

Asher R. J. \& LEHMANN T. 2008. - Dental eruption in afrotherian mammals. BMC Biology 6 (14): 1-11.

Asher R. J. \& Olbricht G. 2009. - Dental ontogeny in Macroscelides proboscideus (Afrotheria) and Erinaceus europeus (Lipotyphla). Journal of Mammalian Evolution 16: 99-115.

Asher R. J., NovaceK M. J. \& Geisler J. H. 2003. — Relationships of endemic African mammals and their fossil relatives based on morphological and molecular evidence. Journal of Mammalian Evolution 10 (1-2): 131-194.

Asher R. J., Meng J., Wible J. R., McKenna M. C., Rougier G.W, Dashzeveg D. \& NovaceK M. J. 2005. - Stem Lagomorpha and the antiquity of Glires. Science 303: 1091-1094.

Asher R. J., Bennett N. \& Lehmann T. 2009. - The new framework for understanding placental mammal evolution. Bioessays 31 (8): 853-864.

Babot J., García López D. A. \& Gaudin T. J. 2012. - The most ancient xenarthran petrosal: morphology and evolutionary significance. Journal of Vertebrate Paleontology 32 (5): 1186-1197.

BARONE R. 1997. - Anatomie comparée des mammiferes domestiques. Tome 1, Ostéologie. Vigot, Paris, 853 p.

BARONE R. 2000. - Anatomie comparée des mammiferes domestiques. Tome 2, Arthrologie et myologie. Vigot, Paris, 1066 p.
Bastos A. C. F. \& Bergqvist L. P. 2007. - A postura locomotora de Protolipterna ellipsodontoides Cifelli, 1983 (Mammalia: Litopterna: Protolipternidae) da bacia de São José de Itaboraí, Rio de Janeiro (Paleoceno superior). Anuário do Instituto de Geociências 30 (1): 52-60.

BAUCHOT R. \& STEPHAN H. 1967. - Encéphales et moulages endocrâniens de quelques insectivores et primates actuels, in Problèmes actuels de Paléontologie (Évolution des Vertébrés), Colloques Internationaux du Centre national de la Recherche scientifique. Paris, France, 6-11 June 1966. Éditions du Centre national de la Recherche scientifique 163: 575-586.

Beck R. M. \& LeE M. S. 2014. - Ancient dates or accelerated rates? Morphological clocks and the antiquity of placental mammals. Proceedings of the Royal Society of London B: Biological Sciences, 281 (1793): 20141278.

BERGQVIST L. P. 1996. - Reassociação dos pós-crânio às espécies de ungulados da bacia de S. J. de Itaboraí (Paleoceno), Estado do Rio de Janeiro, e filogenia dos "Condylarthra" e ungulados sulamericanos com base no pós-crânio. PhD Dissertation, universidade Federal do Rio Grande do Sul.

Bergqvist L. P., Furtado M. R., Souza C. P. \& Powell J. E. 2007. - Colbertia magellanica (bacia de Itaboraí, Brasil) e Colbertia lumbrerense (Grupo Salta, Argentina): a morfologia pós-craniana confrontada, in CARVALHO I. S., CASSAB R. C. T., Schwanke C., Carvalho M. A., Fernandes A. C. S., Rodrigues M. A. C., Carvalho M. S. S., Arai M. \& Oliveira M. E. Q. (eds), Paleontologia: Cenários de Vida. Vol. 1, Editora Interciência: 765-775.

Billet G. 2010. - New observations on the skull of Pyrotherium (Pyrotheria, Mammalia) and new phylogenetic hypotheses on South American ungulates. Journal of Mammalian Evolution 17: 21-59.

BiLlet G. 2011. - Phylogeny of the Notoungulata (Mammalia) based on cranial and dental characters. Journal of Systematic Paleontology 9 (4): 481-497.

Billet G. \& MARTIN T. 2011. — No evidence for an afrotherianlike delayed dental eruption in South American notoungulates. Naturwissenschaften 98: 509-517.

Billet G. \& Muizon C. DE 2013. - External and internal anatomy of a petrosal from the late Paleocene of Itaboraí, Brazil, referred to Notoungulata (Placentalia). Journal of Vertebrate Paleontology 33: 455-469.

Billet G., Muizon C. De \& Mamani B. 2008. - Late Oligocene mesotheriids (Mammalia, Notoungulata) from Salla and Lacayani (Bolivia): implications on stem mesotheriids phylogeny and distribution. Zoological Journal of the Linnean Society 152: 153-200.

Billet G., Muizon C. De \& Patterson B. 2009. - Craniodental anatomy of late Oligocene archaeohyracids (Mammalia, Notoungulata) from Bolivia and Argentina and new phylogenetic hypotheses. Zoological Journal of the Linnean Society 155: 458-509.

Billet G., Hautier L., Muizon C. de \& Valentin X. 2011. - Oldest cingulate skulls provide congruence between morphological and molecular scenarii on armadillo's evolution. Proceedings of the Royal Society B 278: 2791-2797.

Billet G., Hautier L., Asher R. J., Schwartz C., CrumpTON N., MARTin T. \& RUf I. 2012. - High morphological variation of vestibular system accompanies slow and infrequent locomotion in three-toed sloths. Proceedings of the Royal Society B 279: 3932-3939.

Billet G., Germain D., Ruf I., Muizon C. DE \& Hautier L. 2013. - The inner ear of Megatherium and the evolution of the vestibular system of sloths. Journal of Anatomy 223 (6): 557-567.

Billet G., Muizon C. DE, SChellhorn R., RuF I., Ladevèze S. \& BERGQVIST L. 2015. - Petrosal and inner ear anatomy and allometry among specimens referred to Litopterna (Placentalia). Zoological Journal of the Linnean Society 173: 956-987. 
Bininda-Emonds O. R., Beck R., \& MacPhee R. D. 2012. Rocking clocks and clocking rocks: A critical look at divergence time estimation in mammals, in ASHER R. J. \& MÜLLER J. (eds), From Clone to Bone: The Synergy of Morphological and Molecular Tools in Palaeobiology. Cambridge University Press, New York: 38-82.

Bloch J. I. \& Boyer D. M. 2007. — New skeletons of PaleoceneEocene Plesiadapiformes: a diversity of arboreal positional behaviors in early primates, in DAgOsTO M. \& RAVOSA M. J. (eds), Primate Origins: Adaptations and Evolution. Plenum Press, New York: 535-581.

Bloch J. I., Rincón A. F., Head J. J., Herrera F. \& Jaramillo C. A. 2012. - Early Eocene mammals from the hot tropics of Northern South America. Journal of Vertebrate Paleontology 32 (Suppl. to 3): 64.

Bonaparte J. \& Rougier G. 1987. — Mamíferos del Cretácico Inferior de Patagonia. IV Congreso Latinoamericano de Paleontología, Santa Cruz, Bolivia 1: 343-359.

Bonaparte J. F., Van Valen L. M., \& Kramartz A. 1993. - La fauna local de Punta Peligro, Paleoceno inferior, de la Provincia del Chubut, Patagonia, Argentina. Evolutionary monographs 14: 1-61.

Bond M. 1981. - Un nuevo Oldfieldthomasiidae (Mammalia, Notoungulata) del Eoceno inferior (Fm Lumbrera, Grupo Salta) del NW argentino, in Congresso Latino-Americano de Paleontologia, Anais, 2, Porto Alegre: 521-536.

Boyer D. M., Seiffert E. R. \& Simons E. L. 2010. — Astragalar morphology of Afradapis, a large Adapiform primate from the earliest late Eocene of Egypt. American Journal of Physical Anthropology 143: 383-402.

Bryden M. M. 1971. - Myology of the Southern elephant seal Mirounga leonine (L.), in BURT W. H. (ed.), Antarctic Pinnipedia. American Geophysics Union, Washington DC. Antarctic Research Series 18: 109-140.

BUCKLEY M. 2015. - Ancient collagen reveals evolutionary history of the endemic South American 'ungulates'. Proceedings of the Royal Society of London B: Biological Sciences 282 (1806): 20142671

BuTler P. M. 1956. - The skull of Ictops and the classification of Insectivora. Proceedings of the Royal Society of London 126: 453-481.

Cardini A. \& Polly P. D. 2013. - Larger mammals have longer faces because of size-related constraints on skull form. Nature communications 4 (2458): http://dx.doi.org/10.1038/ ncomms3458

Cartmill M. 1974. - Pads and claws in arboreal locomotion, in Jenkins F. A. JR (ed.), Primate Locomotion. Academic Press, New York: 45-83.

Casinos A. 1996. - Bipedalism and quadrupedalism in Megatherium: an attempt at biomechanical reconstruction. Lethaid 29 (1): 87-93.

ChOw M. C. \& WANG B. 1979. - Relationship between the pantodonts and tillodonts and classification of the order Pantodonta. Vertebrata PalAsiatica 17: 37-48.

Ciancio M. R., Castro M. C., Galliari F. C., Carlini A. A. \& AsHer R. J. 2012. - Evolutionary implications of dental eruption in Dasypus (Xenarthra). Journal of Mammalian Evolution 19: 1-8.

Cifelli R. L. 1982. — The petrosal structure of Hyopsodus with respect to that of some other ungulates, and its phylogenetic implications. Journal of Paleontology 56 (3) 795-805.

Cifelli R. L. 1983a. - Eutherian tarsals from the late Paleocene of Brazil. American Museum Novitates 2761: 1-31.

Cifelli R. L. 1983b. - The origin and affinities of the South American Condylarthra and early Tertiary Litopterna (Mammalia). American Museum Novitates 2772: 1-49.

CIFELLI R. L. 1993. - The phylogeny of the native South American ungulates, in Szalay F. S., NovaceK M. J. \& McKenna M. C. (eds), Mammal Phylogeny, Vol. 2: Placentals. Springer Verlag, New York: 195-216.

Conroy G. C. 1976. — Primate postcranial remains from the Oligocene of Egypt. Contributions to Primatology 8: 1-134.

COPE E. D. 1875. - Systematic catalogue of Vertebrata of the Eocene of New Mexico, collected in 1874. Geographical exploration and survey West of 100 th Meridian, 1-37.

Cope E. D. 1877a. - On the brain of Coryphodon. Proceedings of the American Philosophical Society 16 (99): 616-620.

Cope E. D. 1877b. - Report upon the extinct Vertebrata obtained in New Mexico by parties of the expedition of 1874 . Report upon United States Geographical Surveys West of the One Hundredth Meridian, in charge of First Lieut. G.M. Wheeler, Corps of Engineers, U.S. Army 4 (2). Government Printing Office, Washington, D.C.: $1-365$.

Cope E. D. 1882. - The Classification of the Ungulate Mammalia. Proceeding of the American Philosophical Society 20: 438-447.

Cope E. D. 1891. - The Litopterna. American Naturalist 25 (296): 685-693.

Crompton A. W. 1964. — On the skull of Oligokyphus. Bulletin of the Brithish Museum of Natural History, Geology 9: 69-82.

Crompton A. W. \& Sun A.-L. 1985. - Cranial structure and relationships of the liassic mammal Sinoconodon. Zoological Journal of the Linnean Society 85: 99-119.

CROMPTON A. W. \& LUO Z. 1993. - Relationships of early mammals. Sinoconodon, Morganucodon oebleri, and Dinnetherium, in Szalay F. S., NovaceK M. J. \& McKenna M. C. (eds), Mammal Phylogeny. Vol. 1: Mesozoic differentiation, Multituberculates, Monotremes Early Therians and Marsupials. Springer-Verlag, New York: 30-44.

DAUBER W. 2007. - Pocket Atlas of Human Anatomy Founded by Heinz Feneis. $5^{\text {th }}$ revised edition. Georg Thieme Verlag, Stuttgart, 549 p.

DaVIS D. D. 1964. — The Giant Panda, a morphological study of evolutionary mechanisms. Fieldiana: Zoology Memoirs 3: 1-339.

Deaner R. O., Isler K., Burkart J. \& VAN SCHAik C. 2007. Overall brain size, and not encephalization quotient, best predicts cognitive ability across non-human primates. Brain, Behavior and Evolution 70 (2): 115-124.

De BeER G. R. 1937. - The Development of the Vertebrate Skull. Clarendon press, Oxford, $552 \mathrm{p}$.

Dom R., Fisher B. L., \& Martin G. F. 1970. — The venous system of the head and neck of the opossum (Didelphis virginiana). Journal of Morphology 132 (4): 487-496.

Dor M. 1937. - La morphologie de la queue des mammiferes dans ses rapports avec la locomotion. Pierre André, Paris, $184 \mathrm{p}$.

Dos Reyes M., Inoue J., Hashegawa M., Asher R., Donoghue P. \& YANG Z. 2012. - Phylogenomic datasets provide both precision and accuracy in estimating the timescale of placental mammal phylogeny. Proceedings of the Royal Society of London B: Biology 279: 3491-3500.

Dozo M. T. \& MARTinez G. 2015. — First digital cranial endocasts of Late Oligocene Notohippidae (Notoungulata): implications for endemic South American ungulates brain evolution. Journal of Mammalian Evolution 1-16. http://dx.doi.org/10.1007/ s10914-015-9298-5

EDINGER T. 1929. - Die Fossilen Gehirne. Ergebnisse der Anatomie und Entwicklungsgeschichte 28: 1-249.

EDINGER T. 1942. - The pituitary body in giant animals fossil and living: a survey and a suggestion. Quarterly Review of Biology 17: 31-45.

EDINGER T. 1955. - Hearing and smell in cetacean history. Monatsschrift für Psychiatrie und Neurologie 129: 37-58.

EDINGER T. 1956. — Objet et résultats de la paléoneurologie. Annales de Paléontologie 62: 95-116.

EDINGER T. 1964. - Midbrain exposure and overlap in mammals. American Zoologist 4: 5-19.

EISENBERG J. F. 1981. - The Mammalian Radiations. University of Chicago Press, Chicago, 610 p. 
EIsenberG J. F. \& Wilson D. E. 1981. - Relative brain size and demographic strategies in didelphid marsupials. American Naturalist 118: 1-15.

Ekdale E. G., Archibald D. J. \& Averianov A. O. 2004. Petrosal bones of placental mammals from the Late Cretaceous of Uzbekistan. Acta Palaeontologica Polonica 49 (1): 161-176.

EKDALE E. G. \& Rowe T. 2011. - Morphology and variation within the bony labyrinth of zhelestids (Mammalia, Eutheria) and other therian mammals. Journal of Vertebrate Paleontology 31 (3): 658-675.

EMry R. J. \& ThORIngton R. W. JR. 1982. - Descriptive and comparative osteology of rthe oldest fossil squirrel, Protosciurus (Rodentia: Sciuridae). Smithsonian Contributions to Paleobiology 47: 1-35.

Evans H. E. \& DE LahunTa A. 2012. - Miller's Anatomy of the Dog. Saunders, St Louis, 850 p.

FARRIS J. S. 1989a. - The retention index and homoplasy excess. Systematic Biology 38 (4): 406-407.

FARRIS J. S. 1989b. - The retention index and the rescaled consistency index. Cladistics 5 (4): 417-419.

FiSCHER M. S. 1986. - Die Stellung der Schliefer (Hyracoidea) im phylogenetischen System der Eutheria. Zugleich ein Beitrag zur Anpassungsgeschichte der Procaviidae. Courier Forchungsinstitute Senckenberg 84: 1-132.

FISCHER M. S. 1994. - Crouched posture and high fulcrum, a principle in the locomotion of small mammals: the example of the rock hyrax (Procavia capensis) (Mammalia: Hyracoidea). Journal of Human Evolution 26 (5-6): 501-524.

FLEISCHER G. 1973. - Studien am Skelett des Gehororgan der Säugetiere, einschliesslich des Menschen. Säugetierkunde Mitteilungen 21: 131-239.

Flores D. A. 2009. - Phylogenetic analyses of postcranial skeletal morphology in didelphid marsupials. Bulletin of the American Museum of Natural History 320: 1-81.

ForasiePI A. M. 2009. - Osteology of Arctodictis sinclairi (Mammalia, Metatheria, Sparassodonta) and phylogeny of Cenozoic metatherian carnivores from South America. Monografia del Museo Argentino de Ciencias Naturales n.s. 6: 1-174.

Franzen J. L., Gingerich P. D., Habersetzer J., Hurum J. H., Koenigswald W. von \& Smith B. H. 2009. - Complete primate skeleton from the middle Eocene of Messel in Germany: Morphology and paleobiology. PLoS One 4 (5) (e5723): 1-27.

Gannon P. J., Eden A. R. \& Laitman J. T. 1988. - The subarcuate fossa and cerebellum of extant primates: comparative study of a skull-brain interface. American Journal of Physical Anthropology 77: 143-164.

GABBERT S. L. 2004. - The basicranial and posterior cranial anatomy of the families of the Toxodontia. Bulletin of the American Museum of Natural History 285: 177-190.

Garcia-Lopez D. 2011. - Basicranial osteology of Colbertia lumbrerense Bond, 1981 (Mammalia, Notoungulata). Ameghiniana 48 (1): 3-12.

GaUdin T. 2004. - Phylogenetic relationships among sloths (Mammalia, Xenarthra, Tardigrada): the craniodental evidence. Zoological Journal of the Linnean Society 140: 255-305.

Gayet M., Marshall L. G. \& Sempere T. 1992. - The Mesozoic and Palaeocene vertebrates of Bolivia and their stratigraphic context: a review. Revista Técnica de YPFB, 12 (3-4, dic. 1991): 393-433, Santa Cruz.

GAZIN C. L. 1953. - The Tillodontia: an early Tertiary order of mammals. Smithsonian Miscellaneous Collections 121 (10): 1-110.

GazIN C. L. 1956. - Paleocene mammalian faunas of the Bison Basin in south-central Wyoming. Smithsonian Miscellaneous Collections 131 (6): 1-57.

GazIN C. L. 1958. - A review of the middle upper Eocene Primates of North America. Smithsonian Miscellaneous Collections 136 (1): 1-112.
GAZIN C. L. 1965. — A study of the early Tertiary condylarthran mammal Meniscotherium. Smithsonian Miscellaneous Collections 149 (2): 1-98.

GAZIN C. L. 1968 - A study of the Eocene condylarthran mammal Hyopsodus. Smithsonian Miscellaneous Collections 153 (4): 1-90.

Gebo D. L. \& Rose K. D. 1993. - Skeletal morphology and locomotor adaptation in Prolimnocyon atavus, an early Eocene hyaenodontid creodont. Journal of Vertebrate paleontology 13 (1): $125-144$.

GeIsLER J. H. 2001. - New morphological evidence for the phylogeny of Artiodactyla, Cetacea, and Mesonychidae. American Museum Novitates 3344: 1-53.

GeIsler J. H. \& LuO Z-X. 1998 - Relationships of Cetacea to terrestrial ungulates and the evolution of cranial vasculature in Cete, in TheWISSEN J. G. M. (ed.), The Emergences of Whales: Evolutionary Patterns in the Prigin of Cetacea. Plenum press, New York: 163-212.

Geisler J. H. \& Uhen M. D. 2003. - Morphological support for a close relationship between hippos and whales. Journal of Vertebrate Paleontology 23 (4): 991-996.

Gelfo J. N., López G. M. \& Bond M. 2008. - A new Xenungulata (Mammalia) from the Paleocene of Patagonia, Argentina. Journal of Paleontology 82: 329-335.

Gelfo J. N., Goin F. J., Woodburne M. O. \& Muizon C. DE 2009. - Biochronological relationships of the earliest South American Paleogene mammalian faunas. Palaeontology 52 (1): 251-269.

GELDEREN C. vAN 1924. — Die Morphologie der Sinus durae matris. Zweiter Teil. Die vergleichende Ontogenie der neurokraniellen Venen der Vögel und Säugetiere. Zeitschrift für Anatomie und Entwickelungsgeschichte 74: 432-508.

Giannini N. P., Wible J. R. \& Simmons N. B. 2006. - On the cranial osteology of Chiroptera. I. Pteropus (Megachiroptera: Pteropodidae). Bulletin of the American Museum of Natural History 295: 1-134.

GINGERICH P. D. 1981. — Cranial morphology in Eocene Adapidae. I. Sexual dimorphism in Adapis magnus and Adapis parisiensis. American Journal of Physical Anthropology 56: 217-234.

Gingerich P. D. 1989. - New earliest Wasatchian mammalian fauna from the Eocene of northwestern Wyoming: composition and diversity in a rarely sampled high-floodplain assemblage. University of Michigan Papers on Paleontology 28: 1-97.

Gingerich P. D. 1990. - Prediction of body mass in mammalian species from long bone lengths and diameters. Contributions from the Museum of Paleontology, University of Michigan 28: 79-92.

Gingerich P. D. \& GunNell G. F. 1979. - Systematics and evolution of the genus Esthonyx (Mammalia, Tillodontia) in the early Eocene of North America. Contributions from the Museum of Paleontology, University of Michigan 25 (7): 125-157.

GiNGERICH P. D. \& MARTIN R. D. 1981. - Cranial morphology and adaptations in Eocene Adapidae. II. The Cambridge Skull of Adapis parisiensis. American Journal of Physical Anthropology 56: 235-257.

Gingerich P. D. \& GunNell G. F. 1992. - A new skeleton of Plesiadapis cookei, the display case: A Quarterly Newsletter of the University of Michigan Exhibit Museum 6: 1-3.

Gingerich P. D. \& Gunnell G. F. 2005. - Brain of Plesiadapis cookei (Mammalia, Proprimates): surface morphology and encephalization compared to those of primates and Dermoptera. Contributions from the Museum of Paleontology, University of Michigan 31: 185-195.

Gingerich P. D., Houde P. \& Krause D. W. 1983. - A new earliest Tiffanian (late Paleocene) mammalian fauna from Bangtail Plateau, Western Crazy Mountain Basin, Montana. Journal of Paleontology 57 (5): 957-970.

Gingerich P. D., Haq M.-U, Koenigswald W. von, Sanders W. J., \& SMith B. H. 2009 - New protocetid whale from the middle Eocene of Pakistan: birth on land, precocial development, and sexual dimorphism. PlosOne 4 (2): 1-20. 
Godinot M. \& Couette S. 2008. - Morphological diversity in the skulls of large adapines (primates, adapiforms) and its systematic implications, in SARGIS E. J. \& DAGOSTO M. (eds), Mammalian Evolutionary Morphology. Springer Verlag, New York: 285-313.

Goin F. J., Pascual R., Tejedor M., Gelfo J. N., Woodburne M., Case J., Reguero M., Bond M., Cione A., Udrizar Sauthier D., Balarino L., Scasso R., Medina F. A. \& UBALDÓN M. C. 2006. - The earliest Tertiary therian mammal from South America. Journal of Vertebrate Paleontology 26 (2): 505-510.

Goloboff P. A., Farris J. S. \& NixON K. C. 2008. — TNT, a free program for phylogenetic analysis. Cladistics 24 (5): 774-786.

Goswami A., Prasad G. V. R., Upchurch P., Boyer D. M., Seiffert E. R., Verma O., Gheerbrant E. \& Flynn J. J 2011. - A radiation of arboreal basal eutherian mammals beginning in the Late Cretaceous of India. Proceedings of the National Academy of Sciences 108 (39): 16333-16338.

Goswami A., Smaers J. B., Soligo C. \& Polly P. D. 2014. The macroevolutionary consequences of phenotypic integration: from development to deep time. Philosophical Transactions of the Royal Society of London B: Biological Sciences 369 (1649): 20130254.

GrASSÉ P.-P. 1967. — Traité de zoologie: Anatomie, Systématique, Biologie. Vol 16, Fasc. 1. Teguments et Squelette. Masson, Paris, $1170 \mathrm{p}$.

GREgORY W. K. 1910. - The orders of mammals. Bulletin of the American Museum of Natural History 27: 1-524.

Gregory W. K. 1920. - Studies in comparative myology and osteology, no. IV. A review of the evolution of the lacrymal bone of vertebrates with special reference to that of mammals. Bulletin of the American Museum of Natural History 42: 95-263.

GUNNELL G. F. 1989. — Evolutionary history of Microsyopoidea (Mammalia, ?Primates) and the relationships between Plesiadapiformes and Primates. University of Michigan, Papers on Paleontology 27: 1-157.

Gunnell G. F. \& Gingerich P. D. 1987. - Skull and partial skeleton of Plesiadapis cookei from the Clark Fork Basin, Wyoming, American Journal of Physical Anthropology 72: 206A.

HAINES R. W. 1958. - Arboreal or terrestrial ancestry of placental mammals. Quarterly Review of Biology 33: 1-23.

Halliday T. J. D. \& GOSWAMI A. 2013. - Testing the inhibitory cascade model in Mesozoic and Cenozoic mammaliaformes. BMC Evolutionary Biology 13 (79): 1-11.

HeinRich R. E. \& Rose K. D. 1997. — Postcranial morphology and locomotor behavior of two early Eocene miacoid carnivorans, Vulpavus and Didymictis. Palaeontology 40 (2): 279-305.

HershKovitz P. 1982. - The staggered marsupial lower third incisor (I3). Geobios, Mémoire spécial 6: 191-200.

HiCKMAN G. C. 1979. - The mammalian tail: a review of functions. Mammal Reviews 9 (4): 143-157.

Holloway R. L., Broadfield D. C. \& Yuan M. S. 2004. - The Human Fossil Record. Volume 3. Brain Endocasts; the Paleoneurological evidence. New York, Wiley-Liss, 315 p.

Hopson J. A. \& Rougier G. W. 1993. - Braincase structure in the oldest skull of a therian mammal: implications for mammalian systematic and cranial evolution. American Journal of Science 293: 268-299.

Horovitz I. 2000. - The tarsus of Ukhaatherium nessovi (Eutheria, Mammalia) from the Late Cretaceous of Mongolia: an appraisal of the evolution of the ankle in basal therians. Journal of Vertebrate Paleontology 20: 547-560.

Horovitz I. \& SÁNCHEZ-Villagra M. R. 2003. - A morphological analysis of marsupial mammal higher-level phylogenetic relationships. Cladistics 19: 181-212.

Horovitz I., Martin T., Bloch J., Ladevèze S., Kurz C. \& SÁNCHEZ-VILLAGRA M. R. 2009. - Cranial anatomy of the earliest marsupials and the origin of opossums. PlosOne 4 (12): 1-9.
Howell A. B. 1928. - Contribution to the comparative anatomy of the eared and earless seals (genera Zalophus and Phoca). Proceedings of the United States National Museum 73 (15): 1-142.

Hu Y., WANG Y., LUO Z. \& LI C. 1997. — A new symetrodont mammal from China and its implication for mammalian evolution. Nature 390: 137-142.

ItURRALDE-Vinent M. A. 2006. - Meso-Cenozoic Carribean paleogeography: implications for the historical biogeography of the region. International Geology Reviews 48: 791-827.

Jenkins F. A. \& PARRINGTON F. R. 1976. - The postcranial skeletons of the Triassic mammals Eozostrodon, Megazostrodon and Erythrotherium. Philosophical Transactions of the Royal Society of London B, 273: 387-431.

Jenkins F. A. \& Camazine S. M. 1977. - Hip structure and locomotion in ambulatory and cursorial carnivores. Journal of Zoology 181: 351-370.

Jenkins F. A. \& Weijs W. A. 1979. — The functional anatomy of the shoulder in the Virginia opossum (Didelphis virginiana). Journal of Zoology 188: 379-410.

JERISON H. J. 1973. - Evolution of Brain and Intelligence. New York, Academic Press, $482 \mathrm{p}$.

Ji Q., Luo Z. -X., Yuan C. -X., Wible J. R., Zhang J. -P. \& GEORGI J. A. 2002. - The earliest known eutherian mammal. Nature 416: 816-822.

KaAS J. H. (ed.) 2009. - Evolutionary Neuroscience. Academic Press, 1017 p.

KAPPELMAN J. 1988. - Morphology and locomotor adaptations of the bovid femur in relation to habitat. Journal of Morphology 198: 119-130.

Kavanagh K. D., Evans A. R. \& Jernvall J. 2007. — Predicting evolutionary patterns of mammalian teeth from development. Nature 449: 427-432.

Kermack K. A. 1963. - The cranial structure of triconodonts. Philosophical Transactions of the Royal Society, London B 246: 83-102.

Kermack K. A. MussetT F. \& Rigney H. W. 1981. - The skull of Morganucodon. Zoological Journal of the Linnean Society of London 71: 1-158.

Kielan-JaWorowska Z. 1969. - Preliminary data on the Upper Cretaceous eutherian mammals from Bayn Dzack, Gobi Desert. Palaeontologia Polonica 19: 171-191.

Kielan-JaWOROWSKa Z. 1975a. - Preliminary description of two new eutherian genera from the Late Cretaceous of Mongolia. Palaeontologia Polonica 33: 5-16.

KIELAN-JAWOROWSKA Z. 1975b. - Evolution of the therian mammals in the Late Cretaceous of Asia. Part I. Deltatheridiidae. Palaeontologia Polonica 33: 103-132.

KIELAN-JAWOROWSKA Z. 1977. - Evolution of the therian mammals in the Late Cretaceous of Asia. Part II. Postcranial skeleton in Kennalestes and Asioryctes. Palaeontologia Polonica 37: 65-83.

KIELAN-JAWOROWSKA Z. 1978. - Evolution of the therian mammals in the Late Cretaceous of Asia. Part III. Postcranial skeleton in Zalambdalestidae. Palaeontologia Polonica 38: 3-41.

KIELAN-JAWOROWSKA Z. 1981. - Evolution of the therian mammals in the Late Cretaceous of Asia. Part IV. Skull structure of Kennalestes and Asioryctes. Palaeontologia Polonica 42: 25-78.

KIELAN-JAWOROWSKA Z. 1984a. - Evolution of the therian mammals in the Late Cretaceous of Asia. Part VI. Endocranial casts of eutherian mammals. Palaeontologia Polonica 46: 157-171.

KIELAN-JAWOROWSKA Z. 1984b. - Evolution of the therian mammals in the Late Cretaceous of Asia. Part V. Skull structure in Zalambdalestidae. Palaeontologia Polonica 46: 107-117.

KIELAN-JAWOROWSKA Z. 1986. - Brain evolution in Mesozoic mammals, in Flanagan K. M. \& Lillegraven J. A. (eds), Vertebrates, Phylogeny, and Philosophy. Contributions to Geology, University of Wyoming, Special Paper 3: 21-34.

Kielan-Jaworowska Z. \& Trofimov B. A. 1980. - Cranial morphology of the Cretaceous eutherian mammal Barunlestes. Acta Palaeontologica Polonica 25:167-185. 
Kielan-Jaworowska Z. \& Dashzeveg D. 1989. - Eutherian mammals from the Early Cretaceous of Mongolia. Zoologica Scripta 18: 347-355.

Kielan-JaWorowska Z. \& Trofimov B. A. 1986. — Endocranial cast of Cretaceous eutherian mammal Barunlestes. Acta Palaeontologica Polonica 31: 137-149.

Kielan-Jaworowska Z. \& Gambaryan P. P. 1994. — Postcranial anatomy and habits of Asian multituberculate mammals. Fossils and Strata 36: 1-92.

Kielan-Jaworowska Z., Presley R. \& Poplin C. 1986 - The cranial vascular system in taeniolabidoid multituberculate mammals. Philosophical Transactions of the Royal Society of London 313 (1164): 525-602.

Kiley-Worthington M. 1976. - The tail movements of ungulates, canids and felids with particular reference to their causation and function as displays. Behaviour 56: 69-114.

Kirk E. C., Lemelin P., Hamrick M. W., Boyer D. M. \& Bloch J. I. 2008. - Intrinsic hand proportions of euarchontans and other mammals: implications for the locomotor behaviour of plesiadapiforms. Journal of Human Evolution 55: 278-299.

Kirsch J. A. W., Lapointe F.-J. \& Springer M. S. 2007. DNA-hybridisation studies of marsupials and their implications for metatherian classification. Australian Journal of Zoology 45: 211-280.

KLAAUW J. C. VAN DER 1931. - The auditory bulla in some fossil mammals with a general introduction to this region of the skull. Bulletin of the American Museum of Natural History 62: 1-352.

Koenigswald W. von, Ruf I. \& Gingerich P. D. 2009. Cranial morphology of a new apatemyid, Carcinella sigei $\mathrm{n}$. gen. n. sp. (Mammalia, Apatotheria) from the late Eocene of southern France. Palaeontographica Abt. A 288: 53-91.

Koenigswald W. von, Habersetzer J. \& Gingerich P. D. 2011 - Morphology and evolution of the distal phalanges in primates, in LeHMANN T. M. \& SchaAl S. F. K. (eds), The World at the Time of Messel: Puzzles in Palaeobiology, Palaeoenvironment, and the History of Early Primates. Senckenberg Gesellschaft für Naturforschung, Frankfurt on Main: 91-94.

Koenigswald W. von, Habersetzer J. \& Gingerich P. D. 2012. - Pedal distal phalanges of the Eocene adapoids Europolemur and Darwinius compared to phalanges of Notharctus and other primates. Palaeobiology and Palaeoenvironment 92 (4): 539-565.

Kramarz A. G. \& Bond M. 2014. - Critical revision of the alleged delayed dental eruption in South American "ungulates". Mammalian Biology. Zeitschrift für Säugetierkunde 79 (3): $170-175$

Kramarz A. G., Bond M. \& Forasiepi A. M. 2011. - New remains of Astraponotus (Mammalia, Astrapotheria) and considerations on astrapothere cranial evolution. Paläontologische Zeitschrift 85:185-200.

KrebBs B. 1991. - Das Skelett von Henkelotherium guimarotae gen. et sp. nov. (Eupantotheria, Mammalia) aus dem Oberen Jura von Portugal. Berliner Geowissenschaftliche Abhandlungen A, 133: 110 p., Berlin.

KRISHTALKA L. \& STUCKY R. K. 1985. - Revision of the Wind River faunas, early Eocene of central Wyoming. Part7. Revision of Diacodexis (Mammalia, Artiodactyla). Annals of Carnegie Museum 54 (14): 413-486.

Krombach G. A., Schmitz-Rode T., Prescher A., Di Martino E., Weidner J. \& GÜNTher R. W. 2002. — The petromastoid canal on computed tomography. European Radiology 12: 2770-2775.

KuHN H.-J. \& ZelLer U. 1987. - The cavum epiptericum in monotremes and therian mammals, in KUHN H.-J. \& ZELLER U. (eds), Morphogenesis of the mammalian skull. Verlag Paul Parey, Hamburg: 51-70.

LadeVEzze S. \& Muizon C. DE 2007. — The auditory region of early Palaeocene Pucadelphydae (Mammalia, Metatheria) from Tiupampa with phylogenetic implications. Palaeontology 50 (5): 1123-1154.

LadevĖZe S., Missiaen P. \& SMith T. 2010. — First skull of Orthaspidotherium edwarsi (Mammalia, "Condylarthra") from the late Paleocene of Berru (France) and phylogenetic affinities of the enigmatic European family Pleuraspidotheriidae. Journal of Vertebrate Paleontology 30 (5): 1559-1578.

Ladevezze S., Muizon C. De, Beck R., Germain D. \& CésPEDES-PAZ R. 2011. - Earliest evidence of mammalian social behaviour in the basal Tertiary of Bolivia. Nature. 474: 83-86.

LANGER P. 2008. - The phases of maternal investment in eutherian mammals. Zoology 111: 148-162.

LARSON S. G. 1993 - Functionnal morphology of the shouder in Primates, in Gebo D. (ed.), Postcranial Adaptation in NonHuman Primates. Northern Illinois University Press: 45-69.

LeE M. S. Y. \& Camens A. B. 2009. - Strong morphological support for the molecular evolutionary tree of placental mammals. Journal of Evolutionary Biology 22 (11): 2243-2257.

LE Gros Clark W. E. 1932. - The brain of the Insectivora. Proceedings of the Zoological Society of London 1932: 975-1013.

LESSERTISSEUR J. \& SABAN R. 1967. - Squelette apendiculaire, in Grassé P. P. (ed.), Traité de Zoologie. Masson, Paris: 708-1078.

Li C. \& Ting S. 1993 - New cranial and postcranial evidence for the affinities of the eurymylids (Rodentia) and mimotonids (Lagomorpha), in Szalay F. S., NovaceK M. J. \& McKenna M. C. (eds), Mammal Phylogeny, Volume 2: Placentals. Springer Verlag, New York: 151-158.

Lofgren D. L., Lillegraven J. A., Clemens W. A., Gingerich P. D. \& Williamson T. E. 2004. - Paleocene Biochronology: The Puercan through Clarkforkian Land Mammal Ages, in Woodburne M. O. (ed.), Late Cretaceous and Cenozoic Mammals of North America. Biostratigraphy and Geochronology. Columbia University Press, New York: 43-105.

Loomis F. B. 1914 - The Deseado Formation of Patagonia. Rumford Press, Concord, 232 p.

Losos J. B., Hillis D. M. \& Greene H. W. 2012. - Who speaks with a forked tongue. Science 338 (6113): 1428-1429.

LuCAS S. G. 1982. - The phylogeny and composition of the order Pantodonta (Mammalia, Eutheria). Third North American Paleontological Convention, Proceedings 2: 337-342.

LuCAS S. G. 1984. - Systematics, Biostratigraphy and Evolution of Early Cenozoic Coryphodon (Mammalia, Pantodonta). PhD Dissertation, Yale University, New Haven, 649 p.

LUCAS S. G. 1993. - Pantodonts, tillodonts, uintatheres and pyrotheres are not ungulates, in Szalay F. S., NovaceK M. J. \& McKenna M. C. (eds), Mammal Phylogeny, Vol. 2: Placentals. Springer Verlag, New York: 182-194.

LuCAS S. G. 1998a. - Tillodontia, in JANIS M. C., SCOTT K. M. \& JACOBS L. L. (eds), Evolution of Tertiary mammals of North America, Volume 1, Terrestrial Carnivores, Ungulates, and Ungulate-like Mammals. Cambridge University Press, Cambridge: 268-273.

LuCAS S. G. 1998b. - Pantodonta, in Janis M. C., ScotT K. M. \& JACOBS L. L. (eds), Evolution of Tertiary Mammals of North America, Volume 1, Terrestrial Carnivores, Ungulates, and Ungulate-like Mammals. Cambridge University Press, Cambridge: 274-283.

LuO Z.-X. 2007. - Transition and diversification in early mammal evolution. Nature 450: 1011-1019.

Luo Z.-X. \& CROMPTON A. W. 1994. - Transformation of the quadrate (incus) through the transition from non-mammalian cynodonts to mammals. Journal of Vertebrate Paleontology 14 (2): 341-374.

Luo Z. X. \& Wible J. R. 2005. — A Late Jurassic digging mammal and early mammalian diversification. Science 308: 103-107.

Luo Z.-X., Ji Q., Wible J. R. \& Yuan C. X. 2003. - An Early Cretaceous tribosphenic mammal and metatherian evolution. Science 302: 1934-1940. 
Luo Z.-X., Yuan C.-X., Meng Q.-J. \& Ji Q. 2011. — A Jurassic eutherian mammal and divergence of marsupials and placentals. Nature 476: 442-445.

LuO Z.-X., Ruf I. \& MARTIN T. 2012. - The petrosal and inner ear of the Late Jurassic cladotherian mammal Dryolestes leiriensis and implications for ear evolution in therian mammals. Zoological Journal of the Linnean Society 166 (2): 433-463.

MacDonald D. W. 1995. - The Encyclopedia of Mammals. Facts on file, Inc., New York, 895 p.

MacPhee R. D. E. 1981. - Auditory region of primates and eutherian insectivores. Contributions to Primatology 18: 1-282.

MACPHEE R. D. E. 1994. - Morphology, adaptations, and relationships of Plesiorycteropus, and a diagnosis of a new order of eutherian mammals. Bulletin of the American Museum of Natural History 220: 1-214.

MACPheE R. D. E. 2011. - Basicranial morphology and relationships of Antillean Heptaxodontidae (Rodentia, Ctenohystrica, Caviomorpha). Bulletin of the American Museum of Natural History 363: 1-70.

MACPheE R. D. E. 2014. - The serrialis bone, interparietals, "X" elements, entotympanics, and the composition of the notoungulate caudal cranium. Bulletin of the American Museum of Natural History 384: 1-69.

MACRINI T. E. 2006. - The Evolution of Endocranial Space in Mammals and Non-Mammalian Cynodonts. PhD dissertation, University of Texas, Austin, 278 p.

MACRINI T. E. 2012. - Comparative morphology of the internal nasal skeleton of adult marsupials based on X-ray computed tomography. Bulletin of the American Museum of Natural History 365: 1-91.

MaCrini T. E. 2014. - Development of the ethmoid in Caluromys philander (Didelphidae, Marsupialia) with a discussion on the homology of the turbinal elements in marsupials. The Anatomical Record 297: 2007-2017.

Macrini T. E., Rougier G. W. \& Rowe T. 2007a. - Description of a cranial endocast from the fossil mammal Vincelestes neuquenianus (Theriiformes) and its relevance to the evolution of endocranial characters in therians. Anatomical Record 290: 875-892.

Macrini T. E., Rowe T. \& VAndeberg J. L. 2007b. - Cranial endocasts from a growth series of Monodelphis domestica (Didelphidae, Marsupialia): a study of individual and ontogenetic variation. Journal of morphology 268 (10): 844-865.

Macrini T. E., Muizon C. DE, Cifelli R. L. \& Rowe T. 2007c. Digital cranial endocast of Pucadelphys andinus a Paleocene metatherian. Journal of Vertebrate Paleontology 29 (1): 99-107.

Macrini T. E., Flynn J. J., Croft D. A. \& Wyss A. R. 2010. Inner ear of a notoungulate placental mammal: anatomical description and examination of potentially phylogenetically informative characters. Journal of Anatomy 216: 600-610.

MADDISON W. P. \& MAdDISON D. R. 2009 - Mesquite: a modular system for evolutionary analysis. Version 2.71. Available at: http://mesquiteproject.org

MAier W. 2000. - Ontogeny of the nasal capsule in cercopithecoids: a contribution to the comparative and evolutionary morphology of catarrhines, in WHITEHEAD P. F. \& JOLLY C. J. (eds), Old World Monkeys. Cambridge University Press, Cambridge: 99-132.

MAiER W. \& RUF I. 2014. - Morphology of the nasal capsule of Primates - with special reference to Daubentonia and Homo. Anatomical Record 297: 1985-2006.

Marsh O. C. 1876. - On some characters of the genus Coryphodon Owen. American Journal of Science Series 3 (11): 425-428.

Marsh O. C. 1877. - Principal characters of the Coryphodontidae. American Journal of Sciences Series 3 (14): 81-85.

MARSH O. C. 1884. - Dinocerata, a monograph of an extinct order of gigantic mammals. United States Geological Survey Monographs 10: 1-237.
MARSH O. C. 1892. - A new order of mammals (Mesodactyla). American Journal of Science 43 (3): 445-449.

Marshall L. G. \& Muizon C. DE 1988. — The dawn of the age of mammals in South America. National Geographic Research 4 (1): 23-55

Marshall L. G. \& Muizon C. DE 1995. — Part II: the skull, in Muizon C. DE (ed.), Pucadelphys andinus (Marsupialia, Mammalia) from the Early Palaeocene of Bolivia. Mémoires du Muséum national d'Histoire naturelle 165: 21-90.

Marshall L. G., Muizon C. DE \& Sigé B. 1983. — Late cretaceous mammals (Marsupialia) from Bolivia. Geobios 16 (6) 739-745.

Marshall L. G., Muizon C. de \& Sigogneau-Russell D. 1995. - Part I: the locality of Tiupampa: age, taphonomy and mammalian fauna, in Muizon C. DE (ed.), Pucadelphys andinus (Marsupialia, Mammalia) from the Early Palaeocene of Bolivia. Mémoires du Muséum national d'Histoire naturelle 165: 11-20.

Marshall L. G., Sempere T. \& Butler R. F. 1997. - Chronostratigraphy of the mammal-bearing Palaeocene of South America. Journal of South American Earth Sciences 10: 49-70.

MarTin R. D. 1990. - Primate Origins and Evolution. Princeton, NJ, Princeton University Press, 804 p.

MatTHeW W. D. 1904. - The arboreal ancestry of the Mammalia. American Naturalist 38: 811-818.

MatTHEW W. D. 1910. - On the osteology and relationships of Paramys, and the affinities of the Ischyromyidae. Bulletin of the American Museum of Natural History 28: 43-72.

Matthew W. D. 1937. - Paleocene faunas of the San Juan Basin, New Mexico. Transactions of the American Philosophical Society, New Series 30: 1-510.

MCDowELL S. B. JR. 1958. - The greater Antillean insectivores. Bulletin of the American Museum of Natural History 115 (3): 113-214.

McKenna M. C. 1975. - Toward a phylogenetic classification of the Mammalia, in LuCKeTt W. P. \& Szalay F. S. (eds), Phylogeny of the Primates. Plenum Press, New York: 21-46.

MCKenNA MC. 1981. — Early history and biogeography of South America's extinct land mammals, in CiOCHON R. L. \& Chiarelli A. B. (eds), Evolutionnary Biology of the New World Monkeys and Continental Drift. Plenum Press, New York: 43-77.

McKenNa M. C. \& BelL S. K. 1997. - Classification of Mammals above the Species Level. Columbia University Press, New York, $671 \mathrm{p}$.

McKenna M. C., Kielan-Jaworowska Z. \& Meng. J. 2000. Earliest eutherian mammalian skull from the Late Cretaceous (Coniacian) of Uzbekistan. Acta Palaeontologica Polonica 45: 1-54.

MeAd J. G. \& FordyCE R. E. 2009 - The Therian Skull. A lexicon with emphasis on the odontocetes. Smithsonian Contributions to Zoology 627: 1-248.

MENG J. \& Fox R. C. 1995. - Osseous inner ear structure and hearing in early marsupials and placentals. Zoological Journal of the Linnean Society 115: 47-71.

MENG J. \& Wyss A. 2001. - The morphology of Tribosphenomys (Rodentiaformes, Mammalia): phylogenetic implications for basal glires. Journal of Mammal Evolution 8: 1-71.

MeNG J., Hu Y. \& LI C. 2003. - The osteology of Rhombomylus (Mammalia, Glires): implications for phylogeny and evolution of Glires. Bulletin of the American Museum of Natural History 275: 1-247.

Meng J., Bowen G. J., Ye J., Koch P. L., Ting S., Li Q. \& Jin X. 2004. - Gomphos elkema (Glires, Mammalia) from the Erlian Basin: evidence for the early Bumbanian land mammal age in Nei-Mongol, China. American Museum Novitates 3425: 1-24. Meredith R. W., JanečKa J. E., Gatesy J., Ryder O. A., Fisher C. A., Teeling E. C., ... \& Murphy W. J. 2011. — Impacts of the Cretaceous terrestrial revolution and KPg extinction on mammal diversification. Science 334 (6055): 521-524. 
Moodie R. L. 1922. —On the endocranial of some Oligocene and Pleistocene mammals. Journal of Comparative Neurology 34: 343-379.

Morón S., Fox D. L., Feinberg J. M., Jaramillo C., Bayona G., Montes C., Bloch J. I. 2013. - Climate change during the early Paleogene in the Bogotá Basin (Colombia) inferred from paleosol carbon isotope stratigraphy, major oxides, and environmental magnetism. Palaeogeography, Palaeoclimatology, Palaeoecology 388: 115-127.

MuizOn C. DE 1992. — La fauna de mamíferos de Tiupampa (Paleoceno inferior, Formacíon Santa Lucía) Bolivia. Revista Técnica de Yaciminentos Petroliferos Fiscales de Bolivia 12 (3-4, December 1991): 575-624.

Muizon C. DE 1994. - A new carnivorous marsupial from the Palaeocene of Bolivia and the problem of marsupial monophyly. Nature 370: 208-211.

MuizOn C. DE 1998. - Mayulestes ferox, a borhyaenoid, (Metatheria, Mammalia) from the early Palaeocene of Bolivia. Phylogenetic and palaeobiologic implications. Geodiversitas 20 (1): 19-142.

Muizon C. DE \& Marshall L. G. 1987a - Le plus ancien Condylarthre (Mammalia) sud-américain (Crétacé supérieur, Bolivie). Comptes Rendus hebdomadaires des Séances de l'Académie des Sciences, Paris 304: 771-774.

Muizon C. DE \& Marshall L. G. 1987b - Deux nouveaux Condylarthres (Mammalia) du Maestrichtien de Tiupampa (Bolivie). Comptes Rendus hebdomadaires des Séances de l'Académie des Sciences, Paris 304: 947-950.

Muizon C. DE \& Marshall L. G. 1987c - Le plus ancien Pantodonte (Mammalia) du Crétacé supérieur de Bolivie. Comptes Rendus hebdomadaires des Séances de l'Académie des Sciences, Paris 304: 205-208.

Muizon C. DE \& Marshall L. G. 1992. - Alcidedorbignya inopinata (Mammalia: Pantodonta) from the early Paleocene of Bolivia: phylogenetic and paleobiogeographic implications. Journal of Paleontology 66 (3): 499-520.

Muizon C. DE \& Cifeldi R. L. 2000. - The "condylarths" (archaic Ungulata, Mammalia) from the early Palaeocene of Tiupampa (Bolivia): implications on the origin of the South American ungulates. Geodiversitas 22 (1): 47-150.

Muizon C. DE \& Cifelli R. L. 2001. — A new basal "didelphoid" (Marsupialia, Mammalia) from the early Paleocene of Tiupampa (Bolivia). Journal of Vertebrate Paleontology 21 (1): 87-97.

Muizon C. DE \& ARGOT C. 2003. - Comparative anatomy of the didelphimorphs marsupials from the early Palaeocene of Bolivia (Pucadelphys, Andinodelphys, and Mayulestes). Palaeobiologic implications, in JONES M. DICKMAN C. \& ARCHER M. (eds), Predators with Pouches: the Biology of Carnivorous Marsupials. Surrey Beatty \& Sons, Sydney: 42-63.

Muizon C. DE \& CÉSPEDES-PAZ R. in press. - The beginning of the age of therian mammals in South America: Tiupampa, a transition between northern and southern worlds in the basal Paleocene, in Rosenberger A. \& Tejedor M. (eds), Origins and Evolution of Cenozoic South American Mammals. Springer Verlag, New York.

Muizon C. De, Cifelli R. L. \& Céspedes-Paz R. 1997. — The origin of the dog-like borhyaenoid marsupials of South America. Nature 389: 486-489.

Muizon C. De, Cifelli R. L. \& Paglarelli Bergqvist L. 1998. - Eutherian tarsals from the early Paleocene of Bolivia. Journal of Vertebrate Paleontology 18 (3): 655-663.

Nessov L. A., Archibald J. D. \& Kielan-Jaworowska Z. 1998. - Ungulate-like mammals from the Late Cretaceous of Uzbekistan and a phylogenetic analysis of Ungulatomorpha. Bulletin of the Carnegie Museum of Natural History 34: 40-88.

Nishihara H., MARUYAMA S., \& OKADA N. 2009. - Retroposon analysis and recent geological data suggest near-simultaneous divergence of the three superorders of mammals. Proceedings of the National Academy of Sciences 106 (13): 5235-5240.
Nomina Anatomica Veterinaria (5th edition) 2005. - Editorial Committee, Hannover, Columbia, Ghent, Sapporo, 1-166. http://www.wava-amav.org/Downloads/nav_2005.pdf

NOVACEK M. J. 1982. - The brain of Leptictis dakaotensis, an Oligocene leptictid (Eutheria: Mammalia) from North America. Journal of Paleontology 56 (5): 1177-1186.

NOVACEK M. J. 1986. - The skull of leptictid insectivorans and the higher-level classification of eutherian mammals. Bulletin of the American Museum of Natural History 183 (1): 1-112.

NOVACEK M. J. 1993. - Patterns of diversity in the mammalian skull, in Hanken J. \& Hall B. K. (eds), The Skull, Volume II: Patterns of Structural and Systematic Diversity. University of Chicago Press, Chicago: 438-545.

NovaceK M. J. \& Wyss A. R. 1986. — Origin and transformation of the mammalian stapes, in Flanagan K. M. \& LillegraVEN J. A. (eds), Vertebrates, Phylogeny and Philosophy. Contributions to Geology, the University of Wyoming, Special paper 3: 35-53.

NovaceK M. J., Rougier G. W., Wible J. R., McKenna M. C., Dashzeveg D. \& Horovitz I. 1997. - Epipubic bones in eutherian mammals from the Late Cretaceous of Mongolia. Nature 389: 483-486.

NOWAK R. M. 1999 - Walker's Mammals of the World. Sixth edition. Johns Hopkins University Press, Baltimore, 1936 p.

O'LEARY M. A. 2010. - An anatomical and phylogenetic study of the osteology of the petrosal of extant and extinct artiodactylans (Mammalia) and relatives. Bulletin of the American Museum of Natural History 335: 1-206.

O'Leary M. A. \& Geisler J. H. 1999. - The Position of Cetacea Within Mammalia: Phylogenetic Analysis of Morphological Data from Extinct and Extant Taxa. Systematic Biology 48 (3): 455-490.

O'Leary M. A., Bloch J. I., Flynn J. J., Gaudin T. J., Giallombardo A., Giannini N. P., Goldberg S. L., Kraatz B. P., Luo Z.-X., Meng J., Ni X., NovaceK M. J., Perini F. A., Randall Z. J., Rougier G. W., Sargis E. J., Silcox M. T., Simmons N. B., Spaulding M., Velazco P. M., WeKsler M., Wible J. R. \& Cirranello A. L. 2013. - The placental mam$\mathrm{mal}$ ancestor and the post-K-Pg radiation of placentals. Science 339: 662-667. http://dx.doi.org/10.1126/science.1229237

OrLiaC M. J. \& GilisSEN E. 2012. - Virtual endocranial cast of earliest Eocene Diacodexis (Artiodactyla, Mammalia) and morphological diversity of early artiodactyl brains. Proceedings of the Royal Society of London B: Biology 279 (1743): 3670-3677.

OrLiaC M. J. \& O'LEARY M. A. 2014. - Comparative anatomy of the petrosal bones of dichobunids, early members of Artiodactylomorpha (Mammalia). Journal of Mammalian Evolution 21 (3): 299-320.

OrliaC M. J., ARGOt C. \& Gilissen E. 2012a. - Digital cranial endocast of Hyopsodus (Mammalia, "Condylarthra"): a case of Paleogene terrestrial echolocation? PloS One 7 (2): e30000. http://dx.doi.org/10.1371/journal.pone.0030000

Orliac M. J., Benoit J. \& O'Leary M. A. 2012b. - The inner ear of Diacodexis, the oldest artiodactyl mammal. Journal of Anatomy 221: 417-426.

Orliac M. J., Ladevèze S., Gingerich P. D., Lebrun R. \& SMITH T. 2014. - Endocranial morphology of Palaeocene Plesiadapis tricuspidens and evolution of the early primate brain. Proceedings of the Royal Society of London B: Biology 281 (1781): 20132792.

Ortiz-Jaureguizar E. \& Pascual R. 1989. — South American land-mammal faunas during the Cretaceous-Tertiary transition: evolutionary biogeography, in Contribuciones de los simposios sobre Cretacico de América Latina. Parte A: Eventos y registro sedimentario. Buenos Aires: A231-A251.

Pascual R. \& Ortiz-Jaureguizar E. 2007. — The Gondwanan and South American Episodes: Two Major and Unrelated Moments in the History of the South American Mammals. Journal of Mammalian Evolution 14: 75-137. 
Patterson B. 1934. - A contribution to the osteology of Titanoides and the relationships of the Amblypoda. Proceedings of the American Philosophical Society 73: 71-101.

PATTERSON B. 1935. - Second contribution to the osteology and affinities of the paleocene amblypod Titanoides. Proceeedings of the American Philosophical Society 75 (2): 143-162.

PATTERSON B. 1936. - The internal structure of the ear in some notoungulates. Field Museum of Natural History, Geological Series 6: 161-166.

Patterson B. 1937. - Some notoungulate braincasts. Geological Series, Fieldiana Museum of Natural History 6: 273-301.

PATterson B. 1939. - New Pantodonta and Dinocerata from the upper Paleocene of Western Colorado. Field Museum of Natural History, Geological Series 6 (24): 351-384.

PATTERSON B. 1977. - A primitive pyrothere (Mammalia, Notoungulata) from the early Tertiary of northwestern Venezuela. Fieldiana Geology 33 (22): 397-422.

Patterson B. \& Simons E. L. 1958. - A new barylambdid pantodont from the late Paleocene. Breviora, Museum of Comparative Zoology 93: 1-8.

PaUla CouTO C DE 1952. - Fossil mammals from the beginning of the Cenozoic in Brazil: Condylarthra, Litopterna, Xenungulata, and Astrapotheria. Bulletin of the American Museum of Natural History 99: 355-394.

PIÉRARD J. 1971. - Osteology and myology of the Weddell seal Leptonychotes weddelli (Lesson, 1826). Antarctic Research Series 18: 53-108, in BURT W. H. (ed.), Antarctic Pinnipedia. American Geophysics Union, Washington DC.

POLLY P. D. 2007. - Evolutionary biology: development with a bite. Nature 449: 413-415.

Prasad A. B., Allard M. W. \& Green E. D. 2008. - Confirming the phylogeny of mammals by use of large comparative sequence data sets. Molecular Biology and Evolution 25 (9): 1795-1808.

Qiu Z., Li C., Huang X., TANG Y., Xu Q., YAN D. \& ZHANG H. 1977. - Continental Paleocene stratigraphy of Qianshan and Xuancheng Basins, Anhui. Vertebrata PalAsiatica 15 (2): 85-93.

QIU Z. \& LI C. 1977. - Miscellaneous mammalian fossils from the Paleocene of Qianshan Basin, Anhui. Vertebrata PalAsiatica 15 (2): 94-102.

Racicot R. A. \& Colbert M. W. 2013. -Morphology and variation in porpoise (Cetacea: Phocoenidae) cranial endocasts. The Anatomical Record 296 (6): 979-992.

RiCHANY S. F., ANSON B. J. \& BAST T. H. 1975. - The ear and the temporal bone development and adult structure, in ENGLISH G. M. (ed.), Otolaryngology, Vol. I, ch. 1. Harper and Row, Hagerstown: $1-140$.

RADINSKY L. 1978. - Evolution of brain size in carnivores and ungulates. The American Naturalist 112: 815-831.

Romiguier J., Ranwez V., Delsuc F., Galtier N. \& Douzery E. J. 2013. - Less is more in mammalian phylogenomics: AT-rich genes minimize tree conflicts and unravel the root of placental mammals. Molecular Biology and Evolution, mst 116.

Rose K. D. 1987. - Climbing adaptations in the early Eocene mammal Chriacus and the origin of the Artiodactyla. Science 236: 314-316.

Rose K. D. 1999. - Postcranial skeleton of Eocene Leptictidae (Mammalia) and its implications for behavior and relationships. Journal of Vertebrate Paleontology 19: 355-372.

Rose K. D. 2006. - The Beginning of the Age of Mammals. Johns Hopkins University Press, Baltimore, $428 \mathrm{p}$.

Rose K. D. \& CHINNERY B. J. 2004. - The postcranial skeleton of early Eocene rodents. Bulletin of the Carnegie Museum of Natural History 36: 211-244.

RossiE J. B. 2005. - Anatomy of the nasal cavity and paranasal sinuses in Aegyptopithecus and early Miocene African catarrhines. American Journal of Physical Anthropology 126: 250-267.

Rossie J. B. 2006. - Ontogeny and homology of the paranasal sinuses in Platyrrhini (Mammalia: Primates). Journal of Morphology 267: 1-40.
Rougier G.W. 1993. - Vincelestes neuquenianus Bonaparte (Mammalia, Theria) un primitivo mamifero del Cretácico Inferior de la Cuenca Neuquina. PhD dissertation, University of Buenos Aires, 720 p.

Rougier G. W. \& Wible J. R. 2006. - Major changes in the mammalian ear region and basicranium, in CARRANO $\mathrm{M}$. T., Gaudin T. J., Blob R. W. \& Wible J. R. (eds), Amniote Paleobiology: Perspective on the Evolution of Mammals, Birds, and Reptiles. University of Chicago Press, Chicago: 269-311.

Rougier G. W., Wible J. R. \& Hopson J. 1992. — Reconstruction of the cranial vessels in the Early Cretaceous mammal Vincelestes neuquenianus: implications for the evolution of the mammalian cranial vascular system. Journal of Vertebrate Paleontology 12 (2): 188-216.

Rougier G. W., Wible J. R. \& NovaceK M. J. 1998. - Implications of Deltatheridium specimens for early marsupial history. Nature 396: 459-463.

Rougier G. W., Wible J. R., Beck R. M. \& Apesteguía S. 2012. - The Miocene mammal Necrolestes demonstrates the survival of a Mesozoic nontherian lineage into the late Cenozoic of South America. Proceedings of the National Academy of Sciences 109 (49): 20053-20058.

Rowe T. B., Macrini T. E. \& LuO Z. X. 2011 - Fossil evidence on origin of the mammalian brain. Science 332 (6032): 955-957.

Rowe T. B., Eiting T. P., Macrini T. E. \& Ketcham R. A. 2005. - Organisation of the olfactory and respiratory skeleton in the nose of the Gray short-tailed opossum Monodelphis domestica. Journal of Mammalian Evolution 12: 303-336.

RusSELL D. E. 1964. - Les mammifères paléocènes d'Europe. Mémoires du Muséum national d'Histoire naturelle, série C, Géologie 13: 1-324.

Russell D. E. \& Sigogneau D. 1965. — Étude de moulages endocrâniens de mammifères paléocènes. Mémoires du Muséum national d'Histoire naturelle, série C, Géologie 16 (1): 1-34.

Russell D. E., Thewissen J. G. M. \& Sigogneau-Russell D. 1983. - A new dichobunid artiodactyl (Mammalia) from the Eocene of North-West Pakistan. Proceedings of the Koninklijke Nederlandse Akademie van Wetenschappen, Series B 86 (3): 285-300.

SAlOMON M. I. 1930. - Considérations sur l'homologie de l'os lachrymal chez les vertébrés supérieurs. Acta Zoologica 11: 151-183.

SÁNCHEZ-Villagra M. R. 2002. - The cerebellar paraflocculus and the subarcuate fossa in Monodelphis domestica and other marsupial mammals - ontogeny and phylogeny of a brain-skull interaction. Acta Theriologica 47 (1): 1-14.

SÁnchez-Villagra M. R. \& Wible J. R. 2002. — Patterns of evolutionary transformation in the petrosal bone and some basicranial features in marsupial mammals with special reference to didelphids. Journal of Zoological Systematics and Evolutionary Research 40 (1): 26-45.

SANIDES F. 1970. - Functional architecture of motor and sensory cortices in primates in the light of a new concept of neocortex evolution, in Noback R. \& Montagna W. (eds), The Primate Brain: Advances in Primatology. Appleton, New York: 137-201.

SAVAGE R. J. G. \& Long M. R. 1986. - Mammal Evolution, an Illustrated Guide. Facts on File, Inc., New York, 259 p.

SCHALler O. 2007 - Illustrated Veterinary Anatomical Nomenclature 2nd edition. Enke Verlag, Stuttgart, 615 p.

SCOTT W. B. 1910. - Mammalia of the Santa Cruz Beds. Vol. 7 , Paleontology. Part 1. Litopterna, in SCOTT W. B. (ed.), Reports of the Princeton University Expeditions to Patagonia, 1896-1899. Princeton University Press, Princeton, NJ: 1-156.

ScotT W. B. 1912. - Mammalia of the Santa Cruz Beds. Vol. 6, Paleontology. Part 2-3: Toxodonta and Entelonychia, in SсотT W. B. (ed.), Reports of the Princeton University Expeditions to Patagonia, 1896-1899. Princeton University Press, Princeton, NJ: 111-300. 
ScotT W. B. 1928. - Mammalia of the Santa Cruz Beds. Vol. 6, Paleontology. Part 4: Astrapotheria, in ScOTT W. B. (ed.), Reports of the Princeton University Expeditions to Patagonia, 1896-1899. Princeton University Press, Princeton, NJ: 301-342.

ScotT W. B. 1937. - The Astrapotheria. Proceedings of the American Philosophical Society 77: 309-393.

SEGALL W. 1970. - Morphological parallelisms of the bulla and auditory ossicles in some insectivores and marsupials. Fieldiana Zoology 51: 169-205.

Seiffert E. R., Simons E. L. \& Fleagle J. G. 2000. — Anthropoid humeri from the late Eocene of Egypt. Proceedings of National Academy of Sciences 97 (18): 10062-10067.

Sempere T., Butler R. L. \& Marshall L. G. 1997. - Stratigraphy and chronology of the Upper Cretaceous-Lower Paleogene strata in Bolivia and northwest Argentina. Geological Society of America, Bulletin 109 (6): 709-727.

ShOCKEY B. J. \& ANAYA F. 2004. - Pyrotherium macfaddeni, sp. nov. (late Oligocene, Bolivia) and the pedal morphology of pyrotheres. Journal of Vertebrate Paleontology 24: 481-488.

Signogneau-Russell D., Dashzeveg D. \& Russell D. E. 1992. - Further data on Prokennalestes (Mammalia, Eutheria inc. sed.) from the Early Cretaceous of Mongolia. Zoologica Scripta 21: 205-209.

Silcox M. T., Benham A. E. \& Bloch J. I. 2010. — Endocasts of Microsyops (Microsyopsidae, primates) and the evolution of the brain in primitive primates. Journal of Human Evolution 58: 505-521.

Simmons N. B. \& Conway T. M. 2003. - Evolution of ecological diversity, in Kunz T. H. \& Fenton M. B. (eds), Bat Ecology. University of Chicago Press, Chicago, IL: 493-535.

SimOns E. L. 1960. - The Paleocene Pantodonta. Transactions of the American Philosophical Society 50 (6): 1-81.

Simpson G. G. 1933. - Braincasts of Phenacodus, Notostylops, and Rhyphodon. American Museum Novitates 622: 1-19.

Simpson G. G. 1937. - The Fort Union of the Crazy Mountain Field, Montana and its mammalian faunas. Unites States National Museum Bulletin 169: 1-287.

SiMPSON G. G.1945. - The principles of classification and a classification of mammals. Bulletin of the American Museum of Natural History 85: 1-350.

SIMPSON G. G. 1948. - The beginning of the age of mammals in South America. Part I. Bulletin of the American Museum of Natural History 91 (1): 1-232.

SIMPSON G. G. 1967. - The beginning of the age of mammals in South America. Part II. Bulletin of the American Museum of Natural History 137: 1-259.

SinClair W. J. 1909. - Mammalia of the Santa Cruz Beds. Vol. 6, Paleontology. Part. 1: Typotheria of the Santa Cruz Beds, in SCOTT W. B. (ed.), Reports of the Princeton University Expeditions to Patagonia, 1896-1899. Princeton University Press, Princeton, NJ: 1-110.

SLOAN R. E. \& VAN VALEN L. 1965. - Cretaceous mammals from Montana. Science 148: 220-227.

Smith T. D. \& Rossie J. B. 2008. - The nasal fossa of mouse and dwarf lemurs (Primates, Cheirogaleidae). The Anatomical Record 291: 895-915.

SORENSON M. D. 1999. - TreeRot, version 2. Boston University, Boston, MA

Sprain C. J., Renne P. R., Wilson G. P. \& Clemens W. A. 2014. - High-resolution chronostratigraphy of the terrestrial Cretaceous-Paleogene transition and recovery interval in the Hell Creek region, Montana. Geological Society of America Bulletin Published online on 16 September 2014. http://dx.doi. org/10.1130/B31076.1

Springer M. S., Kirsch J. A. W. \& Case J. A. 1997. — The chronicle of marsupial evolution, in GIVNISH T. J. \& SYTSMA K. J. (eds), Molecular Evolution and Radiation. Cambridge University Press, New York: 129-161.
Springer M. S., Meredith R. W., Janecka J. E. \& Murphy W. J. 2011. - The historical biogeography of Mammalia. Philosophical Transactions of the Royal Society of London B: Biological Sciences, 366 (1577): 2478-2502.

Springer M. S., Meredith R. W., Teeling E. C. \& Murphy W. J. 2013. - Technical comment on "The placental mammal ancestor and the post-K-Pg radiation of placentals". Science 341 (6146): 613-613.

STEPHAN H. \& ANDY O. J. 1982. - General brain characteristics and septal areas of the Insectivores, in SCHNiTZLEIN H. N. (ed.), Comparative Correlative Neuroanatomy of the Vertebrate Telencephalon. MacMillan Publication Co. Inc., London: 525-564.

Stephan H., Baron G. \& Frahm H. D. 1991. - Comparative Brain Research in Mammals. Volume 1: Insectivora. Springer Verlag, New York, 573 p.

SwOFFORD D. L. 1993. - Phylogenetic Analysis Using Parsimony (PAUP), Version 3.1.1. University of Illinois, Champaign.

SWOFFORD D. L. 2002. — PAUP*: Phylogenetic analysis using parsimony (and other methods), version 4.0b1.0. Sunderland, Massachusetts: Sinauer.

SZALAY F. S. 1977. - Phylogenetic relationships and a classification of the eutherian Mammalia, in HechT P. C., Goody P. C. \& Hecht B. M. (eds), Major Patterns in Vertebrate Evolution. Plenum press, New York: 315-374.

SZALAY F. S. 1982. - A new appraisal of marsupial phylogeny and classification, in ARCHER M. (ed.), Carnivorous Marsupials. Royal Zoological Society of New South Wales, Sydney: 621-640.

SZALAY F. S. 1984. - Arboreality: is it homologous in metatherian and eutherian mammals?, in HЕCHT M. K. (ed.), Evolutionary Biology, Vol. 6. Plenum Publishing Corp., New York: 215-258.

SZALAY F. S. 1994. - Evolutionary History of the Marsupials and an Analysis of Osteological Characters. Cambridge University Press, New York, $481 \mathrm{p}$.

Szalay F. S. \& Trofimov B. A. 1996. - The Mongolian Late Cretaceous Asiatherium, and the early phylogeny and paleobiogeography of Metatheria. Journal of Vertebrate Paleontology 16 (3): 474-509.

Tassy P. 1981. - Le crâne de Moeritherium (Proboscidea, Mammalia) de l'Éocène de Dor El Talha (Lybie) et le problème de la classification phylogénétique du genre dans les Tethytheria McKenna 1975. Bulletin du Muséum national d'Histoire naturelle, Section C, Sciences de la Terre 1: 87-147.

Templeton A. R. 1983. - Phylogenetic inference from restriction endonuclease cleavage site maps with particular reference to the evolution of humans and the apes. Evolution 37: 221-244.

THEWISSEN J. G. M. 1989. - Mammalian frontal diploic vein and the human foramen caecum. The Anatomical Record 223: 242-244.

Thewissen J. G. M. 1990. — Evolution of Paleocene and Eocene Phenacodontidae (Mammalia, Condylarthra). Papers on Paleontology 29: 1-107.

THEWISSEN J. G. M. 1991. — Limb osteology and function of the primitive Paleocene ungulate Pleuraspidotherium with notes on Tricuspiodon and Dissacus (Mammalia). Geobios 24 (4): 483-495.

TheWissen J. G. M. \& Gingerich P. D. 1989. - Skull and endocranial cast of Eoryctes melanus, a new palaeoryctid (Mammalia: Insectivora) from the early Eocene of western North America. Journal of Vertebrate Paleontology 9: 454-470.

Thewissen J. G. M. \& Domning D. P. 1992. - The role of phenacodontids in the origin of the modern orders of ungulate mammals. Journal of Vertebrate Paleontology 12: 494-504.

Thewissen J. G. M., Williams E. M., Roe L. J. \& Hussain S.T. 2001. - Skeletons of terrestrial cetaceans and the relationship of whales to artiodactyls. Nature 413 (6853): 277-281.

ToBIAS P. V. 1971. - The Brain in Hominid Evolution. Columbia University Press, New York, $170 \mathrm{p}$.

VAN VALEN L. M. 1978. - The beginning of the age of mammals. Evolutionary Theory 4: 45-80. 
VAn Valen L. M. 1988. - Paleocene dinosaurs or Cretaceous ungulates in South America? Evolutionary Monographs 10: 1-79.

Van Valkenburgh B., Smith T. D. \& Craven B. A. 2014. - Tour of a labyrinth: exploring the vertebrate nose. The Anatomical Record 297: 1975-1984.

Villarroel C. 1974. - Les Mésothériinés (Notoungulata, Mammali) du Pliocène de Bolivie. Leurs rapports avec ceux d'Argentine. Annales de Paléontologie 60 (2): 245-281.

VillarRoel C. 1987. — Características y afinidades de Etayoa n. gen., tipo de una nueva familia de Xenungulata (Mammalia) del Paleoceno medio de Colombia. Comunicaciones Paleontológicas del Museo de Historia Natural de Montevideo 19: 241-253.

WANG B. Y. 1979. - A new species of Harpyodus and its taxonomic position, in The Mesozoic and Cenozoic Red Beds of South China. Selected papers from "the field Conference on South China Cretaceous-Early Tertiary red beds" held at Nanxiong, Guandong Province, 24 November-6 December 1976. IVPP Academia Sinica and Nanjing Institute of Geology and Paleontology, Academia Sinica, Kexue Chusbanshe, Beijing: 366-372.

Welker F., Collins M. J., Thomas J. A., Wadsley M., Brace S., Cappellini E., ... \& MacPheE R. D. 2015. - Ancient proteins resolve the evolutionary history of Darwin's South American ungulates. Nature 522: 81-84.

West R. M. 1979. - Paleontology and Geology of the Bridger Formation, Southern Green River Basin, Southwestern Wyoming. Part 3. Notes on Hyopsodus. Contribution in Biology and Geology 25: 1-52.

Wible J. R. 1984. - The Ontogeny and Phylogeny of the Mammalian Cranial Arterial Pattern. PhD Dissertation, Duke University, Durham, $704 \mathrm{p}$.

WibLE J. R. 1986. - Transformations in the extra-cranial course of the internal carotid artery in mammalian phylogeny. Journal of Vertebrate Paleontology 6: 313-325.

Wible J. R. 1987. — The eutherian stapedial artery: character analysis and implications for superordinal relationships. Zoological Journal of the Linnean Society 91:107-135.

WibLE J. R. 1990. — Petrosals of Late Cretaceous marsupials from North America, and a cladistic analysis of the petrosal in therian mammals. Journal of Vertebrate Paleontology 10 (2): 183-205.

Wible J. R. 1993. - Cranial circulation and relationships of the colugo Cynocephalus (Dermoptera, Mammalia). American Museum Novitates 3072: 1-27.

WiBLE J. R. 2003. - On the cranial osteology of the short-tailed opossum Monodelphis brevicaudata (Marsupialia, Didelphidae). Annals of the Carnegie Museum 72 (3): 1-66.

WibLE J. R. 2008. - On the cranial osteology of the Hispaniolan solenodon, Solenodon paradoxus, Brandt, 1833 (Mammalia, Lipotyphla, Solenodontidae). Annals of the Carnegie Museum 77 (3): 321-402.

Wible J. R. 2009. - The ear region of the pen-tailed treeshrew, Ptilocercus lowii Gray 1848 (Placentalia, Scandentia, Ptilocercidae). Journal of Mammalian Evolution 16 (3): 199-234.

Wible J. R. 2010. - Petrosal anatomy of the nine-banded armadillo Dasypus novemcinctus Linnaeus, 1758 (Mammalia, Xenarthra, Dasypodidae). Annals of the Carnegie Museum 79 (1): 1-28.

WibLE J. R. 2011. - On the treeshrew skull (Mammalia, Placentalia, Scandentia). Annals of the Carnegie Museum 79 (3): 149-230.

Wible J. R. 2012. - The ear region of the aardvark, Orycteropus afer (Pallas, 1766) (Mammalia, Placentalia, Tubulidentata). Annals of the Carnegie Museum 80 (2): 115-146.

Wible J. R. \& NovaceK M. J. 1988. - Cranial evidence for the monophyletic origin of bats. American Museum Novitates 2911: 1-19.

Wible J. R. \& Hopson J. A. 1993. - Basicranial evidence for early mammal phylogeny, in Szalay F. S., NovaceK M. J. \&. McKenna M. C. (eds), Mammal Phylogeny. Vol. 1: Mesozoic Differentiation, Multituberculates, Monotremes Early Therians and Marsupials. Springer-Verlag, New York: 45-62.
Wible J. R. \& Zeller U. 1994. - Cranial circulation of the pen-tailed tree shrew Ptilocercus lowii and relationships of Scandentia. Journal of Mammalian Evolution 2: 209-230.

Wible J. R. \& Rougier G. W. 2000. - Cranial anatomy of Kryptobaatar dashzevegi (Mammalia, Multituberculata), and its bearing on the evolution of mammalian characters. Bulletin of the American Museum of Natural History 247: 1-124.

Wible J. R. \& GAUdin T. J. 2004. - On the cranial osteology of the yellow armadillo Euphractus sexcinctus (Dasypodidae, Xenarthra, Placentalia). Annals of the Carnegie Museum 73 (3): 117-196.

Wible J. R. \& SPAULding M. 2013. - On the cranial osteology of the African palm civet, Nandinia binotata (Gray, 1830) (Mammalia, Carnivora, Feliformia). Annals of the Carnegie Museum of Natural History 82 (1): 1-114.

Wible J. R., Rougier G. W., Novacek M. J. \& McKenna M. C. 2001 - Earliest eutherian ear region: a petrosal of Prokennalestes from the Early Cretaceous of Mongolia. American Museum Novitates 3322: 1-44.

Wible J. R., NovaceK M. J. \& Rougier G. W. 2004. - New data on the skull and dentition in the Mongolian Cretaceous eutherian mammal Zalambdalestes. Bulletin of the American Museum of Natural History 281: 1-144.

Wible J. R., Wang Y.-Q., Li J.-K. \& DAWSON M. R. 2005. Cranial anatomy and relationships of a new ctenodactyloid (Mammalia, Rodentia) from the early Eocene of Hubei Province, China. Annals of the Carnegie Museum 74 (2): 91-150.

Wible J. R., Rougier G. W., Novacek M. J. \& Asher R. J. 2007. - Cretaceous eutherians and Laurasian origin for placental mammals near the K/T boundary. Nature 447 (7147): 1003-1006.

Wible J. R., Rougier G. W., Novacek M. J. \& Asher R. J. 2009. - The eutherian mammal Maelestes gobiensis from the Late Cretaceous of Mongolia and the phylogeny of Cretaceous Eutheria. Bulletin of the American Museum of Natural History 327: 1-123.

Wilf P., Cúneo N. R., Escapa I. H., Pol D. \& Woodburne M. O. 2013. - Splendid and seldom isolated: the paleobiogeography of Patagonia. Annual Review of Earth and Planetary Sciences 41 (1): 561.

Williams B. A. 2007. - Comparing levels of homoplasy in the primate skeleton. Journal of Human Evolution 52 (5): 480-489.

Williamson T. E. \& Lucas S. G. 1992. - Meniscotherium (Mammalia, "Condylarthra") from the Paleocene-Eocene of North America. Bulletin of the New Mexico Museum of Natural History 1: 1-75.

Williamson L. A. \& CARR T. D. 2007. - Bomburia and Ellipsodon (Mammalia: Mioclaenidae) from the early Paleocene of New Mexico. Journal of Paleontology 81 (5): 966-985.

WiLson G. P. 2013. - Mammals across the K/Pg boundary in Northeastern Montana, USA: Dental morphology and bodysize patterns reveal extinction selectivity and immigrant-fueled ecospace filling. Paleobiology 39: 429-469.

Wilson G. P. 2014. - Mammalian extinction, survival, and recovery dynamics across the Cretaceous-Paleogene boundary in Northeastern Montana, USA, in WILSON G. P., CLEMens W. A., Horner J. R., \& Hartman J. H. (eds), Through the End of the Cretaceous in the Type Locality of the Hell Creek Formation in Montana and Adjacent Areas. Geological Society of America Special Paper 503: 365-392.

Wilson L. A., Madden R. H., Kay R. F. \& Sánchez-VillaGRA M. R. 2012. - Testing a developmental model in the fossil record: molar proportions in South American ungulates. Paleobiology 38 (2): 308-321.

Woodburne M. O., Goin F. J., Bond M., Carlini A. A., Gelfo J. N., Lopez G. M., Iglesias A. \& ZimicZ A. N. 2014. Paleogene land mammal faunas of South America; a response to climatic changes and indigenous floral diversity. Journal of 
Mammalian Evolution 21 (1): 1-73.

Zack S. P., Penkrot T. A., Bloch J. I. \& Rose K. D. 2005. Affinities of 'hyopsodontids' to elephant shrews and a Holarctic origin of Afrotheria. Nature 434: 497-501.

Zhou M. Z., Zhang Y., Wang B. \& Ting S. 1977. — Mam- malian fauna from the Paleocene of Naxiong Basin, Guandong. Palaeontologia Sinica 153, new series C, 20: 1-100.

Zhou M. Z. \& Wang B. 1979. — Relationship between the pantodonts and tillodonts and classification of the order Pantodonta. Vertebrata PalAsiatica 17: 37-48.

Submitted on 14 January 2015; accepted on 15 September 2015; published on 31 December 2015. 


\section{APPENDICES}

\section{APPENDIX 1. - Character list.}

The character list below is based on the datamatrix of Wible et al. (2009), and the original numbers of the characters in that previous work is noted with a W: for example, the character 150 of Wible et al. (2009) is noted "150W" below. When the character has been modified (in its definition) from the original version of Wible et al., it is indicated: e.g., for the ex-character 193 of Wible et al., the notation "193W modified" indicates that the character has been modified.

In addition to the modifications of some characters, some others have been deleted for the following reasons:

$-28 \mathrm{~W}, 30 \mathrm{~W}, 35 \mathrm{~W}, 103 \mathrm{~W}, 155 \mathrm{~W}, 159 \mathrm{~W}, 229 \mathrm{~W}$ : not informative in our datamatrix and/or difficult/impossible to score confidently for fossils.

-91W: measurement of conular region width not feasible when conules do not have the same extent (e.g., artiodactyls, Pleuraspidotherium).

- 114W: the cuspule is most often absent despite the presence of an anterocingulid, thus we disagree with the scores of Wible et al. (2009) for this character and cast doubts on its informativeness here.

$-143 \mathrm{~W}$ : we disagree with the observations and scores; the observed variation is difficult to score confidently.

- 167W, 184W, 331W, 335W: we disagree with the observations and scores. The criteria to separate the various states are not explicit/clear enough; thus, we were not able to reproduce the scoring of Wible et al. (2009) for these characters.

$-104 \mathrm{~W}$ and 127W: redundant with the newly added characters that compare areas of M1-M3.

For the main analyses (see Material and methods), some multistate characters were treated as ordered (additive) when logical connections between states could be easily established. They are indicated below by the mention "ORDERED".

\section{DENTITION GENERAL}

1 (1W). — Teeth: (0) present; (1) absent (Wible et al. 2009: 1).

$2(2 \mathrm{~W})$. - Teeth: (0) differentiated into morphological type (incisors, canines, premolars, molars) with enamel; (1) homodont and peg-like without enamel (Wible et al. 2009, ch. 2).

$3(3 \mathrm{~W})$. - Number of postcanine tooth loci: (0) eight or more, (1) seven, (2) six, (3) or five or less (Rougier et al. 1998, ch. $7^{*}$ ); ORDERED.

$4(4 \mathrm{~W})$. - Upper diastema: (0) small, between incisors and canine, (1) small, between canine and premolars, (2) enlarged, or (3) absent (Wible et al. 2009, ch. 4).

$5(5 \mathrm{~W})$. - Lower diastema behind incisors: (0) absent or small; (1) enlarged (Wible et al. 2009, ch. 5).

6 (NEW). - Dental eruption timing vs cranial growth: (0) early; (1) late (adult size reached prior to complete eruption of permanent cheek teeth; specimens with $95 \%$ median adult jaw length and less than $60 \%$ of their permanent, occluding premolars and molars erupted; Asher \& Lehmann 2008).

\section{INCISORS}

$7(6 \mathrm{~W})$. - Incisor shape: (0) root and crown are straight and continuous in length or (1) form a continuous curve (Asher $e t$ al. 2005, ch. 3).

8 (7W). - Number of upper incisors: (0) five; (1) four; (2) three; (3) two; (4) one; (5) none Wible et al. 2009, ch. 7); ORDERED.

$9(8 \mathrm{~W})$. - Number of lower incisors: (0) four; (1) three; (2) two; (3) one; (4) none (Wible et al. 2009, ch. 8); ORDERED.

$10(9 \mathrm{~W})$. - Position of anteriormost upper incisors (or alveoli): (0) contacting medially or closely approximated; (1) widely separated (Wible et al. 2009, ch. 9).
11 (NEW SEE 10W). - Upper incisors relative size: (0) subequal; (1) anteriormost is the largest (close to twice the size of following incisors); (2) second anteriormost is the largest (close to twice the size adjacent incisors); (3) third anteriormost is the largest (close to twice the size adjacent incisors) (new character defined on the basis of Wible et al. 2009, ch. 10).

$12(11 \mathrm{~W})$. - Anteriormost upper incisor shape: (0) conical; (1) mediolaterally compressed; (2) anteroposteriorly compressed; (3) cuspate (one major and one minor); (4) spatulate (Wible et al. 2009, ch. 11).

13 (16W). - Anteriormost lower incisor shape: (0) conical; (1) mediolaterally compressed; (2) anteroposteriorly compressed; (3) cuspate (one major and one minor); (4) spatulate (wible et al. 2009, ch. 16).

14 (NEW). - Anteriormost upper incisor procumbent: (0) absent; (1) present.

15 (NEW). - Second anteriormost upper incisor projecting anteriorly: (0) absent; (1) present (Billet 2010, ch. 9, modified).

$16(12 \mathrm{~W}) .-$ Anteriormost upper incisor root: (0) closed; (1) open extending in premaxilla only; (2) open extending in maxilla (Asher et al. 2005, ch. 52; Wible et al. 2009, ch. 12).

$17(13 \mathrm{~W})$. - Anteriormost incisor enamel: (0) surround tooth; (1) discontinuous posteriorly (Asher et al. 2005, ch. 49*; Wible et al. 2009, ch. 13).

18 (14W). - Ultimate upper incisor: (0) in premaxilla; (1) between maxilla and premaxilla; (2) or in maxilla (Wible et al. 2009, ch. 14).

19 (15W). - Anteriormost lower incisor size: (0) small and subequal to subsequent incisors; (1) greatly enlarged; (2) greatly reduced (Archibald et al. 2001, ch. 28*; Wible et al. 2009, ch. 15).

20 (17W). - Procumbent anteriormost lower incisor: (0) absent; (1) present (Archibald et al. 2001, ch. 29; Wible et al. 2009, ch. 17). 
21 (18W). - Anteriormost lower incisor root: (0) closed; (1) open (Archibald et al. 2001, ch. 32; Wible et al. 2009, ch. 18).

22 (19W). - Anteriormost lower incisor root length: (0) not extended posteriorly below $\mathrm{p} 1,(1)$ extending posteriorly below $\mathrm{p} 1$, (2) extending posteriorly below penultimate or ultimate premolar,

(3) or extending posteriorly below molars (Archibald et al. 2001, ch. $\left.32^{*}\right)$; ORDERED.

23 (20W). - Anteriormost lower incisor enamel: (0) covers the whole incisor or (1) discontinous posteriorly (Archibald et al. 2001, ch. 30).

24 (21W). - Procumbent posterior lower incisors: (0) absent; (1) present (Wible et al. 2009, ch. 21).

25 (22W). - Staggered lower i3: (0) absent; (1) present (Hershkovitz 1982). Changes in the scoring of Wible et al. (2009): Rhynchocyon, $(1)>(0)$.

26 (NEW). - Shape of incisor arcade: (0) transverse; (1) U-shaped; (2) sharply angled at I1s (V-shaped) or parallel-sided. Modified from Springer et al. (1997, ch. 25), Horovitz \& Sánchez-Villagra (2003, ch. 161), Ladevèze \& Muizon (2007, ch. 8).

\section{CANINES}

27 (23W). - Upper canine: (0) large; (1) reduced; (2) absent (Meng et al. 2003, ch. 23); ORDERED.

28 (24W). - Roots of upper canines: (0) two roots; (1) one root (Rougier et al. 1998, ch. 10; Wible et al. 2009, ch. 24).

29 (25W). - Lower canine: (0) large; (1) reduced; (2) absent (Meng et al. 2003, ch. 25); ORDERED.

30 (26W). - Roots of lower canines: (0) two roots; (1) one root (Rougier et al. 1998, ch. 44; Wible et al. 2009, ch. 26).

31 (27W). - Procumbent lower canine: (0) absent; (1) present (Wible et al. 2009, ch. 27).

32 (NEW). - Canines diverging externally, uppers and/or lowers: (0) absent; (1) present.

\section{Premolars}

33 (29W). - Number of premolars: (0) five; (1) four; (2) three; (3) less than three (Rougier et al. 1998, ch. 1); ORDERED.

34 (31W). - Tall, trenchant premolar: (0) ultimate premolar; (1) penultimate premolar; (2) absent (Rougier et al. 1998, ch. 3).

35 (32W). - Procumbent first upper premolar: (0) absent; (1) present (Luo \& Wible 2005, ch. 151 modified).

$36(33 \mathrm{~W})$. - First upper premolar (P1 in placentals, cf. Wible et al. 2009 matrix) roots: (0) one; (1) two; (2) three: eutherians with lost P1 are coded non-applicable; ORDERED.

37 (34W). - Diastema posterior to first upper premolar: (0) absent; (1) present (Luo \& Wible 2005, ch. 43; Wible et al. 2009, ch. 34).

38 (NEW). - Shape of the premolar cusps: (0) sharp and uninflated; (1) inflated with apical wear strongly developed (Cifelli 1993; Rougier et al. 1998, ch. 2; Wible et al. 2001, ch. 2).

$39(36 \mathrm{~W})$. - Protocone of the penultimate premolar: (0) absent; (1) lingual bulge; (2) basined (Rougier et al. 1998, ch. 12).

40 (37W). - Metacone of penultimate upper premolar: (0) absent; (1) swelling; (2) large (Wible et al. 2009, ch. 37).
41 (38W). - Penultimate upper premolar parastylar lobe: (0) absent or small; (1) well developed (Wible et al. 2009, ch. 38).

42 (NEW). - Vertically developed cylindrical parastyle separated from paracone by a deep vertical sulcus on labial edge of upper cheek teeth (at least PM): (0) absent; (1) present (modified from Billet 2010, ch. 37).

43 (39W). - Penultimate upper premolars roots: (0) one; (1) two; (2) three; (3) four (Wible et al. 2009, ch. 39); ORDERED.

$44(40 \mathrm{~W})$. - Ultimate upper premolar protocone: (0) absent or narrow cingulum; (1) present but distinctly smaller than paracone; (2) similar in thickness and height to paracone (Rougier et al. 1998; Wible et al. 2009, ch. 40); ORDERED.

45 (41W). - Last upper premolar metacone: (0) absent; (1) swelling (metacone connate to paracone); (2) large (metacone and paracone distinctly separated) (Luo \& Wible 2005, ch. 39).

46 (42W). - Last upper premolar para- and metastylar lobes: (0) absent or insignificant; (1) present and subequal; (2) parastylar lobe larger; (3) metastylar lobe larger (Wible et al. 2009; 42).

47 (43W). - Ultimate premolar precingulum: (0) absent; (1) present (Wible et al. 2009; 43).

48 (44W MODIFIED). - Ultimate premolar postcingulum: (0) absent; (1) present (Wible et al. 2009; 44 modified). Taxa originally scored (2) in $44 \mathrm{~W}$ have been scored (1) here (i.e. with taxa originally scored (1) in $44 \mathrm{~W})$.

49 (45W). - Ultimate premolar conules: (0) absent or very weak; (1) present as a distinct cusp (prominent) (Wible et al. 2009, ch. 45).

$50(46 \mathrm{~W})$. - Ultimate upper premolar size (occlusal surface, L x W) relative to first upper molar: (0) smaller or subequal; (1) larger (Meng et al. 2003, ch. 41).

51 (NEW). - Double V-shaped penultimate and ultimate upper premolars (paracone and protocone V-shaped): (0) absent; (1) present.

$52(47 \mathrm{~W})$. - First lower premolar orientation: (0) in line with the jaw axis; (1) oblique (Rougier et al. 1998, ch. 45; Wible et al. 2009, ch. 47).

53 (48W). - First lower premolar roots: (0) two; (1) one (Wible et al. 2009, ch. 48)

54 (49W). - Diastema separating first and second lower premolars: (0) absent (gap less than one tooth root diameter); (1) present (subequal to or larger than one tooth root diameter) (Luo \& Wible 2005, ch. 152 modified; Wible et al. 2009, ch. 49). Changes in the scorings of Wible et al. (2009): Pucadelphys $(1)>(0,1)$.

55 (50W). - Third lower premolar size compared to second (only scored for taxa with five lower premolars): (0) longer or (1) shorter (Wible et al. 2009, ch. 50).

$56(51 \mathrm{~W})$. - Third lower premolar roots (only scored for taxa with five lower premolars): (0) two or (1) one (Wible et al. 2009, ch. 51).

$57(52 \mathrm{~W})$. - Penultimate lower premolar paraconid: (0) vestigial or absent; (1) distinctive (Luo \& Wible 2005, ch. 52 modified; Wible et al. 2009, ch. 52).

58 (53W). - Penultimate lower premolar metaconid: (0) absent; (1) swelling or metaconid connate to paraconid; (2) metaconid and paraconid distinctly separated (Wible et al. 2009, ch. 53).

59 (54W). - Penultimate lower premolar talonid cusps: (0) one; (1) two; (2) three (Wible et al. 2009, ch. 54); ORDERED. 
60 (55W MODIFIED). - Ultimate lower premolar paraconid: (0) absent (there may be a small swelling at the anterior base of the protoconid but a well-defined cusp is not present); (1) present but low: less than half the height of protoconid (the paraconid is generally a small cusp at the anterior base of the protoconid); (2) present and high, half the height of protoconid or more (Wible et al. 2009, ch. 55 modified); ORDERED.

61 (56W). - Ultimate lower premolar metaconid: (0) absent; (1) metaconid present as a small bulge on posteromedial side of protoconid (swelling); (2) metaconid distinctly individualized (separate, large) (Wible et al. 2009, ch. 56).

62 (57W). - Ultimate lower premolar talonid: (0) narrower than anterior portion of the crown; (1) as wide as anterior portion of the crown; (2) absent (Archibald \& Averianov 2006, ch. 25).

63 (58W). - Ultimate lower premolar talonid cusps: (0) one; (1) two; (2) three (Wible et al. 2009, ch. 58); ORDERED.

$64(59 \mathrm{~W})$. - Length of ultimate lower premolar relative to penultimate premolar: (0) longer; (1) equal or shorter (Archibald \& Averianov 2006, ch. 24).

$65(60 \mathrm{~W})$. - Ultimate lower premolar anterolingual cingulid: (0) absent or (1) present (Wible et al. 2009, ch. 60).

\section{UPPER MOLARS}

66 (61W). - Number of molars: (0) four or more; (1) three; (2) two (Wible et al. 2009, ch. 61); ORDERED.

NB: The four following characters replace $62 \mathrm{~W}$ and are notably based on the studies by Kavanagh et al. (2007), Polly (2007), Wilson et al. (2012) and Halliday \& Goswami (2013).

67 (NEW). - Size (area) of M1 vs M2: (0) M1 much larger (>120\%); (1) subequal $(80 \%<>120 \%)$; (2) M1 much smaller $(80 \%<)$; ORDERED.

Comments on characters 67 to 70: Homology between metatherian and eutherian molars refers to O'Leary et al. (2013). The first molar of metatherians is regarded as a DP5 and the second molar of metatherians is regarded as homologous to the first molar of eutherians. Therefore, in the case of metatherians, comparisons are made between M2 and M3 and between M2 and M4. Furthermore, the scores for the taxa in common with Billet et al. (2015), are scored after the same sources (see references therein).

68 (NEW). - Size (area) of M1 vs M3: (0) M1 much larger (>120\%); (1) subequal $(80 \%<>120 \%)$; (2) M1 much smaller $(80 \%<)$; ORDERED.

69 (NEW). - Size (area) of $\mathrm{m} 1 \mathrm{vs} \mathrm{m} 2:(0) \mathrm{m} 1$ much larger (>120\%); (1) subequal $(80 \%<>120 \%)$; (2) m1 much smaller $(80 \%<)$; ORDERED.

70 (NEW). - Size (area) of $\mathrm{m} 1 \mathrm{vs} \mathrm{m} 3:(0) \mathrm{m} 1$ much larger (>120\%); (1) subequal $(80 \%<>120 \%)$; (2) m1 much smaller $(80 \%<)$; ORDERED.

$71(63 \mathrm{~W})$. - Molar cusps form: (0) sharp and gracile; (1) inflated and robust; (2) crest-like (Wible et al. 2009, ch. 63).

$72(64 \mathrm{~W})$. - Upper molar shape: (0) as long as wide or longer; (1) wider than long (length more than $75 \%$ but less than $99 \%$ of the width); (2) much wider than long (length less than $75 \%$ of the width) (Wible et al. 2009, ch. 64); ORDERED.

73 (65W). - Upper molar stylar shelf maximum width: (0) 50\% or more of total width; (1) between $50 \%$ and $25 \%$ of width; (2) less than 25\%; (3) absent (Wible et al. 2009, ch. 65); ORDERED.
74 (66W). - Labial extent of parastylar and metastylar lobes: (0) parastylar lobe more labial; (1) lobes subequal; (2) metastylar lobe more labial; (3) lobes absent (Wible et al. 2009, ch. 66)

75 (67W). - M1 parastylar lobe relative to paracone: (0) anterolabial to paracone; (1) anterior to paracone (Archibald \& Averianov 2006, ch. 7; Wible et al. 2009, ch. 67) (if parastylar lobe is absent, scored as unapplicable).

$76(68 \mathrm{~W})$. - Length of the parastylar lobe (measured to stylocone or stylocone position) relative to total length on penultimate molar: (0) more than $30 \%$ of the tooth length; (1) between $30 \%$ and $20 \%$; (2) $20 \%$ or less. (Wible et al. 2009, ch. 68); ORDERED.

77 (69W). - Preparastyle: (0) absent; (1) present (Rougier et al. 1998, ch. 21).

78 (70W MODified). - Stylar cusp A: (0) subequal to or larger than stylar cusp B; (1) distinct but smaller than B; (2) very small to indistinct (Wible et al. 2009, ch. 70). Taxa in which both stylar cusps $\mathrm{A}$ and $\mathrm{B}$ are not identifiable are scored inapplicable. Changes in the scorings of Wible et al. (2009): Mayulestes, Pucadelphys: (0) > (1); Zhelestes, Solenodon, Eoryctes, Potamogale: (2) > (1); Cimolestes, Maelestes, Kennalestes, Asioryctes, Kulbeckia, Zalambdalestes, Protungulatum: (2) < (0); Meniscotherium, Phenacodus: (1) > (-); Hyopsodus, Ptilocercus, Plesiadapis, Notharctus, Adapis, Tribosphenomys, Erinaceus: $(2)>(-)$.

79 (71W). - Size of stylar cusp B relatively to paracone: (0) vestigial to absent; (1) smaller but distinct; (2) subequal (Rougier et al. 1998, ch. 22) Taxa scored inapplicable for character 78 are also coded inapplicable for this character. Changes in the scorings of Wible et al. (2009; order of states modified): Mayulestes: (2) > (1); ORDERED.

80 (72W). - Stylar cusp C, mesostyle: (0) absent; (1) present (Rougier et al. 1998, ch. 23). Changes in the scorings of Wible et al. (2009): Mayulestes, Pucadelphys: (0) > (1).

81 (73W MODIFIED). - Stylar cusp D: (0) absent; (1) smaller or subequal to B (Rougier et al. 1998, ch. 24). Changes in the scores of Wible et al. (2009): Potamogale: (0) > (1); Rhynchocyon, Procavia, Moeritherium, $(-)>(0)$.

$82(74 \mathrm{~W})$. - Stylar cusp E: (0) lingual to D or to D position; (1) distal to D; (2) small to indistinct (Rougier et al. 1998, ch. 25). Changes in the scorings of Wible et al. (2009): Rhynchocyon, Procavia, $(-)>(1)$; Moeritherium, $(-)>(2)$.

$83(75 \mathrm{~W})$. - Paracingulum (= preparacingulum of Wible et al. 2009): (0) absent; (1) interrupted between stylar margin and paraconule; (2) continuous between stylar margin and paraconule (taxa with a protoloph with indistinct paraconule are coded 2 as we hypothesize that the labial portion of the protoloph corresponds to the paracingulum) (Wible et al. 2009, ch. 75).

84 (76W). - Deep ectoflexus: (0) present on penultimate molar; (1) present on penultimate and preceding molars; (2) very small or absent (Wible et al. 2009, ch. 76).

85 (77W) MODIFIED. - Relative size of the paracone and metacone on penultimate molar: (0) metacone smaller than paracone; (1) cusps subequal; (2) paracone smaller than metacone (Wible et al. 2009, ch. 77 modified); ORDERED.

86 (78W). - Metacone position relative to paracone: (0) labial; (1) approximately at the same level; (2) lingual (Rougier et al. 1998, ch. 28); ORDERED.

87 (79W). - Paracone and metacone at base: (0) fused at base; (1) distinctly separated; (Muizon \& Marshall 1992, ch. 13). 
88 (80W). - Preparacrista: (0) strong, from labial edge of paracone to stylocone; (1) weak (from base of paracone) or absent (Wible et al. 2009, ch. 80)

89 (NEW). - Orientation of the preparacrista on penultimate and ante-penultimate molars and premolars (in the case of molariform premolars): (0) deflected labially and tend to be aligned with or sub parallel to paracone-protocone axis; (1) shifted anteriorly, forming a distinct angle with paracone-protocone axis. Taxa without a distinct preparacrista are scored inapplicable.

$90(81 \mathrm{~W})$. - Cuspate preparacrista: (0) present; (1) absent (Wible et al. 2009, ch. 81)

91 (82W). - Centrocrista: (0) straight; (1) V-shaped (2) absent (Wible et al. 2009, ch. 82).

$92(83 \mathrm{~W})$. - Postmetacrista: (0) prominent from side of metacone to metastyle; (1) salient (expanded posterolabially); (2) absent or weak (Wible et al. 2009, ch. 83).

93 (84W). - Cuspate postmetacrista: (0) present; (1) absent (Wible et al. 2009, ch. 84).

94 (85W). - Preprotocrista: (0) does not extend labially below the paracone; (1) extends labially below the paracone (double rank prevallum/postvallid shearing); (2) absent (Wible et al. 2009, ch. 85) (taxa with a protoloph are coded (1)).

95 (86W). - Postprotocrista: (0) extends to mid-lingual surface of metacone; (1) extends distal to metacone; (2) absent (Wible et al. 2009, ch. 86). (taxa with a metaloph are coded (1).

96 (87W). - Development of postvallum shear: (0) present, but only by the first rank: postmetacrista; (1) present, with the addition of a second rank (postprotocrista below postmetacrista) but the second rank does not reach labially below the base of the metacone; (2) present, with second rank extending to metastylar lobe: metacingulum; (3) absent (Luo \& Wible 2005, ch. 57*). All taxa with a hypocone subequal to protocone are scored as non applicable.

97 (NEW). - Labial edges of paracone and metacone: (0) Distinctly swollen; (1) slightly convex; (2) flat to concave; ORDERED. Comment: the labial edge of the paracone and metacone on the upper molars of state $(O)$ is strongly convex and presents an anteroposteriorly wide rounded ridge (rib tile-shaped), which extends from the base of the crown to the apex of the cusps; because the convexity is almost identical on both side of the cusps, the latter are roughly conical. On state (1) this rib is present but very weak (at the most the lateral side of the cusps is slightly convex) and the cusps are clearly asymmetrical. On state (2) the lateral side of the cusps is either flat or concave and their cross-section is triangular.

98 (88W). - Paraconule: (0) weak or absent; (1) prominent, closer to protocone; (2) or prominent, mid-way or closer to paracone (Wible et al. 2009, ch. 88).

99 (89W). - Metaconule: (0) weak or absent; (1) prominent, closer to protocone; (2) or prominent, mid-way or closer to metacone (Wible et al. 2009, ch. 89).

100 (90W). - Internal conular cristae: (0) indistinct; (1) distinctive and wing-like (Luo \& Wible 2005, ch. 107). Taxa without prominent conules are scored as unapplicable.

101 (92W). - Protocone: (0) lacking; (1) small; without trigon basin; (2) or with distinct trigon basin (Rougier et al. 1998, ch. 36).

102 (93W). - Protocone anteroposterior expansion: (0) absent and protocone subequal to paracone; (1) present and protocone larger than paracone (Wible et al. 2009, ch. 93).
103 (94W). - Protocone procumbency: (0) absent; (1) present (Rougier et al. 1998:37).

104 (95W). - Protocone labial shift: (0) no shift (10-20\%); (1)* moderate shift (21-30\%); (2) substantial labial shift (more than $31 \%)$; ORDERED.

105 (96W) MODIFIED. - Height of the protocone: (0) low, clearly lower than paracone and/or metacone; (1) approaching, equal or higher than paracone and/or metacone (Wible et al. 2009, ch. 96 modified). Taxa originally scored (2) in 96W have been scored (1) here (i.e. with taxa originally scored (1) in 96W).

106 (97W). - Precingulum: (0) absent or weak; (1) labial edge of the precingulum, lingual to paraconule; (2) labial edge of the precingulum labial to paraconule (= reaching labially past the paraconule or paraconule position) (Wible et al. 2009, ch. 97)

107 (98W). - Postcingulum: (0) absent or weak; (1) labial edge of the postcingulum lingual to metaconule; (2) labial edge of the postcingulum labial to metaconule; (3) postcingulum extending to labial margin (Wible et al. 2009, ch. 98). Changes in the scorings of Wible et al. (2009): Hyopsodus: $(3)>(2,3)$.

108 (99W). - Hypocone on postcingulum: (0) absent; (1) present lower than protocone; (2) present subequal to protocone (Wible et al. 2009, ch. 99); ORDERED.

109 (100W). - Pre- and postcingulum: (0) separated lingually; (1) continuous lingually. If cingula absent coded as non applicable (Wible et al. 2009, ch. 100). Changes in the scorings of Wible et al. (2009): Hyopsodus: $(0,1)>(0)$.

$110(101 \mathrm{~W})$. - Number of roots of upper molars except ultimate molar: (0) three; (1) four; (2) more than four (Wible et al. 2009, ch. 101); ORDERED.

$111(102 \mathrm{~W})$. - Ultimate upper molar number of roots: (0) one; (1) two; (2) three; (3) four or more (Wible et al. 2009, ch. 102); ORDERED.

112 (105W). - Metastylar lobe on ultimate molar: (0) absent; (1) present (Wible et al. 2009, ch. 105).

113 (NEW). - M1-M2 metaconular hypocone: (0) absent; (1) present (Williamson \& Carr 2007: character 22 modified).

114 (NEW). - Presence of a crochet (oblique loph joining the ectoloph (or centrocrista?) to the metaloph (or posterior edge of molar between metacone and protocone): (0) absent; (1) present (Cifelli 1993; Billet 2010, ch. 27 modified).

\section{LOWER MOLARS}

115 (106W). - Paraconid: (0) present (distinctive); (1) absent (or vestigial) (Wible et al. 2009, ch. 106)

116 (107W). - Paraconid height relatively to metaconid: (0) lower; (1) subequal; (2) taller (Wible et al. 2009, ch. 107). Changes in the scorings of Wible et al. (2009): Mayulestes: (0) > (1); ORDERED.

117 (108W). - Paraconid on lingual margin: (0) absent; (1) present (Wible et al. 2009, ch. 108). Changes in the scorings of Wible et al. (2009): Pucadelphys (0) > (1); Meniscotherium (0) > (1).

118 (109W). - Mesiolingual edge of paraconid: (0) rounded; (1) keeled (Wible et al. 2009, ch. 109).

119 (110W). - Paracristid: (0) notched; (1) continuously curved without notch (Wible et al. 2009, ch. 110). 
120 (111W). - Trigonid configuration: (0) open with the paraconid in anteromedial position, paracristid-protocristid angle more than $50^{\circ}$; (1) acute with paraconid more posteriorly placed, paracristidprotocristid angle between $36^{\circ}$ and $49^{\circ}$; (2) anteroposteriorly compressed, paracristid-protocristid angle $35^{\circ}$ or less (Wible et al. 2009 , ch. 111); ORDERED. Original state (3) of $111 \mathrm{~W}$ has been deleted and taxa without paraconid are coded as non-applicable.

121 (112W MODIFIED). — Protoconid height: (0) tallest of trigonid; (1) subequal to lower than other cusps (Wible et al. 2009, ch. 112 modified). Taxa originally scored (2) in 112W have been scored (1) here (i.e. with taxa originally scored (1) in $112 \mathrm{~W}$ ).

122 (113W). - Protocristid orientation: (0) oblique (1) transverse (Wible et al. 2009, ch. 113).

123 (115W). - Talonid: (0) small heel; (1) multicusped basin (Rougier et al. 1998, ch. 49).

124 (116W). - Cristid obliqua: (0) incomplete; (1) complete attaching lingual to notch in protocristid; (2) complete attaching at or labial to notch in protocristid; (3) complete attaching below middle posterior face of protoconid; (4) complete, labially placed (Wible et al. 2009, ch. 116). Original state (5) (absent) has been deleted as no taxon presented this configuration in our data matrix. Changes in the scorings of Wible et al. (2009): Mayulestes: (3) > (2).

125 (117W). - Trigonid height relative to talonid: (0) twice or more; (1) less than twice; (2) subequal (Wible et al. 2009, ch. 117); ORDERED.

$126(118 \mathrm{~W})$. - Anteroposterior shortening of trigonid (at base) relative to talonid: (0) long > 75\% tooth length; (1) moderately long $<75 \%$ and $>50 \%$ tooth length; (2) short $<50 \%$ tooth length (Luo \& Wible 2005, ch. 78); ORDERED.

127 (119W). - Talonid width: (0) talonid very narrow, subequal to base of metaconid, (1) talonid narrower than trigonid, (2) subequal or wider than trigonid (Rougier et al. 1998, ch. 50); ORDERED.

128 (120W). - Hypoconulid: (0) absent; (1) in median position on posterior edge of talonid; (2) lingually placed with slight approximation of entoconid; (3) connate to entoconid (Wible et al. 2009, ch. 120). Changes on the scoring of Wible et al. (2009): Solenodon, Potamogale: $(0)>(2)$.

129 (121W). - Hypoconulid of last lower molar: (0) short and erect; (1) tall and sharply recurved; (2) posteriorly procumbent; (3) absent. (Wible et al. 2009, ch. 121). Changes on the scoring of Wible et al. (2009): Solenodon, Potamogale: (3) > (0).

130 (122W). - Entoconid: (0) absent; (1) smaller than hypoconid and/or hypoconulid; (2) subequal to larger than hypoconid and/or hypoconulid (Wible et al. 2009, ch. 122); ORDERED.

131 (123W). - Postcristid (between entoconid and hypoconulid) taller than hypoconulid and nearly transverse: (0) absent; (1) present (Wible et al. 2009, ch. 123).

132 (124W). - Mesoconid: (0) absent or (1) present. (Meng et al. 2003, ch. 79).

$133(125 \mathrm{~W})$. - Hypolophid (loph joining hypoconid to hypoconulid): (0) absent; (1) present (Meng et al. 2003, ch. 82). Changes in the scores of Wible et al. (2009): Meniscotherium (0) > (-).

134 (126W). - Labial postcingulid: (0) absent; (1) present (Rougier et al. 1998, ch. 55). Changes in the scores of Wible et al. (2009): Meniscotherium (0) > (1).
135 (NEW). - Entoconid transversely expanded into entolophid and not connected to hypoconulid: (0) absent; (1) present (Cifelli 1993; Billet 2010, ch. 55 modified).

\section{DENTARY}

136 (128W). - Number of mental foramina: (0) two or more; (1) one (Meng et al. 2003, ch. 87).

137 (129W). - Anteriormost mental foramen: (0) below incisors; (1) below p1; (2) below p2; (3) more posterior (Wible et al. 2009 , ch. 129). Taxa with only one mental foramen are scored here. Changes on the scorings of Wible et al. (2009): Solenodon (1) > (1 and 2); Potamogale: $(1)>(0)$; ORDERED.

138 (130W). - Posteriormost mental foramen: (0) in canine and anterior premolar region; (1) below penultimate premolar; (2) below ultimate premolar; (3) posterior to ultimate premolar (Wible et al. 2009, ch. 130); ORDERED.

139 (31W). - Depth of dentary body: (0) slender and long; (1) deep and short (Meng et al. 2003, ch. 86).

$140(132 \mathrm{~W})$. - Retromolar space in adults (i.e. in which ultimate molar exhibits wear process): (0) absent; (1) present (Wible et al. 2009, ch. 132).

141 (133W). - Coronoid process height: (0) higher than condyle; (1) even with condyle (Wible et al. 2009, ch. 133).

142 (134W). - Coronoid process width: (0) broad, roughly two molar lengths; (1) narrow, one molar length or less (Wible et al. 2009, ch. 134). Changes in the scorings of Wible et al. (2009): Mayulestes $(0)>(?)$.

143 (135W MODIFIED). - Angle between anterior border of coronoid process and horizontal alveolar border of cheek teeth: (0) distinctly obtuse (anterior border of coronoid process strongly oblique posteriorly); (1) slightly obtuse or more or less right angle (anterior border of coronoid process slightly oblique posteriorly or sub-vertical; (2) acute angle (anterior border of coronoid process shifted anteriorly) (Wible et al. 2009, ch. 135 modified); ORDERED.

144 (136W) MODIFIED. - Coronoid crest (crest of the masseteric fossa along the anterior border of the coronoid process): (0) absent or weakly developed; (1) present and transversely thick; (2) hypertrophied and laterally flaring with a very deep masseteric fossa posterior to it (Luo \& Wible 2005, ch. 21 modified); ORDERED.

145 (137W MODIFIED). - Portion of the mandibular ramus ventral to the masseteric fossa: (0) dorsoventrally narrow crest or ridge with a dorsoventral height less than half that of the mandibular body; (1) dorsoventrally broad ridge (or elevated area) with a dorsoventral height more than half that of the mandibular body. Taxa in which the ventral limit of the masseteric fossa is indistinct are scored as inapplicable (Luo \& Wible 2005, ch. 20 modified).

$146(138 \mathrm{~W}) .-$ Anterior extension of masseteric fossa on the body of the dentary, below the last molars: (0) absent; (1) present (Luo \& Wible 2005, ch. 22). Changes in the scorings of Wible et al. (2009): Hyopsodus (1) > (0); Chaetophractus $(1)>(0)$.

147 (139W). - Labial mandibular foramen: (0) absent; (1) present (Rougier et al. 1998, ch. 70).

148 (140W). - Condyloid crest: (0) absent; (1) present (Wible et al. 2009, ch. 140).

149 (141W). - Posteroventral shelf of the masseteric fossa: (0) absent; (1) present (Rougier et al. 1998, ch. 68). 
150 (142W). - Angular process: (0) process on posterior aspect of dentary ramus; (1) shelf along ventral border of dentary ramus (Wible et al. 2009, ch. 142). Changes in the scorings of Wible et al. (2009): Mayulestes (?) > (1).

151 (144W). - Angular process length: (0) less than dentary ramus length; (1) equal or greater than dentary ramus length (Wible et al. 2009, ch. 144).

152 (145W MODIFIED). - Angular process shape: (0) hook-like with width (in lateral view) of base lesser than length; (1) hook-like with width of base subequal to (or wider than) length; (2) plate-like (no hook distinct), more or less rounded or sub rectangular; (3) triangular (Wible et al. 2009, ch. 145 modified). Changes in the scorings of Wible et al. (2009): Mayulestes (?) > (3).

153 (146W). - Angular process apex vertical position: (0) low: below the level of the alveolar border; (1) elevated: at the level of or above the alveolar border (Luo \& Wible 2005, ch. 9 modified [text only]). Changes in the scorings of Wible et al. (2009): Tamandua (1) > (0).

$154(147 \mathrm{~W}) .-$ - Anteroposterior position of the dorsal root of the angular process: (0) level with or posterior to the level of the condyloid process; (1) anterior to it [Wible et al. 2009, ch. 147 modified (text only)]. Changes in the scorings of Wible et al. (2009): Solenodon (1) > (0); Maelestes, Kennalestes, Zalambdalestes, Erinaceus, Blarina, Potamogale $(0)>(1)$.

155 (148W). - Condylar process: (0) with posteriorly directed peduncle (1) or not. Changes in the scorings of Wible et al. (2009): Chaetophractus $(1)>(0)$.

156 (149W MODIFIED). - Condyle shape: (0) wider than long ovoid; (1) wider than long cylindrical; 2) as long as wide or longer, anteroposteriorly elongate (Rougier et al. 1998, ch. 71 modified and Wible et al. 2009, ch. 149). Changes in the scorings of Wible et al. (2009): Mayulestes (?) > (1); Pucadelphys $(0,1)>(1)$; Tamandua, Orycteropus, Bradypus (0) > (2).

157 (150W MODIFIED). - Condyle position relative to the tooth row: (0) approximately at the same level or slightly above; (1) above by approximately one molar length; (2) above by two molars length or more (Wible et al. 2009, ch. 150 modified); ORDERED.

158 (151W). - Symphysis shape: (0) tapered; (1) deep (Meng et al. 2003, ch. 86).

159 (152W). - Symphysis posterior extent: (0) p1 or more anterior; (1) p2; (2) p3 or more posterior (Wible et al. 2009, ch. 152) ORDERED. Changes in the scorings of Wible et al. (2009): Hyopsodus: (?) > (0) + (1); Meniscotherium $(1)>(2)$; Phenacodus $($ ? $)>(1)$; Plesiadapis (2) > (1); Potamogale (0) > (1); Chaetophractus Bradypus, Tamandua $(0)>(-)$.

160 (153W). - Mandibular symphysis: (0) unfused; (1) fused (Luo \& Wible 2005, ch. 36).

161 (154W). - Meckelian groove: (0) present; (1) absent (Rougier et al. 1998, ch. 75).

162 (156W). — "Coronoid facet": (0) present; (1) absent (Rougier et al. 1998, ch. 76; Wible et al. 2009, ch. 156).

163 (157W MODIFIED). - Mandibular foramen vertical position: (0) near ventral margin of dentary; (1) recessed dorsally from ventral margin; (2) at the level of alveolar plane; (3) above alveolar plane (Wible et al. 2009, ch. 157 modified); ORDERED.

164 (158W). - Mandibular foramen dorsal to prominent longitudinal ridge: (0) present; (1) absent (Wible et al. 2009, ch. 158). Changes in the scorings of Wible et al. (2009): Mayulestes (1) > (?).

\section{SKULL: ROSTRUM}

165 (160W). - Facial process of the premaxilla: (0) does not reach the nasal posterodorsally; (1) reaches the nasal (Rougier et al. 1998, ch. 80). Changes in the scorings of Wible et al. (2009): Orycteropus (0) $>(1)$.

$166(161 \mathrm{~W})$. - Posterior extent of the facial process of the premaxilla: (0) up to the canine (not beyond); (1) between the canine and the frontal (=beyond canine); (2) up to the frontal (Luo \& Wible 2005, ch. 406; Wible et al. 2009, ch. 161 modified). Changes in the scorings of Wible et al. (2009): Pucadelphys $(0)>(0,1)$; ORDERED.

167 (162W). - Facial process of the premaxilla: (0) finger-like process wedged between the maxilla and the nasal posterodorsally; (1) not wedged (no finger-like process) (Wible et al. 2009, ch. 162).

168 (NEW). - Paracanine fossa between posterior incisor and upper canine: (0) absent; (1) distinctly excavated in the tooth row with a crest-like lateral margin; (2) opened laterally but remaining on the tooth row; (3) opened laterally and dorsally expanded on the lateral edge of the rostrum.

169 (163W MODIFIED). - Paracanine fossa: (0) excavated in maxilla; (1) excavated in maxilla and premaxilla; (2) excavated in premaxilla (Rougier et al. 1998, ch. 81 modified; Wible et al. 2009, ch. 163 modified). All taxa coded (0) for character 168 (preceding) are coded as non-applicable.

170 (164W). - Exit(s) of infraorbital canal: (0) multiple; (1) single; (2) absent. (Rougier et al. 1998, ch. 82 modified).

171 (165W MODIFIED). — Infraorbital foramen position: (0) dorsal to penultimate premolar or more anterior; (1) dorsal to ultimate premolar; (2) dorsal to first molar or more posterior (Geisler 2001, ch. 65 modified, Wible et al. 2009, ch. 165 modified). Changes in the scorings of Wible et al. (2009): Meniscotherium, Phenacodus $(1,2)$ $>(0,1)$; ORDERED.

172 (166W). - Infraorbital canal length: (0) long (longer than greater diameter of the anterior opening); (1) short (shorter than or roughly equal to greater diameter of the anterior opening). In the case of multiple openings the diameter is that of the circle with a surface corresponding to the sum of the surfaces of all foramina (Wible et al. 2009, ch. 166 modified).

173 (168W). - Width of the nasals: (0) much wider posteriorly than anteriorly; (1) sides subparallel; (2) widest anteriorly (Gregory 1920); ORDERED.

174 (NEW). - Shape ratio of the nasals (length/mean width): (0) nasals length less than (or equal to) five times their mean width; (1) nasals length more than five times their mean width.

Note: the mean width is the mean of three measurements: maximum width in posterior half, maximum width in anterior half, minimum width between these two measurements.

175 (169W). - Nasals overhang external nasal aperture: (0) present; (1) absent.

176 (NEW). - Nasal of adults fused together but not to surrounding bones: (0) absent; (1) present.

177 (170W). - Anterior processes of the frontals wedged between the nasals: (0) present; (1) absent. Changes in the scorings of Wible et al. (2009): Mayulestes (1) > (0).

178 (171W MODIFIED). - Nasofrontal suture: 0) suture level with or posterior to the anterior edge of the orbit; 1) all points of the suture well anterior to anterior edge of the orbit: (Wible et al. 2009, ch. 171 modified). 
179 (172W). - Nasal foramina: (0) present; (1) absent (Rougier et al. 1998, ch. 84).

180 (173W). - Frontal-maxillary contact on rostrum: (0) absent; (1) present (Gregory 1920).

$181(174 \mathrm{~W})$. - Anterior (maxillary) process of the frontal wedged between the nasal and the maxilla: (0) absent or weak; (1) present, elongated and thin; (Asher et al. 2005, ch. 109).

$182(175 \mathrm{~W})$. - Preorbital length (measured from the anterior edge of the orbit to anterior end of the skull): (0) less than onethird total length; (1) more than one-third (Rougier et al. 1998, ch. 90 modified). Changes in the scorings of Wible et al. (2009): Meniscotherium, Phenacodus (0) > (1).

183 (176W). - Lacrimal: (0) present; (1) absent (Asher et al. 2005, ch. 103). Changes in the scorings of Wible et al. (2009): Eoryctes, Erinaceus $(1)>(0)$

184 (177W). - Facial process of the lacrimal: (0) large external wing on the rostrum at the anterior edge of the orbit; (1) no wing: either the external part of the lacrimal is totally absent or it is reduced to a narrow crescentic rim on the anterior edge of the orbit (Asher $e t$ al. 2005, ch. 105 modified; Wible et al. 2009, ch. 177 modified, text only). Changes in the scorings of Wible et al. (2009): Mayulestes (1) > (0); Chaetophractus $(1)>(0)$.

185 (178W). - Lacrimal tubercle: (0) present; (1) absent. Taxa without lacrimal are scored inapplicable (Rougier et al. 1998, ch. 87). Changes in the scoring of Wible et al. (2009): Erinaceus $(-)>(0)$.

186 (179W). - Lacrimal foramen "on face" (because lateral edge of the foramen is reduced or missing): (0) present; (1) absent (Rougier et al. 1998, ch. 88). Changes in the scoring of Wible et al. (2009): Pucadelphys, Erinaceus $(0)>(0,1)$.

187 (180W). - Lacrimal foramen (0) double; (1) simple (Rougier et al. 1998, ch. 89).

188 (181W). — Lacrimal foramen composition: (0) enclosed within lacrimal; (1) with maxillary contribution; (2) with jugal contribution (Asher et al. 2003, ch. 100; Wible et al. 2009, ch. 181). Changes in the scoring of Wible et al. (2009): Erinaceus (1) > (0).

189 (182W). - Translacrimal canal (see Wible et al. 2004): absent (0) or present (1). Taxa without lacrimal are scored inapplicable.

$190(\mathrm{NEW})$. - Narial processes of the premaxillae (tuberosity at the anterior apices of the premaxillae, on the anterior floor of the narial opening) (Billet 2010, ch. 77) : (0) absent ; (1) present.

\section{SKULL: PALATE}

191 (183W). - Palatal process of the premaxilla: (0) does not reach the alveolus; (1) does reach or nearly reach the canine alveolus (Rougier et al. 1998, ch. 79).

192 (185W MODIFIED). - Incisive foramina length: (0) short, half or less than half the maximal palatal length of the premaxilla along the sagittal plane; (1) long, more than half the length of the premaxilla (Luo \& Wible 2005, ch. 409 modified).

193 (186W). - Incisive foramina composition: (0) between premaxilla and maxilla; (1) within premaxilla (Wible et al. 2009, ch. 186).

$194(187 \mathrm{~W})$. — Palatal vacuities: (0) absent; (1) present (Rougier et al. 1998, ch. 93). Changes in the scoring of Wible et al. (2009): Pucadelphys (?) $>(0)$.
195 (188W MODIFIED). - Major palatine foramina: (0) wellindividualized, within maxilla and/or palatine; (1) multiple small foramina in palatine and/or maxilla (Wible et al. 2009, ch. 188 modified). Taxa without major palatine foramina are scored NA. Changes in the scoring of Wible et al. (2009): Pucadelphys (?) > (0).

196 (189W). - Anterior extent of palatine: (0) posterior to M1; (1) level with M1; (2) more anterior (Wible et al. 2009, ch. 189). Changes in the scoring of Wible et al. (2009; order of states modified): Pucadelphys (?) > (0); Solenodon: (0) > (1); ORDERED.

197 (190W). - Palatal expansion with regard to ultimate molar: (0) posterior; (1) even with; (2) anterior (Wible et al. 2009, ch. 190). Change in the scoring of Wible et al. (2009), order of states modified: Miacis (2) > (0); ORDERED.

198 (191W). - Postpalatine torus: (0) absent; (1) present (Rougier et al. 1998, ch. 95). Change in the scoring of Wible et al. (2009): Meniscotherium $(0)>(1)$.

199 (192W). - Posterior palatine spine: (0) weak or absent; (1) prominent (Wible et al. 2009, ch. 192). Change in the scoring of Wible et al. (2009): Mayulestes (0) > (1).

200 (193W MODIFIED). - Passageway of the minor palatine artery (minor palatine foramen or groove): (0) totally enclosed in the minor palatine foramen; (1) in a groove on the posteromedial edge of the palate as a result of the posterior opening of the minor palatine foramen (no posterior bony bridge); (2) absent (no groove nor foramen); (Wible et al. 2009, ch. 193 modified).

Note: Xenarthra, which have multiple small foramina and no wellidentified minor palatine foramen or notch, are scored NA here (as scoring again the presence of multiple foramina would be redundant with character 198 [188W]). Tenrecs have multiple small foramina but also a well-identified minor palatine foramen and therefore present an applicable condition for this character.

201 (194W). - Minor palatine foramen composition: (0) palatine or maxilla-palatine or (1) palatine-pterygoid. (Wible et al. 2009, ch. 194). When a distinct palatine notch is present as a consequence of the opening of the minor palatine foramen the character is scored. Changes in the scorings of Wible et al. (2009): Plesiadapis and Adapis, $(-)>(0)$; Rhynchocyon, (0) > (-); Chaetophractus, Bradypus, and Tamandua, $(0)>(-)$.

202 (195W). - Maxilla with shelf-like expansion posterior to ultimate molar with a length at least equal to half a tooth and lateral to minor palatine notch or foramen: (0) absent; (1) present (Wible et al. 2009, ch. 195, modified [text only]).

\section{SKULL: ZYGOMA}

203 (196W MODIFIED). - Posterior edge of the anterior zygomatic root aligned: (0) with last molar or more posterior; (1) with more anterior molars; (2) with premolars (Meng et al. 2003, ch. 123; Wible et al. 2009, ch. 196 modified); ORDERED.

204 (197W). - Zygomatic process of the maxilla: (0) present; (1) vestigial; (Wible et al. 2009, ch. 197).

205 (198W). - Jugal: (0) present; (1) absent; (Wible et al. 2009, ch. 198).

206 (199W). - Jugal contribution to anteroventral edge of the orbit: (0) present; (1) absent (Wible et al. 2009, ch. 199 modified text only).

207 (200W). - Mx-Ju suture bifurcated: (0) absent; (1) present (Rougier et al. 1998, ch. 91). 
208 (201W). - Jugal-lacrimal contact: (0) present; (1) absent (Meng et al. 2003, ch. 137).

209 (202W). - Zygomatic arch: (0) stout; (1) delicate; (2) incomplete (Wible et al. 2009, ch. 199). Change in the scoring of Wible et al. (2009): Pucadelphys (0) > (1); ORDERED.

210 (NEW). - Orientation of the anterior root of zygoma in anterior view: (0) oblique, extends dorsally; (1) transverse, roughly horizontal; (2) extends ventrally; ORDERED.

211 (NEW). - Vertical position of posterior root of zygoma relative to foramen magnum: (0) even; (1) clearly higher.

212 (NEW). - Shape of zygomatic arches in dorsal view: (0) not protruding much laterally, smoothly curved or parallel to sagittal plane; (1) protruding laterally (largely exceeding other parts of skull laterally), with a large posterior elbow. See comparison.

\section{SKULL: ORBIT}

213 (203W). - Roots of molars exposed in orbit floor: (0) absent; (1) present (Asher et al. 2005, ch. 126). Change in the scoring of Wible et al. (2009): Pucadelphys (0) > (1).

$214(204 \mathrm{~W})$. - Palatine enters the posterior opening of the infraorbital canal (maxillary foramen): (0) present; (1) absent (Rougier et al. 1998, ch. 98).

215 (205W). - Lacrimal contributes to maxillary foramen: (0) present; (1) absent (Luo \& Wible 2005, ch. 376 modified).

216 (206W). - Groove connects maxillary to sphenopalatine foramina: (0) absent; (1) present (Asher et al. 2005, ch. 97). Changes in the scorings of Wible et al., (2009): Pucadelphys: (0) > (1); Erinaceus: $(0)>(1)$; Procavia: $(0)>(1)$.

217 (207W) MODIFIED. - Sphenopalatine foramen: (0) within palatine; (1) between palatine and maxilla; (2) between palatine, maxilla and frontal (Asher et al. 2005, ch. 133 modified).

218 (208W). - Sphenopalatine foramen: proximal (= approximated) to maxillary foramen: (0) absent; (1) present (Wible et al. 2009, ch. 208)

219 (209W). - Maxilla in the orbit: (0) no participation in orbital wall; (1) large dorsal process participating in medial orbital wall (Wible et al. 2009, ch. 209 modified - text only). Changes in the scoring of Wible et al. (2009): Chaetophractus (1) > (0).

220 (210W). - Frontal and maxilla contact in medial orbital wall: (0) absent; (1) present (Geisler 2001, ch. 52). It is noteworthy that this character is partially redundant with character $204 \mathrm{~W}$ (aproximately 66\%) and with character $210 \mathrm{~W}$ (approximately 80\%). (\% are from original matrix of Wible et al. 2009).

$221(211 \mathrm{~W})$. - Orbital process of the palatine: (0) present; (1) absent (Asher et al. 2005:127 modified). Change in the scoring of Wible et al. (2009): Chaetophractus (0) > (1).

222 (212W). - Ethmoid exposure in medial orbital wall: (0) absent or (1) present (Wible et al. 2009, ch. 212).

223 (213W). - Ethmoidal foramen: (0) between frontal and orbitosphenoid; (1) within frontal (Wible et al. 2009:213).

224 (214W). - Foramina for the frontal diploic vein: (0) absent; (1) present (Wible et al. 2009, ch. 214).
225 (NEW). - Position of frontal diploic foramina vs postorbital process or tuberosity: (0) posterior; (1) anterior or even. Taxa lacking a postorbital process or tuberosity and/or frontal diploic foramina are coded unapplicable.

226 (215W MODIFIED). - Supraorbital foramina (also named: frontal - Wible et al. 2009 - or postorbital): (0) absent; (1) present on the skull roof, often extending anteriorly as a groove on the maxilla and/or nasal; (2) present more laterally on the dorsal orbital edge and/or postorbital process, groove variably present (Thewissen $e t$ al. 2001, ch. 41 modified).

227 (216W). - Postorbital process of the frontal: (0) present and prominent; (1) present weak (tuberosity); (2) absent (Wible et al. 2009, ch. 216). Changes in the scorings of Wible et al. (2009): Phenacodus $(2)>(1)$; Chaetophractus $(2)>(1)$; ORDERED.

228 (217W). - Postorbital process (main) composition: (0) frontal; (1) parietal (Wible et al. 2005, ch. 67 modified). Changes in the scoring of Wible et al. (2009): Chaetophractus (-) > (0).

229 (218W). - Postorbital bar: (0) absent; (1) present (Meng et al. 2003, ch. 145).

$230(219 \mathrm{~W})$. - Dorsal process of the jugal: (0) absent or weak (hump); (1) strong (Meng et al. 2003, ch. 142).

231 (220W). - Optic foramen:(0) confluent with sphenorbital fissure; (1) well separated from sphenorbital fissure (Rougier $e t$ al. 1998, ch. 102).

232 (221W MODIFIED). - Optic foramen position: (0) narrowly separated from sphenorbital fissure; (1) broadly separated from sphenorbital fissure (Wible et al. 2009, ch. 221, modified).

233 (222W). - Orbitosphenoid: (0) expanded anteriorly from optic foramen (or with anterior process for forms without optic foramen); (1) expanded dorsally from optic foramen (or with dorsal process for forms without optic foramen); (2) not expanded anteriorly or dorsally (Wible et al. 2009, ch. 222).

234 (223W). - Suboptic foramen: (0) absent; (1) present (Wible et al. 2009, ch. 223). Changes in the scorings of Wible et al. (2009): Leptictis, Hyopsodus, Phenacodus, Plesiadapis (0) > (1).

235 (224W). - Orbitotemporal canal: (0) present; (1) absent (Rougier et al. 1998:103).

236 (225W). - Frontal-alisphenoid contact: (0) present: small contact at the anterodorsal corner of the alisphenoid;(1) present: more extensive contact on approximately $50 \%$ of the dorsal margin of the alisphenoid; (2) absent (Luo \& Wible 2005, ch. 382 modified). Changes in the scorings of Wible et al. (2009): Mayulestes, Pucadelphys $(1)>(0)$.

237 (NEW). - Sphenorbital fissure confluent with foramen rotundum (i.e. there is no foramen rotundum per se and the V2 exits with the V1 through the sphenorbital fissure): (0) absent; (1) present.

See Geisler \& Uhen (2003, ch. 50), also scored by Ladevèze et al. (2010, ch. 15): (modified from Novacek 1986 [ch. 31, table 3]; Novacek \& Wyss 1986; Thewissen \& Domning 1992; O'Leary \& Geisler 1999).

\section{SKULL: BRAINCASE}

238 (226W). - Frontal length on midline: (0) less than half that of the parietal; (1) subequal to slightly smaller than parietal; (2) more than 50\% longer than parietal (Wible et al. 2009, ch. 226). Change in the scoring of Wible et al (2009); order of states modified): Mayulestes (0) > (1); ORDERED. 
239 (227W). - Frontoparietal suture: (0) transverse; (1) with anterior process of the parietal off the midline; (2) with anterior process of the parietal on the midline (Wible et al. 2009, ch. 227).

240 (228W). - Temporal lines meet on midline to form a sagittal crest: (0) present; (1) absent (Geisler 2001, ch. 33 modified).

241 (230W). - Nuchal crest: (0) level with or anterior to foramen magnum; (1) posterior to foramen magnum (Wible et al. 2009, ch. 230).

242 (NEW). - Nuchal crest in dorsal view: (0) deeply notched on medial line: bilobate morphology and V-shaped opening posteriorly; (1) transverse: roughly straight; (2) median point posterior to lateral parts of the crest: V-shaped morphology opening anteriorly; ORDERED.

243 (231 W MODIFIED). - Anterior lamina of the petrosal: (0) present and exposed on lateral braincase wall; (1) internal to braincase and small to vestigial; (2) absent (Wible et al. 2009, ch. 231 modified).

244 (232W). - Squama of the squamosal: (0) absent; (1) present (Rougier et al. 1998:113).

245 (233W). - Foramina for temporal rami (including suprameatal foramen when present) of the stapedial artery: (0) in the petrosal; (1) in parietal and/or squama of the squamosal; (2) absent (Rougier et al. 1998, ch. 143).

\section{SKULL: MESOCRANIUM}

$246(234 \mathrm{~W})$. - Choanae: (0) almost as wide as posterior palate; (1) distinctly narrower (Wible et al. 2009, ch. 234). Change in the scoring of Wible et al. (2009): Pucadelphys (0) > (1).

247 (235W). - Vomer contacts pterygoids: (0) present; (1) absent (Wible et al. 2009, ch. 235). Change in the scoring of Wible et al. (2009): Pucadelphys (?) > (0).

248 (236W). - Pterygoids contact in midline: (0) present; (1) absent (Rougier et al. 1998, ch. 99). Changes in the scoring of Wible et al. (2009): Pucadelphys (?) > (0); Potamogale: (1) > (0).

249 (237W). - Pterygopalatine crests: (0) present; (1) absent (Rougier et al. 1998, ch. 100).

250 (238W). - Midline crest in basipharyngeal canal: (0) absent; (1) present (Wible et al. 2009, ch. 238). Change in the scoring of Wible et al. (2009): Pucadelphys (?) > (0).

251 (239W). - Entopterygoid process (posterior extension): (0) absent; (1) ends at anterior basisphenoid; (2) approaches ear region (Wible et al. 2009, ch. 239).

252 (240W). - Midline rod-shaped eminence on basisphenoid: (0) absent; (1) present (Wible et al. 2009, ch. 240).

253 (241W). - Ectopterygoid process of the alisphenoid: (0) absent (or fused with entopterygoid crest); (1) present: ends at anterior basisphenoid; (2) present: approaches ear region (Rougier et al. 1998, ch. 101 modified).

254 (242W). - Ectopterygoid process shape: (0) long crest or triangular blade; (1) narrow process, spine or tubercle (Wible et al. 2009, ch. 242).

255 (NEW). - Ectopterygoid crests (blades) or tubercles orientation: (0) sub-parallel and facing ventromedially; (1) diverging and facing mostly posteriorly (Billet 2010, ch. 74 modified).
256 (243W). - Transverse canal: (0) absent; (1) present (Rougier et al. 1998, ch. 104). Change in the scoring of Wible et al. (2009): Pucadelphys (1) > (0).

$257(244 \mathrm{~W})$. - Exit for maxillary nerve (V2) (foramen rotundum) relative to alisphenoid: (0) between the posterior edge of the alisphenoid anteriorly and the anterior lamina of the petrosal posteriorly; (1) within the alisphenoid; (2) at the anterior edge of the alisphenoid (Rougier et al. 1998, ch. 110 modified [text only]). Changes in the scorings of Wible et al. (2009): Meniscotherium $(1,2)$ $>$ (2); ORDERED.

258 (245W). - Number of exits for the mandibular branch of the trigeminal nerve (V3): (0) two or more; (1) one (Luo \& Wible 2005, ch. 317).

259 (246W). - Foramen ovale composition: (0) in anterior lamina of the petrosal; (1) between petrosal and alisphenoid; (2) in alisphenoid; (3) between alisphenoid and squamosal (Rougier et al. 1998, ch. 111 modified).

260 (247W MODIFIED). — Foramen ovale position: (0) on lateral wall of the braincase; (1) on ventral surface of the skull facing ventrally; (2) on the ventral side of the skull, facing anteroventrally (Rougier et al. 1998 , ch. 112 modified).

261 (248W). - Alisphenoid canal: (0) absent; (1) present (Rougier et al. 1998, ch. 107).

262 (249W). - Posterior opening of alisphenoid canal: (0) separated from foramen ovale or (1) in common depression with foramen ovale. [Taxa without alisphenoid canal are scored inapplicable] (Wible et al. 2009, ch. 249).

263 (NEW). - Posterior border of hypophyseal fossa (located just anterior to basisphenoid-basioccipital suture) position relative to posterior edge of foramen ovale: (0) anterior to or roughly even with; (1) well posterior to.

Note: the hypophyseal fossa is located on the dorsal side of the basisphenoid. It is often limited posteriorly by a thin vertical wall, the dorsum sellae. This wall is located on the posterior suture of the basisphenoid with the basioccipital. Therefore, the posterior edge of the hypophyseal fossa approximately corresponds to the suture; it is in fact slightly anterior to it. In ventral view of the skull the basioccipital-basisphenoid suture therefore provides a reasonable indication of the posterior edge of the hypophyseal fossa.

\section{SKULL: BASICRANIUM}

$264(250 \mathrm{~W})$. - Position of the dentary articulation relative to fenestra vestibuli: (0) at the same level; (1) anterior (Rougier et al. 1998, ch. 114).

265 (251W). - Glenoid fossa position: (0) on zygoma; (1) partly below braincase (Wible et al. 2009, ch. 251).

266 (252W). - Glenoid fossa shape: (0) concave (or concave posteriorly and flat anteriorly), open anteriorly; (1) trough-like; (2) anteroposteriorly elongate, with the major axis of fossa directed anteroposteriorly; (3) anteroposteriorly short, with the major axis of fossa directed anteroposteriorly or flat; (4) concavo-convex (Rougier et al. 1998, ch. 115; Archibald et al. 2001, ch. 137; Wible et al. 2009 , ch. 252 modified) Changes in the scorings of Wible et al. (2009): Miacis, Vulpavus (0) > (1); Tamandua, Bradypus (0) > (2).

267 (253W). - Glenoid fossa dorsoventral position relative to roof of basipharyngeal canal: (0) approximately level with; (1) dorsal to it (Wible et al. 2009, ch. 253). 
$268(254 \mathrm{~W})$. - Glenoid process of the jugal: (0) present with articular facet; (1) present without facet; (2) absent (Wible et al. 2009, ch. 254).

269 (255W). - Glenoid process of the alisphenoid: (0) absent; (1) present (Rougier et al. 1998, ch. 117).

270 (256W MODIFIED). — Postglenoid process: (0) absent or very small; (1) present: subequal in width to (or slightly narrower than) glenoid cavity; (2) present: approximately half the width of the glenoid cavity. (Rougier et al. 1998, ch. 118 modified).

271 (257W). - Postglenoid foramen: (0) absent; (1) present (Wible et al. 2009, ch. 257).

272 (258W). - Postglenoid foramen position: (0) posterior to postglenoid process (or glenoid fossa if postglenoid process is absent); (1) medial or anteromedial to the postglenoid process (within glenoid fossa); (2) on lateral aspect of braincase (Rougier et al. 1998, ch. 120 modified).

273 (NEW). - Postglenoid eminence of the squamosal: (0) absent; (1) present (see description).

Note: The postglenoid eminence (new term, this paper) is a small tuberosity on the squamosal posteromedial to the postglenoid foramen and anterolateral to the epitympanic recess and which receives the lateral articulation of the anterior crus of the ectotympanic. Taxa without a postglenoid foramen are scored as inapplicable. Taxa with a large tympanic bulla and crista meatus (e.g., notoungulates) are also scored as inapplicable.

274 (259W). Postglenoid foramen composition: (0) within squamosal or (1) on posterior edge of squamosal. [Taxa without postglenoid foramen are scored inapplicable] (Wible et al. 2009, ch. 259 modified, text only).

275 (260W). - Suprameatal foramen: (0) absent; (1) present (Wible et al. 2009, ch. 260). Change in the scoring of Wible et al. (2009): Mayulestes (?) > (1).

276 (261W). - Entoglenoid process of the squamosal: (0) absent; (1) present, separated from the postglenoid process; (2) present, continuous with the postglenoid process (Luo \& Wible 2005, ch. 284). Changes in the scorings of Wible et al. (2009): Procavia, (2) > (1). Note: both processes are separated by the Glaserian fissure, which conveys the chorda tympani. When processes are merged, the Glaserian fissure is obliterated and the chorda tympani runs on the posterior edge of the common process. It often forms a distinct groove on the posterior wall of the common process as is observed in Solenodon (Wible 2008: fig. 26B).

277 (262W). - Posttympanic crest of the squamosal: (0) absent; (1) present (Wible et al. 2009, ch. 262). Change in the scoring of Wible et al. (2009): Solenodon, (1) > (0).

Note: The postympanic crest is a medial extension of the posterior wall of the external auditory meatus (the posttympanic process), which is adjacent to (sometimes closely appressed against) the anteroventral edge of the tympanohyal. It is well developed in Zalambdalestes (Wible et al. 2004: fig. 37C), where it is separated from the posttympanic process by a deep notch.

278 (263W). - Carotid foramen: (0) within basisphenoid; (1) between petrosal and basisphenoid; (2) absent (Rougier et al. 1998, ch. 105 modified)

279 (264W). - Floor of cavum epiptericum composition: (0) petrosal; (1) petrosal and/or alisphenoid; (2) primarily or exclusively squamosal; (3) primarily open as pyriform fenestra (Rougier et al. 1998, ch. 109 modified).
280 (265W). - Posterodorsal extension of the tympanic process of the alisphenoid, which forms the roof of the anterior region of the tympanic cavity: (0) absent; (1) present (Rougier et al. 1998, ch. 121 modified).

281 (266W). - Basisphenoid tympanic process: (0) absent; (1) present (Wible et al. 2009, ch. 266).

282 (267W). - Basicochlear fissure: (0) closed; (1) patent (Thewissen et al. 2001, ch. 59 modified)

283 (268W MODIFIED). - Medial flange of petrosal: (0) absent; (1) present; (Rougier et al. 1998, ch. 122 modified). Changes in the scorings of Wible et al. (2009): Pucadelphys, Mayulestes, Leptictis (1) $>(0)$; Plesiadapis, Notharctus, Adapis (0) > (1); Orycteropus (2) > (0). Note: The medial flange of the petrosal is a thin shelf-like annular blade on the medial edge of the promontorium. It is separated from the promontorium by a distinct break in slope and does not simply represent a thin medial edge of the promontorium. Following this definition, we consider that a medial flange is absent in Orycteropus and therefore removed state (2) of Wible et al. (2009).

284 (269W). - Rostral tympanic process of petrosal: (0) absent or low ridge; (1) moderate ridge contributing to posterodorsomedial bulla; (2) tall ridge contributing to ventral bulla (Rougier et al. 1998, ch. 130 modified). Change in the scoring of Wible et al. (2009): Solenodon: $(0)>(0,1)$.

285 (270W MODIFIED). - Course of the internal carotid artery: (0) lateral (transpromontorial); (1) medial (perbullar or extrabullar) (Wible et al. 2009, ch. 270 modified). Change in the scoring of Wible et al. (2009): Phenacodus: (1) > (0).

Note: State (2) of 270W has been deleted. The absence of course indication is scored as (?) because it does not represent a true alternative to the other two states and does not correspond to a specific course of the internal carotid artery. In this case, any of the two other states are possible.

286 (271W + 288W MODIFIED). - Bony canals for intratympanic carotid system (transpromontorial internal carotid and $+/$ - stapedial artery): (0) absent; (1) present (Wible et al. 2009, ch. 271). Taxa lacking a transpromontorial internal carotid artery are scored non-applicable.

$287(272 \mathrm{~W})$. - Deep groove for the internal carotid artery excavated in the apex of the promontorium: (0) absent; (1) present (Rougier et al. 1998, ch. 148).

288 (273W). - Perbular carotid canal (for medial internal carotid): (0) absent; (1) present (Wible et al. 2009, ch. 273). Taxa with transpromotorial course are scored non-applicable. Change in the scoring of Wible et al. (2009): Phenacodus (1) > (-).

289 (274W MODIFIED). - Sulcus or canal for stapedial artery on promontorium: (0) present; (1) absent (Asher et al. 2005, ch. 161 modified).

$290(275 \mathrm{~W})$. - Stapedial ratio (length/width fenestra vestibuli): (0) rounded less than 1.8 ; (1) elliptical more than 1.8 (Rougier et al. 1998, ch. 127).

291 (276W MODIFIED). - C Coiling of the cochlea: (0) less than $360^{\circ}$; (1) more than $360^{\circ}$ (Rougier et al. 1998, ch. 129).

292 (277W). - Pars cochlearis length: (0) more than $13 \%$ of skull length; (1) less than $10 \%$ of skull length (Wible et al. 2009, ch. 277).

293 (278W). - Promontorium shape: (0) flat; (1) globose (Wible et al. 2009, ch. 278). Change in the scoring of Wible et al. (2009): Potamogale $(0)>(1)$. 
294 (NEW). - Kidney (or bean-) -shaped promontorium: (0) absent; (1) present (Billet 2010, ch. 127 modified).

295 (279W). - Promontorium depth (most ventral point) relative to basioccipital: (0) level or ventral; (1) dorsal (Wible et al. 2009, ch. 279). Change in the scoring of Wible et al. (2009): Hyopsodus: (1) $>(0)$.

296 (280W). - Intratympanic course of facial nerve: (0) open in sulcus; (1) open anteriorly, canal posteriorly; (2) in canal (Meng et al. 2003, ch. 169 modified).

297 (281W MODified). - Aperture of hiatus Fallopii: (0) on cerebral face of petrosal; (1) at anterior edge of petrosal; (2) on tympanic face of petrosal; (3) via fenestra semilunaris: (Rougier et al. 1998, ch. 123 modified, Sánchez-Villagra \& Wible (2002, ch. 12) modified). Changes in the scorings of Wible et al. (2009): Mayulestes, Pucadelphys (1) > (0); Maelestes (2) > (?); Leptictis (1) $>$ (2); Protungulatum (0) > (2); Erinaceus (1) > (2); Solenodon (1) $>(0,1,2)$; Chaetophractus (absent) > (2); Bradypus (absent) > (?); Tamandua (?) > (2).

Note: on the scoring of Maelestes by Wible et al. (2009): On the single specimen of Maelestes the roof of the cavum supracochleare is missing. Wible et al. (2009, ch. 40) suggest that it was originally missing (it has not been broken post mortem) and that, therefore, there is no hiatus Fallopii. They suggest that the greater petrosal nerve would have emerged directly from the geniculate ganglion towards the pterygoid canal following the anterolateral edge of the promontorium. However, observation of the specimen has not convinced us on the original absence of the floor of the cavum supracochleare and we do not discard the possibility that this structure may have been destroyed post-mortem. Given this uncertainty, we score Maelestes (?). The new scoring of Solenodon takes into account the observations of Wible (2008), who mentions that the three states exist in the sample available to him.

298 (282W). - Prootic canal: (0) present; (1) absent (Rougier et al. 1998, ch. 124 modified).

299 (283W). - Prootic canal length and orientation: (0) long and vertical; (1) short and vertical; (2) short and horizontal. (Rougier et al. 1998, ch. 124 modified).

300 (284W). - Lateral flange: (0) parallels length of the promontorium, (1) greatly reduced or absent (Rougier et al. 1998, ch. 126 modified).

$301(285 \mathrm{~W})$. - Anterior extension of bony shelf lateral to promontorium (lateral trough or tegmen tympani): (0) does not reach the apex of the promontorium anteriorly and confined posterolaterally; (1) extends as far anteriorly as the apex of the promontorium; (2) extends further anteriorly than the apex of the promontorium (Wible et al. 2009, ch. 285 modified [original states 0 and 1 have been inverted]); ORDERED.

302 (286W). - Width of bony shelf lateral to promontorium (lateral trough or tegmen tympani): (0) uniform; (1) expanded anteriorly (Wible et al. 2009, ch. 286).

303 (287W). - Inflation of bony shelf lateral to promontorium (lateral trough or tegmen tympani): (0) absent; (1) present (Cifelli 1982; Geisler \& Luo 1998; Thewissen et al. 2001, ch. 52).

304 (289W). - Tensor tympani fossa on petrosal: (0) shallow; (1) deep circular fossa (Wible et al. 2009, ch. 289 modified [text only]. Changes in the scoring of Wible et al. (2009): Leptictis, Procavia (0) $>(1)$.

305 (NEW). - Area occupied by tensor tympani fossa: (0), small and/or poorly excavated; (1) large.
306 (NEW). - Location of tensor tympani fossa: (0) mostly medial (or ventromedial) to the cavum supracochleare; (1) strictly ventral or lateral to it, not medial (Billet \& Muizon 2013, ch. 138).

307 (290W). - Medial process of squamosal in tympanic cavity: (0) absent; (1) present (Muizon 1994).

308 (291W). - Hypotympanic sinus: (0) absent; (1) formed by squamosal, petrosal and alisphenoid; (2) formed by alisphenoid and petrosal; (3) formed by petrosal (Muizon 1994 modified).

309 (292W). - Epitympanic recess vs fossa incudis: (0) subequal; (1) epitympanic recess larger; (2) no visible depression for epitympanic recess (Wible et al. 2009, ch. 292). Change in the scoring of Wible et al. (2009): Hyopsodus: (2) > (0).

310 (293W). - Epitympanic recess lateral wall: (0) with small contribution of squamosal to posterolateral wall; (1) with extensive contribution of squamosal; (2) with no squamosal contribution (Rougier et al. 1998, ch. 138 modified).

311 (294W). - Fossa incudis relationship with epitympanic recess: (0) continuous with; (1) separated from (Rougier et al. 1998, ch. 137).

312 (NEW). - Squamosal epitympanic sinus: (0) absent; (1) present (Billet 2010: character 124). Several taxa have been scored from Klaauw (1931).

Note: The squamosal epitympanic sinus is a lateral extension of the epitympanic recess, which forms a deep epitympanic sinus in posterodorsal part of squamosal.

313 (295W MODIFIED). - Floor ventral to fossa incudis: (0) absent (the fossa is open ventrally); (1) formed by squamosal; (2) formed by ectotympanic; (3) formed by the petrosal (Wible et al. 2009, ch. 295 modified).

$314(296 \mathrm{~W})$. - Fossa incudis position relative to fenestra vestibuli: (0) lateral; (1) anterolateral (Wible et al. 2009, ch. 296).

315 (297W). - Foramen for superior ramus of the stapedial artery: (0) in petrosal; (1) in petrosal-squamosal suture; (2) absent (Rougier et al. 1998, ch. 145).

$316(298 \mathrm{~W})$. - Position of the ramus superior foramen relative to fenestra vestibuli: (0) posterior or lateral; (1) anterior (Luo \& Wible 2005, ch. 326).

$317(299 \mathrm{~W})$. - Ascending canal (passage for the ramus superior of the stapedial artery): (0) intramural in the petrosal, (1) intracranial, or (2) absent. (Rougier et al. 1998, ch. 152).

318 (300W). - Diameter of the stapedius fossa: (0) approximately twice the size of that of the fenestra vestibuli; (1) distinctly less than twice the size of the diameter of the fenestra vestibuli (= small and shallow) (Rougier et al. 1998, ch. 139 modified [text only]). Changes in the scorings of Wible et al. (2009): Mayulestes (1) > (0); Phenacodus: $(1)>(0,1)$.

319 (301W). - Cochlear canaliculus visible in middle ear space: (0) absent; (1) present (Wible et al. 2009, ch. 301).

320 (NEW). - Stapedial fossa and postpromontorial tympanic sinus: (0) distinct, separated by a break in slope or a crest; (1) merged, almost indistinct (Billet \& Muizon 2013: character 139; replaces $302 \mathrm{~W}$ in a modified version).

$321(303 \mathrm{~W})$. - External aperture of cochlear fossula (fenestra cochleae in Wible et al. 2009, ch. 303) position relative to fenestra vestibuli: (0) posteromedial, (1) posterior (Wible et al. 2009, ch. 303). 
$322(304 \mathrm{~W})$. - Posterior septum shields fenestra cochleae: (0) absent; (1) present (Wible et al. 2009, ch. 304).

$323(305 \mathrm{~W})$. - Mastoid process (= paroccipital process of Wible et al. 2009; see appendix 5): (0) distinctly present and vertical; (1) slanted, projecting anteroventrally; (2) indistinct to absent (Wible et al. 2009, ch. 305). Changes in the scorings of Wible et al. (2009): Hyopsodus, Meniscotherium, Phenacodus (2) > (0).

324 (306W + 310W MODIFIED). - Posterior border of the middle ear cavity medial to the stylomastoid notch and lateral to the lateral edge of the jugular foramen: (0) straight or convex anteriorly; (1) concave or deeply notched (redefined from Wible et al. 2009, ch. 306 and 310 modified).

Comments: in many therian taxa the medial edge of the stylomastoid notch is formed by the lateral caudal tympanic process, when present. This is the case in, e.g., Vincelestes, Pucadelphys, Mayulestes, Alcidedorbignya, Pantolambda, Azygonyx, Hyopsodus, Meniscotherium, Tenrec, Erinaceus, Solenodon, Potamogale (see Appendix 5 for our interpretation of this process in Vincelestes and in basal metatherians and eutherians). However, it is noteworthy that, in eutherians, the lateral caudal tympanic process is extremely variable interspecifically and intraspecifically; this process may in some cases be distinct on one side of a skull and hardly present on the other. That is why in this analysis, the presence of a lateral caudal tympanic process is not scored as a separate character as, because of this high level of variability, it is not possible to confidently score it for fossil species known by only a few specimens. In Vincelestes and in metatherians (e.g., Pucadelphys, Andinodelphys, Mayulestes), the portion of the caudal tympanic process medial to the lateral caudal tympanic process is a continuous crest (or ridge), which extends medially toward the lateral edge of the jugular foramen. This ridge (caudal tympanic process of Wible 1990; Rougier et al. 1998) is either roughly rectilinear or slightly convex anteriorly and vanishes on the posterolateral angle of the jugular foramen. This condition is the character state (0). In many eutherians, between the lateral caudal tympanic process (on the medial edge of the stylomastoid notch) and the lateral edge of the jugular foramen is a distinct notch and, anteromedial to this notch, is the medial caudal tympanic process. In other words, the lateral and medial caudal tympanic processes are separated by a deep notch. This condition is the character state (1). When the lateral caudal tympanic process is distinct, this notch forms, with the stylomastoid notch, a distinct $\omega$-shaped aperture on the posterior opening of the middle ear. When the lateral caudal tympanic process is reduced or absent the posterior opening of the middle ear is a large roughly circular aperture.

$325(307 \mathrm{~W})$. - Crista interfenestralis and lateral caudal tympanic process of petrosal (= caudal tympanic process of Wible et al. 2009, ch. 307) connected by curved ridge: (0) absent; (1) present (Rougier et al. 1998, ch. 133 modified).

Changes in Wible et al. (2009) scorings: Blarina (1) > (?); Erinaceus, Solenodon, Eoryctes $(1)>(0)$.

326 (308W). - Medial caudal tympanic process (sensu MacPhee 1981 ) of the petrosal (= "tympanic process" of Wible et al. 2009, ch. 308): (0) absent; (1) present (low); (2) present (high) (e.g., inflated into bullar wall) (Rougier et al. 1998, ch. 134 modified); ORDERED.

327 (309W). - Medial caudal tympanic process (sensu MacPhee 1981) of the petrosal (= "tympanic process" of Wible et al. 2009, ch. 309) composition: (0) petrosal; (1) petrosal and exoccipital (Wible et al. 2009, ch. 309).

328 (311W MODIFIED). - Inferior petrosal sinus: (0) intrapetrosal; (1) horizontal between petrosal and basioccipital; (2) intracranial: entering cranial cavity on anterior edge of the jugular foramen; (3) intracranial: entering cranial cavity via a foramen at mid-length of the medial border of the promontorium (Rougier et al. 1998, ch. 151 modified).

$329(312 \mathrm{~W})$. - Size of the jugular foramen: (0) smaller or subequal to external aperture of cochlear fossula; (1) larger (Rougier et al. 1998, ch. 149).

$330(313 \mathrm{~W})$. - Jugular foramen and inferior petrosal sinus: (0) confluent; (1) separated (Rougier et al. 1998, ch. 150). Change in the scoring of Wible et al. (2009): Solenodon (1) > (0).

331 (314W). - Hypoglossal foramen: (0) two or more; (1) one; (2) absent (Luo \& Wible 2005, ch. 349).

$332(315 \mathrm{~W})$. - Hypoglossal foramen housed in opening larger than jugular foramen (0) absent; (1) present (Wible et al. 2009, ch. 315).

333 (NEW). - Hypoglossal foramen ventral opening on exoccipitals: (0) in a fossa; (1) on a flat to convex bony surface.

334 (316W). - Paroccipital (paracondylar of Wible et al. 2009) process of the exoccipital: (0) weak or absent; (1) prominent vertical; (2) prominent posteriorly directed (Rougier et al. 1998, ch. 135 modified).

335 (317W). - Ectotympanic: (0) phaneric or visible in ventral view; (1) aphaneric, hidden by auditory bulla (Wible et al. 2009, ch. 317). Change in the scoring of Wible et al. (2009): Pucadelphys (?) > (0).

336 (318W). - Ectotympanic: (0) ringlike; (1) fusiform; (2) transversely expanded (Rougier et al. 1998, ch. 142). Change in the scoring of Wible et al. (2009): Pucadelphys (?) > (0).

337 (319W). - Anterior crus of ectotympanic broad articulation with squamosal: (0) absent; (1) present (Wible et al. 2009, ch. 319).

338 (NEW). - Anterior crus of ectotympanic articulation with petrosal: (0) absent; (1) present (this paper).

339 (320W). - Elongate ossified external acoustic canal: (0) absent; (1) present (Wible et al. 2009, ch. 320). Changes in the scorings of Wible et al. (2009): Hyopsodus, Meniscotherium (?) > (0).

340 (321W). - Roof of external acoustic meatus: (0) petrosal; (1) squamosal (Wible et al. 2009, ch. 321).

$341(322 \mathrm{~W})$. - Entotympanic distinct in adults: (0) absent; (1) present (Luo \& Wible 2005, ch. 363).

342 (323W MODIFIED). - Pit for hyoid on ectotympanic (or between ectotympanic and exoccipital): (0) absent; (1) present (Wible et al. 2009, ch. 323 modified). Change in the scoring of Wible et al. (2009): Procavia $(0)>(1)$.

343 (324W). - Hyoid arch contributes to bullar floor: (0) absent; (1) present (Wible et al. 2009, ch. 324).

344 (325W). - Dorsum sellae: (0) tall; (1) low (Rougier et al. 1998, ch. 106).

345 (326W). - Posterior clinoid process contacts apex of the promontorium: (0) absent; (1) present (Wible et al. 2004).

346 (327W). - Position of sulcus for anterior distributary of transverse sinus relative to fossa subarcuata: (0) anterolateral; (1) posterolateral; (2) absent (Rougier et al. 1998, ch. 125).

$347(328 \mathrm{~W})$. - Wall separating the cavum supracochleare from the cavum epiptericum: (0) absent; (1) incomplete with fenestra semilunaris; (2) complete (Rougier et al. 1998, ch. 128 modified). 
348 (329W). - Crista petrosa: (0) vestigial or absent; (1) tall and thin crest (Wible et al. 2009, ch. 329).

349 (330W MODIFIED). — Subarcuate fossa morphology: (0) subspherical with constricted aperture: diameter of aperture is smaller than maximum diameter of the fossa; (1) cylindrical, diameter more or less constant; (2) conical or shallow depression: aperture is the widest diameter (Wible et al. 2009, ch. 330 modified) ORDERED.

$350(332 \mathrm{~W})$. - Internal acoustic meatus: (0) deep with thick prefacial commissure; (1) shallow with thin prefacial commissure (Wible et al. 2009, ch. 332).

\section{SKULL OCCIPUT}

351 (333W MODIFIED). - Posttemporal canal: (0) present; (1) absent (Wible et al. 2009, ch. 333 modified).

$352(334 \mathrm{~W})$. - Posttemporal canal composition (posterior opening): (0) between petrosal and squamosal; (1) within petrosal (Wible et al. 2009, ch. 334)

353 (336W MODIFIED). - Mastoid foramen l: (0) absent; (1) present (one or more) (Meng et al. 2003, ch. 114 modified). Changes in the scorings of Wible et al. (2009): Rhynchocyon (2) > (0); Bradypus (0) > (1). Note: because we have observed a great deal of individual variation of this (these) foramen (foramina) in their number and in their position, sometimes on the same specimen on right and left petrosal, we have simplified (present vs absent) the character as defined by Meng et al. (2003). Rhynchocyon has been scored (0) contra Wible et al. (2009) but following O'Leary et al. (2013).

354 (337W). - Amastoidy or lack of occipital exposure of the mastoid: (0) absent; (1) present (Geisler 2001, ch. 38).

355 (338W). - Dorsal margin of the foramen magnum: (0) formed by the exoccipitals; (1) formed by the exoccipitals and the supraoccipital (Rougier et al. 1998, ch. 156).

356 (NEW). - Dorsal relief of lambdoid crest continuous with dorsal margin of zygomatic arch: (0) absent; (1) present (Billet 2010, ch. 95).

\section{POSTCRANIAL CHARACTERS}

357 (339W). - atlantal foramen (Horovitz \& Sánchez-Villagra 2003:1 modified): (0) present; (1) absent.

358 (340W). - Atlas neural hemiarches fused (Luo \& Wible 2005, ch. 169 modified): (0) absent; (1) present. Changes in the scorings of Wible et al. (2009): Mayulestes, Pucadelphys (0) > (1).

$359(341 \mathrm{~W})$. - Atlas neural arch and intercentrum fused (Luo \& Wible 2005, ch. 167): (0) absent; (1) present.

$360(342 \mathrm{~W})$. - Suture between atlantal and axial parts of the axis (Luo \& Wible 2005, ch. 169 modified): (0) present; (1) absent.

$361(343 \mathrm{~W})$. - Axis with extra pair of transverse processes on ventral surface of body (Horovitz \& Sánchez-Villagra 2003, ch. 11 modified): (0) present; (1) absent.

262 (344W). - Axis anterior facets (prezygapophyses) and dens connection (Horovitz \& Sánchez-Villagra 2003, ch. 12 modified): (0) not linked; (1) linked; facets extend ventral to dens.

363 (345W). - Inferior lamellae on posterior cervical vertebrae (essentially on C6 and occasionally on C5) (Wible et al. 2009, ch. 345): (0) present; (1) absent. Change in the scoring of Wible et al. (2009): Mayulestes (?) > (0).
364 (346W). - C7 transverse foramen (Horovitz \& Sánchez-Villagra 2003, ch. 231 modified): (0) present; (1) absent.

365 (347W). - Number of thoracic vertebrae (Luo \& Wible 2005, ch. 172): (0) 13 or fewer; (1) 15 or more.

366 (348W). - Number of lumbar vertebrae Wible et al. 2009, ch. 348): (0) 6 or more; (1) 5 or fewer.

367 (349W). - Xenarthrous articulations on lumbar vertebrae (Luo \& Wible 2005, ch. 176): (0) absent; (1) present. Changes in the scorings of Wible et al. (2009): Mayulestes, Pucadelphys (?) > (0).

368 (350W). - number of sacral vertebrae (Geisler 2001, ch. 131 modified): (0) 2; (1) 3; (2) 4 or more. Change in the scoring of Wible et al. (2009): Pucadelphys (?) > (0); ORDERED.

369 (351W). - Sacral vertebrae fused to pelvis: (0) absent; (1) present (Wible et al. 2009, ch. 351). Changes in the scorings of Wible et al. (2009): Mayulestes, Pucadelphys (?) > (0).

$370(352 \mathrm{~W})$. - Infraspinous fossa position to supraspinous fossa (Rougier 1993, ch. 13 modified): (0) different planes (in part medial to); (1) coplanar.

371 (353W). - Suprascapular incisure (Luo \& Wible 2005, ch. 196): (0) absent; (1) present.

$372(354 \mathrm{~W})$. - Apex of acromion (Asher et al. 2005, ch. 174 modified): (0) distal to glenoid articulation; (1) proximal; (2) absent.

373 (355W). - Metacromion (Wible et al. 2009): (0) weak or absent; (1) well-developed process.

$374(356 \mathrm{~W})$. - Elevation of the greater tubercle of the humerus relatively to the humeral head (Asher et al. 2005, ch. 175): (0) ventral (distal); (1) even or dorsal (proximal).

375 (357W). - Extension of deltopectoral crest (Horovitz \& Sánchez-Villagra 2003, ch. 50): (0) limited to proximal half of the humerus; (1) reaches distal half.

$376(358 \mathrm{~W})$. - Epicondylar crest (= sigmoidal shelf for supinator ridge extending proximally from ectepicondyle (Luo \& Wible 2005, ch. 206): (0) weak or absent; (1) present and patent.

377 (359W). - Entepicondyle (Geisler 2001, ch. 134): (0) Robust and projecting medially; (1) weak or absent.

378 (360W). - Entepicondylar foramen (Geisler 2001, ch. 135): (0) present; (1) absent.

379 (361W). - Supratrochlear foramen (Asher et al. 2005 178): (0) absent; (1) present.

$380(362 \mathrm{~W})$. - Ulnar articulation of the humerus (Luo \& Wible 2005, ch. 203 modified): (0) cylindrical trochlea in posterior view with a vestigial ulnar condyle in anterior view; (1) cylindrical trochlea without an ulnar condyle (cylindrical trochlea extending to the anterior/ventral side).

381 (363W). - Radial articulation (Luo \& Wible 2005, ch. 204 modified): (0) rounded radial condyle (= capitulum) anteriorly but cylindrical posteriorly; (1) capitulum forming a continuous synovial surface with the ulnar trochlea (cylindrical in both anterior and posterior views).

$382(364 \mathrm{~W})$. - Humeral articulation on radius (Geisler 2001, ch. 141 modified): (0) single fossa; (1) two fossae.

383 (365W). - Central process of radial head (Asher et al. 2005, ch. 181): (0) small or absent; (1) present. 
384 (366W). - Radius and ulna distal fusion (Thewissen $e t$ al. 2001, ch. 81): (0) absent; (1) present.

385 (367W). - Radial articulation with carpals (Thewissen et al. 200180 ): (0) single fossa; (1) two fossae.

386 (368W). - Scaphoid and lunate (Asher et al. 2005, ch. 183): (0) separate; (1) fused.

387 (369W). - Os centrale (Asher et al. 2005, ch. 184): (0) present; (1) absent.

388 (370W). - Pubic symphysis (Meng et al. 2003, ch. 22): (0) extensive; (1) narrow.

389 (371W). — Epipubic bone (Luo \& Wible 2005, ch. 218): (0) present; (1) absent.

390 (372W). - Articular surface of femoral head (Asher et al. 2005, ch. 186): (0) extended posterolaterally; (1) limited to the sphere of the head.

$391(373 \mathrm{~W})$. - Fovea for ligamentum teres on femoral head (MacPhee 1994, ch. 27): (0) surrounded by articular surface; (1) interrupt margin of articular surface; (2) absent.

$392(374 \mathrm{~W})$. - Greater trochanter relative to femoral head (Horovitz \& Sánchez-Villagra 2003, ch. 79): (0) lower; (1) even; (2) higher. Changes in the scorings of Wible et al. (2009): Mayulestes, Pucadelphys $(0)>(2)$; ORDERED.

393 (375W). - Size of lesser trochanter of femur (Luo \& Wible 2005, ch. 228; Flores 2009, ch. 93) (0) large, surpassing half of the medial extension of the femoral head; (1) small. Changes in the scorings of Wible et al. (2009): Mayulestes, Pucadelphys (1) > (0).

394 (376W). - Third trochanter of femur (Asher et al. 2005:188): (0) absent; (1) present. Changes in the scorings of Wible et al. (2009): Mayulestes, Pucadelphys $(1)>(0)$.

395 (377W). - Pectineal tubercle (see Lessertisseur \& Saban 1967): (0) absent or vestigial; (1) distinct.

396 (378W). - Proportions of the distal extremity of the femur (Asher et al. 2005, ch. 189): (0) as long as wide; (1) longer than wide.

397 (379W). - Patellar facet of the femur (Luo \& Wible 2005, ch. 230 modified): (0) weakly developed; (1) broad and shallow; (2) narrow and elevated (deep).

398 (380W). - Ossified patella (Luo \& Wible 2005, ch. 273): (0) absent; (1) present.

399 (381 W). - Articulation between femur and fibula (Horovitz \& Sánchez-Villagra 2003, ch. 84): (0) absent; (1) present. Change in the scoring of Wible et al. (2009): Pucadelphys (?) > (1).

400 (382W). - Tibia and fibula proximal fusion (Asher et al. 2005 , ch. 190): (0) absent; (1) present.

$401(383 \mathrm{~W})$. - Tibia and fibula distal fusion (Horovitz \& SánchezVillagra 2003, ch. 87): (0) absent; (1) present.

$402(384 \mathrm{~W})$. - Depth of trochlear groove of the astragalus (Zack et al. 2005, ch. 40 modified): (0) Shallow (slightly concave articular surface); (1) moderately deep (section of the groove U-shaped); (2) deep (V-shaped); ORDERED.

403 (385W). - Angle between medial and lateral facet for the tibia on the astragalar body (Horovitz \& Sánchez-Villagra 2003, ch. 94 modified): (0) $180^{\circ}$; (1) intermediate; (2) $90^{\circ}$. Changes in the scorings of Wible et al. (2009): Mayulestes, Pucadelphys (0) > (1); ORDERED.

$404(386 \mathrm{~W})$. - Angle between facet for the fibula and the lateral facet for the tibia (Horovitz \& Sánchez-Villagra 2003, ch. 99): (0) $180^{\circ}$; (1) intermediate; (2) $90^{\circ}$. Change in the scoring of Wible et al. (2009): Pucadelphys (?) > (0); ORDERED.

405 (387W). - Radius of curvature of the lateral trochlear ridge of the astragalus relatively to the medial trochlear ridge (Zack et al. 2005, ch. 41): (0) greater; (1) subequal.

$406(388 \mathrm{~W})$. - Cotylar fossa on the medial edge of the astragalus trochlea (Zack et al. 2005, ch. 44 modified): (0) absent; (1) present.

407 (389W). - Contact between sustentacular and navicular facets of the astragalus (Asher et al. 2005 204): (0) absent; (1) present.

$408(390 \mathrm{~W})$. - Medial extension of the astragalar sustentacular facet (Horovitz \& Sánchez-Villagra 2003, ch. 102): (0) reaches the medial edge of the neck; (1) does not reach the medial edge.

409 (391W). - Astragalar medial plantar tuberosity (Horovitz \& Sánchez-Villagra 2003, ch. 98 modified): (0) weak or absent; (1) protruding. Changes in the scorings of Wible et al. (2009): Mayulestes, Pucadelphys (0) > (1).

$410(392 \mathrm{~W})$. - Astragalar neck (Horovitz \& Sánchez-Villagra 2003, ch. 100): (0) absent; (1) present shorter than body width; (2) present and similar in length to body width. Changes in the scorings of Wible et al. (2009): Mayulestes, Pucadelphys (1) > (0).

411 (393W). - Convex astragalar head (Thewissen et al. 2001, ch. 92 modified): (0) absent; (1) present.

$412(394 \mathrm{~W})$. - Facet on astragalus for cuboid (Asher et al. 2005, ch. 208): (0) absent; (1) present.

413 (395W). - Astragalar canal (Horovitz \& Sánchez-Villagra 2003, ch. 104 modified): (0) present; (1) dorsal foramen only; (2) absent.

$414(396 \mathrm{~W})$. - Posterior trochlear shelf of the astragalus (Asher et al. 2005, ch. 198) (0) weak or absent; (1) strong.

415 (397W). - Calcaneal width (Asher et al. 2005, ch. 210): (0) Broad with sustentacular and ectal facet extending from body; (1) narrow with sustentacular and ectal facets in line with long axis.

$416(398 \mathrm{~W})$. - Ectal (or posterior calcaneoastragalar) facet orientation of longest axis (Horovitz \& Sánchez-Villagra 2003, ch. 113 modified): (0) anteromedial to posterolateral, (1) anteroposterior (proximodistal), (2) posteromedial to anterolateral; ORDERED.

417 (399W). - Anteroposterior overlap between calcaneal ectal and sustentacular facets (Zack et al. 2005, ch. 32 modified): (0) no overlap; (1) partial overlap; (2) nearly complete overlap; ORDERED.

418 (400W). - Calcaneal sustentacular facet orientation (Horovitz \& Sánchez-Villagra 2003): (0) medial; (1) dorsal.

$419(401 \mathrm{~W})$. - Calcaneal sustentacular facet expanded onto body (Wible et al. 2009, ch. 401): (0) absent; (1) present.

$420(402 W)$. - Calcaneal peroneal process (Horovitz \& SánchezVillagra 2003, ch. 117): (0) Protruding distally beyond calcaneocuboid facet; (1) distal but non-protruding; (2) at a distance from distal end of calcaneum; (3) absent.

$421(403 \mathrm{~W})$. - Calcaneal plantar tubercle (Horovitz \& SánchezVillagra 2003, ch. 122 modified): (0) absent; (1) present at distal margin; (2) present more proximal than distal margin. 
$422(404 \mathrm{~W})$. - Tuber calcis ventral curvature (Horovitz \& SánchezVillagra 2003, ch. 125 modified): (0) present; (1) absent. Change in the scoring of Wible et al. (2009): Pucadelphys (0) > (1).

$423(405 \mathrm{~W})$. - Calcaneal facet for the fibula (Horovitz \& SánchezVillagra 2003, ch. 125 modified): (0) present; (1) absent.

$424(406 \mathrm{~W})$. - Orientation of mediolateral axis of the cuboid facet relative to the long axis of the calcaneum (Zack et al. 2005, ch. 37): (0) less than $70^{\circ}$; (1) c. $70^{\circ}-80^{\circ}$; (2) c. $90^{\circ}$; ORDERED.
$425(407 \mathrm{~W})$. - proportions of the cuboid facet on the calcaneum (Zack et al. 2005, ch. 38): (0) facet much deeper (dorsoplantar) than wide (mediolateral); (1) facet depth and width subequal; (2) facet much wider (mediolateral) than deep (dorsoplantar); ORDERED.

$426(408 \mathrm{~W})$. - - deep groove for the tendon of the flexor hallucis longus (= flexor fibularis) on the posterior edge of the sustentaculum calcaneum (Wible et al. 2009, ch. 408): (0) absent; (1) present. 
Appendix 2. - Taxon list, specimens, and reference.

Vincelestes neuquenianus: cast of MACN-N04; photographs the ear region of this specimen, MACN-N05, and MACNN09 kindly provided by G. Rougier; Rougier et al. (1992); Bonaparte \& Rougier (1987).

Deltatheridium praetrituberculare: casts of ZPAL MgMI/102, ZPAL MgM-I/91; Kielan-Jaworowska (1975b) and Rougier et al. (1998).

Mayulestes ferox: MHNC 1249 (holotype).

Pucadelphys andinus: MHNC 8265, 8266, 8365, 8376-8395 (all specimens are complete or partial skulls and mandible).

Eomaia scansoria: Ji et al. (2002) and Wible et al. (2009).

Prokennalestes trofimovi: PSS-MAE 136; Kielan-Jaworowska \& Dashzeveg (1989); Sigogneau-Russell et al. (1992); Wible et al. (2001).

Zhelestes temikazyk: Nessov et al. 1998; Ekdale et al. (2004); Archibald \& Averianov (2005); Wible et al. (2009).

Cimolestes spp.: Scoring follows Wible et al. (2009), cast of UCMP 36658.

Maelestes gobiensis: PPS-MAE-607, Wible et al., (2009).

Kennalestes gobiensis: Kielan-Jaworowska (1969, 1977, 1981); Wible et al. (2009), casts of ZPALMgM-I/ 2 and I/3.

Asioryctes nemegtensis: Kielan-Jaworowska (1975a, 1977, 1981); Horovitz (2000); Horovitz \& Sánchez-Villagra (2003); Wible et al. (2009), cast of ZPAL MgM-I/56 and I/98.

Kulbeckia kulbecke: Archibald et al. (2001); Archibald \& Averianov (2003); Archibald \& Averianov (2005); Ekdale et al. (2004); Ekdale \& Rowe (2011).

Zalambdalestes lechei: PSS-MAE 108, 130, Kielan-Jaworowska (1978, 1984b); Kielan-Jaworowska \& Trofimov (1980); Novacek et al. (1997); Wible et al. (2004, 2009); Ekdale \& Rowe (2011).

Leptictis dakotensis: AMNH 80213; AMNH FAM 87458; cast of AMNH 108194; Novacek (1986); Rose (1999); Rose (2006); Wible et al. (2009).

Didelphodus altidens: USNM 18369; D. absarokae casts of USGS 303, 2318, 3709, 3951, 5972.

Alcidedorbignya inopinata: MHNC 1210, 8282, 8287, 8290, 8295, 8298, 8300, 8315, 8359-8363, 8371, 8372, 8373, 8399-8463, 13830-13845.

Pantolambda bathmodon: AMNH 16663, 16664, 16665, 15934, 3957, Matthew 1937; Simons 1960.

Coryphodon spp.: MNHN.F.ARP8, ARP9, ARP10, ARP17, ARP18, ARP48, ARP49, ARP58, ARP74, ARP137; Cope (1877a, b ; 1882).

Bemalambda nanhsiungensis and B. pachyoesteus: casts of teeth of IVPP 4124, 4126, 4132; Zhou et al. (1977); Lucas (1993).
Harpyodus euros, H. decorus: cast of IVPP 4316 (holotype of $H$. euros), and photos of IVPP V 5035 (holotype skull of $H$. decorus) kindly provided by S. Lucas; Wang (1979).

Esthonyx-Azygonyx: Esthonyx xenicus: AMNH 15111, 16065, 66166; Azygonyx ancylion: UM 68511; Gazin (1953).

Trogosus grangeri: USNM 17886, AMNH 17008; Gazin (1953).

Protongulatum donnae: scoring follows Wible et al. (2009), UCMP 134558.

Vulpavus profectus, V. ovatus, V. canatus: Scoring follows Wible et al. (2009).

Miacis parvivorus, M. Sylvestris: Scoring follows Wible et al. (2009).

Cynodictis sp.: MNHN uncatalogued specimen.

Diacodexis: Wible et al. (2009); for inner ear (Orliac et al. 2012b); Krishtalka \& Stucky (1985).

Dichobune leporina: MNHN.F.QU16586; Orliac \& O’Leary (2014).

Acotherulum saturninum: MNHN.F.QU16366, Orliac \& O'Leary (2014).

Hyopsodus spp.: AMNH FM 2301, 11877, 95495, 95496 143783, USNM 17311, 17980, 23745, 23747, cast of AMNH FM 48668, cast of UCMP 92907; Gazin (1968); West (1979); Cifelli (1982).

Meniscotherium spp.: AMHN FM 2560, 4447, 48082, 48083, 48121, 48122, 48129; USNM 18260, 22672, 22673, 22918; cast of AMNH FM 4410; Gazin (1965); Williamson \& Lucas (1992).

Phenacodus spp.: AMNH FM 20070; PU 14864; cast of USNM 20068, 44262, YPM 20524; Thewissen (1990).

Pleuraspidotherium aumonieri: MNHN.F.CRL250, CRL251, MNHN.F.CR645, CR760, CR941, CR962, CR963, CR964, CR1128, CR1312, MNHN.F.BR12534, BR14058, BR14059, BR17498, BR17500; Russell (1964), Thewissen (1991).

Maiorana noctiluca: YPM-PU 14171, 16667, 21124.

Baioconodon nordicum: AMNH 27714, 96406, YPM-PU 14234 casts of YPM-PU 14473, 14475,16720, 16870,16854, $21011,21123$.

Arctocyonides arenae: MNHN.F.CR144, CR145, CR146, CR733; MNHN.F.BR1065, BR17501, BR17503; Russell (1964).

Arctocyon primaevus: MNHN.F.CR700, CR706; MNHN.F.BR12669, BR17499; Russell (1964).

Ptilocercus lowii: scoring follows Wible et al. (2009); Wible (2011). 
Plesiadapis tricuspidens: MNHN.F.CR125; Russell (1964), Wible et al. (2009), Orliac et al. (2014).

Adapis-Magnadapis: MNHN.F.QU10870, QU10872; PLV 14; Wible et al. (2009), Gingerich (1981), Gingerich \& Martin (1981).

Notharctus tenebrosus: scoring follows Wible et al. (2009); Gazin (1958).

Tribosphenomys minutus: scoring follows Wible et al. (2009); Meng \& Wyss (2001) used for new characters.

Paramys spp.: scoring follows Wible et al. (2009); Matthew (1910); Rose \& Chinnery (2004).

Rhombomylus turpanensis: scoring follows Wible et al. (2009), Meng et al. (2003) used for new characters.

Gomphos elkema: scoring follows Wible et al. (2009), Meng et al. (2004) used for new characters.

Mimotona wana: scoring follows Wible et al. (2009), Li \& Ting (1993) used for new characters.

Blarina brevicauda: MNHN-AC-1865-206, CG 1962 2597, CG 1962-2899, CG2006-364; scoring follows Wible et al. (2009).

Erinaceus europeus: 10 uncatalogued specimens; Asher \& Olbricht (2009) for character 6 on dental eruption.

Solenodon paradoxus: MNHN-AC-14487, 1902-156, 1932-195, 2006-341; Wible (2008).

Eoryctes melanus: cast of UM68074; Thewissen \& Gingerich (1989).

Potamogale velox: MNHN-AC-1952-422, 1947-866, 1947-864, 2006-344, A1274; Asher \& Olbricht (2009) for character 6 on dental eruption.

Tenrec ecaudatus: MNHN-AC-6997, 1932-3433, 1935-47, 1931-157, 1951-5, 1963-387 and Asher \& Lehmann (2008) for character 6 on dental eruption.

Orycteropus afer: MNHN-AC-CG 1951-435, CG 1970581913-101, 2007-388; Wible (2012); Asher \& Lehmann (2008).

Rhynchocyon spp.: MNHN-AC-CG 1891- 265, CG $1897-$ 623 and 624, CG 1897-622; and Asher \& Olbricht (2009) for character 6 on dental eruption.

Procavia capensis: MNHN-AC-1972-429 and three uncatalogued specimens; Asher \& Lehmann (2008) for character 6 on dental eruption.

Moeritherium trigodon: Wible et al. (2009); Tassy (1981); Andrews (1906) and MNHN LBE 1.

Chaetophractus villosus: MNHN-AC-10451; Wible et al. (2009); Babot et al. (2012).

Dasypus novemcinctus: RH 16, 165 ; Ciancio et al. (2012).

Bradypus spp.: RH 7 et 80.
Tamandua tetradactyla: RH 25, 26, 164, Babot et al. (2012), further aspects of inner ear and petrosal scored from UMZC E581 (Billet et al. 2012).

Colbertia spp.: AMNH 49873, 49867, 49868, 49874, 49871 and 49887; PVL 4294, 6218, 4607, 4183, PVL-5-11 and PVL5-2 (photographs sent by Daniel Garcia-López University of Tucumán, Argentina) and Cifelli (1983a), Bergqvist (1996), Bergqvist et al. (2007), Bond (1981); Paula-Couto (1952), Garcia-López (2011).

Plesiotypotherium achirense: Villarroel (1974); MNHN.F.ACH18, ACH20, ACH23, ACH24, ACH25, ACH26, ACH36, ACH40.

Protypotherium spp.: MNHN.F.SCZ178, SCZ179, SCZ184; MACN A-4001; FMNH P13002;

AMNH 9534, YPM-PU 15161, 15828, 15386, 15340, 15665; Sinclair (1909); Billet \& Martin (2011).

Adinotherium spp.: MNHN.F.SCZ5, SCZ13, SCZ14; FMNH P 13110, YPM-PU 15131, 15978, 15482, 15466, 15989, 15382, 15118, 16032, 15288, 15986, 15114, 15159; Scott (1912), Gabbert (2004), Billet \& Martin (2011).

Notostylops spp.: MNHN.F.CAS96, CAS97, CAS637, CAS647 (and one right lower jaw annotated 1903-3); MACN A-10499 and 10498; FMNH P13319; AMNH 28581, 28604, 28614, 28634, 28758 and 28956; Simpson (1948), Billet \& Martin (2011).

Pyrotherium: P. romeroi, ACM 3207; FMNH 13515; MNHN.F.DES1238, DES1239, DES1240, DES1243; Loomis (1914), Patterson (1977), Billet (2010). Pyrotherium macfaddeni, MNHN.F.SAL21, SAL22, SAL181, SAL182, several uncatalogued isolated postcranial elements.

Trigonostylops spp.: AMNH 28700; MNHN.F.CAS187, CAS188, CAS1200; Simpson (1967).

Astrapotherium spp.: MNHN.F.SCZ8, SCZ11; AMNH 9278, YPM-PU 15261, 15332, 15143, 15984, 15255; other uncatalogued specimens from the MNHN.F.SCZ collection (from the Santa Cruz Beds of Patagonia); Scott (1928, 1937), Kramarz \& Bond (2014).

Miguelsoria: UFRJ-DGM 330M, DGM 305M, DGM 966M, DGM 1505M, DGM 1504M, DGM 402M, DGM 397M, UFRJ-C isolated petrosals (Billet et al. 2015), Cifelli (1983b).

Proterotherium: AMNH 9245, MNHN.F.SCZ uncatalogued specimens (skulls and mandibles annotated 1902-6), YPM-PU 15368, 15107, 15712, 15732, 15996, 15718, Scott (1910).

Macrauchenia: MNHN.F.PAM69, PAM70-PAM75, PAM78, PAM79, PAM329, PAM337, PAM339, PAM375, PAM379, PAM380, MNHN.F.TAR738; MNHN.F.PAM uncatalogued lower jaw fragments; MNHN.F.TAR uncatalogued isolated incisors; Cope (1891).

Carodnia vierai: Paula-Couto (1952), UFRJ-DG 333M, 334M. 


\section{APPENDiX 3. - Description of the auditory region of Pantolambda bathmodon.}

One of the morphologically closest taxa to Alcidedorbignya inopinata is Pantolambda bathmodon from the Torrejonian of New Mexico (Muizon \& Marshall 1992 and this paper). Superficial descriptions have been provided by Matthew (1937) but the best-preserved specimen (AMNH 16663) was incompletely prepared at the time of its first description. Especially the ventral part of the skull, which still retained the lower jaw in connection, has not been described. Further preparation of the specimen with mechanic tools has allowed a separation of the skull and lower jaw. Further preparation (by one of us, $\mathrm{CM}$ ) of the skull by acid etching has permitted observation of a perfectly preserved left auditory region. The right ear had a slightly damaged promontorium and tympanohyal prior to acid preparation. Furthermore, the well-preserved left ectotympanic and attached malleus have been separated from the petrosal. Because the basicranium of AMNH 16663 is the best northern pantodont basicranium available for this study, it was continuously used for comparison in the description of Alcidedorbignya. Therefore, because this auditory region is undescribed, we present below a thorough description of the ventral view of the ear region of this basal pantodont (Figs 124-126). The specimen has been CT scanned but the presence of metallic oxides (probably hematite) in the intracranial ear region precluded reconstruction of the dorsal face of the petrosal an adjacent bones.

The most immediately striking characteristic of the petrosal of Pantolambda is the large anterior fossa for the carotid foramen and pyriform fenestra. In fact, the two apertures are completely merged and form a vast triangular opening at the apex of the promontorium. The anterior angle of the pyriform fenestra was probably the passage of the internal carotid artery and is in fact a carotid notch resulting from the opening of the internal carotid foramen totally enclosed in the basisphenoid, as is present in basal eutherians (e.g., Zalambdalestes Asioryctes, Maelestes). The anterior edge of the promontorium has a bizarre vertically cut morphology, which strongly resembles an artificial break. However, the other petrosal has the same morphology and both petrosals were prepared with no bone breakage. On the medial edge of the promontorium a continuous basicochlear fissure connects the pyriform fenestra to the elongated jugular foramen posteriorly. The anterior angle and anterior part of the lateral edge of the pyriform fenestra are formed by the basisphenoid, the posterior end of the lateral edge is formed by the alisphenoid and the medial edge is formed by the basioccipital. Several foramina pierce the basisphenoid in the bottom of the carotid notch at the anterolateral edge of the pyriform fenestra. Three foramina are present on the left side of AMNH 16663 and a single very large slit-like foramen on the right. These foramina open in a similar position as the foramen described by Wible (2008) in Solenodon as the posterior opening of the pterygoid canal and could therefore have had a similar function in Pantolambda. However, a CT scan of the skull reveals that these foramina extend in the bone in a short canal, which quickly disappears anteriorly. Therefore, we interpret these structures as nutrient foramina. In other respects, on the dorsolateral angles of the basipharyngeal canal, along the basisphenoid-pterygoid suture, delicate but distinct grooves run anteriorly from the carotid notch (observable c. $5 \mathrm{~mm}$ anterior to the bottom of the notch) for approximately $15 \mathrm{~mm}$ and penetrate the pterygoid-basisphenoid suture (observable on the right side of the specimen). We interpret these grooves as the passageway of the nerve of the pterygoid canal, which ran some distance in the basipharyngeal canal before entering the pterygoid canal anteriorly. The pterygoid canal transmits the nerve of the pterygoid canal anteriorly to the pterygopalatine ganglion, from which stem three branches for the innervation of the lacrimal, nasal, and palatine glands. The promontorium of Pantolambda is very globular and inflated in its posterolateral region. It does not present any obvious sulcus for the stapedial artery. Anterolateral to the globular part of the promontorium is a large and shallow fossa for the musculus tensor tympani sloping anterodorsolaterally. The medial side of the pars cochlearis is very thick and is as high as the two-thirds of the greatest width of the promontorium. The surface of the medial face of the pars cochlearis is irregular and puffy. It bears no distinct sulcus for the inferior petrosal sinus. On the posterolateral side of the promontorium is a strongly ovalshaped fenestra vestibuli. It is at least three times longer than wide, a condition observed on both sides of the specimen. On the anterolateral side of the promontorium is a relatively wide tegmen tympani. On its medial side it is perforated by the facial canal (the floor of which is partly broken on both sides of the specimen), which conveys anteriorly the greater petrosal nerve and posteriorly the facial nerve sensu stricto. Both nerve are issued from the geniculate ganglion located above the canal. The anterior opening through which exits the greater petrosal nerve is the hiatus Fallopii. It is located at the anterior border of the ventral face of the tegmen tympani. The other extremity of the canal is the secondary facial foramen, which conveys the facial nerve to the posterior end of the facial sulcus. On the lateral side of the tegmen tympani is a large dorsally-oriented foramen. It conveyed the superior ramus of the stapedial artery dorsal to the ear region. Apparently the foramen is not totally enclosed in the petrosal. Its posterior wall is a deep groove in the petrosal and its anterior wall is formed by the alisphenoid. Posterolateral to the tegmen tympani and lateral to the fenestra vestibuli is the epitympanic recess. It is large and deep and extends posterodorsally. Its divided in its middle by the petrosal-squamosal suture. The medial portion, excavated in the petrosal, is bordered medially by a sharp and thin crest, the crista parotica. At the anterior end of the crista parotica is a small elongate and lenticular facet, which received the medial articulation of the anterior crus of the ectotympanic. At the posterior end of the medial wall of the recess the crista parotica bears another articular facet, which extends medially and floors the posteromedial quarter of the recess. This facet extends on the anterior face of the 
tympanohyal, the distal element of the hyoid, which is fused to the crista petrosa. This facet is for the posterior crus of the ectotympanic. This posterior tympanic facet hides the bottom of the epitympanic recess and it is not possible to observe the fossa incudis. The lateral portion of the recess, excavated in the squamosal, opens anteroventrally in a wide groove, the Glaserian fissure, which conveyed anteriorly the chorda tympani to the lingual nerve, a branch of the mandibular nerve (CN V3). The medial wall of the fissure is formed by the entoglenoid process of the squamosal and the lateral by the postglenoid eminence (new term: see above in the cranial description of Alcidedorbignya). On the medial edge of the fissure is an anteroventral extension of the petrosal articular facet for the medial articulation of the anterior crus of the ectotympanic, which extends on the entoglenoid process of the squamosal. This medial articulation received the styliform process and the blade-like rostral process of the malleus, which is wedged between the petrosal and the ectotympanic (Fig. 126C). On the medial edge of the postglenoid eminence is a deep elongated subvertical fossula, which received the lateral articular facet of the anterior crus of the ectotympanic. The postglenoid eminence is relatively large and prismatic having a distinctly triangular section.

Posterior to the epitympanic recess, the tympanohyal is hook-like and strongly bent medially. It almost contacts medially the medial caudal tympanic process and forms the ventral edge of the stylomastoid notch, through which the facial nerve exits the skull. Posterior to the stylomastoid notch the passageway of the facial nerve extends posterolaterally in a shallow groove, which reaches the petrosal-squamosal suture at a point just anterior to the mastoid process. There the facial nerve probably followed the suture on the posterior edge of the external auditory meatus.

The other large opening, which opens in the posterior side of the promontorium, is the external aperture of the cochlear fossula, which opens internally on the fenestra cochleae. The external aperture of the cochlear fossula is large, subtriangular, and faces posterolateroventrally. Posterolateral to the external aperture of the cochlear fossula is a large medial caudal tympanic process. It is laterally bent and its truncated and flat apex closely approximates to the tympanohyal. Because of this orientation, its posterolateral edge is deeply notched. Its ventromedial edge is an elongated sharp subhorizontal crest, which originates on the lateral edge of the jugular foramen. Anterior to this crest, is a wide and deep groove, which exits from the medial edge of the jugular foramen. This groove passes between the external aperture of the cochlear fossula and the medial caudal tympanic process in the postpromontorial sinus. In fact, this sinus is not well defined in Pantolambda and is partly invaded by the groove mentioned above. This groove is likely for the tympanic nerve, an offshoot of the glossopharyngeal nerve (CN IX), which exits the skull at the jugular foramen. The tympanic nerve runs anteriorly on the crista interfenestralis (which separates the promontorium fenestrae), passes medial to the fenestra vestibuli, and ventral to the tegmen tympani (where it continues its course anteriorly as the minor petrosal nerve), and joins the otic ganglion, which in turn is connected to the mandibular branch of the trigeminal nerve (CN V3) (MacPhee 1981; Evans \& de Lahunta 2012). Posterolateral to the medial caudal tympanic process is the lateral caudal tympanic process. This lateral process is distinctly notched and bears a groove, which was probably the passageway of a posterior ramus of the stapedial artery. Because of the presence of a notch for the posterior ramus, the lateral caudal tympanic process is subdivided into two crests, lateral and medial. The medial crest of the lateral caudal tympanic process almost contacts the medial caudal tympanic process. The space between the two processes is a deep groove, which excavates the posterolateral edge of the medial caudal tympanic process. This groove exits from the jugular foramen posterior to that for the tympanic nerve. It probably conveyed the auricular branch of the vagus nerve (CN X), which connects to the facial nerve. The orientation of this groove indicates that the auricular branch clearly entered the middle ear, passed below the stapedial fossa and turned posteriorly anterior to the lateral caudal tympanic process. Therefore, the connection with the facial nerve was probably inside the middle ear, before its exit from the skull through the stylomastoid notch. This condition in Pantolambda is one of the reasons why in the above description we favored the interpretation that, in Alcidedorbignya, the junction between the auricular nerve and facial nerve probably occurred within the tympanic cavity. The space between the medial and lateral tympanic process presents, posterolateral to the deep groove for the auricular nerve, a small crest, which materialises a shallow groove on the anteromedial edge of the lateral caudal tympanic process. We have no interpretation for the structure (nerve of vessel), which was conveyed by this sulcus.

Posterior to the notch for the posterior ramus is a groove extending posteriorly on the ventral side of the mastoid exposure and which probably conveyed the artery outside of the skull. Lateral to this groove and posterior to the posttympanic process is a robust mastoid process, which forms the posterolateral angle of the skull. The medial edge of the mastoid exposure bears a small tubercle, which is adjacent to the small and low paroccipital process of the exoccipital. It is likely that the two tuberosities received the origin of the digastric muscle. However this part of the petrosal is relatively encrusted in hematite and difficult to prepare and therefore the structures are not easy to observe.

The ectotympanic and partial malleus were preserved in situ on the left auditory region of AMNH 16663, which provides confidential empirical basis for identification of its articular facet with the petrosal and squamosal.

The ectotympanic is roughly horseshoe-shaped but is not a simple ring as observed on the neonate of Alcidedorbignya (MHNC 8373) and on the juvenile skull (MHNC 8423). The bone is composed of the anterior crus anteriorly, for the articulation with the petrosal and squamosal; the posterior crus posteriorly, for articulation with the petrosal and tympanohyal, and the intermediary part, for which we proposed above the term "arc of the ectotympanic".

In ventral view, the arc presents a medially-expanded blade of bone, which is slightly convex on most of its surface, but 


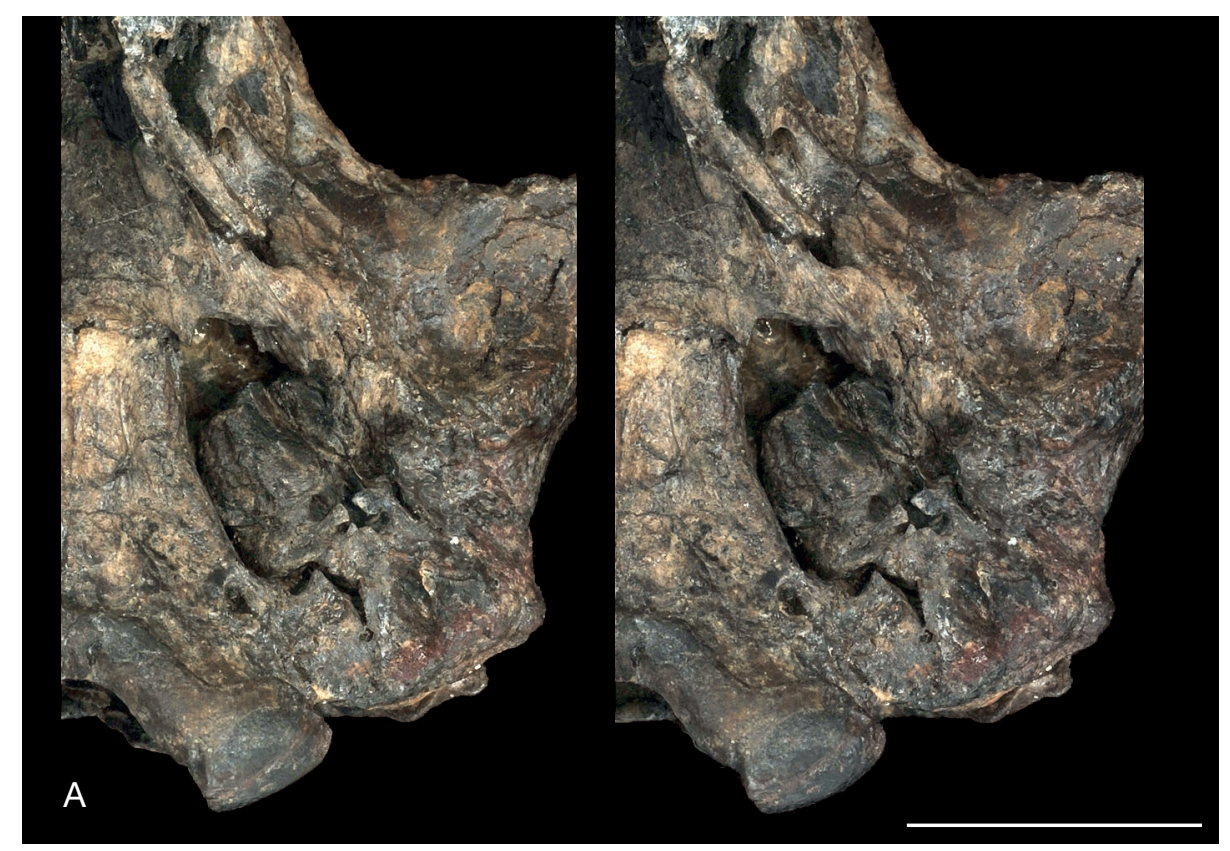

B

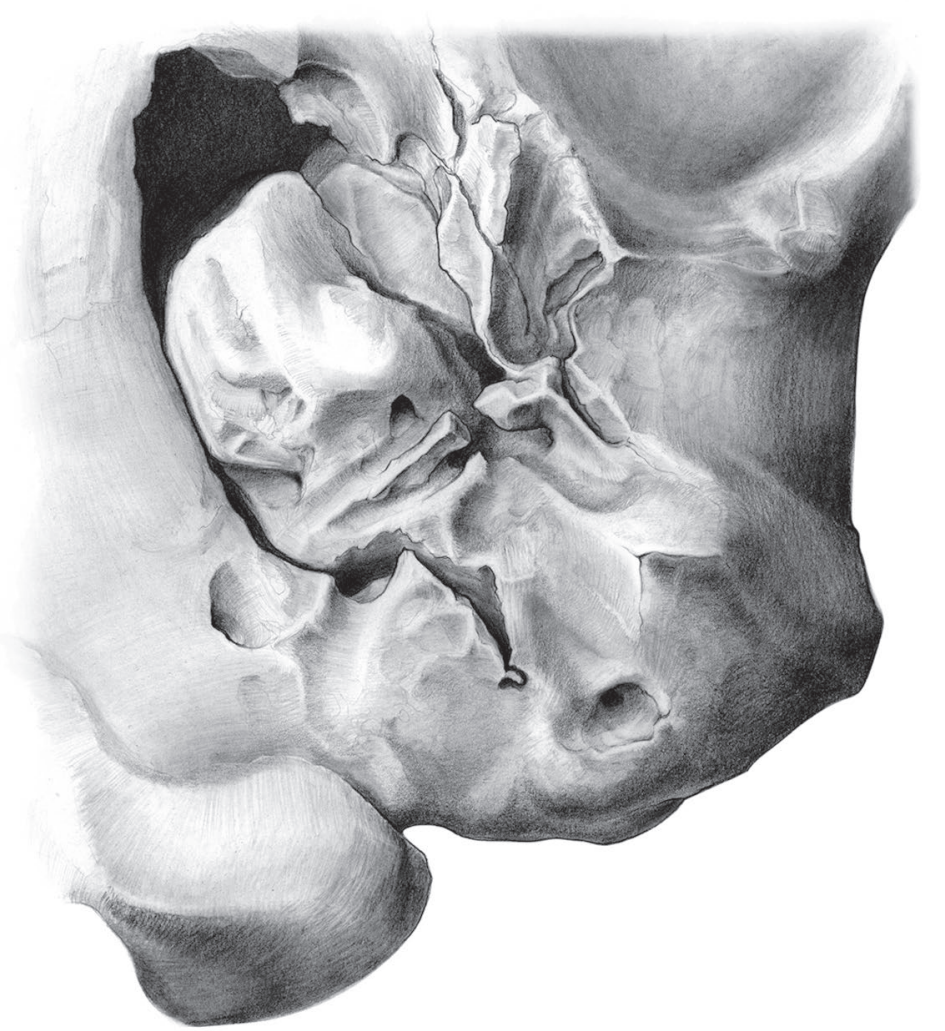

FIG. 124. - Left auditory region of Pantolambda bathmodon (AMNH 16663) in ventral view: A, stereophotographs; B, pencil drawing (cracks and breakages of the specimen have been omitted); Scale bar: A, $2 \mathrm{~cm}$.

which is slightly concave on its anterior and posterior margins. The bone of the arc becomes thinner medially and its medial margin is very thin. This is an indication that probably little is missing of the bone medially and that the width of the arc, as preserved, is close to what it was during the life of the specimen. The anteromedial angle of the arc is more expanded medially than the posteromedial. However, a small part of this angle has been broken, which indicates that it was slightly longer in life. This expansion of the anteromedial angle, in Solenodon, is called anteromesial spine of the tympanic by McDowell (1958) and styliform process by Wible (2008), whose term, we shall use here. In Orycteropus, 


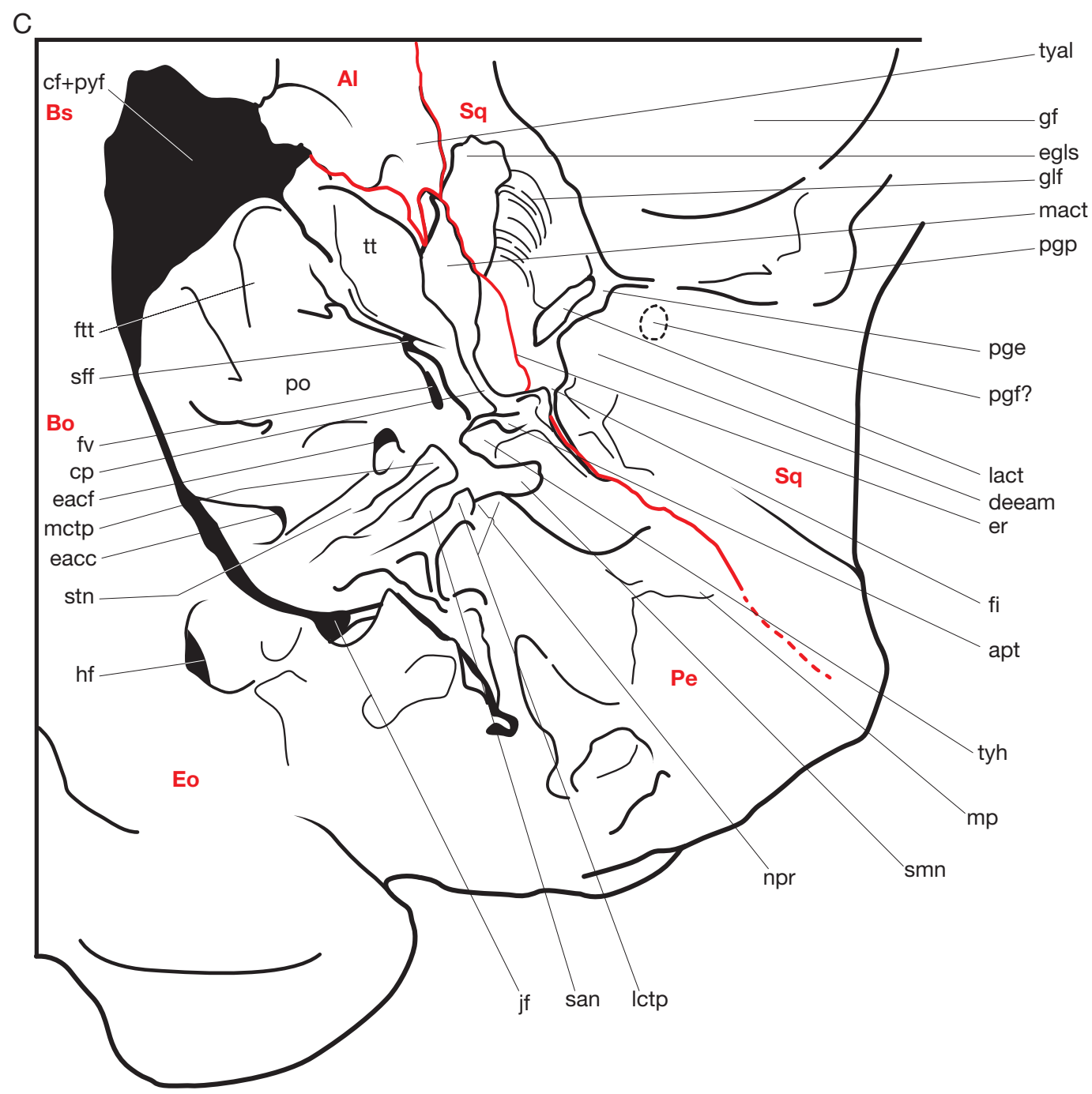

FIG. 124 (continuation). - Left auditory region of Pantolambda bathmodon (AMNH 16663) in ventral view: C, line drawings with captions. Abbreviations: Al, alisphenoid; apt, articular facet for the posterior crus of the ectotympanic; Bo, basioccipital, Bs, basisphenoid; cf, carotid foramen; cp, crista parotica; deeam, dorsal edge of the external acoustic meatus; eacc, external aperture of the cochlear canaliculus; eacf, external aperture of the cochlear fossula; egls, entoglenoid process of the squamosal; Eo, exoccipital; er, epitympanic recess; fi, fossa incudis; ftt, fossa for tensor tympani muscle; fv, fenestra vestibuli; gf, glenoid fossa; glf, Glaserian fissure; hf, hypoglossal foramen; jf, jugular foramen; lact, facet for the lateral articulation of the anterior crus of the ectotympanic; Ictp, lateral caudal tympanic process; mact, facet for the medial articulation of the anterior crus of the ectotympanic; mctp, medial caudal tympanic process; mp, mastoid process; npr, notch for the posterior ramus of the stapedial artery; Pe, petrosal; po, promontorium; pge, postglenoid eminence; pgf?, postglenoid foramen; pgp, postglenoid process; pr, promontorium; pyf, pyriform fenestra; san, sulcus for the auricular nerve (auricular branch of the X); sff, secondary facial foramen; smn, stylomastoid notch; Sq, squamosal; stn, sulcus for the tympanic nerve; tt, tegmen tympani; tyal, tympanic process of the alisphenoid; tyh, tympanohyal.

Wible (2012) described a styliform process, which bears the rostral process of the malleus. We have some doubts that it could be homologous to that described in Solenodon (Wible 2008) since, as described below, both structures are present in Pantolambda.

The lateral edge of the arc is the ventral edge of the external auditory meatus. It is deeply concave laterally and its margin is irregular, granulose, and puffy. This condition is especially obvious in dorsal view. On this side of the ectotympanic, the puffy lateral edge of the arc, is bordered medially by a sharp crest, which borders laterally a deep sulcus tympanicus. The sulcus tympanicus (or tympanic annulus) receives the thickened fibrocartilagineous ring, which encircles the tympanum. It extends from the anterior face of the posterior crus to the base of the posterior face of the anterior crus. Anteriorly, the sulcus widens, and is subdivided into two "sub-sulci" by a small eminence. Posteriorly it extends up to the extremity of the ventral edge of the external auditory meatus, on its internal side. Contrary to the condition observed on the anterior crus, the sulcus strongly tappers at its posterior extremity. The medial edge of the sulcus tympanicus is bordered by a sharp crest, which overhang the sulcus: it is the crista tympanica.

The anterior crus is a large plate, anterodorsally oriented and transversely expanded. It bears two articulations with the skull. The lateral articulation is oval-shaped, elongated and resembles an olive stone. It fits in the elongated fossa, which excavates the posteromedial edge of the postglenoid eminence described above. The medial articulation is more elongated and bears the rostral process (Folian process of McDowell 1958) of the malleus, which is firmly imbricated in a groove 


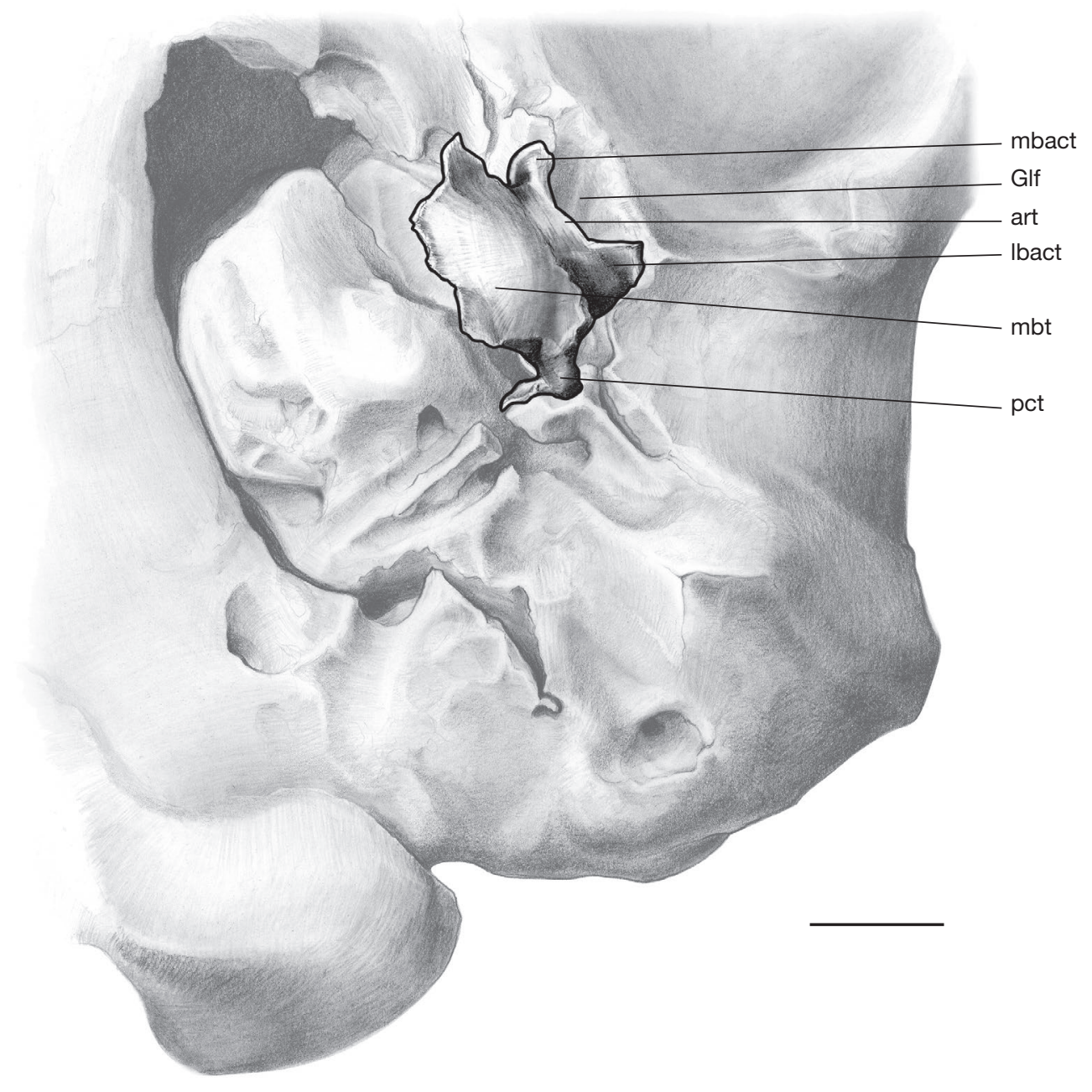

FIG. 125. - Left auditory region of Pantolambda bathmodon (AMNH 16663) in ventral view, with the ectotympanic in situ. Abbreviations: art, arc of the ectotympanic; Glf, Glaserian fissure; Ibact; lateral branch of the anterior crus of the tympanic; mbact, medial branch of the anterior crus of the tympanic; mbt, medial blade of the tympanic; pct, posterior crus of the tympanic. Scale bar: $5 \mathrm{~mm}$.

of the anterior crus of the ectotympanic. The anterodorsal face of the process is also very concave and articulates with the articular facet on the anteromedial edge of the petrosal portion of the epitympanic recess (see above). In fact, at the level of the medial articulation of the anterior crus with the skull, the rostral process of the malleus is tightly wedged between the petrosal dorsally and the tympanic ventrally. This articulation extends anteroventrally in an elongated sharp process that also articulates with the posterior edge of the entoglenoid process of the squamosal on the medial side of the Glaserian fissure. This process is in the same position as that described by Wible (2012) as the styliform process in Orycteropus. Here we call it the medial articular process of the anterior crus of the tympanic, a structure different from the styliform process. Between the two articulations of the anterior crus is a distinct groove excavated in the tympanic, constituting the floor of the Glaserian fissure, which conveys the chorda tympani. The posterior crus articulates with the tympanohyal and the petrosal. The articular facet is a large plate, which is partially preserved on AMNH 16663. In fact, the preserved portion of this specimen is that, which articulates with the tympanohyal. The large portion of the facet, which articulated with the facet of the petrosal, which partially floors the epitympanic recess (see above in the description of the middle ear), is not preserved on the ectotympanic of AMNH 16663. At the lateralmost angle of the facet on its anterodorsal face is a distinct notch, which probably conveyed the chorda tympani on its way to the Glaserian fissure anteriorly, dorsal to the anterior crus. Because the articular facet of the posterior crus is broken, it is difficult to evaluate the width of the tympanic incisure (the space, which separates the distal ends of the anterior and posterior crura). However, evaluating the size of the posterior facet (by comparison with its symmetrical facet on the petrosal), it is likely that the whole articular facet was at least twice larger than what is preserved. Therefore, it is clear that the gap between the crura was no larger than one millimetre.

The malleus is partially preserved and is missing the manubrium, its base and the process for the tensor tympani muscle. The head, which bears the incudal articulation, is well 

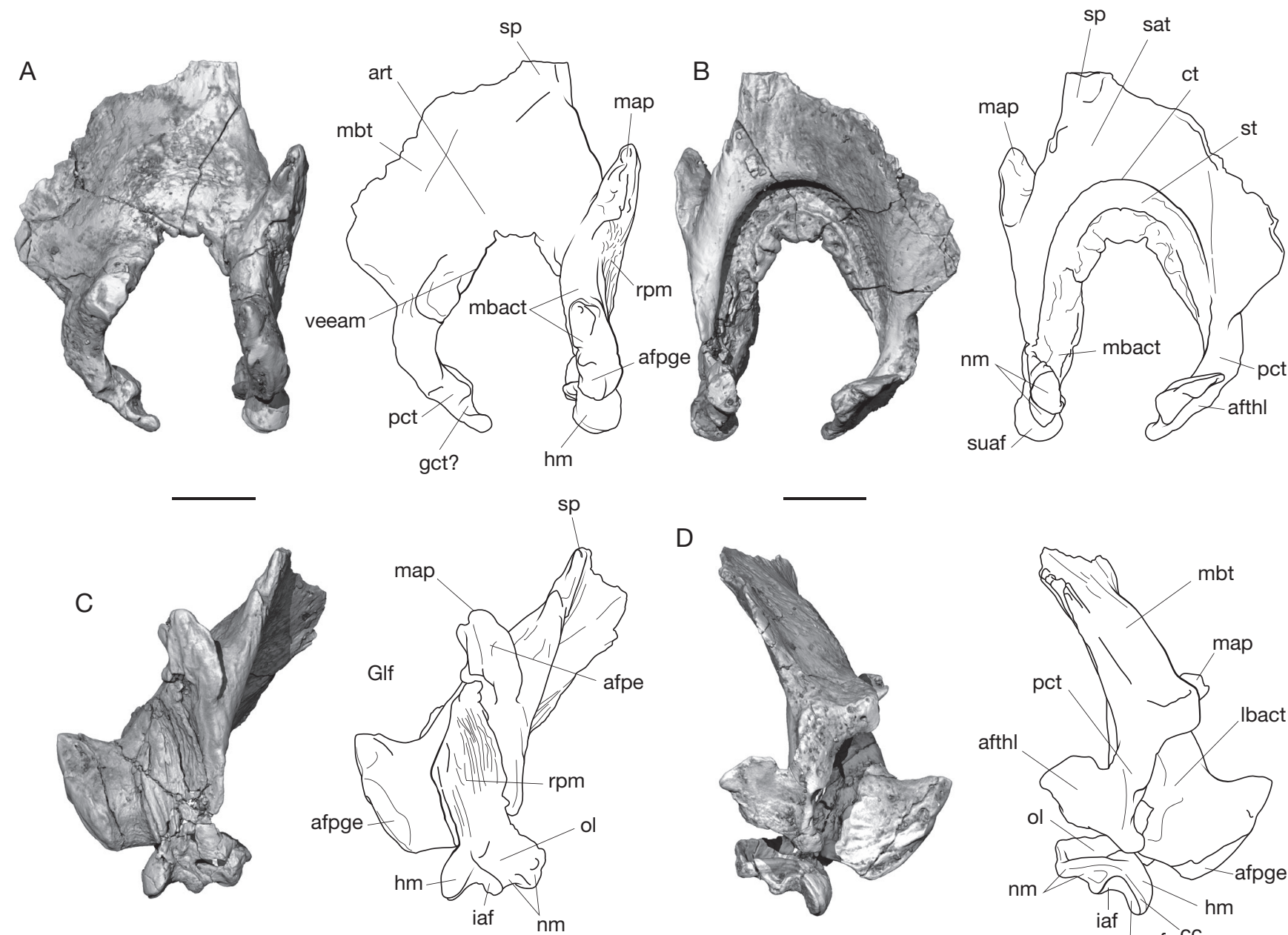

D
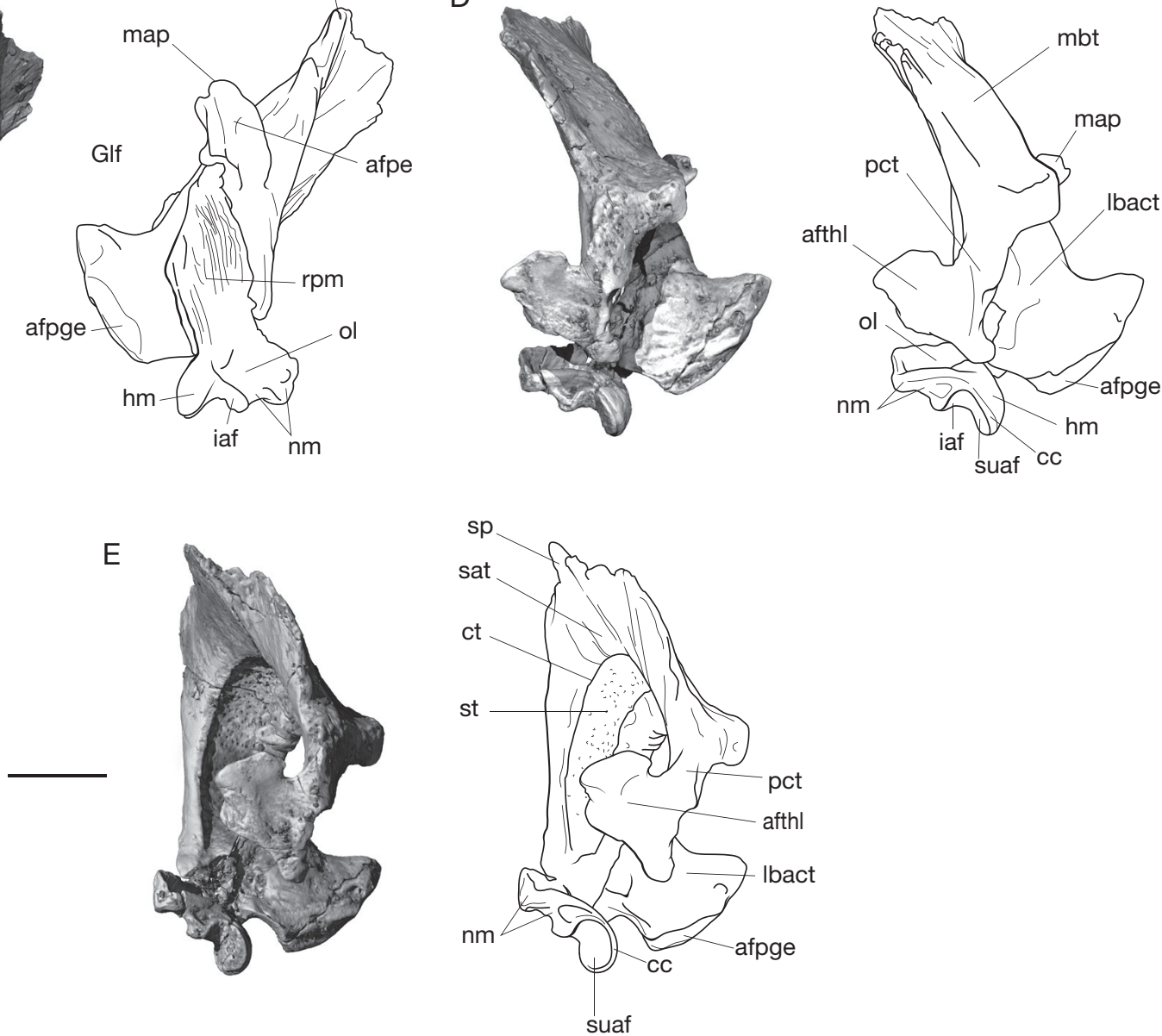

FIG. 126. - Digital rendering based on CT data (left) and schematic drawing (right) of the left ectotypmpanic and partial malleus of Pantolambda bathmodon (AMNH 16663). Cracks and breakages of the specimen have been omitted on the drawings. A, lateral view; B, medial view; C, anterior view; D, posterior view; E, posteromedial view. Abbreviations: afpe, articular facet for the petrosal; afpge, articular facet for the postglenoid eminence; afthl; articular facet for the tympanohyal; art, arc of the ectotympanic; cc, capitular crest; ct, crista tympanica; gct?, possible groove for the passage of the chorda tympani; Glf, Glaserian fissure; hm, head of the malleus; iaf, inferior articular facet of the malleus for the incus; lbact, lateral branch of the anterior crus of the tympanic; map, medial articular process of the anterior crus of the tympanic; mbact, medial branch of the anterior crus of the tympanic; mbt, medial blade of the tympanic; nm, neck of the manubrium; ol, osseous lamina; pct, posterior crus of the tympanic; rpm, rostral process of the malleus; sat, sulcus for auditory tube; sp, styliform process; st, sulcus tympanicus; suaf, superior articular facet of the malleus for the incus; veeam, ventral edge of the external acoustic meatus. Scale bars: $2 \mathrm{~mm}$. The 3D rendering of CT data is available in pdf format at the address http://sciencepress. mnhn.fr/en/periodiques/geodiversitas/37/4/alcidedorbignya-inopinata-additional-files. 
preserved; as usual, it presents a large superior articular facet and a small inferior one. The latter is a cylindrical odontoid process, with its long axis perpendicular to that of the large facet. The latter is relatively flat and subcircular. The lateral part of the head, which bears the large articular facet, is inflated and strongly concave. In anterodorsal view of the malleus the small cylindrical facet is at an angle of approximately $120^{\circ}$ with the long axis of the rostral process. The neck of the malleus separates the head from the manubrium and the anterior process. In anterodorsal view, at the angle between the groove of the Glaserian fissure and the convex lateral part of the head, is a deep notch, which probably transmitted the chorda tympani. Because the malleus is fragmented and presents numerous cracks, it is difficult to observe the foramen and sulcus for the chorda tympani, which are mentioned in Solenodon by Wible (2008). However, a possible foramen is observable in the chorda tympani notch mentioned above, but it is uncertain that it actually represents a natural structure. 
APPENDIX 4. - List of genus and species names cited in the text with authorship and year.

Acotherulum Gervais, 1850

Acristatherium Hu, Meng, Li \& Wang, 2010

Adapis Cuvier, 1831

Adinotherium Ameghino, 1887

Agouti Lacépède, 1799

Ailurus Cuvier, 1825

Ailurus fulgens Cuvier, 1825

Alcidedorbignya Muizon \& Marshall, 1987

Alcidedorbignya inopinata

Muizon \& Marshall, 1987

Alkoqirus Muizon \& Marshall, 1988

Allqokirus australis Marshall \& Muizon, 1988

Amphicynodon Filhol, 1881

Andinodelphys Marshall \& Muizon, 1988

Andinodelphys cochabambensis

Marshall \& Muizon, 1988

Andinodus Muizon \& Marshall, 1987

Andinodus boliviensis Muizon \& Marshall, 1987

Aphronorus Simpson, 1935

Archaeohyrax Ameghino, 1897

Archaeolemur Filhol, 1895

Arctocyon Blainville, 1841

Arctocyonides Lemoine, 1891

Asiatherium Trofimov \& Szalay, 1994

Asiocorpyphodon Xu, 1976

Asioryctes Kielan-Jaworowska, 1975

Astraponotus Ameghino, 1901

Astrapotherium Burmeister, 1879

Azygonyx Gingerich, 1989

Azygonyx ancylion Gingerich \& Gunnell, 1979

Baioconodon Gazin, 1941

Barunlestes Kielan-Jaworowska, 1975

Barylambda Patterson and Simons, 1958

Barylambda faberi (Patterson, 1933)

Bemalambda Zhou, Zhang, Wang \& Ting, 1973

Bemalambda nanbsiungensis Zhou, Zhang, Wang \& Ting, 1973

Bemalambda pachyoesteus Zhou, Zhang, Wang \& Ting, 1973

Bobolestes Nessov, 1985

Bomburia Van Valen, 1978

Borhyaena Ameghino, 1887

Bos Linnaeus, 1758

Bradypus Linnaeus, 1758

Caenolambda Gazin, 1956

Caenolambda pattersoni Gazin, 1956

Callistoe Babot, Powell \& Muizon, 2002

Callosciurus Gray, 1867

Callosciurus flavimanus (Geoffroy, 1831)

Caluromys Allen, 1900

Canis Linnaeus, 1758

Capreolus Gray, 1821

Carcinella Koenigswald, Ruf \& Gingerich, 2009

Carodnia Simpson, 1935
Castor Linnaeus, 1758

Catopsalis Cope, 1884

Cavia Pallas, 1766

Ceratotherium Gray, 1868

Cervus Linnaeus, 1758

Chaetophractus Fitzinger, 1871

Choloepus Illiger, 1811

Chrysochloris Lacépède, 1799

Chulsanbaatar Kielan-Jaworowska, 1974

Cimolestes Simpson, 1927

Cladosictis Ameghino, 1887

Cocomys Dawson, Li \& Qi, 1984

Coelodonta Blumenbach, 1799

Coendou Lacépède, 1799

Coryphodon Owen, 1845

Coryphodon elephantopus Cope, 1874

Coryphodon hamatus Marsh, 1876

Coryphodon testis (Cope, 1882)

Crocidura Wagler, 1832

Cynodictis Bravard \& Pomel, 1850

Dama Frisch, 1775

Dasyprocta Illiger, 1811

Dasypus Linnaeus, 1758

Daulestes Trofimov \& Nessov, 1979

Deltatheridium Gregory \& Simpson, 1926

Deltatherium Cope, 1881

Dendrohyrax Gray, 1868

Dendrohyrax arboreus (Smith, 1827)

Diacodexis Cope, 1882

Diadiaphorus Ameghino, 1887

Dichobune Cuvier, 1822

Didelphis Linnaeus, 1758

Didelphis virginiana Kerr, 1792

Didelphodus Cope, 1882

Dipus Zimmermann, 1780

Dolichotis Desmarest, 1820

Echinops Martin, 1838

Echinosorex Blainville, 1838

Ectocion Cope, 1882

Ectoconus Cope, 1884

Elephas Linnaeus, 1758

Eomaia Ji, Luo, Yuan, Wible, Zhang \&

Georgi, 2002

Eoryctes Thewissen \& Gingerich, 1989

Equus Linnaeus, 1758

Erinaceus Linnaeus, 1758

Esthonyx Cope, 1874

Etayoa Villarroel, 1987

Etayoa batacensis Villarroel, 1987

Euphractus Wagler, 1830

Felis Linnaeus, 1758 
Genetta Cuvier, 1816

Geogale Milne-Edwards \& Grandidier, 1872

Giraffa Brisson, 1762

Glyptodon Owen, 1839

Gomphos Shevyreva, 1975

Hapalemur I. Geoffroy Saint-Hilaire, 1851

Hapalops Ameghino, 1887

Haplolambda Patterson, 1939

Haplolambda quinni Patterson, 1939

Harpyodus Qiu \& Li, 1977

Harpyodus decorus Wang, 1979

Harpyodus euros Qiu \& Li, 1977

Hegetotherium Ameghino, 1887

Hemicentetes Mivart, 1871

Herpestes Illiger, 1811

Hexaprotodon Falconer \& Cautley, 1836

Homacodon Marsh, 1872

Homalodotherium Trouessart, 1898

Hyaena Brisson, 1862

Hyopsodus Leidy, 1870

Ictidomys Allen, 1877

Incadelphys antiquus, Marshall \& Muizon, 1988

Jaskhadelphys minutus Marshall \& Muizon, 1988

Juramaia Luo, Yuan, Meng \& Ji, 2011

Kennalestes Kielan-Jaworowska, 1968

Khasia cordillierensis Marshall \& Muizon, 1988

Kryptobaatar Kielan-Jaworowska, 1970

Kulbeckia Nessov, 1993

Kuntinaru Billet, Hautier, Muizon \& Valentin, 2011

Lagothrix É Geoffroy Saint-Hilaire, 1812

Lainodon Gheerbrant \& Astibia, 1994

Leontinia Ameghino, 1895

Lepilemur I. Geoffroy Saint-Hilaire, 1851

Leptictis Leidy, 1868

Leptolambda Patterson \& Simons, 1958

Leptolambda schmidti Patterson \& Simons, 1958

Lepus Linnaeus, 1758

Limnogale Major, 1896

Lofochaius Zhou, Zhang, Wang \& Ting, 1973

Loris É. Geoffroy Saint-Hilaire, 1796

Loxolophus Cope, 1885

Lutra Brünnich, 1771

Lynx Kerr, 1792

Macrauchenia Owen, 1838

Maelestes Wible, Rougier Novacek \& Asher, 2007

Magnadapis Godinot \& Couette, 2008

Maiorana Van Valen, 1978

Maiorana noctiluca Van Valen, 1978

Manis Linnaeus, 1758

Marmota Blumenbach, 1779

Marmota monax (Linnaeus, 1758)

Martes Pinel, 1792

Mayulestes Muizon, 1994

Mayulestes ferox Muizon, 1994
Megatherium Cuvier, 1796

Meles Brisson, 1762

Meniscotherium Cope, 1874

Metachirus Burmeister, 1854

Metachirus nudicaudatus

(É. Geoffroy Saint-Hilaire, 1803)

Miacis Cope, 1872

Microcebus É. Geoffroy Saint-Hilaire, 1834

Microgale Thomas, 1882

Micropotamogale Haime de Balsac, 1954

Miguelsoria Cifelli, 1983

Mizquedelphys pilpinensis Marshall \& Muizon, 1988

Moeritherium Andrews, 1901

Molinodus Muizon \& Marshall, 1987

Molinodus suarezi Muizon \& Marshall, 1987

Monodelphis Burnett, 1830

Monodelphis domestica Wagner, 1842

Montanalestes Cifelli, 1999

Mustela Linnaeus, 1758

Myocastor Kerr, 1792

Nasua Storr, 1780

Nasua rufa Goldman, 1932

Nemegtbaatar Kielan-Jaworowska, 1974

Nesodon Owen, 1846

Nesophontes Antony, 1916

Notharctus Leidy, 1870

Notopithecus Ameghino, 1901

Notostylops Ameghino, 1897

Nycticebus É Geoffroy Saint-Hilaire, 1812

Oldfieldthomasia Ameghino, 1901

Ornithorhynchus Blumenbach, 1800

Orthaspidotherium Lemoine, 1885

Orycteropus Cuvier, 1798

Oryctolagus Lilljeborg, 1874

Oryzorictes Grandidier, 1870

Pachyrukhos Ameghino, 1885

Paedotherium Burmeister, 1888

Palaeoryctes Matthew, 1913

Palaeosinopa Matthew, 1901

Palaeotherium Cuvier, 1804

Pantolambda Cope, 1882

Pantolambda bathmodon Cope, 1882

Pantolambda cavirictus Cope, 1883

Pantolestes Cope, 1872

Paraceratherium Forster Cooper, 1911

Paramys Leidy, 1871

Pararyctes Van Valen, 1966

Parechinus Trouessart, 1879

Peradectes Matthew \& Granger, 1921

Periphragnis Roth, 1899

Periptychus Cope, 1881

Phascolarctos Blainville, 1816

Phenacodus Cope, 1873

Platymastus Van Valen, 1978 
Plesiadapis Gervais, 1877

Plesiadapis tricuspidens Gervais, 1877

Plesiotypotherium Villarroel, 1974

Pleuraspidotherium Lemoine, 1878

Posnanskytherium Liendo Lazarte, 1943

Potamogale Du Chaillu, 1860

Potos É Geoffroy Saint-Hilaire \& F. G. Cuvier, 1795

Procavia Storr, 1780

Procavia capensis (Pallas, 1766)

Prokennalestes Kielan-Jaworowska \& Dashzeveg, 1989

Prolagus Pomel, 1853

Promioclaenus Touessart, 1904

Proterotherium Ameghino, 1883

Prothylacinus Ameghino, 1891

Protictis Matthew, 1937

Protictis schaffi Gingerich \& Winkler, 1985

Protolipterna Cifelli, 1983

Protungulatum Sloan \& Van Valen, 1965

Protypotherium Ameghino, 1882

Pteropus Erxleben, 1777

Ptilocercus Gray, 1848

Pucadelphys Marshall \& Muizon, 1988

Pucadelphys andinus Marshall \& Muizon, 1988

Pucanodus gagnieri Muizon \& Marshall, 1991

Puelia Roth, 1902

Pyrotherium Ameghino, 1888

Rattus Waldenheim, 1803

Rhombomylus Zhai, 1978

Rhynchippus Ameghino, 1897

Rhynchocyon Petters, 1847

Rhyphodon Roth, 1899

Roberthoffstetteria nationalgeographica Marshall, Muizon \&

Sigé, 1983

Sallacyon Villarroel \& Marshall, 1982

Scarrittia Simpson, 1934

Sciurus Linnaeus, 1758

Sciurus carolinensis Gmelin, 1788

Sciurus igniventris Wagner, 1842

Sciurus niger Linnaeus, 1758

Setifer Froriep, 1806

Simoclaenus Muizon \& Cifelli, 2000
Simoclaenus sylvaticus Muizon \& Cifelli, 2000

Simpsonotus Pascual, Vucetich \& Fernandez, 1979

Solenodon Brandt, 1833

Solenodon paradoxus Brandt, 1833

Suncus Ehrenberg, 1832

Suricata Desmarest, 1804

Sus Linnaeus, 1758

Szalinia gracilis Muizon \& Cifelli, 2001

Tachyglossus Illiger, 1811

Talpa Linnaeus, 1758

Tamandua Gray, 1925

Tapirus Brünnich, 1771

Tenrec Lacépède, 1799

Tenrec ecaudatus Schreber, 1777

Tetheopsis Cope, 1885

Thalarctos Gray, 1825

Tillodon Gazin, 1953

Titanoides Gidley, 1917

Titanoides primaevus Gidley, 1917

Tiuclaenus, Muizon \& Marshall, 1987

Tiuclaenus cotasi Muizon \& Cifelli, 2000

Tiuclaenus minutus Muizon \& Marshall, 1987

Tiuclaenus robustus Muizon \& Cifelli, 2000

Tiulordia floresi Marshall \& Muizon, 1988

Tolypeutes Illiger, 1811

Tragulus Pallas, 1779

Trigonostylops Ameghino, 1897

Trogosus Leidy, 1871

Tupaia Raffles, 1821

Typotheriopsis Cabrera \& Kraglievich, 1930

Uchkudukodon Archibald \& Averianov, 2006

Uintatherium Leidy, 1872

Ukhaatherium Novacek, Rougier, Wible, McKenna,

Dashzeveg \& Horovitz, 1997

Ursus Linnaeus, 1758

Vincelestes Bonaparte, 1986

Vulpavus Marsh, 1871

Wanglia Van Valen, 1988

Zalambdalestes Gregory \& Simpson, 1926

Zhelestes Nessov, 1985 
APPENDIX 5. - Comments on the terms paroccipital process, lateral caudal tympanic process and mastoid process.

\section{1) PARoccipital PRocess and Lateral CAUDAl TyMPANiC PROCESS OF THE PETROSAL}

The structure that we interpret as the lateral caudal tympanic process of Alcidedorbignya (Fig. 36) is located in a position similar to the structure described by Rougier et al. (1992) in Vincelestes, as the paroccipital process of the petrosal. In these taxa, both processes form the medial wall of the stylomastoid notch. As a matter of fact, Wible et al. (2001: 7) regard the two terms as synonyms.

Furthermore, the paroccipital process of Zalambdalestes, as described by Wible et al. (2004), and that of Maelestes (Wible et al. 2009) are not in the same position as in Vincelestes because they do not form the medial wall of the stylomastoid notch but are in position well lateral to the exit of the facial nerve, which casts doubts on the homology of the "paroccipital processes" of the three genera. In return, the "paroccipital processes" (sensu Wible et al. 2004, 2009) of Zalambdalestes and Maelestes are in the same position as the mastoid process of Alcidedorbignya and as the structure called mastoid eminence by Rougier et al. (1992) in Vincelestes, i.e. posterolateral to the tympanohyal rather than posteromedial or medial. Furthermore, like the mastoid process of Alcidedorbignya, the paroccipital process (sensu Wible et al. 2004, 2009) of Zalambdalestes and Maelestes are closely appressed to the posterior edge of the posttympanic process of the squamosal. We therefore, strongly suspect that the paroccipital process of Vincelestes (sensu Rougier et al. 1992) is not homologous to those of Zalambdalestes (Wible et al. 2004) and Maelestes (Wible et al. 2009). In Zalambdalestes, Wible et al. (2004) do not mention a lateral caudal tympanic process. In fact, the small crest, which forms the posteromediodorsal wall of the stylomastoid notch as figured by Wible et al. (2004: fig. 37A), could represent an equivalent of the lateral caudal tympanic process (as also for Maelestes), although no well-developed process is observed.

Rougier et al. (1992) employed the term "paroccipital process of the petrosal" in their description of the auditory region of Vincelestes because it resembles that of morganucodonts, Sinoconodon, triconodontids, and multituberculates. Rougier \& Wible (2006) defined the paroccipital process as "a prominent process that projects ventrally from the posterolateral corner of the petrosal among Mesozoic mammaliaforms". According to these authors the paroccipital process "is homologous with the portion of the petrosal providing support for the quadrate among basal nonmammalian cynodonts...". We can accept this definition and homology. However, the condition of the Zatheria (i.e Vincelestes + Theria [sensu McKenna \& Bell 1997]) is confusing because Rougier et al. (1992) describe, in Vincelestes, a paroccipital process, that lies in a very different position (in relation with the stylomastoid notch) from that they describe in Zalambdalestes and Maelestes, the former being medial to the facial nerve and the latter being lateral to it. The paroccipital process (sensu Rougier et al. 1992) of Vincelestes seems to be homologous to the crest, which, in
Zalambdalestes and Maelestes, forms the dorsomedial wall of the stylomastoid notch and which we regard as a lateral caudal tympanic process. Furthermore, what Rougier $e t$ al. (1992) called mastoid eminence in Vincelestes is in the same position as what Wible et al. (2004) called the paroccipital process in Zalambdalestes and Maelestes, which we regard as the mastoid process (see below).

It seems, therefore, that both the paroccipital process + mastoid eminence (sensu Rougier et al. 1992) of Vincelestes could represent what is identified as the posterior paroccipital process (or paroccipital process according to the authors) in Oligokyphus (Crompton 1964; Crompton \& Luo 1993), Morganucodon (Crompton \& Sun 1985; Wible \& Hopson 1993), Dinnetherium (Crompton \& Luo 1993), Sinoconodon (Crompton \& Sun 1985; Crompton \& Luo 1993), Trioracodon, Triconodon (Kermack 1963), Catopsalis, Nemegtbaatar (Kielan-Jaworowska et al. 1986), Kryptobaatar (Wible \& Rougier 2000), and Ornithorhynchus (Wible \& Hopson 1993; Luo \& Crompton 1994). It is noteworthy, however, that the paroccipital process of Zhangheotherium (Hu et al. 1997) is similar in position and morphology to that of Vincelestes (sensu Rougier et al. 1992). Nevertheless, as is observed in Vincelestes and in the taxa mentioned above, the paroccipital process of Zhangheotherium is closely related to what $\mathrm{Hu}$ et al. (1997) called the mastoid exposure of the petrosal. Therefore, although homologies are never easy to establish especially when dealing with taxa, belonging to distantly related groups, it seems clear that the paroccipital process of stem mammaliaforms is closely related to (if not part of) the pars mastoidea of the petrosal. As a matter of fact, in Zalambdalestes, Wible et al. (2004) called paroccipital process what is called here a mastoid process. It is clear that what Wible et al. (2004) called the paroccipital process sensu Rougier et al. (1992) (our mastoid process) in Zalambdalestes is different from the lateral caudal tympanic process (MacPhee 1981 and above), a structure, which Wible et al. (2001: 20) regarded as equivalent to the paroccipital process. To conclude, if the term paroccipital process of the petrosal is relatively well understood in stem-mammaliaforms (excluding Zatheria) and in non-mammalian cynodonts, there is hence an ambiguity in the use of this term among Zatheria.

Therefore, because of this ambiguity and also because MacPhee (1981: 16-19) clearly demonstrated that both medial and lateral caudal tympanic processes belong to the same structure of bone that arises in cartilage (contrary to the rostral tympanic process), we rather use MacPhee's terminology here, which seems more appropriate to Theria. As mentioned by this author, not all the elements of the caudal tympanic process are necessarily present in all mammals.

In Vincelestes, a lateral caudal tympanic process (sensu this paper) is present as a well-developed tubercle medial to the stylomastoid notch. This lateral caudal tympanic process extends medially as a crest, which borders posteriorly the stapedial fossa and is oriented toward the jugular foramen. In contrast, a medial caudal tympanic process is not clearly 
(or not at all) differentiated in Vincelestes. However, it is unclear whether or not it could be part of the medial region of Rougier et al.'s caudal tympanic process. A medial caudal tympanic process may also simply be absent in Vincelestes. Rougier et al.'s caudal tympanic process may also be regarded as a medial extension of the lateral caudal tympanic process. The mastoid eminence of Rougier et al. (1992) (= mastoid process herein), in Vincelestes, is located posterolateral and adjacent to the lateral caudal tympanic process.

The condition in basal metatherians is reminiscent of that of Vincelestes. In Pucadelphys, Andinodelphys or Mayulestes a caudal tympanic process (sensu Wible 1990) is present as a transverse crest extending from the medial edge of the stylomastoid notch to the petrosal-exoccipital suture on the posterolateral region of the jugular foramen, forming the posterior wall of the stapedial fossa. The lateral part of this crest, just medial to the stylomastoid notch, is slightly inflated and forms a small protruding hump adjacent to and almost continuous with the mastoid process, which is located on the posterior edge of the stylomastoid notch. This hump is probably equivalent to the lateral caudal tympanic process of Vincelestes (= paroccipital process of Rougier et al. 1992), although clearly less developed. We therefore suggest that, in Vincelestes and stem metatherians, the process (or hump) located medial to the stylomastoid notch and the crest medial to it represent the lateral caudal tympanic process. Because of the probable absence of the medial caudal tympanic process, this structure could understandably be called a caudal tympanic process. In eutherians, a medial caudal tympanic process is present, on the posterior edge of the post-promontorial sinus, being well separated from the lateral caudal tympanic process. The latter is reduced to its lateral tubercle (more a tuberosity) on the medial edge of the stylomastoid notch and the medial crest is obliterated by a notch (see character list, number 324), which excavates the lateral face of the crista interfenestralis. Because the lateral caudal tympanic process is located between the two notches, when present, it confers to the posterior opening of the middle ear a distinctive $\omega$-shaped morphology. When very reduced or absent the opening is roughly circular. An $\omega$ shaped morphology is present in Alcidedorbignya (see above).

In early eutherians (e.g., Asioryctes, Kennalestes, Zalambdalestes, Maelestes, early ungulates) a medial caudal tympanic process (tympanic process of Kielan-Jaworowska 1981; "tympanic process" of Wible et al. 2009) is present and often well-developed, while the lateral caudal tympanic process is generally absent or very small. For instance, a lateral caudal tympanic process is apparently absent in the early ungulates, Maiorana and Baioconodon, in Asioryctes, Kennalestes, Zalambdalestes (probably) (Wible et al. 2004: fig. 37A), and in Kulbeckia (Ekdale et al. 2004: fig. 3C). In Maelestes a small lateral caudal tympanic process is distinctly observed on the left petrosal of PSS-MAE 607 (Wible et al. 2009: fig. 15), which provides a moderate $\omega$-shaped morphology to the posterior opening of the middle ear. A distinct lateral caudal tympanic process is variably present in some archaic ungulates (Pleuraspidotherium, Phenacodus) and in some Recent Lipotyphla (Tenrec, and Echinosorex).
In Prokennalestes, because this region is broken on the petrosal described by Wible et al. (2001), it is not possible to identify a medial and a lateral caudal tympanic process. It is nevertheless possible to observe that the paroccipital process (sensu Rougier et al. 1992) described in Prokennalestes is posterolateral to the stylomastoid notch and therefore cannot be homologous to the lateral caudal tympanic process, which is medial to the exit for the facial nerve. The paroccipital process (sensu Wible et al. 2001) of Prokennalestes is therefore probably not homologous to the paroccipital process (sensu Rougier et al. 1992) of Vincelestes but homologous to our mastoid process (see below). Similarly, the paroccipital processes (sensu Wible et al. 2004, 2009) of Maelestes and Zalambdalestes are located posterolateral to the stylomastoid notch and are regarded here as homologous to our mastoid process (see below).

To conclude, we believe that: 1) the paroccipital process (sensu Rougier et al. 1992) of Vincelestes is the lateral caudal tympanic process, which possibly also includes the medial crest (their caudal tympanic process); and 2) the paroccipital process of Prokennalestes, Maelestes, Zalambdalestes is the mastoid process.

\section{2) MASTOID PROCESS}

MacPhee (1981) recommended avoiding the term mastoid process because "it is used in a great variety of senses", and uses instead the term mastoid eminence. However, the term processus mastoideus is present in the nomina anatomica veterinaria. According to its use in anatomical treatises (Davis 1964; Barone 1997; Evans \& de Lahunta 2012; Dauber 2007; Schaller 2007; Mead \& Fordyce 2009), it represents a thickening of the anteroventrolateral area of the pars mastoidea of the petrosal, which is in contact with the squamosal (posterior edge of the posttympanic process) and/or with the ectotympanic, most commonly closely appressed against this (these) bone(s). The mastoid process is posterolateral to lateral to the stylomastoid notch or foramen and posterior to posteromedial to the posttympanic process of the squamosal. A mastoid process is absent in amastoid taxa (e.g., proboscideans, hyracoids). The mastoid process receives the insertion (or part of the insertion) of the depressors muscles of the head. These muscles are (or part of) the brachiocephalicus (sternocleidomastoideus, cleidomastoideus) (Howell 1928; Davis 1964; Barone 2000; Evans \& de Lahunta 2012). Concerning the non-therian Vincelestes, Rougier et al. (1992) used the term mastoid eminence to define a thickening of the pars mastoidea posterolateral to the stylomastoid notch and contacting the squamosal because they regarded as uncertain its homology with the human mastoid process. In this work we strongly suspect that their mastoid eminence is homologous with the mastoid process of therians because it is probable that the mastoid eminence described in Vincelestes received the insertion of the depressors of the head. Therefore, in this paper the term mastoid process is used in the sense given above. It is noteworthy, however, that in some cases the depressor function of the process is displaced on the strongly thickened posttympanic process of the squamosal (e.g., Zalambdalestes, Pteropus, Setifer, and in amastoid taxa such as Procavia). 

TABLE OF CONTENTS

INTRODUCTION 399

FAUNAL LIST 400

MATERIAL AND METHODS 401

INSTITUTIONAL ABBREVIATIONS 401

DESCRIPTION AND COMPARISON 401

Phylogenetic ANALYSIS 403

Taxonomic sample 403

Character sampling and scoring

Phylogenetic analysis

404 404

MEASUREMENTS 404

SUPPLEMENTARY DATA 404

PARSIMONY ANALYSIS FILES 404

3D PDF FILES 405

SPECIMENS, TAPHONOMY AND BIOLOGY .... 405

THE NEW PANTODONT SPECIMENS 405

TAPHONOMY AND PALAEOBIOLOGY 406

SEXUAL DIMORPHISM 408

SYSTEMATIC PALAEONTOLOGY 410

EMENDED DIAGNOSIS 410 General morphology ...... 410

Teeth 410

Skull 411

Dentary 411

Postcranial skeleton 411

DifFERENTIAL DIAGNOSIS 412

UNAMBIGUOUS APOMORPHIES OF THE TAXON ALCIDEDORBIGNYA 413

\section{COMPARATIVE DESCRIPTION} 415

SKULL 415

Dentition 415

Bony skull 415

General features ............................................... 415

Premaxilla ............................................................ 420

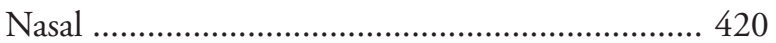

Maxilla ............................................................. 421

Turbinals and nasal cavity .................................... 424

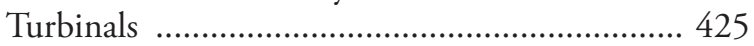

Paranasal recesses and turbinal fossae ................ 427

Palatine ............................................................ 428

Lacrimal ......................................................... 430

Frontal ............................................................ 430

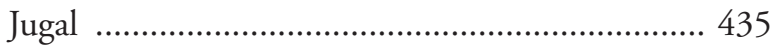

Parietal ........................................................... 435

Squamosal ............................................................ 439

Alisphenoid ....................................................... 443

Orbitosphenoid .................................................... 445

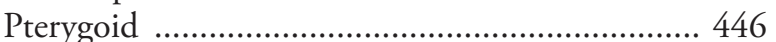

Presphenoid ........................................................... 446

Basisphenoid .......................................................... 447

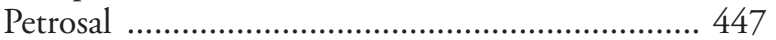

Ventral (tympanic) face of the petrosal .............. 447

Dorsal (endocranial) surface of the petrosal ....... 464

Squamosal surface of the petrosal ....................... 471

Inner ear ....................................................... 472

Cochlear canal ................................................. 472

Vestibule and semicircular canals ...................... 474

Ectotympanic, entotympanic, and ear ossicles ..... 474

Basioccipital, exoccipital, and supraoccipital ....... 476

Braincase endocast .............................................. 477

Overall morphology ......................................... 478

Forebrain region …………………………….... 478

Venous system ................................................... 480

Midbrain region ................................................. 481

Hindbrain region ............................................... 481

Midventral surface of the endocast ..................... 481

Endocast volume and encephalization quotient ... 482

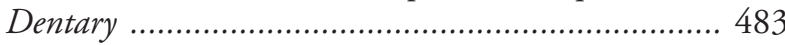

POSTCRANIAL SKELETON ………................................ 485

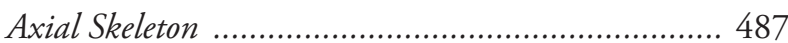

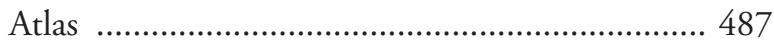

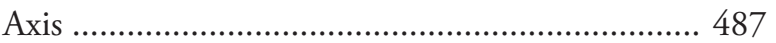

Posterior cervical vertebrae (C3-C7) .................... 489

Thoracic vertebrae .................................................. 490

Lumbar vertebrae .............................................. 492

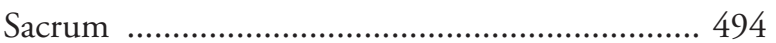

Caudal vertebrae ............................................... 496

Ribs and sternum ................................................ 497

Pectoral girdle and Forelimb ................................. 498

Scapula and clavicle …………………………..... 498

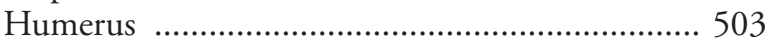

Ulna ……..................................................... 507

Radius ……………………………………..... 509

Carpal bones ....................................................... 510

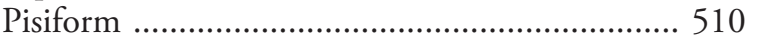

Cuneiform …................................................... 510

Unciform …………........................................ 511

Trapezoid .......................................................... 511

Trapezium ............................................................. 511

Metacarpals and phalanges .................................. 513

Pelvic girdle and Hind limb ................................. 516

Os Coxae ...................................................... 516

Femur and Patella _................................................ 519

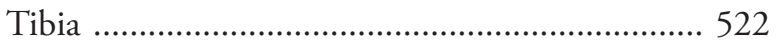

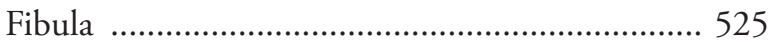

Tarsal bones .......................................................... 526 


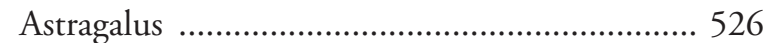

Calcaneus …………………………………... 528

Cuboid ............................................................ 528

Navicular ........................................................... 529

Ectocuneiform ............................................... 530

Mesocuneiform ……………………………..... 530

Entocuneiform …………………………….... 530

Metatarsals and phalanges ...................................... 531

\section{DISCUSSION}

COMPARISON AND ANALYSIS OF CRANIAL CHARACTERS ... 535

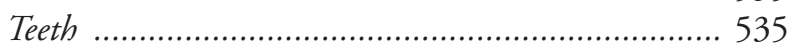

Incisors .............................................................. 535

Molars relative size ……………………………... 535

Bony skull .......................................................... 535

General morphology of the skull ......................... 535

Premaxilla ........................................................... 536

Nasal ............................................................... 536

Maxilla ............................................................. 537

Turbinals and nasal cavity .................................. 538

Palatine .............................................................. 539

Lacrimal ...................................................... 540

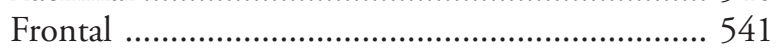

Pterygoid ............................................................. 543

Squamosal, orbitosphenoid, and alisphenoid ...... 544

Parietal ............................................................ 547

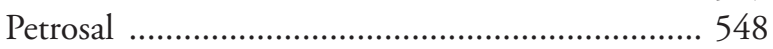

Ectotympanic ……………………………….... 554

Basisphenoid ........................................................ 555

Basioccipital, exoccipital ....................................... 555

Supraoccipital .................................................... 555

Brain endocast ................................................... 556

Olfactory bulbs, circular fissure ......................... 556

Lissencephalic brain .......................................... 556

Fissuration: rhinal fissure and neocortical sulcus .... 556

Midbrain exposure ……………………………... 557

Hindbrain - cerebellum ...................................... 558

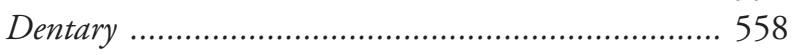

DISCUSSION OF POSTCRANIAL CHARACTERS .............. 559

Axial skeleton ………………………………....... 559

Cervical vertebrae .............................................. 559

Thoracolumbar vertebrae ...................................... 560

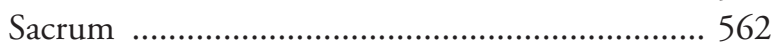

Caudal vertebrae ................................................ 562

Pectoral girdle and Forelimb ................................. 563

Scapula …………………………………….... 563

Humerus ........................................................... 564

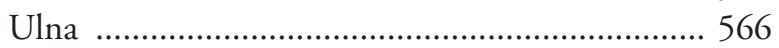

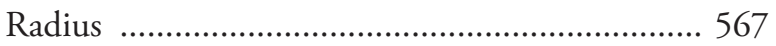

Manus ……………………………………....... 568

Conclusion on the forelimb ................................ 568

Pelvic girdle and hind limb ................................... 569

Innominate ....................................................... 569

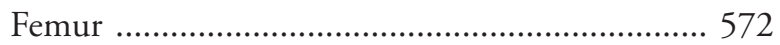

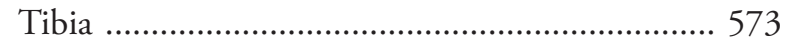

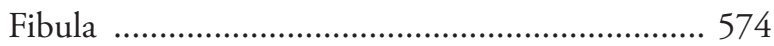

Pes ……….................................................... 574

Conclusions on the hind limb ............................. 575

General conclusions on the postcranial skeleton ......... 576

Climbing vs digging or both? ................................... 578

PHYLOGENETIC ANALYSIS .......................................... 580

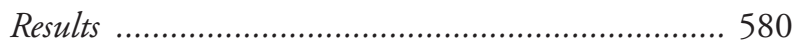

Unconstrained analysis ......................................... 580

Analysis with backbone constraint ....................... 580

Discussion .......................................................... 582

Morphological analyses of placental phylogeny ...... 582

Alcidedorbignya, Pantodonta and Xenungulata relationships .................................................... 584

Potential paleobiogeographic and temporal implications on the history of Pantodonta .................. 586

GENERAL CONCLUSIONS ............................................... 587

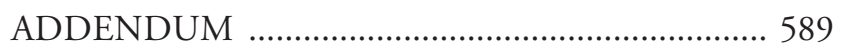

REFERENCES ……………………………….... 592

APPENDICES …………………………………..... 603

ApPENDIX 1, Character list ......................................... 603

Dentition general .................................................. 603

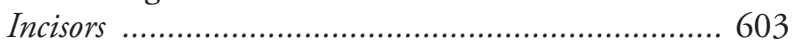

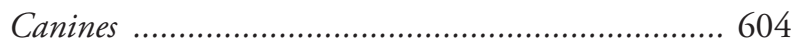

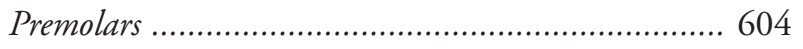

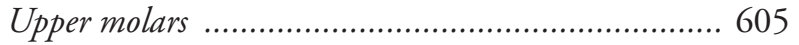

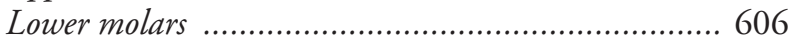

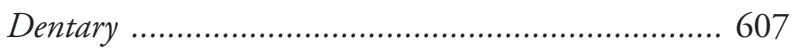

Skull: rostrum ..................................................... 608

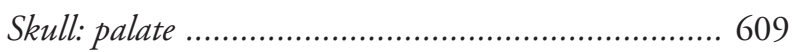

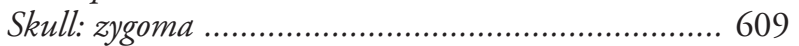

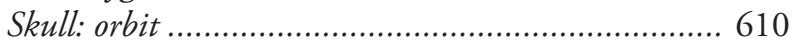

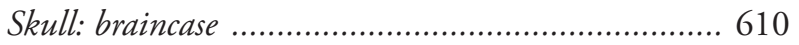

Skull: mesocranium …………………………........ 611

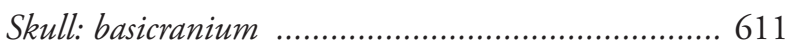

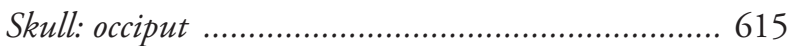

Postcranial characters ............................................. 615

ApPEndix 2, Taxon list, specimens, and reference .... 618

APPENDIX 3, Description of the auditory region of

Pantolambda bathmodon

APPENDIX 4, List of genus and species names cited in the text with authorship and year 627

APPENDIX 5, Comments on the terms paroccipital process, lateral caudal tympanic process and mastoid process .... 630 1) Paroccipital process and lateral caudal tympanic process of the petrosal ............................................ 630

2) Mastoid process ……………………………....... 631 